Ivan Francisco Ruiz Torres

\title{
DESENVOLVIMENTO E APLICAÇÃO DO MÉTODO DOS ELEMENTOS FINITOS GENERALIZADOS EM ANÁLISE TRIDIMENSIONAL NÃO-LINEAR DE SÓLIDOS
}

Tese apresentada à Escola de Engenharia de São Carlos da Universidade de São Paulo, como parte dos requisitos para a obtenção do título de Doutor em Engenharia de Estruturas

Orientador: Prof. Dr. Sérgio Persival Baroncini Proença

São Carlos 2003 


\section{FOLHA DE JULGAMENTO}

\section{Candidato: Engenheiro IVAN FRANCISCO RUIZ TORRES}

Tese defendida e julgada em 26-09-2003 perante a Comissão Julgadora:

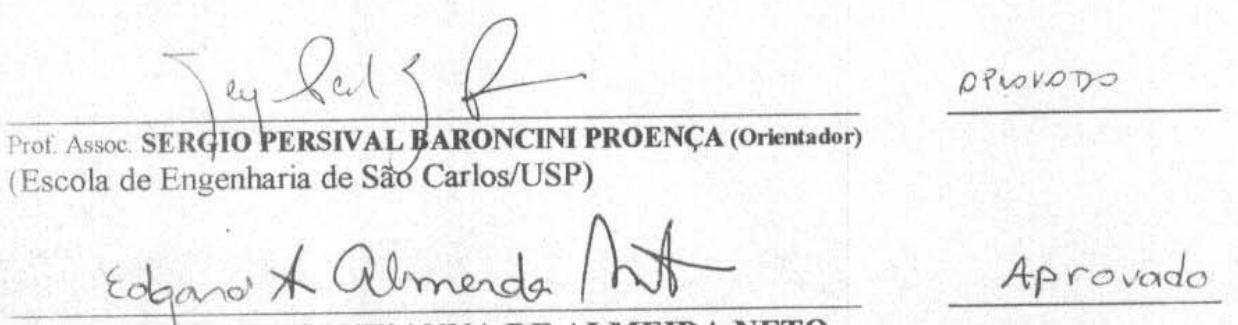

Prof. Dr. EDGARD SANT'ANNA DE ALMEIDA NETO

(Escola Politécnica/USP)

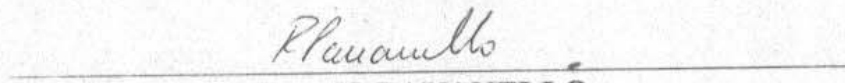

APROVADO

Prof. Liv. Doc. RENATO PAVANELLO

(Universidade Estadual de Campinas/UNICAMP)

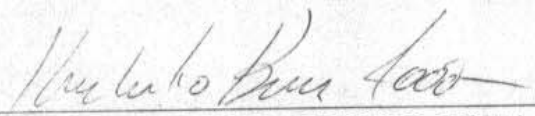

Aprounc'o

Prof. Assoc. HUMBERTO BREVES CODA

(Escola de Engenharia de São Carlos/USP)

$$
\text { Adair R. Aguiar }
$$

APROVADO

Prof. Dr. ADAIR(ROBERTIO AGUIAR

(Escola de Engenharia de São Carlos/USP)

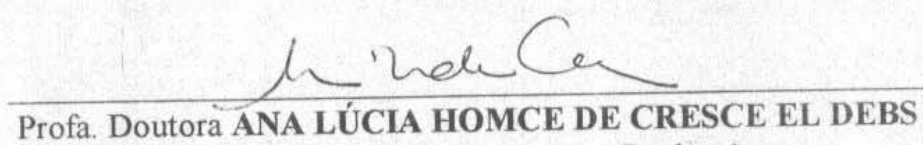

Coordenadora do Programa de Pós-Graduação em

Engenharia Civil (Engenharia de Estruturas)

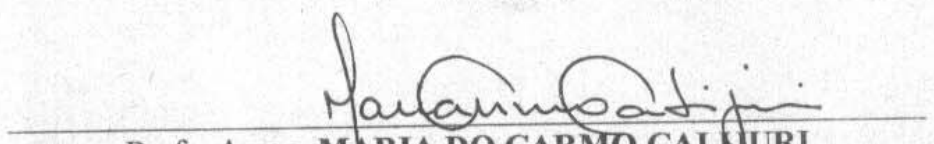

Profa. Assoc. MARIA do CARMO CALNURI

Presidente da Comissão de Pós-Graduação 
À querida Andréia 


\section{AGRADECIMENTOS}

Ao Prof. Dr. Sérgio Persival Baroncini Proença, pela orientação segura e competente, pela dedicação e por continuamente auxiliar-me na busca do conhecimento.

Aos funcionários do Departamento de Estruturas da Escola de Engenharia de São Carlos, especialmente a Maria Nadir Minatel, pela disposição permanente em ajudar.

Ao colega Felício Bruzzi Barros pela generosidade incomum em todo tipo de auxílio que me concedeu.

À CAPES, pela bolsa de estudo concedida.

Aos meus Pais, pelo apoio integral ao longo deste trabalho, e de toda a vida.

À minha esposa Andréia, pela atenção, apoio e compreensão.

A Deus. 


\section{RESUMO}

TORRES, I. F. R. (2003). Desenvolvimento e aplicação do método dos elementos finitos generalizados em análise tridimensional não-linear de sólidos. Tese (Doutorado) - Escola de Engenharia de São Carlos, Universidade de São Paulo, São Carlos.

Este trabalho apresenta uma contribuição ao emprego do Método dos Elementos Finitos Generalizados (MEFG) na análise tridimensional não-linear de sólidos. A análise numérica em campo não-linear, com modelos de dano e plasticidade, é original. O MEFG é uma formulação não-convencional do Método dos Elementos Finitos (MEF), que resulta da incorporação a este último de conceitos e técnicas dos denominados Métodos sem Malha, especialmente o enriquecimento da aproximação inicial (partição de unidade) por funções convenientes. Apresenta-se uma breve revisão bibliográfica dos Métodos sem Malha e do Método dos Elementos Finitos Generalizados, bem como suas principais características. Apresenta-se, com base no MEFG, a formulação de elementos tetraédricos e hexaédricos. Três modelos constitutivos são considerados visando análises não-lineares: o de plasticidade (perfeita ou com encruamento isótropo linear) com critério de plastificação de von Mises; o de dano frágil em concreto sob carregamento monótono crescente (modelo de Mazars) e o de dano e plasticidade acoplados (modelo de Lemaitre), próprio para materiais metálicos. São apresentados detalhes do código computacional, baseado no MEFG e nos modelos constitutivos acima mencionados, bem como resultados de análises numéricas. Esses resultados ressaltam algumas das vantagens do MEFG aplicado à análise não-linear, tais como: o enriquecimento da aproximação inicial limitado a regiões de interesse no domínio, como por exemplo, as que exibem elevados gradientes de deformação e tensão; uma definição mais precisa da distribuição de grandezas como a variável de dano e a tensão equivalente de von Mises, evitando a necessidade de alterações na malha; e a superação do travamento volumétrico associado a modelos de plasticidade.

Palavras-chave: Método dos Elementos Finitos; Métodos Numéricos; Análise Não-linear; Plasticidade; Mecânica do Dano. 


\section{ABSTRACT}

TORRES, I. F. R. (2003). Development and employment of generalized finite element method in three-dimensional nonlinear analysis of solids. Ph.D. Thesis - São Carlos School of Engineering, University of São Paulo, São Carlos.

This work presents a contribution to the Generalized Finite Element Method (GFEM) employment in three-dimensional nonlinear analysis of solids. The nonlinear numerical analysis conduced with damage and plasticity models is original. GFEM is a nonconventional formulation of Finite Element Method (FEM) which results from the addition to the latter of concepts and techniques of the so called Meshless Methods, specially the enrichment of the initial approximations (partition of unity) by customized functions. A brief review of Meshless Methods and Generalized Finite Element Method bibliography is presented, as well as their main features. Based on GFEM, the formulation of tetrahedral and hexahedral elements is shown. Three material laws are considered aiming nonlinear analysis: plasticity (perfectly plastic or linear isotropic hardening), with von Mises yield criterion; brittle damage on concrete under monotonic increasing loading (Mazars model) and damage coupled with plasticity (Lemaitre model), a suitable model for metals. Details of the computational code, based on GFEM and material laws mentioned above, are presented, as well as results of numerical analysis. These results emphasize some of the advantages of GFEM applied to nonlinear analysis, such as: enrichment of the basic approximations limited to some regions of interest in the domain, for instance, those exhibiting high strain and stress gradients; an accurated definition of the distributions of quantities like damage variable and von Mises equivalent stress, avoiding remeshing; and overcoming of volumetric locking associated to plasticity models.

Keywords: Finite Element Method; Numerical Methods; Nonlinear Analysis; Plasticity; Damage Mechanics. 


\section{LISTA DE FIGURAS}

Figura 1 - Aproximação para o campo de deslocamentos no MEF ………..................... 25

Figura 2 - Nuvens de influência num domínio $\Omega \in R^{2}$................................................. 34

Figura 3 - Nuvens de influência no MEFG (caso bidimensional) ................................. 42

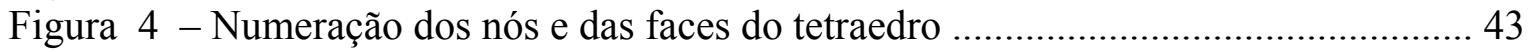

Figura 5 - Estrutura da matriz de rigidez do elemento tetraédrico ................................. 46

Figura 6 - Expansão da submatriz $3 \times 3$ em função do enriquecimento .......................... 51

Figura 7 - Elemento hexaédrico - nós, faces e sistema de eixos adimensionais ............. 52

Figura 8 - Estrutura da matriz de rigidez do elemento hexaédrico ................................. 55

Figura 9 - Sistemas de eixos no plano das faces do hexaedro ...................................... 57

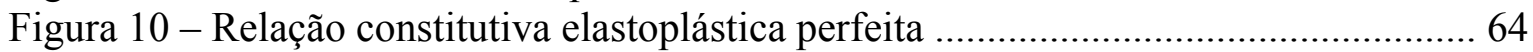

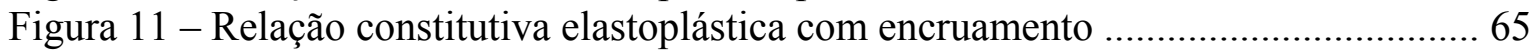

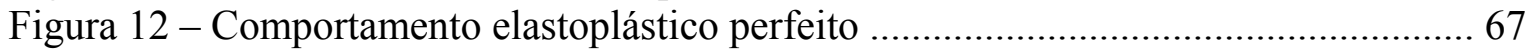

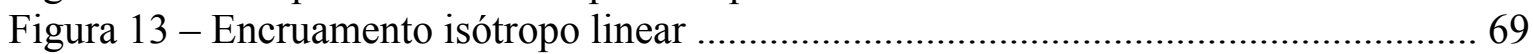

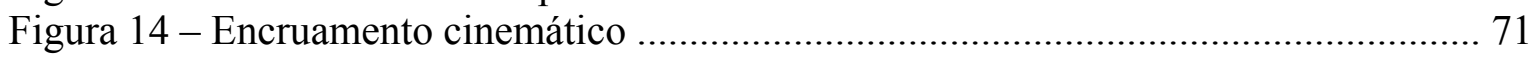

Figura 15 - Superfície de plastificação no espaço das tensões principais .......................... 73

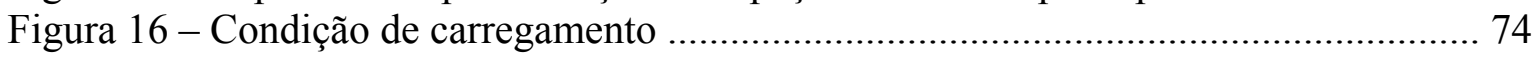

Figura 17 - Superfície de plastificação de von Mises .................................................... 76

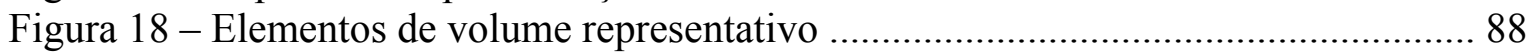

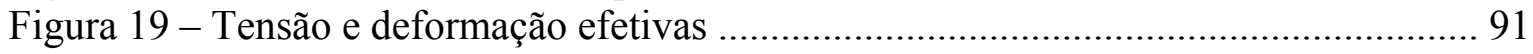

Figura 20 - Características geométricas da viga analisada ........................................ 118

Figura 21 - Convergência da energia de deformação (elementos tetraédricos) ................ 119

Figura 22 - Convergência da energia de deformação (elementos hexaédricos) ............... 120

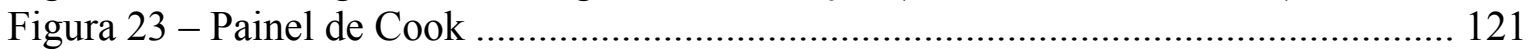

Figura 24 - Convergência da energia de deformação .................................................. 122

Figura 25 - Dimensões da barra e das regiões enfraquecidas ....................................... 124

Figura 26 - Malha de elementos e faixa central enriquecida .......................................... 124

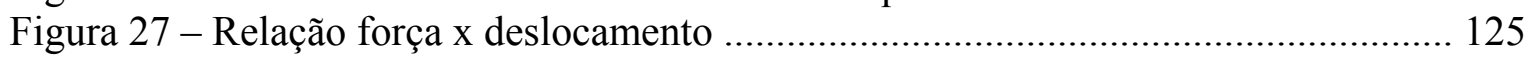

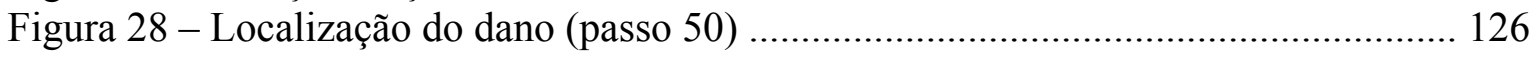

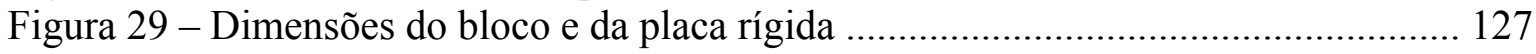

Figura 30 - Estrutura analisada utilizando dupla simetria .......................................... 128

Figura 31 - Relação Força x Deslocamento para malha de 64 elementos ........................ 129

Figura 32 - Relação Força x Deslocamento para malha de 384 elementos ..................... 129

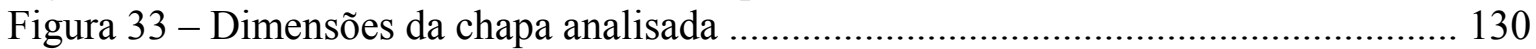

Figura 34 - Estrutura efetivamente analisada - vínculos de simetria ............................. 131

Figura 35 - Malhas de elementos utilizadas na análise ................................................... 132

Figura 36 - Nós da faixa central com enriquecimento polinomial de segundo grau ........ 133

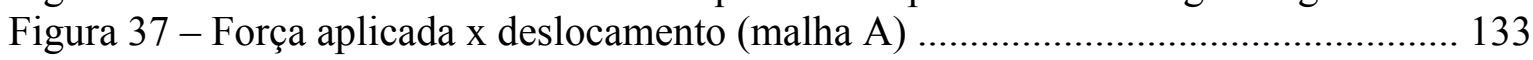

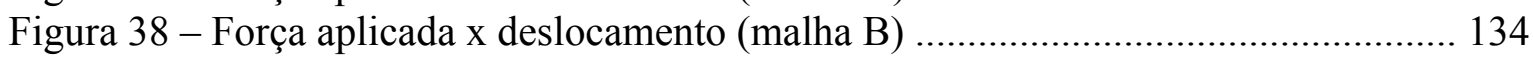

Figura 39 - Mapa da tensão equivalente (malha A), em $\mathrm{N} / \mathrm{mm}^{2}$.................................. 135

Figura 40 - Mapa da tensão equivalente (malha B), em $\mathrm{N} / \mathrm{mm}^{2}$................................... 136

Figura 41 - Força aplicada x deslocamento (malha A) ................................................ 137

Figura 42 - Força aplicada x deslocamento (malha B) ............................................... 137

Figura 43 - Malha A - Tensão equivalente em $\mathrm{N} / \mathrm{mm}^{2}$ - Passo 27 ............................. 139

Figura 44 - Malha A - Dano - Passo 27 ...................................................................... 140

Figura 45 - Malha A - Tensão equivalente em $\mathrm{N} / \mathrm{mm}^{2}$ - Passo 74 ............................. 141 
Figura 46 - Malha A - Dano - Passo 74 142

Figura 47 - Malha B - Tensão equivalente em $\mathrm{N} / \mathrm{mm}^{2}$ - Passo 43.

Figura 48 - Malha B - Dano - Passo 43 144

Figura 49 - Malha B - Tensão equivalente em $\mathrm{N} / \mathrm{mm}^{2}$ - Passo 71 ............................... 145

Figura 50 - Malha B - Dano - Passo 71 ........................................................................... 146

Figura B.1 - Significado geométrico das coordenadas adimensionais de volume 159

Figura C.1 - Significado geométrico das coordenadas adimensionais de área 161 


\section{LISTA DE TABELAS}

Tabela 1 - Correspondência de índices dos deslocamentos (nas faces e no elemento) ..... 58

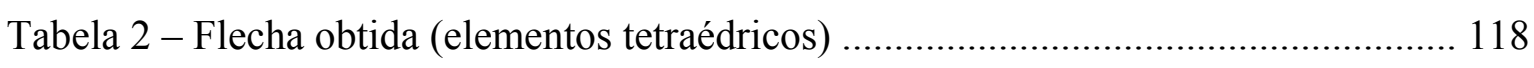

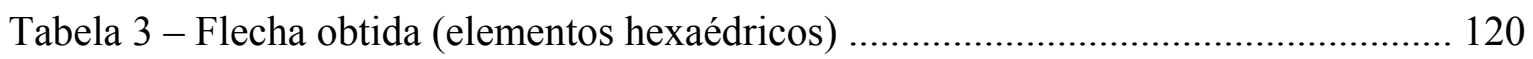

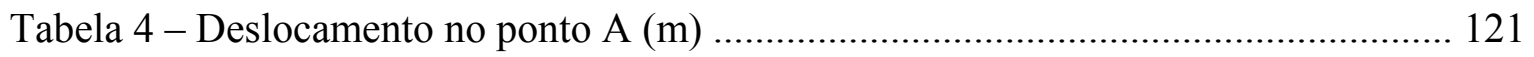




\section{LISTA DE SÍMBOLOS}

$\tilde{u}(x)$ - aproximação para o campo de deslocamentos, em problema unidimensional.

$\phi_{i}(x)$ - função de forma associada ao nó $i$.

$\tilde{u}_{i}$ - valor discreto da função aproximadora $\widetilde{u}(x)$ no nó $i$.

$x, y$ e $z$ - coordenadas do sistema cartesiano triortogonal de referência.

$\Omega$ - domínio de um sólido.

$L$ - operador diferencial de divergência.

$\sigma$ - tensor de tensões (segunda ordem).

$\{\sigma\}_{6 x 1}$ - vetor das componentes de tensão.

$\bar{p}_{V}$ - vetor de componentes da força de volume.

$\bar{p}$ - vetor de componentes da força de superfície.

$S_{p}$ - região do contorno do sólido sujeita a forças de superfície.

$d$ - vetor que reúne os deslocamentos $u, v$ e $w$.

$u, v$ e $w$ - deslocamentos segundo as direções $x, y$ e $z$, respectivamente.

$\varepsilon$ - tensor de deformações (segunda ordem)

$\{\varepsilon\}_{6 x 1}$ - vetor das componentes de deformação.

$[D]_{6 \times 6}$ - no Cap. 2, matriz da relação constitutiva elástica linear.

$\lambda, \mu$ - constantes de Lamé.

$E$ - módulo de elasticidade longitudinal.

$v$ - coeficiente de Poisson.

$d_{e}$ - vetor de deslocamentos nodais do elemento finito.

$n_{d}$ - dimensão do vetor $d_{e}$.

$[\phi]$ - matriz de aproximação dos deslocamentos.

$\bar{p}_{e}^{V}$ - vetor de parâmetros nodais referentes às forças de volume do elemento.

$n_{p v}$ - dimensão do vetor $\bar{p}_{e}^{V}$.

$\left\lfloor\phi^{p_{V}}\right\rfloor$ - matriz de interpolação das forças de volume.

$\bar{p}_{e}$ - vetor de parâmetros nodais referentes às forças de superfície do elemento.

$n_{p}$ - dimensão do vetor $\bar{p}_{e}$.

$\left[\phi^{p}\right]$ - matriz de interpolação das forças de superfície.

$\Omega_{e}$ - domínio de um elemento finito.

$S_{p e}$ - superfície carregada de um elemento finito.

$R_{e}$ - matriz de rigidez do elemento finito.

$f_{e}$ - vetor de forças nodais do elemento finito.

$B$ - matriz de aproximação das deformações.

$R_{g}$ - matriz de rigidez global.

$f_{g}$ - vetor de forças nodais global. 
$d_{g}$ - vetor de deslocamentos globais.

$N_{d}$ - dimensão do sistema linear global.

$Q$ - no Cap. 2, conjunto de nós utilizados na discretização do domínio $\Omega$ (Métodos sem Malha).

$x_{j}$ - nó que compõe o conjunto $Q, j=1, \ldots, n_{\text {nos }}$

$\omega_{j}$ - região de influência, ou nuvem, de um nó $x_{j}$.

$r_{j}$ - raio que define a nuvem nodal $\omega_{j}$.

$\mathfrak{I}$ - região formada pela união de todas as nuvens nodais $\omega_{j}$.

$W_{j}$ - função ponderadora associada à nuvem $\omega_{j}$.

$J(x)$ - funcional quadrático do erro da aproximação - Método dos Mínimos Quadrados Móveis (MMQM).

$u_{j}$ - valor do parâmetro nodal associado ao nó $x_{j}$.

$\hat{u}(x)$ - função aproximadora global (MMQM).

$\alpha(x)$ - vetor das constantes da combinação linear que define a aproximação $\hat{u}(x)$.

$p(x)$ - vetor que reúne a base de funções adotadas na aproximação $\hat{u}(x)$.

$A(x)$ - matriz utilizada na geração das funções de forma $\phi_{j}$, no MMQM.

$B_{j}(x)$ - vetor utilizado na geração da função de forma $\phi_{j}$, no MMQM.

$\phi_{j}$ - função de forma associada ao nó $x_{j}$.

$C_{0}^{q}$ - conjunto de funções cujas derivadas até a ordem $q$ apresentam suporte compacto.

$C^{k}$ - conjunto de funções cujas derivadas até a ordem $k$ são contínuas.

$P_{k}$ - base polinomial de ordem $k$ (caso unidimensional)

$P_{p}$ - base polinomial de ordem $p$ (caso unidimensional), $p>k$.

$\mathfrak{J}_{n_{\text {nos }}}^{k, p}$ - Família de funções do Método das Nuvens hp: funções de forma geradas com a base $P_{k}$ e enriquecidas com a base $P_{p}-P_{k}$.

$F_{j \alpha}$ - funções de índice $\alpha$ que enriquecem as funções de forma associadas ao nó de índice $j$.

$I(j)$ - contador para o número de funções adicionadas a cada nó de índice $j$.

$\xi_{i}$ - coordenada adimensional de volume do tetraedro, referente ao nó $i, i=1, \ldots, 4$.

$V_{0}^{i}$ - termo utilizado no cálculo da coordenada adimensional $\xi_{i}$.

$a_{i}, b_{i}, c_{i}$ - derivadas parciais de $\xi_{i}$ em relação às coordenadas cartesianas $x, y$ e $z$, respectivamente.

$x_{i}, y_{i}$ e $z_{i}, i=1, \ldots, 4$ - coordenadas cartesianas dos nós do tetraedro.

$V$ - volume do tetraedro.

$u_{i}, v_{i}$ e $w_{i}, i=1, \ldots, 4$ - deslocamentos dos nós do tetraedro.

$[A]_{3 \times 3}$ - submatriz que compõe as matrizes de rigidez do tetraedro e do hexaedro.

$c t_{1}, c t_{2}$ e $c t_{3}$ - termos que formam a matriz constitutiva $[D]_{6 \times 6}$. 
$\zeta_{i}$ - coordenada adimensional de área do triângulo, referente ao nó $i, i=1, \ldots, 3$.

$a_{0}^{i}, \omega_{i}, \eta_{i}$ - termos utilizados no cálculo da coordenada $\zeta_{i}$.

$(r, s)$ - sistema de coordenadas cartesianas no plano das faces dos elementos (tetraedro e hexaedro).

$A$ - área de face do tetraedro.

$u_{i}^{j}, \quad v_{i}^{j}$ e $w_{i}^{j}$ - parâmetros adicionais de índice $j$, associados, respectivamente, aos deslocamentos $u_{i}, v_{i}$ e $w_{i}$.

$f_{u_{i}}^{j}, f_{v_{i}}^{j}$ e $f_{w_{i}}^{j}$ - funções de índice $j$ que enriquecem os deslocamentos $u_{i}, v_{i}$ e $w_{i}$, respectivamente.

$n f\left(u_{i}\right), n f\left(v_{i}\right)$ e $n f\left(w_{i}\right)$ - número de funções que enriquecem os deslocamentos $u_{i}, v_{i}$ e $w_{i}$, respectivamente.

npar - total de parâmetros nodais adicionados a cada elemento.

$(\xi, \eta, \psi)$ - sistema de coordenadas adimensionais do hexaedro.

$N_{i}, i=1, \ldots, 8$ - funções de forma trilineares lagrangianas do hexaedro.

$x_{i}, y_{i}$ e $z_{i}, i=1, \ldots, 8$ - coordenadas cartesianas dos nós do hexaedro.

$u_{i}, v_{i}$ e $w_{i}, i=1, \ldots, 8$ - deslocamentos dos nós do hexaedro.

$[J]_{3 \times 3}$ - Jacobiano da transformação de coordenadas no hexaedro.

$N_{i}, i=1, \ldots, 4$ - funções de forma bilineares lagrangianas das faces do hexaedro.

$(\zeta, \vartheta)$ - sistema de coordenadas adimensionais das faces do hexaedro.

$u_{i}^{f}, v_{i}^{f}$ e $w_{i}^{f}, i=1, \ldots, 4, f=1, \ldots, 6$ - deslocamentos do hexaedro com referência à face do mesmo.

$J_{2}$ - invariante das tensões desviadoras.

$\varepsilon^{e}$ - parcela elástica do tensor de deformações.

$\varepsilon^{p}$ - parcela plástica do tensor de deformações.

$\sigma_{y}$ - tensão de plastificação.

$f$ - função de plastificação.

$\dot{\lambda}$ - módulo da taxa de deformação plástica.

$K$ - módulo plástico de encruamento isótropo.

$\alpha$ - medida da deformação plástica acumulada.

$q$ - deslocamento do intervalo elástico inicial no encruamento cinemático.

$H$ - módulo plástico do encruamento cinemático.

$C$ - tensor constitutivo elástico linear de quarta ordem.

I - tensor identidade de segunda ordem.

$\Pi$ - tensor identidade de quarta ordem.

$R$ - vetor associado ao encruamento.

$h$ - vetor que dá a direção de $R$.

$r$ - tensor de segunda ordem que estabelece a direção do fluxo plástico.

$D$ - no item 3.3.2, Cap. 3, matriz de módulos plásticos de encruamento.

$\sigma_{t}$ - tensão de escoamento do material num ensaio de tração simples. 
$S$ - no Cap. 3 e no item 4.3 do Cap. 4, tensor desviador de tensões.

$\sigma_{m}$ - tensão média ou hidrostática.

$\sigma_{e q}$ - tensão equivalente ou tensão de von Mises.

$n$ - tensor de norma unitária que dá a direção do fluxo plástico.

$\varepsilon_{n}, \varepsilon_{n+1}$ - tensores de deformação, nos passos $n$ e $n+1$, respectivamente.

$\Delta \varepsilon_{n}$ - incremento na deformação do passo $n$ para o $n+1$.

$\sigma_{n+1}$ - tensor de tensões, no passo $n+1$.

$\varepsilon_{n}^{p}, \varepsilon_{n+1}^{p}$ - tensores de deformação plástica, nos passos $n$ e $n+1$.

$\Delta \lambda$ - magnitude da evolução das variáveis associadas à plastificação.

$n_{n+1}$ - tensor de norma unitária $n$, no passo $n+1$.

$S_{n+1}$ - tensor desviador de tensões, no passo $n+1$.

$\alpha_{n}, \alpha_{n+1}$ - variáveis relacionadas à deformação plástica, nos passos $n$ e $n+1$, respectivamente.

$f_{n+1}$ - função de plastificação, no passo $n+1$

$\varepsilon_{n+1}^{p, p r}$ - deformação plástica de previsão, no passo $n+1$.

$\alpha_{n+1}^{p r}$ - valor de previsão da variável $\alpha$, no passo $n+1$.

$\sigma_{n+1}^{p r}$ - tensão de previsão, no passo $n+1$.

$S_{n+1}^{p r}$ - tensão desviadora de previsão, no passo $n+1$.

$f_{n+1}^{p r}$ - função de plastificação de previsão, no passo $n+1$.

$e_{n+1}$ - parcela desviadora do tensor de deformações, no passo $n+1$.

$n_{n+1}^{p r}$ - tensor de norma unitária $n$ de previsão, no passo $n+1$.

$C_{n+1}^{e p}$ - tensor elastoplástico algorítmico tangente, no passo $n+1$.

$D^{e p}$ - matriz da relação constitutiva elastoplástica tangente.

$r_{D}$ - raio que define a região de influência para o dano não-local.

$N$ - força axial.

$S$ - no item 4.2 do Cap. 4, seção transversal de um elemento de volume representativo.

$S_{D}$ - área de defeitos na seção $S$.

$\sigma_{e f}$ - tensão efetiva.

$D_{n}^{S_{D}}$ - dano associado ao conceito de área de defeitos.

$l_{0}$ - comprimento de um elemento de volume representativo.

$\Delta l$ - aumento do comprimento $l_{0}$.

$\Delta d$ - parcela do aumento do comprimento $\Delta l$ devida à abertura de descontinuidades internas.

$\varepsilon_{e f}$ - deformação efetiva.

$D_{n}^{\Delta d}$ - dano associado ao conceito de abertura de descontinuidades internas.

$\Delta V_{D}$ - acréscimo no volume associado aos defeitos. 
$D_{n}$ - dano segundo uma direção definida pelo versor $n$.

$D$ - dano escalar (itens 4.2.1 e 4.2.2 do Cap. 4).

$E_{D}$ - módulo de elasticidade longitudinal do meio com dano.

$D$ - tensor de dano de quarta ordem (item 4.2.3 do Cap. 4)

$C_{D}$ - tensor constitutivo de quarta ordem com efeito de dano.

$C_{D}^{e q . d e f}$. - tensor constitutivo de quarta ordem com efeito de dano, calculado utilizando o princípio de equivalência em deformação.

$C_{D}^{\text {eq. tens. }}$ - tensor constitutivo de quarta ordem com efeito de dano, calculado utilizando o princípio de equivalência em tensão.

$f(d)$ - função de valor escalar que varia com a danificação.

$Q$ - no Cap. 4, tensor constante de anisotropia.

$\widetilde{\varepsilon}$ - deformação equivalente.

$D_{T}, D_{C}$ - níveis de dano associados a estados uniaxiais de tração e compressão, respectivamente.

$\alpha_{T}, \alpha_{C}$ - ponderadores dos níveis de dano $D_{T}$ e $D_{C}$.

$A_{T}, B_{T}, A_{C}, B_{C}, \varepsilon_{d 0}$ - parâmetros característicos do material segundo o modelo de

Mazars.

$\varepsilon^{\text {princ }}$ - vetor de deformações principais.

$\sigma^{\text {princ }}$ - vetor de tensões principais.

$\sigma^{+}$- parte positiva de $\sigma^{\text {princ }}$.

$\sigma^{-}$- parte negativa de $\sigma^{\text {princ }}$.

$\varepsilon^{T}$ - parte do vetor $\varepsilon^{\text {princ }}$ relacionada à tração.

$\varepsilon^{C}$ - parte do vetor $\varepsilon^{\text {princ }}$ relacionada à compressão.

$\varepsilon^{T,+}, \varepsilon^{T,-}-$ partes positiva e negativa do vetor $\varepsilon^{T}$.

$\varepsilon^{C,+}, \varepsilon^{C,-}-$ partes positiva e negativa do vetor $\varepsilon^{C}$.

$\varepsilon_{V}^{+}$- variável representativa do estado de extensão do material.

$W_{e}$ - energia de deformação elástica.

$W_{e}^{S}$ - energia de deformação elástica relacionada à distorção.

$W_{e}^{H}$ - energia de deformação elástica relacionada à deformação volumétrica.

$\varepsilon_{e}^{S}$ - parcela desviadora do tensor de deformações elásticas.

$\varepsilon_{e}^{H}$ - deformação elástica média ou volumétrica.

$\sigma^{*}$ - tensão equivalente generalizada.

$R_{v}$ - fator de triaxialidade.

$\psi$ - no item 4.3.2.2, potencial termodinâmico de energia livre.

$\psi_{e}$ - parcela recuperável do potencial termodinâmico de energia livre $\psi$.

$\psi_{\alpha}$ - parcela irrecuperável do potencial termodinâmico de energia livre $\psi$.

$\rho$ - densidade do material. 
$Y$ - variável associada ao dano.

$W_{e}^{*}$ - energia de deformação elástica do meio danificado.

$\widetilde{\sigma}^{*}$ - tensão equivalente efetiva.

$\varphi^{*}$ - potencial de dissipação convexo.

$\varphi_{p}^{*}$ - parcela de $\varphi^{*}$ relativa aos efeitos de plastificação e encruamento.

$\varphi_{D}^{*}$ - parcela de $\varphi^{*}$ relativa ao efeito da danificação.

$S_{0}, s_{0}$ - coeficientes característicos do material no modelo de Lemaitre.

$\varepsilon_{p}^{*}$ - norma da deformação plástica acumulada.

$C_{K}$ - constante do modelo de Lemaitre.

$\varepsilon_{D}$ - deformação plástica uniaxial que inicia o dano.

$\varepsilon_{p, D}$ - norma da deformação plástica que inicia o dano (caso tridimensional).

$D_{C}$ - dano crítico.

$\varepsilon_{R}$ - valor da deformação plástica (caso unidimensional), correspondente ao dano crítico.

$\varepsilon_{p, R}$ - norma da deformação plástica (caso tridimensional), correspondente ao dano crítico.

$D_{n}, D_{n+1}$ - dano nos passos $n$ e $n+1$, respectivamente.

$f_{D}$ - variável relacionada à evolução do dano.

$D_{n+1}^{p r}$ - valor de previsão do dano no passo $n+1$.

$k_{1}, k_{2}$ - constantes utilizadas no modelo de Lemaitre.

$C_{n+1}^{e p, d}$ - tensor algorítmico tangente do modelo de Lemaitre.

$K N_{1}, K N_{2}$ e $K N_{3}$ - valores utilizados no cálculo de $\frac{\partial n_{n+1}}{\partial \varepsilon_{n+1}}$

$K L 1, K L 2$ - valores utilizados no cálculo de $\frac{\partial \Delta \lambda}{\partial \varepsilon_{n+1}}$

$K D 1, K D 2$ - valores utilizados no cálculo de $\frac{\partial D_{n+1}}{\partial \varepsilon_{n+1}}$

$C_{n+1}^{e l, d}$ - tensor que dá o efeito do dano sobre o tensor elástico linear.

$C_{n+1}^{p, d}$ - tensor relacionado ao efeito do acoplamento entre o dano e a plasticidade.

$D P_{1}, D P_{2}, D P_{3}, D P_{4}, D P_{5}, D P_{6}, D P_{7}, D P_{8}$ e $D P_{9}$ - variáveis utilizadas no cálculo do tensor $C_{n+1}^{p, d}$.

$w_{\text {ref }}$ - deslocamento de referência.

$U_{\text {ref }}$ - energia de deformação de referência.

$q$ - força de superfície uniformemente distribuída. 


\section{Operadores}

$\dot{f}=\frac{\partial f}{\partial t}-$ taxa da função $f$

$\operatorname{sign}(\sigma)= \begin{cases}+1, & \text { se } \sigma>0 \\ -1, & \text { se } \sigma<0\end{cases}$

$f_{\alpha}$ - derivada parcial da função $f$ em relação à variável $\alpha$.

$a \otimes b$ - produto tensorial.

$a \cdot b$ - produto interno.

$\|A\|$ - norma do tensor $A$.

$\operatorname{tr}(A)$ - traço do tensor $A$.

\section{Abreviaturas}

MEF - Método dos Elementos Finitos.

MEFG - Método dos Elementos Finitos Generalizados.

PTV - Princípio dos Trabalhos Virtuais.

MMQM - Método dos Mínimos Quadrados Móveis.

MED - Método dos Elementos Difusos.

MGLE - Método de Galerkin Livre de Elementos.

MPF - Método dos Pontos Finitos.

PU - Partição de Unidade.

MEFPU - Método dos Elementos Finitos Partição de Unidade

$\mathrm{NE}$ - aproximação não-enriquecida

P1, P2, P3 e P4 - enriquecimento da aproximação do campo de deslocamentos por polinômios de primeiro ao quarto graus, respectivamente. 


\section{SUMÁRIO}

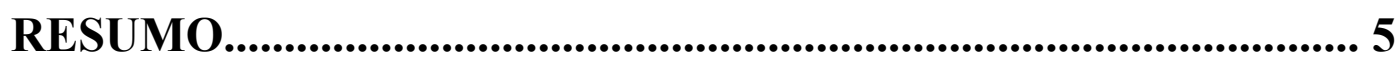

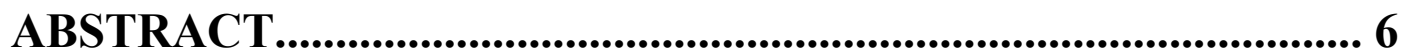

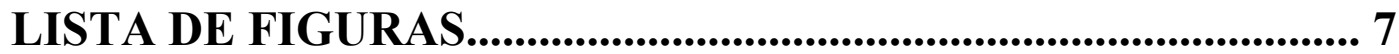

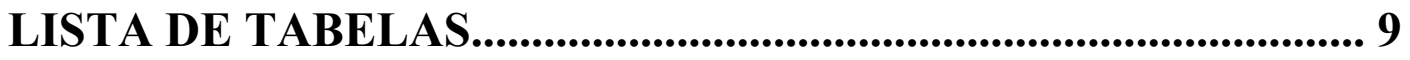

LISTA DE SÍMBOLOS..................................................................... 10

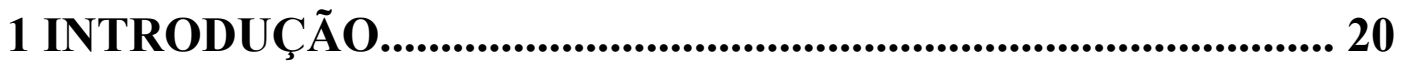

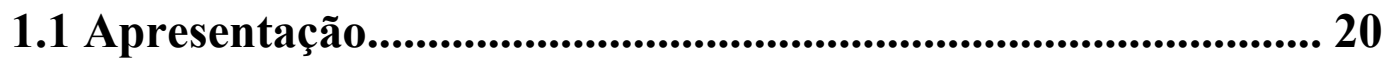

1.2 Justificativa e Objetivos...................................................................... 21

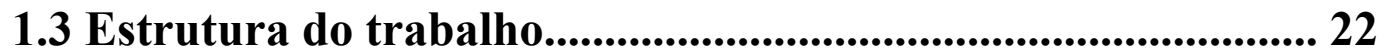

\section{O MÉTODO DOS ELEMENTOS FINITOS}

GENERALIZADOS (MEFG) ....................................................... 24

2.1 O Método dos Elementos Finitos (MEF)................................... 24

2.2 Os Métodos sem Malha.............................................................. 30

2.2.1 Revisão Bibliográfica............................................................................. 30

2.2.2 Formulação geral dos Métodos sem Malha

com base no MMQM..................................................................... 32

2.3 Síntese do Método dos Elementos Finitos Generalizados....... 39

2.3.1 Revisão Bibliográfica........................................................................... 39

2.3.2 Formulação geral....................................................................... 41

2.3.3 Formulação de elementos tridimensionais no MEFG............................. 43

2.3.3.1 Formulação do tetraedro..................................................................... 43

2.3.3.1.1 Tetraedro sem enriquecimento....................................................... 44

2.3.3.1.2 Tetraedro com enriquecimento........................................................ 49

2.3.3.2 Formulação do hexaedro..................................................................... 52

2.3.3.2.1 Hexaedro sem enriquecimento...................................................... 52

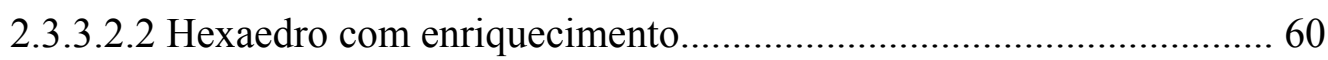

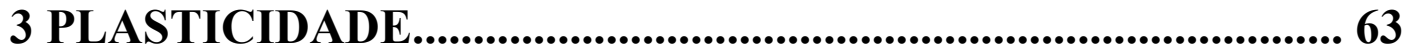

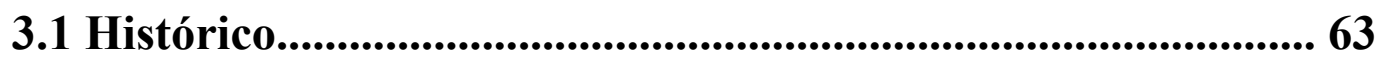


3.2 Conceitos Básicos.......................................................................... 64

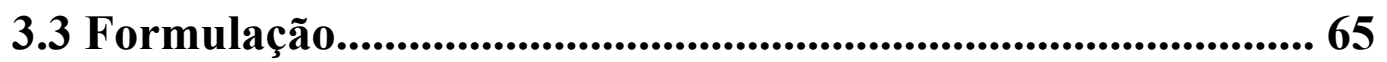

3.3.1 Comportamento elastoplástico unidimensional............................. 65

3.3.1.1 Comportamento elastoplástico perfeito................................................. 66

3.3.1.2 Comportamento elastoplástico com encruamento............................... 67

3.3.2 Comportamento elastoplástico tridimensional..................................... 71

3.3.2.1 Modelo elastoplástico perfeito - critério de von Mises........................ 75

3.3.2.2 Modelo elastoplástico com encruamento isótropo linear critério de von Mises.......................................................................... 77

3.4 Travamento volumétrico.................................................................. 84

4 MECÂNICA DO DANO CONTÍNUO............................................... 86

4.1 Introdução e Revisão Bibliográfica............................................ 86

4.2 Conceitos fundamentais da Mecânica do Dano........................... 88

4.2.1 Tensão e deformação efetivas - caso unidimensional............................. 89

4.2.2 Princípios de equivalência de resposta - caso unidimensional.............. 91

4.2.3 Representação tensorial do dano................................................................ 93

4.3 Modelos de dano utilizados neste trabalho............................... 95

4.3.1 Modelo de MAZARS (1984)................................................................... 95

4.3.2 Modelo constitutivo de dano e plasticidade - LEMAITRE (1985)...... 98

4.3.2.1 Energia de deformação elástica e tensão equivalente generalizada...... 98

4.3.2.2 Potencial termodinâmico de energia livre e variáveis associadas......... 99

4.3.2.3 Potencial de dissipação e leis de evolução da deformação plástica, do dano e do encruamento 101

4.3.2.4 Resumo das equações do Modelo de Lemaitre................................... 105

4.3.2.5 Algoritmo de integração em passo finito.............................................. 106

5 O CÓDIGO DE CÁLCULO DESENVOLVIDO.......................... 114

6 EXEMPLOS DE APLICAÇÃO NUMÉRICA............................ 117

6.1 Exemplo 1..................................................................................................... 117

6.2 Exemplo 2.............................................................................................. 120

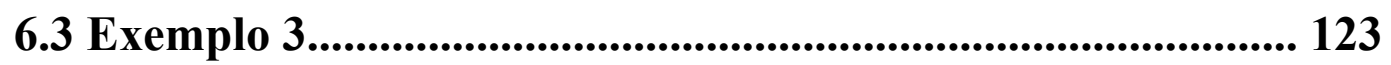

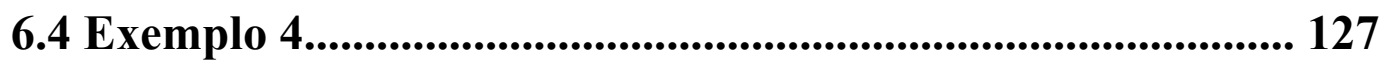




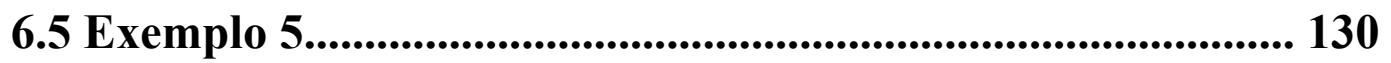

7 CONCLUSÕES E CONSIDERAÇÕES FINAIS.......................... 147

REFERÊNCIAS BIBLIOGRÁFICAS.......................................... 150

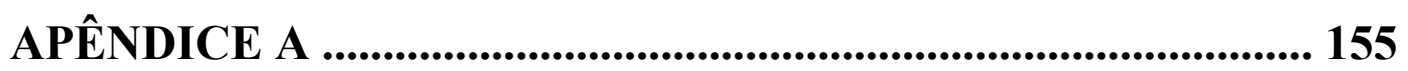

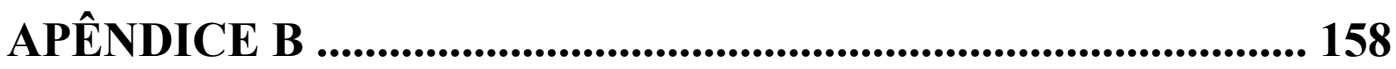

APÊNDICE C ........................................................................ 160

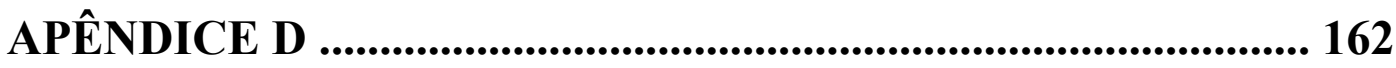

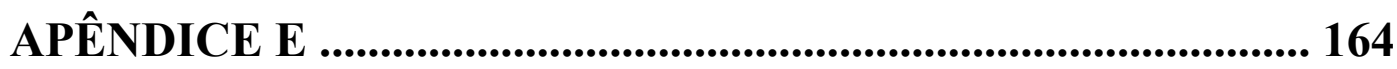

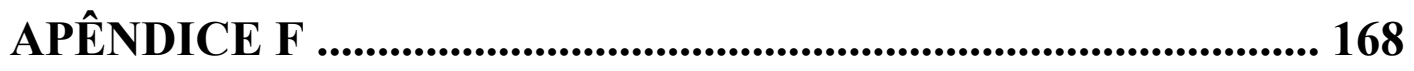

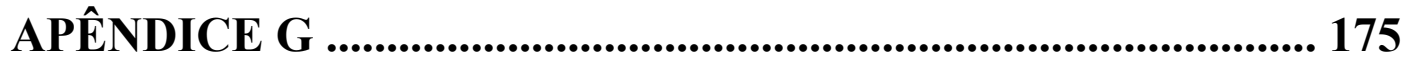

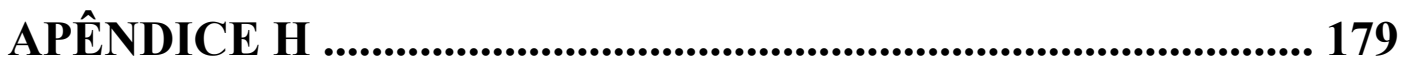

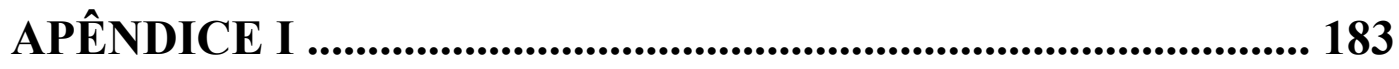

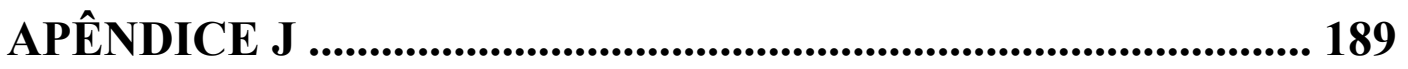

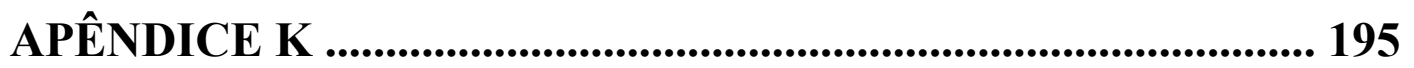

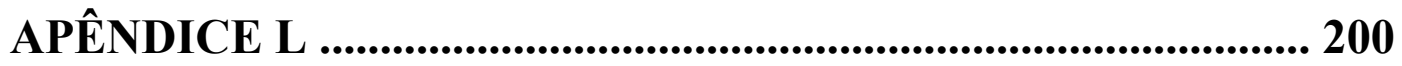

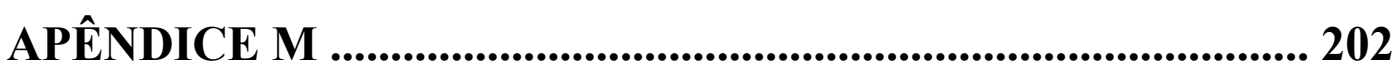

APÊNDICE N .................................................................................. 204

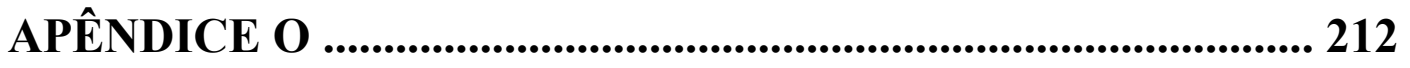




\section{INTRODUÇÃO}

\subsection{Apresentação.}

Este trabalho trata de uma formulação não-convencional do Método dos Elementos Finitos (MEF), denominada Método dos Elementos Finitos Generalizados (MEFG), e da análise de estruturas sujeitas ao efeito da não-linearidade física. Mais especificamente, realiza-se a implementação computacional do MEFG, visando análises tridimensionais, e sua aplicação à simulação numérica de problemas estruturais não-lineares, em que se manifestam os efeitos do dano e da plasticidade (associados ou não) no comportamento do material. Trabalhos nesse sentido já vêm sendo realizados em São Carlos, limitados a análises planas, como em BARROS (2002). Neste sentido, este trabalho é uma extensão natural daquelas pesquisas.

O MEFG resulta de uma combinação do MEF em sua forma tradicional baseada no emprego de uma malha de elementos com conceitos e técnicas típicas dos métodos sem malha (notadamente do Método das Nuvens hp, de DUARTE \& ODEN (1996)), especialmente quanto à adição de funções enriquecedoras (e parâmetros a elas associados) atrelados a pontos nodais do domínio, visando a melhorar a qualidade da aproximação no entorno desses pontos. O método preserva a estrutura básica do MEF (subdivisão do domínio em elementos, aproximação dos deslocamentos no interior dos mesmos por funções de forma do tipo lagrangianas, etc), permitindo o enriquecimento local da aproximação com funções (associadas aos nós) que se julguem mais apropriadas, sem que haja perda de conformidade entre os elementos.

Quanto à não-linearidade física, são objeto de estudo, implementação e aplicação à simulação numérica os seguintes modelos constitutivos:

- Modelo elastoplástico (perfeito ou com encruamento linear isótropo) com função de plastificação de von Mises. Em modelos como este, nos quais a deformação plástica é isocórica, ou seja, ocorre sem variação de volume, é freqüente, na simulação numérica, a ocorrência do chamado travamento volumétrico, especialmente se a aproximação utilizada for de baixa ordem polinomial. A viabilidade de superar esse problema utilizando-se do enriquecimento da aproximação nos moldes do MEFG é mostrada através de exemplo numérico.

- Modelo de dano evolutivo frágil (MAZARS (1984)), formulado visando a descrever o comportamento do concreto sob carregamento monótono crescente. Por este 
modelo, o dano é representado por uma variável escalar. Além disso, não são consideradas deformações plásticas e considera-se que o surgimento e a evolução do dano estejam relacionados às extensões segundo as direções principais de deformação.

- Modelo de LEMAITRE (1985), que considera o acoplamento entre o dano e a plasticidade. Este modelo permite descrever o comportamento de materiais metálicos, nos quais a danificação ocorre após um processo de encruamento causado pelas deformações plásticas. Empregando-se este modelo, a possibilidade de se realizar o enriquecimento da aproximação apenas em regiões de interesse, permitida pelo MEFG, é explorada em exemplo numérico, visando a obter-se uma melhor definição da distribuição do dano e de tensões na estrutura.

\subsection{Justificativa e Objetivos.}

A busca de melhoria na qualidade dos métodos de simulação numérica tem sido objeto de um grande número de trabalhos publicados nos últimos anos, com destaque para aqueles que exploram formulações alternativas ao Método dos Elementos Finitos (MEF), em sua forma convencional. Embora o MEF seja o método mais amplamente utilizado na Mecânica dos Sólidos, dispondo de uma fundamentação teórica muito bem estabelecida e de uma ampla experimentação e validação de resultados, podem ser notadas limitações neste método em determinadas aplicações, notadamente no campo não-linear.

Uma dessas limitações relaciona-se ao fato do método gerar aproximações com base em funções interpoladoras polinomiais, cuja qualidade resulta diretamente dependente da geometria e do tipo de elemento. Essa característica pode mostrar-se particularmente ineficaz na simulação de fenômenos que implicam na ocorrência de elevados gradientes de tensão e de deformação, como os de localização (por exemplo, decorrente do comportamento de softening devido à danificação), de grande concentração de tensões (causada por singularidades no comportamento do material) e daqueles que, de uma forma geral, implicam em grande distorção da geometria dos elementos.

Esta limitação pode ser parcialmente superada recorrendo-se a técnicas que exploram procedimentos normalmente onerosos de refinamento h-adaptativo da malha, as quais são concebidas de modo a assegurar níveis aceitáveis de qualidade da resposta numérica. Como alternativa pode-se recorrer à adaptabilidade da base de aproximação polinomial (processos p-adaptativos). Porém, apesar das vantagens que apresenta, em particular nas regiões de solução regular, a adaptabilidade polinomial é também onerosa e relativamente pouco eficaz no contexto da formulação convencional do Método dos 
Elementos Finitos, por não ser fácil conceber e programar elementos p-adaptativos compatíveis.

Uma outra alternativa, que mais recentemente vem sendo explorada, é o investimento em formulações não-convencionais do Método dos Elementos Finitos, como o Método dos Elementos Finitos Generalizados (MEFG), que oferecem condições bastante favoráveis à superação das limitações do MEF referidas anteriormente, como se comenta a seguir:

- No MEFG, faz-se o enriquecimento após o mapeamento de coordenadas entre o elemento mestre e o escravo (no caso de elementos isoparamétricos), não havendo a perda de qualidade aproximadora verificada no MEF convencional no caso de distorção da malha.

- Em regiões de singularidade, o MEFG permite a utilização de funções enriquecedoras que correspondam às soluções analíticas conhecidas de problemas com essas características, reduzindo muito o erro da aproximação.

- O enriquecimento polinomial pode ser realizado apenas em regiões específicas do domínio, mantendo a conformidade entre os elementos. Essa característica é de grande utilidade na simulação de processos localizados de danificação ou plastificação.

As potencialidades exibidas por esta nova ferramenta justificam o interesse pelo seu estudo e, particularmente, a investigação de sua aplicação em problemas não-lineares segundo uma abordagem tridimensional; este é o aspecto original deste trabalho.

\subsection{Estrutura do trabalho.}

O capítulo 2 trata do Método dos Elementos Finitos Generalizados, enfocando basicamente os seguintes tópicos:

- Breve revisão bibliográfica e principais conceitos e características dos Métodos sem Malha, em especial: a Partição da Unidade e o enriquecimento local de uma aproximação.

- O Método dos Elementos Finitos Generalizados é apresentado como um método híbrido, resultado da fusão do MEF convencional com conceitos e técnicas dos Métodos sem malha, especialmente do Método das Nuvens hp.

- Apresentação da formulação de elementos finitos tridimensionais (tetraedro e hexaedro) baseados no MEFG.

O capítulo 3 trata da Teoria da Plasticidade, sendo apresentada uma breve revisão bibliográfica e os conceitos gerais da formulação de relações constitutivas elastoplásticas. 
O modelo utilizado neste trabalho, o de von Mises, é apresentado segundo uma formulação em passo finito que utiliza algoritmo do tipo implícito. A questão do travamento volumétrico na plasticidade é abordada, mostrando-se a aplicabilidade do MEFG para superá-la.

O capítulo 4 trata da Mecânica do Dano Contínuo. Apresenta-se uma breve revisão bibliográfica desta teoria, bem como os conceitos fundamentais a ela associados como os de tensão e deformação efetivas e os princípios de equivalência da resposta constitutiva. São apresentados os dois modelos constitutivos utilizados neste trabalho: o de dano frágil para o concreto (modelo de Mazars) e do de dano dúctil (modelo de Lemaitre), próprio para material metálico ou ligas.

O capítulo 5 descreve as principais características do código computacional desenvolvido, com base nas formulações descritas nos capítulos 2, 3 e 4, para a simulação numérica de problemas estruturais tridimensionais não-lineares.

No capítulo 6 são apresentados alguns exemplos numéricos, que visam a demonstrar a viabilidade e vantagens da aplicação do MEFG em problemas de análise nãolinear.

No capítulo 7, apresentam-se algumas conclusões que puderam ser extraídas dos experimentos numéricos realizados, bem como sugestões para trabalhos futuros nessa linha de pesquisa.

Ao final do trabalho, apresentam-se as obras que foram objeto de referência, bem como os apêndices mencionados ao longo do texto. 


\section{O MÉTODO DOS ELEMENTOS FINITOS GENERALIZADOS (MEFG)}

Como já citado, o MEFG é um método híbrido que utiliza a estrutura básica do Método dos Elementos Finitos, incorporando técnicas e recursos dos chamados Métodos sem Malha para melhorar a aproximação no domínio do problema. Portanto, faz-se nos itens 2.1 e 2.2 uma breve revisão das principais características dos métodos que servem de base para o MEFG, sendo este último descrito em maior detalhe no item 2.3.

\subsection{O Método dos Elementos Finitos (MEF)}

Método mais amplamente utilizado na Mecânica dos Sólidos, o MEF, baseando-se na subdivisão do domínio estudado em elementos limitados por nós, propõe a construção de aproximações para as grandezas incógnitas de interesse do problema por interpolação de valores nodais. Em campo unidimensional, uma aproximação para o campo de deslocamentos, por exemplo, pode ser escrita na forma:

$$
\tilde{u}(x)=\alpha_{1} \phi_{1}(x)+\alpha_{2} \phi_{2}(x)+\ldots+\alpha_{i} \phi_{i}(x)+\ldots+\alpha_{n} \phi_{n}(x)
$$

onde $\phi_{i}(x)$, com $i=1, \ldots, n$ são denominadas funções de forma e devem ter continuidade suficiente para que as integrais que aparecem na forma fraca do problema de valor de contorno possam existir.

Uma característica do MEF é que as constantes $\alpha_{i}$ coincidem com valores discretos da função $\widetilde{u}(x)$ nos pontos nodais, isto é:

$$
\alpha_{i}=\tilde{u}_{i}
$$

Outra característica do método é que as funções $\phi_{i}(x)$ têm valor unitário no nó $i$ e nulo nos outros nós. Essas funções podem ser construídas mediante uma técnica de interpolação lagrangiana, que no caso linear fornece:

$$
\phi_{i}(x)=\left\{\begin{array}{lll}
\frac{\left(x-x_{i-1}\right)}{\left(x_{i}-x_{i-1}\right)} & \text { se } x_{i-1} \leq x \leq x_{i} \\
\frac{\left(x_{i+1}-x\right)}{\left(x_{i+1}-x_{i}\right)} & \text { se } x_{i} \leq x \leq x_{i+1}
\end{array}\right.
$$

As figuras 1-a e 1-b ilustram, respectivamente, a aproximação descrita pela eq. (1) e a interpolação dada na eq. (3). 


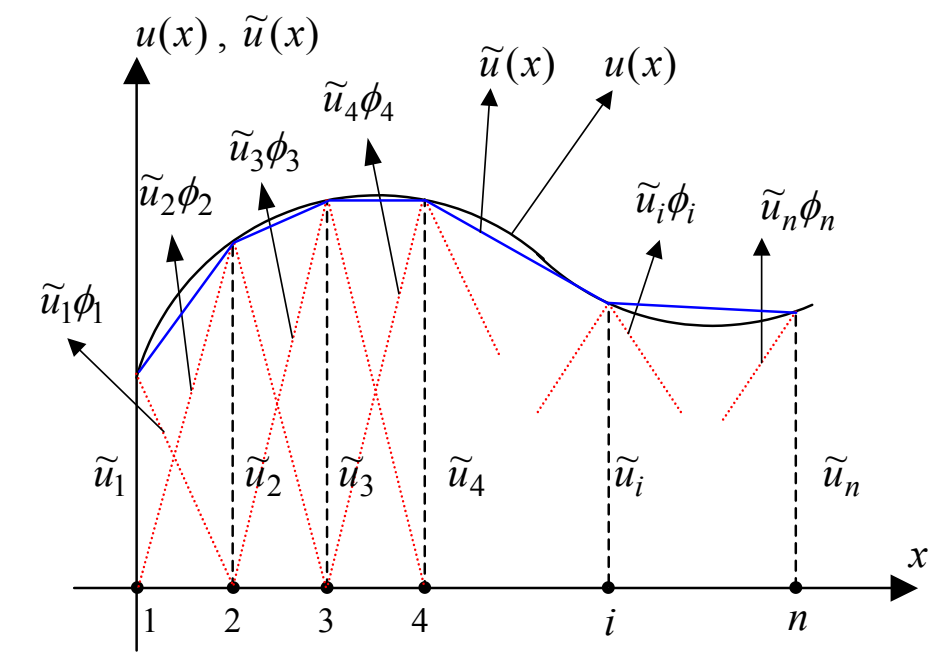

a)

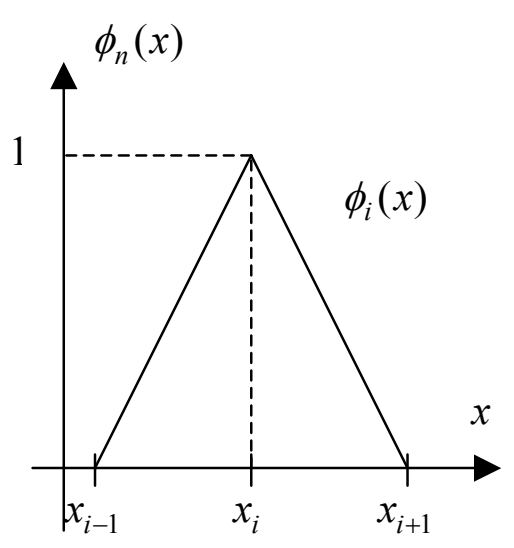

b)

Figura 1 - Aproximação para o campo de deslocamentos no MEF

O método aplica-se à obtenção de soluções aproximadas de problemas de valor de contorno formulados em forma fraca pelo Método dos Resíduos Ponderados (Método de Galerkin, por exemplo) ou pelo Princípio dos Trabalhos Virtuais (PTV). Em problemas lineares, a aplicação da função aproximadora à forma fraca gera um sistema de equações lineares tendo por incógnitas os valores nodais. A matriz dos coeficientes desse sistema é dita matriz de rigidez, enquanto que o vetor independente é denominado vetor de forças nodais equivalentes.

Outra característica do método é que tomando-se por base a malha de elementos adotada "a priori", a forma fraca, normalmente expressa por integrais sobre o domínio de interesse, passa a ser representada pela soma de integrais sobre o domínio de cada elemento.

Tal característica coloca em destaque os campos de aproximação no interior dos elementos e permite identificar um procedimento automático de montagem da matriz de rigidez e do vetor de forças equivalentes da estrutura a partir das contribuições dos elementos.

Na seqüência, apresenta-se o processo de obtenção da matriz de rigidez e do vetor de forças nodais para o caso tridimensional, utilizando-se o Princípio dos Trabalhos Virtuais (PTV). O sistema de referência é o cartesiano triortogonal com eixos $x, y$ e $z$. 
Seja o equilíbrio num corpo sujeito a forças de volume definido pela equação diferencial mostrada abaixo, válida em todos os pontos de seu domínio $\Omega$ :

$$
L^{T} \sigma+\bar{p}_{V}=0
$$

A representação matricial é a mais adequada para uma sistematização do processo de obtenção da matriz de rigidez e do vetor de forças nodais equivalentes.

Seja, então, $\{\sigma\}_{6 x 1}$ o vetor que agrupa as componentes do tensor de tensões $[T]_{3 \times 3}$, sendo estes dados por:

$$
\begin{aligned}
\sigma^{T} & =\left\{\begin{array}{llllll}
\sigma_{x} & \sigma_{y} & \sigma_{z} & \tau_{x y} & \tau_{x z} & \tau_{y z}
\end{array}\right\} \\
T & =\left[\begin{array}{lll}
\sigma_{x} & \tau_{x y} & \tau_{x z} \\
\tau_{x y} & \sigma_{y} & \tau_{y z} \\
\tau_{x z} & \tau_{y z} & \sigma_{z}
\end{array}\right]
\end{aligned}
$$

$L^{T}$ é um operador de divergência, de modo que vale a igualdade $L^{T} \sigma=\operatorname{div}(T)$.

O vetor $\left\{\bar{p}_{V}\right\}_{3 x 1}$ contém as componentes da força de volume:

$$
\bar{p}_{V}^{T}=\left\{\begin{array}{lll}
\bar{p}_{V x} & \bar{p}_{V y} & \bar{p}_{V z}
\end{array}\right\}
$$

Já o vetor $\{\bar{p}\}_{3 x 1}$ contém as componentes das forças distribuídas por unidade de superfície do sólido:

$$
\bar{p}^{T}=\left\{\begin{array}{lll}
\bar{p}_{x} & \bar{p}_{y} & \bar{p}_{z}
\end{array}\right\}
$$

Indica-se por $S_{p}$ a região do contorno do corpo sob efeito de forças de superfície.

O vetor $\{d\}_{3 x 1}$ reúne as componentes do campo de deslocamentos:

$d^{T}=\left\{\begin{array}{lll}u & v & w\end{array}\right\}$

O vetor $\{\varepsilon\}_{6 \times 1}$ contém as componentes do tensor de deformações, sendo dado por:

$$
\varepsilon^{T}=\left\{\begin{array}{llllll}
\varepsilon_{x} & \varepsilon_{y} & \varepsilon_{z} & \gamma_{x y} & \gamma_{x z} & \gamma_{y z}
\end{array}\right\}
$$

O tensor de deformações $[\varepsilon]_{3 \times 3}$ tem por expressão:

$$
\varepsilon=\left[\begin{array}{ccc}
\varepsilon_{x} & \varepsilon_{x y} & \varepsilon_{x z} \\
\varepsilon_{x y} & \varepsilon_{y} & \varepsilon_{y z} \\
\varepsilon_{x z} & \varepsilon_{y z} & \varepsilon_{z}
\end{array}\right]
$$

Valem as seguintes relações entre as componentes relativas à distorção:

$\gamma_{x y}=2 \varepsilon_{x y}, \gamma_{x z}=2 \varepsilon_{x z}$ e $\gamma_{y z}=2 \varepsilon_{y z}$. 
Entre os vetores de deformação e de deslocamento, dados, respectivamente, pelas eqs. (10) e (9), vale a relação:

$$
\varepsilon=L d
$$

sendo $L$ o operador diferencial definido pela matriz $[L]_{6 \times 3}$ dada em seguida:

$$
L=\left[\begin{array}{ccc}
\partial_{x} & 0 & 0 \\
0 & \partial_{y} & 0 \\
0 & 0 & \partial_{z} \\
\partial_{y} & \partial_{x} & 0 \\
\partial_{z} & 0 & \partial_{x} \\
0 & \partial_{z} & \partial_{y}
\end{array}\right]
$$

O Princípio dos Trabalhos Virtuais pode ser enunciado, segundo PILKEY \& WUNDERLICH (1994) por:

“Um sistema deformável está em equilíbrio se a soma do trabalho virtual interno com o trabalho virtual externo é nula para deslocamentos virtuais $\delta d$ que satisfazem as equações de compatibilidade e são homogêneos nas condições de contorno essenciais em deslocamento, ou seja, para os valores de $\delta d$ cinematicamente admissíveis".

Finalmente, o Princípio dos Trabalhos Virtuais escreve-se na forma:

$$
\int_{\Omega} \delta \varepsilon^{T} \sigma d \Omega+\left(-\int_{\Omega} \delta d^{T} \bar{p}_{V} d \Omega-\int_{S_{p}} \delta d^{T} \bar{p} d S\right)=0
$$

As integrais da eq. (14) são expressões de trabalho virtual, pois $\delta d$ é um vetor de deslocamentos virtuais e $\delta \varepsilon$ um vetor de deformações virtuais compatíveis com $\delta d$. A primeira integral da igualdade corresponde ao trabalho virtual interno, enquanto as demais ao trabalho virtual das forças externas.

Como se vê na eq. (14), o princípio é independente das propriedades do material. Além disso, está escrito tanto em função de deslocamentos (e suas derivadas, as deformações) como de tensões. Para que se possa expressar exclusivamente em função de deslocamentos, característica básica do chamado Método dos Deslocamentos do MEF, é preciso fazer uso da relação constitutiva (tensão-deformação), definida pela matriz $[D]_{6 \times 6}$ :

$$
\sigma=D \varepsilon
$$

Para material elástico, linear e isotrópico, a matriz $D$ tem por expressão: 


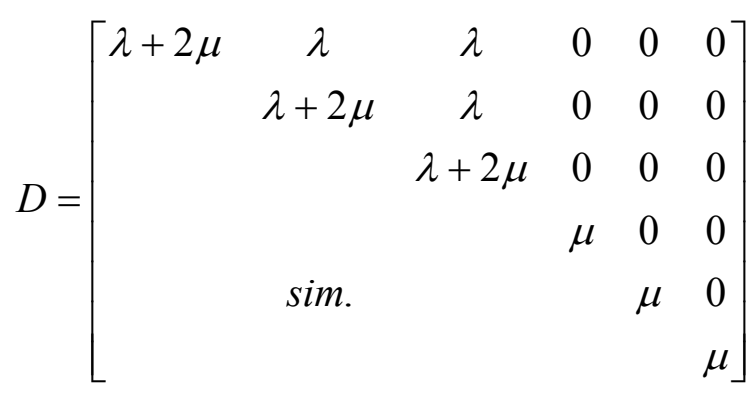

sendo $\lambda$ e $\mu$ as constantes de Lamé dadas por:

$$
\begin{aligned}
& \lambda=\frac{E v}{(1+v)(1-2 v)} \\
& \mu=\frac{E}{2(1+v)}
\end{aligned}
$$

Nas eqs. (17a) e (17b), E é módulo de elasticidade longitudinal e $v$ é o coeficiente de Poisson do material.

Aplicando-se a eq. (15) à eq. (14) e utilizando a eq. (12), escreve-se:

$$
\int_{\Omega} \delta d^{T}\left(L^{T} D L\right) d d \Omega=\int_{\Omega} \delta d^{T} \bar{p}_{V} d \Omega+\int_{S_{p}} \delta d^{T} \bar{p} d S
$$

A equação (18) implica na imposição da igualdade de trabalho interno e externo para todo o domínio $\Omega$. Como já ressaltado, no MEF essa igualdade é imposta a subdivisões do domínio (elementos finitos). Dentro dos limites de cada elemento, conforme ilustrado na fig. (1), pode-se interpretar que os deslocamentos e as forças aplicadas são aproximados em função dos valores nodais dessas grandezas.

Nos mesmos moldes da eq. (1), a aproximação para os deslocamentos pode ser indicada como se segue:

$$
d=\phi d_{e}
$$

sendo $[\phi]_{3 x n_{d}}$ a matriz de aproximação dos deslocamentos, que contém as funções interpoladoras, $\left\{d_{e}\right\}_{n_{d} x 1}$ o vetor de deslocamentos nodais de um elemento e $n_{d}$ o número de deslocamentos nodais do elemento.

A interpolação usada para o vetor de forças de volume é:

$$
\bar{p}_{V}=\phi^{p_{V}} \bar{p}_{e}^{V}
$$

sendo $\left[\phi^{p_{V}}\right\rfloor_{3 x n_{p v}}$ a matriz de interpolação das forças de volume, $\left\{\bar{p}_{e}^{V}\right\}_{n_{p v} x 1}$ o vetor de parâmetros nodais referentes às forças de volume de um dado elemento e $n_{p v}$ o número desses parâmetros.

Quanto às forças aplicadas à superfície, a aproximação é: 


$$
\bar{p}=\phi^{p} \bar{p}_{e}
$$

sendo $\left[\phi^{p}\right]_{3 x n_{p}}$ a matriz de interpolação das forças de superfície, $\left\{\bar{p}_{e}\right\}_{n_{p} x 1}$ o vetor de parâmetros nodais referentes às forças aplicadas à superfície de um dado elemento e $n_{p} \mathrm{o}$ número desses parâmetros.

Aplicando-se as aproximações definidas nas eqs. (19), (20) e (21) à eq. (18), chegase à expressão:

$$
\int_{\Omega_{e}} \delta d_{e}^{T}\left[(L \phi)^{T} D(L \phi)\right] d_{e} d \Omega=\int_{\Omega_{e}} \delta d_{e}^{T} \phi^{T} \phi^{p_{v}} \bar{p}_{e}^{V} d \Omega+\int_{S_{p e}} \delta d_{e}^{T} \phi^{T} \phi^{p} \bar{p}_{e} d S
$$

na qual $\Omega_{e}$ e $S_{p e}$ são, respectivamente, o domínio e a parte do contorno sujeita a cargas de superfície de cada elemento.

Como o vetor $\delta d_{e}{ }^{T}$ é formado por constantes em relação às integrais de volume e de área da eq. (22), o mesmo pode ser extraído dessas integrais e colocado em evidência. A equação resultante é utilizada para cálculo da matriz de rigidez e do vetor de forças nodais do elemento, pois pode ser expressa da seguinte forma:

$$
R_{e} d_{e}=f_{e}
$$

Na eq. (23), $\left[R_{e}\right]_{n_{d} x n_{d}}$ é a matriz de rigidez do elemento, dada por:

$$
R_{e}=\int_{\Omega_{e}} B^{T} D B d \Omega
$$

sendo que a matriz de aproximação das deformações $[B]_{6 x n_{d}}$ é dada por:

$$
B=L \phi
$$

e $\left\{f_{e}\right\}_{n_{d} x 1}$ é o vetor de forças nodais do elemento, dado por:

$$
f_{e}=\int_{\Omega_{e}} \phi^{T} \phi^{p_{V}} \bar{p}_{e}^{V} d \Omega+\int_{S_{p e}} \phi^{T} \phi^{p} \bar{p}_{e} d S
$$

Seja $N_{d}$ o número total de parâmetros de deslocamento (graus de liberdade) no domínio $\Omega$, quando da montagem do sistema global. Para todos os elementos em que a estrutura está dividida, calculam-se $R_{e}$ e $f_{e}$, compatibilizando-se seus valores, respectivamente, na matriz de rigidez $\left[R_{g}\right\rfloor_{N_{d} x N_{d}}$ e no vetor de forças nodais $\left\{f_{g}\right\}_{N_{d} x 1}$ globais, em correspondência aos graus de liberdade globais. Para isso, é necessário conhecer a correspondência que existe entre as posições dos deslocamentos no vetor $\left\{d_{e}\right\}$ do elemento e no vetor $\left\{d_{g}\right\}$ que reúne os graus de liberdade globais. 
Monta-se o sistema linear global, dado por:

$$
R_{g} d_{g}=f_{g}
$$

Impõem-se as condições de contorno estáticas, ou seja, impõem-se valores prescritos às posições correspondentes do vetor de deslocamentos globais $\left\{d_{g}\right\}$.

A resolução do sistema linear pode tirar partido do fato de que a matriz é simétrica (a aproximação do campo de deslocamentos virtuais é a mesma do campo real), positiva definida (devido ao fato da matriz $D$ ter essa característica) e esparsa (cada nó da estrutura conecta-se com um número limitado de outros nós). Uma vez calculado o vetor $\left\{d_{g}\right\}_{N_{d} x 1}$, podem-se obter os vetores $\left\{d_{e}\right\}_{n_{d} x 1}$ de cada elemento, novamente utilizando-se a correspondência entre a numeração local (do elemento) e global (de todo o corpo) de um dado deslocamento nodal.

Conhecidos os deslocamentos nodais de cada elemento, pode-se obter o campo de deformações, fazendo-se uso das eqs. (12) e (19). O campo de tensões é obtido na seqüência, utilizando-se a eq. (15).

\subsection{Os Métodos sem Malha}

\subsubsection{Revisão Bibliográfica}

A motivação principal para o desenvolvimento dos métodos sem malha foi superar alguns inconvenientes apresentados pelo Método dos Elementos Finitos (MEF), na sua formulação em deslocamentos. Segundo BELYTSCHKO et al (1994), entre esses inconvenientes podem ser citados:

- O custo computacional da geração da malha de elementos, fato ainda mais notável na análise de estruturas tridimensionais.

- O refinamento do tipo $\mathbf{h}$ numa região exige a construção de uma nova malha de elementos, ou a imposição de restrições cinemáticas aos novos nós.

- Quando se trata da simulação de grandes deformações, para evitar que a presença de elementos excessivamente distorcidos comprometa a qualidade da solução, pode ser necessária uma repetitiva e custosa alteração na malha.

- O método não favorece a resolução de problemas que envolvem propagação de fratura, visto que estas não podem ser simuladas no interior dos elementos e, portanto, a sua evolução também requer uma alteração contínua da malha, com grandes inconvenientes. 
Nos métodos sem malha, a aproximação é feita utilizando-se um conjunto de pontos dispersos no domínio, sem que a conectividade entre eles seja previamente definida (não se gera, portanto, uma malha de elementos).

Na maior parte dos métodos ditos sem malha, uma aproximação para a função incógnita é determinada pelo Método dos Mínimos Quadrados Móveis (MMQM), introduzido e estudado por LANCASTER \& SAULKAUSKAS (1981). Basicamente, a aproximação global num ponto do domínio é construída com base numa ponderação sobre valores conhecidos da função incógnita associados a nós que estejam numa vizinhança daquele ponto, definida por uma região de influência, ou nuvem.

Os passos iniciais para o desenvolvimento dos métodos sem malha foram dados por autores que trabalhavam com diferenças finitas (LISZKA \& ORKISZ (1980), PERRONE \& KAO (1975)), ao criarem esquemas para possibilitar análises com disposição irregular de nós.

Entretanto, considera-se usualmente o procedimento proposto por NAYROLES et al. (1992), denominado Método dos Elementos Difusos (MED), como referência pioneira dos métodos sem malha, tendo sido utilizado por esses autores essencialmente o Método dos Mínimos Quadrados Móveis na construção da aproximação. Naquele trabalho, a aplicação do método para a solução de um problema de valor de contorno segue a metodologia da abordagem variacional usual, isto é, introduz-se a aproximação (ou, quando necessário, suas derivadas) no funcional de energia correspondente, cuja condição de minimização leva a um sistema de equações lineares envolvendo as incógnitas do problema.

BELYTSCHKO et al (1994) introduzem algumas modificações, descritas a seguir, no método proposto por NAYROLES et al (1992) e denominam sua proposta de Método de Galerkin Livre de Elementos (MGLE). As principais alterações introduzidas são:

- Uso de células auxiliares de integração sobre o domínio. As células de integração não devem ser confundidas com uma malha de elementos finitos, sendo utilizadas apenas para facilitar a integração numérica.

- Consideração das derivadas completas da aproximação, levando-se em conta termos que foram omitidos por NAYROLES et al;

- Uso de multiplicadores de Lagrange para a imposição de condições de contorno essenciais, visto que a aproximação gerada pelo MMQM não se constitui numa interpolação e, portanto, valores prescritos no contorno não podem ser verificados de modo exato. 
OÑATE et al (1996) também deduzem a aproximação por mínimos quadrados móveis e propõem o seu uso na resolução de problemas de valor de contorno expressos em forma forte, isto é, mediante imposição da equação diferencial por colocação em pontos do domínio. O processo resultante é denominado pelos autores Método dos Pontos Finitos (MPF).

A partir dos trabalhos de DUARTE (1996) e DUARTE \& ODEN (1996), surge outro método sem malha: o Método das Nuvens hp. Sua principal característica é a possibilidade de enriquecer a aproximação polinomial original, obtida a partir do MMQM, sem acréscimo no número de pontos nodais, mas adicionando novos parâmetros a esses nós.

Esta característica representa uma grande vantagem em relação ao MGLE, pois, neste último, quando se deseja construir uma aproximação polinomial de grau mais elevado, faz-se necessário adicionar um número muito grande de pontos, o que pode elevar demais o custo computacional. No Método das Nuvens, pode-se optar por gerar uma aproximação inicial de ordem mais baixa, exigindo-se, assim, um pequeno número de nós contribuintes. $\mathrm{O}$ enriquecimento processa-se, então, multiplicando-se a aproximação local inicial por funções de enriquecimento, introduzindo-se novos parâmetros aos nós existentes. Tais parâmetros correspondem às constantes multiplicadoras das parcelas polinomiais enriquecedoras de grau mais elevado introduzidas.

A denominação hp do Método das Nuvens faz referência à grande maleabilidade do mesmo para combinar os enriquecimentos $\mathbf{h}$ (introduzindo-se novos pontos nodais no domínio) e p (introduzindo-se funções de grau superior com novos parâmetros nodais a elas associados).

Direta, ou indiretamente, todos os métodos sem malha citados envolvem o conceito de Partição da Unidade (PU), a ser descrito mais adiante, uma vez que as funções de forma geradas a partir do MMQM apresentam esta característica. No Método das Nuvens exigese explicitamente que o enriquecimento seja feito sobre funções que se constituam numa PU, o que irá garantir que qualquer função polinomial de grau igual ou inferior ao máximo grau das funções de enriquecimento possa ser exatamente reproduzida pela aproximação.

\subsubsection{Formulação geral dos Métodos sem Malha com base no MMQM.}

Como já mencionado, nos métodos sem malha a aproximação num certo ponto do domínio é construída com base nos valores da função em nós cujas áreas de influência 
incluem o ponto em questão. Ao contrário dos métodos “com malha”, o domínio não é subdividido em elementos.

O conjunto de nós escolhidos para discretizar o domínio $\Omega$ é indicado por $Q_{n_{\text {nos }}}=\left\{x_{1}, x_{2}, \ldots, x_{n_{\text {nos }}}\right\}, x_{j} \in \Omega$. Centrada em cada nó $x_{j}$, define-se uma região de influência $\omega_{j}$, também denominada nuvem nodal, formada pelos pontos do espaço no qual se situa o domínio, cuja distância ao nó é igual ou inferior a um dado raio $r_{j}$. De modo formal, escreve-se:

$$
\omega_{j}=\left\{x \in R^{n}:\left\|x-x_{j}\right\|_{R^{n}} \leq r_{j}\right\}
$$

$\mathrm{Na}$ expressão acima, $x \in R^{n}$ indica um ponto qualquer do espaço $R^{n}$. A distribuição de pontos e nuvens é tal que a união de todas as nuvens resultará na região $\chi_{n_{n o s}}$ que deverá conter o domínio $\Omega$ e seu contorno. Essa região é caracterizada como:

$$
\chi_{n_{n o s}}=\bigcup_{j=1}^{n_{n o s}} \omega_{j}, \quad \chi_{n_{n o s}} \supset \bar{\Omega}
$$

sendo que $\bar{\Omega}$ inclui o interior e o contorno da região $\Omega$.

A cada nuvem $\omega_{j}$ associa-se uma função ponderadora contínua $W_{j}$, de valor máximo sobre o nó $x_{j}$ e que decresce progressivamente com a distância ao mesmo até anular-se nos pontos limites da região. Como se verá, uma aproximação local atrelada ao nó $x_{j}$ e que venha a ser definida na nuvem $\omega_{j}$, mediante uma condição de mínimos quadrados, sofrerá a influência da função ponderadora, de modo que sua precisão num certo ponto da nuvem será tanto maior quanto mais próximo estiver o ponto do nó $x_{j}$.

Em geral, um ponto $x$ qualquer do domínio estará coberto por mais de uma nuvem nodal. Assim sendo, nesse ponto qualquer pode-se também associar uma nuvem, definida pela envoltória das nuvens nodais que o contém, como ilustrado na figura 2. Por conseqüência, seguindo o mesmo raciocínio anterior, também uma aproximação poderá ser construída para o ponto em questão, assumindo valores não-nulos dentro dos limites da sua nuvem. Neste caso, a aproximação no ponto será determinada pela combinação das funções de aproximação locais das nuvens nodais em que ele esteja incluído. Como se verá na seqüência, em função da base de funções utilizada na aproximação, a nuvem associada ao ponto deverá envolver um número mínimo de nuvens nodais.

A seguir, apresenta-se a construção, por um processo de mínimos quadrados móveis, da aproximação para uma função incógnita. Inicialmente, para cada ponto onde se 
deseja construir a aproximação $\widehat{u}(x)$, monta-se o funcional quadrático do erro da aproximação $(J)$, definido por:

$$
J(x)=\sum_{j=1}^{n_{n o s}} W_{j}\left(x_{j}-x\right)\left[u_{j}-\hat{u}(x)\right]^{2}
$$

$\mathrm{Na}$ eq. (28), o índice $j$ é o contador para todos os nós da discretização (em número $\left.n_{n o s}\right) ; W_{j}\left(x_{j}-x\right)$ é o valor que a função ponderadora com centro no nó $x_{j}$ assume no ponto $x$ e $u_{j}$ é o valor do parâmetro nodal associado a $x_{j}$.

Por outro lado, à função aproximadora global $\hat{u}(x)$ pode-se dar a seguinte representação geral:

$$
\hat{u}(x)=p^{T}(x) \alpha(x)
$$

na qual:

$$
\alpha^{T}(x)=\left\{\alpha_{1}(x), \alpha_{2}(x), \ldots, \alpha_{m}(x)\right\} \text { é o vetor de dimensão } m \text { dos multiplicadores da }
$$
combinação linear que define a aproximação associada ao ponto $x$.

$p^{T}(x)=\left\{p_{1}(x), p_{2}(x), \ldots, p_{m}(x)\right\}$ é o vetor que reúne a base de funções adotada (geralmente polinomial), também de dimensão $m$.

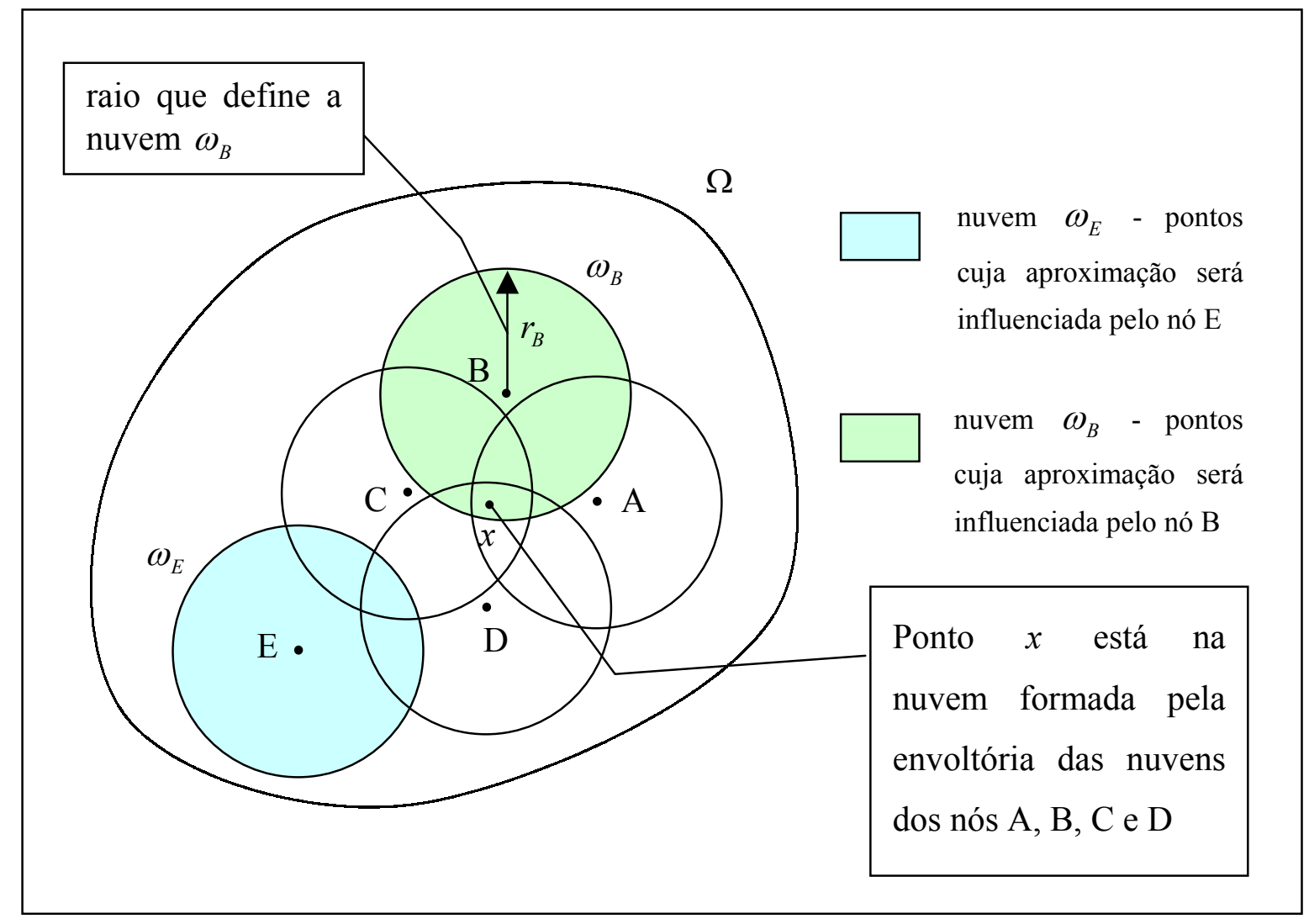

Figura 2 - Nuvens de influência num domínio $\Omega \in R^{2}$. 
Introduzindo-se a eq. (29) no funcional dado por (28), chega-se a:

$$
J(x)=\sum_{j=1}^{n_{n o s}} W_{j}\left(x_{j}-x\right)\left[u_{j}-p^{T}(x) \alpha(x)\right]^{2}
$$

As componentes do vetor $\alpha$ resultam da minimização do funcional $J$. Para realizar a minimização deste funcional, impõe-se, como condição necessária, a nulidade de sua derivada parcial em relação a cada coeficiente $\alpha_{i}, i=1, \ldots, m$ :

$$
\frac{\partial J(x)}{\partial \alpha_{i}}=\frac{\partial \sum_{j=1}^{n_{n o s}} W_{j}\left(x_{j}-x\right)\left[u_{j}-p^{T}(x) \alpha(x)\right]^{2}}{\partial \alpha_{i}}=0
$$

Resolvendo-se o sistema linear formado pelas $m$ equações obtidas da expressão (31), obtém-se a seguinte expressão para o cálculo do vetor $\alpha(x)$ :

$$
\alpha(x)=A^{-1}(x) \sum_{j=1}^{n_{\text {nos }}} B_{j}(x) u_{j}
$$

na qual a matriz $A(x)$ é dada por:

$$
A(x)=\sum_{j=1}^{n_{n o s}} W_{j}\left(x_{j}-x\right) p\left(x_{j}\right) p^{T}\left(x_{j}\right)
$$

e o vetor $B_{j}(x)$ por:

$$
B_{j}(x)=W_{j}\left(x_{j}-x\right) p\left(x_{j}\right)
$$

Substituindo-se a eq. (32) na eq. (29), obtém-se finalmente:

$$
\hat{u}(x)=\sum_{j=1}^{n_{\text {nos }}} \phi_{j}(x) u_{j}
$$

sendo as funções de forma $\phi_{j}(x)$ dadas por:

$$
\phi_{j}(x)=p^{T}(x) A^{-1}(x) B_{j}(x)
$$

Uma condição necessária (mas não suficiente) para que a matriz $A(x)$ dada por (33) seja inversível é que o número $n_{n o s}$ de nós que contribuem para a aproximação seja maior ou igual ao número $m$ de funções da base $p$.

A função ponderadora $W_{j}$ exerce um papel muito importante na geração da aproximação, especialmente quanto à sua continuidade, como se verá na seqüência. $\mathrm{Na}$ situação mais comum de nuvens com forma circular (no $R^{2}$ ) ou esférica (no $R^{3}$ ), ela deve ter valor máximo no nó ao qual está associada $\left(x_{j}\right)$ e decrescer até anular-se nos pontos 
cuja distância ao nó sejam iguais ao raio $\left(r_{j}\right)$ definido para aquela nuvem. Formalmente, escreve-se:

$$
W_{j}\left(x-x_{j}\right)=\left\{\begin{array}{cl}
f\left(x-x_{j}\right) \geq 0 & \forall\left\|x-x_{j}\right\|_{R^{n}} \leq r_{j} \\
0 & \forall\left\|x-x_{j}\right\|_{R^{n}}>r_{j}
\end{array}\right.
$$

Evidentemente, podem-se definir nuvens $\omega_{j}$ de geometria não-circular. De modo a englobar o caso geral, a condição anterior pode ser escrita na forma:

$$
W_{j}\left(x-x_{j}\right)=\left\{\begin{array}{cc}
f\left(x-x_{j}\right) \geq 0 & \forall x \in \omega_{j} \\
0 & \forall x \notin \omega_{j}
\end{array}\right.
$$

Funções como $W_{j}$, ou seja, que apresentam valor não-nulo apenas numa dada região do domínio, são denominadas funções com suporte compacto $\left(C_{0}\right)$. Se as derivadas de $W_{j}$ até uma ordem $q$ também exibirem essa propriedade, diz-se que $W_{j} \in C_{0}^{q}\left(\omega_{j}\right)$.

Uma característica básica associada às funções de forma $\phi_{j}$ obtidas pelo MMQM, considerando-se funções peso com suporte compacto, é que elas constituem uma partição de unidade (PU).

Formalmente, num ponto $x$ o conjunto de funções de forma $\phi_{j}(x)$, associadas a subdomínios $\omega_{j}$ que o contém, é uma partição de unidade se atende a quatro condições:

1) $\phi_{j}(x) \in C_{0}^{\infty}\left(\omega_{j}\right)$ : as funções de forma pertencem ao conjunto de funções (incluindo-se suas derivadas até a ordem infinita) para as quais a região $\omega_{j}$ é um suporte compacto. Isto significa que $\phi_{j}$ e suas derivadas assumem valores não-nulos apenas na região $\omega_{j}$.

2) $\sum_{j=1}^{n_{\text {nos }}} \phi_{j}(x)=1$ : esta condição é necessária para assegurar que as funções de forma sejam capazes de reproduzir de modo exato uma função constante. Por exemplo, admita-se que a função a ser aproximada seja $u(x)=c$, de modo que todos os valores nodais sejam $u_{j}=c$. A condição em questão é necessária pois:

$$
\hat{u}(x)=c=\sum_{j=1}^{n_{\text {nos }}} \phi_{j} u_{j}=\sum_{j=1}^{n_{\text {nos }}} \phi_{j} c \Rightarrow \sum_{j=1}^{n_{\text {nos }}} \phi_{j}=1
$$

3) $\phi_{j}(x) \geq 0$ em $\Omega$. 
4) Todo sub-conjunto compacto de $\Omega$ intersecta apenas um número finito de suportes $\omega_{j}$.

Deve-se notar que a condição (1) exige suporte compacto até ordem infinita nas derivadas, porém para fins práticos ela é comumente relaxada, de modo que as funções de suporte compacto até uma ordem finita de derivada são ainda consideradas uma PU.

Uma aproximação gerada a partir de funções de forma $\phi_{j}(x)$ que sejam uma PU apresenta duas características de grande importância:

- Se as funções de forma forem geradas por uma base $P$ tal que as derivadas até ordem $k$ das funções desta base são contínuas em $\omega_{j}$, isto é, $P \in C^{k}\left(\omega_{j}\right)$, e por funções ponderadoras $W \in C_{0}^{q}\left(\omega_{j}\right)$, ou seja, as derivadas até a ordem $q$ em $\omega_{j}$ têm suporte compacto, demonstra-se que $\phi_{j} \in C_{0}^{\min (k, q)}\left(\omega_{j}\right)$. Portanto, a aproximação no domínio terá suporte compacto até a ordem de derivada igual ao menor entre os valores de $k$ e $q$. Isto demonstra a importância da função peso na qualidade da aproximação, particularmente quanto à sua continuidade e suavidade.

- Qualquer função que seja uma combinação linear dos monômios da base $P$ pode ser exatamente reproduzida pela aproximação.

\section{O Método das Nuvens hp.}

No Método dos Mínimos Quadrados Móveis, quando se deseja criar uma aproximação do tipo polinomial de ordem mais elevada num ponto $x$ do domínio, é necessário assegurar a contribuição de um número considerável de nós na sua nuvem de influência, de modo a garantir que a matriz $A(x)$ seja inversível. O número de nós participantes deve ser ainda maior em domínios bi e tridimensionais. Como resultado, a matriz $A(x)$ poderá ter uma dimensão tal que torne o processo de inversão muito custoso computacionalmente, além de induzir a problemas de imprecisão numérica.

Para superar essa dificuldade, DUARTE \& ODEN (1996) propuseram gerar a partição de unidade a partir de uma base polinomial de ordem mais baixa e enriquecer essa aproximação, até a ordem polinomial desejada, sem um aumento do número de nós, apenas com o acréscimo de novos parâmetros aos nós já existentes.

Para ilustrar essa proposta, admita-se, para o caso unidimensional, que as funções de forma tenham sido geradas segundo o procedimento padrão (MMQM) a partir de uma base polinomial $P_{k}=\left\{1, x, x^{2}, \ldots, x^{k}\right\}$ e de funções ponderadoras $W \in C_{0}^{q}\left(\omega_{j}\right)$, tal que 
$q \geq k$. Portanto, qualquer polinômio de ordem menor ou igual a $k$ pode ser exatamente reproduzido pela aproximação gerada. Para elevar a ordem da aproximação até uma ordem $p$, tal que $p>k$, passando então a corresponder a uma nova base $P_{p}=\left\{1, x, x^{2}, \ldots, x^{k}, \ldots, x^{p}\right\}$, define-se uma família de funções formada pela soma das funções de forma originais com as funções resultantes do produto de cada uma das funções originais pelas componentes do conjunto $P_{p}-P_{k}=\left\{x^{k+1}, \ldots, x^{p}\right\}$. A família assim definida denomina-se família de funções de forma do Método das Nuvens hp e apresenta a representação seguinte:

$$
\mathfrak{J}_{n_{\text {nos }}}^{k, p}=\left\{\left\{\phi_{j}(x)\right\}_{j=1}^{n_{n o s}} \cup\left\{\phi_{j}(x) x^{i}\right\}_{j=1}^{n_{\text {nos }}}: i=k+1, \ldots, p\right\}
$$

A função aproximadora resultante terá então como expressão:

$$
\hat{u}(x)=\sum_{j=1}^{n_{\text {nos }}} \phi_{j}(x)\left[u_{j}+\sum_{i=k+1}^{p} x^{i} b_{j(i-k)}\right]
$$

Na expressão anterior, $b_{j(i-k)}$ são os parâmetros adicionados aos nós em função do enriquecimento realizado.

Ainda conforme os autores, quando se utilizam as funções aproximadoras da família do Método das Nuvens hp a taxa de convergência da resposta é dependente apenas da base final $P_{p}$, ou seja, a base original $P_{k}$ não tem influência sobre ela. Dessa forma, é usual escolher para $P_{k}$ a base mais simples possível, $P_{k=0}=\{1\}$, o que elimina a necessidade de inverter a matriz $A(x)$. Nessa condição, as expressões de $A(x), B_{j}(x)$ e das funções de forma $\phi_{j}(x)$ (denominadas, nesse caso, funções de Shepard) resultam em:

$$
\begin{aligned}
& A(x)=\sum_{j=1}^{n_{\text {nos }}} W_{j}\left(x_{j}-x\right)\{1\}\{1\} \Rightarrow A^{-1}(x)=\frac{1}{\sum_{j=1}^{n_{\text {nos }}} W_{j}\left(x_{j}-x\right)} \\
& B_{j}(x)=W_{j}\left(x_{j}-x\right)\{1\}=W_{j}\left(x_{j}-x\right) \\
& \phi_{j}(x)=\frac{W_{j}\left(x_{j}-x\right)}{\sum_{s=1}^{n_{\text {nos }}} W_{s}\left(x_{s}-x\right)}
\end{aligned}
$$

Embora o uso das funções de Shepard enriquecidas com funções polinomiais de ordem superior leve a um aumento do número de variáveis, comparando-se com o emprego de uma base polinomial inicial de mesma ordem, este fato é mais do que compensado pela redução do esforço computacional que seria despendido pela inversão da matriz $A(x)$. 


\subsection{Síntese do Método dos Elementos Finitos Generalizados}

\subsubsection{Revisão Bibliográfica}

Como já citado, há alguns trabalhos que foram precursores do que é hoje denominado Método dos Elementos Finitos Generalizados e são descritos a seguir.

O emprego de funções de forma típicas do MEF para evitar a inversão da matriz $A(x)$ foi proposto originalmente nos trabalhos de MELENK (1995) e MELENK \& BABUSKA (1996), dando origem ao chamado Método dos Elementos Finitos Partição da Unidade (MEFPU). Este método apresenta as seguintes características básicas:

- o método é apresentado como uma forma de obter boas condições de aproximação local e, além disso, assegurar a conformidade da solução global por meio do uso da partição da unidade. Enquanto no MEF convencional a escolha de um espaço de funções quaisquer (não necessariamente polinomiais) mas com boas qualidades aproximadoras locais pode levar a que não se consiga impor a conformidade, ou seja, a continuidade entre elementos, no MEFPU não há esse inconveniente, pois o espaço de aproximação gerado combina a partição da unidade (que garante a conformidade) com funções enriquecedoras que produzem uma boa aproximação local.

- além das funções de Shepard, podem-se adotar funções "chapéu" lineares e bilineares de elementos finitos uni ou bidimensionais como uma possível escolha para a partição da unidade. A nuvem, ou suporte da partição atrelada a um dado nó, é formada pelo conjunto de elementos para os quais o vértice comum é o nó em questão.

- há um paralelo entre as versões $\mathbf{h}$ e $\mathbf{p}$ do MEF e o MEFPU (se o espaço das funções enriquecedoras é polinomial). Se o espaço das aproximações locais é enriquecido e os suportes das funções partição de unidade são mantidos, tem-se uma situação equivalente ao refinamento $\mathbf{p}$ do MEF; por outro lado, se o espaço das aproximações locais não é enriquecido mas a área de influência de cada suporte é reduzida, tem-se um processo semelhante ao MEF com refinamento $\mathbf{h}$.

Em ODEN et al (1998), apresenta-se um método híbrido entre o Método das Nuvens hp e a forma convencional do Método dos Elementos Finitos. Segundo os autores, os trabalhos de MELENK \& BABUSKA (1996) e o Método das Nuvens de DUARTE \& ODEN (1996) levaram à idéia de adicionar refinamentos hierárquicos a um conjunto de funções de forma, como as interpolações lagrangianas usadas originalmente no MEF, as quais satisfazem os requisitos de partição da unidade (PU). Propuseram ainda empregar a própria malha de elementos finitos para a definição das nuvens e dos nós. Como principais 
vantagens do método proposto, os autores citam a fácil implementação das condições de contorno e a robustez do método mesmo sob forte distorção da malha (já que o enriquecimento é feito sobre as coordenadas após o mapeamento).

As principais características do Método dos Elementos Finitos Generalizados (MEFG) já estavam, portanto, bastante nítidas tanto no MEFPU quanto no método híbrido de ODEN et al (1998), ou seja: a utilização das funções de forma dos elementos finitos (que podem ser consideradas como uma partição de unidade, se houver um relaxamento dos critérios que a definem) e seu enriquecimento com funções polinomiais de grau superior, adicionando-se novos parâmetros aos nós. A região de influência de um certo nó, denominada nuvem ou suporte, é definida pelo conjunto de elementos finitos localizados no entorno do nó em questão.

Quando disponíveis, pode-se também realizar o enriquecimento escolhendo-se funções que representam a solução exata do problema, o que permite obter melhor taxa de convergência da resposta.

Portanto, no MEFG têm-se reunidas características positivas do MEF com recursos bastante vantajosos típicos dos métodos sem malha.

Entre as características positivas do MEF, destacam-se: a simplicidade na geração da partição de unidade devida ao uso da interpolação lagrangiana, o fato da malha dos elementos já servir como domínio para a integração numérica, o fato de ser um método robusto para o qual já existe uma grande base de códigos que podem ser adaptados ao MEFG e a facilidade na imposição das condições de contorno essenciais.

Os recursos típicos dos métodos sem malha são: a possibilidade de modelar a ocorrência de trincas ou regiões de maior concentração de tensões através da introdução de funções especiais, a maior facilidade de realizar o refinamento p (basta acrescentar novos parâmetros aos nós já existentes - no MEF é necessário criar nós nos lados ou mesmo no interior dos elementos) e a possibilidade de enriquecer a aproximação apenas numa região do domínio, sem comprometer, como no MEF convencional, a conformidade entre elementos.

Uma aplicação bastante interessante do MEFG, apresentada em DUARTE et al (2000) e STROUBOULIS et al (2000) e limitada ao campo linear, é a análise de estruturas tridimensionais complexas, com cantos reentrantes que se constituem em pontos de singularidade quanto à concentração de tensões. A discretização dessas regiões usualmente requer um número muito elevado de elementos para capturar os elevados gradientes de tensão; com o enriquecimento utilizando funções que representam a solução exata nos nós 
próximos aos cantos, podem-se utilizar malhas menos refinadas e reduzir significativamente o erro na aproximação.

Outro campo de aplicação do método, que vem sendo bastante explorado, é a análise de problemas de fratura. $\mathrm{O}$ uso do MEFG abre a possibilidade de utilizar funções enriquecedoras que simulem a descontinuidade no campo de deslocamentos dentro de um mesmo elemento, tornando desnecessária qualquer mudança na malha. Além disso, podese enriquecer a aproximação na região próxima à ponta da fratura (zona de processo) com funções que representam a solução analítica do problema, no caso de análise elásticalinear. Tal procedimento permite um ganho muito substancial na qualidade da resposta, quando comparada à aproximação de base polinomial usualmente empregada no Método dos Elementos Finitos.

Entre os trabalhos que utilizam a metodologia acima descrita podem-se citar DOLBOW et al (2000), no qual se realizam análises em elasticidade bidimensional e em placas de Reissner-Mindlin, e BELYTSCHKO \& MÖES (2002), no qual se analisa a evolução da fratura em vigas de concreto, sendo que neste último os autores denominam o processo empregado Método dos Elementos Finitos Extendidos.

Em WELLS \& SLUYS (2001), também se utiliza o enriquecimento da partição de unidade dos elementos finitos para simular a evolução da fratura, utilizando um modelo de fratura coesiva. Os autores ressaltam a habilidade do modelo em permitir a propagação da fratura independentemente da estrutura da malha, a insensibilidade dos resultados ao tamanho do elemento e a possibilidade de modelar uma fratura curva. A eficiência computacional é assegurada pelo fato de que apenas os nós próximos à fratura devem ser enriquecidos, de modo que a dimensão global do sistema não é aumentada de modo muito significativo.

Uma aplicação do MEFG à análise de estruturas de concreto sob efeito de dano encontra-se em BARROS (2002). Em sua tese, o autor também desenvolve um estimador de erro aplicável ao MEFG, tanto para análises lineares como não-lineares.

\subsubsection{Formulação geral}

Enquanto no Método das Nuvens hp a região de influência $\left(\omega_{j}\right)$ de um nó é definida como uma região circular (ou poligonal) em torno daquele nó, no MEFG, aproveitando-se da malha de elementos, a região de influência (nuvem nodal) será o conjunto de elementos que têm aquele nó como vértice, como ilustra a figura 3. 
Em cada nuvem, a partição da unidade (PU) fica determinada pelo conjunto das funções de forma dos elementos finitos que compõem a nuvem e associadas ao nó base. Com isso, a continuidade entre elementos será da mesma ordem das funções de forma adotadas. Por sua vez, o enriquecimento da aproximação em cada nuvem é realizado pela multiplicação da PU associada a cada nó base por funções de interesse, polinomiais ou especiais, com vistas a definir uma melhor aproximação local.

Portanto, também no MEFG define-se uma família de funções atreladas aos nós:

$$
\mathfrak{I}_{n}^{p}=\left\{\phi_{j} \cup \phi_{j} f_{j}^{\alpha}, j=1, \ldots, n ; \alpha=1, \ldots, I(j)\right\}
$$

na qual:

$n$ é o número total de nós.

$p$ é o grau polinomial da família de funções.

$\phi_{j}$ são as funções de forma (PU) referentes aos nós $j=1, \ldots, n$.

$f_{j}^{\alpha}$ é a função de índice $\alpha$ que multiplica (ou enriquece) a função de forma atrelada a cada nó de índice $j$.

$I(j)$ é o contador para o número de funções adicionadas a cada nó de índice $j$.

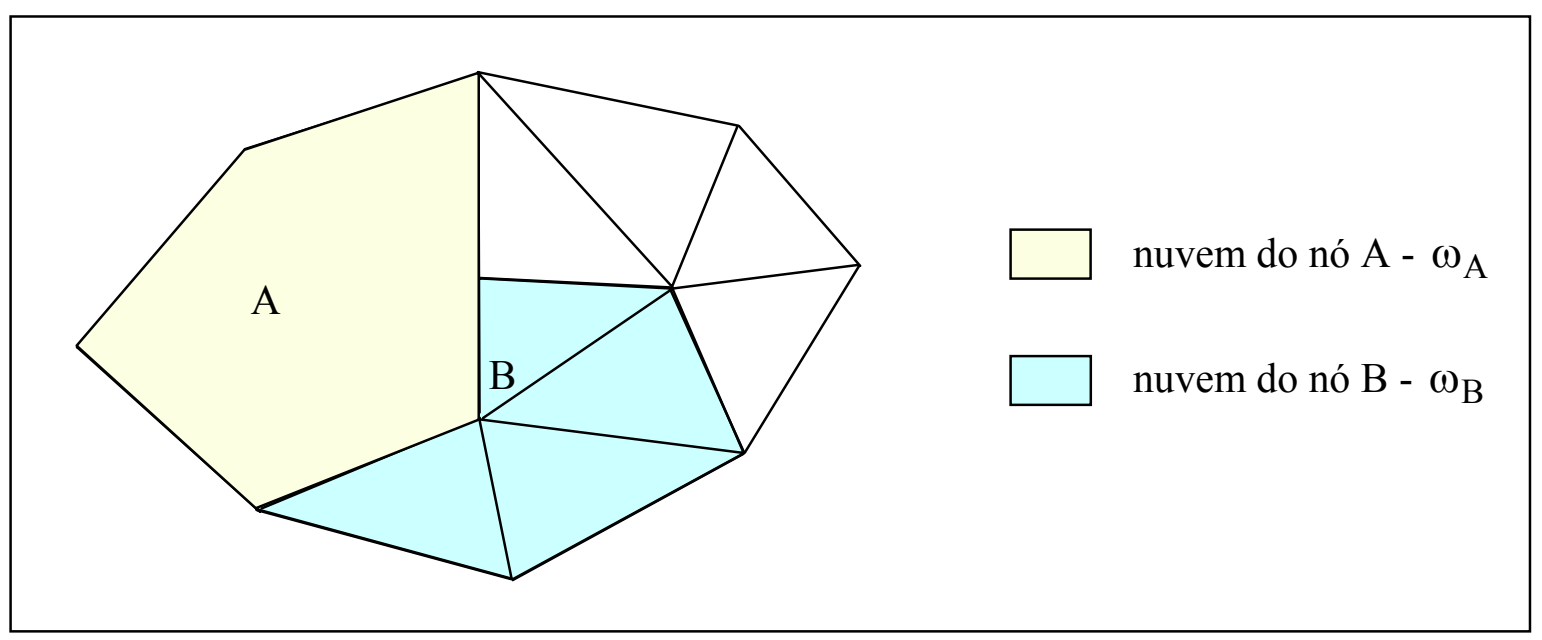

Figura 3 - Nuvens de influência no MEFG (caso bidimensional)

Nota-se, porém, que quando as funções de forma (partição de unidade) dos elementos finitos são polinomiais (o mais usual) e as funções enriquecedoras da aproximação também o são, há uma grande probabilidade de que as funções da família gerada sejam linearmente dependentes. A demonstração da dependência linear num elemento tetraédrico com funções de forma cuja base é linear e as funções enriquecedoras também o são é realizada no Apêndice A. O uso de uma família de funções linearmente 
dependentes na aproximação dos deslocamentos faz com que as matrizes de rigidez dos elementos também o sejam, assim como a matriz de rigidez global.

Pode-se evitar a ocorrência de dependência linear na família de funções enriquecidas escolhendo-se funções PU que não sejam polinomiais. Caso isso não seja possível, ou mesmo desejável, há duas abordagens possíveis para resolver um sistema linear $K u=f$, onde $K$ é uma matriz positiva semi-definida:

- Usar um algoritmo direto para sistemas simétricos indefinidos, como o de DUFF \& REID (1983).

- Usar um algoritmo iterativo, como sugerido por STROUBOULIS et al (2000).

Segundo DUARTE et al (2000), para uma mesma malha e uma mesma ordem de aproximação, as matrizes de rigidez geradas quando se utiliza o MEFG apresentam um grau de esparsidade menor do que aquelas geradas pelo MEF convencional, ou seja, apresentam uma maior proporção de termos não-nulos. Entretanto, a menor dimensão das matrizes do MEFG comparada às correspondentes matrizes do MEF compensa a desvantagem do maior número de termos não-nulos, fazendo com que o tempo de solução do sistema seja menor quando se utiliza o MEFG.

\subsubsection{Formulação de elementos tridimensionais no MEFG}

No presente trabalho foi desenvolvida a formulação de dois dos principais tipos de elementos tridimensionais: o tetraedro e o hexaedro. A formulação do tetraedro é apresentada no item 2.3.3.1 e a do hexaedro, no item 2.3.3.2. Para cada um desses elementos, apresenta-se inicialmente a formulação considerando a aproximação linear para os deslocamentos e, posteriormente, a resultante do enriquecimento da mesma. As forças de volume foram desconsideradas na presente formulação.

\subsubsection{Formulação do tetraedro.}

A figura 4 apresenta a numeração adotada para os nós e as faces do tetraedro. As funções de forma adotadas são as mesmas que representam as coordenadas adimensionais de volume $\xi_{i}, i=1, \ldots, 4$, também chamadas coordenadas naturais. Elas são utilizadas para aproximar tanto a geometria quanto o campo de deslocamentos, o que significa que os elementos desenvolvidos são isoparamétricos. A dedução da expressão das coordenadas adimensionais é feita no Apêndice $\mathrm{B}$, sendo sua forma geral a seguinte, sendo $x, y$ e $z$ as 
coordenadas de um ponto qualquer do elemento segundo um sistema cartesiano triortogonal:

$$
\xi_{i}=V_{0}^{i}+a_{i} x+b_{i} y+c_{i} z, \quad i=1, \ldots, 4
$$

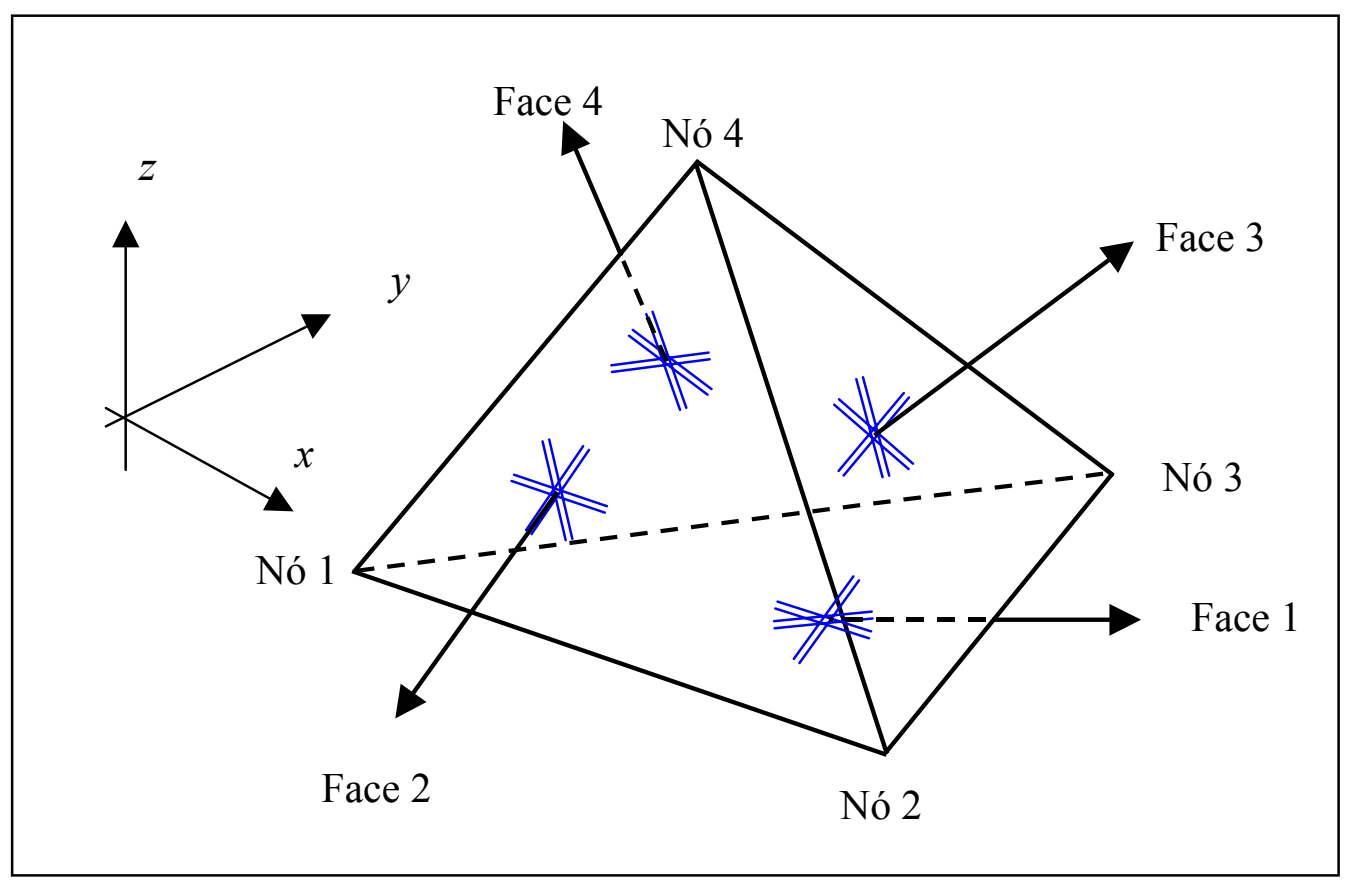

Figura 4 - Numeração dos nós e das faces do tetraedro.

Os termos $V_{0}^{i}, a_{i}, b_{i}$ e $c_{i}$ da equação (45) são dados pelas expressões seguintes, fazendo-se uma permutação cíclica dos índices $i, j, k$ e $l$, correspondentes aos números $1,2,3$ e 4 .

$$
\begin{aligned}
& V_{0}^{i}=\frac{1}{6 V}\left|\begin{array}{lll}
x_{j} & x_{k} & x_{l} \\
y_{j} & y_{k} & y_{l} \\
z_{j} & z_{k} & z_{l}
\end{array}\right| \\
& a_{i}=\frac{-1}{6 V}\left|\begin{array}{lll}
1 & 1 & 1 \\
y_{j} & y_{k} & y_{l} \\
z_{j} & z_{k} & z_{l}
\end{array}\right| \\
& b_{i}=\frac{1}{6 V}\left|\begin{array}{lll}
1 & 1 & 1 \\
x_{j} & x_{k} & x_{l} \\
z_{j} & z_{k} & z_{l}
\end{array}\right| \\
& c_{i}=\frac{-1}{6 V}\left|\begin{array}{ccc}
1 & 1 & 1 \\
x_{j} & x_{k} & x_{l} \\
y_{j} & y_{k} & y_{l}
\end{array}\right|
\end{aligned}
$$


Nas eqs. (46), $x_{i}, y_{i}$ e $z_{i}$, para $i=1, \ldots, 4$, são as coordenadas dos nós do tetraedro e $6 V$ corresponde a seis vezes o volume desse elemento, o que se obtém calculando o determinante dado a seguir:

$$
6 V=\left|\begin{array}{cccc}
1 & 1 & 1 & 1 \\
x_{1} & x_{2} & x_{3} & x_{4} \\
y_{1} & y_{2} & y_{3} & y_{4} \\
z_{1} & z_{2} & z_{3} & z_{4}
\end{array}\right|
$$

\subsection{Tetraedro sem enriquecimento}

\section{Aproximação da geometria:}

Sendo $x_{i}, y_{i}$ e $z_{i}$ as coordenadas cartesianas dos nós e $\xi_{i}$ as coordenadas adimensionais de volume, com $i=1, \ldots, 4$, as coordenadas de um ponto qualquer do tetraedro são dadas por:

$$
\begin{aligned}
& x=\sum_{i=1}^{4} x_{i} \xi_{i} \\
& y=\sum_{i=1}^{4} y_{i} \xi_{i} \\
& z=\sum_{i=1}^{4} z_{i} \xi_{i}
\end{aligned}
$$

Aproximação dos deslocamentos no interior do elemento:

Os deslocamentos $u, v$ e $w$ (nas direções $x, y$ e $z$ de um sistema de eixos ortogonais, respectivamente), que formam o vetor $\{d\}^{T}=\left\{\begin{array}{lll}u & v & w\end{array}\right\}$, são aproximados no interior do elemento por:

$$
\begin{gathered}
u=\sum_{i=1}^{4} u_{i} \xi_{i} \\
v=\sum_{i=1}^{4} v_{i} \xi_{i} \\
w=\sum_{i=1}^{4} w_{i} \xi_{i}
\end{gathered}
$$

Os parâmetros nodais $u_{i}, v_{i}$ e $w_{i}(i=1, \ldots, 4)$ constituem o vetor de deslocamentos nodais do elemento $\left\{d_{e}\right\}_{12 x 1}$, que é dado abaixo: 
$d_{e}^{T}=\left\{\begin{array}{llllllllllll}u_{1} & v_{1} & w_{1} & u_{2} & v_{2} & w_{2} & u_{3} & v_{3} & w_{3} & u_{4} & v_{4} & w_{4}\end{array}\right\}$

A matriz da aproximação do campo de deslocamentos $[\phi]_{3 \times 12}$ é, portanto, a seguinte:

$$
\phi=\left[\begin{array}{cccccccccccc}
\xi_{1} & 0 & 0 & \xi_{2} & 0 & 0 & \xi_{3} & 0 & 0 & \xi_{4} & 0 & 0 \\
0 & \xi_{1} & 0 & 0 & \xi_{2} & 0 & 0 & \xi_{3} & 0 & 0 & \xi_{4} & 0 \\
0 & 0 & \xi_{1} & 0 & 0 & \xi_{2} & 0 & 0 & \xi_{3} & 0 & 0 & \xi_{4}
\end{array}\right]
$$

A matriz $[B]_{6 \times 12}$, que reúne as derivadas parciais das funções de forma, é dada por:

$$
B=\left[\begin{array}{cccccccccccc}
a_{1} & 0 & 0 & a_{2} & 0 & 0 & a_{3} & 0 & 0 & a_{4} & 0 & 0 \\
0 & b_{1} & 0 & 0 & b_{2} & 0 & 0 & b_{3} & 0 & 0 & b_{4} & 0 \\
0 & 0 & c_{1} & 0 & 0 & c_{2} & 0 & 0 & c_{3} & 0 & 0 & c_{4} \\
b_{1} & a_{1} & 0 & b_{2} & a_{2} & 0 & b_{3} & a_{3} & 0 & b_{4} & a_{4} & 0 \\
c_{1} & 0 & a_{1} & c_{2} & 0 & a_{2} & c_{3} & 0 & a_{3} & c_{4} & 0 & a_{4} \\
0 & c_{1} & b_{1} & 0 & c_{2} & b_{2} & 0 & c_{3} & b_{3} & 0 & c_{4} & b_{4}
\end{array}\right]
$$

Como se pode ver na eq. (45), os termos $a_{i}, b_{i}$ e $c_{i}$ correspondem às derivadas das funções de forma $\left(\xi_{i}\right)$ em relação às coordenadas cartesianas $x, y$ e $z$, respectivamente:

$$
\begin{aligned}
& a_{i}=\frac{\partial \xi_{i}}{\partial x}, \quad i=1, \ldots, 4 \\
& b_{i}=\frac{\partial \xi_{i}}{\partial y}, \quad i=1, \ldots, 4 \\
& c_{i}=\frac{\partial \xi_{i}}{\partial z}, \quad i=1, \ldots, 4
\end{aligned}
$$

Como se observa pelas eqs. (46b), (46c) e (46d), esses termos são constantes no domínio do elemento. Desse modo a matriz $[\mathrm{B}]_{6 \times 12}$ é constante e a expressão de cálculo da matriz de rigidez do elemento, dada pela eq. (24), pode ser escrita dispensando-se a integração:

$$
\left[R_{e}\right]_{12 \times 12}=[B]^{T} D[B] V
$$

A matriz de rigidez calculada pela eq. (54) é formada por um conjunto de 16 submatrizes (correspondentes às combinações de $i=1, \ldots, 4$ e $j=1, \ldots, 4$ ), de dimensão $3 \times 3$, como ilustrado na figura 5 .

Os termos de uma submatriz $3 \times 3$ genérica têm por expressão:

$$
A(3 i-2,3 j-2)=\left(a_{i} a_{j} c t_{1}+b_{i} b_{j} c t_{3}+c_{i} c_{j} c t_{3}\right) V
$$




$$
\begin{aligned}
& A(3 i-2,3 j-1)=\left(a_{i} b_{j} c t_{2}+b_{i} a_{j} c t_{3}\right) V \\
& A(3 i-2,3 j)=\left(a_{i} c_{j} c t_{2}+c_{i} a_{j} c t_{3}\right) V \\
& A(3 i-1,3 j-2)=\left(b_{i} a_{j} c t_{2}+a_{i} b_{j} c t_{3}\right) V \\
& A(3 i-1,3 j-1)=\left(a_{i} a_{j} c t_{3}+b_{i} b_{j} c t_{1}+c_{i} c_{j} c t_{3}\right) V \\
& A(3 i-1,3 j)=\left(b_{i} c_{j} c t_{2}+c_{i} b_{j} c t_{3}\right) V \\
& A(3 i, 3 j-2)=\left(c_{i} a_{j} c t_{2}+a_{i} c_{j} c t_{3}\right) V \\
& A(3 i, 3 j-1)=\left(c_{i} b_{j} c t_{2}+b_{i} c_{j} c t_{3}\right) V \\
& A(3 i, 3 j)=\left(a_{i} a_{j} c t_{3}+b_{i} b_{j} c t_{3}+c_{i} c_{j} c t_{1}\right) V
\end{aligned}
$$

sendo $c t_{1}, c t_{2}$ e $c t_{3}$ dados por:

$$
\begin{aligned}
& c t_{1}=(\lambda+2 \mu)=\frac{E(1-v)}{(1+v)(1-2 v)} \\
& c t_{2}=\lambda=\frac{E v}{(1+v)(1-2 v)} \\
& c t_{3}=\mu=\frac{E}{2(1+v)}
\end{aligned}
$$

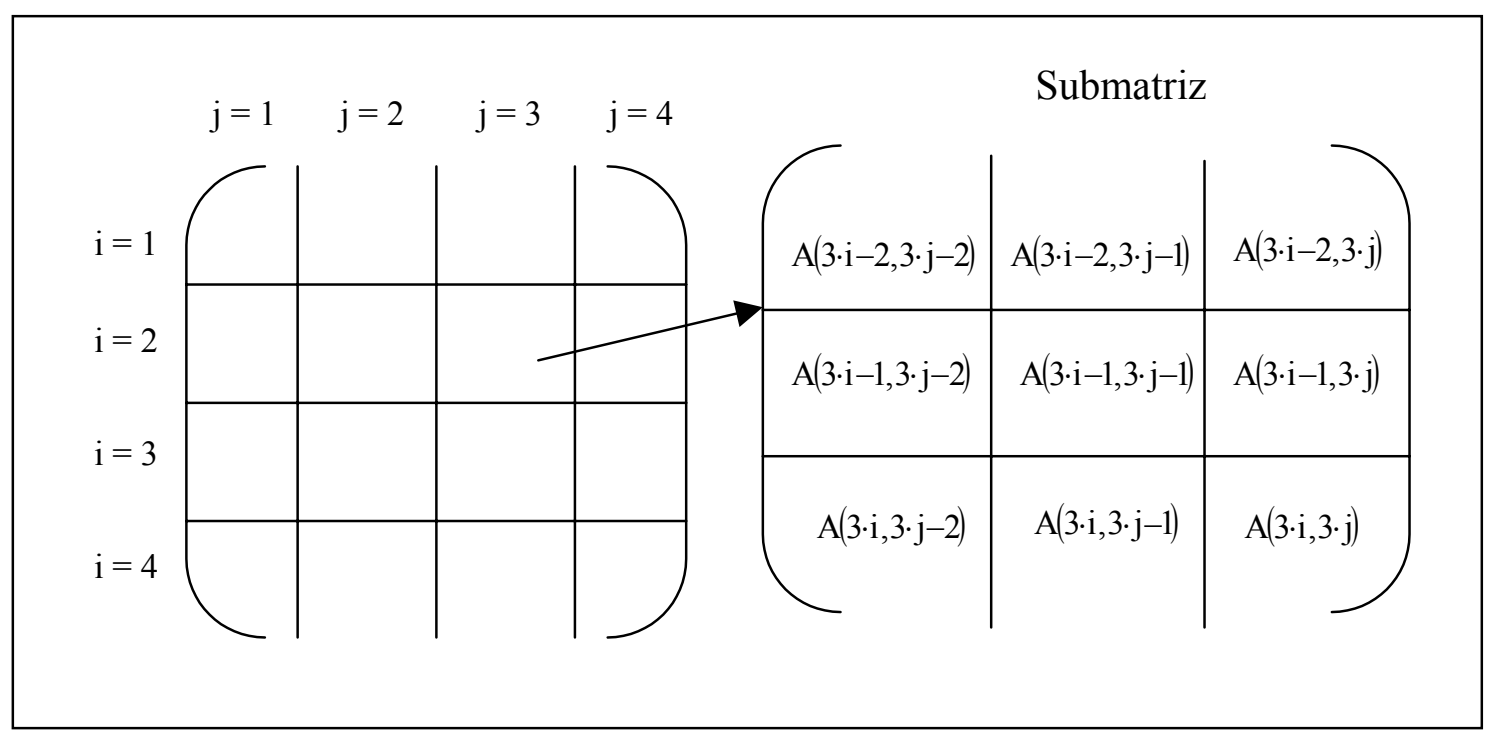

Figura 5 - Estrutura da matriz de rigidez do elemento tetraédrico.

Aproximação dos deslocamentos e do carregamento na superfície:

A aproximação, na superfície carregada (correspondente a um triângulo), dos deslocamentos e das forças distribuídas aplicadas será feita com base em coordenadas 
adimensionais de área $(\zeta)$. A dedução da expressão das coordenadas adimensionais de área é feita no Apêndice $C$, sendo sua forma final:

$$
\zeta_{i}=a_{i}^{0}+\omega_{i} r+\eta_{i} s, \quad(i=1, \ldots, 3)
$$

Na eq. (57), $r$ e $s$ são as coordenadas dos pontos da face do tetraedro segundo um sistema cartesiano ortogonal, de localização arbitrária no plano da face.

Os demais termos da eq. (57) são dados a seguir, fazendo-se uma permutação cíclica dos índices $i, j$ e $k$, correspondentes aos números 1,2 e 3.

$$
\begin{aligned}
& a_{i}^{0}=\left(r_{j} s_{k}-r_{k} s_{j}\right) / 2 A \\
& \omega_{i}=\left(s_{j}-s_{k}\right) / 2 A \\
& \eta_{i}=\left(r_{k}-r_{j}\right) / 2 A
\end{aligned}
$$

sendo $2 A$ o dobro da área do triângulo, calculado pelo determinante:

$$
2 A=\left|\begin{array}{lll}
1 & 1 & 1 \\
r_{1} & r_{2} & r_{3} \\
S_{1} & S_{2} & S_{3}
\end{array}\right|
$$

Existe a necessidade de se definir quatro aproximações para os deslocamentos $(u$, $v$ e $w)$ e os carregamentos $\left(p_{x}, p_{y}\right.$ e $\left.p_{z}\right)$, correspondentes a cada uma das quatro faces. Para cada face, apresentam-se a seguir a matriz de aproximação dos deslocamentos $[\phi]_{3 \times 12}$ e do carregamento $[\phi]_{3 \times 12}^{p}$, que são iguais, e o vetor de parâmetros nodais de carregamento do elemento $\left\{\bar{p}_{e}\right\}_{12 x 1}$. A contribuição do carregamento de cada face ao vetor de forças nodais do elemento é calculada pela eq. (26), desconsiderando-se a parcela referente às forças de volume.

Face 1:

$$
\begin{aligned}
& \phi=\phi^{p}=\left[\begin{array}{cccccccccccc}
\zeta_{1} & 0 & 0 & \zeta_{2} & 0 & 0 & \zeta_{3} & 0 & 0 & 0 & 0 & 0 \\
0 & \zeta_{1} & 0 & 0 & \zeta_{2} & 0 & 0 & \zeta_{3} & 0 & 0 & 0 & 0 \\
0 & 0 & \zeta_{1} & 0 & 0 & \zeta_{2} & 0 & 0 & \zeta_{3} & 0 & 0 & 0
\end{array}\right] \\
& \left\{\bar{p}_{e}\right\}_{\text {face } 1}^{T}=\left\{\begin{array}{llllllllllll}
\bar{p} x_{1} & \bar{p} y_{1} & \bar{p} z_{1} & \bar{p} x_{2} & \bar{p} y_{2} & \bar{p} z_{2} & \bar{p} x_{3} & \bar{p} y_{3} & \bar{p} z_{3} & 0 & 0 & 0
\end{array}\right.
\end{aligned}
$$

Face 2:

$$
\phi=\phi^{p}=\left[\begin{array}{cccccccccccc}
\zeta_{1} & 0 & 0 & \zeta_{2} & 0 & 0 & 0 & 0 & 0 & \zeta_{3} & 0 & 0 \\
0 & \zeta_{1} & 0 & 0 & \zeta_{2} & 0 & 0 & 0 & 0 & 0 & \zeta_{3} & 0 \\
0 & 0 & \zeta_{1} & 0 & 0 & \zeta_{2} & 0 & 0 & 0 & 0 & 0 & \zeta_{3}
\end{array}\right]
$$


$\left\{\bar{p}_{e}\right\}_{\text {face } 2}^{T}=\left\{\begin{array}{llllllllllll}\bar{p} x_{1} & \bar{p} y_{1} & \bar{p} z_{1} & \bar{p} x_{2} & \bar{p} y_{2} & \bar{p} z_{2} & 0 & 0 & 0 & \bar{p} x_{4} & \bar{p} y_{4} & \bar{p} z_{4}\end{array}\right\}$

Face 3:

$$
\begin{aligned}
\phi=\phi^{p} & =\left[\begin{array}{cccccccccccc}
0 & 0 & 0 & \zeta_{1} & 0 & 0 & \zeta_{2} & 0 & 0 & \zeta_{3} & 0 & 0 \\
0 & 0 & 0 & 0 & \zeta_{1} & 0 & 0 & \zeta_{2} & 0 & 0 & \zeta_{3} & 0 \\
0 & 0 & 0 & 0 & 0 & \zeta_{1} & 0 & 0 & \zeta_{2} & 0 & 0 & \zeta_{3}
\end{array}\right] \\
\left\{\bar{p}_{e}\right\}_{\text {face } 3}^{T} & =\left\{\begin{array}{llllllllllll}
0 & 0 & 0 & \bar{p} x_{2} & \bar{p} y_{2} & \bar{p} z_{2} & \bar{p} x_{3} & \bar{p} y_{3} & \bar{p} z_{3} & \bar{p} x_{4} & \bar{p} y_{4} & \bar{p} z_{4}
\end{array}\right\}
\end{aligned}
$$

Face 4:

$$
\begin{aligned}
& \phi=\phi^{p}=\left[\begin{array}{cccccccccccc}
\zeta_{1} & 0 & 0 & 0 & 0 & 0 & \zeta_{2} & 0 & 0 & \zeta_{3} & 0 & 0 \\
0 & \zeta_{1} & 0 & 0 & 0 & 0 & 0 & \zeta_{2} & 0 & 0 & \zeta_{3} & 0 \\
0 & 0 & \zeta_{1} & 0 & 0 & 0 & 0 & 0 & \zeta_{2} & 0 & 0 & \zeta_{3}
\end{array}\right] \\
& \left\{\bar{p}_{e}\right\}_{\text {face } 4}^{T}=\left\{\begin{array}{llllllllllll}
\bar{p} x_{1} & \bar{p} y_{1} & \bar{p} z_{1} & 0 & 0 & 0 & \bar{p} x_{3} & \bar{p} y_{3} & \bar{p} z_{3} & \bar{p} x_{4} & \bar{p} y_{4} & \bar{p} z_{4}
\end{array}\right\}
\end{aligned}
$$

O cálculo e a expressão final do vetor de forças nodais do elemento são apresentados no Apêndice D.

\subsection{Tetraedro com enriquecimento}

\section{Aproximação da geometria:}

A interpolação das coordenadas dentro do elemento é feita da mesma forma que para o elemento não-enriquecido, sendo dada, portanto, pelas eqs. (48).

Aproximação dos deslocamentos no interior do elemento:

A aproximação para o campo de deslocamentos é enriquecida pela adição aos nós de parâmetros associados a funções de enriquecimento que multiplicam as funções de forma básicas (PU), como mostrado abaixo:

$$
\begin{aligned}
& u=\xi_{1}\left(u_{1}+\sum_{j=1}^{n f\left(u_{1}\right)} u_{1}^{j} f_{u_{1}}^{j}\right)+\xi_{2}\left(u_{2}+\sum_{j=1}^{n f\left(u_{2}\right)} u_{2}^{j} f_{u_{2}}^{j}\right)+\xi_{3}\left(u_{3}+\sum_{j=1}^{n f\left(u_{3}\right)} u_{3}^{j} f_{u_{3}}^{j}\right)+\xi_{4}\left(u_{4}+\sum_{j=1}^{n f\left(u_{4}\right)} u_{4}^{j} f_{u_{4}}^{j}\right) \\
& v=\xi_{1}\left(v_{1}+\sum_{j=1}^{n f\left(v_{1}\right)} v_{1}^{j} f_{v_{1}}^{j}\right)+\xi_{2}\left(v_{2}+\sum_{j=1}^{n f\left(v_{2}\right)} v_{2}^{j} f_{v_{2}}^{j}\right)+\xi_{3}\left(v_{3}+\sum_{j=1}^{n f\left(v_{3}\right)} v_{3}^{j} f_{v_{3}}^{j}\right)+\xi_{4}\left(v_{4}+\sum_{j=1}^{n f\left(v_{4}\right)} v_{4}^{j} f_{v_{4}}^{j}\right) \\
& w=\xi_{1}\left(w_{1}+\sum_{j=1}^{n f\left(w_{1}\right)} w_{1}^{j} f_{u_{1}}^{j}\right)+\xi_{2}\left(w_{2}+\sum_{j=1}^{n f\left(w_{2}\right)} w_{2}^{j} f_{w_{2}}^{j}\right)+\xi_{3}\left(w_{3}+\sum_{j=1}^{n f\left(w_{3}\right)} w_{3}^{j} f_{w_{3}}^{j}\right)+\xi_{4}\left(w_{4}+\sum_{j=1}^{n f\left(w_{4}\right)} w_{4}^{j} f_{w_{4}}^{j}\right)
\end{aligned}
$$

Admitindo-se que $d$ se refira genericamente às deslocabilidades $u, v$ ou $w$ e $i$ aos nós do elemento $(i=1, \ldots, 4)$, são válidas as seguintes convenções nas eqs. (64): 
$n f\left(d_{i}\right)$ : número de funções enriquecedoras associadas ao deslocamento $d$ do nó $i$. $d_{i}^{j}$ : parâmetro adicional de índice $j$, associado ao deslocamento $d$ do nó $i$. $f_{d_{i}}^{j}$ : função enriquecedora de índice $j$, associada ao deslocamento $d$ do nó $i$.

Sendo o número total de parâmetros adicionais $\left(d_{i}^{j}\right)$ associados aos deslocamentos dos nós $\left(d_{i}\right)$ que definem um certo elemento indicado por npar, o vetor de deslocamentos nodais do elemento $\left\{d_{e}\right\}_{(12+n p a r) x 1}$ tem a seguinte estrutura:

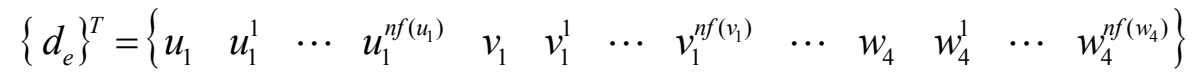

A matriz de aproximação dos deslocamentos $[\phi]_{3 x(12+n p a r)}$ tem seu trecho relativo ao nó $i, i=1, \ldots, 4$, apresentado abaixo:

$$
\phi=\left[\begin{array}{cccccccccccccc} 
& \xi_{i} & \xi_{i} f_{u_{i}}^{1} & \cdots & \xi_{i} f_{u_{i}}^{n f\left(u_{i}\right)} & 0 & 0 & \cdots & 0 & 0 & 0 & \cdots & 0 & \\
\cdots & 0 & 0 & \cdots & 0 & \xi_{i} & \xi_{i} f_{v_{i}}^{1} & \cdots & \xi_{i} f_{v_{i}}^{n f\left(v_{i}\right)} & 0 & 0 & \cdots & 0 & \cdots \\
& 0 & 0 & \cdots & 0 & 0 & 0 & \cdots & 0 & \xi_{i} & \xi_{i} f_{w_{i}}^{1} & \cdots & \xi_{i} f_{w_{i}}^{n f\left(w_{i}\right)} &
\end{array}\right]
$$

Já o trecho relativo ao nó $i$ da matriz $[B]_{6 \times(12+n p a r)}$ é:

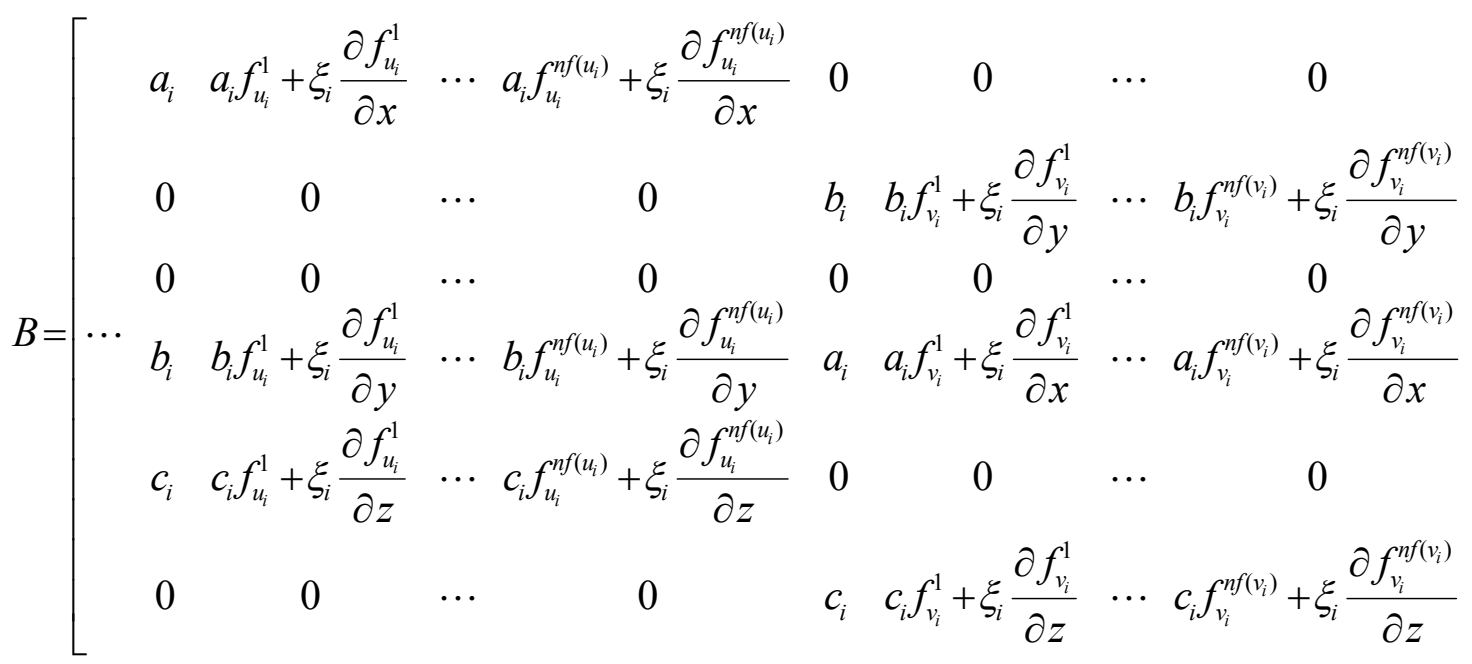

$$
\left[\begin{array}{ccccc}
0 & 0 & \cdots & 0 \\
0 & 0 & \cdots & 0 \\
c_{i} & c_{i} f_{w_{i}}^{1}+\xi_{i} \frac{\partial f_{w_{i}}^{1}}{\partial z} & \cdots & c_{i} f_{w_{i}}^{n f\left(w_{i}\right)}+\xi_{i} \frac{\partial f_{w_{i}}^{n f\left(w_{i}\right)}}{\partial z} & \\
0 & 0 & \cdots & 0 & \cdots \\
a_{i} & a_{i} f_{w_{i}}^{1}+\xi_{i} \frac{\partial f_{w_{i}}^{1}}{\partial x} & \cdots & a_{i} f_{w_{i}}^{n f\left(w_{i}\right)}+\xi_{i} \frac{\partial f_{w_{i}}^{n f\left(w_{i}\right)}}{\partial x} \\
b_{i} & b_{i} f_{w_{i}}^{1}+\xi_{i} \frac{\partial f_{w_{i}}^{1}}{\partial y} & \cdots & b_{i} f_{w_{i}}^{n f\left(w_{i}\right)}+\xi_{i} \frac{\partial f_{w_{i}}^{n f f\left(w_{i}\right)}}{\partial y}
\end{array}\right]
$$


Calcula-se a matriz de rigidez do elemento enriquecido pela equação (24), sendo sua dimensão $(12+$ npar $)$. Ela também é formada a partir de um conjunto de 16 submatrizes (correspondentes às combinações de $i=1, \ldots, 4$ e $j=1, \ldots, 4$ ), como ilustrado na figura 5. Porém, em função do enriquecimento, cada submatriz $3 \times 3$ do elemento nãoenriquecido irá gerar uma nova submatriz expandida pela inclusão de linhas e colunas referentes aos novos parâmetros nodais. Essa expansão é mostrada na figura 6, na qual, a título de exemplo, o número de funções enriquecedoras relativas aos deslocamentos $u_{i}, v_{i}$ e $w_{i}$ é, respectivamente, 1,2 e 0 e o número de funções enriquecedoras relativas aos deslocamentos $u_{j}, v_{j}$ e $w_{j}$ é, respectivamente, 2,1 e 0 .

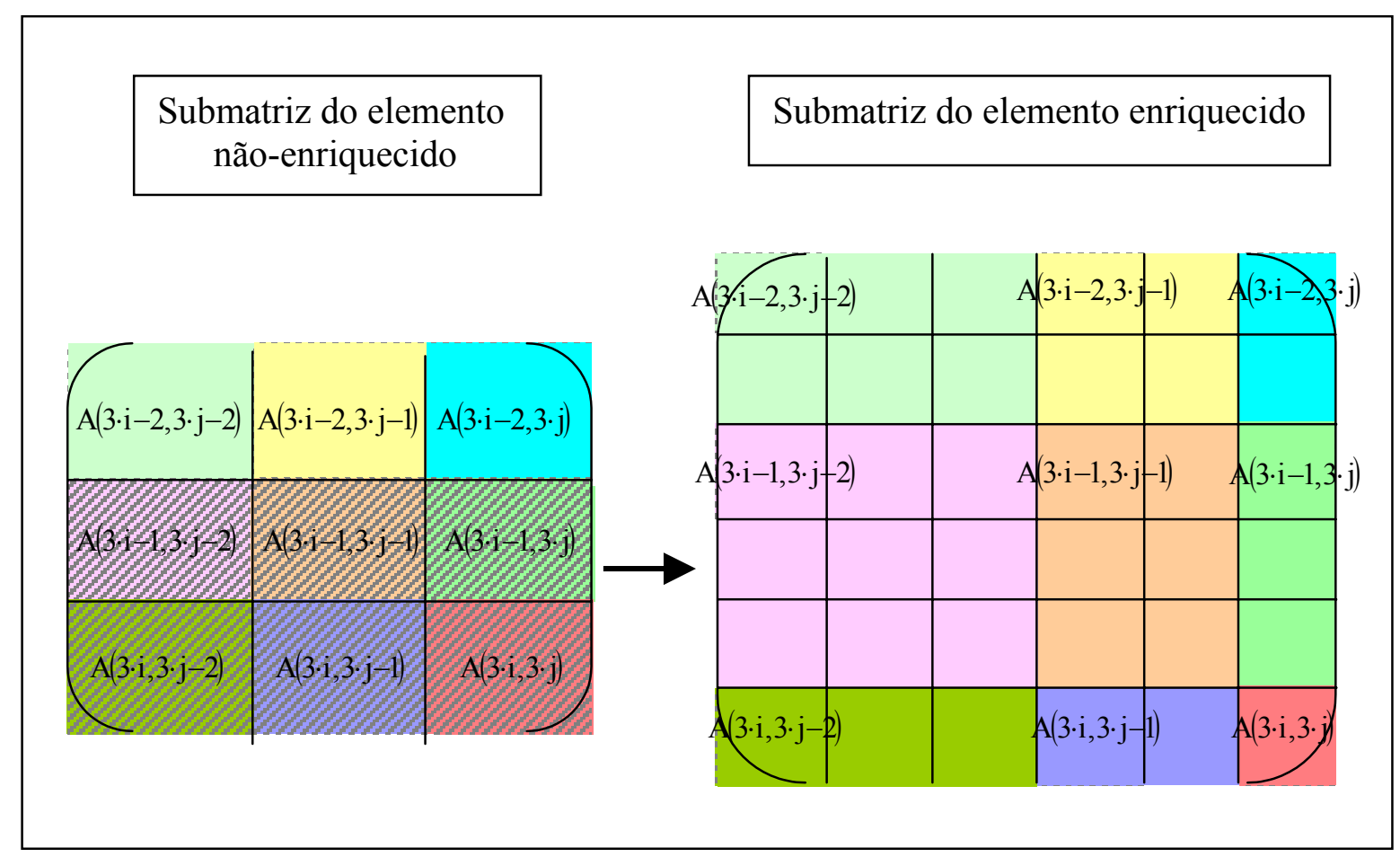

Figura 6 - Expansão da submatriz $3 \times 3$ em função do enriquecimento.

Cada termo da submatriz do elemento não-enriquecido, cujas expressões são dadas pelas eqs. (55), dá origem, portanto, à sua própria submatriz enriquecida, o que se indica pelo esquema de cores da figura 6. Esses termos têm todos eles uma mesma expressão geral, que é apresentada no Apêndice E.

Aproximação dos deslocamentos e do carregamento na superfície:

Utilizam-se as coordenadas adimensionais de superfície, definidas pelas eqs. (57), (58) e (59), sendo necessário definir aproximações distintas para cada face. A aproximação do carregamento é exatamente a mesma adotada para o elemento não-enriquecido. Para as 
faces contadas de 1 a 4 , a matriz $[\phi]_{3 \times 12}^{p}$ é dada, respectivamente, pelas eqs. (60a), (61a), (62a) e (63a) e o vetor de parâmetros nodais de carregamento $\left\{\bar{p}_{e}\right\}_{12 \times 1}$, pelas eqs. (60b), (61b), (62b) e (63b).

A matriz da aproximação dos deslocamentos $[\phi]_{3 x(12+n p a r)}$ referente a cada face do elemento é apresentada no Apêndice F, onde também se apresenta a contribuição de cada face ao vetor de forças nodais, utilizando a eq. (26) .

A integração no volume do tetraedro é feita com base em pontos de Gauss definidos especialmente para esse elemento, como em JINYUN (1984). A integração na superfície é feita com base em pontos de Gauss dados por COWPER (1973).

\subsubsection{Formulação do hexaedro.}

A figura 7 apresenta a numeração adotada para os nós e as faces do elemento hexaédrico, assim como a orientação do sistema local de eixos do mesmo, com base em coordenadas adimensionais $(\xi, \eta, \psi)$.

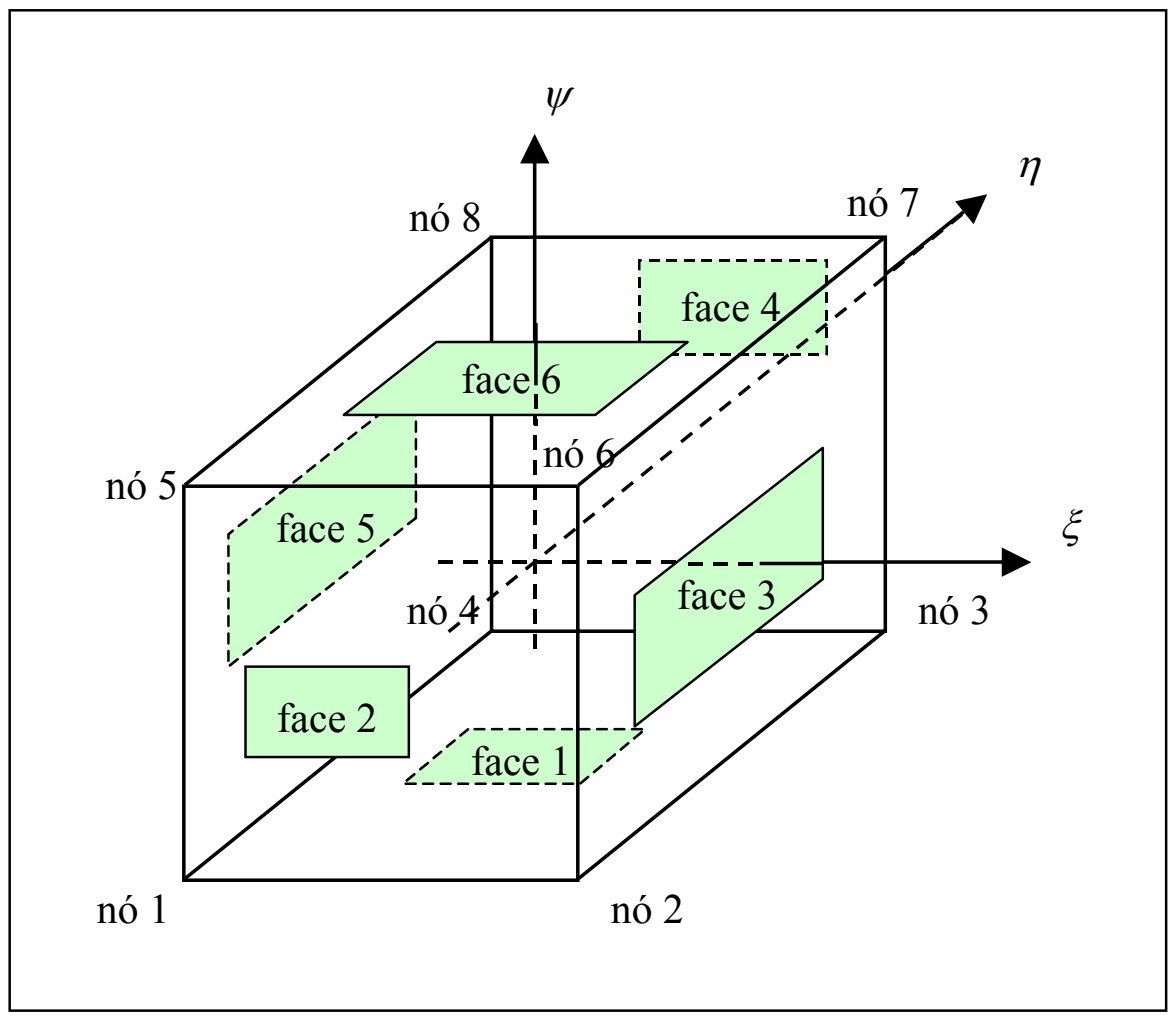

Figura 7 - Elemento hexaédrico - nós, faces e sistema de eixos adimensionais.

2.3.3.2.1 Hexaedro sem enriquecimento.

Aproximação da geometria e dos deslocamentos no interior do elemento: 
Adota-se como sistema de referência o cartesiano triortogonal de eixos $x, y$ e $z$. O elemento hexaédrico desenvolvido é também isoparamétrico, pois como se pode ver nas equações abaixo, as funções de forma utilizadas na aproximação das coordenadas cartesianas e dos deslocamentos são as mesmas:

$$
\begin{aligned}
& x=\sum_{i=1}^{8} N_{i} x_{i} \\
& y=\sum_{i=1}^{8} N_{i} y_{i} \\
& z=\sum_{i=1}^{8} N_{i} z_{i} \\
& u=\sum_{i=1}^{8} N_{i} u_{i} \\
& v=\sum_{i=1}^{8} N_{i} v_{i} \\
& w=\sum_{i=1}^{8} N_{i} w_{i}
\end{aligned}
$$

Nas eqs. (68), $x_{i}, y_{i}$ e $z_{i}$, para $i=1, \ldots, 8$, são as coordenadas dos nós do hexaedro em relação ao sistema de eixos cartesianos, enquanto nas eqs. (69), $u_{i}, v_{i}$ e $w_{i}$, com $i=1, \ldots, 8$, são os parâmetros nodais de deslocamento.

As funções de forma utilizadas na aproximação são as trilineares lagrangianas, dadas abaixo:

$$
\begin{aligned}
& N_{1}=(1-\xi)(1-\eta)(1-\psi) / 8 \\
& N_{2}=(1+\xi)(1-\eta)(1-\psi) / 8 \\
& N_{3}=(1+\xi)(1+\eta)(1-\psi) / 8 \\
& N_{4}=(1-\xi)(1+\eta)(1-\psi) / 8 \\
& N_{5}=(1-\xi)(1-\eta)(1+\psi) / 8 \\
& N_{6}=(1+\xi)(1-\eta)(1+\psi) / 8 \\
& N_{7}=(1+\xi)(1+\eta)(1+\psi) / 8 \\
& N_{8}=(1-\xi)(1+\eta)(1+\psi) / 8
\end{aligned}
$$

Nas eqs. (70), $-1 \leq \xi \leq+1,-1 \leq \eta \leq+1$ e $-1 \leq \psi \leq+1$ são as coordenadas adimensionais do hexaedro, mostradas na figura 7. 
Os deslocamentos $u, v$ e $w$ dados na eq. (69) compõem o vetor de deslocamentos $\{d\}_{3 \times 1}$, sendo o vetor de deslocamentos nodais do elemento $\left\{d_{e}\right\}_{24 x 1}$ dado por:

$$
d_{e}^{T}=\left\{\begin{array}{llllllllll}
u_{1} & v_{1} & w_{1} & u_{2} & v_{2} & w_{2} & \cdots & u_{8} & v_{8} & w_{8}
\end{array}\right\}
$$

A matriz da aproximação dos deslocamentos $[\phi]_{3 \times 24}$ é dada, portanto, por:

$$
\phi=\left[\begin{array}{cccccccccc}
N_{1} & 0 & 0 & N_{2} & 0 & 0 & \cdots & N_{8} & 0 & 0 \\
0 & N_{1} & 0 & 0 & N_{2} & 0 & \cdots & 0 & N_{8} & 0 \\
0 & 0 & N_{1} & 0 & 0 & N_{2} & \cdots & 0 & 0 & N_{8}
\end{array}\right]
$$

E a matriz de aproximação das deformações, $[B]_{6 \times 24}$, é dada a seguir:

$$
B=\left[\begin{array}{cccccccccc}
\frac{\partial N_{1}}{\partial x} & 0 & 0 & \frac{\partial N_{2}}{\partial x} & 0 & 0 & \cdots & \frac{\partial N_{8}}{\partial x} & 0 & 0 \\
0 & \frac{\partial N_{1}}{\partial y} & 0 & 0 & \frac{\partial N_{2}}{\partial y} & 0 & \cdots & 0 & \frac{\partial N_{8}}{\partial y} & 0 \\
0 & 0 & \frac{\partial N_{1}}{\partial z} & 0 & 0 & \frac{\partial N_{2}}{\partial z} & \cdots & 0 & 0 & \frac{\partial N_{8}}{\partial z} \\
\frac{\partial N_{1}}{\partial y} & \frac{\partial N_{1}}{\partial x} & 0 & \frac{\partial N_{2}}{\partial y} & \frac{\partial N_{2}}{\partial x} & 0 & \cdots & \frac{\partial N_{8}}{\partial y} & \frac{\partial N_{8}}{\partial x} & 0 \\
\frac{\partial N_{1}}{\partial z} & 0 & \frac{\partial N_{1}}{\partial x} & \frac{\partial N_{2}}{\partial z} & 0 & \frac{\partial N_{2}}{\partial x} & \cdots & \frac{\partial N_{8}}{\partial z} & 0 & \frac{\partial N_{8}}{\partial x} \\
0 & \frac{\partial N_{1}}{\partial z} & \frac{\partial N_{1}}{\partial y} & 0 & \frac{\partial N_{2}}{\partial z} & \frac{\partial N_{2}}{\partial y} & \cdots & 0 & \frac{\partial N_{8}}{\partial z} & \frac{\partial N_{8}}{\partial y}
\end{array}\right]
$$

Para obter as derivadas parciais das funções de forma (que são dadas em coordenadas adimensionais) em relação às coordenadas globais, utiliza-se a relação:

$$
\left\{\begin{array}{l}
\frac{\partial N_{i}}{\partial x} \\
\frac{\partial N_{i}}{\partial y} \\
\frac{\partial N_{i}}{\partial z}
\end{array}\right\}=[J]^{-1}\left\{\begin{array}{l}
\frac{\partial N_{i}}{\partial \xi} \\
\frac{\partial N_{i}}{\partial \eta} \\
\frac{\partial N_{i}}{\partial \psi}
\end{array}\right\} \quad, i=1, \ldots, 8
$$

sendo $[J]_{3 \times 3}$ o Jacobiano da transformação de coordenadas, dado por:

$$
J=\left[\begin{array}{lll}
\frac{\partial x}{\partial \xi} & \frac{\partial y}{\partial \xi} & \frac{\partial z}{\partial \xi} \\
\frac{\partial x}{\partial \eta} & \frac{\partial y}{\partial \eta} & \frac{\partial z}{\partial \eta} \\
\frac{\partial x}{\partial \psi} & \frac{\partial y}{\partial \psi} & \frac{\partial z}{\partial \psi}
\end{array}\right]
$$

A matriz de rigidez do elemento é calculada utilizando a eq. (24). Ela é constituída por 64 submatrizes (correspondentes às combinações de $i=1, \ldots, 8$ e $j=1, \ldots, 8$ ), possuindo 
cada uma dessas submatrizes dimensão $3 \times 3$. A figura 8 ilustra a estrutura da matriz de rigidez.

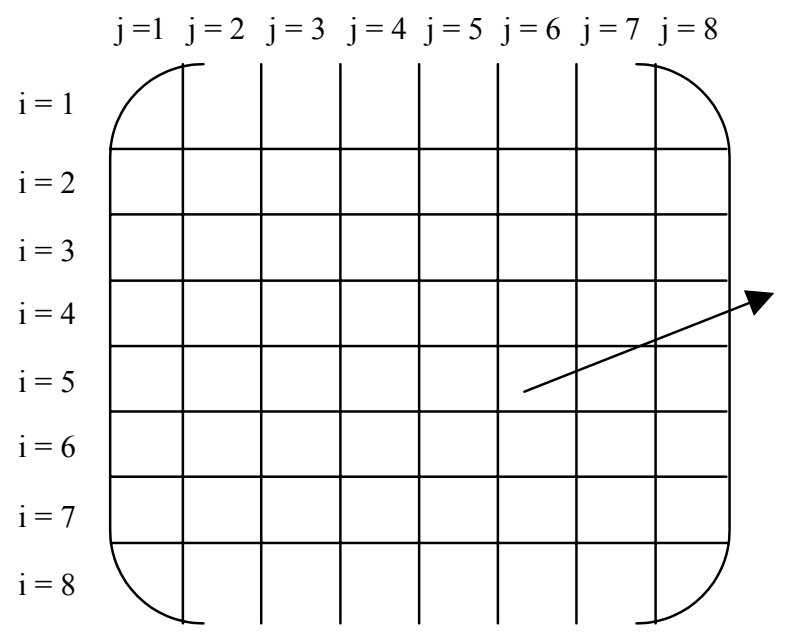

Submatriz $3 \times 3$

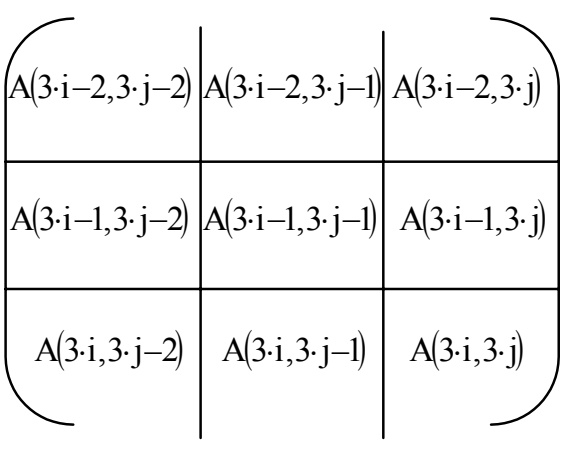

Figura 8 - Estrutura da matriz de rigidez do elemento hexaédrico.

A submatriz $3 \times 3$ genérica mostrada na figura 8 é formada pelos seguintes termos:

$$
\begin{aligned}
& A(3 i-2,3 j-2)=\int_{V}\left(\frac{\partial N_{i}}{\partial x} \frac{\partial N_{j}}{\partial x} c t_{1}+\frac{\partial N_{i}}{\partial y} \frac{\partial N_{j}}{\partial y} c t_{3}+\frac{\partial N_{i}}{\partial z} \frac{\partial N_{j}}{\partial z} c t_{3}\right) d V \\
& A(3 i-2,3 j-1)=\int_{V}\left(\frac{\partial N_{i}}{\partial x} \frac{\partial N_{j}}{\partial y} c t_{2}+\frac{\partial N_{i}}{\partial y} \frac{\partial N_{j}}{\partial x} c t_{3}\right) d V \\
& A(3 i-2,3 j)=\int_{V}\left(\frac{\partial N_{i}}{\partial x} \frac{\partial N_{j}}{\partial z} c t_{2}+\frac{\partial N_{i}}{\partial z} \frac{\partial N_{j}}{\partial x} c t_{3}\right) d V \\
& A(3 i-1,3 j-2)=\int_{V}\left(\frac{\partial N_{i}}{\partial y} \frac{\partial N_{j}}{\partial x} c t_{2}+\frac{\partial N_{i}}{\partial x} \frac{\partial N_{j}}{\partial y} c t_{3}\right) d V \\
& A(3 i-1,3 j-1)=\int_{V}\left(\frac{\partial N_{i}}{\partial x} \frac{\partial N_{j}}{\partial x} c t_{3}+\frac{\partial N_{i}}{\partial y} \frac{\partial N_{j}}{\partial y} c t_{1}+\frac{\partial N_{i}}{\partial z} \frac{\partial N_{j}}{\partial z} c t_{3}\right) d V \\
& A(3 i-1,3 j)=\int_{V}\left(\frac{\partial N_{i}}{\partial y} \frac{\partial N_{j}}{\partial z} c t_{2}+\frac{\partial N_{i}}{\partial z} \frac{\partial N_{j}}{\partial y} c t_{3}\right) d V \\
& A(3 i, 3 j-2)=\int_{V}\left(\frac{\partial N_{i}}{\partial z} \frac{\partial N_{j}}{\partial x} c t_{2}+\frac{\partial N_{i}}{\partial x} \frac{\partial N_{j}}{\partial z} c t_{3}\right) d V \\
& A(3 i, 3 j-1)=\int_{V}\left(\frac{\partial N_{i}}{\partial z} \frac{\partial N_{j}}{\partial y} c t_{2}+\frac{\partial N_{i}}{\partial y} \frac{\partial N_{j}}{\partial z} c t_{3}\right) d V
\end{aligned}
$$




$$
A(3 i, 3 j)=\int_{V}\left(\frac{\partial N_{i}}{\partial x} \frac{\partial N_{j}}{\partial x} c t_{3}+\frac{\partial N_{i}}{\partial y} \frac{\partial N_{j}}{\partial y} c t_{3}+\frac{\partial N_{i}}{\partial z} \frac{\partial N_{j}}{\partial z} c t_{1}\right) d V
$$

sendo $c t_{1}, c t_{2}$ e $c t_{3}$ dados respectivamente pelas eqs. (56a), (56b) e (56c).

As integrais acima são calculadas numericamente. Para permitir a utilização de pontos de Gauss, alteram-se as variáveis de integração, passando do sistema global $(x, y, z)$ para o adimensional $(\xi, \eta, \psi)$. A expressão utilizada para calcular a matriz de rigidez, dada em (24), passa a ser a seguinte:

$$
R_{e}=\int_{-1}^{+1} \int_{-1}^{+1+1} \int^{T} B^{T} D B|J| d \xi d \eta d \psi
$$

sendo $|J|$ o determinante do Jacobiano dado pela eq. (75).

\section{Aproximação dos deslocamentos e do carregamento na superfície.}

Faz-se uma aproximação distinta para cada face do hexaedro. Em cada uma delas, adota-se um sistema de eixos bidimensionais $(r, s)$ no plano da face, de origem arbitrária, em função do qual definem-se as coordenadas nodais $\left(r_{i}\right.$ e $\left.s_{i}, i=1, \ldots, 4\right)$. Também para cada face define-se um sistema de eixos adimensionais $(\zeta, \vartheta)$, ao qual se associam funções de forma bilineares lagrangianas, dadas abaixo:

$$
\begin{aligned}
& N_{1}=\frac{1}{4}(1-\zeta)(1-\vartheta) \\
& N_{2}=\frac{1}{4}(1+\zeta)(1-\vartheta) \\
& N_{3}=\frac{1}{4}(1+\zeta)(1+\vartheta) \\
& N_{4}=\frac{1}{4}(1-\zeta)(1+\vartheta)
\end{aligned}
$$

A aproximação para as coordenadas $r$ e $s$ é, portanto:

$$
\begin{aligned}
& r=\sum_{i=1}^{4} r_{i} N_{i} \\
& s=\sum_{i=1}^{4} s_{i} N_{i}
\end{aligned}
$$

Os sistemas de eixos $(r, s)$ e $(\zeta, \vartheta)$ são mostrados na figura 9. O Jacobiano da transformação entre esses sistemas de coordenadas é dado por: 


$$
J_{p}=\left[\begin{array}{ll}
\frac{\partial r}{\partial \zeta} & \frac{\partial s}{\partial \zeta} \\
\frac{\partial r}{\partial \vartheta} & \frac{\partial s}{\partial \vartheta}
\end{array}\right]
$$

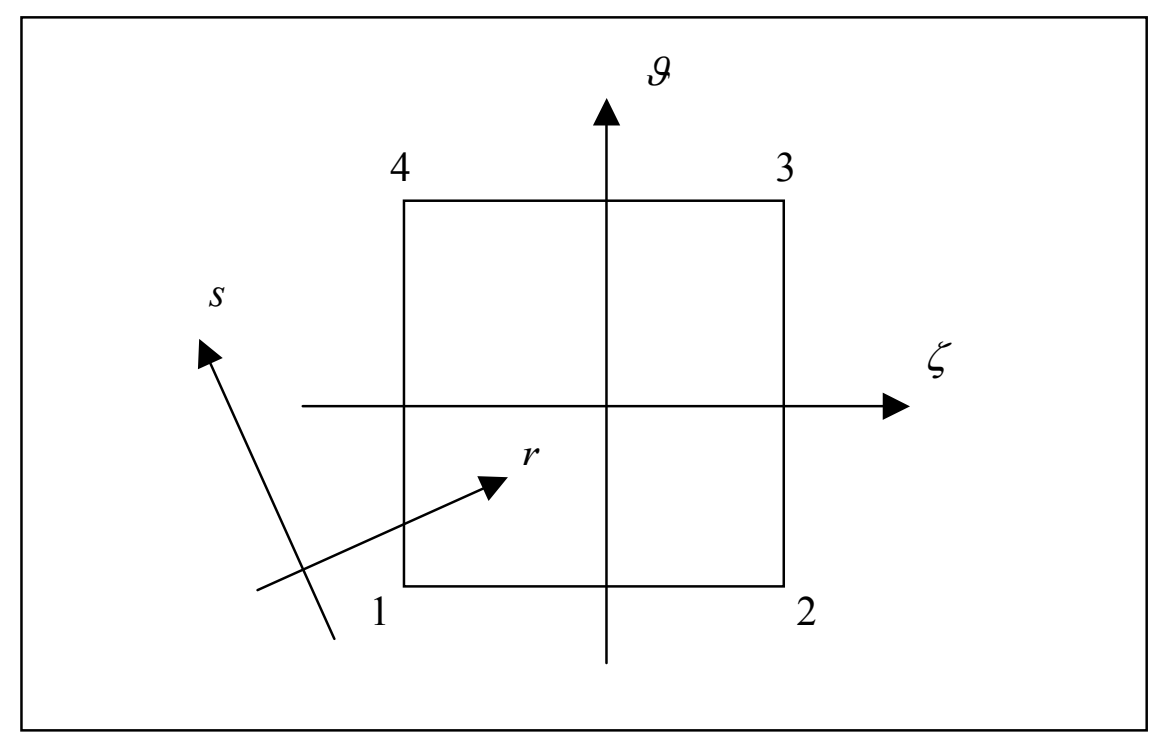

Figura 9 - Sistemas de eixos no plano das faces do hexaedro

Os deslocamentos $u, v$ e $w$ são aproximados, em função dos valores nodais de deslocamento de cada face $\left(u_{i}^{f}, v_{i}^{f}\right.$ e $w_{i}^{f}, i=1, \ldots, 4$ e $\left.f=1, \ldots, 6\right)$ e das funções de forma dadas pelas eqs. (78):

$$
\begin{aligned}
& u=\sum_{i=1}^{4} u_{i}^{f} N_{i} \\
& v=\sum_{i=1}^{4} v_{i}^{f} N_{i} \\
& w=\sum_{i=1}^{4} w_{i}^{f} N_{i}
\end{aligned}
$$

Os deslocamentos referidos às faces são os próprios deslocamentos nodais que formam o vetor $d_{e}$ dado em (71). A tabela seguinte apresenta, para cada face do hexaedro, a correspondência entre o índice referente à face e o índice referente ao elemento. 
Tabela 1 - Correspondência de índices dos deslocamentos (nas faces e no elemento)

\begin{tabular}{|c|c|c|c|c|c|}
\hline \multicolumn{2}{|c|}{} & \multicolumn{5}{|c|}{ Índice relativo à face } \\
\cline { 2 - 6 } \multicolumn{2}{c|}{} & 1 & 2 & 3 & 4 \\
\hline \multirow{2}{*}{$\begin{array}{c}\text { Para cada } \\
\text { face, } \\
\text { índice } \\
\text { relativo ao } \\
\text { elemento }\end{array}$} & 1 & 1 & 2 & 3 & 4 \\
\cline { 2 - 6 } & 2 & 1 & 2 & 6 & 5 \\
\cline { 2 - 6 } & 5 & 2 & 3 & 7 & 6 \\
\cline { 2 - 6 } & 6 & 5 & 6 & 7 & 8 \\
\hline
\end{tabular}

Apresentam-se a seguir para cada face do hexaedro:

- As matrizes de aproximação dos deslocamentos $[\phi]_{3 \times 24}$ e do carregamento $\left[\phi^{p}\right]_{3 \times 24}$, que são as mesmas.

- O vetor de parâmetros nodais de carregamento $\left\{\bar{p}_{e}\right\}_{24 \times 1}$.

Face 1:

$$
\begin{aligned}
\phi=\phi^{p}=\left[\begin{array}{cccccccccccc:}
N_{1} & 0 & 0 & N_{2} & 0 & 0 & N_{3} & 0 & 0 & N_{4} & 0 & 0 \\
0 & N_{1} & 0 & 0 & N_{2} & 0 & 0 & N_{3} & 0 & 0 & N_{4} & 0 \\
0 & 0 & N_{1} & 0 & 0 & N_{2} & 0 & 0 & N_{3} & 0 & 0 & N_{4} \\
0 & 0 & 0 & 0 & 0 & 0 & 0 & 0 & 0 & 0 & 0 & 0 \\
0 & 0 & 0 & 0 & 0 & 0 & 0 & 0 & 0 & 0 & 0 & 0 \\
0 & 0 & 0 & 0 & 0 & 0 & 0 & 0 & 0 & 0 & 0 & 0
\end{array}\right]
\end{aligned}
$$

$\left\{\bar{p}_{e}\right\}_{\text {face } 1}=\left\{\begin{array}{llllllllllll}p x_{1} & p y_{1} & p z_{1} & p x_{2} & p y_{2} & p z_{2} & p x_{3} & p y_{3} & p z_{3} & p x_{4} & p y_{4} & p z_{4}\end{array}\right.$

$$
\left.\begin{array}{llllllllllll}
0 & 0 & 0 & 0 & 0 & 0 & 0 & 0 & 0 & 0 & 0 & 0
\end{array}\right\}
$$

Face 2:

$$
\begin{aligned}
\phi=\phi^{p}= & {\left[\begin{array}{cccccccccccc:c}
N_{1} & 0 & 0 & N_{2} & 0 & 0 & 0 & 0 & 0 & 0 & 0 & 0 & \\
0 & N_{1} & 0 & 0 & N_{2} & 0 & 0 & 0 & 0 & 0 & 0 & 0 & \vdots \\
0 & 0 & N_{1} & 0 & 0 & N_{2} & 0 & 0 & 0 & 0 & 0 & 0 & \vdots \\
N_{4} & 0 & 0 & N_{3} & 0 & 0 & 0 & 0 & 0 & 0 & 0 & 0 \\
0 & N_{4} & 0 & 0 & N_{3} & 0 & 0 & 0 & 0 & 0 & 0 & 0 \\
0 & 0 & N_{4} & 0 & 0 & N_{3} & 0 & 0 & 0 & 0 & 0 & 0
\end{array}\right] } \\
\left\{\bar{p}_{e}\right\}_{\text {face } 2}= & \left\{\begin{array}{llllllllllll}
p x_{1} & p y_{1} & p z_{1} & p x_{2} & p y_{2} & p z_{2} & 0 & 0 & 0 & 0 & 0 & 0 \\
1 p x_{5} & p y_{5} & p z_{5} & p x_{6} & p y_{6} & p z_{6} & 0 & 0 & 0 & 0 & 0 & 0
\end{array}\right\}
\end{aligned}
$$


Face 3:

$$
\begin{aligned}
\phi=\phi^{p}= & {\left[\begin{array}{cccccccccccc:c}
0 & 0 & 0 & N_{1} & 0 & 0 & N_{2} & 0 & 0 & 0 & 0 & 0 & \\
0 & 0 & 0 & 0 & N_{1} & 0 & 0 & N_{2} & 0 & 0 & 0 & 0 & \\
0 & 0 & 0 & 0 & 0 & N_{1} & 0 & 0 & N_{2} & 0 & 0 & 0 & \\
& {\left[\begin{array}{cccccccccccc}
0 & 0 & 0 & N_{4} & 0 & 0 & N_{3} & 0 & 0 & 0 & 0 & 0 \\
0 & 0 & 0 & 0 & N_{4} & 0 & 0 & N_{3} & 0 & 0 & 0 & 0 \\
0 & 0 & 0 & 0 & 0 & N_{4} & 0 & 0 & N_{3} & 0 & 0 & 0
\end{array}\right]} \\
\left\{\bar{p}_{e}\right\}_{\text {face } 3} & =\left\{\begin{array}{lllllllllllll}
0 & 0 & 0 & p x_{2} & p y_{2} & p z_{2} & p x_{3} & p y_{3} & p z_{3} & 0 & 0 & 0
\end{array}\right. \\
0 & 0 & 0 & p x_{6} & p y_{6} & p z_{6} & p x_{7} & p y_{7} & p z_{7} & 0 & 0 & 0
\end{array}\right\} }
\end{aligned}
$$

Face 4:

$$
\begin{aligned}
& \phi=\phi^{p}=\left[\begin{array}{cccccccccccc:}
0 & 0 & 0 & 0 & 0 & 0 & N_{1} & 0 & 0 & N_{2} & 0 & 0 \\
0 & 0 & 0 & 0 & 0 & 0 & 0 & N_{1} & 0 & 0 & N_{2} & 0 \\
0 & 0 & 0 & 0 & 0 & 0 & 0 & 0 & N_{1} & 0 & 0 & N_{2}
\end{array}\right. \\
& {\left[\begin{array}{cccccccccccc}
0 & 0 & 0 & 0 & 0 & 0 & N_{4} & 0 & 0 & N_{3} & 0 & 0 \\
0 & 0 & 0 & 0 & 0 & 0 & 0 & N_{4} & 0 & 0 & N_{3} & 0 \\
0 & 0 & 0 & 0 & 0 & 0 & 0 & 0 & N_{4} & 0 & 0 & N_{3}
\end{array}\right]} \\
& \left\{\bar{p}_{e}\right\}_{\text {face } 4}=\left\{\begin{array}{llllllllllll}
0 & 0 & 0 & 0 & 0 & 0 & p x_{3} & p y_{3} & p z_{3} & p x_{4} & p y_{4} & p z_{4}
\end{array}\right. \\
& \left.\begin{array}{llllllllllll}
0 & 0 & 0 & 0 & 0 & 0 & p x_{7} & p y_{7} & p z_{7} & p x_{8} & p y_{8} & p z_{8}
\end{array}\right\}
\end{aligned}
$$

Face 5:

$$
\begin{aligned}
& \phi=\phi^{p}=\left[\begin{array}{cccccccccccc:}
N_{2} & 0 & 0 & 0 & 0 & 0 & 0 & 0 & 0 & N_{1} & 0 & 0 \\
0 & N_{2} & 0 & 0 & 0 & 0 & 0 & 0 & 0 & 0 & N_{1} & 0 \\
0 & 0 & N_{2} & 0 & 0 & 0 & 0 & 0 & 0 & 0 & 0 & N_{1}
\end{array}\right. \\
& \left.\begin{array}{llllllllllll}
N_{3} & 0 & 0 & 0 & 0 & 0 & 0 & 0 & 0 & N_{4} & 0 & 0
\end{array}\right] \\
& \begin{array}{llllllllllll}
0 & N_{3} & 0 & 0 & 0 & 0 & 0 & 0 & 0 & 0 & N_{4} & 0
\end{array} \\
& \left.\begin{array}{llllllllllll}
0 & 0 & N_{3} & 0 & 0 & 0 & 0 & 0 & 0 & 0 & 0 & N_{4}
\end{array}\right] \\
& \left\{\bar{p}_{e}\right\}_{\text {face } 5}=\left\{\begin{array}{llllllllllll}
p x_{1} & p y_{1} & p z_{1} & 0 & 0 & 0 & 0 & 0 & 0 & p x_{4} & p y_{4} & p z_{4}
\end{array}\right. \\
& \left.\begin{array}{llllllllllll}
p x_{5} & p y_{5} & p z_{5} & 0 & 0 & 0 & 0 & 0 & 0 & p x_{8} & p y_{8} & p z_{8}
\end{array}\right\}
\end{aligned}
$$

Face 6:

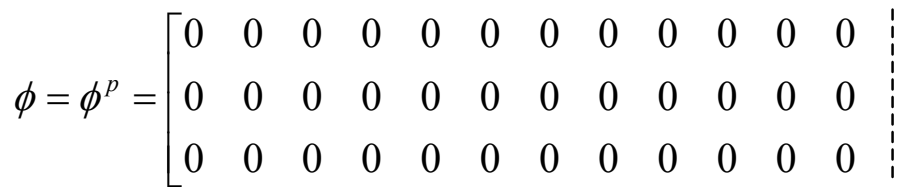




$$
\begin{aligned}
& {\left[\begin{array}{cccccccccccc}
N_{1} & 0 & 0 & N_{2} & 0 & 0 & N_{3} & 0 & 0 & N_{4} & 0 & 0 \\
0 & N_{1} & 0 & 0 & N_{2} & 0 & 0 & N_{3} & 0 & 0 & N_{4} & 0 \\
0 & 0 & N_{1} & 0 & 0 & N_{2} & 0 & 0 & N_{3} & 0 & 0 & N_{4}
\end{array}\right]} \\
& \left\{\bar{p}_{e}\right\}_{\text {face } 6}=\left\{\begin{array}{llllllllllll:}
0 & 0 & 0 & 0 & 0 & 0 & 0 & 0 & 0 & 0 & 0 & 0
\end{array}\right. \\
& \left.\begin{array}{llllllllllll}
p x_{5} & p y_{5} & p z_{5} & p x_{6} & p y_{6} & p z_{6} & p x_{7} & p y_{7} & p z_{7} & p x_{8} & p y_{8} & p z_{8}
\end{array}\right\}
\end{aligned}
$$

O vetor de forças nodais do elemento é calculado com a eq. (26), desconsiderandose as forças de volume. A contribuição de cada face para o vetor de forças nodais é apresentada no Apêndice G.

\subsection{Hexaedro com enriquecimento.}

\section{Aproximação da geometria:}

As coordenadas no interior do elemento são interpoladas do mesmo modo que no caso do hexaedro não-enriquecido, fazendo-se uso, portanto, das eqs. (68).

\section{Aproximação dos deslocamentos no interior do elemento:}

O enriquecimento do campo de deslocamentos é feito de forma análoga ao que foi realizado com o tetraedro, ou seja, adicionando-se novos parâmetros vinculados aos deslocamentos dos nós, associados a funções que multiplicam as funções de forma originais:

$$
\begin{aligned}
& u=\sum_{i=1}^{8} N_{i}\left(u_{i}+\sum_{j=1}^{n f\left(u_{i}\right)} u_{i}^{j} f_{u_{i}}^{j}\right) \\
& v=\sum_{i=1}^{8} N_{i}\left(v_{i}+\sum_{j=1}^{n f\left(v_{i}\right)} v_{i}^{j} f_{v_{i}}^{j}\right) \\
& w=\sum_{i=1}^{8} N_{i}\left(w_{i}+\sum_{j=1}^{n f\left(w_{i}\right)} w_{i}^{j} f_{w_{i}}^{j}\right)
\end{aligned}
$$

A convenção adotada nas eqs (88a), (88b) e (88c) é a mesma adotada nas eqs. (64a), (64b) e (64c) para o tetraedro enriquecido. Também para o hexaedro se denomina npar ao número de novos parâmetros (em cada elemento) relativos ao enriquecimento.

$\mathrm{O}$ vetor de deslocamentos nodais $\left\{d_{e}\right\}_{(24+n p a r) x 1}$ do elemento tem a seguinte estrutura:

$$
d_{e}^{T}=\left\{\begin{array}{lllllllllllll}
u_{1} & u_{1}^{1} & \cdots & u_{1}^{n f\left(u_{1}\right)} & v_{1} & v_{1}^{1} & \cdots & v_{1}^{n f\left(v_{1}\right)} & \cdots & w_{8} & w_{8}^{1} & \cdots & w_{8}^{n f\left(w_{8}\right)}
\end{array}\right\}
$$

sendo o trecho referente ao nó $i, i=1, \ldots, 8$, da matriz da aproximação dos deslocamentos $[\phi]_{3 x(24+n p a r)}$ dado abaixo: 


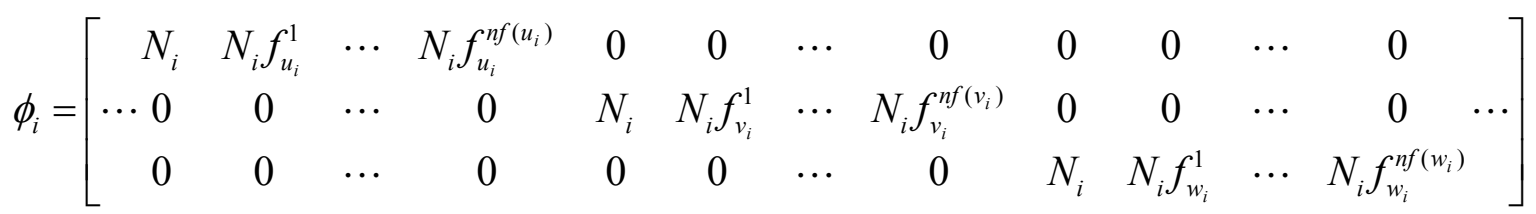

O trecho relativo ao nó $i, i=1, \ldots, 8$, da matriz de aproximação das deformações $[B]_{6 x(24+n p a r)}$, é dado abaixo:

$$
B_{i}=\left[\begin{array}{cccc}
\frac{\partial N_{i}}{\partial x} & \frac{\partial N_{i}}{\partial x} f_{u_{i}}^{1}+N_{i} \frac{\partial f_{u_{i}}^{1}}{\partial x} & \cdots & \frac{\partial N_{i}}{\partial x} f_{u_{i}}^{n f\left(u_{i}\right)}+N_{i} \frac{\partial f_{u_{i}}^{n f\left(u_{i}\right)}}{\partial x} \\
0 & 0 & \cdots & 0 \\
\cdots & 0 & \cdots & 0 \\
\frac{\partial N_{i}}{\partial y} & \frac{\partial N_{i}}{\partial y} f_{u_{i}}^{1}+N_{i} \frac{\partial f_{u_{i}}^{1}}{\partial y} & \cdots & \frac{\partial N_{i}}{\partial y} f_{u_{i}}^{n f\left(u_{i}\right)}+N_{i} \frac{\partial f_{u_{i}}^{n f\left(u_{i}\right)}}{\partial y} \\
\frac{\partial N_{i}}{\partial z} & \frac{\partial N_{i}}{\partial z} f_{u_{i}}^{1}+N_{i} \frac{\partial f_{u_{i}}^{1}}{\partial z} & \cdots & \frac{\partial N_{i}}{\partial z} f_{u_{i}}^{n f\left(u_{i}\right)}+N_{i} \frac{\partial f_{u_{i}}^{n f\left(u_{i}\right)}}{\partial z} \\
0 & 0 & \cdots & 0
\end{array}\right.
$$

$$
\begin{array}{ccccc}
0 & 0 & \cdots & 0 \\
\frac{\partial N_{i}}{\partial y} & \frac{\partial N_{i}}{\partial y} f_{v_{i}}^{1}+N_{i} \frac{\partial f_{v_{i}}^{1}}{\partial y} & \cdots & \frac{\partial N_{i}}{\partial y} f_{v_{i}}^{n f\left(v_{i}\right)}+N_{i} \frac{\partial f_{v_{i}}^{n f\left(v_{i}\right)}}{\partial y} \\
0 & 0 & \cdots & 0 \\
\frac{\partial N_{i}}{\partial x} & \frac{\partial N_{i}}{\partial x} f_{v_{i}}^{1}+N_{i} \frac{\partial f_{v_{i}}^{1}}{\partial x} & \cdots & \frac{\partial N_{i}}{\partial x} f_{v_{i}}^{n f\left(v_{i}\right)}+N_{i} \frac{\partial f_{v_{i}}^{n f\left(v_{i}\right)}}{\partial x} \\
0 & 0 & \cdots & 0 \\
\frac{\partial N_{i}}{\partial z} & \frac{\partial N_{i}}{\partial z} f_{v_{i}}^{1}+N_{i} \frac{\partial f_{v_{i}}^{1}}{\partial z} & \cdots & \frac{\partial N_{i}}{\partial z} f_{v_{i}}^{n f\left(v_{i}\right)}+N_{i} \frac{\partial f_{v_{i}}^{n f\left(v_{i}\right)}}{\partial z}
\end{array}
$$

$$
\left.\begin{array}{ccccc}
0 & 0 & \cdots & 0 \\
0 & 0 & \cdots & 0 & \\
\frac{\partial N_{i}}{\partial z} & \frac{\partial N_{i}}{\partial z} f_{w_{i}}^{1}+N_{i} \frac{\partial f_{w_{i}}^{1}}{\partial z} & \cdots & \frac{\partial N_{i}}{\partial z} f_{w_{i}}^{n f\left(w_{i}\right)}+N_{i} \frac{\partial f_{w_{i}}^{n f\left(w_{i}\right)}}{\partial z} \\
0 & 0 & \cdots & 0 & \cdots \\
\frac{\partial N_{i}}{\partial x} & \frac{\partial N_{i}}{\partial x} f_{w_{i}}^{1}+N_{i} \frac{\partial f_{w_{i}}^{1}}{\partial x} & \cdots & \frac{\partial N_{i}}{\partial x} f_{w_{i}}^{n f\left(w_{i}\right)}+N_{i} \frac{\partial f_{w_{i}}^{n f\left(w_{i}\right)}}{\partial x} & \cdots \\
\frac{\partial N_{i}}{\partial y} & \frac{\partial N_{i}}{\partial y} f_{w_{i}}^{1}+N_{i} \frac{\partial f_{w_{i}}^{1}}{\partial y} & \cdots & \frac{\partial N_{i}}{\partial y} f_{w_{i}}^{n f\left(w_{i}\right)}+N_{i} \frac{\partial f_{w_{i}}^{n f\left(w_{i}\right)}}{\partial y}
\end{array}\right]
$$

Assim como ocorre com o elemento não-enriquecido, a matriz de rigidez do hexaedro enriquecido, calculada pela eq. (24), é também formada por um conjunto de 64 submatrizes (correspondentes às combinações de $i=1, \ldots, 8$ e $j=1, \ldots, 8$ ), como ilustrado pela figura 8 . 
De modo análogo ao mostrado para o tetraedro com enriquecimento na figura 6, cada uma dessas submatrizes é formada a partir da expansão de uma matriz $3 \times 3$ original, dada pelas eqs. (76), pela inclusão de linhas e colunas referentes aos novos parâmetros nodais. Cada termo da submatriz original gera, portanto, a sua própria submatriz, formada por termos que possuem uma mesma expressão geral, o que se mostra no Apêndice H.

\section{Aproximação dos deslocamentos e do carregamento na superfície:}

A aproximação é feita separadamente para cada face do hexaedro. Adotam-se um sistema auxiliar de coordenadas $(r, s)$ e um sistema de coordenadas adimensionais $(\zeta, \vartheta)$, conforme mostrado na figura 9. As funções de forma relativas às coordenadas adimensionais $\left(N_{i}, i=1, \ldots, 4\right)$ são dadas pelas eqs. (78), enquanto a aproximação das coordenadas $r$ e $s$ no plano das faces é definida pelas eqs. (79).

A aproximação para o carregamento nas faces é idêntica àquela apresentada para 0 elemento não-enriquecido. Para as faces do hexaedro de 1 a 6 , as matrizes $\phi^{p}$ são dadas, respectivamente, pelas eqs. (82a), (83a), (84a), (85a), (86a) e (87a); também para as faces de 1 a 6 , os vetores dos parâmetros de carregamento $\bar{p}_{e}$ são dados pelas eqs. (82b), (83b), (84b), (85b), (86b) e (87b), respectivamente.

Já a aproximação para os deslocamentos é enriquecida, como mostrado abaixo para uma face $f, f=1, \ldots, 6$ :

$$
\begin{aligned}
& u=\sum_{i=1}^{4} N_{i}\left(u_{i}^{f}+\sum_{j=1}^{n f\left(u_{i}^{f}\right)} u_{i}^{j} f_{u_{i}^{f}}^{j}\right) \\
& v=\sum_{i=1}^{4} N_{i}\left(v_{i}^{f}+\sum_{j=1}^{n f\left(v_{i}^{f}\right)} v_{i}^{j f} f_{v_{i}^{j}}^{j}\right) \\
& w=\sum_{i=1}^{4} N_{i}\left(w_{i}^{f}+\sum_{j=1}^{n f\left(w_{i}^{f}\right)} w_{i}^{j f} f_{w_{i}^{j}}^{j}\right)
\end{aligned}
$$

Nas eqs. (92), verifica-se que os índices dos deslocamentos nodais ( $i$ ) estão referidos à numeração dos nós em cada face, que variam de 1 a 4, como na figura 9. Para que se obtenham os índices dos deslocamentos com referência ao elemento, deve-se consultar a tabela 1. A partir daí, podem-se obter as matrizes de aproximação dos deslocamentos nas faces $[\phi]_{3 x(24+n p a r)}$, que são apresentadas no Apêndice I.

No Apêndice J, calcula-se a contribuição de cada face ao vetor de forças nodais do elemento, utilizando-se a eq. (26). 


\section{PLASTICIDADE}

\subsection{Histórico}

Considera-se usualmente como marco inicial da teoria da plasticidade o artigo de TRESCA (1864) sobre a extrusão de metais, em que o seguinte critério de plastificação foi pela primeira vez proposto: um metal deforma-se plasticamente quando a máxima tensão de cisalhamento atinge um valor crítico.

SAINT-VENANT (1870) introduziu as relações constitutivas, no estado plano, de um material rígido-plástico. Em seu artigo, propõe-se uma regra de fluxo através da qual os incrementos de deformação se dão segundo as direções dos eixos principais de tensão.

VON MISES (1913) apresenta um critério de plastificação que tem como base a limitação sobre a tensão octaédrica de cisalhamento (ou o invariante de tensões desviadoras $J_{2}$ ). Em 1928, este mesmo autor fez uma generalização de seu trabalho anterior para permitir a adoção de uma função de plastificação arbitrária. Além disso, apresentou uma relação entre a direção da deformação plástica e a superfície de plastificação, definindo a regra de fluxo plástico.

PRANDTL (1924) incluiu nas equações desenvolvidas por Saint-Venant/Von Mises a componente elástica da deformação, conceituando o modelo elastoplástico, e REUSS (1930) fez a extensão para o caso tridimensional.

Nos trabalhos citados anteriormente, a ênfase recaiu sobre o comportamento rígido ou elastoplástico perfeito. Relações constitutivas para materiais que apresentam o fenômeno do encruamento positivo (hardening) foram desenvolvidas mais lentamente, podendo-se citar a contribuição de MELAN (1938).

As décadas de 1940 e 1950 foram um período de intenso desenvolvimento de conceitos básicos da chamada Teoria Clássica da Plasticidade em Metais.

PRAGER (1949), independente do trabalho de Melan, estabeleceu relações constitutivas para materiais com encruamento e superfícies de plastificação regulares. Foram formulados e discutidos os conceitos de função de carregamento (ou plastificação), de condição de carregamento ou de descarregamento, a condição de consistência e a de irreversibilidade da deformação plástica. A inter-relação entre a convexidade da superfície de carregamento, a regra da normalidade em relação ao incremento de deformação plástica e a unicidade da solução do problema de valor de contorno associado foi claramente estabelecida. 
A partir desses conceitos, DRUCKER (1951) propôs o chamado Postulado da Estabilidade de um material, o que permitiu provar a unicidade da solução tanto para materiais elastoplásticos perfeitos como para os que sofrem encruamento positivo.

\subsection{Conceitos básicos}

Em nível macroscópico, pode-se caracterizar o comportamento plástico de um material pela ocorrência de deformações irreversíveis, ou seja, não recuperáveis, observadas num ciclo de carregamento e descarregamento. Usualmente, o material apresenta um nível de tensão denominado tensão de plastificação, que uma vez atingido pode levar à ocorrência de deformações plásticas.

Materiais elastoplásticos perfeitos são aqueles nos quais a tensão jamais excede a tensão de plastificação e uma vez que esta seja atingida e mantida arbitrariamente, toda a deformação adicional que passa a ocorrer é exclusivamente de natureza plástica. É o que se mostra na figura 10, no caso de tração uniaxial.

Materiais cuja tensão pode aumentar além da tensão de plastificação (usualmente denominada nesse caso de limite elástico) apresentam o fenômeno do encruamento positivo, como mostrado na figura 11.

Nos materiais em regime elastoplástico, a deformação total é composta por parcelas plástica e elástica. Nesse regime não há uma correspondência unívoca entre tensão e deformação, sendo necessário o conhecimento da história de carregamento, caracterizada pelo nível de deformação plástica acumulada.

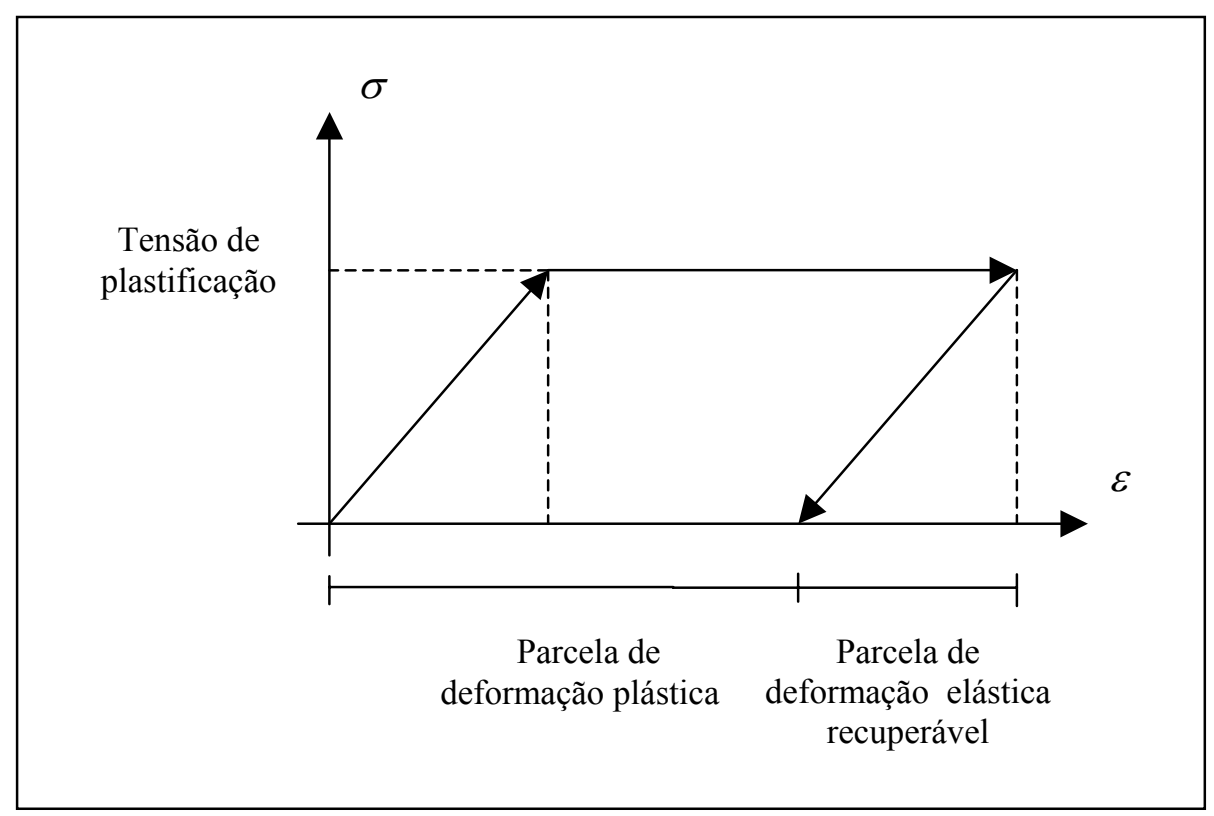

Figura 10 - Relação constitutiva elastoplástica perfeita 


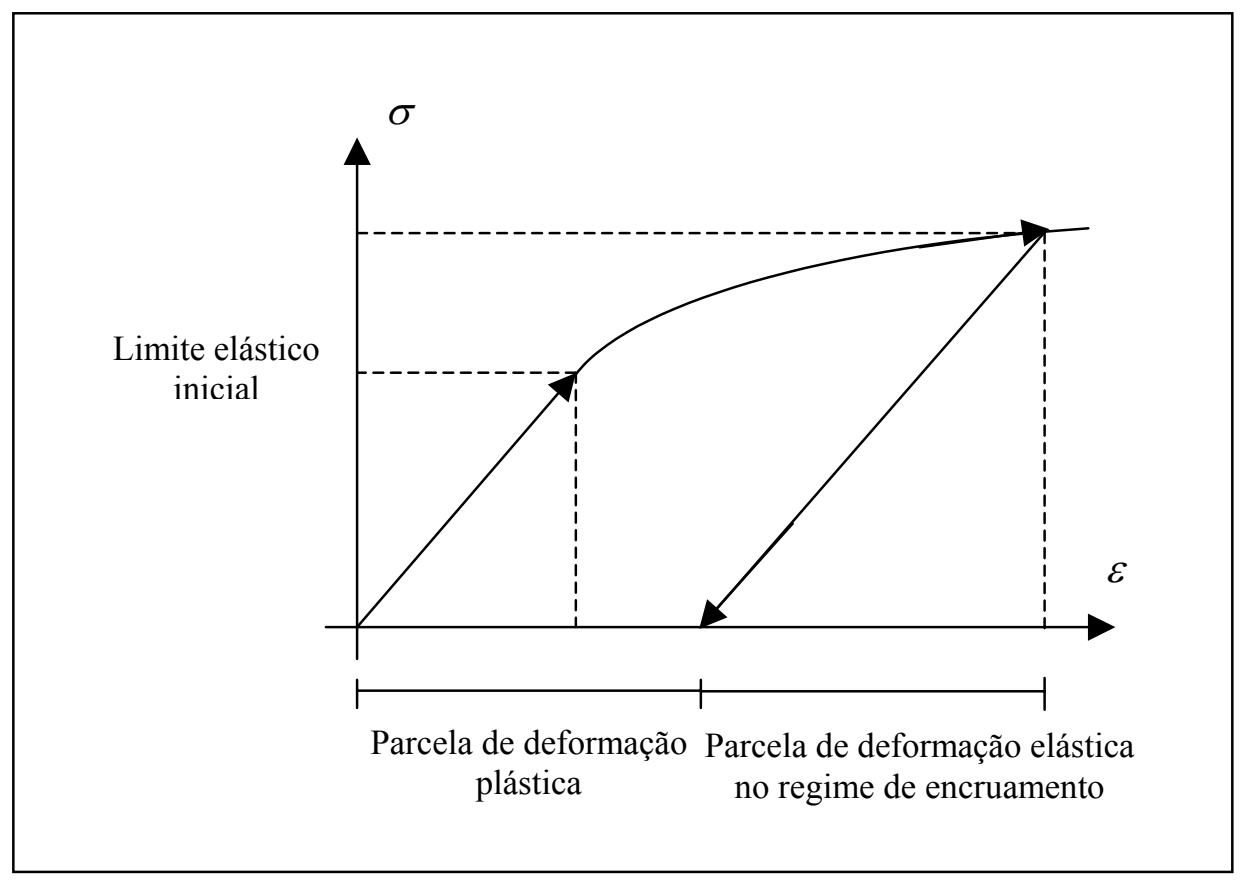

Figura 11 - Relação constitutiva elastoplástica com encruamento.

\subsection{Formulação}

Apresenta-se inicialmente a formulação do comportamento elastoplástico para o caso unidimensional. São apresentados os dois modelos básicos desse comportamento - o elastoplástico perfeito e o elastoplástico com encruamento. A generalização para o caso tridimensional é feita na seqüência. Uma referência para os desenvolvimentos aqui apresentados é SIMO \& HUGHES (1997).

\subsubsection{Comportamento elastoplástico unidimensional.}

Admite-se que a deformação total $(\varepsilon)$ possa ser desmembrada em duas parcelas: elástica $\left(\varepsilon^{e}\right)$ e plástica $\left(\varepsilon^{p}\right)$, conforme mostra a eq. (93).

$$
\varepsilon^{e}=\varepsilon-\varepsilon^{p}
$$

Sendo a tensão dada por $\sigma$ e sendo $E$ o módulo de elasticidade longitudinal do material, a relação constitutiva é dada por:

$$
\sigma=E \varepsilon^{e}=E\left(\varepsilon-\varepsilon^{p}\right)
$$

A relação acima pode ser escrita em termos de taxas, admitindo-se que a tensão e as deformações plástica e elástica sejam funções contínuas no tempo:

$$
\dot{\sigma}=E \dot{\varepsilon}^{e}=E\left(\dot{\varepsilon}-\dot{\varepsilon}^{p}\right)
$$

sendo $\dot{\sigma}=\frac{d \sigma}{d t}, \dot{\varepsilon}=\frac{d \varepsilon}{d t}$ e $\dot{\varepsilon}^{p}=\frac{d \varepsilon^{p}}{d t}$ 


\subsubsection{Comportamento elastoplástico perfeito}

No modelo elastoplástico perfeito, a tensão não pode exceder, em valor absoluto, o valor da tensão de plastificação $\sigma_{y}$. Isso se exprime através do seguinte critério de plastificação, que define o intervalo de tensões admissíveis:

$$
f(\sigma)=|\sigma|-\sigma_{y} \leq 0
$$

A evolução da plastificação $\left(\dot{\varepsilon}^{p} \neq 0\right)$ ocorre apenas se $f(\sigma)=0$, ou seja, se $|\sigma|=\sigma_{y}$. Caso $f(\sigma)<0$, ou seja, se $|\sigma|<\sigma_{y}$ a resposta imediata do material é exclusivamente elástica ( $\dot{\varepsilon}=\dot{\varepsilon}^{e}, \dot{\varepsilon}^{p}=0$ ). Usualmente, denomina-se $\dot{\lambda} \geq 0$ ao valor do módulo da taxa de deformação plástica. Portanto, valem as relações:

$$
\begin{aligned}
& \dot{\varepsilon}^{p}=\dot{\lambda}, \text { caso } \sigma=\sigma_{y} \\
& \dot{\varepsilon}^{p}=-\dot{\lambda}, \text { caso } \sigma=-\sigma_{y}
\end{aligned}
$$

De modo compacto, pode-se escrever:

$$
\dot{\varepsilon}^{p}=\dot{\lambda} \operatorname{sign}(\sigma)
$$

sendo $\operatorname{sign}(\sigma)=+1$, se $\sigma>0$ e $\operatorname{sign}(\sigma)=-1$, se $\sigma<0$.

Entre $\dot{\lambda}$ e $f$ há claramente uma condição de complementariedade, expressa na forma:

$$
\dot{\lambda} f=0
$$

Porém, um estado de tensão tal que $f(\sigma)=0$ não implica obrigatoriamente em evolução da plastificação. É necessário verificar a variação da função $f$ no tempo $\left(\dot{f}=\frac{\partial f}{\partial t}\right)$. Caso $f=0$ e $\dot{f}=0$, haverá evolução da plastificação pois o novo estado de tensão se manterá sobre o nível limite $\sigma_{y}$ e, portanto, $\dot{\lambda}>0$. Caso $f=0$ e $\dot{f}<0$, configura-se uma situação de descarregamento e, portanto, $\dot{\lambda}=0$ (não há variação da deformação plástica). A figura 12 ilustra as possibilidades descritas. Com isso se estabelece a chamada condição de consistência, dada por (100):

$$
\dot{\lambda} \dot{f}=0
$$

A condição de consistência permite obter uma expressão para $\dot{\lambda}$ impondo-se $\dot{f}=0$. Utilizando-se as eqs. (95), (96) e (98), tem-se:

$$
\dot{f}=\frac{\partial f}{\partial \sigma} \frac{\partial \sigma}{\partial t}=\frac{\partial|\sigma|}{\partial \sigma} \dot{\sigma}=\operatorname{sign}(\sigma) E\left(\dot{\varepsilon}-\dot{\varepsilon}^{p}\right)=\operatorname{sign}(\sigma) E \dot{\varepsilon}-\operatorname{sign}(\sigma) E \dot{\lambda} \operatorname{sign}(\sigma)=0
$$


Uma vez que $[\operatorname{sign}(\sigma)]^{2}=1$, obtém-se:

$\dot{\lambda}=\operatorname{sign}(\sigma) \dot{\varepsilon}$

Substituindo-se a eq. (101) na (98), chega-se à expressão da deformação plástica:

$\dot{\varepsilon}^{p}=\dot{\varepsilon}$

A eq. (102) indica que, no escoamento, todo o acréscimo de deformação é plástico.

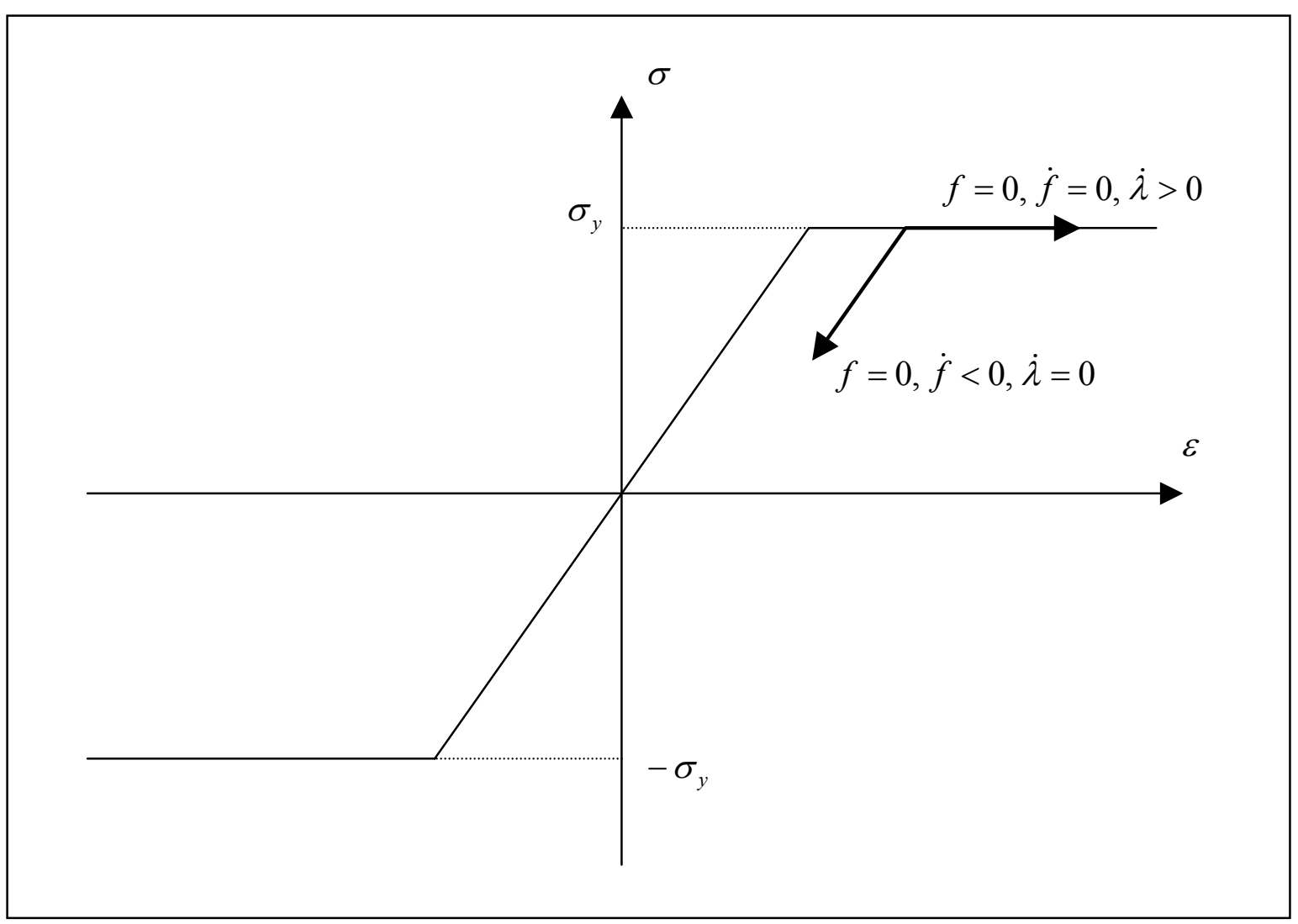

Figura 12 - Comportamento elastoplástico perfeito

\subsubsection{Comportamento elastoplástico com encruamento}

Neste caso o intervalo elástico inicial se altera com a evolução da plastificação, seja em tamanho, posição ou numa combinação de ambos.

O encruamento é chamado isótropo quando ocorre uma expansão do intervalo elástico de modo simétrico ao seu centro. Normalmente, postula-se que o encruamento seja uma função da deformação plástica acumulada (encruamento por deformação). Caso seja diretamente proporcional ao módulo da deformação plástica, o encruamento isótropo é dito linear, como ilustrado na figura 13. Para este caso, a função de plastificação tem por expressão:

$$
f(\sigma, \alpha)=|\sigma|-\left(\sigma_{y}+K \alpha\right) \leq 0
$$


Na eq. (103), $K$ é chamado módulo plástico de encruamento isótropo e $\alpha$ é uma medida da deformação plástica acumulada, definida conforme a expressão:

$$
\alpha=\int_{0}^{t}\left|\dot{\varepsilon}^{p}\right| d t
$$

O módulo utilizado na eq. (104) implica que a evolução da plastificação tanto na compressão como na tração produz encruamento, ou seja, um aumento do intervalo elástico inicial.

De modo análogo às eqs. (97a) e (97b), as condições sobre $\dot{\varepsilon}^{p}$ são agora expressas por:

$$
\begin{aligned}
& \dot{\varepsilon}^{p}=\dot{\lambda}, \text { caso } \sigma=\left(\sigma_{y}+K \alpha\right) \\
& \dot{\varepsilon}^{p}=-\dot{\lambda}, \text { caso } \sigma=-\left(\sigma_{y}+K \alpha\right)
\end{aligned}
$$

A eq. (98) é válida também neste caso.

Pode-se concluir das eqs (105a) e (105b) que $\dot{\lambda}=\left|\dot{\varepsilon}^{p}\right|$. E pode-se concluir da eq. (104) que $\dot{\alpha}=\left|\dot{\varepsilon}^{p}\right|$. Portanto, chega-se à igualdade:

$$
\dot{\alpha}=\dot{\lambda}
$$

Utilizando-se agora a relação de consistência $(\dot{\lambda}>0$ apenas se $\dot{f}=0)$ e as eqs. (95), (98), (103) e (106), é possível obter-se uma expressão para $\dot{\lambda}$ :

$$
\dot{f}=\frac{\partial f}{\partial \sigma} \dot{\sigma}+\frac{\partial f}{\partial \alpha} \dot{\alpha}=\operatorname{sign}(\sigma) E[\dot{\varepsilon}-\dot{\lambda} \operatorname{sign}(\sigma)]-K \dot{\lambda}=0
$$

Isolando-se $\dot{\lambda}$, obtém-se:

$$
\dot{\lambda}=\frac{\operatorname{sign}(\sigma) E}{(E+K)} \dot{\varepsilon}
$$

Combinando-se as eqs. (95), (98) e (107), calculam-se:

$$
\begin{aligned}
& \dot{\varepsilon}^{p}=\frac{E}{(E+K)} \dot{\varepsilon} \\
& \dot{\sigma}=\frac{E K}{(E+K)} \dot{\varepsilon}
\end{aligned}
$$

A relação $\frac{E K}{(E+K)}$ define o módulo elastoplástico tangente, no trecho de encruamento, como se vê na figura 13. Além disso, utilizando-se as eqs. (108) e (109) chega-se à expressão que dá o significado físico do módulo plástico $K$ : 


$$
K=\frac{\dot{\sigma}}{\dot{\varepsilon}^{p}}=\frac{\partial \sigma}{\partial \varepsilon^{p}}
$$

Portanto $K$ é a variação da tensão em relação à deformação plástica.

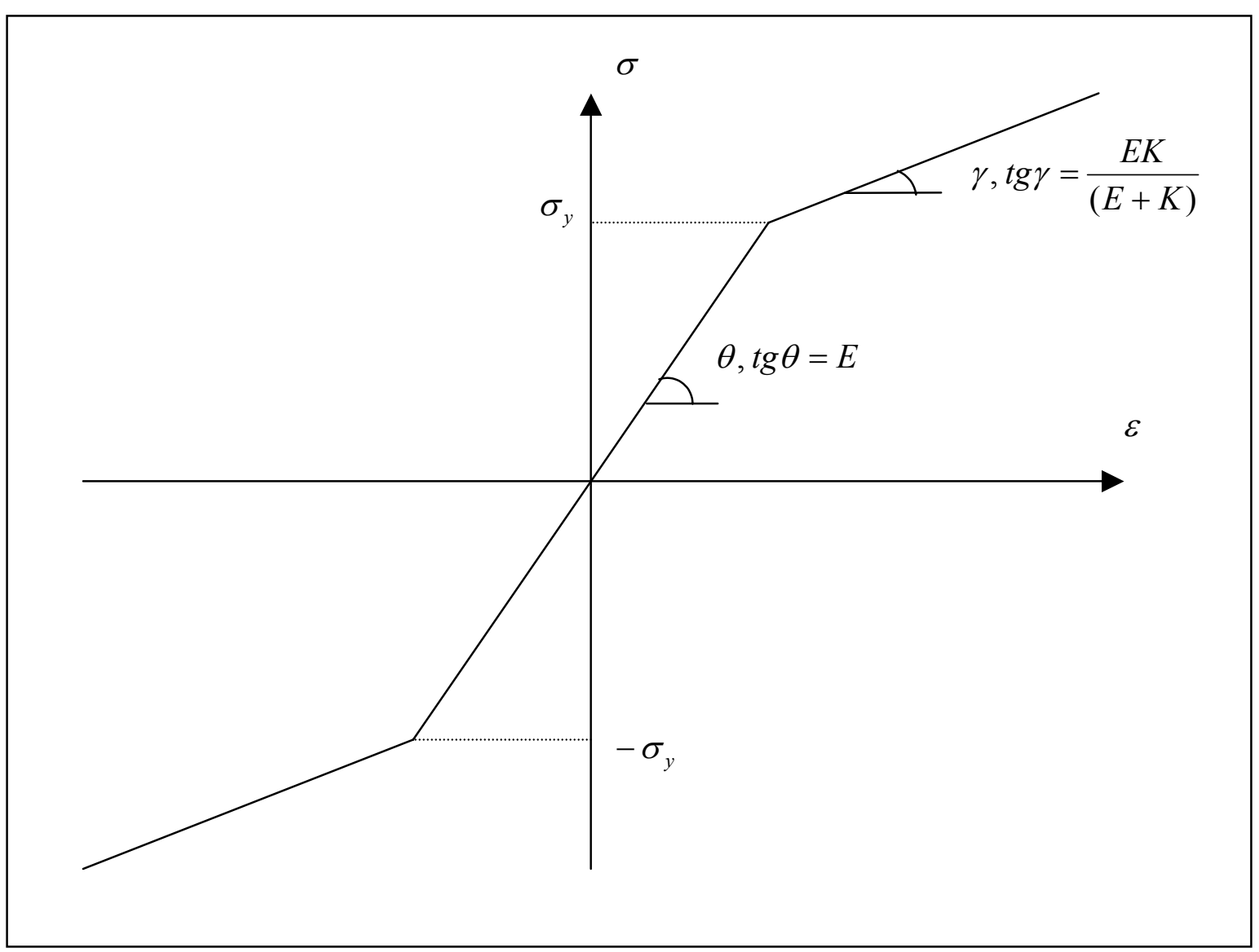

Figura 13 - Encruamento isótropo linear

O encruamento é denominado cinemático quando o intervalo elástico inicial tem seu tamanho mantido, mas sua origem no espaço das tensões é modificada pelo processo de plastificação. Neste caso, a função de plastificação assume a forma:

$$
f(\sigma, q)=|\sigma-q|-\sigma_{y} \leq 0
$$

A variável $q$ dá o deslocamento do intervalo elástico em relação à sua posição original, sendo, também, uma função da deformação plástica. No caso de uma relação linear entre ambas, a lei de evolução do parâmetro $q$ é:

$$
\dot{q}=H \dot{\varepsilon}^{p}
$$

sendo a constante $H$ denominada módulo plástico de encruamento cinemático.

Deve-se observar que o sentido da variação do parâmetro $q$ é definido pelo sentido do fluxo plástico. Como exemplificado na figura 14, uma plastificação devida à tração altera o limite elástico à tração para $\left(\sigma_{y}+q_{1}\right)$ e reduz (em módulo) o limite elástico à 
compressão. Essa "assimetria" induzida pelo modelo de encruamento cinemático permite reproduzir o chamado efeito BAUSCHINGER (1886).

Seguindo um procedimento análogo ao anterior, a taxa de deformação plástica é definida a partir das seguintes condições:

$$
\begin{aligned}
& \dot{\varepsilon}^{p}=\dot{\lambda}, \text { caso } \sigma-q=\sigma_{y} \\
& \dot{\varepsilon}^{p}=-\dot{\lambda}, \text { caso } \sigma-q=-\sigma_{y}
\end{aligned}
$$

Resumidamente, escreve-se:

$$
\dot{\varepsilon}^{p}=\dot{\lambda} \operatorname{sign}(\sigma-q)
$$

sendo $\operatorname{sign}(\sigma-q)=+1$, se $(\sigma-q)>0$ e $\operatorname{sign}(\sigma-q)=-1$, se $(\sigma-q)<0$.

Combinando-se as eqs. (112) e (114), pode-se escrever que:

$$
\dot{q}=\dot{\lambda} H \operatorname{sign}(\sigma-q)
$$

A expressão para $\dot{\lambda}$ é obtida impondo-se $\dot{f}=0$. Fazendo-se, então, uso das eqs. (95), (111), (114) e (115):

$$
\dot{f}=\frac{\partial f}{\partial \sigma} \dot{\sigma}+\frac{\partial f}{\partial q} \dot{q}=\operatorname{sign}(\sigma-q) E[\dot{\varepsilon}-\dot{\lambda} \operatorname{sign}(\sigma-q)]-\operatorname{sign}(\sigma-q) H \dot{\lambda} \operatorname{sign}(\sigma-q)=0
$$

e isolando-se $\dot{\lambda}$, obtém-se:

$$
\dot{\lambda}=\frac{\operatorname{sign}(\sigma-q) E}{(E+H)} \dot{\varepsilon}
$$

Finalmente, com as eqs. (95), (114) e (116), calculam-se:

$$
\begin{aligned}
& \dot{\varepsilon}^{p}=\frac{E}{(E+H)} \dot{\varepsilon} \\
& \dot{\sigma}=\frac{E H}{(E+H)} \dot{\varepsilon}
\end{aligned}
$$

sendo $\frac{E H}{(E+H)}$ o módulo elastoplástico tangente, cujo significado está ilustrado na figura 14. Por outro lado, utilizando-se as eqs (117) e (118), encontra-se a seguinte expressão para o módulo plástico $H$ :

$$
H=\frac{\dot{\sigma}}{\dot{\varepsilon}^{p}}=\frac{\partial \sigma}{\partial \varepsilon^{p}}
$$




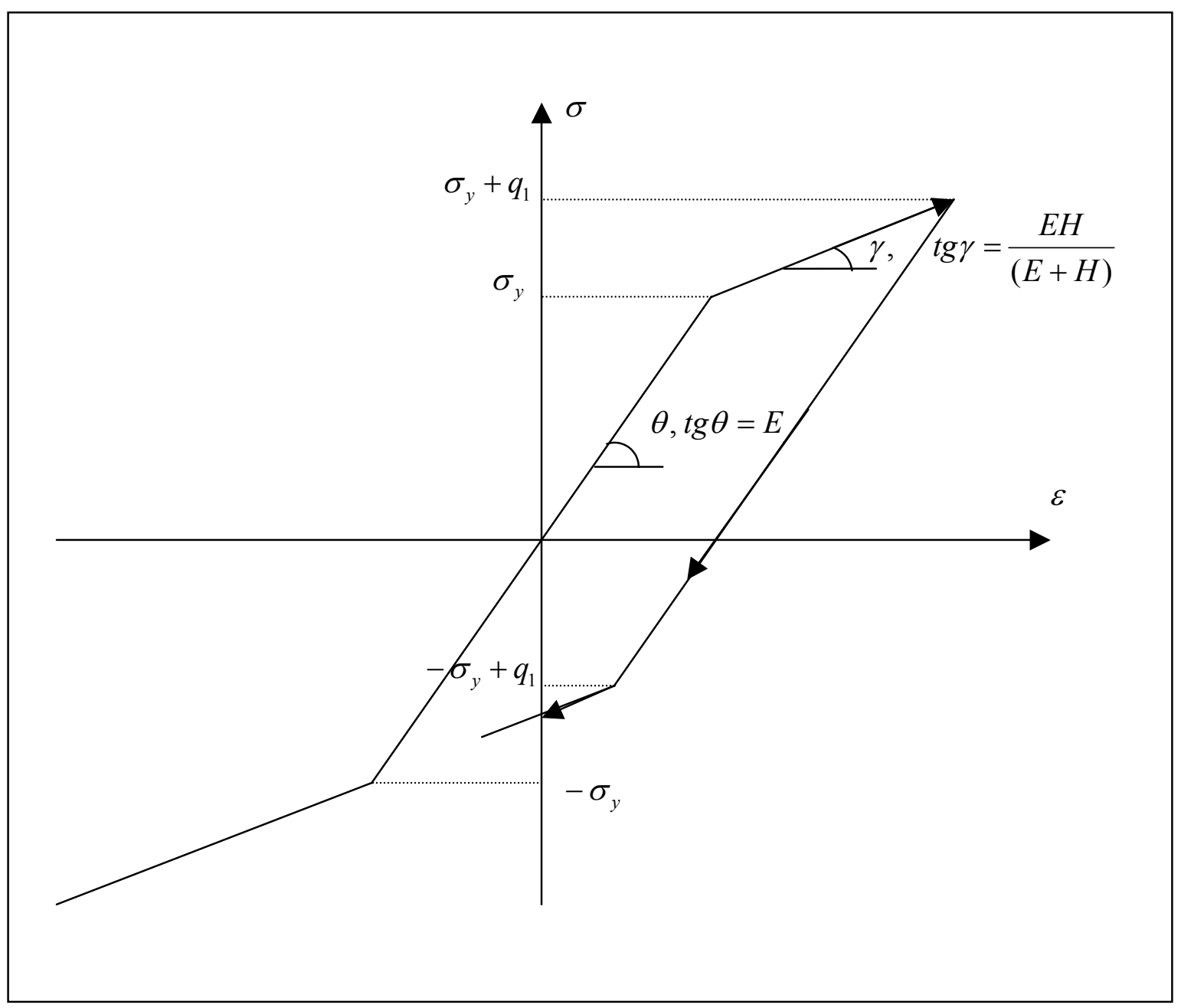

Figura 14 - Encruamento cinemático

\subsubsection{Comportamento elastoplástico tridimensional.}

O modelo apresentado a seguir é baseado numa generalização daquele desenvolvido para o caso unidimensional. Admite-se a decomposição aditiva do tensor taxa de deformação total $(\dot{\varepsilon})$ em parcelas elástica $\left(\dot{\varepsilon}^{e}\right)$ e plástica $\left(\dot{\varepsilon}^{p}\right)$.

$$
\dot{\varepsilon}=\dot{\varepsilon}^{e}+\dot{\varepsilon}^{p}
$$

A relação constitutiva é expressa (em termos de taxas) por:

$$
\dot{\sigma}=C \dot{\varepsilon}^{e}=C\left(\dot{\varepsilon}-\dot{\varepsilon}^{p}\right)
$$

$\mathrm{Na}$ eq. (121), $\sigma$ é o tensor de tensões e $C$ é o tensor constitutivo linear de quarta ordem, dado por:

$$
C=\lambda(\mathrm{I} \otimes \mathrm{I})+2 \mu \Pi
$$

$\mathrm{Na}$ eq. (122), I e $\Pi$ são os tensores identidade de segunda e quarta ordens, respectivamente, e $\lambda$ e $\mu$ são as constantes de Lamé, relacionadas com o módulo de elasticidade longitudinal $E$ e com o coeficiente de Poisson $v$ por: 


$$
\begin{aligned}
& \lambda=\frac{E v}{(1+v)(1-2 v)} \\
& \mu=\frac{E}{2(1+v)}
\end{aligned}
$$

Considera-se que o encruamento seja determinado por um vetor $R$. Este vetor é função da deformação plástica (ou alguma medida associada) e envolve parâmetros do material (como os módulos plásticos $H$ e $K$ ). Sua dimensão varia conforme os tipos de encruamento e os modelos considerados. Genericamente, tem-se:

$$
\begin{aligned}
& R=R\left(\varepsilon^{p} ; H, K\right) \\
& \dot{R}=\dot{R}\left(\dot{\varepsilon}^{p} ; H, K\right)
\end{aligned}
$$

O critério de plastificação é definido por uma função $f$ tal que:

$$
f(\sigma, R) \leq 0
$$

Para permitir uma representação geométrica do critério de plastificação, é usual escrevê-lo em termos das componentes principais $\left(\sigma_{1}, \sigma_{2}, \sigma_{3}\right)$ do tensor de tensões. Tomando-se como referência eixos associados às tensões principais e considerando-se um certo nível de encruamento caracterizado por $\bar{R}$, pode-se então representar a chamada superfície de plastificação, ou seja, a região que reúne os pontos para os quais $f(\sigma, \bar{R})=0$. É o que mostra a figura 15 . Nota-se que pontos tais que $f<0$ estão no interior da região definida pela superfície de plastificação e representam estados de tensão correspondentes ao regime elástico para um certo nível de encruamento.

$\mathrm{Na}$ generalização em questão, admite-se que a lei de evolução do tensor de deformações plásticas seja dada por:

$$
\dot{\varepsilon}^{p}=\dot{\lambda} r(\sigma, R)
$$

sendo $r(\sigma, R)$ um tensor de segunda ordem que estabelece a direção do fluxo plástico e $\dot{\lambda} \geq 0$ a variável que dá a "velocidade" da plastificação.

Adota-se para o vetor $R$ uma lei de evolução do tipo:

$$
\dot{R}=-\dot{\lambda} h(\sigma, R)
$$

que por incluir $\dot{\lambda}$ representa uma forma particular da eq. (124b). Na eq. (127), $h(\sigma, R)$ é o vetor que define uma direção para $R$ no seu espaço.

Caso se tenha $f(\sigma, R)<0$, sabe-se, pela condição de complementariedade, que $\dot{\lambda}=0$. Nessas condições, conclui-se, de (126) e (127), que tanto a deformação plástica 
quanto o encruamento não evoluem ( $\dot{\varepsilon}^{p}=0$ e $\dot{R}=0$ ), ou seja, o ponto em questão está no domínio elástico.

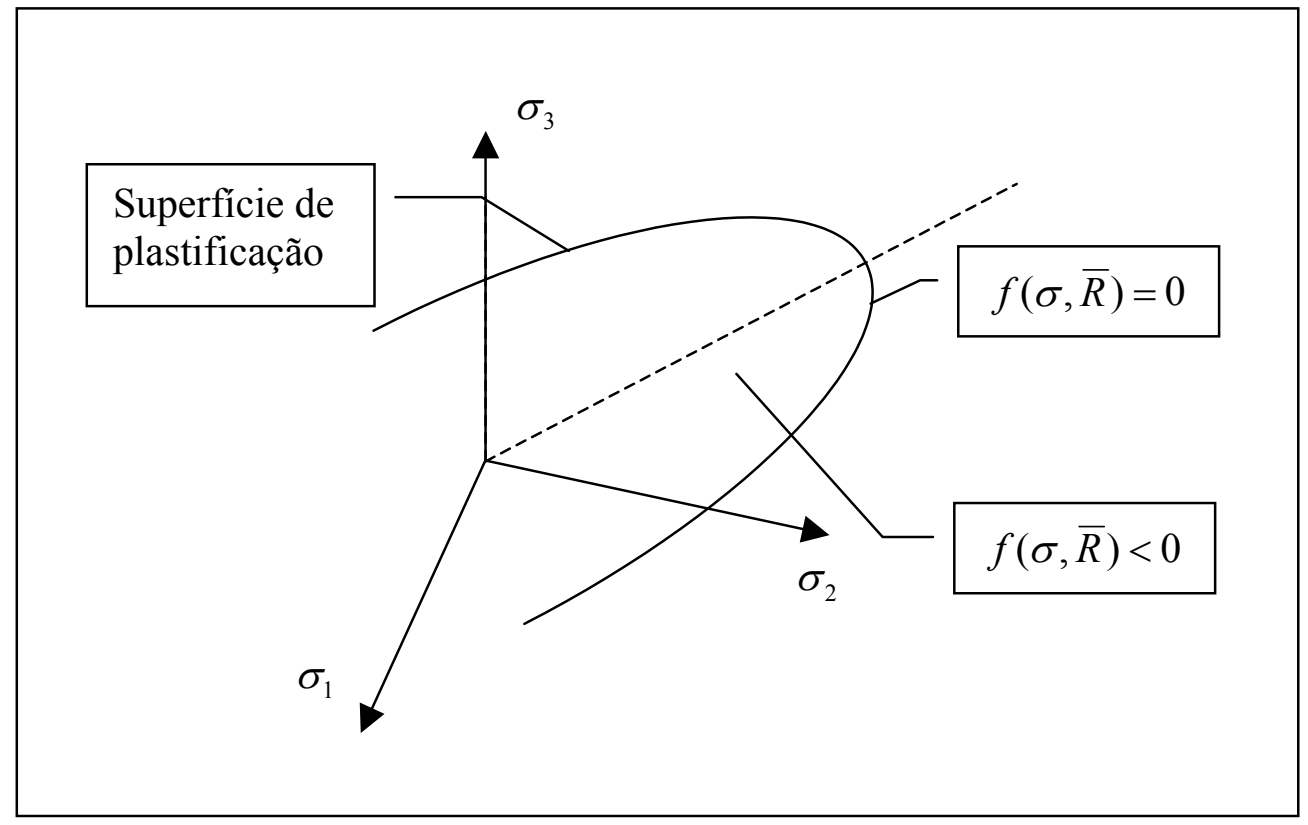

Figura 15 - Superfície de plastificação no espaço das tensões principais.

Caso se tenha $f(\sigma, R)=0$, tem-se que $\dot{\lambda} \geq 0$, ou seja, a plastificação pode estar, ou não, ocorrendo. Caso $\dot{f}(\sigma, R)<0$, a condição de consistência impõe que $\dot{\lambda}=0$, ou seja, está ocorrendo um descarregamento elástico. Caso $\dot{f}(\sigma, R)=0$, há duas situações possíveis:

- $\dot{\lambda}>0$ - há evolução da plastificação e do encruamento.

- $\dot{\lambda}=0$ - não há variação na deformação plástica e no encruamento. As componentes de tensão mudam para uma outra posição na superfície de plastificação, constituindo um estado de carregamento neutro.

Da relação de consistência, obtém-se uma expressão para $\dot{\lambda}>0$ :

$\dot{f}=f_{\sigma} \cdot \dot{\sigma}+f_{R} \cdot \dot{R}=0$

Utilizando-se as eqs. (121), (126) e (127), obtém-se:

$\dot{\lambda}=\frac{f_{\sigma} \cdot C \dot{\varepsilon}}{\left(f_{\sigma} \cdot C r+f_{R} \cdot h\right)}$

Substituindo-se a eq. (128) em (126), obtém-se:

$$
\dot{\varepsilon}^{p}=\frac{\left(f_{\sigma} \cdot C \dot{\varepsilon}\right) r}{\left(f_{\sigma} \cdot C r+f_{R} \cdot h\right)}=\frac{\left(r \otimes C f_{\sigma}\right)}{\left(f_{\sigma} \cdot C r+f_{R} \cdot h\right)} \dot{\varepsilon}
$$

E com as eqs. (128) e (127), obtém-se: 


$$
\dot{R}=-\frac{\left(f_{\sigma} \cdot C \dot{\varepsilon}\right) h}{\left(f_{\sigma} \cdot C r+f_{R} \cdot h\right)}
$$

Utilizando-se a eq. (129) e substituindo-a na eq. (121) é possível obter a relação $\dot{\sigma}=C^{e p} \dot{\varepsilon}$, onde $C^{e p}$ é o tensor elastoplástico tangente de quarta ordem. Sua expressão é:

$$
C^{e p}= \begin{cases}C & \text { para } \dot{\lambda}=0 \\ C-\frac{\left(C r \otimes C f_{\sigma}\right)}{\left(f_{\sigma} \cdot C r+f_{R} \cdot h\right)} & \text { para } \dot{\lambda}>0\end{cases}
$$

O tensor $C^{e p}$ não é, em geral, simétrico. Pode-se obter a sua simetria adotando-se as seguintes condições, também denominadas regras de associatividade:

1) $r=f_{\sigma}$ : é também chamada de regra da normalidade, pois implica em que o fluxo de deformação plástica tenha a direção da normal à superfície de plastificação.

2) $h=D f_{R}$, sendo $D$ a matriz dos módulos plásticos de encruamento.

Com a adoção das condições referidas, garante-se que o denominador da eq. (128) seja sempre positivo, visto que o tensor $C$ é positivo definido, bem como a matriz dos módulos plásticos de encruamento $D$. Por outro lado, como $\dot{\lambda} \geq 0$, impõe-se que $f_{\sigma} \cdot C \dot{\varepsilon} \geq 0$. Isso implica que no espaço das tensões principais o ângulo entre os vetores $f_{\sigma}$ e $C \dot{\varepsilon}$ seja menor ou igual a $90^{\circ}$ (conforme mostrado na figura 16 ).

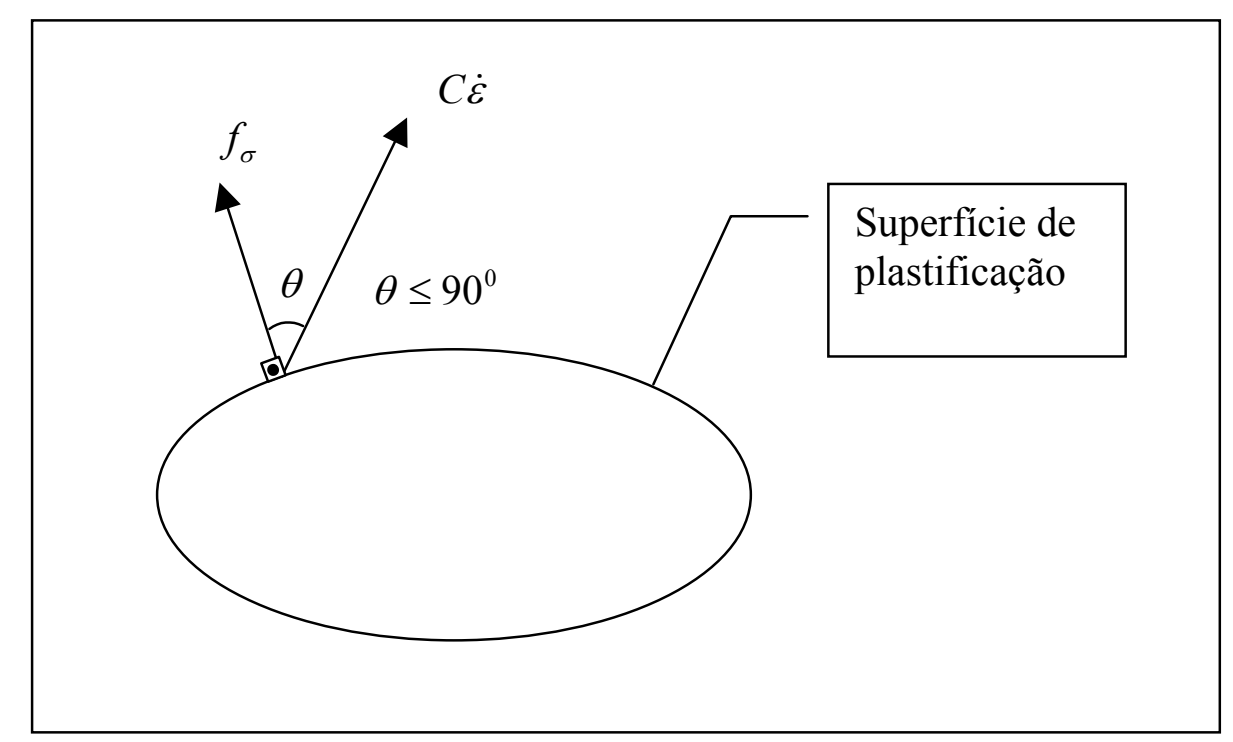

Figura 16 - Condição de carregamento

As expressões deduzidas até aqui podem ser aplicadas a diferentes modelos constitutivos elastoplásticos, visto que nenhuma particularização foi feita a respeito da 
função de plastificação adotada. Nos itens seguintes, apresentam-se dois dos modelos elastoplásticos mais comumente utilizados, especialmente no caso de materiais metálicos, que são o elastoplástico perfeito com função de plastificação de von Mises e o modelo de encruamento isótropo linear, também com critério de plastificação de von Mises.

\subsubsection{Modelo elastoplástico perfeito - critério de von Mises}

Adota-se a função de plastificação de von Mises, sem considerar o encruamento, cuja expressão é:

$$
f=\sqrt{2 J_{2}}-\sqrt{\frac{2}{3}} \sigma_{t} \leq 0
$$

onde $\sigma_{t}$ é o valor da tensão de escoamento do material num ensaio da tração simples.

Na eq. (131), $J_{2}$ é o segundo invariante do tensor desviador de tensões $(S)$ e dado, em função deste, por:

$$
J_{2}=\frac{\|S\|^{2}}{2}=\frac{(S \cdot S)}{2}
$$

O tensor $S$ é obtido quando se extrai do tensor de tensões $\sigma$ a sua parcela hidrostática $\left(\sigma_{m} \mathrm{I}\right)$ :

$$
S=\sigma-\sigma_{m} \mathrm{I}
$$

sendo $\sigma_{m}$ dado pela equação:

$$
\sigma_{m}=\frac{\left(\sigma_{x}+\sigma_{y}+\sigma_{z}\right)}{3}=\frac{\operatorname{tr} \sigma}{3}
$$

Uma característica do tensor desviador $S$ é que seu traço é nulo.

Em termos de componentes genéricas do tensor de tensões, o invariante $J_{2}$ tem a seguinte expressão:

$$
J_{2}=\frac{1}{6}\left[\left(\sigma_{x}-\sigma_{y}\right)^{2}+\left(\sigma_{x}-\sigma_{z}\right)^{2}+\left(\sigma_{y}-\sigma_{z}\right)^{2}\right]+\tau_{x y}^{2}+\tau_{x z}^{2}+\tau_{y z}^{2}
$$

No espaço das tensões principais, a superfície de plastificação definida pelo critério de von Mises é um cilindro de raio $\sqrt{\frac{2}{3}} \sigma_{t}$ cujo eixo corresponde ao eixo hidrostático $\left(\sigma_{1}=\sigma_{2}=\sigma_{3}=\sigma_{m}\right)$, como se mostra na figura 17 . 
Observa-se que é bastante usual que a expressão do critério de plastificação de von Mises seja dada pela função $f=\sigma_{e q}-\sigma_{t} \leq 0$, na qual $\sigma_{e q}$ é denominada tensão equivalente ou tensão de von Mises, dada por:

$$
\sigma_{e q}=\sqrt{3 J_{2}}
$$

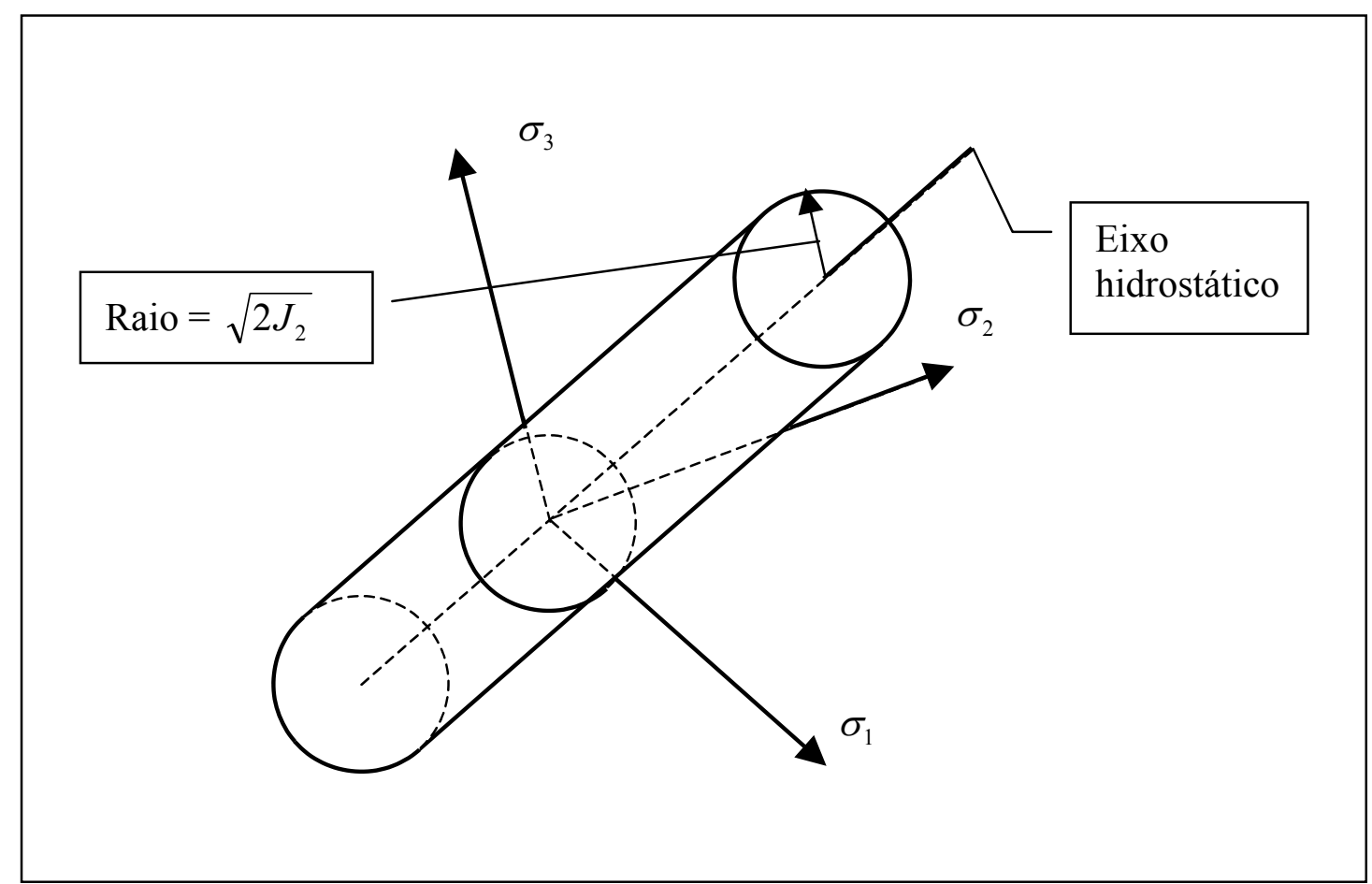

Figura 17 - Superfície de plastificação de von Mises.

Considerando-se as relações (132), (133) e (134), o invariante $J_{2}$ pode ser escrito em função do tensor de tensões $\sigma$ como mostrado abaixo:

$$
J_{2}=\frac{\|\sigma\|^{2}}{2}-\frac{1}{6}(\operatorname{tr} \sigma)^{2}
$$

Substituindo-se a eq. (137) na (131), obtém-se:

$$
f=\sqrt{\|\sigma\|^{2}-\frac{1}{3}(\operatorname{tr} \sigma)^{2}}-\sqrt{\frac{2}{3}} \sigma_{t} \leq 0
$$

Valendo a regra da normalidade, a evolução do tensor de deformações plásticas fica expressa na forma:

$$
\dot{\varepsilon}^{p}=\dot{\lambda} f_{\sigma}=\dot{\lambda} \frac{\sigma-\frac{1}{3}(\operatorname{tr} \sigma) I}{\sqrt{\|\sigma\|^{2}-\frac{1}{3}(\operatorname{tr} \sigma)^{2}}}=\dot{\lambda} \frac{S}{\|S\|}=\dot{\lambda}_{n}
$$


Na equação (139) o tensor $n$ tem norma unitária e dá a direção do fluxo plástico. Além disso, o tensor de deformações plásticas apresenta traço nulo, o que implica em que a deformação plástica ocorre sem provocar variação de volume (é um processo isocórico).

Para a obtenção de $\dot{\lambda}$, parte-se da eq. (128), adotando-se $r=f_{\sigma}=n$ (normalidade) e $h=0$ (elastoplasticidade perfeita):

$$
\dot{\lambda}=\frac{n \cdot C \dot{\varepsilon}}{n \cdot C n}
$$

Aplicando-se à eq. (140) o tensor constitutivo dos módulos elásticos dado pela eq. (122) e fazendo-se uso do fato que $\operatorname{tr} n=0$ e que $(n \cdot n)=\|n\|^{2}=1$, chega-se a:

$$
\dot{\lambda}=n \cdot \dot{\varepsilon}
$$

Substituindo-se, então, a eq. (141) na (139), tem-se a expressão da taxa de deformação plástica:

$$
\dot{\varepsilon}^{p}=\dot{\lambda} n=(n \cdot \dot{\varepsilon}) n=(n \otimes n) \dot{\varepsilon}
$$

A expressão da taxa do tensor de tensões é obtida substituindo-se a eq. (142) na (121):

$$
\dot{\sigma}=C[\dot{\varepsilon}-(n \otimes n) \dot{\varepsilon}]=C[\Pi-(n \otimes n)] \dot{\varepsilon}
$$

válida apenas caso $\dot{\lambda}>0$

Finalmente, a expressão do módulo elastoplástico tangente, tal que $\dot{\sigma}=C^{e p} \dot{\varepsilon}$, resulta:

$$
C^{e p}= \begin{cases}C, & \text { para } \dot{\lambda}=0 \\ C\left(\prod-n \otimes n\right)=\lambda(I \otimes I)+2 \mu(\Pi-n \otimes n), & \text { para } \dot{\lambda}>0\end{cases}
$$

3.3.2.2 Modelo elastoplástico com encruamento isótropo linear - critério de von Mises

O critério de plastificação de von Mises, considerando-se somente o encruamento isótropo, passa a ser dado por:

$$
f=\sqrt{2 J_{2}}-\sqrt{\frac{2}{3}}\left(\sigma_{t}+R\right) \leq 0
$$

No caso de uma única forma de encruamento, como o isótropo linear, o vetor $R$ passa a apresentar uma única componente, de modo que assume uma natureza de variável escalar, dada por: 


$$
R=K \alpha
$$

sendo que $K$ tem o mesmo significado do caso unidimensional e $\alpha$ é um parâmetro relacionado à evolução da deformação plástica.

Utilizando-se a eq. (127), adotando-se a associatividade no encruamento ( $\left.h=D f_{R}\right)$ e sendo $D=K$, pode-se definir a lei de evolução de $R$ :

$$
\dot{R}=-\dot{\lambda} K\left(-\sqrt{\frac{2}{3}}\right)=\dot{\lambda} \sqrt{\frac{2}{3}} K
$$

Comparando-se a eq. (146) com a expressão da eq. (145) na forma de taxa no tempo ( $\dot{R}=K \dot{\alpha}$ ), obtém-se a expressão da lei de evolução do parâmetro $\alpha$ :

$$
\dot{\alpha}=\dot{\lambda} \sqrt{\frac{2}{3}}
$$

A lei de evolução do tensor de deformações plásticas $\dot{\varepsilon}^{p}$ é a mesma do caso elastoplástico perfeito e é dada pela eq. (139).

O cálculo de $\dot{\lambda}$ irá agora incluir os termos referentes ao encruamento. Utilizando-se a eq. (144) e as condições de normalidade do fluxo plástico $\left(r=f_{\sigma}=n\right)$ e a associatividade no encruamento $\left(h=D f_{R}\right)$, chega-se, por meio da eq. (128), a:

$$
\dot{\lambda}=\frac{n \cdot C \dot{\varepsilon}}{n \cdot C n+\frac{2}{3} K}
$$

Aplicando-se à eq. (148) o valor do tensor constitutivo elástico de quarta ordem $C$, dado por (122), e sendo válidas as relações $\operatorname{tr} n=0$ e $(n \cdot n)=\|n\|^{2}=1$, tem-se:

$$
\dot{\lambda}=\frac{n \cdot \dot{\varepsilon}}{\left(1+\frac{K}{3 \mu}\right)}
$$

Com a eq. (149) na eq. (139), chega-se à expressão da taxa de deformação plástica:

$$
\dot{\varepsilon}^{p}=\frac{(n \otimes n) \dot{\varepsilon}}{\left(1+\frac{K}{3 \mu}\right)}
$$

A expressão da taxa do tensor de tensões é obtida substituindo-se (150) em (121):

$$
\dot{\sigma}=C\left[\dot{\varepsilon}-\frac{(n \otimes n)}{\left(1+\frac{K}{3 \mu}\right)} \dot{\varepsilon}\right]=C\left[\Pi-\frac{(n \otimes n)}{\left(1+\frac{K}{3 \mu}\right)}\right] \dot{\varepsilon}
$$

E a expressão do módulo elastoplástico tangente resulta, portanto: 


$$
C^{e p}= \begin{cases}C, & \text { para } \dot{\lambda}=0 \\ C\left[\Pi-\frac{(n \otimes n)}{\left(1+\frac{K}{3 \mu}\right)}\right]=\lambda(I \otimes I)+2 \mu\left(\Pi-\frac{(n \otimes n)}{\left(1+\frac{K}{3 \mu}\right),}\right. & \text { para } \dot{\lambda}>0\end{cases}
$$

\section{Algoritmo para análise incremental-iterativa.}

As expressões apresentadas anteriormente são dadas em termos de taxas. Apresentam-se agora as expressões em forma de passo finito, mais adaptadas a uma análise não-linear incremental-iterativa. Adota-se um algoritmo de resolução do tipo implícito, constituído de uma etapa de previsão e outra de correção.

Por este algoritmo, uma vez conhecidas as variáveis que definem o modelo constitutivo num passo $n$, admite-se que, no passo $n+1$, essas variáveis tenham evoluído segundo um processo puramente elástico. É essa a etapa de previsão. Caso se verifique que houve evolução da plastificação é preciso corrigir as variáveis correspondentes ao passo $n+1$ (etapa de correção).

As expressões que definem o modelo constitutivo na sua forma incremental implícita são:

$$
\begin{aligned}
& \varepsilon_{n+1}=\varepsilon_{n}+\Delta \varepsilon_{n} \\
& \sigma_{n+1}=C\left(\varepsilon_{n+1}-\varepsilon_{n+1}^{p}\right) \\
& \varepsilon_{n+1}^{p}=\varepsilon_{n}^{p}+\Delta \lambda n_{n+1}, \text { sendo } n_{n+1}=\frac{S_{n+1}}{\left\|S_{n+1}\right\|} \\
& S_{n+1}=\sigma_{n+1}-\frac{1}{3} \operatorname{tr} \sigma_{n+1} \mathrm{I} \\
& \alpha_{n+1}=\alpha_{n}+\sqrt{\frac{2}{3}} \Delta \lambda \\
& f_{n+1}=\left\|S_{n+1}\right\|-\sqrt{\frac{2}{3}}\left(\sigma_{t}+K \alpha_{n+1}\right)
\end{aligned}
$$

Na expressão de $f_{n+1}$ utiliza-se a relação $\|S\|=\sqrt{2 J_{2}}$, obtida da eq. (132).

Nas equações acima, $\Delta \lambda$ dá a magnitude da evolução das variáveis associadas à plastificação. 
Sendo conhecidos no passo $n$ os valores de $\varepsilon_{n}, \Delta \varepsilon_{n}, \varepsilon_{n}^{p}$ e $\alpha_{n}$, tem-se os seguintes valores das variáveis no passo $n+1$, admitindo-se previsão elástica $(\Delta \lambda=0)$.

$$
\begin{aligned}
& \varepsilon_{n+1}^{p, p r}=\varepsilon_{n}^{p} \\
& \alpha_{n+1}^{p r}=\alpha_{n} \\
& \sigma_{n+1}^{p r}=C\left(\varepsilon_{n+1}-\varepsilon_{n+1}^{p, p r}\right)=C\left(\varepsilon_{n+1}-\varepsilon_{n}^{p}\right) \\
& S_{n+1}^{p r}=\sigma_{n+1}^{p r}-\frac{1}{3} \operatorname{tr} \sigma_{n+1}^{p r} \mathrm{I} \\
& f_{n+1}^{p r}=\left\|S_{n+1}^{p r}\right\|-\sqrt{\frac{2}{3}}\left(\sigma_{t}+K \alpha_{n+1}^{p r}\right)=\left\|S_{n+1}^{p r}\right\|-\sqrt{\frac{2}{3}}\left(\sigma_{t}+K \alpha_{n}\right)
\end{aligned}
$$

Caso se tenha $f_{n+1}^{p r} \leq 0$, os valores de previsão das variáveis estão corretos para o passo $n+1$. Porém, se $f_{n+1}^{p r}>0$, passa-se à etapa de correção dos valores previstos, uma vez que essa condição não é compatível devido à convexidade da função $f$ e indica evolução da plastificação nesse passo. O que se faz basicamente é calcular o valor de $\Delta \lambda$ de tal modo que se tenha $f_{n+1}=0$. Conhecido o valor de $\Delta \lambda$, atualizam-se os valores de todos os demais parâmetros.

Inicialmente deve-se obter uma relação entre os tensores desviadores $S_{n+1}$ e $S_{n+1}^{p r}$. Aplicando-se o tensor constitutivo elástico, dado pela eq. (122), à expressão de $\sigma_{n+1}^{p r}$, obtém-se:

$$
\sigma_{n+1}^{p r}=\lambda\left(\operatorname{tr} \varepsilon_{n+1}\right) \mathrm{I}+2 \mu \varepsilon_{n+1}-2 \mu \varepsilon_{n}^{p}
$$

Substituindo-se a eq. (152) na expressão de $S_{n+1}^{p r}$ e sendo $\operatorname{tr} \varepsilon_{n}^{p}=0$, obtém-se:

$$
S_{n+1}^{p r}=2 \mu\left(e_{n+1}-\varepsilon_{n}^{p}\right)
$$

sendo $e_{n+1}$ a parcela desviadora do tensor de deformações, dada por:

$$
e_{n+1}=\varepsilon_{n+1}-\frac{1}{3} \operatorname{tr} \varepsilon_{n+1} \mathrm{I}
$$

Substitui-se agora o tensor constitutivo dado por (122) na expressão de $\sigma_{n+1}$ :

$$
\sigma_{n+1}=\lambda\left(\operatorname{tr} \varepsilon_{n+1}\right) \mathrm{I}+2 \mu \varepsilon_{n+1}-2 \mu \varepsilon_{n+1}^{p}
$$

sendo também nulo o traço de $\varepsilon_{n+1}^{p}$.

Aplica-se então a eq. (155) à expressão de $S_{n+1}$, obtendo-se:

$$
S_{n+1}=2 \mu\left(e_{n+1}-\varepsilon_{n+1}^{p}\right)
$$


sendo $e_{n+1}$ dado pela eq. (154).

Substituindo-se a expressão de $\varepsilon_{n+1}^{p}$ na eq. (156), obtém-se:

$$
S_{n+1}=2 \mu\left(e_{n+1}-\varepsilon_{n}^{p}-\Delta \lambda n_{n+1}\right)
$$

Da comparação das eqs. (153) e (157), obtém-se a igualdade:

$$
S_{n+1}=S_{n+1}^{p r}-2 \mu \Delta \lambda n_{n+1}
$$

Da definição de $n_{n+1}$ obtém-se a relação: $S_{n+1}=\left\|S_{n+1}\right\| n_{n+1}$. Substituindo-se esta relação na eq. (158), pode-se escrever:

$$
\left(\left\|S_{n+1}\right\|+2 \mu \Delta \lambda\right) n_{n+1}=\left\|S_{n+1}^{p r}\right\| n_{n+1}^{p r}
$$

De modo análogo à definição de $n_{n+1}$, adota-se $n_{n+1}^{p r}=\frac{S_{n+1}^{p r}}{\left\|S_{n+1}^{p r}\right\|}$. A partir da eq. (159) podem-se extrair as seguintes relações:

$$
\begin{gathered}
n_{n+1}=n_{n+1}^{p r}=\frac{S_{n+1}^{p r}}{\left\|S_{n+1}^{p r}\right\|} \\
\left\|S_{n+1}\right\|=\left\|S_{n+1}^{p r}\right\|-2 \mu \Delta \lambda
\end{gathered}
$$

Impõe-se agora a condição $f_{n+1}=0$, substituindo-se na expressão de $f_{n+1}$ a eq. (161) e a expressão de $\alpha_{n+1}$ :

$$
\left\|S_{n+1}^{p r}\right\|-2 \mu \Delta \lambda-\sqrt{\frac{2}{3}}\left(\sigma_{t}+K \alpha_{n}\right)-\frac{2}{3} K \Delta \lambda=0
$$

O primeiro e o terceiro termos da eq. (162) equivalem à expressão de $f_{n+1}^{p r}$. Isolando-se $\Delta \lambda$, tem-se :

$$
\Delta \lambda=\frac{f_{n+1}^{p r}}{\left(2 \mu+\frac{2}{3} K\right)}
$$

Conhecido o valor de $\Delta \lambda$, atualizam-se $\alpha_{n+1}$ e $\varepsilon_{n+1}^{p}$; uma vez conhecido $\varepsilon_{n+1}^{p}$, atualiza-se o valor de $\sigma_{n+1}$.

Outra relação de interesse, na medida em que se usa um processo incrementaliterativo do tipo Newton-Raphson, é a do tensor elastoplástico algorítmico tangente $C_{n+1}^{e p}$, dado por:

$$
C_{n+1}^{e p}=\frac{\partial \sigma_{n+1}}{\partial \varepsilon_{n+1}}
$$


A partir das expressões de $\sigma_{n+1}$ e $\varepsilon_{n+1}^{p}$, escreve-se:

$$
\sigma_{n+1}=C \varepsilon_{n+1}-C \varepsilon_{n}^{p}-\Delta \lambda C n_{n+1}
$$

A derivada parcial indicada na eq. (164) deve ser feita em relação a cada um dos três termos da eq. (165).

Em relação ao primeiro termo:

$$
\frac{\partial\left(C \varepsilon_{n+1}\right)}{\partial \varepsilon_{n+1}}=C
$$

Em relação ao segundo termo:

$$
\frac{\partial\left(-C \varepsilon_{n}^{p}\right)}{\partial \varepsilon_{n+1}}=0
$$

Antes de aplicar a derivada ao terceiro termo é conveniente substituir no mesmo a expressão do tensor $C$. O fato de que $\operatorname{tr}\left(n_{n+1}\right)=0$ implica em que $C n_{n+1}=2 \mu n_{n+1}$. Assim, obtém-se:

$$
\frac{\partial\left(-2 \mu \Delta \lambda n_{n+1}\right)}{\partial \varepsilon_{n+1}}=-2 \mu\left(\Delta \lambda \frac{\partial n_{n+1}}{\partial \varepsilon_{n+1}}+n_{n+1} \otimes \frac{\partial \Delta \lambda}{\partial \varepsilon_{n+1}}\right)
$$

É preciso calcular os tensores $\frac{\partial n_{n+1}}{\partial \varepsilon_{n+1}}$ e $\frac{\partial \Delta \lambda}{\partial \varepsilon_{n+1}}$. Utilizando-se a eq. (160), pode-se escrever o primeiro na forma:

$$
\frac{\partial\left(\frac{S_{n+1}^{p r}}{\left\|S_{n+1}^{p r}\right\|}\right)}{\partial \varepsilon_{n+1}}=\frac{1}{\left\|S_{n+1}^{p r}\right\|} \frac{\partial S_{n+1}^{p r}}{\partial \varepsilon_{n+1}}-\frac{1}{\left\|S_{n+1}^{p r}\right\|^{2}} S_{n+1}^{p r} \otimes \frac{\partial\left\|S_{n+1}^{p r}\right\|}{\partial \varepsilon_{n+1}}
$$

A partir das eqs. (153) e (154), pode-se escrever, fazendo-se uso de tensores de quarta ordem:

$$
S_{n+1}^{p r}=2 \mu\left[\Pi-\frac{1}{3}(\mathrm{I} \otimes \mathrm{I})\right] \varepsilon_{n+1}-2 \mu \Pi \varepsilon_{n}^{p}
$$

De (170) resulta:

$$
\frac{\partial S_{n+1}^{p r}}{\partial \varepsilon_{n+1}}=2 \mu\left[\Pi-\frac{1}{3}(\mathrm{I} \otimes \mathrm{I})\right]
$$

Sendo $\left\|S_{n+1}^{p r}\right\|=\left(S_{n+1}^{p r} \cdot S_{n+1}^{p r}\right)^{1 / 2}$, pode-se escrever:

$$
\frac{\partial\left\|S_{n+1}^{p r}\right\|}{\partial \varepsilon_{n+1}}=\frac{1}{2} \frac{1}{\left\|S_{n+1}^{p r}\right\|}\left(2 \frac{\partial S_{n+1}^{p r}}{\partial \varepsilon_{n+1}} S_{n+1}^{p r}\right)=\frac{2 \mu}{\left\|S_{n+1}^{p r}\right\|}\left[\Pi-\frac{1}{2}(\mathrm{I} \otimes \mathrm{I})\right] S_{n+1}^{p r}
$$


Uma vez que $t r S_{n+1}^{p r}=0$, a eq. (172) resulta em:

$$
\frac{\partial\left\|S_{n+1}^{p r}\right\|}{\partial \varepsilon_{n+1}}=2 \mu n_{n+1}
$$

Substituindo-se as eqs. (171) e (173) na eq. (169) e fazendo uso da eq. (160), chega-se a:

$$
\frac{\partial n_{n+1}}{\partial \varepsilon_{n+1}}=\frac{2 \mu}{\left\|S_{n+1}^{p r}\right\|}\left[\Pi-\frac{1}{3}(\mathrm{I} \otimes \mathrm{I})-n_{n+1} \otimes n_{n+1}\right]
$$

Utilizando-se agora a eq. (163), a expressão de $f_{n+1}^{p r}$ e a eq. (173), chega-se à seguinte relação para o tensor $\frac{\partial \Delta \lambda}{\partial \varepsilon_{n+1}}$ :

$$
\frac{\partial \Delta \lambda}{\partial \varepsilon_{n+1}}=\frac{1}{\left(2 \mu+\frac{2}{3} K\right)} \frac{\partial f_{n+1}^{p r}}{\partial \varepsilon_{n+1}}=\frac{1}{\left(2 \mu+\frac{2}{3} K\right)} \frac{\partial \mid S_{n+1}^{p r} \|}{\partial \varepsilon_{n+1}}=\frac{1}{\left(1+\frac{K}{3 \mu}\right)} n_{n+1}
$$

Substituindo-se as eqs. (174) e (175) na equação (168), obtém-se o terceiro termo do tensor elastoplástico:

$$
\frac{\partial\left(-2 \mu \Delta \lambda n_{n+1}\right)}{\partial \varepsilon_{n+1}}=-\frac{4 \mu^{2} \Delta \lambda}{\left\|S_{n+1}^{p r}\right\|} \Pi+\frac{4 \mu^{2} \Delta \lambda}{3\left\|S_{n+1}^{p r}\right\|}(\mathrm{I} \otimes \mathrm{I})+\left[\frac{4 \mu^{2} \Delta \lambda}{\left\|S_{n+1}^{p r}\right\|}-\frac{2 \mu}{\left(1+\frac{K}{3 \mu}\right)}\right] n_{n+1} \otimes n_{n+1}
$$

O tensor elastoplástico algorítmico tangente é dado pela soma das eqs. (166), (167) e (176):

$$
C_{n+1}^{e p}=C-\frac{4 \mu^{2} \Delta \lambda}{\left\|S_{n+1}^{p r}\right\|} \Pi+\frac{4 \mu^{2} \Delta \lambda}{3\left\|S_{n+1}^{p r}\right\|}(\mathrm{I} \otimes \mathrm{I})+\left[\frac{4 \mu^{2} \Delta \lambda}{\left\|S_{n+1}^{p r}\right\|}-\frac{2 \mu}{\left(1+\frac{K}{3 \mu}\right)}\right] n_{n+1} \otimes n_{n+1}
$$

O tensor de quarta ordem dado na eq. (177) define a relação incremental entre os tensores de tensão e de deformação. Por conveniência, agrupam-se os incrementos nas componentes dos tensores de tensão e de deformação em vetores $\delta \sigma^{T}=\left\{\begin{array}{llllll}\delta \sigma_{x} & \delta \sigma_{y} & \delta \sigma_{z} & \delta \tau_{x y} & \delta \tau_{x z} & \delta \tau_{y z}\end{array}\right\}$ e $\delta \varepsilon^{T}=\left\{\begin{array}{llllll}\delta \varepsilon_{x} & \delta \varepsilon_{y} & \delta \varepsilon_{z} & \delta \gamma_{x y} & \delta \gamma_{x z} & \delta \gamma_{y z}\end{array}\right\}$, respectivamente. Assim, pode-se deduzir uma matriz da relação constitutiva elastoplástica tangente $D_{6 x 6}^{e p}$, tal que $\delta \sigma=D^{e p} \delta \varepsilon$; é o que se apresenta no Apêndice K. 


\subsection{Travamento volumétrico}

Denomina-se travamento volumétrico à ocorrência de uma rigidez excessiva em elementos finitos tridimensionais de baixo grau de aproximação em situações de quase incompressibilidade. Este travamento ocorre devido à combinação de uma restrição cinemática imposta pelo modelo constitutivo com a pouca capacidade de deformação dos elementos de baixa ordem. As situações mais freqüentes de travamento volumétrico são:

- em análises elásticas, quando o coeficiente de Poisson se aproxima de 0,5.

- quando há fluxo plástico. Sabe-se que o efeito do travamento volumétrico se dá tanto em modelos em que a deformação plástica é um fenômeno isocórico (como o de von Mises) como naqueles em que há contração ou dilatação volumétrica (como o modelo de Drucker-Prager).

Em razão do efeito de travamento quando o modelo constitutivo adotado é o elastoplástico perfeito, a resposta excessivamente rígida do elemento finito leva a que a carga de colapso seja superestimada ou que, em muitos casos, ela simplesmente não seja encontrada - neste caso, a curva força x deslocamento aumenta indefinidamente.

Segundo WELLS et al (2002), diferentes abordagens têm sido propostas para superar o travamento volumétrico em elementos de baixa ordem. As principais são apresentadas a seguir:

- Uso de arranjos especiais de elementos triangulares com aproximação linear, o que é bastante eficiente em problemas planos de geometria mais simples.

- Interpolar separadamente a tensão hidrostática e os deslocamentos, mediante uma formulação mista. No entanto, elementos triangulares lineares com interpolação de tensão robustos são difíceis de gerar.

- Expandir o campo de deformações segundo uma abordagem denominada Enhanced Assumed Strain, adicionando-se novas variáveis ao elemento, o que pode ocasionar o surgimento de modos incompatíveis.

Ainda segundo os autores, nenhum dos métodos acima pode ser aplicado a todos os elementos com geometria triangular ou tetraédrica, o que é uma grande desvantagem quando se lida com estruturas de geometria complexa, visto que tais elementos são os mais facilmente adaptáveis a elas.

Efetivamente, o método mais eficiente para evitar o travamento volumétrico é o enriquecimento polinomial de ordem superior em todo o elemento ou o enriquecimento da aproximação nos moldes do MEFG. 
Esta última opção tem como vantagem em relação ao uso de elementos finitos convencionais uma maior facilidade para gerar uma aproximação de ordem mais elevada apenas nas regiões onde isso seja mais necessário, permitindo assim maior eficiência computacional. 


\section{MECÂNICA DO DANO CONTÍNUO}

\subsection{Introdução e Revisão Bibliográfica.}

Pode-se considerar o trabalho de KACHANOV (1958) como referência pioneira no desenvolvimento da Mecânica do Dano em meios contínuos, tendo este autor introduzido o conceito de dano (na verdade, de integridade do material) para modelar a ocorrência de ruptura frágil em metais após um período de deformação lenta (fluência). Pela primeira vez, o processo físico de danificação, inerentemente discreto, foi modelado por meio de uma abordagem típica de meio contínuo. Ainda na modelagem de problemas de dano e fluência, podem-se citar os trabalhos de RABOTNOV (1969), MURAKAMI \& OHNO (1981) e KACHANOV (1984).

A utilização da Mecânica do Dano em meio contínuo como base de modelos constitutivos para o concreto pode ser encontrada em trabalhos como os de MAZARS (1984), LA BORDERIE et al (1991) e CIPOLLINA et al (1995).

Em MAZARS (1984), propõe-se um modelo para o concreto submetido a carregamento monotonamente crescente, no qual o dano (definido por um escalar) é quantificado em função de uma variável, a deformação equivalente, que caracteriza o estado local de alongamento do material. A lei de evolução do dano requer a identificação experimental de cinco parâmetros, por meio de ensaios uniaxiais de tração e compressão. O modelo não considera a ocorrência de deformações permanentes.

Uma aplicação do modelo de Mazars na análise de vigas de concreto armado, com o emprego do Método dos Elementos Finitos, pode ser encontrada em ÁLVARES (1993), no qual se apresenta um confronto entre resultados de ensaios e respostas obtidas numericamente. DRIEMEIER (1995) apresenta uma extensão do modelo de Mazars para considerar solicitações cíclicas em vigas de concreto armado.

Já o modelo de LA BORDERIE et al (1991) utiliza duas variáveis que representam a danificação, referentes a estados predominantes de tração e de compressão. Este modelo considera a ocorrência de deformações anelásticas, bem como o efeito do fechamento de fissuras no caso da inversão do sinal de estados inicialmente de tração para compressão.

CIPOLLINA et al (1995) propõem um modelo voltado à análise de estruturas reticuladas, tendo desenvolvido um elemento finito de barra no qual os efeitos não-lineares do dano e da plasticidade se concentram em "rótulas" nas extremidades, adotando-se para o restante da barra um comportamento elástico. 
Entre os trabalhos que utilizam modelos constitutivos no qual o dano ocorre associado a uma deformação plástica significativa (dano dúctil) podem ser citados os de GURSON (1977), LEMAITRE (1985), BENALLAL et al (1988) e DOGHRI (1995).

GURSON (1977) desenvolve um modelo constitutivo - critério de plastificação, regra de fluxo plástico, critério de encruamento e de ruptura - para materiais porosos e dúcteis. O trabalho ressalta o papel da tensão hidrostática no crescimento de vazios e no fluxo plástico.

LEMAITRE (1985) apresenta um modelo de dano plástico dúctil isótropo baseado no conceito de tensão efetiva, derivado de uma formulação termodinâmica. A influência da razão de triaxialidade (razão entre as tensões média e a equivalente de von Mises) é demonstrada e são apresentadas comparações de resultados com aqueles obtidos experimentalmente e utilizando modelos de dano já existentes.

A formalização da Mecânica do Dano Contínuo em termos da Termodinâmica dos Processos Irreversíveis ocorre em LEMAITRE \& CHABOCHE (1985).

Em muitos dos modelos constitutivos baseados na Mecânica do Dano, ocorre o fenômeno do encruamento negativo (softening), ou seja, a redução de resistência do material após um certo nível de deformação. Desse modo, o tensor que define a relação constitutiva tangente deixa de ser positivo-definido, ocasionando um problema de perda da objetividade da resposta, ou seja, os resultados tornam-se dependentes da malha adotada, divergindo com o refinamento da mesma (ou com a elevação do grau da aproximação). Na realidade, com a evolução das deformações há uma localização do dano numa região progressivamente menor até o surgimento de uma descontinuidade.

Uma das técnicas adotadas para regularizar o problema numérico é o uso da formulação não-local, como descrito em BAŽANT et al (1984) e em PIJAUDIER-CABOT \& BAŽANT (1987). Basicamente, a formulação não-local consiste em adotar para o dano num ponto um valor médio obtido sobre uma região do domínio no entorno daquele ponto (definida por um raio $r_{D}$ ). Alternativamente, pode-se tomar a média nessa região de grandezas associadas ao dano - como a deformação equivalente no modelo de Mazars. Dessa forma, resulta que a região (banda) de localização do dano fica restrita a um valor mínimo próximo ao raio $r_{D}$, mesmo que se utilize uma malha bem refinada (ou grau polinomial mais alto na região); esse fato recupera a objetividade da resposta numérica.

Outra técnica semelhante à abordagem não-local e que pode ser utilizada na regularização da resposta numérica consiste na introdução, no modelo constitutivo, de 
gradientes de ordens superiores das variáveis relacionadas ao dano, como em FRÉMOND \& NEDJAR (1996), COMI (1999) e DRIEMEIER (1999).

\subsection{Conceitos fundamentais da Mecânica do Dano}

De modo geral, pode-se conceituar a danificação como um conjunto de fenômenos mecânicos ou não-mecânicos que alteram a microestrutura dos materiais e que provocam, em nível macroscópico, uma redução de suas propriedades mecânicas, como a rigidez e a resistência.

Em função do tipo de material analisado (e das características de sua microestrutura), diferentes mecanismos estão envolvidos nos processos de danificação. $\mathrm{O}$ dano no concreto, por exemplo, está relacionado à evolução e união de microfissuras que se iniciam no contorno dos grãos e avançam para o interior da pasta de cimento e areia. Já o dano em materiais metálicos é gerado a partir do acúmulo de discordâncias (desalinhamentos no arranjo dos átomos numa malha cristalina) em processos de plastificação.

Seja qual for o tipo de defeito (fissuras, discordâncias, etc) e sua dimensão, sua distribuição real, em geral, é difusa no volume total do corpo. Para que se possa estender a hipótese de continuidade ao caso dos meios com dano, usa-se o conceito de elemento de volume representativo, como ilustrado na figura 18.
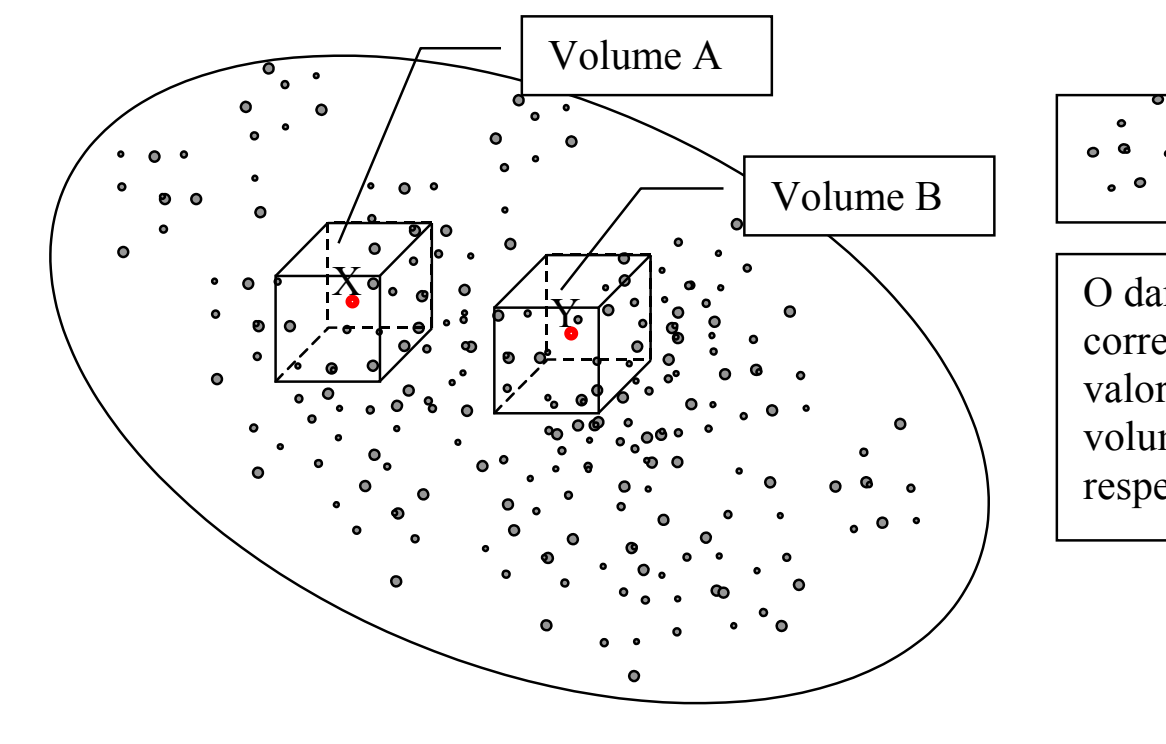

defeitos

O dano em X e Y corresponde ao valor médio nos volumes A e B, respectivamente

Figura 18 - Elementos de volume representativo 
O elemento representativo deve ter uma dimensão grande o suficiente para que se possa admitir uma distribuição homogênea de defeitos em seu interior e pequena o suficiente para que se evitem gradientes elevados de variáveis de interesse. Portanto, a dimensão desse elemento é função do tipo de material e do processo de danificação a ele associado.

Em função da continuidade, a cada ponto material pode-se associar um valor de dano e, nessas condições, é possível descrever esse fenômeno utilizando-se funções contínuas no volume do sólido.

Apresentam-se a seguir, para o caso de solicitação uniaxial, os conceitos de tensão e deformação efetivas (item 4.2.1) e os princípios de equivalência de resposta (item 4.2.2). Esses conceitos são fundamentais na Mecânica do Dano Contínuo, sendo sua extensão para o caso multiaxial apresentada no item 4.2.3.

\subsubsection{Tensão e deformação efetivas - caso unidimensional.}

Os conceitos de tensão efetiva e de deformação efetiva são apresentados tendo-se em vista o caso de solicitação uniaxial segundo a direção do versor $n$ de um elemento de volume representativo, como mostrado na figura 19. Um corte segundo um plano perpendicular ao versor $n$ define a seção transversal do elemento, de área $S$.

Para a solicitação indicada, a tensão nominal na seção transversal do elemento é:

$$
\sigma=\frac{N}{S}
$$

sendo $N$ a força axial aplicada.

Designando por $S_{D}$ a área de defeitos na seção, define-se a tensão efetiva admitindo-se que a força normal $N$ atue exclusivamente sobre a área integra da seção, ou seja:

$$
\sigma_{e f}=\frac{N}{\left(S-S_{D}\right)}
$$

Associado ao conceito de área de defeitos, propõe-se a seguinte definição para o dano segundo o plano de normal $n$ :

$$
D_{n}^{S_{D}}=\frac{S_{D}}{S}
$$

Nota-se que a tensão efetiva é sempre igual (quando $S_{D}=0$ ) ou superior $\left(0<S_{D}<S\right)$ à tensão nominal, podendo-se estabelecer a seguinte relação entre ambas, fazendo-se uso da eq. (180): 


$$
\sigma_{e f}=\frac{\sigma}{\left(1-D_{n}^{S_{D}}\right)}
$$

Define-se agora o conceito de deformação efetiva. Seja $l_{0}$ o comprimento do elemento segundo a direção definida pelo versor $n$ e $\Delta l$ o aumento desse comprimento pelo efeito da solicitação (figura 19). A deformação nominal é dada por:

$$
\varepsilon=\frac{\Delta l}{l_{0}}
$$

Uma vez que o material apresenta descontinuidades internas, parte do aumento de comprimento pode ser associado à abertura das mesmas $(\Delta d)$. Assim, a deformação efetiva, que desconsidera a contribuição dessa parcela de deslocamentos, é definida por:

$$
\varepsilon_{e f}=\frac{\Delta l-\Delta d}{l_{0}}
$$

Nesse caso, o dano é definido por:

$$
D_{n}^{\Delta d}=\frac{\Delta d}{\Delta l}
$$

Fazendo-se uso da eq. (184), obtém-se uma relação entre as deformações efetiva e nominal:

$$
\varepsilon_{e f}=\left(1-D_{n}^{\Delta d}\right) \varepsilon
$$

$\mathrm{O}$ acréscimo no volume associado aos defeitos $\Delta V_{d}$ pode ser calculado, tendo-se em vista as definições anteriores, de duas formas:

- Considerando-se a variação do comprimento total $\Delta l$ multiplicada pela área da seção de defeitos $S_{D}$.

- Considerando-se apenas a parcela de variação de deslocamento relacionada ao dano $(\Delta d)$ multiplicada pela seção total $S$. Portanto, obtém-se a igualdade:

$$
\Delta V_{D}=\Delta l S_{D}=\Delta d S
$$

E, da eq. (186), conclui-se que $D_{n}^{S_{D}}=D_{n}^{\Delta d}=D_{n}$, ou seja, as definições de dano dadas pelas eqs. (180) e (184) podem ser unificadas.

Por definição, a variável $D_{n}$ assume valores limitados ao intervalo $0 \leq D_{n} \leq 1$, sendo a situação de material íntegro definida por $D_{n}=0$ e a de material totalmente danificado por $D_{n}=1$.

Além disso, admitindo-se uma distribuição uniforme de defeitos no volume representativo, pode-se considerar que segundo qualquer plano de normal $n$ o valor da 
variável de dano $D_{n}$ seja o mesmo. Nessa situação, uma única variável escalar de dano $D$ é capaz de representar o estado de deterioração do material no ponto.

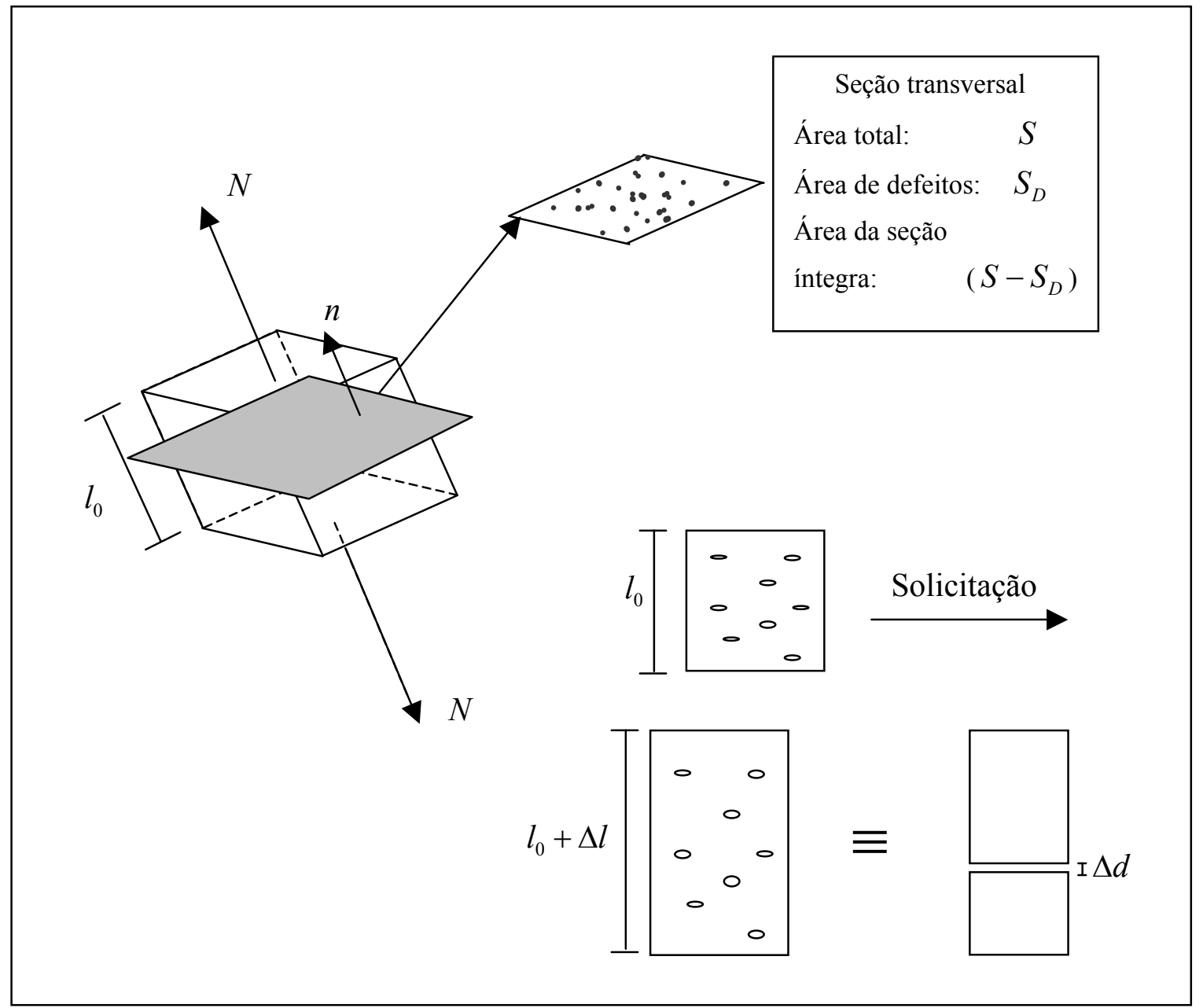

Figura 19 - Tensão e deformação efetivas

\subsubsection{Princípios de equivalência de resposta - caso unidimensional.}

A formulação de relações constitutivas para meios contínuos com dano está baseada num princípio geral de equivalência de resposta, que estabelece que a lei constitutiva do meio danificado contínuo equivalente pode ser obtida a partir da lei constitutiva da parte íntegra do meio real danificado, considerando-se as tensões e deformações efetivas.

Assim sendo, a relação constitutiva da parte íntegra do meio real danificado pode ser expressa por:

$$
\sigma_{e f}=E \varepsilon_{e f}
$$

sendo $E$ o módulo de elasticidade longitudinal do meio íntegro. 
Para o caso unidimensional, a relação constitutiva para o meio danificado contínuo equivalente pode então ser obtida substituindo-se em (187) as definições de $\sigma_{e f}$ e $\varepsilon_{e f}$, assumindo a forma:

$$
\sigma=E_{D} \varepsilon
$$

sendo $E_{D}$ o módulo de elasticidade longitudinal do meio com dano.

O cálculo do módulo de elasticidade $E_{D}$ passa a depender do critério a ser utilizado para definir a equivalência entre os meios real e danificado contínuo equivalente, como se mostra a seguir.

a) Princípio de equivalência de deformação.

Estabelece que o meio íntegro e o meio danificado equivalente, cada qual com o seu módulo de elasticidade, apresentam o mesmo estado de deformação:

$$
\varepsilon=\frac{\sigma}{E_{D}}=\frac{\sigma_{e f}}{E}=\frac{\frac{\sigma}{(1-D)}}{E}
$$

Portanto, da eq. (189) obtém-se que o módulo elástico do meio danificado vale:

$$
E_{D}=(1-D) E
$$

b) Princípio da equivalência de tensão.

Estabelece que o meio íntegro e o meio danificado contínuo equivalente, cada qual com o seu módulo de elasticidade, apresentam o mesmo estado de tensão:

$$
\sigma=E_{D} \varepsilon=E \varepsilon_{e f}=E(1-D) \varepsilon
$$

O módulo elástico do meio danificado fica também expresso pela eq. (190).

c) Princípio da equivalência de energia.

Estabelece que a energia de deformação é a mesma quer se considere a parte íntegra do meio real com dano ou o meio danificado contínuo equivalente. Esse princípio pode ser estabelecido através da equação:

$$
\sigma_{e f} \varepsilon_{e f}=\sigma \varepsilon
$$

Substituindo-se na eq. (192) os valores de $\sigma$ e $\sigma_{e f}$ dados pelas eqs. (187) e (188), respectivamente, chega-se a:

$$
E \varepsilon_{e f}{ }^{2}=E_{D} \varepsilon^{2}
$$

E, da eq. (185), tem-se que $\varepsilon_{e f}=(1-D) \varepsilon$, obtendo-se para o módulo elástico do meio danificado o valor: 


$$
E_{D}=(1-D)^{2} E
$$

\subsubsection{Representação tensorial do dano.}

Os conceitos da Mecânica do Dano enunciados anteriormente para o caso uniaxial, como o de tensão e deformação efetivas, e os princípios de equivalência da resposta constitutiva podem ser extendidos para o caso multiaxial. A relação constitutiva envolve um tensor de dano de quarta ordem, cuja expressão dependerá do tipo de equivalência adotada entre o meio real e o meio danificado contínuo equivalente. Sendo, agora, $\sigma$ o tensor de tensões e $\varepsilon$ o de deformações, ambos de segunda ordem, e $D$ o tensor de dano (de quarta ordem), as expressões dos correspondentes tensores de tensão e deformação efetivas são dadas a seguir:

$$
\begin{aligned}
& \sigma_{e f}=(\Pi-D)^{-1} \sigma \\
& \varepsilon_{e f}=(\Pi-D) \varepsilon
\end{aligned}
$$

Pelo princípio geral de equivalência da resposta constitutiva, escreve-se:

$$
\sigma_{e f}=C \varepsilon_{e f}
$$

sendo $C$ o tensor constitutivo do meio íntegro.

A relação constitutiva do meio danificado contínuo é dada por:

$$
\sigma=C_{D} \varepsilon
$$

sendo $C_{D}$ o tensor de quarta ordem que considera o efeito da danificação.

Para o cálculo do tensor $C_{D}$, podem-se adotar os três princípios básicos de equivalência:

a) Equivalência em deformação:

Por um lado, a deformação efetiva, expressa em função do tensor nominal de tensões, é obtida substituindo-se na eq. (197) o valor de $\sigma_{e f}$ dado pela eq. (195):

$$
\varepsilon_{e f}=C^{-1} \sigma_{e f}=C^{-1}(\Pi-D)^{-1} \sigma
$$

Por outro lado, a deformação resultante da aplicação da tensão nominal ao meio danificado, conforme a eq. (198) é:

$$
\varepsilon=C_{D}^{-1} \sigma
$$

Impondo-se a igualdade das eqs. (199) e (200), obtém-se:

$$
C_{D}{ }^{\text {eq.def. }}=(\Pi-D) C
$$

b) Equivalência em tensão: 
A tensão efetiva expressa em função da deformação nominal é obtida da eq. (197), substituindo-se nela o valor de $\varepsilon_{e f}$ dado pela eq. (196).

$$
\sigma_{e f}=C \varepsilon_{e f}=C(\Pi-D) \varepsilon
$$

Sendo a tensão num meio danificado dada pela eq. (198), obtém-se, ao igualar as equações (198) e (202):

$$
C_{D}^{\text {eq.tens. }}=C(\Pi-D)
$$

c) Equivalência em energia de deformação:

A energia de deformação, considerando-se os conceitos de tensão e deformação efetivas e utilizando-se a eq. (197), é dada por:

$$
\sigma_{e f} \cdot \varepsilon_{e f}=\left(C \varepsilon_{e f}\right) \cdot \varepsilon_{e f}
$$

Já a energia de deformação considerando-se o meio danificado contínuo fica expressa, fazendo-se uso da eq. (198), por:

$$
\sigma \cdot \varepsilon=\left(C_{D} \varepsilon\right) \cdot \varepsilon
$$

Substituindo-se na eq. (204) o valor da deformação efetiva dada pela eq. (196), obtém-se:

$$
\left(C \varepsilon_{e f}\right) \cdot \varepsilon_{e f}=[C(\Pi-D) \varepsilon] \cdot(\Pi-D) \varepsilon=\left[(\Pi-D)^{T} C(\Pi-D) \varepsilon\right] \cdot \varepsilon
$$

Da comparação das eqs. (205) e (206), obtém-se a seguinte expressão para o tensor constitutivo que leva em conta o dano:

$$
C_{D}=(\Pi-D)^{T} C(\Pi-D)
$$

Entre os três tipos de equivalência mencionados, o de deformação é o mais freqüentemente utilizado.

A formulação acima apresentada não impõe qualquer forma particular ao tensor de dano $D$. Uma expressão deste tensor que representa um processo de danificação em que se preserva a isotropia do meio é a seguinte:

$$
D=d \Pi
$$

onde $d$ é um escalar. Aplicando-se a eq. (208) à eq. (201) e substituindo-se o valor do tensor constitutivo obtido na eq. (198), obtém-se o seguinte tensor de tensões:

$$
\sigma=(1-d) C \varepsilon
$$

Verifica-se na eq. (209) que as componentes do tensor de tensões são igualmente penalizadas pelo fator $(1-d)$.

Outra expressão para o tensor $D$ que permite preservar a isotropia do meio é a seguinte: 


$$
D=d(\mathrm{I} \otimes \mathrm{I})
$$

Aplicando-se a eq. (210) à eq. (201) e substituindo-se o tensor constitutivo obtido na eq. (198), tem-se o tensor de tensões dado por:

$$
\sigma=C \varepsilon-d(\operatorname{tr} C \varepsilon) \mathrm{I}
$$

Uma vez que $\operatorname{tr}(C \varepsilon) \mathrm{I} / 3$ é a parcela hidrostática do tensor de tensões no meio íntegro, verifica-se que, neste caso, o dano dado pela eq. (210) penaliza igualmente apenas as componentes de tensão normal. As direções principais do tensor não são alteradas e por isso trata-se de um caso de dano isótropo.

Podem-se também propor expressões para o tensor de dano que produzam anisotropia no meio, como a mostrada abaixo:

$$
D=f(d) Q
$$

Na eq. (212), $Q$ é um tensor constante de anisotropia e $f(d)$ uma função de valor escalar que varia com a danificação. Nota-se que neste caso a evolução do dano não altera a "direção" do tensor de dano, mas apenas sua norma.

A expressão mais geral para o dano anisótropo é:

$$
D=Q(d)
$$

na qual $Q$ não é mais constante, mas função da variável $d$, que também pode ser de natureza tensorial (primeira ou segunda ordem).

\subsection{Modelos de dano utilizados neste trabalho.}

Os modelos adotados neste trabalho são conhecidos pelos nomes de seus autores: o de dano para o concreto (Mazars) e o de dano dúctil (Lemaitre). Suas principais características são apresentadas a seguir.

\subsubsection{Modelo de MAZARS (1984)}

Segundo ÁLVARES (1993), este modelo é indicado para o concreto submetido a carregamento proporcional crescente. As principais hipóteses adotadas são:

1) O concreto em processo de dano evolutivo é considerado um meio elástico sem deformações residuais.

2) O processo de evolução do dano é causado exclusivamente pela existência de extensões segundo as direções principais de deformação.

3) A isotropia inicial do meio é preservada. 
4) O surgimento do dano se dá inicialmente quando uma grandeza denominada deformação equivalente, indicada por $\widetilde{\varepsilon}$ e cujo cálculo é apresentado na seqüência, exceder um valor de referência $\left(\varepsilon_{d 0}\right)$, obtido experimentalmente. A partir daí, o dano evolui se $\widetilde{\varepsilon}$ exceder o maior valor para esta grandeza já calculado num passo de carga ou iteração anterior.

O modelo de Mazars é dito escalar pois o dano é representado por uma única variável $D$, sendo $0 \leq D \leq 1$.

O dano $D$ penaliza de modo uniforme todas as componentes do tensor elástico de rigidez correspondente ao material íntegro $(C)$. A relação constitutiva secante é descrita, portanto, pela expressão:

$$
\sigma=(1-D) C \varepsilon
$$

O modelo de Mazars propõe que o dano $D$ seja determinado como resultado da combinação linear de outras duas variáveis $\left(D_{T}\right.$ e $\left.D_{C}\right)$, tomando-se como ponderadores as variáveis $\alpha_{T}$ e $\alpha_{C}$, como se vê abaixo:

$$
D=\alpha_{T} D_{T}+\alpha_{C} D_{C}
$$

As variáveis $D_{T}$ e $D_{C}$ representam níveis de dano associados a estados uniaxiais de tração e compressão, respectivamente, sendo quantificadas pelas seguintes expressões:

$$
\begin{aligned}
& D_{T}=1-\frac{\varepsilon_{d 0}\left(1-A_{T}\right)}{\widetilde{\varepsilon}}-\frac{A_{T}}{e^{B_{T} \cdot\left(\widetilde{\varepsilon}-\varepsilon_{d 0}\right)}} \\
& D_{C}=1-\frac{\varepsilon_{d 0}\left(1-A_{C}\right)}{\widetilde{\varepsilon}}-\frac{A_{C}}{e^{B_{C} \cdot\left(\widetilde{\varepsilon}-\varepsilon_{d 0}\right)}}
\end{aligned}
$$

Nas eqs. (216) e (217), $A_{T}, B_{T}, A_{C}, B_{C}$ e $\varepsilon_{d 0}$ são parâmetros característicos do material identificáveis em ensaios uniaxiais. Uma análise detalhada do significado dos parâmetros acima mencionados é apresentada em ÁLVARES (1993).

E a deformação equivalente do material $(\widetilde{\varepsilon})$ é dada pela expressão:

$$
\widetilde{\varepsilon}=\sqrt{\sum_{i=1}^{3}\left(\varepsilon_{i}^{+}\right)^{2}}
$$

na qual $\varepsilon_{i}^{+}=\left(\varepsilon_{i}+\left|\varepsilon_{i}\right|\right) / 2$, sendo $\varepsilon_{i}, i=1, \ldots, 3$, as deformações principais. Isto equivale a incorporar no somatório indicado na eq. (218) somente as deformações principais positivas. 
Os ponderadores $\alpha_{T}$ e $\alpha_{C}$ definem a contribuição das parcelas de tração e de compressão do estado de tensão dado para a danificação. Tais variáveis são calculadas seguindo os passos abaixo:

1) Calcula-se o vetor de deformações principais: $\varepsilon^{\text {princ }}=\left\{\begin{array}{lll}\varepsilon_{1} & \varepsilon_{2} & \varepsilon_{3}\end{array}\right\}^{T}$.

2) Utilizando-se a relação constitutiva elástica isótropa, define-se um estado de tensões principais fictícias, agrupadas no vetor $\sigma^{\text {princ }}=\left\{\begin{array}{lll}\sigma_{1} & \sigma_{2} & \sigma_{3}\end{array}\right\}^{T}$. Suas componentes são obtidas pela relação:

$$
\sigma_{i}=\frac{E}{(1+v)} \varepsilon_{i}+\frac{E v}{(1+v)(1-2 v)} \sum_{j=1}^{3} \varepsilon_{j}
$$

3) $\mathrm{O}$ vetor de tensões principais $\sigma^{\text {princ }}$ é separado em partes positiva $\left(\sigma^{+}\right)$e negativa $\left(\sigma^{-}\right)$, cujas componentes são dadas abaixo:

$$
\begin{aligned}
& \sigma_{i}^{+}=\frac{1}{2}\left(\sigma_{i}+\left|\sigma_{i}\right|\right) \\
& \sigma_{i}^{-}=\frac{1}{2}\left(\sigma_{i}-\left|\sigma_{i}\right|\right)
\end{aligned}
$$

4) Utilizando-se os valores de $\sigma^{+}$e $\sigma^{-}$dados pelas eqs. (220a) e (220b), separa-se o vetor de deformações principais $\left(\varepsilon^{\text {princ }}\right)$ em duas partes sendo a primeira relacionada à tração $\left(\varepsilon^{T}\right)$ e a segunda, à compressão $\left(\varepsilon^{C}\right)$ :

$$
\begin{aligned}
& \varepsilon_{i}^{T}=\frac{(1+v)}{E} \sigma_{i}^{+}-\frac{v}{E} \sum_{j=1}^{3} \sigma_{j}^{+} \\
& \varepsilon_{i}^{C}=\frac{(1+v)}{E} \sigma_{i}^{-}-\frac{v}{E} \sum_{j=1}^{3} \sigma_{j}^{-}
\end{aligned}
$$

5) Separam-se os vetores $\varepsilon^{T}$ e $\varepsilon^{C}$ em partes positiva e negativa, de forma análoga à separação do tensor de tensões principais, mostrada nas eqs. (220):

$$
\begin{aligned}
& \varepsilon^{T}=\varepsilon^{T,+}+\varepsilon^{T,-} \\
& \varepsilon^{C}=\varepsilon^{C,+}+\varepsilon^{C,-}
\end{aligned}
$$

6) Calcula-se a variável $\varepsilon_{V}^{+}$, representativa do estado de extensão do material, dada por:

$$
\varepsilon_{V}^{+}=\sum_{i=1}^{3}\left(\varepsilon_{i}^{T,+}+\varepsilon_{i}^{C,+}\right)
$$

7) Finalmente, os ponderadores $\alpha_{T}$ e $\alpha_{C}$ são calculados por: 


$$
\begin{aligned}
& \alpha_{T}=\frac{\sum_{i=1}^{3} \varepsilon_{i}^{T,+}}{\varepsilon_{V}^{+}} \\
& \alpha_{C}=\frac{\sum_{i=1}^{3} \varepsilon_{i}^{C,+}}{\varepsilon_{V}^{+}}
\end{aligned}
$$

Como se vê pelas equações (224a) e (224b), sempre se terá $\alpha_{T}+\alpha_{C}=1$.

\subsubsection{Modelo constitutivo de plasticidade e dano - LEMAITRE (1985)}

Este modelo aplica-se basicamente a materiais dúcteis (especialmente aos metais), nos quais a danificação decorre de processos associados à plastificação (como o encruamento por acúmulo de discordâncias e a conseqüente localização de defeitos na rede cristalina). Suas principais características são:

- O dano é modelado por uma variável escalar;

- Admite-se a associatividade com o critério de plastificação: na regra da normalidade do fluxo plástico e na lei de encruamento;

- Dano e plastificação ocorrem de modo cinematicamente acoplado, ou seja, o dano interfere na velocidade da deformação plástica.

A seguir, apresentam-se inicialmente os conceitos fundamentais utilizados na formulação do modelo: a tensão equivalente generalizada e a tensão equivalente efetiva, os potenciais termodinâmico e de dissipação e as leis de evolução da deformação plástica, da variável de encruamento e da variável de dano. Por fim, reúnem-se as equações que definem o modelo constitutivo do material segundo a presente formulação.

4.3.2.1 Energia de deformação elástica e a tensão equivalente generalizada.

A energia de deformação elástica $\left(W_{e}\right)$ pode ser decomposta em duas parcelas: uma relacionada à distorção $\left(W_{e}^{S}\right)$ e outra relacionada à deformação volumétrica $\left(W_{e}^{H}\right)$. As expressões dessas parcelas são dadas abaixo:

$$
\begin{aligned}
& W_{e}^{S}=\int_{0}^{\varepsilon_{e}^{S}} S \cdot d \varepsilon_{e}^{S} \\
& W_{e}^{H}=3 \int_{0}^{\varepsilon_{e}^{H}} \sigma_{H} d \varepsilon_{e}^{H}
\end{aligned}
$$

Na eq. (225), $S$ é o tensor desviador de tensões, dado pela eq. (133), e $\varepsilon_{e}^{S}$ a parcela desviadora do tensor de deformações elásticas; enquanto que na eq. (226) $\sigma_{H}$ é a tensão 
média ou hidrostática, dada por (134), e $\varepsilon_{e}^{H}$ é a deformação elástica média ou volumétrica. Em elasticidade linear valem as seguintes relações entre os tensores $S$ e $\varepsilon_{e}^{S}$ e entre $\sigma_{H}$ e $\varepsilon_{e}^{H}:$

$$
\begin{aligned}
& \varepsilon_{e}^{S}=\frac{(1+v)}{E} S \\
& \varepsilon_{e}^{H}=\frac{(1-2 v)}{E} \sigma_{H}
\end{aligned}
$$

Substituindo-se as eqs. (227) e (228) nas eqs. (225) e (226), respectivamente, obtém-se para a energia de deformação elástica a expressão:

$$
W_{e}=\frac{1}{2 E}\left[(1+v) S \cdot S+3(1-2 v) \sigma_{H}^{2}\right]
$$

Das eqs. (132) e (136), verifica-se que a tensão equivalente de von Mises pode ser expressa na forma $\sigma_{e q}=\sqrt{\frac{3}{2}(S \cdot S)}$. Portanto, pode-se escrever a eq. (229) na forma:

$$
W_{e}=\frac{\left(\sigma^{*}\right)^{2}}{2 E}
$$

onde a tensão $\sigma^{*}$ é denominada tensão equivalente generalizada, dada por:

$$
\begin{aligned}
& \sigma^{*}=\sigma_{e q} \sqrt{\frac{2}{3}(1+v)+3(1-2 v)\left(\frac{\sigma_{H}}{\sigma_{e q}}\right)^{2}}=\sigma_{e q} R_{v}^{1 / 2} \\
& \text { O fator } R_{v}=\frac{2}{3}(1+v)+3(1-2 v)\left(\frac{\sigma_{H}}{\sigma_{e q}}\right)^{2} \text { é denominado fator de triaxialidade. }
\end{aligned}
$$

\subsubsection{Potencial termodinâmico de energia livre e variáveis associadas.}

$\mathrm{Na}$ formulação termodinâmica geral para materiais com dano e plasticidade, incluindo-se o efeito da temperatura, postula-se a existência de um potencial termodinâmico $(\psi)$, referenciado à unidade de massa e função das variáveis de estado, entre as quais o dano definido pelo escalar $D$. Tem-se, portanto:

$$
\psi=\psi(\varepsilon, T, D, \alpha)
$$

O símbolo $T$ refere-se à temperatura absoluta e $\alpha$ é a variável interna associada a uma medida do encruamento. Neste trabalho, desconsidera-se o efeito da temperatura.

Admite-se, ainda, que o potencial termodinâmico para o meio com dano e plasticidade refira-se à energia livre que o sistema poderia disponibilizar para realizar 
trabalho e que esta seja composta por uma parcela recuperável e outra irrecuperável. A parcela recuperável é função da deformação elástica e do dano; já a parcela irrecuperável depende do encruamento. Nessas condições, vale a relação:

$$
\psi=\psi_{e}\left(\varepsilon^{e}, D\right)+\psi_{\alpha}(\alpha)
$$

Sendo $\rho$ a densidade do material, pode-se propor a seguinte expressão para a energia elástica recuperável (ou o potencial elástico) de um material, sob efeito do dano, por unidade de volume:

$$
\rho \psi_{e}=\frac{1}{2}(1-D) C \varepsilon^{e} \cdot \varepsilon^{e}
$$

onde $(1-D) C$ é o tensor constitutivo de rigidez elástica com dano.

Por definição, a variável associada à deformação elástica, a tensão, é obtida por derivação do potencial dado em (234):

$$
\sigma=\rho \frac{\partial \psi_{e}}{\partial \varepsilon^{e}}=(1-D) C \varepsilon^{e}
$$

Define-se também a variável associada ao dano, o escalar $Y$, por:

$$
Y=\rho \frac{\partial \psi_{e}}{\partial D}=-\frac{1}{2} C \varepsilon^{e} \cdot \varepsilon^{e}
$$

A variável $Y$ corresponde, a menos do sinal, à metade da variação da energia de deformação elástica do meio danificado $\left(W_{e}^{*}\right)$, causada por uma variação no dano, sob tensão constante:

$$
Y=-\left.\frac{1}{2} \frac{d W_{e}^{*}}{d D}\right|_{\sigma=c t e}
$$

Para demonstrar a eq. (237), escreve-se a expressão diferencial da energia de deformação elástica do meio danificado, fazendo-se uso da eq. (235):

$$
d W_{e}^{*}=\sigma \cdot d \varepsilon^{e}=(1-D) C \varepsilon^{e} \cdot d \varepsilon^{e}
$$

A condição de tensão constante é expressa por: $d \sigma=0$. Utilizando-se a relação (235), chega-se à expressão: $d \sigma=(1-D) C d \varepsilon^{e}-C \varepsilon^{e} d D=0$, da qual se obtém:

$$
d \varepsilon^{e}=\frac{\varepsilon^{e}}{(1-D)} d D
$$

Substituindo-se (239) em (238), obtém-se finalmente:

$$
\left.\frac{d W_{e}^{*}}{d D}\right|_{\sigma=c t e}=C \varepsilon^{e} \cdot \varepsilon^{e}
$$


Comparando-se as eqs. (236) e (240), verifica-se a eq. (237).

Em meio danificado, as expressões da parcela desviadora do tensor de deformações elásticas $\left(\varepsilon_{e}^{S}\right)$ e da deformação volumétrica $\left(\varepsilon_{e}^{H}\right)$ são:

$$
\begin{aligned}
& \varepsilon_{e}^{S}=\frac{(1+v)}{E(1-D)} S \\
& \varepsilon_{e}^{H}=\frac{(1-2 v)}{E(1-D)} \sigma_{H}
\end{aligned}
$$

Substituindo-se as eqs. (241) e (242) na eq. (240), e lembrando-se que $\varepsilon^{e}=\varepsilon_{e}^{S}+\varepsilon_{e}^{H} \mathrm{I}$ e $\operatorname{tr}\left(\varepsilon_{e}^{S}\right)=0$, chega-se a:

$$
\left.\frac{d W_{e}^{*}}{d D}\right|_{\sigma=c t e}=\frac{S}{(1-D)} \cdot \frac{(1+v) S}{E(1-D)}+3 \sigma_{H}^{2} \frac{(1-2 v)}{E(1-D)^{2}}
$$

Utilizando-se a expressão da tensão equivalente generalizada $\sigma^{*}$, dada pela eq. (231), pode-se reescrever a equação (243) da seguinte forma:

$$
\left.\frac{d W_{e}^{*}}{d D}\right|_{\sigma=c t e}=\frac{\left(\sigma^{*}\right)^{2}}{E(1-D)^{2}}
$$

Substituindo-se a eq. (244) na eq. (237), chega-se a uma expressão para a variável associada ao dano $Y$ :

$$
Y=-\frac{\left(\widetilde{\sigma}^{*}\right)^{2}}{2 E}
$$

na qual $\tilde{\sigma}^{*}=\frac{\sigma^{*}}{(1-D)}$ é denominada tensão equivalente efetiva.

Por fim, define-se a variável $R$, associada ao encruamento $\alpha$, dada pela expressão:

$$
R=\rho \frac{\partial \psi_{\alpha}}{\partial \alpha}
$$

4.3.2.3 Potencial de dissipação e leis de evolução da deformação plástica, do dano e do encruamento.

A desigualdade de Clausius-Duhem, obtida a partir da combinação da primeira e da segunda leis da Termodinâmica, expressa a condição para que um processo seja termodinamicamente admissível: processos dissipativos dispendem parte da energia de deformação específica $(\sigma \cdot \varepsilon)$. Sendo desconsiderado o efeito de variação de temperatura, sua expressão é a seguinte: 


$$
\sigma \cdot \dot{\varepsilon}-\rho \dot{\psi} \geq 0
$$

Utilizando-se a eq. (233), pode-se obter a variação no tempo do potencial termodinâmico (em relação à unidade de volume):

$$
\rho \dot{\psi}=\rho\left(\frac{\partial \psi_{e}}{\partial \dot{\varepsilon}^{e}} \cdot \dot{\varepsilon}^{e}+\frac{\partial \psi_{e}}{\partial D} \dot{D}\right)+\rho \frac{\partial \psi_{\alpha}}{\partial \alpha} \dot{\alpha}
$$

$\mathrm{Na}$ eq. (248), estão presentes as expressões que definem as variáveis associadas à deformação elástica, ao dano e ao encruamento, dadas respectivamente pelas eqs. (235), (236) e (246); substituindo-se essas variáveis na equação anterior, obtém-se:

$$
\rho \dot{\psi}=\sigma \cdot \dot{\varepsilon}^{e}+Y \dot{D}+R \dot{\alpha}
$$

Substituindo-se a eq. (249) na eq. (247) e admitindo-se a decomposição da deformação total em parcelas elástica e plástica $\left(\dot{\varepsilon}=\dot{\varepsilon}^{e}+\dot{\varepsilon}^{p}\right)$, obtém-se:

$$
\sigma \cdot \dot{\varepsilon}^{p}-Y \dot{D}-R \dot{\alpha} \geq 0
$$

$\mathrm{Na}$ eq. (250), tem-se a desigualdade de Clausius-Duhem escrita exclusivamente em função de processos dissipativos; a restrição que ela expressa significa, portanto, que a dissipação deve ser sempre positiva.

De modo que a eq. (250) seja obedecida (LEMAITRE (1990)), postula-se a existência de um potencial de dissipação convexo $\varphi^{*}$, função das variáveis associadas $\sigma$, $Y$ e $R$, do qual derivam as leis de evolução para $\dot{\varepsilon}^{p}, \dot{D}$ e $\dot{\alpha}$.

A regra da normalidade em relação a $\varphi^{*}$ pode ser considerada válida e as regras para $\dot{\varepsilon}^{p}, \dot{D}$ e $\dot{\alpha}$ são dadas pelas expressões seguintes:

$$
\begin{gathered}
\dot{\varepsilon}^{p}=\dot{\lambda} \frac{\partial \varphi^{*}}{\partial \sigma} \\
\dot{D}=-\dot{\lambda} \frac{\partial \varphi^{*}}{\partial Y} \\
\dot{\alpha}=-\dot{\lambda} \frac{\partial \varphi^{*}}{\partial R}
\end{gathered}
$$

De modo análogo ao realizado para o potencial termodinâmico, admite-se a decomposição do potencial de dissipação $\varphi^{*}$ numa parcela relativa aos efeitos de plastificação e encruamento $\varphi_{p}^{*}(\sigma, D, R)$ - em função da qual se definem as leis de evolução de $\dot{\varepsilon}^{p}$ e de $\dot{\alpha}$ - e outra relativa à danificação $\varphi_{D}^{*}(Y, D)$ - em função da qual se 
define a lei de evolução de $\dot{D}$. Em cada uma das parcelas a variável $D$ aparece como um parâmetro.

Adota-se como potencial de dissipação $\varphi_{p}^{*}$ a função de plastificação de von Mises com encruamento isótropo, utilizando-se a tensão efetiva de modo a levar em conta a danificação:

$$
\varphi_{p}^{*}=\frac{\|S\|}{(1-D)}-\sqrt{\frac{2}{3}}\left(\sigma_{t}+R\right)
$$

Substituindo-se a eq. (252) na eq. (251a), obtém-se a seguinte expressão para a lei de evolução da deformação plástica:

$$
\dot{\varepsilon}^{p}=\dot{\lambda} \frac{\partial \varphi_{p}^{*}}{\partial \sigma}=\frac{\dot{\lambda}}{(1-D)} n
$$

sendo $n=\frac{S}{\|S\|}$ o versor normal à superfície de plastificação definida pela função $\varphi_{p}^{*}$.

Por outo lado, substituindo-se a eq. (252) na eq. (251c), chega-se à lei de evolução da variável de encruamento $\alpha$ :

$$
\dot{\alpha}=-\dot{\lambda}\left(-\sqrt{\frac{2}{3}}\right)=\dot{\lambda} \sqrt{\frac{2}{3}}
$$

Observa-se que no caso de encruamento isótropo linear, a variável associada ao encruamento é dada por uma relação na forma $R=K \alpha$, sendo $K$ denominado módulo plástico de encruamento isótropo.

Com relação ao potencial de dissipação referente à danificação $\varphi_{D}^{*}$, escolhe-se uma expressão de modo a ser uma função não-linear em $-Y$ :

$$
\varphi_{D}^{*}=\frac{S_{0}}{\left(s_{0}+1\right)} \frac{1}{(1-D)}\left(-\frac{Y}{S_{0}}\right)^{s_{0}+1}
$$

onde $S_{0}$ e $s_{0}$ são coeficientes característicos do material a serem identificados mediante ensaios experimentais.

Substituindo-se a expressão de $\varphi_{D}^{*}$ dada pela eq. (255) na eq. (251b), obtém-se a seguinte lei para a evolução do dano:

$$
\dot{D}=\frac{\dot{\lambda}}{(1-D)}\left(-\frac{Y}{S_{0}}\right)^{s_{0}}
$$


Define-se também uma grandeza $\left(\varepsilon_{p}^{*}\right)$, a norma da deformação plástica acumulada, cuja lei de evolução é dada, fazendo-se uso da eq. (253), por:

$$
\dot{\varepsilon}_{p}^{*}=\left(\dot{\varepsilon}^{p} \cdot \dot{\varepsilon}^{p}\right)^{\frac{1}{2}}=\frac{\dot{\lambda}}{(1-D)}
$$

Substituindo-se a eq. (257) na eq. (256), pode-se expressar a lei de evolução do dano em função da norma da deformação plástica acumulada:

$$
\dot{D}=\left(-\frac{Y}{S_{0}}\right)^{S_{0}} \dot{\varepsilon}_{p}^{*}
$$

Substituindo-se a expressão de $Y$ definida pela eq. (245) na eq. (258), fazendo-se uso da eq. (231), obtém-se:

$$
\dot{D}=\left(\frac{\sigma_{e q}{ }^{2}}{2 E S_{0}(1-D)^{2}}\right)^{s_{0}}\left[\frac{2}{3}(1+v)+3(1-2 v)\left(\frac{\sigma_{H}}{\sigma_{e q}}\right)^{2}\right]^{s_{0}} \dot{\varepsilon}_{p}^{*}
$$

Pode-se considerar que o dano dúctil ocorra quando o encruamento está saturado, ou seja, que $R$ tenha atingido um valor: $R=R_{\infty}$. Neste caso, a eq. (252) permite tomar como constante o termo $\sigma_{e q} /(1-D)=C_{K}$. Se forem considerados apenas carregamentos radiais, para os quais a razão $\sigma_{H} / \sigma_{e q}$ é constante, então a eq. (259) passa a ser diretamente integrável e o dano resulta linearmente variável com a norma da deformação plástica acumulada $\left(\varepsilon_{p}^{*}\right)$.

O modelo, porém, permite considerar que o dano evolua somente após ser atingido um certo valor de deformação plástica acumulada, que seria uma condição inicial para a integração da eq. (259). Este valor, no caso unidimensional, é representado por $\varepsilon_{D}$, sendo característico de cada material e podendo ser determinado em ensaios simples. Este conceito é extendido para o caso tridimensional, admitindo-se que o início do dano se dê uma vez que a norma da deformação plástica atinja o valor $\varepsilon_{p, D}$. A partir das considerações anteriores, pode-se escrever a eq. (259) integrada na seguinte forma:

$$
D=\left(\frac{C_{K}{ }^{2}}{2 E S_{0}}\right)^{s_{0}}\left[\frac{2}{3}(1+v)+3(1-2 v)\left(\frac{\sigma_{H}}{\sigma_{e q}}\right)^{2}\right]^{s_{0}}\left(\varepsilon_{p}{ }^{*}-\varepsilon_{p, D}\right)
$$

Por outro lado, pode-se considerar, também, a existência de um valor crítico para o dano $\left(D_{C}\right)$, em correspondência ao início da fratura em nível local, ao qual corresponde, 
no caso unidimensional, um valor limite $\left(\varepsilon_{R}\right)$ para a deformação plástica acumulada. Ambos os parâmetros $\left(D_{C}\right.$ e $\left.\varepsilon_{R}\right)$ podem ser determinados em ensaios uniaxiais. No caso tridimensional, o dano crítico $\left(D_{C}\right)$ é atingido quando a norma da deformação plástica acumulada atinge o valor máximo $\varepsilon_{p, R}$. Substituindo-se esses valores na eq. (260), obtémse:

$$
\frac{D_{C}}{\varepsilon_{p, R}\left(1-\frac{\varepsilon_{p, D}}{\varepsilon_{p, R}}\right)}=\left(\frac{C_{K}{ }^{2}}{2 E S_{0}}\right)^{s_{0}}\left[\frac{2}{3}(1+v)+3(1-2 v)\left(\frac{\sigma_{H}}{\sigma_{e q}}\right)^{2}\right]^{s_{0}}
$$

Por simplificação, pode-se admitir que as normas de deformação plástica no início do dano e na ruptura $\left(\varepsilon_{p, D}\right.$ e $\left.\varepsilon_{p, R}\right)$ - definidas para o caso multiaxial - sejam as mesmas do caso unidimensional, definidas por $\varepsilon_{D}$ e $\varepsilon_{R}$, respectivamente. Além disso, considera-se que quando $\varepsilon_{p}=\varepsilon_{p, R}$, a razão de triaxialidade seja unitária $\left(R_{v}=1\right)$. Essa consideração é razoável, em modelos de dano e plasticidade acoplados, quando o critério de plastificação está relacionado às tensões desviadoras (RIZZI (1995), PITUBA (2003)). A partir das considerações anteriores, pode-se, finalmente, estabelecer a igualdade:

$$
\left(\frac{C_{K}^{2}}{2 E S_{0}}\right)^{s_{0}}=\frac{D_{C}}{\left(\varepsilon_{R}-\varepsilon_{D}\right)}
$$

Substituindo-se, então, a eq. (262) na eq. (259) e o valor de $\dot{\varepsilon}_{p}{ }^{*}$ por aquele dado na eq. (257), a expressão da lei de evolução do dano assume a seguinte forma:

$$
\dot{D}=\dot{\lambda} \frac{D_{C}}{\left(\varepsilon_{R}-\varepsilon_{D}\right)(1-D)}\left[\frac{2}{3}(1+v)+3(1-2 v)\left(\frac{\sigma_{H}}{\sigma_{e q}}\right)^{2}\right]
$$

Na eq. (263), admitiu-se $s_{0}=1$.

\subsubsection{Resumo das equações do modelo de Lemaitre.}

Neste trabalho, com o objetivo de se obter uma expressão incremental explícita para o multiplicador $\dot{\lambda}$, optou-se por introduzir uma forma não-associativa para o modelo original de Lemaitre. Dessa forma, o critério de plastificação $(f)$ difere do potencial $\varphi_{p}^{*}$, do qual se derivam $\dot{\varepsilon}^{p}$ e $\dot{\alpha}$. As novas expressões são as seguintes:

$$
\varphi_{p}^{*}=\frac{\|S\|}{(1-D)}-\sqrt{\frac{2}{3}}\left(\sigma_{t}+K \alpha\right)
$$




$$
\begin{aligned}
& f=\|S\|-\sqrt{\frac{2}{3}}\left(\sigma_{t}+K \alpha\right)(1-D) \\
& \dot{\sigma}=(1-D) C\left(\dot{\varepsilon}-\dot{\varepsilon}^{p}\right) \\
& \dot{\varepsilon}^{p}=\frac{\dot{\lambda}}{(1-D)} n \\
& \dot{\alpha}=\frac{\dot{\lambda}}{(1-D)} \sqrt{\frac{2}{3}} \\
& \dot{D}=\dot{\lambda} \frac{D_{C}}{\left(\varepsilon_{R}-\varepsilon_{D}\right)(1-D)}\left[\frac{2(1+v)}{3}+3(1-2 v)\left(\frac{\sigma_{H}}{\sigma_{e q}}\right)^{2}\right]
\end{aligned}
$$

Pode-se constatar a partir da análise das equações do modelo que o encruamento adotado é do tipo isótropo linear. Essa opção foi adotada neste trabalho por conveniência em relação à maior facilidade de implementação e, a rigor, não é totalmente consistente com as hipóteses que conduziram à expressão (263). Para diminuir a influência dessa consideração sobre a precisão das respostas, as simulações numéricas limitam-se a valores de dano inferiores ao dano crítico.

\subsubsection{Algoritmo de integração em passo finito}

As expressões do modelo constitutivo de Lemaitre dadas no item 4.3.2.4 são agora apresentadas em passo finito. Como mencionado no item 4.3.2.3, o dano inicia-se somente quando a norma da deformação plástica $\left(\varepsilon_{p}^{*}\right)$ excede o valor característico do material $\left(\varepsilon_{D}\right)$. Caso já tenha ocorrido a plastificação e $\varepsilon_{p}^{*}<\varepsilon_{D}$, as equações apresentadas no item 4.3.2.4 reduzem-se àquelas apresentadas no item 3.3.2.2; o algoritmo de integração a ser utilizado nesta situação é o apresentado naquele item.

Por simplicidade, neste item apresenta-se o algoritmo de integração apenas para a situação em que a plastificação ocorre acompanhada de evolução do dano, ou seja, quando $\varepsilon_{p}^{*} \geq \varepsilon_{D}$

O algoritmo adotado é do tipo implícito, constituído de etapas de previsão e correção. Em resumo, admitem-se conhecidas as variáveis que definem o modelo constitutivo num passo $n$. A etapa de previsão consiste num passo elástico sem evolução da plastificação e da danificação. Se as variáveis de previsão levarem a que se tenha função de plastificação negativa ou nula, elas são confirmadas como às correspondentes ao passo $n+1$. Caso as variáveis do estado de previsão levem a função de plastificação a exibir um 
valor positivo, é preciso passar à fase de correção para encontrar os valores efetivos das variáveis no passo $n+1$.

As expressões que definem o modelo constitutivo na sua forma incremental são:

$$
\begin{aligned}
& \varepsilon_{n+1}=\varepsilon_{n}+\Delta \varepsilon_{n} \\
& \sigma_{n+1}=\left(1-D_{n+1}\right) C\left(\varepsilon_{n+1}-\varepsilon_{n+1}^{p}\right) \\
& \varepsilon_{n+1}^{p}=\varepsilon_{n}^{p}+\frac{\Delta \lambda}{\left(1-D_{n+1}\right)} n_{n+1}, \text { sendo } n_{n+1}=\frac{S_{n+1}}{\left\|S_{n+1}\right\|} \\
& S_{n+1}=\sigma_{n+1}-\frac{1}{3} \operatorname{tr} \sigma_{n+1} \mathrm{I} \\
& \alpha_{n+1}=\alpha_{n}+\sqrt{\frac{2}{3}} \frac{\Delta \lambda}{\left(1-D_{n+1}\right)} \\
& f_{n+1}=\left\|S_{n+1}\right\|-\sqrt{\frac{2}{3}}\left(\sigma_{t}+K \alpha_{n+1}\right)\left(1-D_{n+1}\right) \\
& D_{n+1}=D_{n}+\Delta \lambda f_{D}, \text { sendo } f_{D}=\frac{k_{1}}{\left(1-D_{n}-\Delta \lambda f_{D}\right)}+\frac{k_{2}\left(1-D_{n}-\Delta \lambda f_{D}\right)\left(\operatorname{tr} \varepsilon_{n+1}\right)^{2}}{\left\|S_{n+1}\right\|^{2}}
\end{aligned}
$$

A transformação da lei de evolução do dano do modelo de Lemaitre na expressão anterior é mostrada no Apêndice L. Nas equações acima, $\Delta \lambda$ dá a magnitude da evolução das variáveis associadas à plastificação e à danificação.

Sendo no passo $n$ conhecidos os valores de $\varepsilon_{n}, \Delta \varepsilon_{n}, \varepsilon_{n}^{p}, \alpha_{n}$ e $D_{n}$, tem-se os seguintes valores das variáveis no passo $n+1$, admitindo-se que na previsão elástica $\Delta \lambda=0$.

$$
\begin{aligned}
& \varepsilon_{n+1}^{p, p r}=\varepsilon_{n}^{p} \\
& \alpha_{n+1}^{p r}=\alpha_{n} \\
& D_{n+1}^{p r}=D_{n} \\
& \sigma_{n+1}^{p r}=\left(1-D_{n+1}^{p r}\right) C\left(\varepsilon_{n+1}-\varepsilon_{n+1}^{p, p r}\right)=\left(1-D_{n}\right) C\left(\varepsilon_{n+1}-\varepsilon_{n}^{p}\right) \\
& S_{n+1}^{p r}=\sigma_{n+1}^{p r}-\frac{1}{3} t r \sigma_{n+1}^{p r} \mathrm{I} \\
& f_{n+1}^{p r}=\left\|S_{n+1}^{p r}\right\|-\sqrt{\frac{2}{3}}\left(\sigma_{t}+K \alpha_{n+1}^{p r}\right)\left(1-D_{n+1}^{p r}\right)=\left\|S_{n+1}^{p r}\right\|-\sqrt{\frac{2}{3}}\left(\sigma_{t}+K \alpha_{n}\right)\left(1-D_{n}\right)
\end{aligned}
$$

Caso se tenha $f_{n+1}^{p r} \leq 0$, os valores de previsão das variáveis estão corretos para o passo $n+1$. Porém, se $f_{n+1}^{p r}>0$, passa-se à etapa de correção desses valores, uma vez que 
houve evolução da plastificação (e também do dano, a partir de um certo nível de deformação) nesse processo.

$\mathrm{Na}$ etapa de correção, é necessário calcular o valor das variáveis $\Delta \lambda$ e $f_{D}$ expressas em função das variáveis no estado de previsão, de modo a que se tenha $f_{n+1}=0$. Calculados os valores de $\Delta \lambda$ e de $f_{D}$, torna-se possível atualizar os valores de todas as variáveis do modelo.

Inicialmente deve-se obter uma relação entre os tensores de tensões desviadoras $S_{n+1}$ e $S_{n+1}^{p r}$. Aplicando-se o tensor constitutivo elástico dado pela eq. (122) à expressão do tensor de tensões de previsão $\sigma_{n+1}^{p r}$, obtém-se:

$$
\sigma_{n+1}^{p r}=\left(1-D_{n}\right)\left[\lambda\left(\operatorname{tr} \varepsilon_{n+1}\right) \mathrm{I}+2 \mu \varepsilon_{n+1}-2 \mu \varepsilon_{n}^{p}\right]
$$

E, aplicando-se a eq. (264) à expressão de $S_{n+1}^{p r}$, obtém-se:

$$
S_{n+1}^{p r}=\left(1-D_{n}\right) 2 \mu\left(e_{n+1}-\varepsilon_{n}^{p}\right)
$$

sendo $e_{n+1}$ a parcela desviadora do tensor de deformações, dada pela eq. (154).

Aplica-se agora o tensor constitutivo elástico dado por (122) à expressão do tensor de tensões $\sigma_{n+1}$ :

$$
\sigma_{n+1}=\left(1-D_{n+1}\right)\left[\lambda\left(\operatorname{tr} \varepsilon_{n+1}\right) \mathrm{I}+2 \mu \varepsilon_{n+1}-2 \mu \varepsilon_{n+1}^{p}\right]
$$

Aplica-se então a eq. (266) à expressão de $S_{n+1}$, obtendo-se:

$$
S_{n+1}=\left(1-D_{n+1}\right) 2 \mu\left(e_{n+1}-\varepsilon_{n+1}^{p}\right)
$$

Substituindo-se as expressões de $D_{n+1}$ e $\varepsilon_{n+1}^{p}$ na eq. (267), obtém-se:

$$
S_{n+1}=\left(1-D_{n}-\Delta \lambda f_{D}\right) 2 \mu\left(e_{n+1}-\varepsilon_{n}^{p}-\frac{\Delta \lambda n_{n+1}}{\left(1-D_{n}-\Delta \lambda f_{D}\right)}\right)
$$

Rearranjando-se os termos da eq. (268), escreve-se:

$$
S_{n+1}=\left(1-D_{n}\right) 2 \mu\left(e_{n+1}-\varepsilon_{n}^{p}\right)-\Delta \lambda f_{D} 2 \mu\left(e_{n+1}-\varepsilon_{n}^{p}\right)-2 \mu \Delta \lambda n_{n+1}
$$

Substituindo-se a eq. (265) na eq. (269) e simplificando-a, obtém-se:

$$
S_{n+1}=\frac{\left(1-D_{n}-\Delta \lambda f_{D}\right)}{\left(1-D_{n}\right)} S_{n+1}^{p r}-2 \mu \Delta \lambda n_{n+1}
$$

Definindo-se os tensores de norma unitária $n_{n+1}$ e $n_{n+1}^{p r}$ tais que sejam válidas as relações $S_{n+1}=\left\|S_{n+1}\right\| n_{n+1}$ e $S_{n+1}^{p r}=\left\|S_{n+1}^{p r}\right\| n_{n+1}^{p r}$ e aplicando-as à eq. (270), chega-se a:

$$
\left(1-D_{n}\right)\left[\left\|S_{n+1}\right\|+2 \mu \Delta \lambda\right] n_{n+1}=\left(1-D_{n}-\Delta \lambda f_{D}\right)\left\|S_{n+1}^{p r}\right\| n_{n+1}^{p r}
$$


Da eq. (271), conclui-se que $n_{n+1}=n_{n+1}^{p r}$ e que vale a seguinte relação entre as normas dos tensores desviadores efetivo e de previsão:

$$
\left\|S_{n+1}\right\|=\frac{\left(1-D_{n}-\Delta \lambda f_{D}\right)}{\left(1-D_{n}\right)}\left\|S_{n+1}^{p r}\right\|-2 \mu \Delta \lambda
$$

Impõe-se agora a condição $f_{n+1}=0$, substituindo-se na expressão de $f_{n+1}$ a eq. (272) e as expressões que definem $\alpha_{n+1}$ e $D_{n+1}$ :

$$
\frac{\left(1-D_{n}-\Delta \lambda f_{D}\right)}{\left(1-D_{n}\right)}\left\|S_{n+1}^{p r}\right\|-2 \mu \Delta \lambda-\sqrt{\frac{2}{3}}\left(\sigma_{t}+K \alpha_{n}\right)\left(1-D_{n}-\Delta \lambda f_{D}\right)-\frac{2}{3} K \Delta \lambda=0
$$

Fatorando-se o primeiro e o terceiro termos da eq. (273) e isolando-se os termos em $\Delta \lambda$, obtém-se:

$$
\frac{\left(1-D_{n}-\Delta \lambda f_{D}\right)}{\left(1-D_{n}\right)}\left[\left\|S_{n+1}^{p r}\right\|-\sqrt{\frac{2}{3}}\left(\sigma_{t}+K \alpha_{n}\right)\left(1-D_{n}\right)\right]=\Delta \lambda\left(2 \mu+\frac{2}{3} K\right)
$$

O termo entre colchetes corresponde à expressão de $f_{n+1}^{p r}$. Substituindo-se este termo na eq. (274) e isolando-se a variável $f_{D}$, obtém-se, finalmente, a equação:

$$
f_{D}=\frac{\left(1-D_{n}\right)}{\Delta \lambda}-\frac{\left(1-D_{n}\right)\left(2 \mu+\frac{2}{3} K\right)}{f_{n+1}^{p r}}
$$

Para o cálculo de $\Delta \lambda$, retoma-se a expressão de $f_{D}$ que aparece na definição de $D_{n+1}$ (pág. 107), nela substituindo-se a expressão de $\left\|S_{n+1}\right\|$ dada pela eq. (272):

$$
f_{D}=\frac{k_{1}}{\left(1-D_{n}-\Delta \lambda f_{D}\right)}+\frac{k_{2}\left(\operatorname{tr} \varepsilon_{n+1}\right)^{2}}{\left(1-D_{n}-\Delta \lambda f_{D}\right)\left[\frac{\left\|S_{n+1}^{p r}\right\|}{\left(1-D_{n}\right)}-\frac{2 \mu \Delta \lambda}{\left(1-D_{n}-\Delta \lambda f_{D}\right)}\right]^{2}}
$$

Fazendo-se uso da eq. (275), calcula-se o termo $\left(1-D_{n}-\Delta \lambda f_{D}\right)$ :

$$
\left(1-D_{n}-\Delta \lambda f_{D}\right)=\frac{\left(1-D_{n}\right)\left(2 \mu+\frac{2}{3} K\right) \Delta \lambda}{f_{n+1}^{p r}}
$$

Substituindo-se então na eq. (276) a expressão de $f_{D}$ dada pela eq. (275) e a expressão de $\left(1-D_{n}-\Delta \lambda f_{D}\right)$ dada por (277), é possível chegar a uma expressão para $\Delta \lambda$ em função de valores das variáveis do modelo exclusivamente no estado de previsão: 


$$
\Delta \lambda=\frac{f_{n+1}^{p r}}{\left(2 \mu+\frac{2}{3} K\right)}-\frac{k_{1}\left(f_{n+1}^{p r}\right)^{2}}{\left(1-D_{n}\right)^{2}\left(2 \mu+\frac{2}{3} K\right)^{2}}-\frac{k_{2}\left(t r \varepsilon_{n+1}\right)^{2}\left(f_{n+1}^{p r}\right)^{2}}{\left(2 \mu+\frac{2}{3} K\right)^{2}\left[\left\|S_{n+1}^{p r}\right\|-\frac{2 \mu f_{n+1}^{p r}}{\left(2 \mu+\frac{2}{3} K\right)}\right]^{2}}
$$

Utilizando-se a eq. (277) e sendo $\left(1-D_{n}-\Delta \lambda f_{D}\right)=\left(1-D_{n+1}\right)$, obtém-se:

$$
D_{n+1}=1-\frac{\left(1-D_{n}\right)\left(2 \mu+\frac{2}{3} K\right) \Delta \lambda}{f_{n+1}^{p r}}
$$

Substituindo-se a eq. (278) na eq. (279), tem-se a expressão incremental para o dano no passo $n+1$ :

$$
D_{n+1}=D_{n}+\frac{k_{1} f_{n+1}^{p r}}{\left(1-D_{n}\right)\left(2 \mu+\frac{2}{3} K\right)}+\frac{k_{2}\left(1-D_{n}\right)\left(\operatorname{tr} \varepsilon_{n+1}\right)^{2} f_{n+1}^{p r}}{\left(2 \mu+\frac{2}{3} K\right)\left[\left\|S_{n+1}^{p r}\right\|-\frac{2 \mu f_{n+1}^{p r}}{\left(2 \mu+\frac{2}{3} K\right)}\right]^{2}}
$$

Conhecidos $\Delta \lambda$ e $D_{n+1}$, atualizam-se $\alpha_{n+1}, \varepsilon_{n+1}^{p}$ e $\sigma_{n+1}$.

Apresenta-se agora o cálculo do tensor algorítmico $C_{n+1}^{e p, d}$, o qual leva em consideração o acoplamento do dano e da plasticidade, cujo conhecimento é necessário para o cálculo da matriz de rigidez tangente usada no método de Newton-Raphson de solução de sistemas não-lineares.

$$
C_{n+1}^{e p, d}=\frac{\partial \sigma_{n+1}}{\partial \varepsilon_{n+1}}
$$

Fazendo-se uso das expressões de $\sigma_{n+1}$ e $\varepsilon_{n+1}^{p}$, escreve-se:

$$
\sigma_{n+1}=C \varepsilon_{n+1}-D_{n+1} C \varepsilon_{n+1}-C \varepsilon_{n}^{p}+D_{n+1} C \varepsilon_{n}^{p}-\Delta \lambda C n_{n+1}
$$

A derivada indicada na eq. (281) deve ser feita em relação a cada um dos cinco termos da eq. (282), o que é apresentado no Apêndice M.

A expressão resultante do tensor algorítmico é:

$$
\begin{aligned}
C_{n+1}^{e p, d} & =\left(1-D_{n+1}\right) C-\lambda\left(\operatorname{tr} \varepsilon_{n+1}\right) \mathrm{I} \otimes \frac{\partial D_{n+1}}{\partial \varepsilon_{n+1}}-2 \mu \varepsilon_{n+1} \otimes \frac{\partial D_{n+1}}{\partial \varepsilon_{n+1}}+2 \mu \varepsilon_{n}^{p} \otimes \frac{\partial D_{n+1}}{\partial \varepsilon_{n+1}}- \\
& -2 \mu \Delta \lambda \frac{\partial n_{n+1}}{\partial \varepsilon_{n+1}}-2 \mu n_{n+1} \otimes \frac{\partial \Delta \lambda}{\partial \varepsilon_{n+1}}
\end{aligned}
$$


Da análise da eq. (283), verifica-se a necessidade de calcular os tensores $\frac{\partial n_{n+1}}{\partial \varepsilon_{n+1}}$, $\frac{\partial \Delta \lambda}{\partial \varepsilon_{n+1}}$ e $\frac{\partial D_{n+1}}{\partial \varepsilon_{n+1}}$. A dedução da expressão desses tensores é feita no Apêndice $\mathrm{N}$, sendo sua forma final apresentada a seguir:

$$
\frac{\partial n_{n+1}}{\partial \varepsilon_{n+1}}=K N_{1} \Pi+K N_{2}(\mathrm{I} \otimes \mathrm{I})+K N_{3}\left(n_{n+1} \otimes n_{n+1}\right)
$$

sendo os valores das constantes da eq. (284):

$$
\begin{aligned}
& K N_{1}=\frac{2 \mu\left(1-D_{n}\right)}{\left\|S_{n+1}^{p r}\right\|} \\
& K N_{2}=-\frac{2 \mu\left(1-D_{n}\right)}{3\left\|S_{n+1}^{p r}\right\|}=-\frac{1}{3} K N_{1} \\
& K N_{3}=-\frac{2 \mu\left(1-D_{n}\right)}{\left\|S_{n+1}^{p r}\right\|}=-K N_{1} \\
& \frac{\partial \Delta \lambda}{\partial \varepsilon_{n+1}}=K L_{1} \mathrm{I}+K L_{2} n_{n+1}
\end{aligned}
$$

sendo $K L_{1}$ e $K L_{2}$ dados por:

$$
\begin{aligned}
& K L_{1}=\frac{-2 k_{2}\left(\operatorname{tr} \varepsilon_{n+1}\right)\left(f_{n+1}^{p r}\right)^{2}}{\left(2 \mu+\frac{2}{3} K\right)^{2}\left[\left\|S_{n+1}^{p r}\right\|-\frac{2 \mu f_{n+1}^{p r}}{\left(2 \mu+\frac{2}{3} K\right)}\right]^{2}} \\
& K L_{2}=\frac{2 \mu}{\left(2 \mu+\frac{2}{3} K\right)}\left[\left(1-D_{n}\right)-\frac{2 k_{1} f_{n+1}^{p r}}{\left(1-D_{n}\right)\left(2 \mu+\frac{2}{3} K\right)}\right]+ \\
& +\frac{4 \mu k_{2}\left(t r \varepsilon_{n+1}\right)^{2} f_{n+1}^{p r}\left(1-D_{n}\right)}{\left[2 \mu+\frac{2}{3} K\right)^{2}\left[\left\|S_{n+1}^{p r}\right\|-\frac{2 \mu f_{n+1}^{p r}}{\left(2 \mu+\frac{2}{3} K\right)}\right]^{2}}\left[-1+\frac{2 K f_{n+1}^{p r}}{3\left(2 \mu+\frac{2}{3} K\right)\left[\left\|S_{n+1}^{p r}\right\|-\frac{2 \mu f_{n+1}^{p r}}{\left(2 \mu+\frac{2}{3} K\right)}\right]}\right]
\end{aligned}
$$




$$
\frac{\partial D_{n+1}}{\partial \varepsilon_{n+1}}=K D_{1} \mathrm{I}+K D_{2} n_{n+1}
$$

sendo:

$$
\begin{aligned}
K D_{1}= & \frac{2 k_{2}\left(1-D_{n}\right)\left(\operatorname{tr} \varepsilon_{n+1}\right) f_{n+1}^{p r}}{\left(2 \mu+\frac{2}{3} K\right)\left[\left\|S_{n+1}^{p r}\right\|-\frac{2 \mu f_{n+1}^{p r}}{\left(2 \mu+\frac{2}{3} K\right)}\right]^{2}} \\
K D_{2}= & \frac{2 \mu k_{1}}{\left(2 \mu+\frac{2}{3} K\right)}+\frac{2 \mu k_{2}\left(1-D_{n}\right)^{2}\left(\operatorname{tr} \varepsilon_{n+1}\right)^{2}}{\left(2 \mu+\frac{2}{3} K\right)\left[\left\|S_{n+1}^{p r}\right\|-\frac{2 \mu f_{n+1}^{p r}}{\left(2 \mu+\frac{2}{3} K\right)}\right]^{2}}- \\
& -\frac{8 \mu K k_{2}\left(1-D_{n}\right)^{2}\left(\operatorname{tr} \varepsilon_{n+1}\right)^{2} f_{n+1}^{p r}}{\left.2 \mu+\frac{2}{3} K\right)^{2}\left[\left\|S_{n+1}^{p r}\right\|-\frac{2 \mu f_{n+1}^{p r}}{\left(2 \mu+\frac{2}{3} K\right)}\right]^{3}}
\end{aligned}
$$

Substituindo-se na eq. (283) os valores de $\frac{\partial n_{n+1}}{\partial \varepsilon_{n+1}}$, $\frac{\partial \Delta \lambda}{\partial \varepsilon_{n+1}}$ e $\frac{\partial D_{n+1}}{\partial \varepsilon_{n+1}}$, dados respectivamente pelas eqs. (284), (285) e (286), obtém-se para o tensor algorítmico tangente a seguinte expressão:

$$
C_{n+1}^{e p, d}=C_{n+1}^{e l, d}-C_{n+1}^{p, d}
$$

O tensor $C_{n+1}^{e l, d}$ está relacionado ao efeito do dano sobre o tensor elástico linear $C$, sendo dado por:

$$
C_{n+1}^{e l, d}=\left(1-D_{n+1}\right) C
$$

E o tensor $C_{n+1}^{p, d}$ está relacionado ao efeito do acoplamento entre o dano e a plasticidade, sendo dado por:

$$
\begin{aligned}
C_{n+1}^{p, d}= & D P_{1} \Pi+D P_{2}(\mathrm{I} \otimes \mathrm{I})+D P_{3}\left(\varepsilon_{n+1} \otimes \mathrm{I}\right)+D P_{4}\left(\varepsilon_{n}^{p} \otimes \mathrm{I}\right)+D P_{5}\left(\mathrm{I} \otimes n_{n+1}\right)+ \\
& +D P_{6}\left(n_{n+1} \otimes \mathrm{I}\right)+D P_{7}\left(\varepsilon_{n+1} \otimes n_{n+1}\right)+D P_{8}\left(\varepsilon_{n}^{p} \otimes n_{n+1}\right)+D P_{9}\left(n_{n+1} \otimes n_{n+1}\right)
\end{aligned}
$$

sendo:

$$
\begin{aligned}
& D P_{1}=2 \mu \Delta \lambda K N_{1} \\
& D P_{2}=\lambda\left(\operatorname{tr} \varepsilon_{n+1}\right) K D_{1}+2 \mu \Delta \lambda K N_{2} \\
& D P_{3}=2 \mu K D_{1}
\end{aligned}
$$




$$
\begin{aligned}
& D P_{4}=-2 \mu K D_{1} \\
& D P_{5}=\lambda\left(\operatorname{tr} \varepsilon_{n+1}\right) K D_{2} \\
& D P_{6}=2 \mu K L_{1} \\
& D P_{7}=2 \mu K D_{2} \\
& D P_{8}=-2 \mu K D_{2} \\
& D P_{9}=2 \mu \Delta \lambda K N_{3}+2 \mu K L_{2}
\end{aligned}
$$

A dedução da expressão da matriz tangente deste modelo (obtida utilizando-se o tensor $C_{n+1}^{e p, d}$ ) é apresentada no Apêndice O. 


\section{O CÓDIGO DE CÁLCULO DESENVOLVIDO}

Apresentam-se a seguir as principais características do código desenvolvido:

1) Escrito em linguagem Fortran 90.

2) Pede-se ao usuário que realize, inicialmente, as seguintes escolhas:

a) Tipo de elemento tridimensional a ser adotado: tetraedro ou hexaedro.

b) Forma de armazenamento da matriz de rigidez: integralmente ou em banda.

c) Modelo constitutivo a ser utilizado: elástico-linear, dano elástico (Mazars), elastoplástico (von Mises), ou dano dúctil (Lemaitre).

d) No caso de análise não-linear, deve-se optar entre o controle de carga aplicada ou de deslocamento aplicado.

3) A leitura de dados, realizada através de arquivo, está dividida em cinco partes: uma leitura preliminar, uma leitura geral, uma leitura relativa ao enriquecimento da aproximação, uma leitura relativa ao modelo constitutivo adotado (dano de Mazars, plasticidade ou modelo de Lemaitre) e uma leitura relativa ao processo incrementaliterativo de solução do sistema de equações.

3.1) Na leitura preliminar, lêem-se o número de nós da estrutura, o número de elementos em que a estrutura foi discretizada, o número de faces de elementos carregadas e o número de nós com ao menos um deslocamento prescrito.

3.2) Na leitura geral, lêem-se: as coordenadas dos nós da estrutura segundo um sistema cartesiano global $x y z$, a numeração global dos nós dos vértices de cada elemento, o módulo de elasticidade longitudinal e o coeficiente de Poisson, o valor das forças distribuídas aplicadas às faces dos elementos (e sua direção) e o valor dos deslocamentos prescritos.

3.3) O código permite enriquecer de forma independente os deslocamentos de um mesmo nó. Dessa forma, a leitura referente ao enriquecimento consiste nos seguintes passos: lê-se um enriquecimento geral, que é adotado para todos os deslocamentos de todos os nós; lê-se a seguir o número de nós que terá pelo menos um deslocamento com enriquecimento distinto do geral; por fim, lê-se para cada um desses nós qual o tipo de enriquecimento adotado para seus deslocamentos.

3.4) Em função do modelo constitutivo adotado, podem-se ter três tipos de leitura: 
3.4.1) Modelo de Mazars: lêem-se os cinco parâmetros obtidos em ensaios de identificação do material $\left(\varepsilon_{d 0}, A_{T}, B_{T}, A_{C}, B_{C}\right)$. Permite-se a especificação de valores distintos de $\varepsilon_{d 0}$ para cada elemento.

3.4.2) Modelo elastoplástico de von Mises: lêem-se o módulo plástico de encruamento isótropo $(K)$ e a tensão de plastificação $\left(\sigma_{t}\right)$, sendo permitida a determinação de valores distintos dessas grandezas para cada elemento. Caso se atribua ao parâmetro $K$ valor nulo, tem-se análise do tipo elastoplástica perfeita.

3.4.3) Modelo de Lemaitre: lêem-se o módulo plástico de encruamento isótropo $(K)$, a tensão de plastificação $\left(\sigma_{t}\right)$ e os parâmetros de identificação do material relativos a este modelo $\left(\varepsilon_{D}, \varepsilon_{R}\right.$ e $\left.D_{C}\right)$. Também neste caso, podem-se especificar valores de $K$ e $\sigma_{t}$ distintos para cada elemento; valor nulo atribuído a $K$ implica em elastoplasticidade perfeita.

3.5) Em relação ao processo incremental-iterativo, lêem-se o número de passos de carga (ou de deslocamento), o número limite de iterações em cada passo de carga ou de deslocamento e o valor da tolerância da norma de energia (critério de parada das iterações).

4) Para permitir a realização do enriquecimento, faz-se necessário o cálculo de alguns parâmetros, vetores e matrizes, indicados a seguir:

a) Cálculo do vetor que associa a cada deslocamento o número de funções enriquecedoras, em função do tipo de enriquecimento adotado.

b) Cálculo de um parâmetro que guarda a máxima dimensão entre as matrizes de rigidez dos elementos.

c) Cálculo do vetor que permite corrigir a numeração global dos deslocamentos, da situação não-enriquecida para a enriquecida.

d) Cálculo do total de novos parâmetros (ou funções) de enriquecimento, necessário para a alocação da matriz de rigidez e do vetor de forças nodais globais.

e) Cálculo de vetores que armazenam para cada elemento (tetraédrico ou hexaédrico), o número de pontos de Gauss utilizados na integração na área e no volume dos mesmos. O número de pontos de integração é definido automaticamente em função do enriquecimento dos deslocamentos dos nós dos vértices do elemento.

5) O código permite realizar o enriquecimento da aproximação inicial com funções associadas a bases polinomiais de primeiro até o sexto graus. A título de exemplo, apresentam-se a seguir as bases enriquecedoras de primeiro, segundo e terceiro graus: 


$$
\begin{aligned}
& P_{1}=\{1, x, y, z\} \\
& P_{2}=\left\{1, x, y, z, x^{2}, x y, x z, y^{2}, y z, z^{2}\right\} \\
& P_{3}=\left\{1, x, y, z, x^{2}, x y, x z, y^{2}, y z, z^{2}, x^{3}, x^{2} y, x^{2} z, x y^{2}, x y z, x z^{2}, y^{3}, y^{2} z, y z^{2}, z^{3}\right\}
\end{aligned}
$$

Deve-se ressaltar que as funções utilizadas no enriquecimento, correspondentes às bases polinomiais, são tais que seu valor é nulo no nó cujos deslocamentos são enriquecidos (sendo as coordenadas cartesianas nodais $x_{n o}, y_{n o}$ e $z_{n o}$ ). Por exemplo, a função enriquecedora correspondente ao monômio $x y z$ é dada por:

$$
f^{(x y z)}=\frac{\left(x-x_{n o}\right)}{l_{x}} \frac{\left(y-y_{n o}\right)}{l_{y}} \frac{\left(z-z_{n o}\right)}{l_{z}}
$$

sendo $l_{x}, l_{y}$ e $l_{z}$ parâmetros relacionados às dimensões dos elementos.

6) A escolha do método de solução do sistema linear depende do tipo de armazenamento escolhido para a matriz de rigidez global. Caso se tenha escolhido armazenamento em banda, há duas opções: pelo método de redução de Gauss ou por um processo direto com correção iterativa (STROUBOULIS et al (2000)). Caso se tenha escolhido armazenamento integral da matriz, são quatro as opções de resolução: pelo método de Gauss, pelo método sugerido por STROUBOULIS et al (2000), por um algoritmo especial para resolução de sistemas lineares esparsos simétricos, denominado MA27 (DUFF \& REID (1983)), ou por uma subrotina de solução de sistemas do programa FORTRAN POWERSTATION, que utiliza fatorização LU.

7) O código permite ao usuário definir pontos no domínio nos quais se deseja obter valores de resposta, em termos de deslocamentos, deformações, tensões, dano e deformações plásticas. Definindo-se as coordenadas cartesianas dos pontos, a determinação do elemento em que está o ponto e de suas coordenadas no sistema local do elemento (coordenadas de volume do tetraedro ou as adimensionais do hexaedro) é realizada de modo automático. 


\section{EXEMPLOS DE APLICAÇÃO NUMÉRICA}

A apresentação dos exemplos 1 e 2 tem como finalidade principal verificar a convergência da resposta numérica para o resultado exato. Admite-se em ambos os exemplos uma relação constitutiva elástica linear.

O exemplo 3 consiste na análise estrutural de uma barra de concreto, utilizando-se o modelo de Mazars.

O exemplo 4 tem como objetivo mostrar a aplicação do Método dos Elementos Finitos Generalizados a um problema de plasticidade sujeito ao travamento volumétrico, enquanto o exemplo 5 apresenta uma aplicação do modelo de Lemaitre.

\subsection{Exemplo 1.}

Este exemplo foi extraído de BUSSAMRA (1999). Analisa-se uma viga biengastada, cujas características geométricas são apresentadas na figura 20, sendo suas propriedades mecânicas as seguintes:

$E=1,0$ (módulo de elasticidade longitudinal).

$v=0,2$ (coeficiente de Poisson).

O carregamento consiste numa força uniformemente distribuída sobre a face superior da viga, de valor $q=1,0$.

Neste trabalho, adota-se como valor de referência para o deslocamento no centro da face inferior $w_{\text {ref }}=5,5061$ e para a energia de deformação $U_{\text {ref }}=0,32705$. Estes valores foram obtidos utilizando-se o programa ANSYS 5.4, com 5.000 elementos hexaédricos de 20 nós, totalizando 68.277 graus de liberdade.

Neste exemplo, nos processamentos com o MEFG, foram utilizados tanto elementos tetraédricos como hexaédricos. Utilizando-se elementos tetraédricos, foram realizadas análises com quatro discretizações da estrutura - com 26, 66, 105 e 285 elementos. Para cada discretização, foram utilizados cinco casos de aproximação do campo de deslocamentos: a linear, indicada como não-enriquecida (NE) e a linear enriquecida por polinômios de primeiro ao quarto graus (indicadas, respectivamente, por P1, P2, P3 e P4). Os nós situados nas faces engastadas não tiveram seus deslocamentos enriquecidos em nenhuma das aproximações, de modo a respeitar as condições de contorno do problema. A tabela 2 apresenta a flecha para cada uma das vinte análises realizadas, sendo que os 
termos entre parênteses indicam o número de graus de liberdade. A convergência da energia de deformação para o valor de referência é mostrada na figura 21.

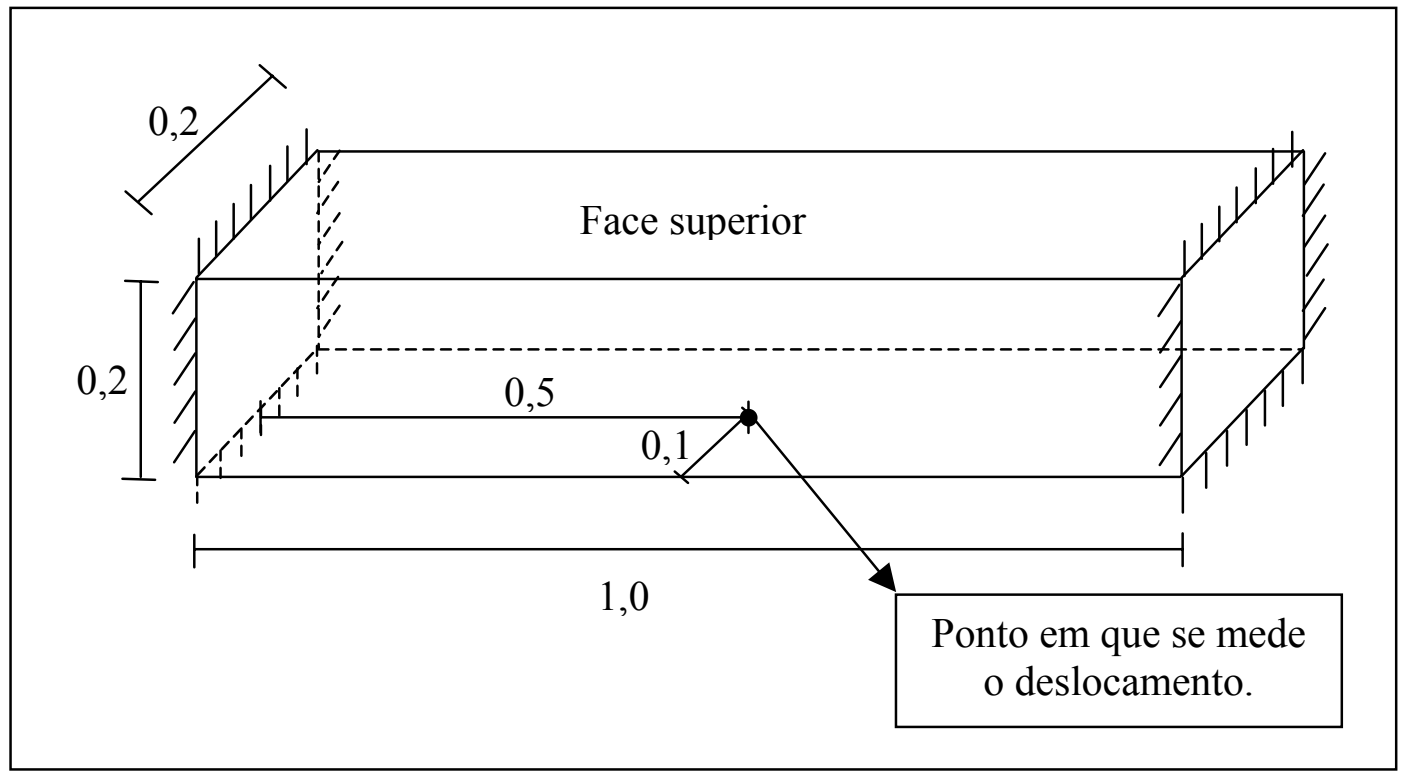

Figura 20 - Características geométricas da viga analisada.

Tabela 2 - Flecha obtida (elementos tetraédricos).

\begin{tabular}{|c|c|c|c|c|}
\hline & \multicolumn{4}{|c|}{ Número de elementos em cada discretização } \\
\hline Enriquecimento & 26 & 66 & 105 & 285 \\
\hline $\mathrm{NE}$ & $0,060499 \quad(15)$ & $2,715043 \quad(75)$ & $2,949964(102)$ & $3,845828(276)$ \\
\hline $\mathrm{P} 1$ & $0,061498 \quad(60)$ & $4,895195(300)$ & $5,066694(408)$ & $5,355138(1104)$ \\
\hline $\mathrm{P} 2$ & $0,078377(150)$ & $5,439094(750)$ & $5,469855(1020)$ & $5,477777(2760)$ \\
\hline P3 & $0,099243(300)$ & $5,477753(1500)$ & $5,491350(2040)$ & $5,498717(5520)$ \\
\hline $\mathrm{P} 4$ & $0,138355(525)$ & $5,497050(2625)$ & $5,501308(3570)$ & $5,504660(9660)$ \\
\hline
\end{tabular}

Da análise dos resultados, percebe-se que uma discretização muito grosseira (como a de 26 elementos) não permite a obtenção de uma boa resposta, exigindo, para sua melhoria, um enriquecimento de ordem muito elevada. Por outro lado, com uma discretização um pouco mais fina, mas mesmo assim com um número de elementos ainda pequeno, como na discretização em 66 elementos, pode-se notar que o enriquecimento polinomial proposto assegura uma convergência para a solução desejada. 


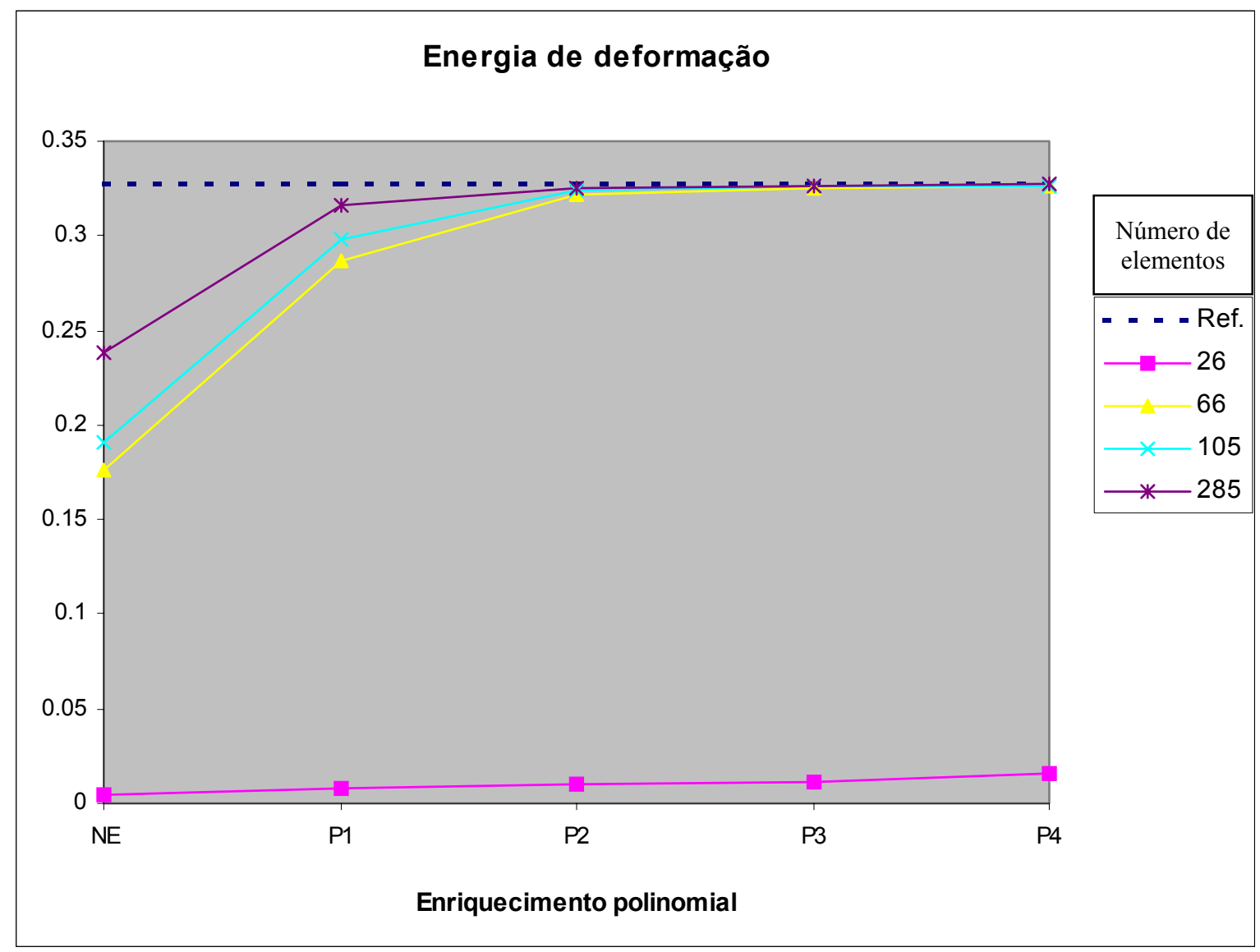

Figura 21 - Convergência da energia de deformação (elementos tetraédricos)

Utilizando-se elementos hexaédricos, foram realizadas análises com três discretizações da estrutura - com 24, 40 e 80 elementos. Para cada discretização, foram utilizadas quatro aproximações: a linear, indicada como não-enriquecida (NE), e com enriquecimentos polinomiais de primeiro ao terceiro graus (indicados, respectivamente, por P1, P2 e P3). Assim como na discretização em elementos tetraédricos, não foram enriquecidos os deslocamentos relativos aos nós das faces engastadas. A tabela 3 apresenta a flecha para cada uma das doze análises realizadas, enquanto a convergência da energia de deformação para o valor de referência é mostrada na figura 22. Os termos entre parênteses na tabela 3 dão o número de graus de liberdade em cada caso.

Da análise dos resultados, pode-se notar a obtenção de uma boa resposta para os deslocamentos mesmo com discretizações relativamente pouco refinadas. Além disso, é possível notar que para números semelhantes de graus de liberdade, a resposta é melhor para a situação de grau polinomial mais elevado. É o que se percebe comparando-se, por exemplo, a discretização em 40 elementos com enriquecimento de terceiro grau com a de 80 elementos enriquecida com polinômio de segundo grau. 
Tabela 3 - Flecha obtida (elementos hexaédricos).

\begin{tabular}{|c|c|c|c|}
\hline & \multicolumn{3}{|c|}{ Número de elementos em cada discretização } \\
\hline Enriquecimento & 24 & 40 & 80 \\
\hline NE & $4,001485(135)$ & $4,679543(243)$ & $5,048318(513)$ \\
\hline P1 & $5,375747(540)$ & $5,432852(972)$ & $5,460098(2052)$ \\
\hline P2 & $5,429627(1350)$ & $5,478777(2430)$ & $5,495093(5130)$ \\
\hline P3 & $5,484458(2700)$ & $5,495207(4860)$ & $5,502209(10260)$ \\
\hline
\end{tabular}

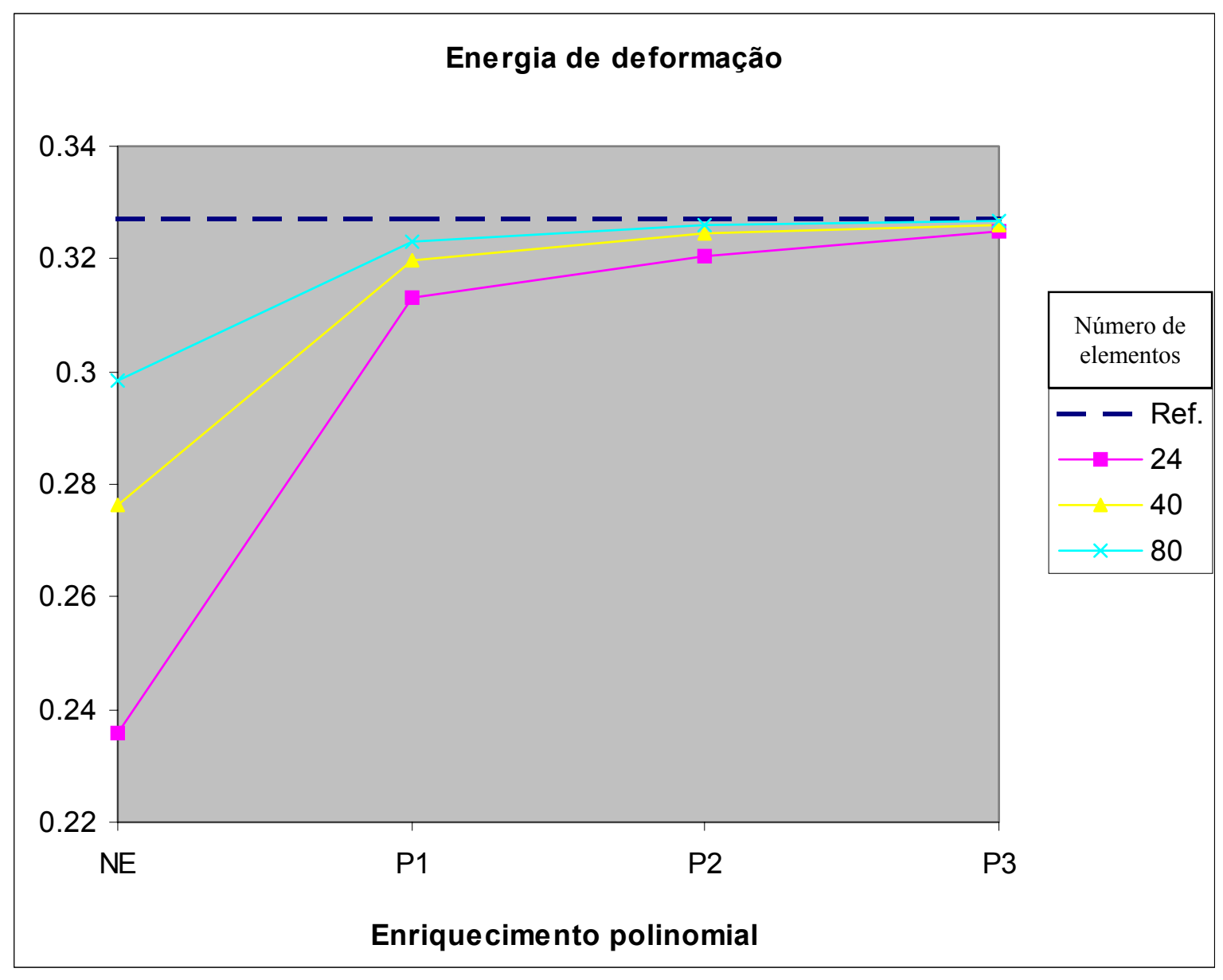

Figura 22 - Convergência da energia de deformação (elementos hexaédricos)

\subsection{Exemplo 2.}

Este exemplo foi extraído de NÓBREGA (1997). Trata-se de uma chapa engastada numa extremidade e sujeita a um carregamento uniformemente distribuído na outra. As dimensões da chapa, suas propriedades mecânicas e o valor do carregamento são 
apresentados na figura 23. Este exemplo foi apresentado originalmente por COOK (1987), constituindo-se atualmente em exemplo de referência para testes numéricos. $\mathrm{O}$ valor de confronto para a flecha no ponto A é $0,239 \mathrm{~m}$.

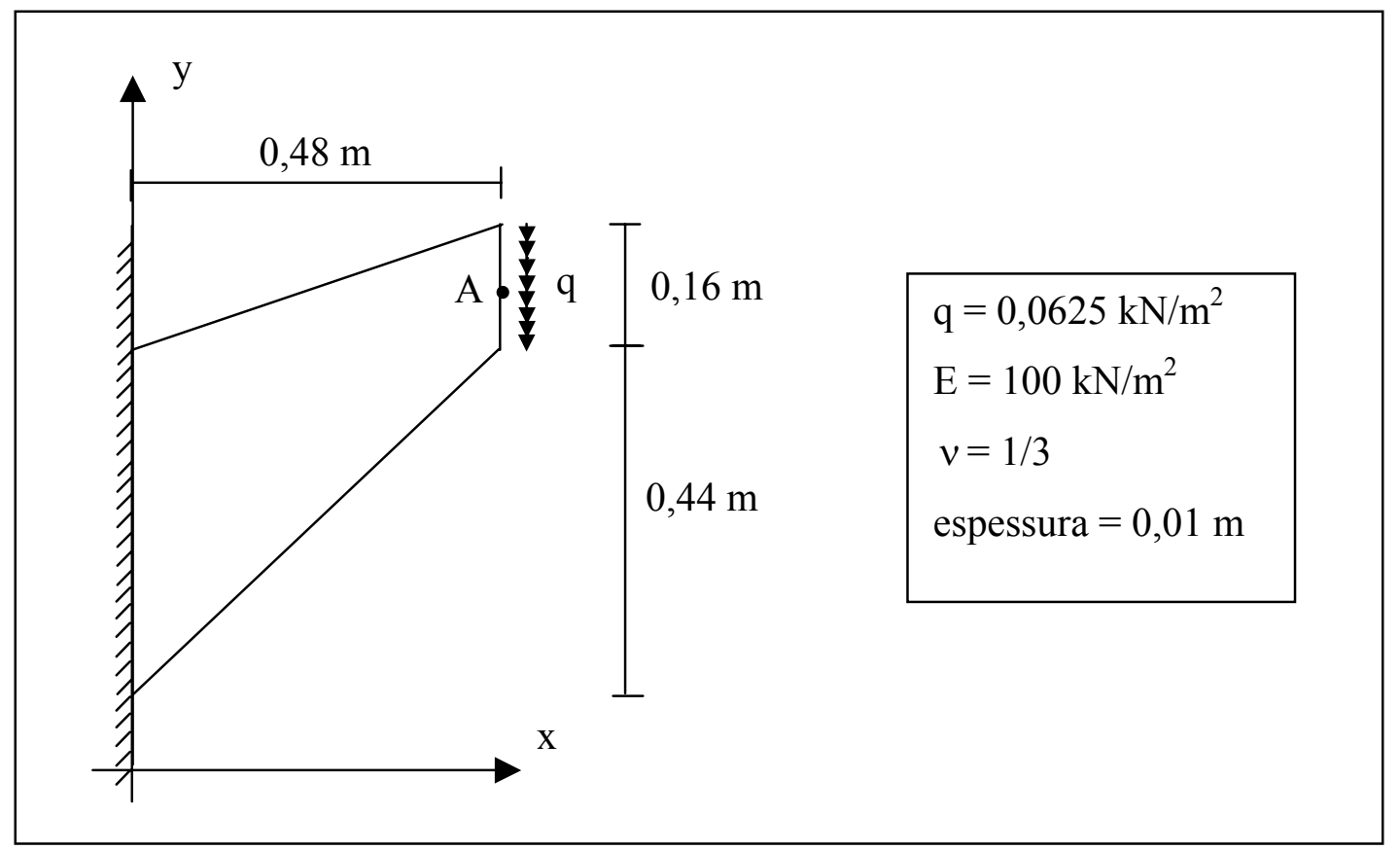

Figura 23 - Painel de Cook

Neste exemplo, utilizaram-se elementos hexaédricos em quatro discretizações: com 6, 24, 54 e 96 elementos. Para cada discretização, foram testadas quatro aproximações: a linear (indicada por NE) e as enriquecidas com polinômios de primeiro, segundo e terceiro graus (indicadas respectivamente por P1, P2 e P3). Os deslocamentos associados aos nós situados na extremidade engastada não foram enriquecidos em nenhuma das aproximações. $\mathrm{Na}$ tabela 4, apresentam-se os valores de deslocamento no ponto A obtidos com as dezesseis análises realizadas, sendo que os valores entre parênteses indicam o número de graus de liberdade da análise. A convergência da energia de deformação é apresentada na figura 24.

Tabela 4 - Deslocamento no ponto A (m)

\begin{tabular}{|c|c|c|c|c|}
\hline \multirow{2}{*}{$\begin{array}{c}\text { Enriquecimento } \\
\text { da aproximação }\end{array}$} & 6 & 24 & 54 & 96 \\
\cline { 2 - 5 } & $0,140414(54)$ & $0,196250(180)$ & $0,216331(378)$ & $0,225111(648)$ \\
\hline NE & $0,230846(216)$ & $0,236610(720)$ & $0,237791(1512)$ & $0,238318(2592)$ \\
\hline P1 & $0,236809(540)$ & $0,238598(1800)$ & $0,238979(3780)$ & $0,239139(6480)$ \\
\hline P2 & $0,237975(1080)$ & $0,238955(3600)$ & $0,239173(7560)$ & $0,239269(12960)$ \\
\hline P3 & & & & \\
\hline
\end{tabular}




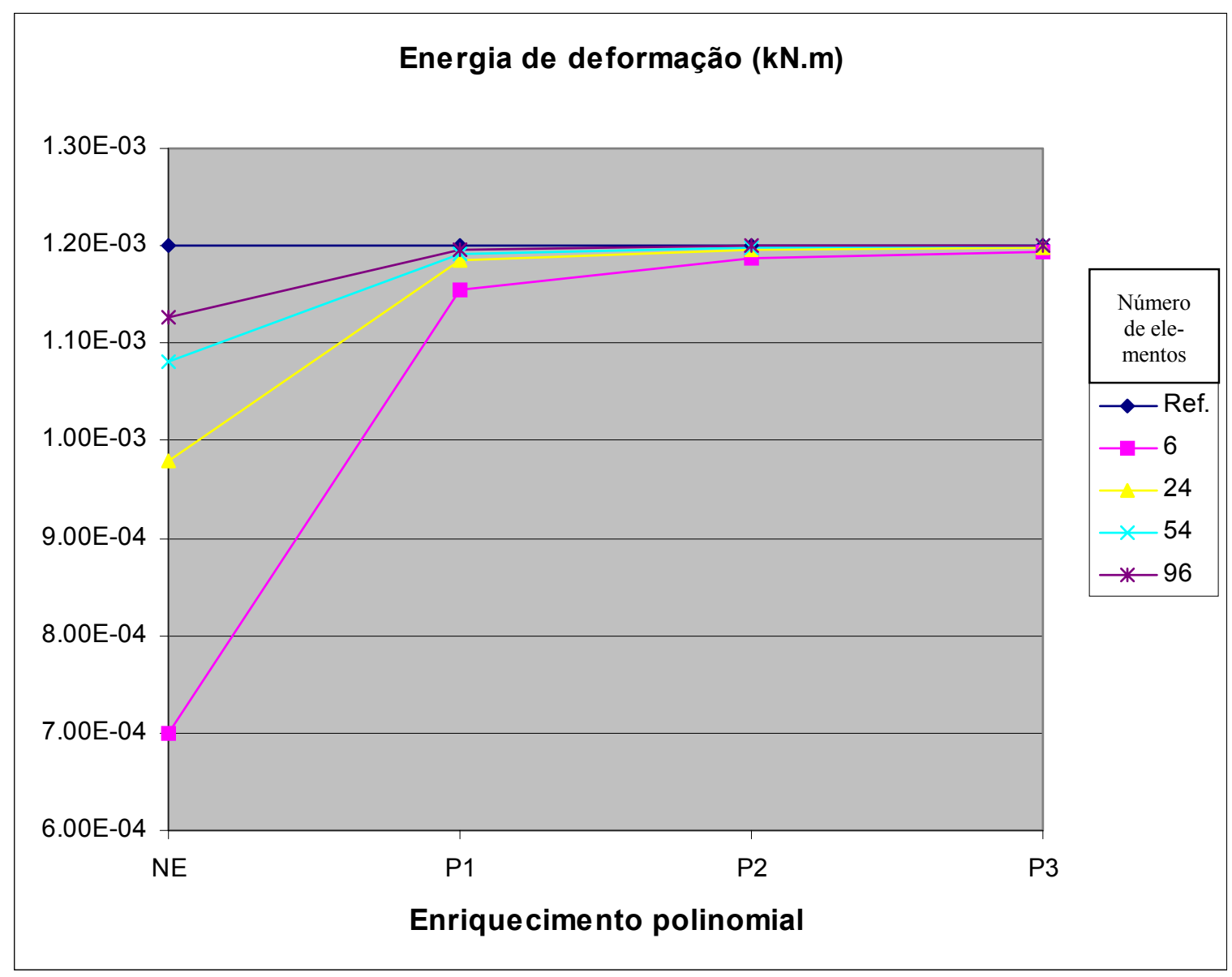

Figura 24 - Convergência da energia de deformação.

Da análise dos resultados é possível extrair algumas conclusões, expostas a seguir:

- Nota-se que a convergência da resposta para o valor de referência é muito lenta, exigindo um refinamento considerável, quando se utilizam elementos com aproximação linear (não-enriquecidos).

- Entretanto, utilizando-se enriquecimentos polinomiais de ordem mais elevada é possível obter-se boas respostas mesmo com discretizações grosseiras (como é a discretização em 6 elementos).

- Também neste exemplo é possível perceber a maior eficiência do refinamento $\mathbf{p}$ quando comparado ao $\mathbf{h}$ - verifica-se uma melhor resposta da discretização em 6 elementos com enriquecimento de terceiro grau (com 1080 graus de liberdade) do que a discretização em 54 elementos com enriquecimento de primeiro grau (com 1512 graus de liberdade). 


\subsection{Exemplo 3}

Neste exemplo, a estrutura analisada é uma barra de concreto sujeita a carregamento axial de tração em sua face superior, tendo sido adotado para o material o modelo constitutivo de Mazars, descrito no item 4.3.1. Admite-se que a barra apresente duas regiões de maior fragilidade, as quais apresentam parâmetro $\varepsilon_{d 0}$, usado para controlar o início da danificação, menor que o do restante da peça. As dimensões da barra e da região enfraquecida são mostradas na figura 25. O carregamento é aplicado na face superior através de deslocamentos controlados na direção do eixo $Z$, enquanto que, na face inferior, prescrevem-se deslocamentos nulos naquela direção.

As propriedades mecânicas do material idealizado são:

$$
\begin{aligned}
& E=25.000 \mathrm{MPa}=2,5 \cdot 10^{7} \mathrm{kN} / \mathrm{m}^{2} \\
& v=0,25 \\
& \varepsilon_{d 0}=6,7 \cdot 10^{-5}\left(6,0 \cdot 10^{-5} \text { nas regiões de maior fragilidade }\right) . \\
& A_{T}=0,7 \\
& B_{T}=8.000 \\
& A_{C}=1,13 \\
& B_{C}=1.250
\end{aligned}
$$

Discretizou-se a estrutura em 20 elementos hexaédricos, conforme mostrado na figura 26. Utilizaram-se, além da aproximação linear para os deslocamentos (aqui referida como NE), os seguintes tipos de enriquecimento:

- P1: enriquecimento polinomial de primeiro grau em todos os nós.

- P1P2: Os nós da faixa central (mostrada na figura 26) são enriquecidos com funções polinomiais de segundo grau, enquanto os demais, com funções de primeiro grau.

- P2P3: Os nós da faixa central são enriquecidos com funções polinomiais de terceiro grau, enquanto os demais, com funções de segundo grau.

Nas aproximações P1, P1P2 e P2P3, os deslocamentos na direção do eixo $Z$ dos nós situados nas faces inferior e superior não são enriquecidos, de modo a assegurar as condições de contorno em deslocamento previstas. 


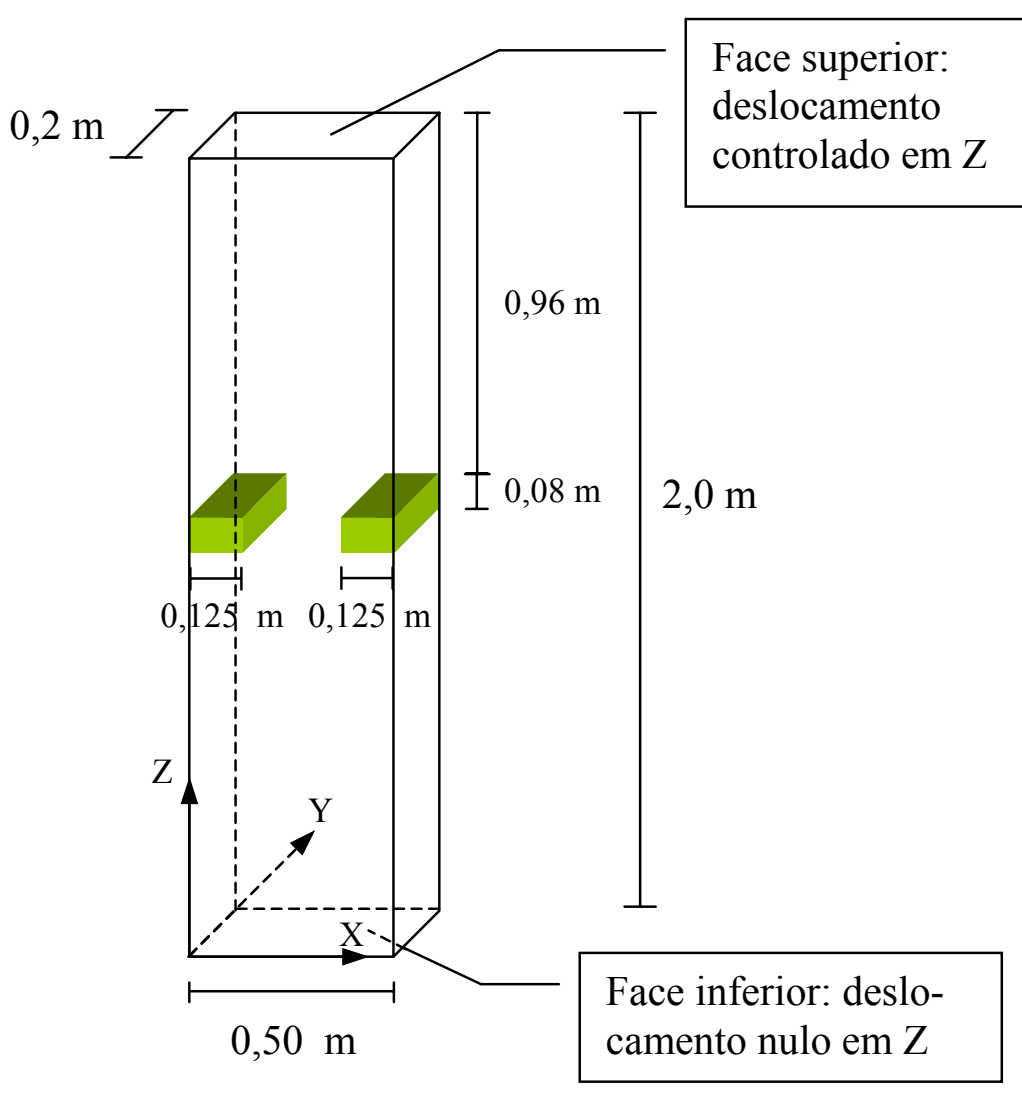

Figura 25 - Dimensões da barra e das regiões enfraquecidas.

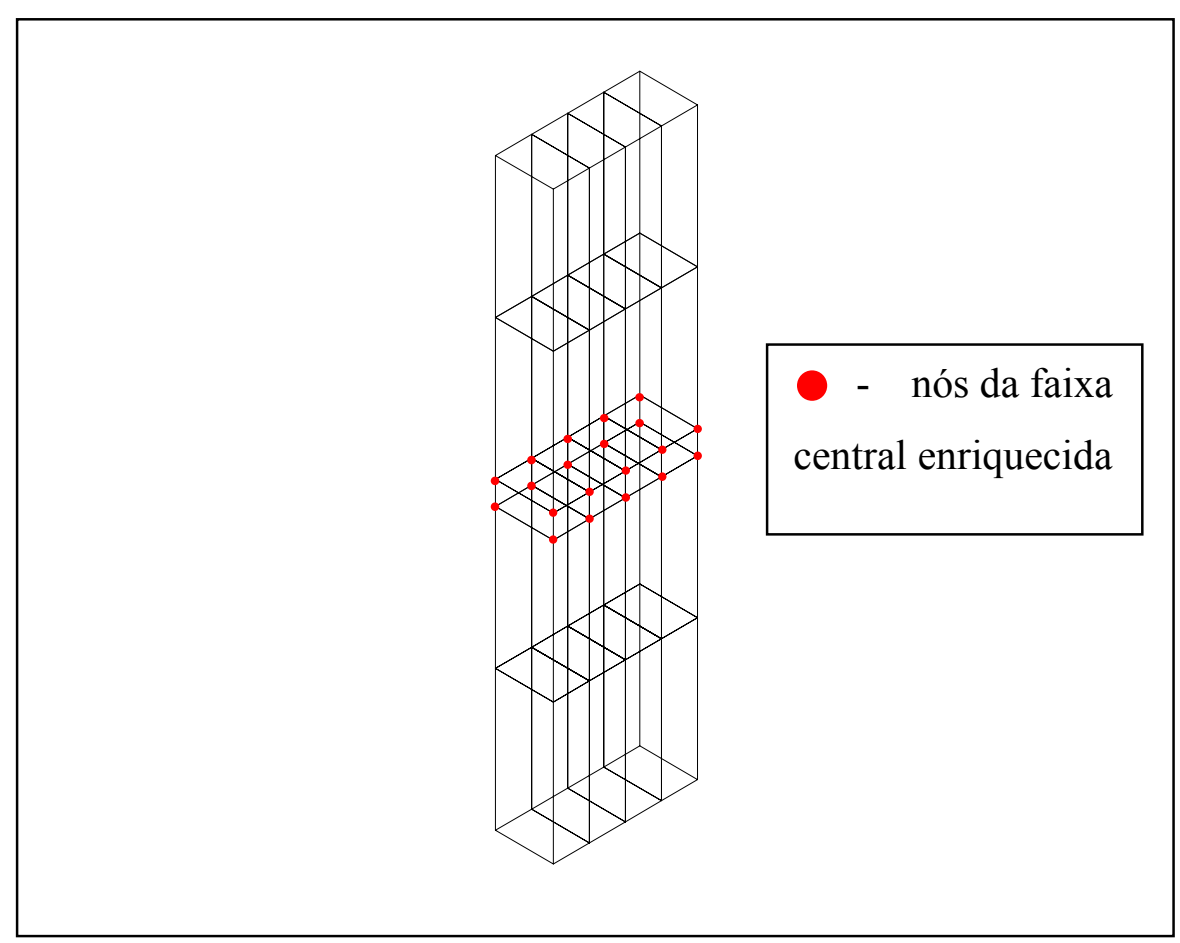

Figura 26 - Malha de elementos e faixa central enriquecida 
Impõe-se à face superior um deslocamento total de $2,425.10^{-4} \mathrm{~m}$, ao longo de cinqüenta passos. O primeiro deles consiste num deslocamento de $1,2.10^{-4} \mathrm{~m}$ e os demais, de $2,5.10^{-6} \mathrm{~m}$. Na figura 27 , apresentam-se as curvas força $\mathrm{x}$ deslocamento para os quatro tipos de enriquecimento utilizados na análise.

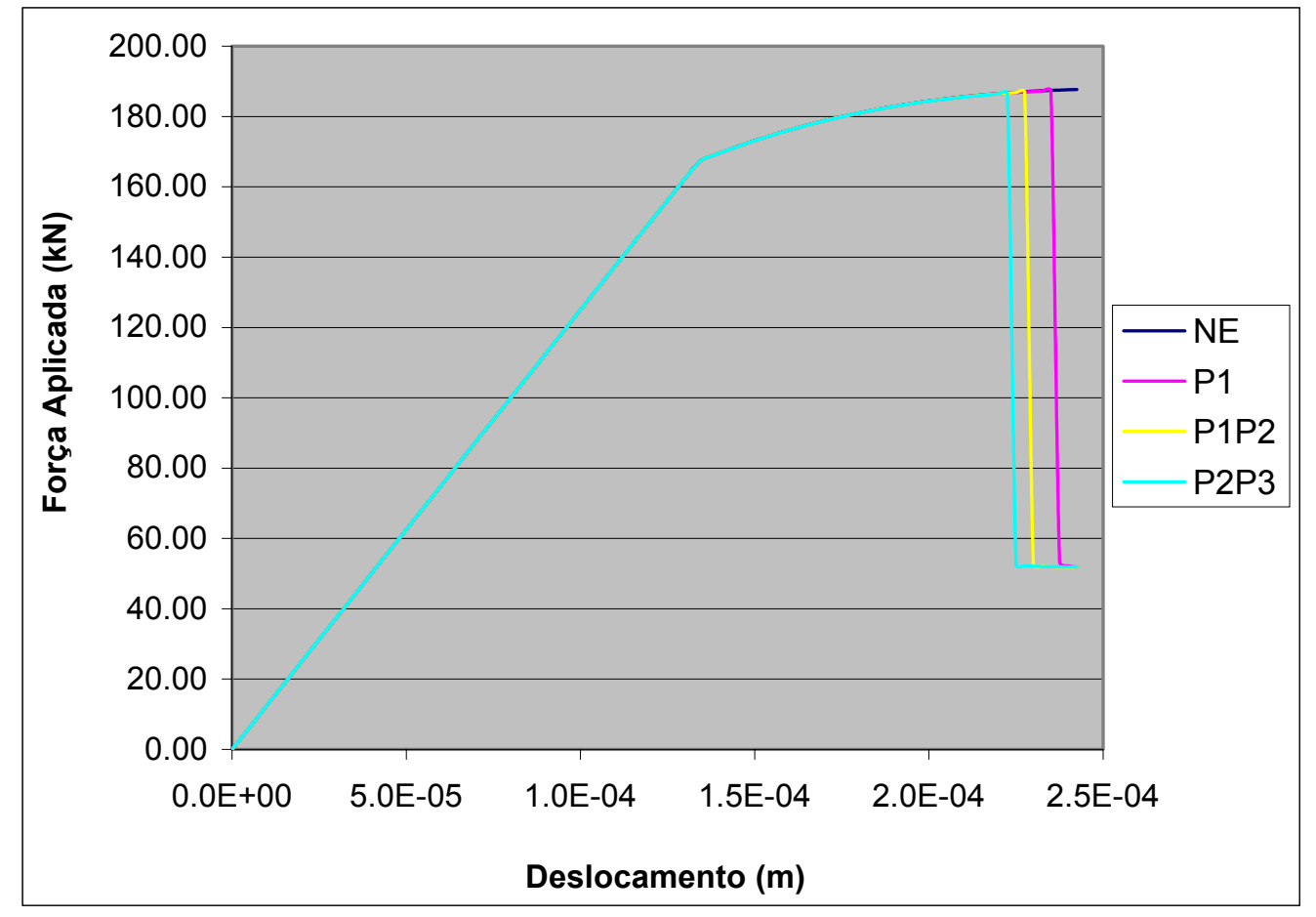

Figura 27 - Relação força x deslocamento

Verifica-se que o fenômeno da localização do dano (ao qual corresponde, na figura 27, a queda abrupta na força aplicada) ocorre em passos distintos de acordo com o tipo de enriquecimento adotado. Para a aproximação NE, a localização não chega a ocorrer. Para os enriquecimentos P1, P1P2 e P2P3, a localização ocorre, respectivamente, quando se impõem à peça os seguintes deslocamentos: $2,375.10^{-4} \mathrm{~m}, 2,3.10^{-4} \mathrm{~m}$ e $2,25.10^{-4} \mathrm{~m}$. Nota-se, além disso, que as curvas força $\mathrm{x}$ deslocamento para os diferentes tipos de enriquecimento, até o início da localização, praticamente se sobrepõem.

A figura 28 apresenta o dano num plano perpendicular ao eixo $Y$, na metade da espessura da peça, no último passo de deslocamento aplicado, para os enriquecimentos $\mathrm{P} 1$, P1P2 e P2P3. Percebe-se nitidamente como a localização do dano ocorre de forma distinta de acordo com o enriquecimento adotado: na medida em que a aproximação é enriquecida, a zona de localização tende a expandir-se no centro da peça. 


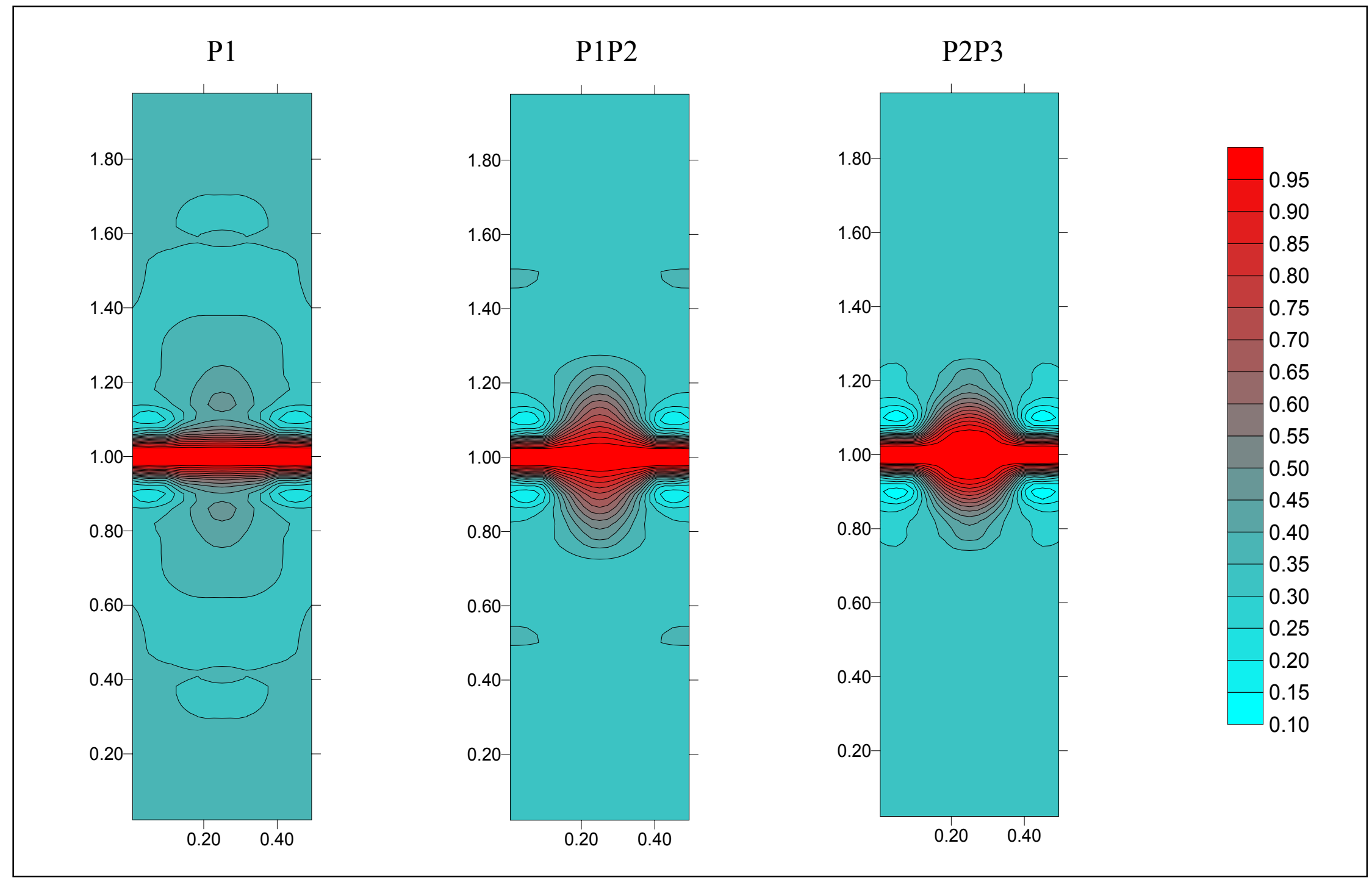

Figura 28 - Localização do dano (passo 50). 
Naturalmente, não é o objetivo desta pesquisa abordar em profundidade o problema da localização de deformações. O objetivo aqui é o de avaliar se o enriquecimento proporcionado pelo MEFG permite definir de modo mais claro a região de evolução mais localizada do dano, o que parece, de fato, acontecer.

\subsection{Exemplo 4}

Este exemplo foi extraído de WELLS et al (2002). A estrutura analisada é um bloco, em cuja face superior está uma placa infinitamente rígida sobre a qual se aplica a carga. As dimensões do bloco e da placa são mostradas na figura 29. As faces inferior e laterais do bloco têm deslocamento prescrito em todas as direções.

O modelo constitutivo do material do bloco é o elastoplástico perfeito com critério de plastificação de von Mises. As propriedades mecânicas do material idealizado são:

$$
\begin{aligned}
& E=1,0 M P a=1,0 \mathrm{~N} / \mathrm{mm}^{2} \\
& v=0,49 \\
& \sigma_{t}=0,01 \mathrm{MPa}=0,01 \mathrm{~N} / \mathrm{mm}^{2}
\end{aligned}
$$

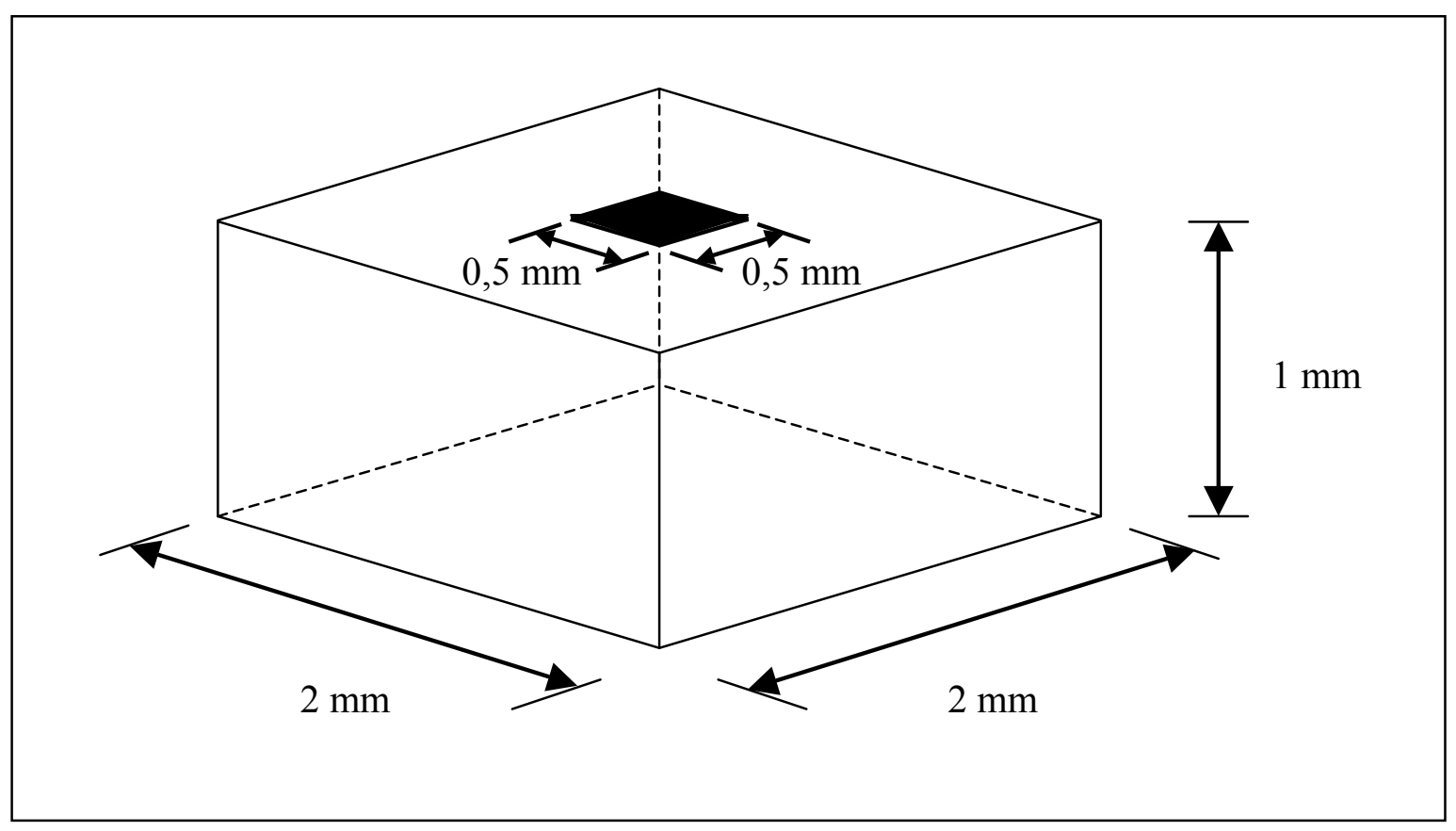

Figura 29 - Dimensões do bloco e da placa rígida.

Fazendo-se uso da dupla simetria da estrutura, analisa-se apenas uma quarta parte da mesma. A carga é aplicada de modo indireto, por meio de deslocamento vertical prescrito na região sob a placa. A figura 30 mostra a estrutura analisada e as respectivas condições de contorno. 
Foram utilizadas duas malhas de elementos hexaédricos: a primeira com 64 elementos e a segunda com 384. Para ambas as discretizações apresenta-se a relação obtida entre o deslocamento na direção $\mathrm{Z}$ na região sob a placa e a força necessária para produzilo (ambos em valor absoluto).

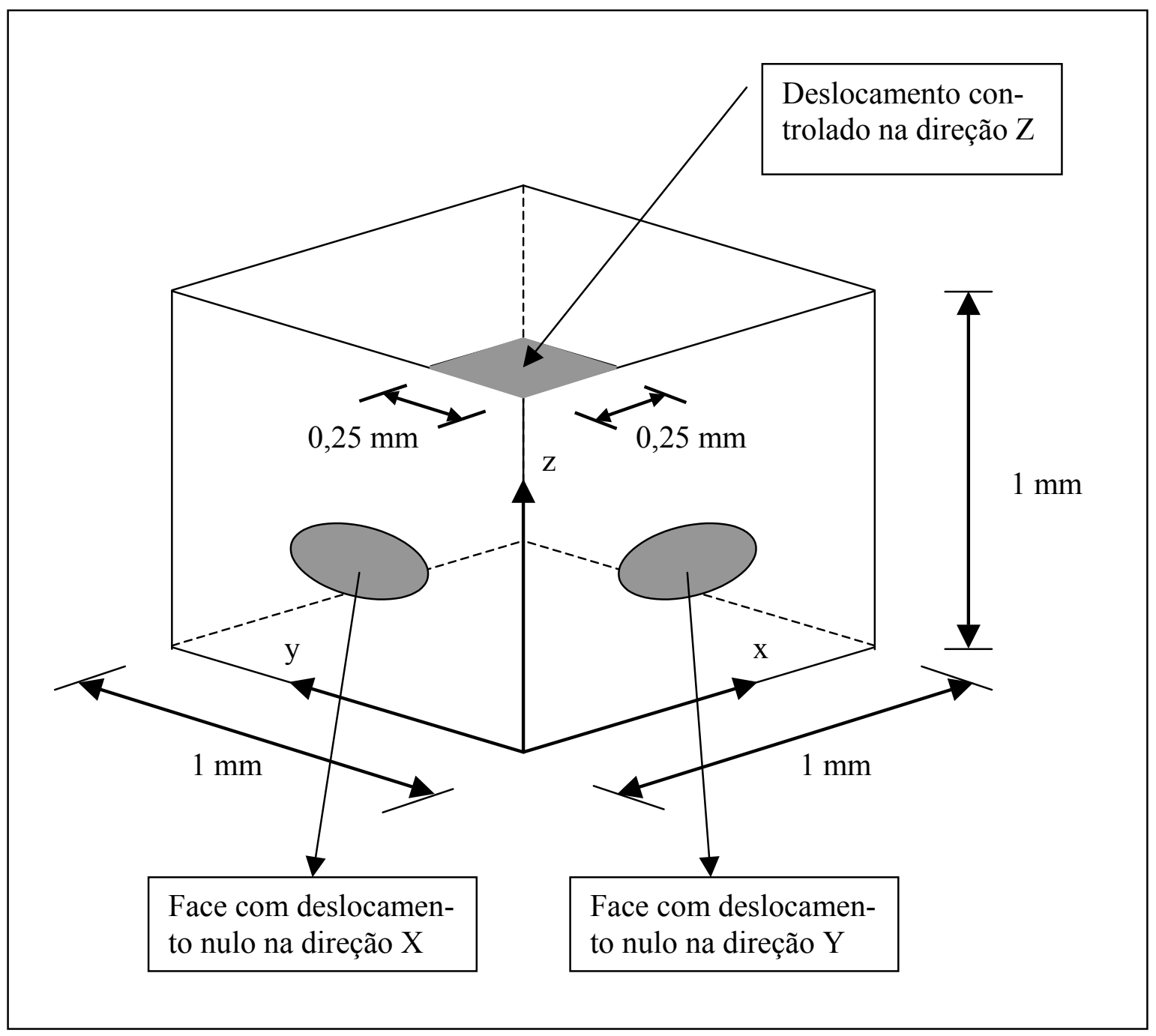

Figura 30 - Estrutura analisada utilizando dupla simetria.

Nas figuras 31 e 32 é possível perceber nitidamente o efeito do travamento volumétrico, quando se usa baixo grau de aproximação (a sigla NE indica que a aproximação linear para os deslocamentos não foi enriquecida), pois em ambos os gráficos, a carga limite não é atingida.

Verifica-se também que o enriquecimento tal como proposto neste trabalho permite superar esse efeito, uma vez que já para o enriquecimento polinomial de primeiro grau (P1) a estrutura atinge a carga limite. Além disso, nota-se uma boa eficiência do método de enriquecimento polinomial, visto que mesmo para uma malha pouco refinada (como a de 64 elementos), o valor da carga limite obtida aproxima-se bastante do apresentado por 
WELLS et al (2002), na medida em que se utilizam enriquecimentos polinomiais de segundo e terceiro graus.

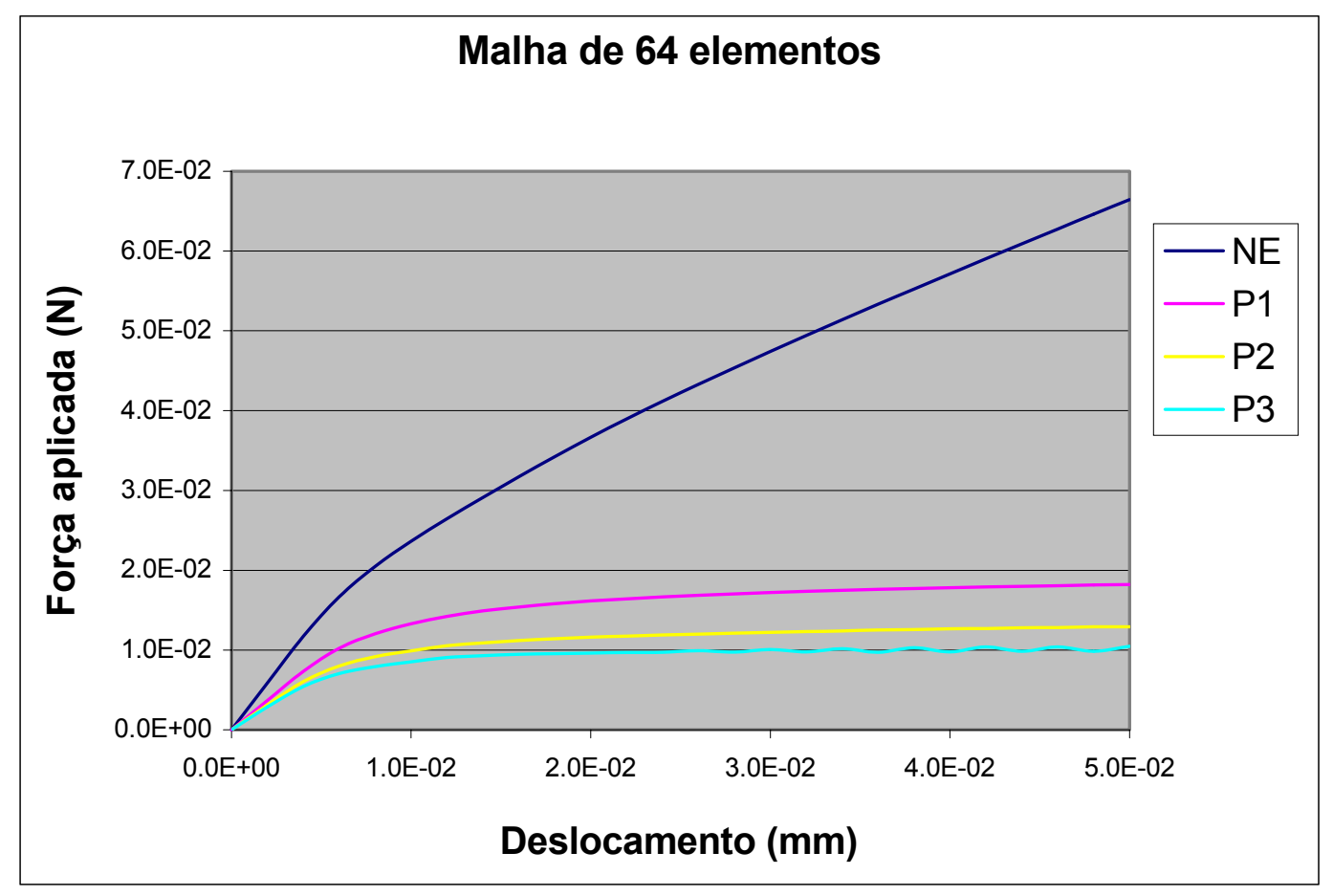

Figura 31 - Relação Força x Deslocamento para malha de 64 elementos.

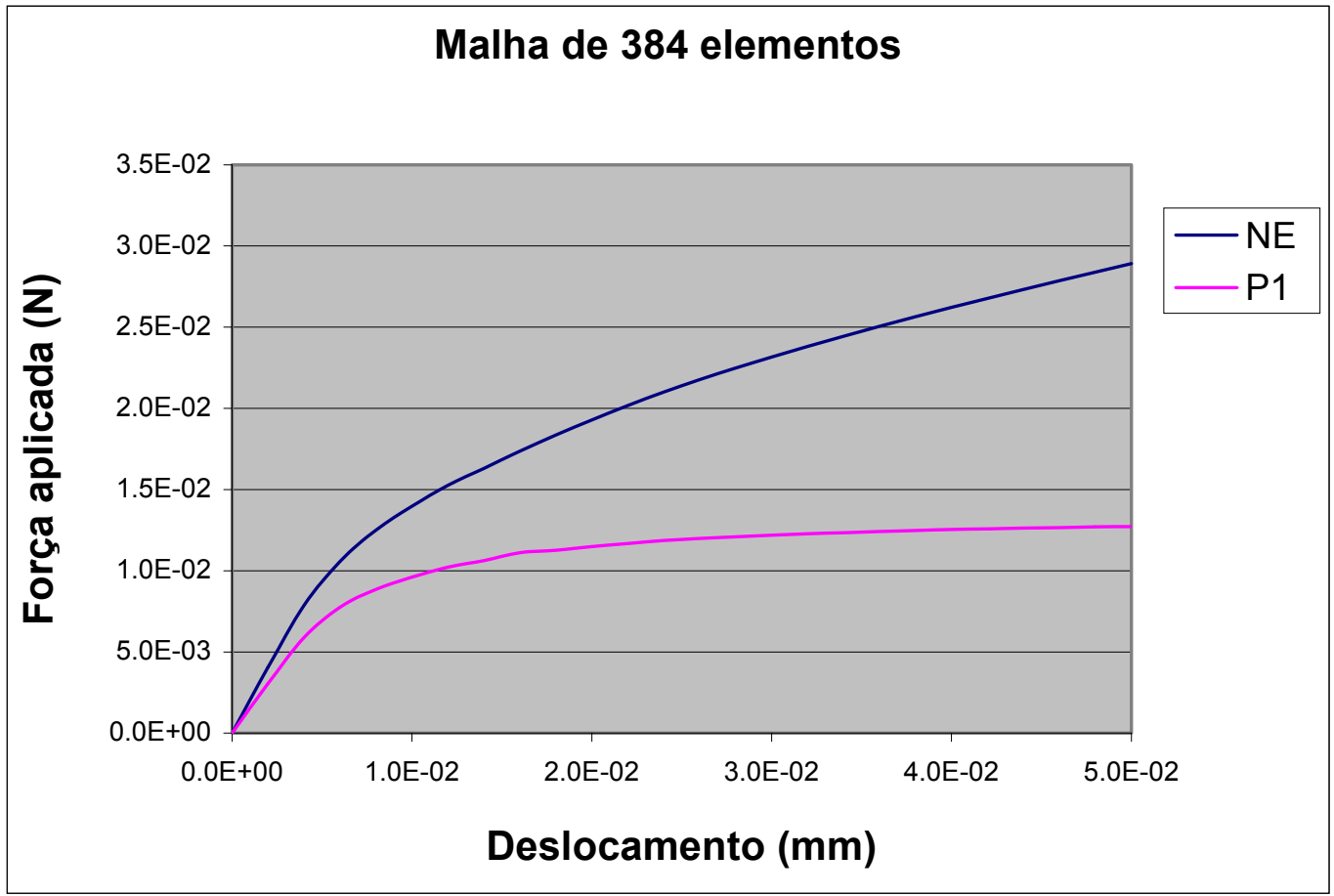

Figura 32 - Relação Força x Deslocamento para malha de 384 elementos. 


\subsection{Exemplo 5}

A estrutura analisada é uma chapa de espessura unitária com dois recortes cilíndricos, mostrada na figura 33. Indicam-se também na figura as faces nas quais se impõem deslocamentos controlados na direção do eixo $y$.

O modelo constitutivo do material que forma a chapa é o de Lemaitre - com dano e plasticidade, conforme apresentado no item 4.3.2 do capítulo 4. As propriedades mecânicas do material são:

$$
\begin{aligned}
& E=210 \mathrm{GPa}=2,1 \cdot 10^{5} \mathrm{~N} / \mathrm{mm}^{2} \\
& v=0,3 \\
& K=10,5 \mathrm{GPa}=1,05 \cdot 10^{4} \mathrm{~N} / \mathrm{mm}^{2} \\
& \sigma_{t}=620 \mathrm{MPa}=620 \mathrm{~N} / \mathrm{mm}^{2} \\
& D_{C}=0,40, \varepsilon_{D}=0,0 \text { e } \varepsilon_{R}=0,2
\end{aligned}
$$

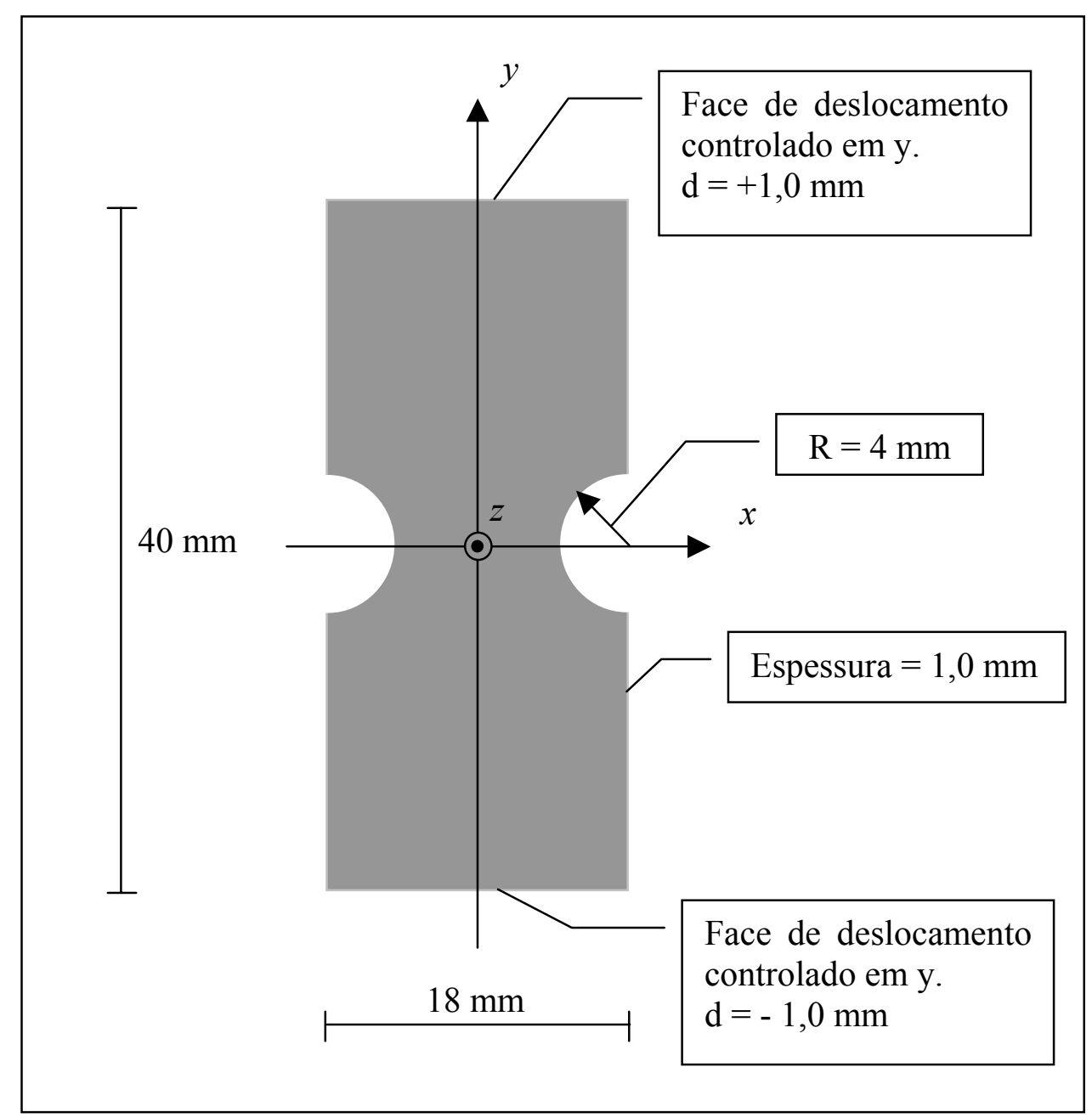

Figura 33 - Dimensões da chapa analisada. 
A análise estrutural é realizada apenas sobre um quarto da estrutura - utilizando-se os seguintes vínculos de simetria:

- num plano normal ao eixo $z$ (na metade da espessura), impõe-se deslocamento nulo na direção do eixo $z$.

- num plano normal ao eixo $x$, passando por $x=0$, impõe-se deslocamento nulo na direção desse eixo.

A figura 34 mostra a estrutura analisada efetivamente, num plano $x-y$ em $z=0$, fazendo-se uso dos vínculos de simetria.

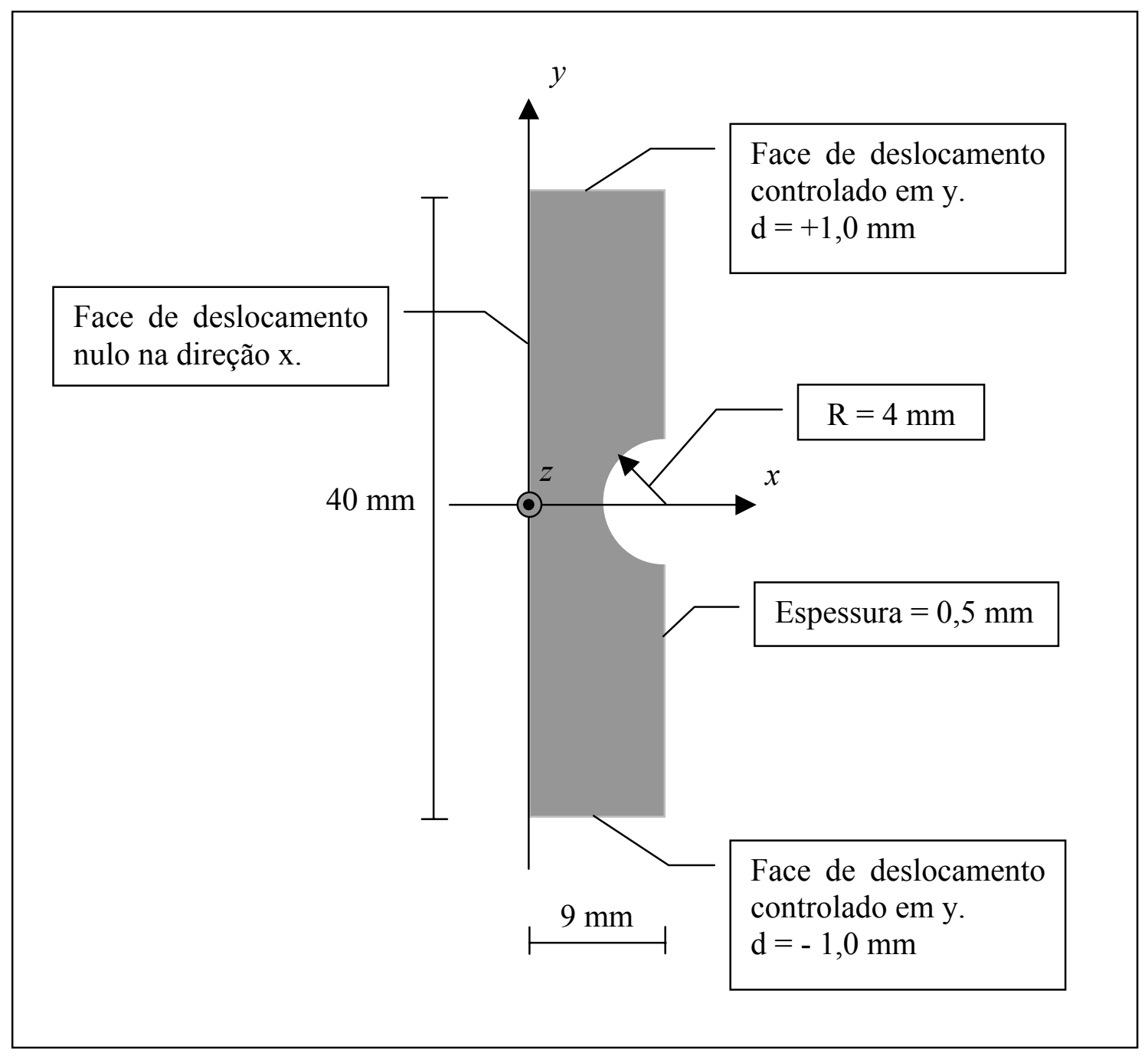

Figura 34 - Estrutura efetivamente analisada - vínculos de simetria

O deslocamento controlado é aplicado ao longo de 100 passos. Foram utilizadas duas malhas de elementos hexaédricos: a primeira com 62 elementos (malha A) e a segunda com 82 (malha B). As malhas utilizadas são mostradas na figura 35, sendo que na espessura há apenas uma camada de elementos. Como se pode ver nessa figura, a malha B 
é mais refinada na região central da peça (que apresenta os mais elevados gradientes de tensão), em comparação à malha $\mathrm{A}$ : com isso, podem-se confrontar resultados levando-se em conta também o efeito do refinamento $\mathbf{h}$.

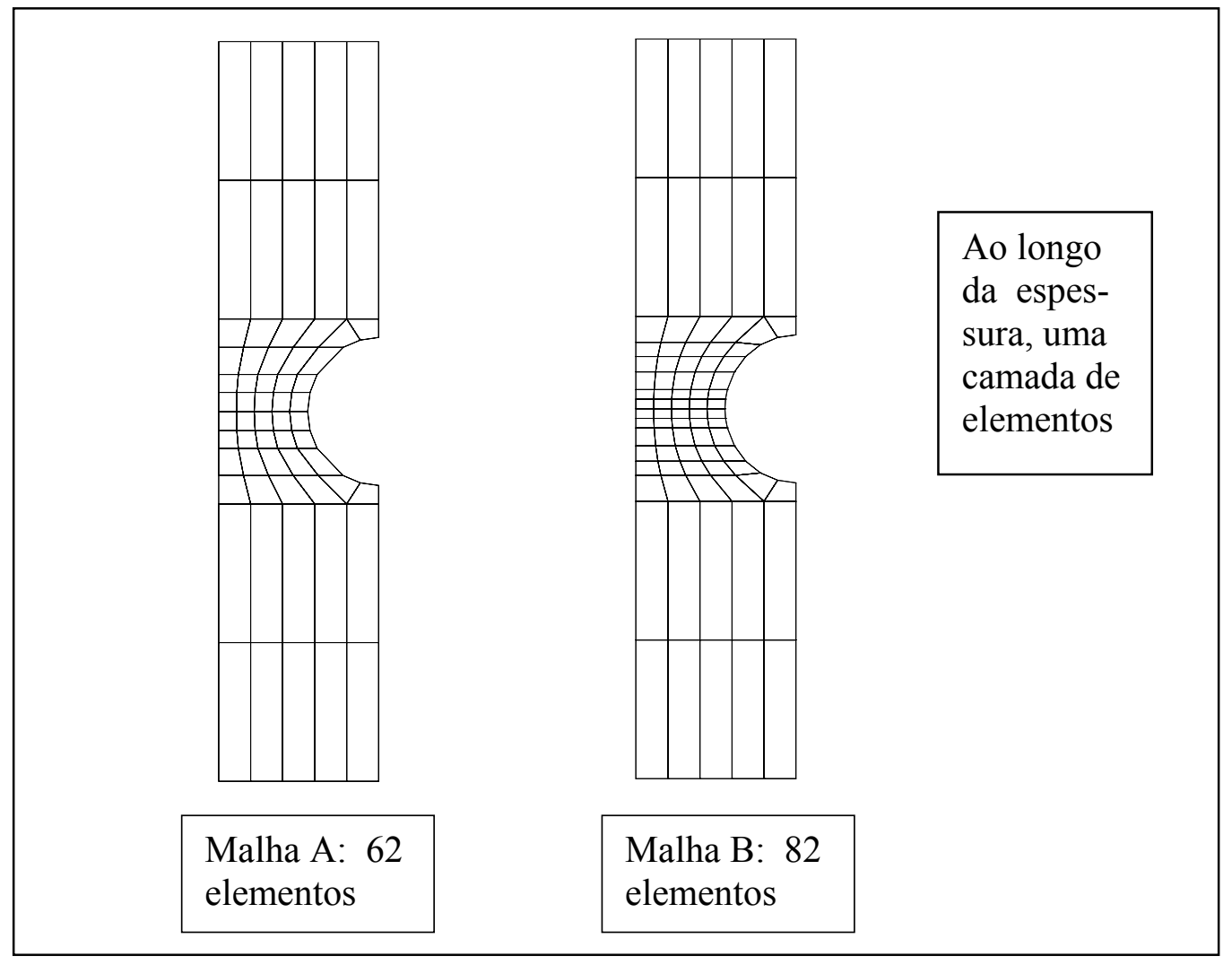

Figura 35 - Malhas de elementos utilizadas na análise.

Duas simulações foram realizadas: na primeira, não se considerou o dano, apenas a plasticidade, adotando-se o modelo constitutivo descrito no item 3.3.2.2 do capítulo 3; na segundo, utilizou-se o modelo de Lemaitre.

$1^{\text {a }}$ simulação - Elastoplasticidade com encruamento isótropo linear.

Para ambas as malhas mostradas na figura 35, a análise foi realizada utilizando-se três tipos de aproximação:

- Sem enriquecimento (NE).

- Com enriquecimento polinomial de primeiro grau em todos os nós (P1).

- Com enriquecimento polinomial de segundo grau nos nós da faixa central da chapa (mostrados na figura 36) e de primeiro grau nos demais nós (adotando-se a sigla P2 para esta aproximação).

Nas aproximações P1 e P2, nas faces de deslocamento prescrito (nulo ou controlado) numa determinada direção, os deslocamentos nessas direções não são enriquecidos. 


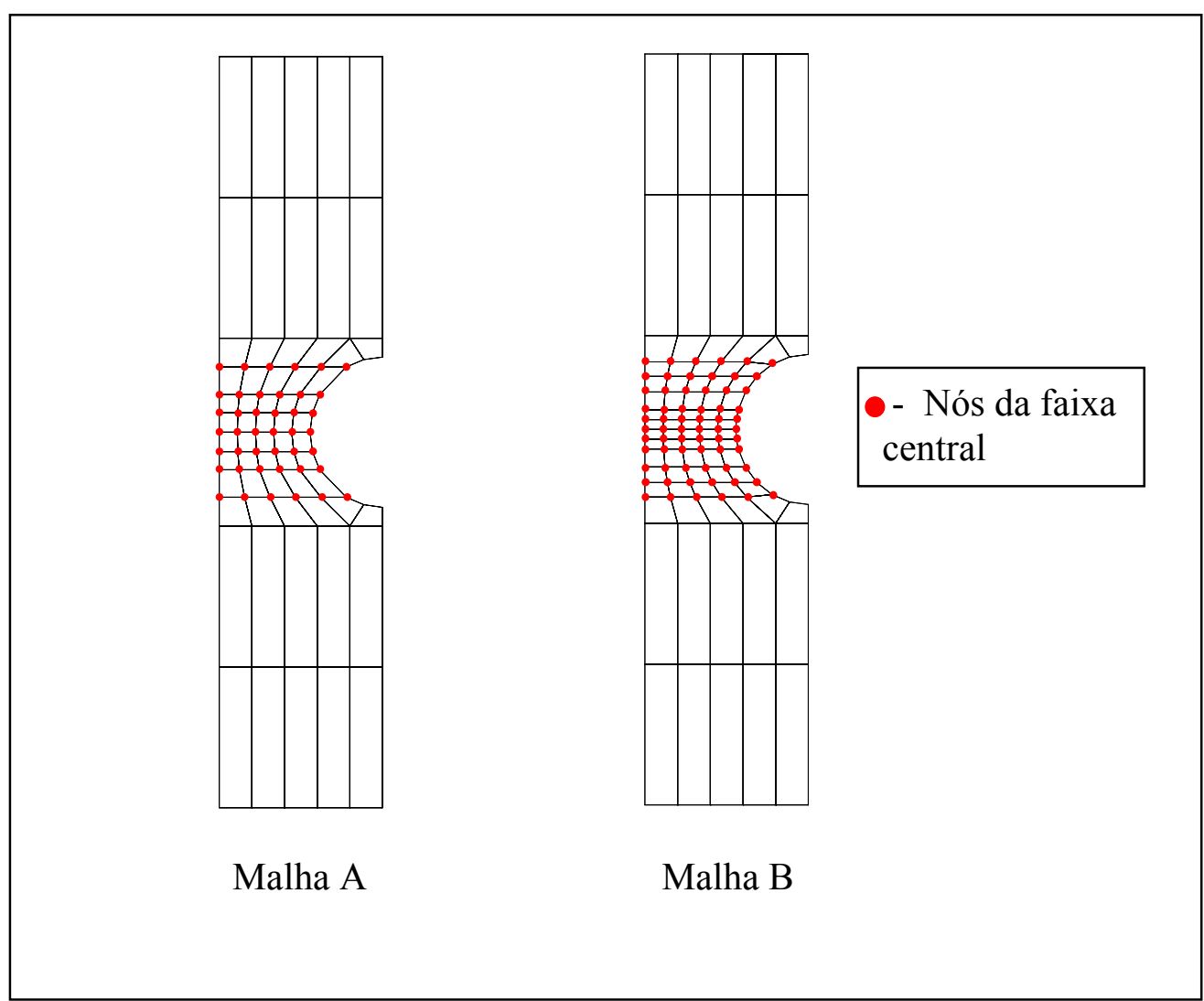

Figura 36 - Nós da faixa central com enriquecimento polinomial de segundo grau

Apresentam-se a seguir os gráficos: valor da força aplicada nas faces contra deslocamento prescrito na direção $y$.

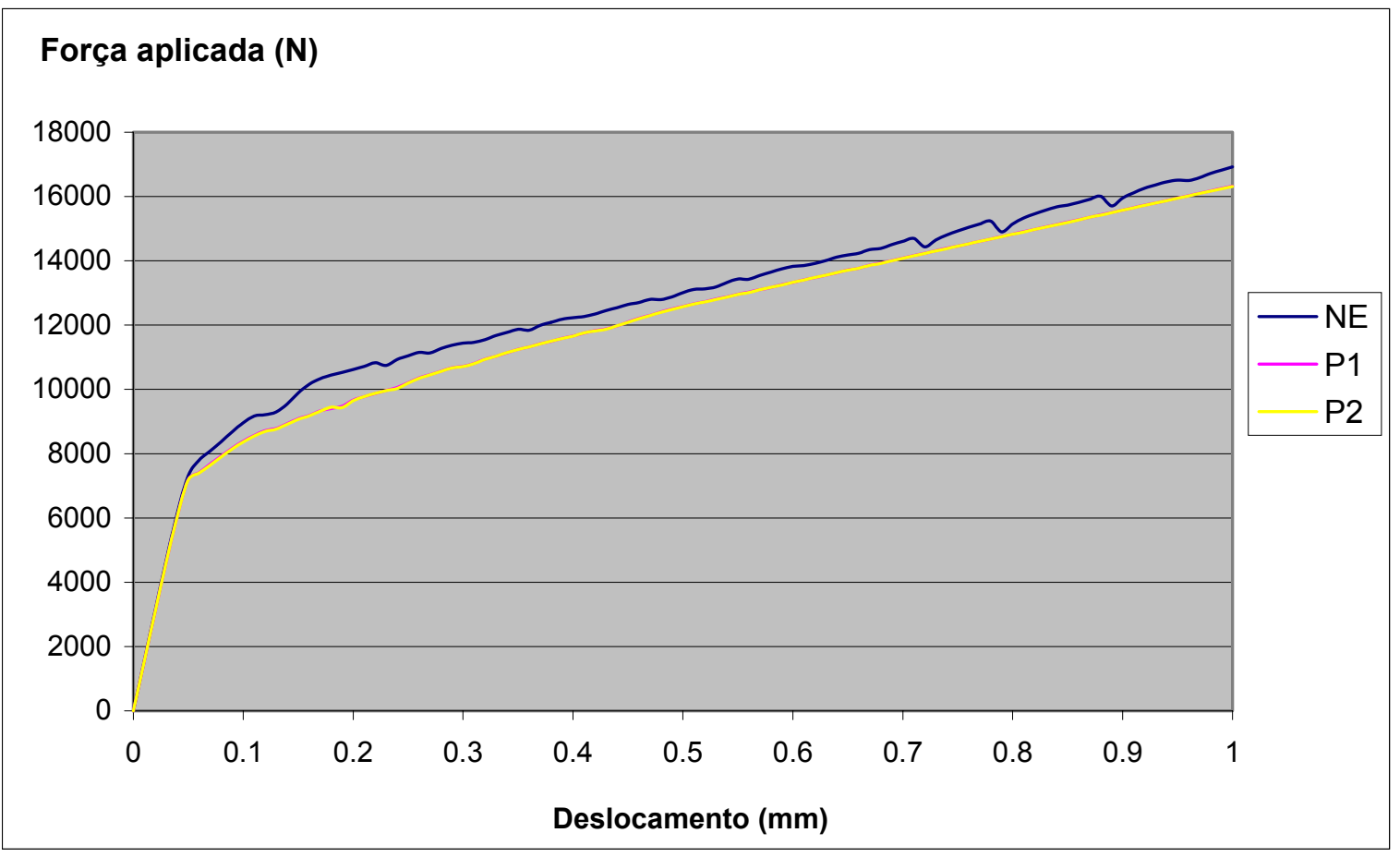

Figura 37 - Força aplicada x deslocamento (malha A) 


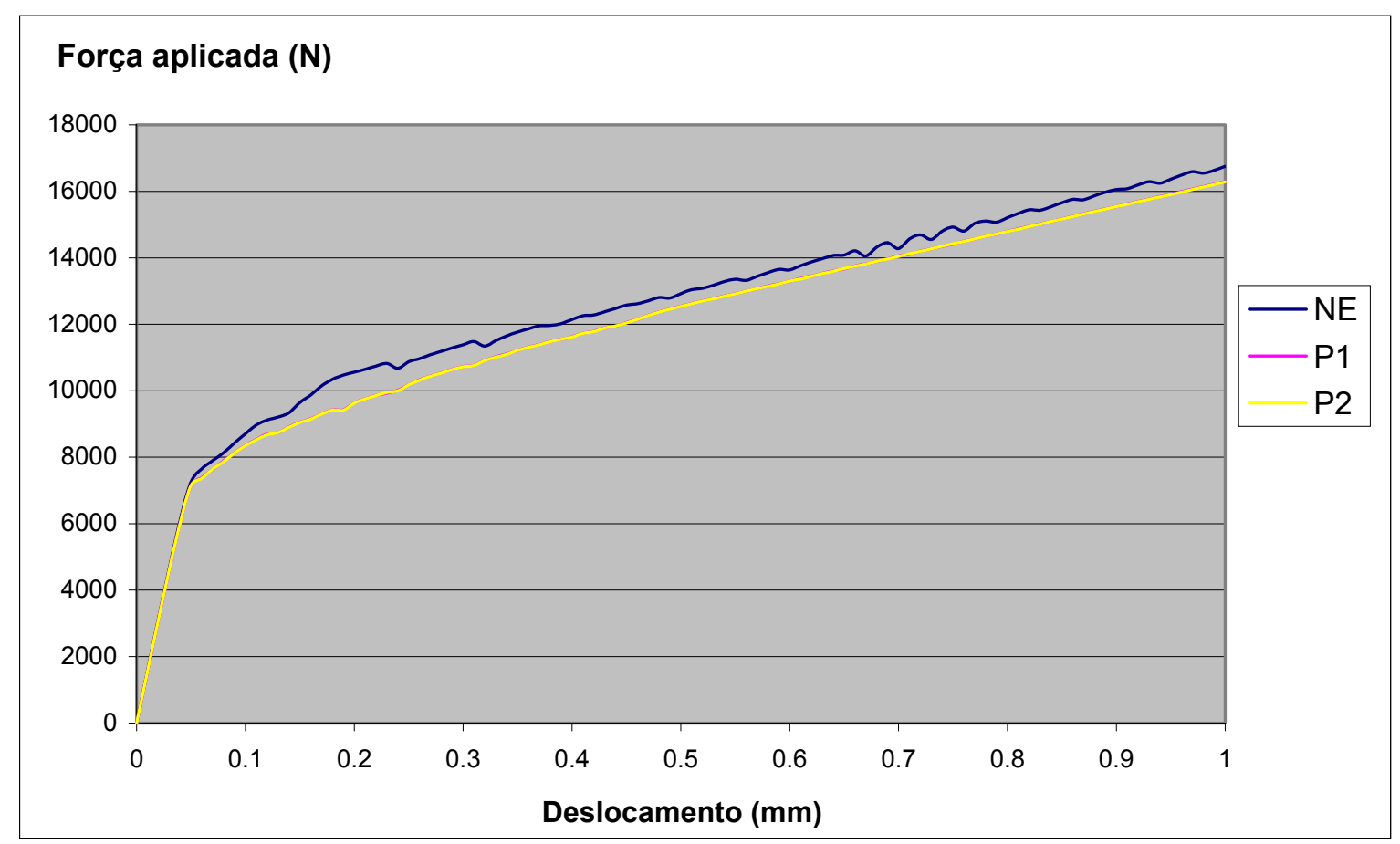

Figura 38 - Força aplicada x deslocamento (malha B)

Nas figuras 37 e 38, pode-se ver nitidamente o efeito de regularização da resposta global (carga x deslocamento) que se obtém com o enriquecimento da aproximação (tanto para a malha menos refinada como para a mais refinada). Nota-se que há pouca alteração na resposta global ao se melhorar a aproximação na faixa central (passando do enriquecimento $\mathrm{P} 1$ para o $\mathrm{P} 2$ ).

Nas figuras seguintes, apresentam-se (para ambas as malhas) os mapas da distribuição da tensão de von Mises (tensão equivalente) no $100^{\circ}$ passo (nível de deslocamento: 1,0 mm), considerando-se os três tipos de aproximação. Esses valores correspondem aos obtidos num plano normal a $z$ que dista $2,346.10^{-2} \mathrm{~mm}$ do plano que passa pela metade da espessura.

Das figuras 39 e 40, pode-se constatar que, para ambas as malhas, há uma diferença muito acentuada na resposta obtida sem enriquecimento (NE), de um lado, e com enriquecimentos P1 e P2, de outro. As tensões mínimas e máximas obtidas com enriquecimentos P1 e P2 são muito próximas entre si e divergem bastante dos respectivos valores obtidos quando não se faz enriquecimento (NE). Provavelmente a resposta sem enriquecimento sofre dos efeitos de travamento já discutidos anteriormente. Embora discreto, o efeito do enriquecimento P2 sobre o P1 faz-se notar pela maior regularização das curvas de isotensão na região central, que é exatamente a de maiores gradientes de tensão. 


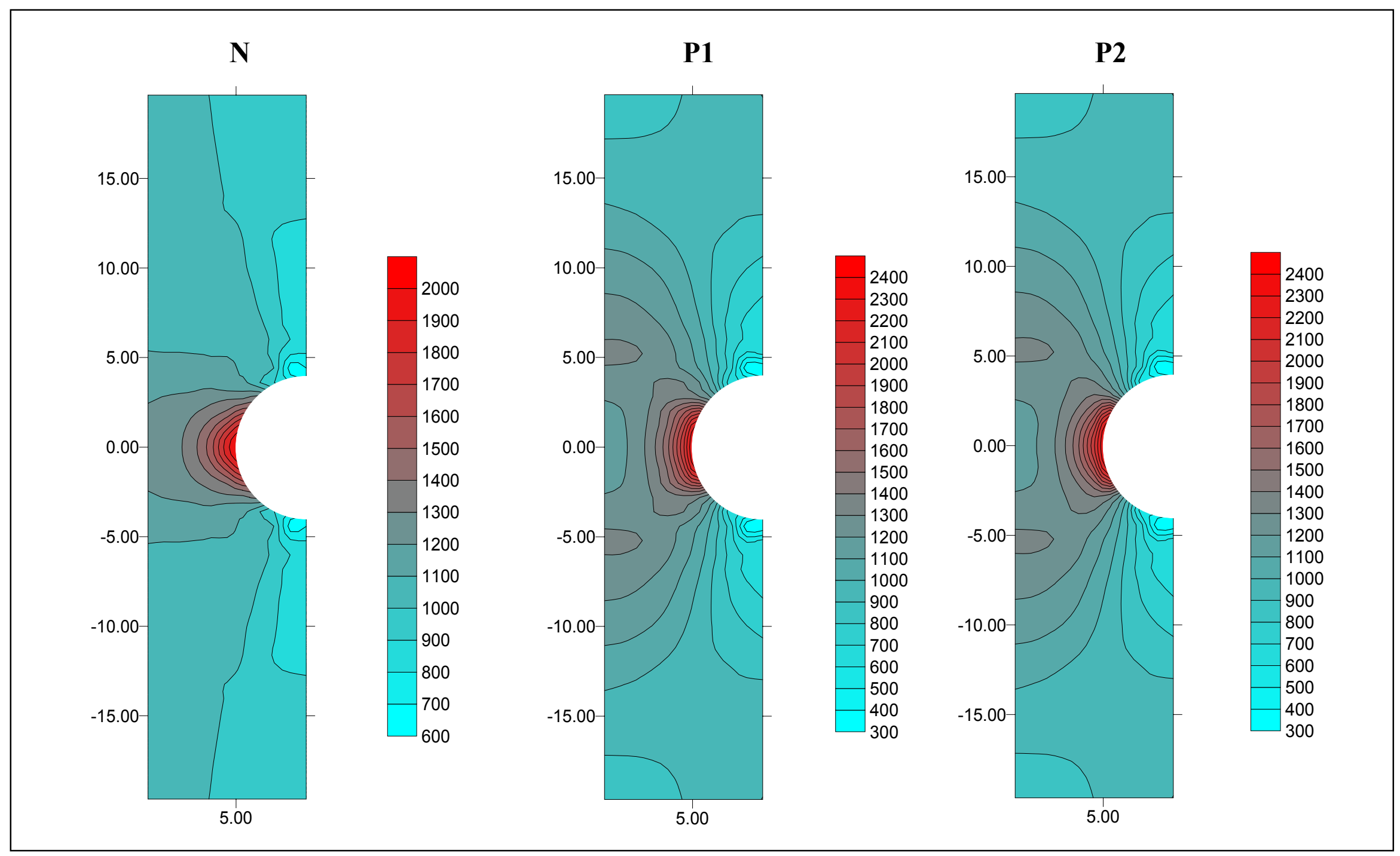

Figura 39 - Mapa da tensão equivalente (malha A), em $\mathrm{N} / \mathrm{mm}^{2}$. 


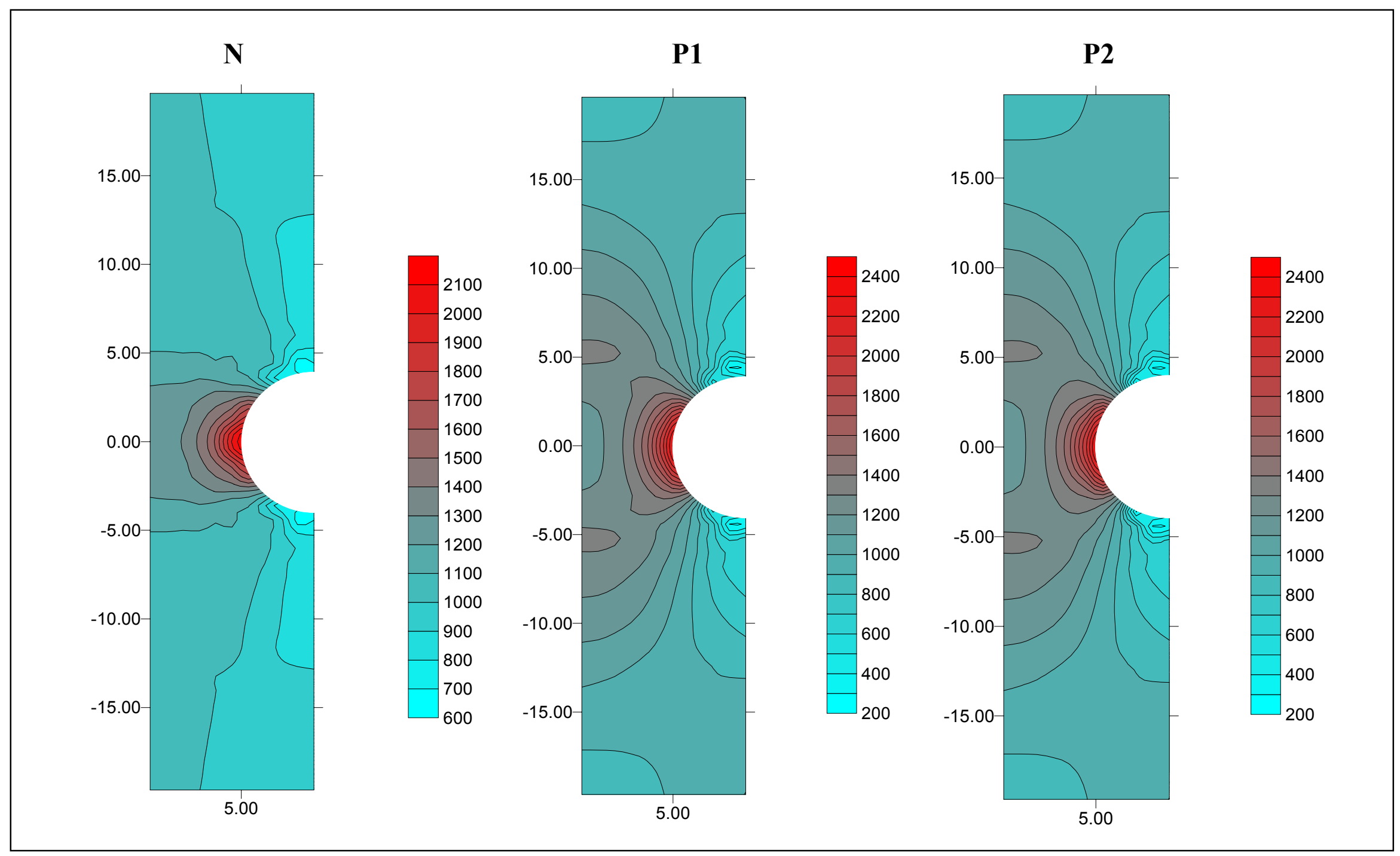

Figura 40 - Mapa da tensão equivalente (malha B), em $\mathrm{N} / \mathrm{mm}^{2}$. 
$2^{\mathrm{a}}$ simulação - Modelo de Lemaitre.

Para ambas as malhas mostradas na figura 35, a análise foi realizada utilizando-se os três tipos de aproximação descritos na primeira etapa.

Os gráficos com o valor da força aplicada nas faces de deslocamento prescrito na direção $y$ são apresentados a seguir.

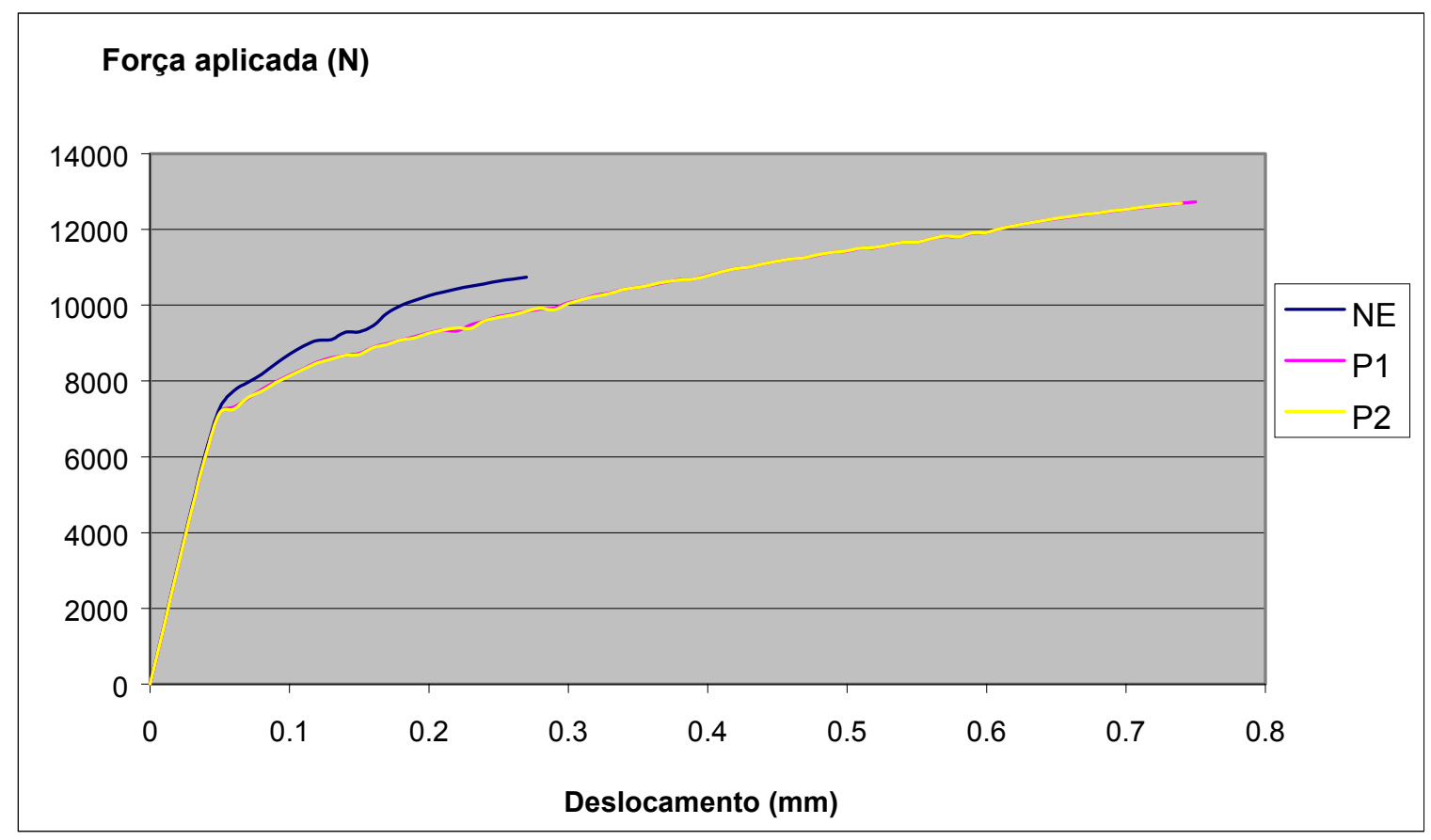

Figura 41 - Força aplicada x deslocamento (malha A)

\section{Força aplicada (N)}

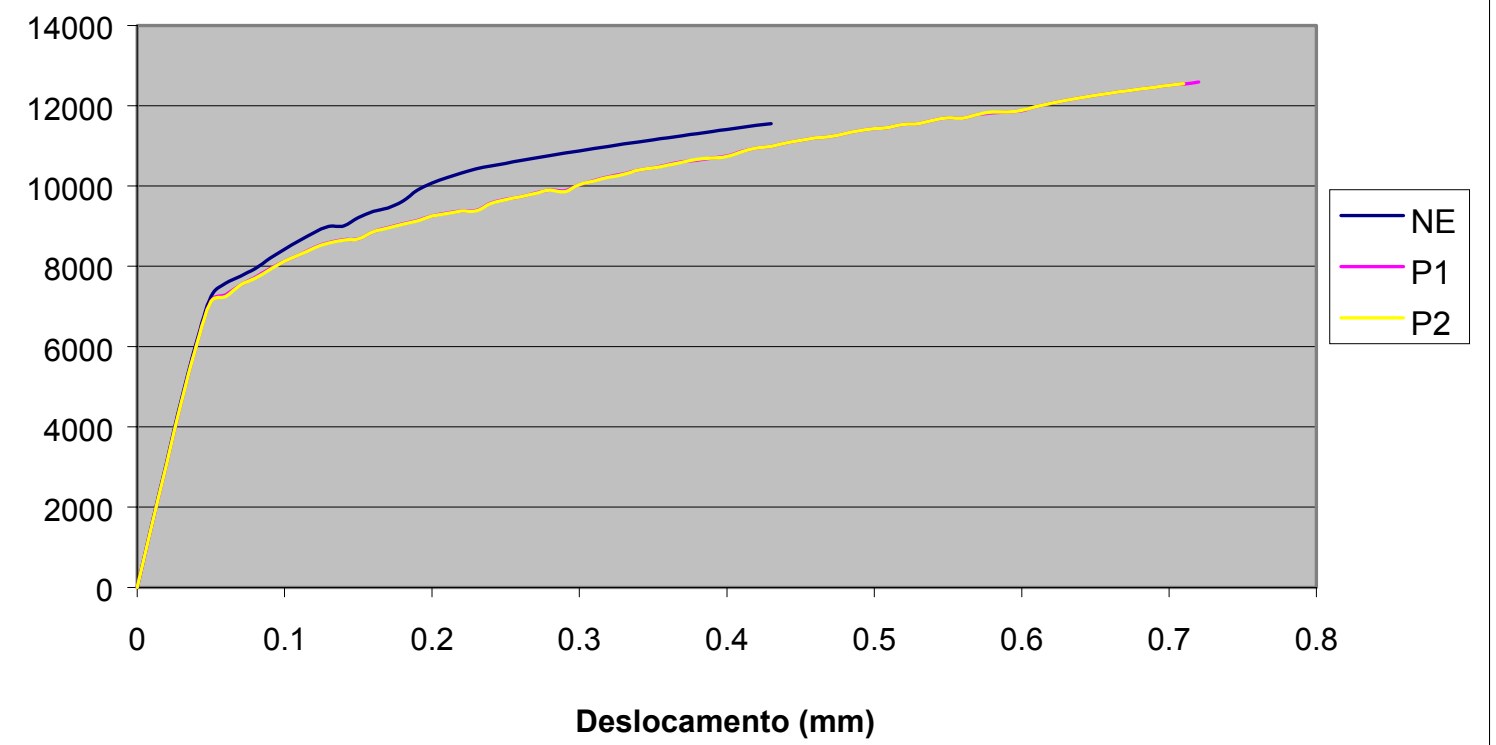

Figura 42 - Força aplicada x deslocamento (malha B) 
Da análise das figuras 41 e 42, percebe-se que, para ambas as malhas, o dano crítico é atingido mais rapidamente quando se usa a aproximação polinomial mais pobre (NE). Isso ocorre pois nesse caso a estrutura apresenta uma rigidez excessiva, com conseqüente aumento das deformações plásticas e do dano. Utilizando-se a malha A (menos refinada), o dano atingiu o valor máximo (limitado a 0,99) nos passo 27 (aproximação NE), 75 (P1) e 74 (P2). Já com a utilização da malha B (mais refinada), o valor máximo do dano foi atingido nos passos 43 (NE), 72 (P1) e 71 (P2).

Relativamente à malha $\mathrm{A}$, os mapas de tensão equivalente e de dano são apresentados nas figuras 43 e 44, correspondentes ao passo 27, para as três aproximações e nas figuras 45 e 46, correspondentes ao passo 74, apenas para os enriquecimentos P1 e P2.

Nas figuras 43 e 44, nota-se uma qualidade muito ruim da resposta quando se utiliza aproximação não-enriquecida $(\mathrm{NE})$, tanto em termos da tensão equivalente como do dano, quando comparada à obtida com o uso das aproximações enriquecidas P1 e P2. Na figura 44, verifica-se inclusive a obtenção de dano máximo em posições incorretas.

A melhoria da qualidade da resposta obtida ao se utilizar o enriquecimento $\mathrm{P} 2$, em relação ao P1, pode ser vista nas figuras 45 e 46 . Na região central, percebe-se claramente a melhor definição das curvas de tensão de von Mises e de dano, respectivamente.

Os mapas de tensão equivalente e de dano referentes à malha B são apresentados nas figuras 47 e 48 , no passo 43 para as três aproximações e nas figuras 49 e 50, no passo 71, apenas com os enriquecimentos P1 e P2.

Os mesmos comentários feitos com relação à malha A são válidos: a aproximação NE conduz a resultados insatisfatórios (figuras 47 e 48) e há uma regularização da resposta em termos de tensões e do dano ao se passar do enriquecimento P1 para o P2 (figuras 49 e $50)$.

Quando se usa a malha B, porém, nota-se que o efeito de regularização da resposta pelo uso de aproximação com enriquecimento P2, comparado ao uso do enriquecimento $\mathrm{P} 1$, é bem menos pronunciado do que o verificado ao se utilizar a malha A. Isso provavelmente deve-se ao fato de a malha B já apresentar maior refinamento na região central (do tipo h), tornando o efeito do enriquecimento polinomial (do tipo p) mais notável na malha menos refinada (A). 


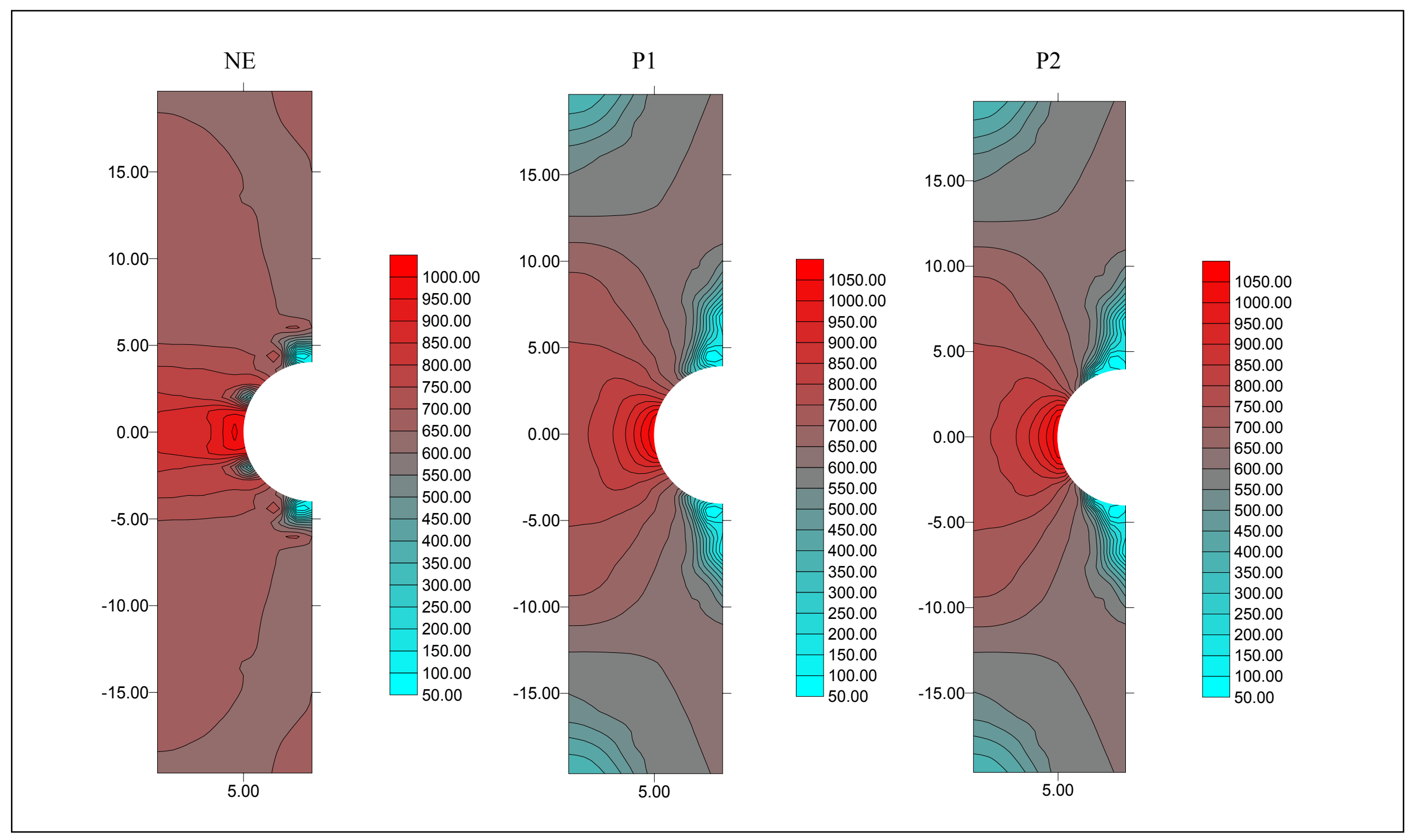

Figura 43 - Malha A - Tensão equivalente em $\mathrm{N} / \mathrm{mm}^{2}$ - Passo 27 


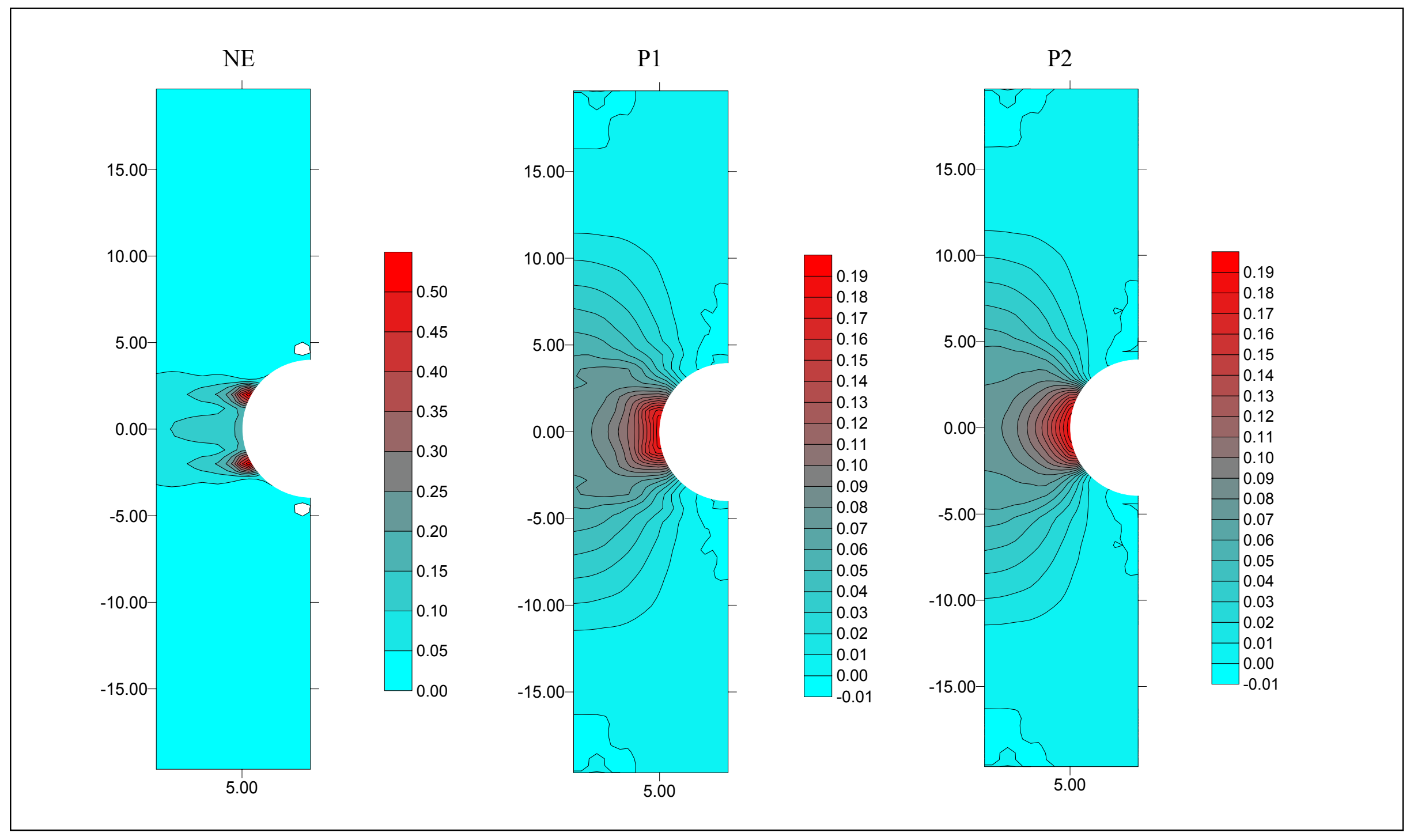

Figura 44 - Malha A - Dano - Passo 27 


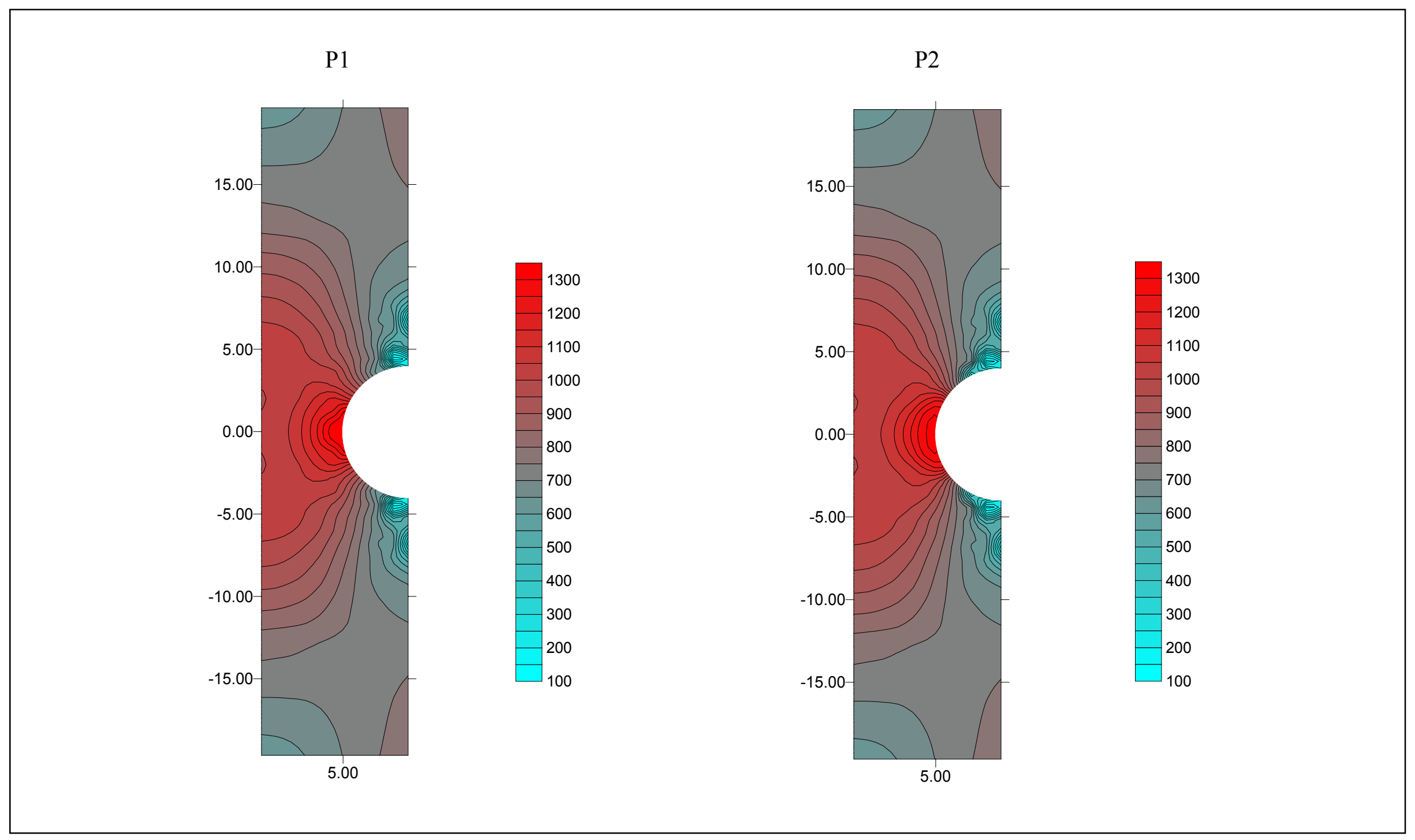

Figura 45 - Malha A - Tensão equivalente em $\mathrm{N} / \mathrm{mm}^{2}$ - Passo 74 


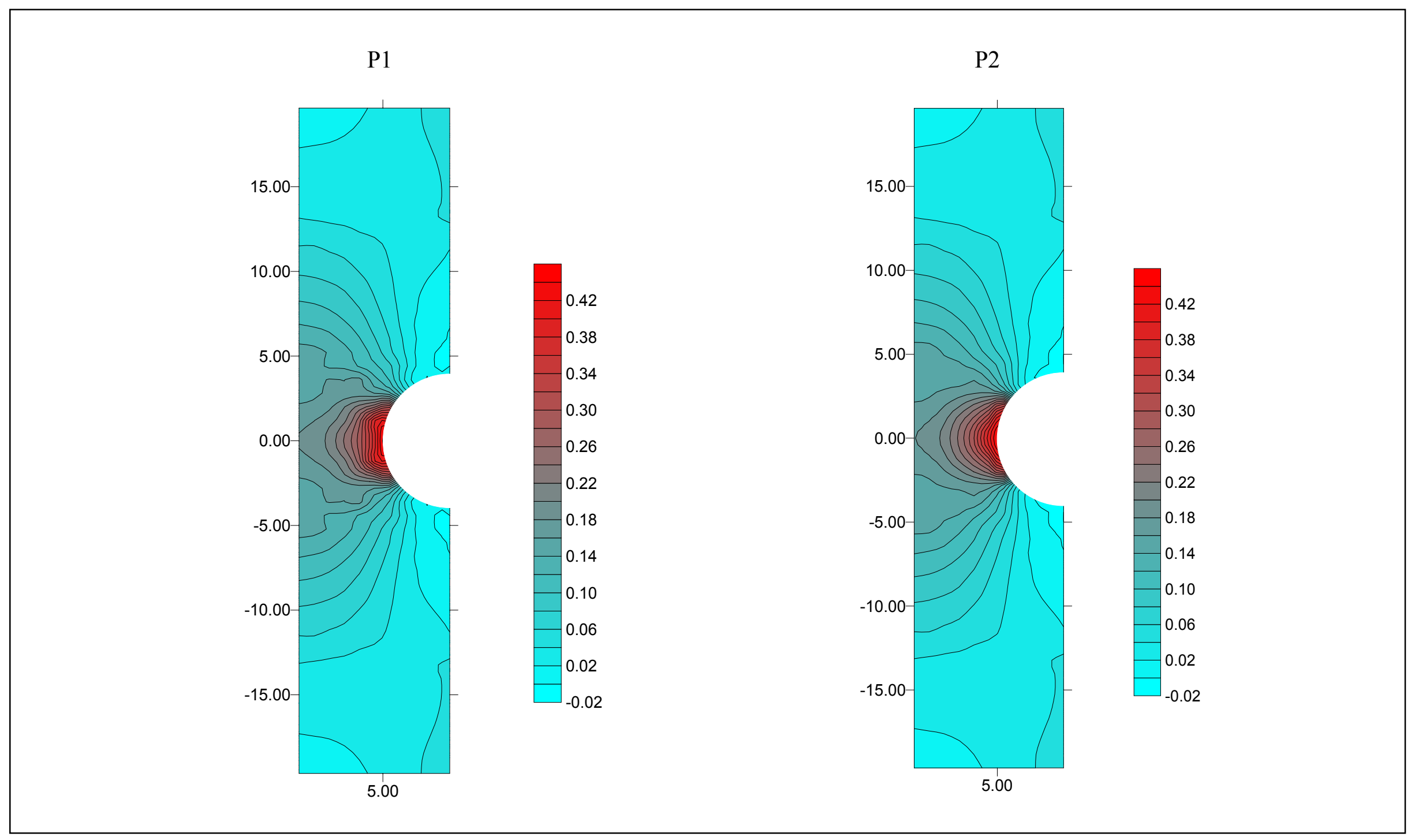

Figura 46 - Malha A - Dano - Passo 74 


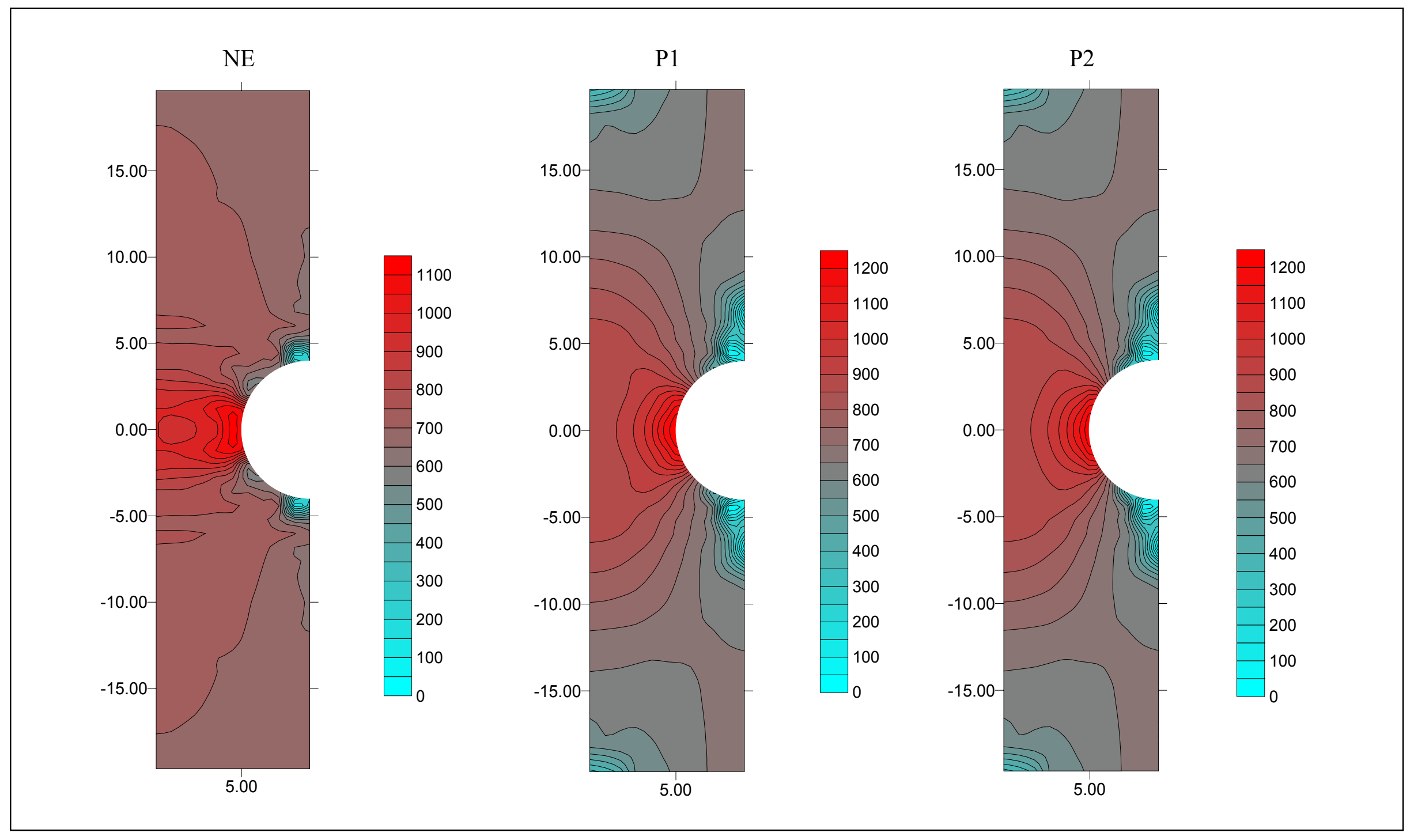

Figura 47 - Malha B - Tensão equivalente em $\mathrm{N} / \mathrm{mm}^{2}$ - Passo 43 


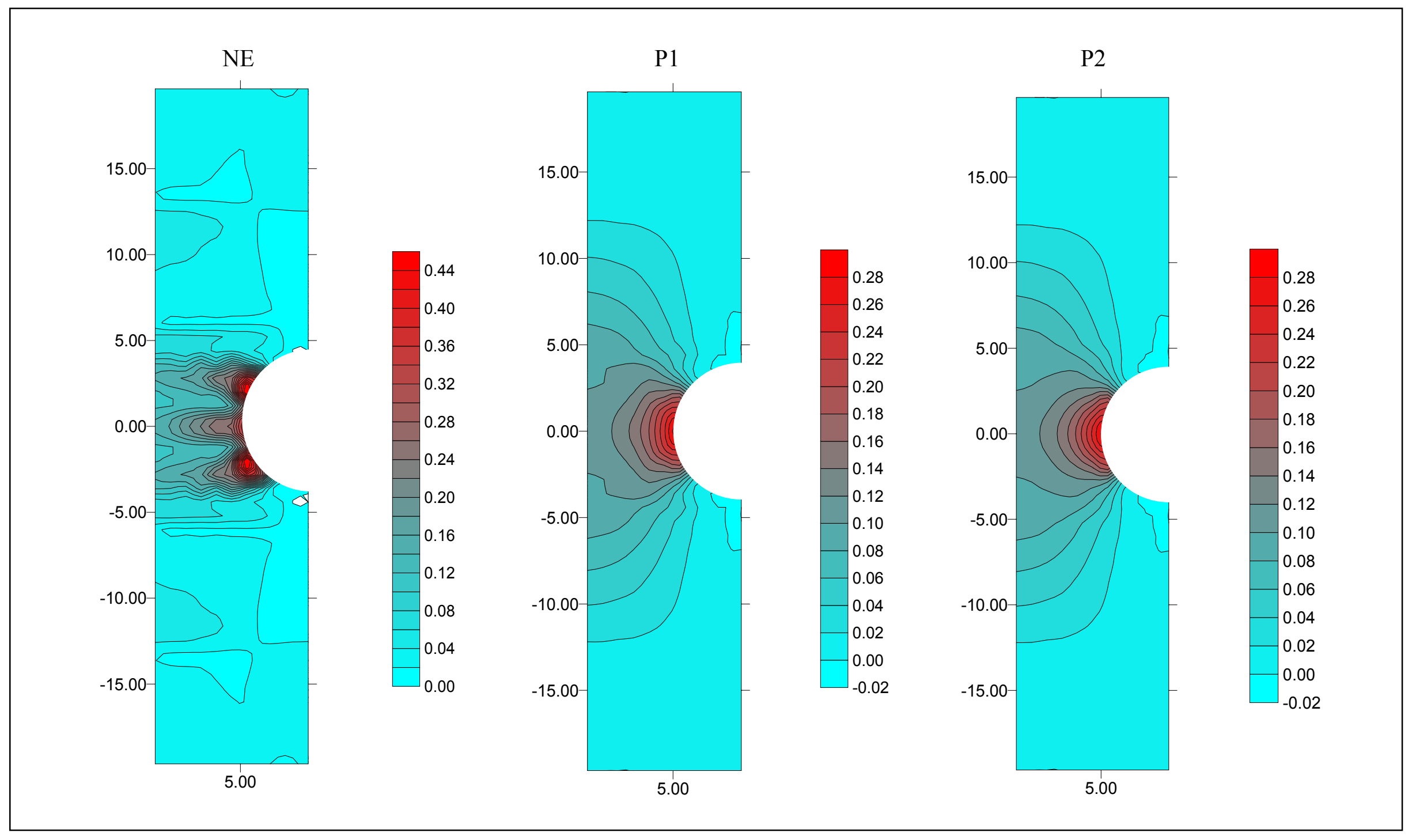

Figura 48 - Malha B - Dano - Passo 43 


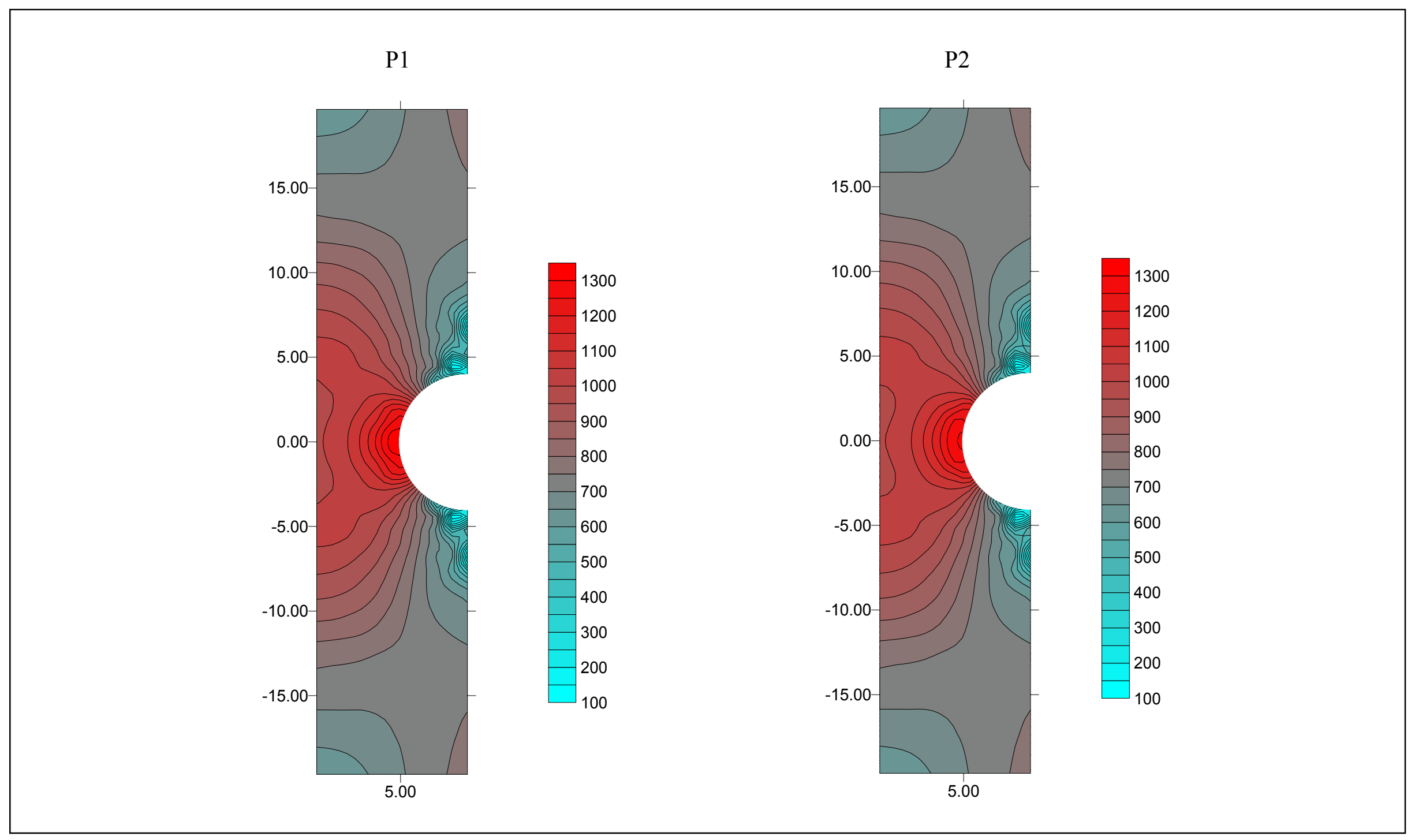

Figura 49 - Malha B - Tensão equivalente em $\mathrm{N} / \mathrm{mm}^{2}$ - Passo 71 


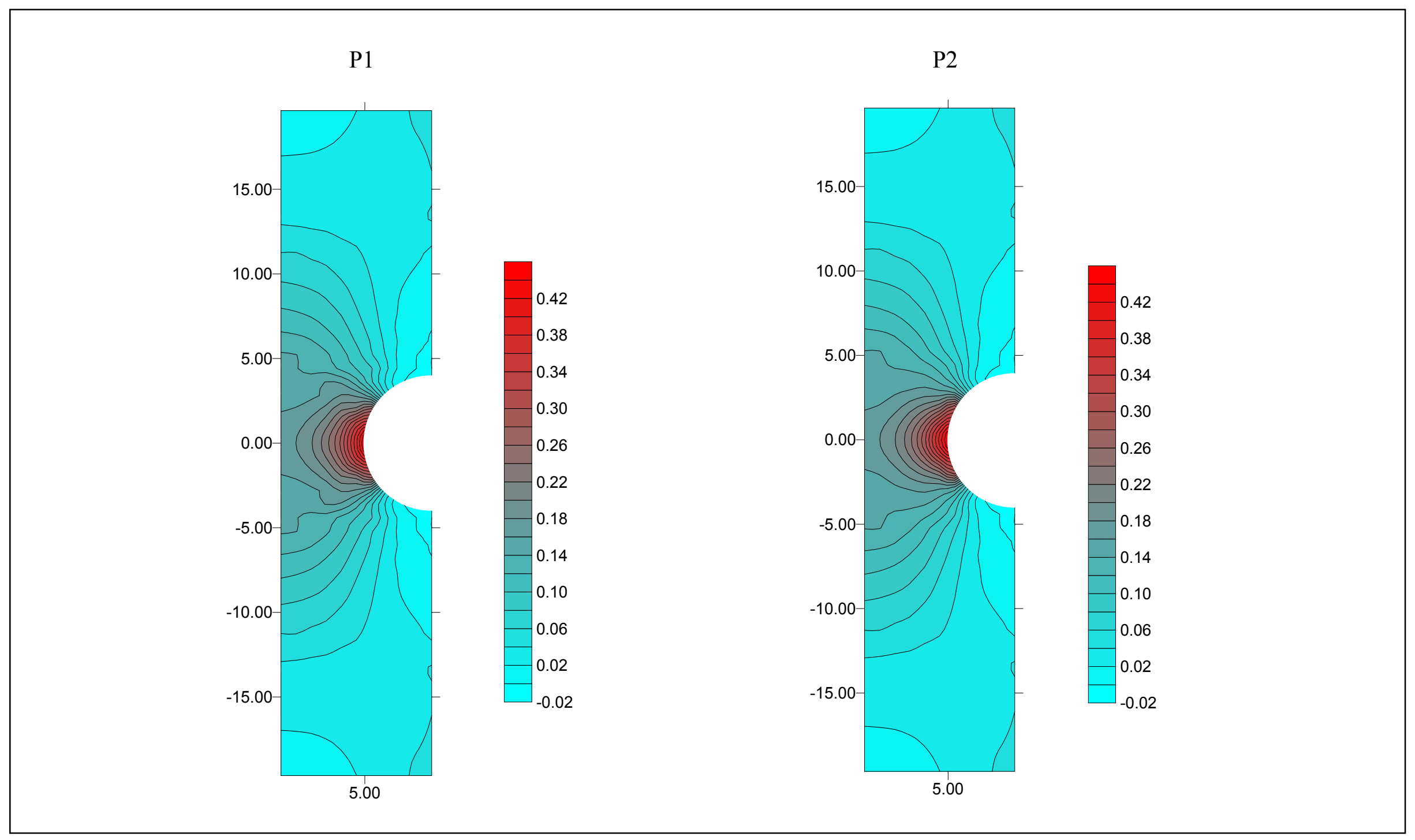

Figura 50 - Malha B - Dano - Passo 71 


\section{EXEMPLOS DE APLICAÇÃO NUMÉRICA}

A apresentação dos exemplos 1 e 2 tem como finalidade principal verificar a convergência da resposta numérica para o resultado exato. Admite-se em ambos os exemplos uma relação constitutiva elástica linear.

O exemplo 3 consiste na análise estrutural de uma barra de concreto, utilizando-se o modelo de Mazars.

O exemplo 4 tem como objetivo mostrar a aplicação do Método dos Elementos Finitos Generalizados a um problema de plasticidade sujeito ao travamento volumétrico, enquanto o exemplo 5 apresenta uma aplicação do modelo de Lemaitre.

\subsection{Exemplo 1.}

Este exemplo foi extraído de BUSSAMRA (1999). Analisa-se uma viga biengastada, cujas características geométricas são apresentadas na figura 20, sendo suas propriedades mecânicas as seguintes:

$E=1,0$ (módulo de elasticidade longitudinal).

$v=0,2$ (coeficiente de Poisson).

O carregamento consiste numa força uniformemente distribuída sobre a face superior da viga, de valor $q=1,0$.

Neste trabalho, adota-se como valor de referência para o deslocamento no centro da face inferior $w_{\text {ref }}=5,5061$ e para a energia de deformação $U_{\text {ref }}=0,32705$. Estes valores foram obtidos utilizando-se o programa ANSYS 5.4, com 5.000 elementos hexaédricos de 20 nós, totalizando 68.277 graus de liberdade.

Neste exemplo, nos processamentos com o MEFG, foram utilizados tanto elementos tetraédricos como hexaédricos. Utilizando-se elementos tetraédricos, foram realizadas análises com quatro discretizações da estrutura - com 26, 66, 105 e 285 elementos. Para cada discretização, foram utilizados cinco casos de aproximação do campo de deslocamentos: a linear, indicada como não-enriquecida (NE) e a linear enriquecida por polinômios de primeiro ao quarto graus (indicadas, respectivamente, por P1, P2, P3 e P4). A tabela 2 apresenta a flecha para cada uma das vinte análises realizadas, sendo que os termos entre parênteses indicam o número de graus de liberdade. A convergência da energia de deformação para o valor de referência é mostrada na figura 21. 


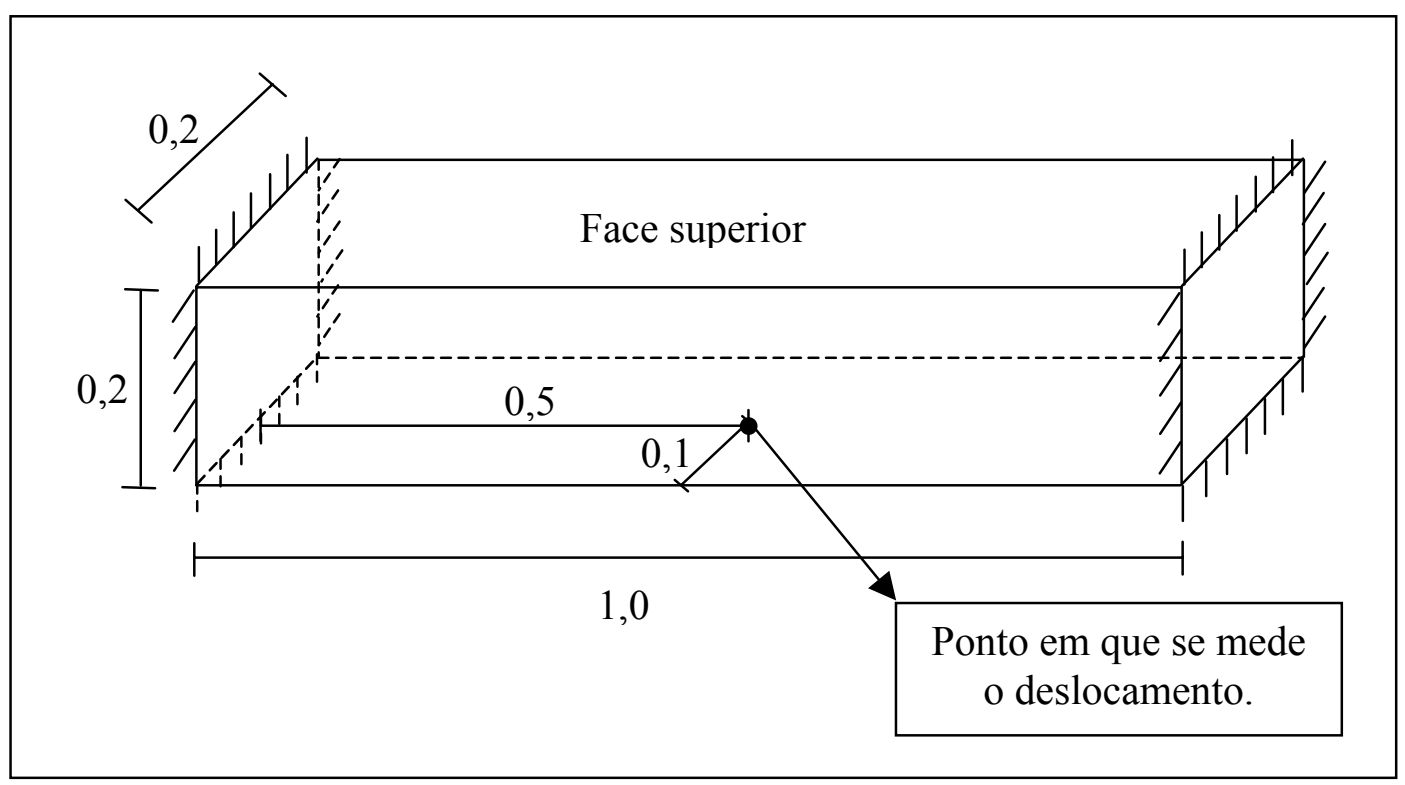

Figura 20 - Características geométricas da viga analisada.

Tabela 2 - Flecha obtida (elementos tetraédricos).

\begin{tabular}{|c|c|c|c|c|}
\hline & \multicolumn{5}{|c|}{ Número de elementos em cada discretização } \\
\hline Enriquecimento & 26 & 66 & 105 & 285 \\
\hline NE & $0,060499(15)$ & $2,715043(75)$ & $2,949964(102)$ & $3,845828(276)$ \\
\hline P1 & $0,061498(60)$ & $4,895195(300)$ & $5,066694(408)$ & $5,355138(1104)$ \\
\hline P2 & $0,078377(150)$ & $5,439094(750)$ & $5,469855(1020)$ & $5,477777(2760)$ \\
\hline P3 & $0,099243(300)$ & $5,477753(1500)$ & $5,491350(2040)$ & $5,498717(5520)$ \\
\hline P4 & $0,138355(525)$ & $5,497050(2625)$ & $5,501308(3570)$ & $5,504660(9660)$ \\
\hline
\end{tabular}

Da análise dos resultados, percebe-se que uma discretização muito grosseira (como a de 26 elementos) não permite a obtenção de uma boa resposta, exigindo, para sua melhoria, um enriquecimento de ordem muito elevada. Por outro lado, com uma discretização um pouco mais fina, mas mesmo assim com um número de elementos ainda pequeno, como na discretização em 66 elementos, pode-se notar que o enriquecimento polinomial proposto assegura uma convergência para a solução desejada. 


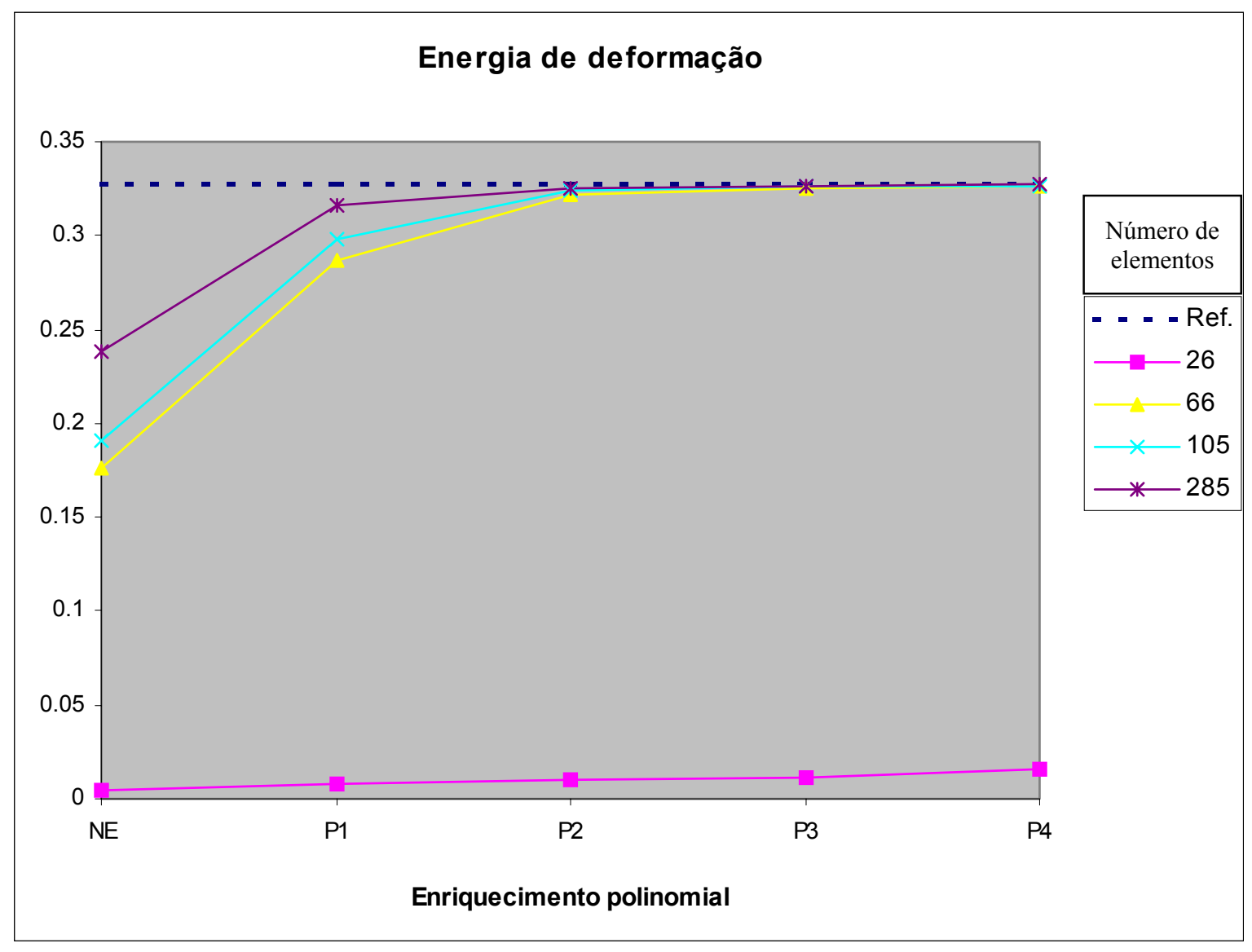

Figura 21 - Convergência da energia de deformação (elementos tetraédricos)

Utilizando-se elementos hexaédricos, foram realizadas análises com três discretizações da estrutura - com 24, 40 e 80 elementos. Para cada discretização, foram utilizadas quatro aproximações: a linear, indicada como não-enriquecida (NE), e com enriquecimentos polinomiais de primeiro ao terceiro graus (indicados, respectivamente, por P1, P2 e P3). A tabela 3 apresenta a flecha para cada uma das doze análises realizadas, enquanto a convergência da energia de deformação para o valor de referência é mostrada na figura 22. Os termos entre parênteses na tabela 3 dão o número de graus de liberdade em cada caso.

Da análise dos resultados, pode-se notar a obtenção de uma boa resposta para os deslocamentos mesmo com discretizações relativamente pouco refinadas. Além disso, é possível notar que para números semelhantes de graus de liberdade, a resposta é melhor para a situação de grau polinomial mais elevado. É o que se percebe comparando-se, por exemplo, a discretização em 40 elementos com enriquecimento de terceiro grau com a de 80 elementos enriquecida com polinômio de segundo grau. 
Tabela 3 - Flecha obtida (elementos hexaédricos).

\begin{tabular}{|c|c|c|c|}
\hline & \multicolumn{3}{|c|}{ Número de elementos em cada discretização } \\
\hline Enriquecimento & 24 & 40 & 80 \\
\hline NE & $4,001485(135)$ & $4,679543(243)$ & $5,048318(513)$ \\
\hline P1 & $5,375747(540)$ & $5,432852(972)$ & $5,460098(2052)$ \\
\hline P2 & $5,429627(1350)$ & $5,478777(2430)$ & $5,495093(5130)$ \\
\hline P3 & $5,484458(2700)$ & $5,495207(4860)$ & $5,502209(10260)$ \\
\hline
\end{tabular}

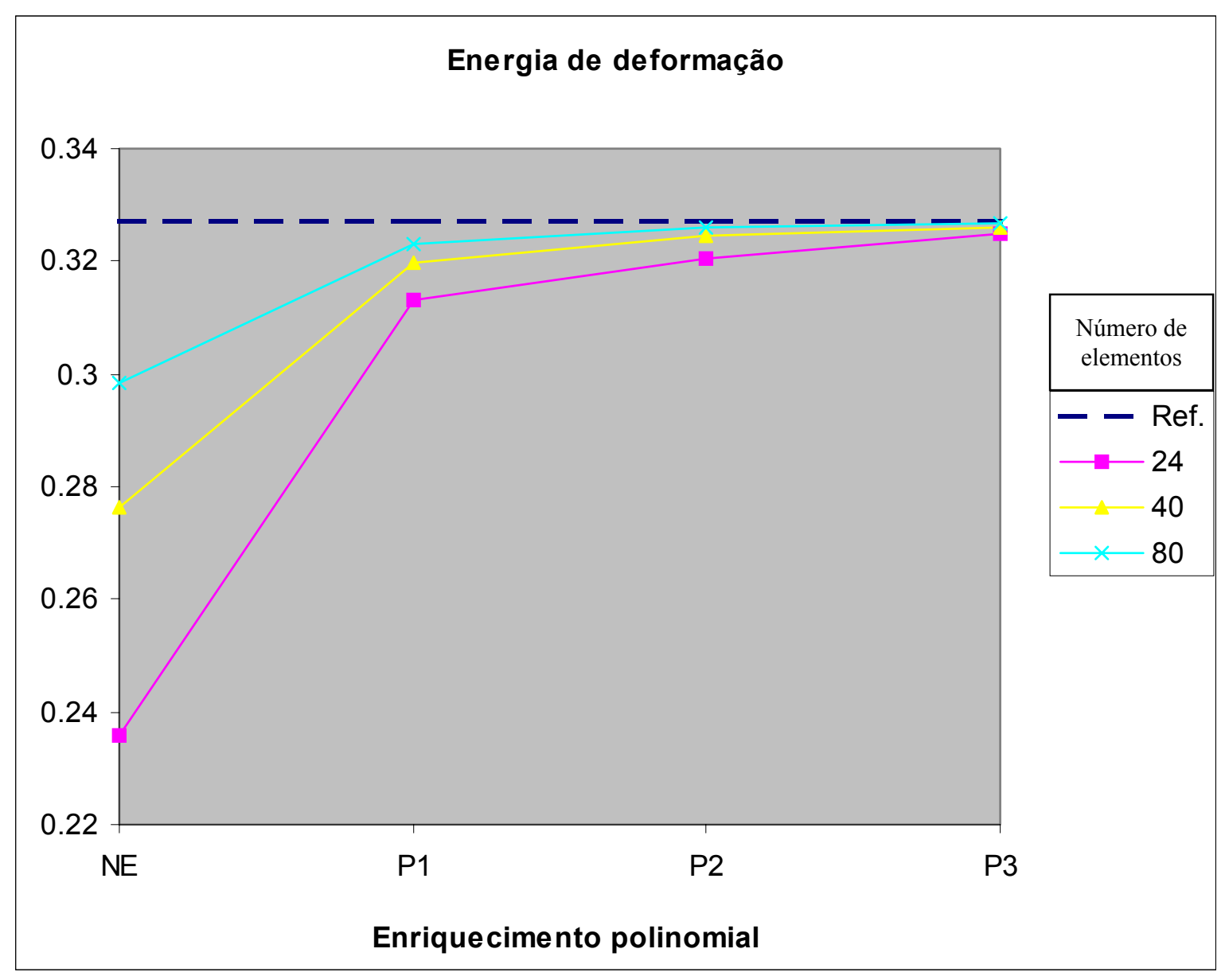

Figura 22 - Convergência da energia de deformação (elementos hexaédricos)

\subsection{Exemplo 2.}

Este exemplo foi extraído de NÓBREGA (1997). Trata-se de uma chapa engastada numa extremidade e sujeita a um carregamento uniformemente distribuído na outra. As dimensões da chapa, suas propriedades mecânicas e o valor do carregamento são 
apresentados na figura 23. Este exemplo foi apresentado originalmente por COOK (1987), que dá como referência o valor de $0,239 \mathrm{~m}$ para a flecha no ponto A.

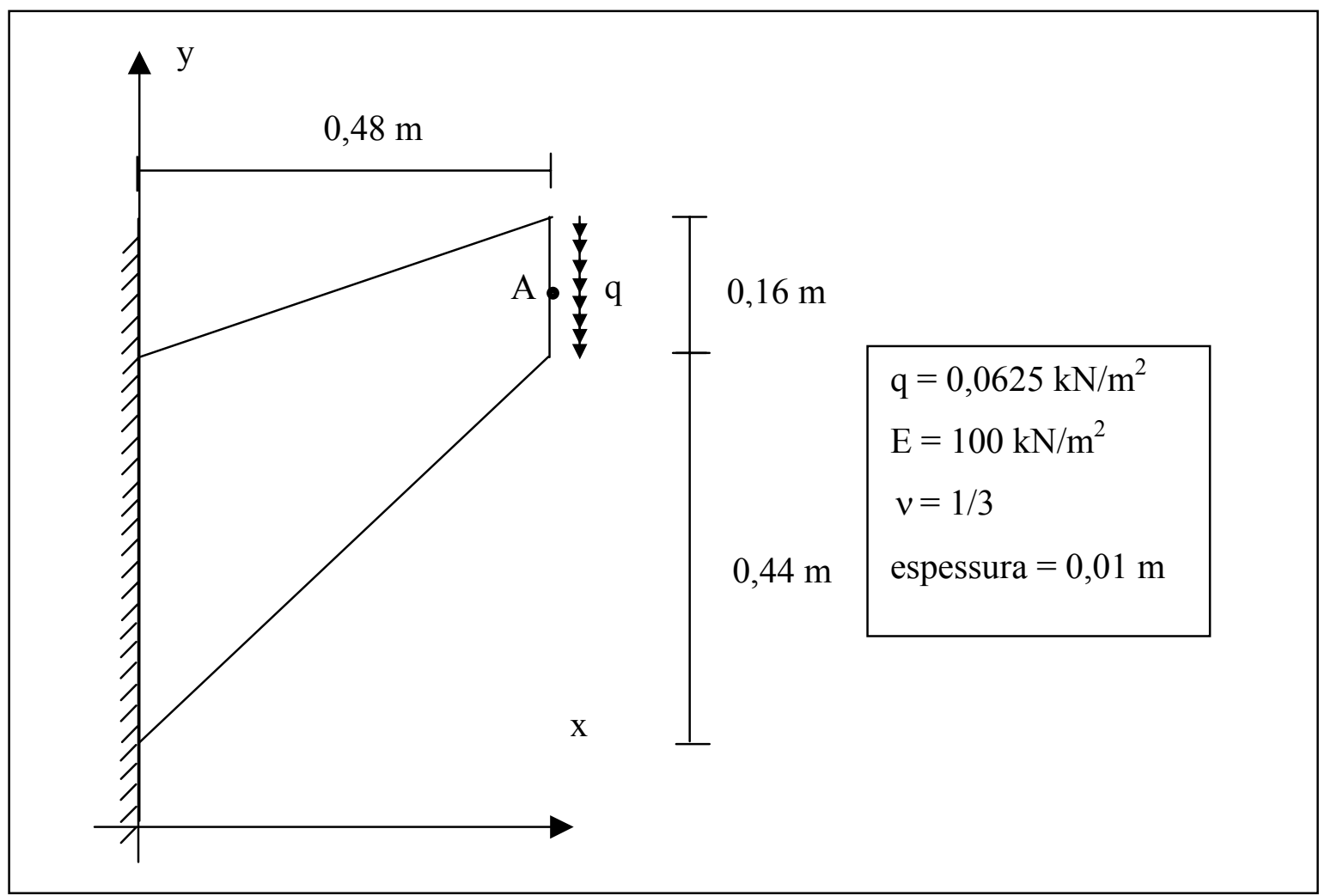

Figura 23 - Painel de Cook

Neste exemplo, utilizaram-se elementos hexaédricos em quatro discretizações: com 6, 24, 54 e 96 elementos. Para cada discretização, foram testadas quatro aproximações: a linear (indicada por NE) e as enriquecidas com polinômios de primeiro, segundo e terceiro graus (indicadas respectivamente por P1, P2 e P3). Na tabela 4, apresentam-se os valores de deslocamento em A obtidos com as dezesseis análises realizadas, sendo que os valores entre parênteses indicam o número de graus de liberdade da análise. A convergência da energia de deformação é apresentada na figura 24.

Tabela 4 - Deslocamento no ponto A (m)

\begin{tabular}{|c|c|c|c|c|}
\hline \multirow{2}{*}{$\begin{array}{c}\text { Enriquecimento } \\
\text { da aproximação }\end{array}$} & \multicolumn{5}{|c|}{ Número de elementos de cada discretização } \\
\cline { 2 - 5 } & 6 & 24 & 54 & 96 \\
\hline NE & $0,140414(54)$ & $0,196250(180)$ & $0,216331(378)$ & $0,225111(648)$ \\
\hline P1 & $0,230846(216)$ & $0,236610(720)$ & $0,237791(1512)$ & $0,238318(2592)$ \\
\hline P2 & $0,236809(540)$ & $0,238598(1800)$ & $0,238979(3780)$ & $0,239139(6480)$ \\
\hline P3 & $0,237975(1080)$ & $0,238955(3600)$ & $0,239173(7560)$ & $0,239269(12960)$ \\
\hline
\end{tabular}




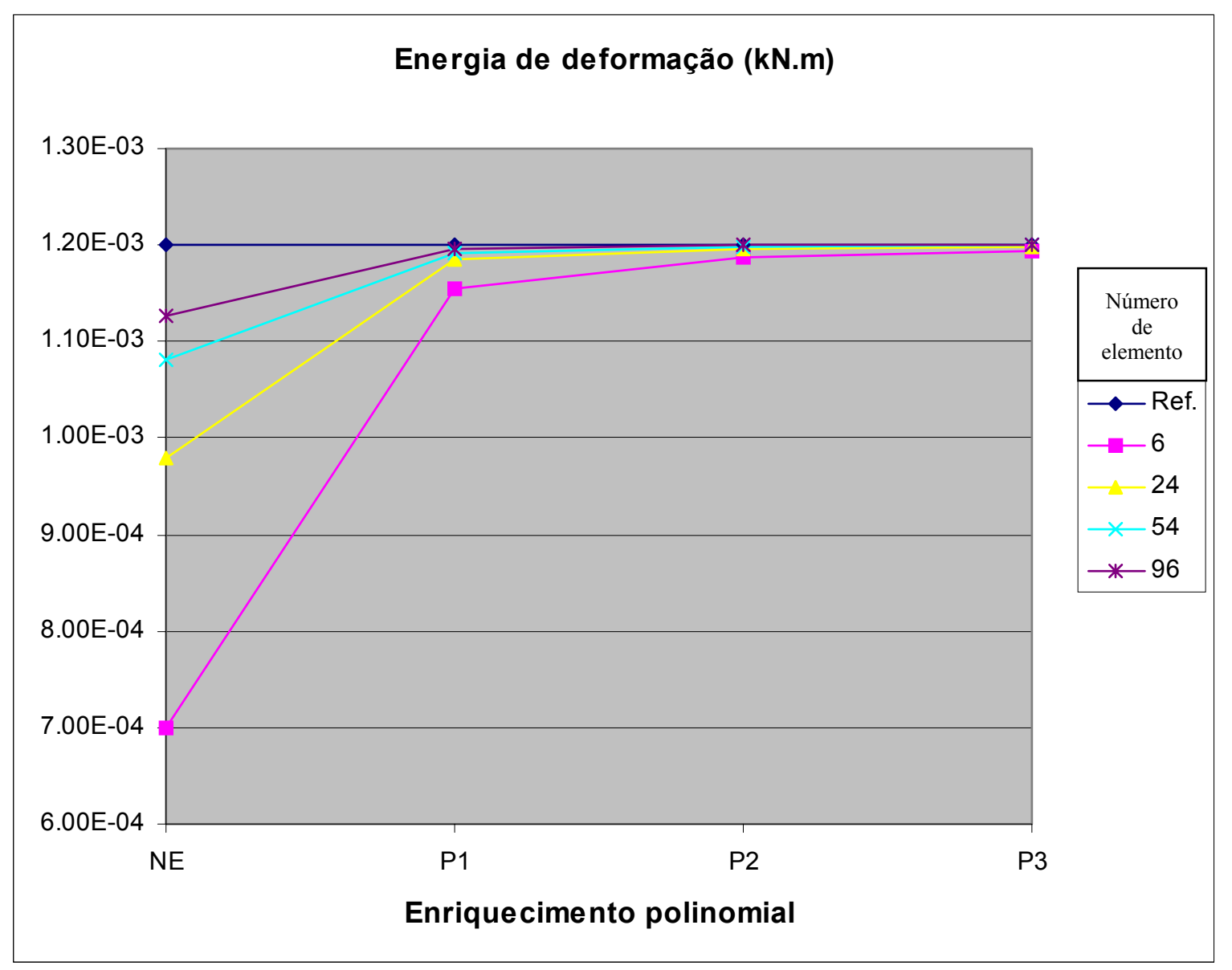

Figura 24 - Convergência da energia de deformação.

Da análise dos resultados é possível extrair algumas conclusões, expostas a seguir:

- Nota-se que a convergência da resposta para o valor de referência é muito lenta, exigindo um refinamento considerável, quando se utilizam elementos com aproximação linear (não-enriquecidos).

- Entretanto, utilizando-se enriquecimentos polinomiais de ordem mais elevada é possível obter-se boas respostas mesmo com discretizações grosseiras (como é a discretização em 6 elementos).

- Também neste exemplo é possível perceber a maior eficiência do refinamento $\mathbf{p}$ quando comparado ao $\mathbf{h}$ - verifica-se uma melhor resposta da discretização em 6 elementos com enriquecimento de terceiro grau (com 1080 graus de liberdade) do que a discretização em 54 elementos com enriquecimento de primeiro grau (com 1512 graus de liberdade). 


\subsection{Exemplo 3}

Neste exemplo, a estrutura analisada é uma barra de concreto sujeita a carregamento axial de tração em sua face superior, tendo sido adotado para o material o modelo constitutivo de Mazars, descrito no item 4.3.1. Admite-se que a barra apresente duas regiões de maior fragilidade, as quais apresentam parâmetro $\varepsilon_{d 0}$, usado para controlar o início da danificação, menor que o do restante da peça. As dimensões da barra e da região enfraquecida são mostradas na figura 25 . O carregamento é aplicado na face superior através de deslocamentos controlados na direção do eixo $Z$, enquanto que, na face inferior, prescrevem-se deslocamentos nulos naquela direção.

As propriedades mecânicas do material idealizado são:

$$
\begin{aligned}
& E=25.000 \mathrm{MPa}=2,5 \cdot 10^{7} \mathrm{kN} / \mathrm{m}^{2} \\
& v=0,25 \\
& \varepsilon_{d 0}=6,7.10^{-5}\left(6,0.10^{-5} \text { nas regiões de maior fragilidade }\right) . \\
& A_{T}=0,7 \\
& B_{T}=8.000 \\
& A_{C}=1,13 \\
& B_{C}=1.250
\end{aligned}
$$

Discretizou-se a estrutura em 20 elementos hexaédricos, conforme mostrado na figura 26. Utilizaram-se, além da aproximação linear para os deslocamentos (aqui referida como NE), os seguintes tipos de enriquecimento:

- P1: enriquecimento polinomial de primeiro grau em todos os nós.

- P1P2: Os nós da faixa central (mostrada na figura 26) são enriquecidos com funções polinomiais de segundo grau, enquanto os demais, com funções de primeiro grau.

- P2P3: Os nós da faixa central são enriquecidos com funções polinomiais de terceiro grau, enquanto os demais, com funções de segundo grau. 


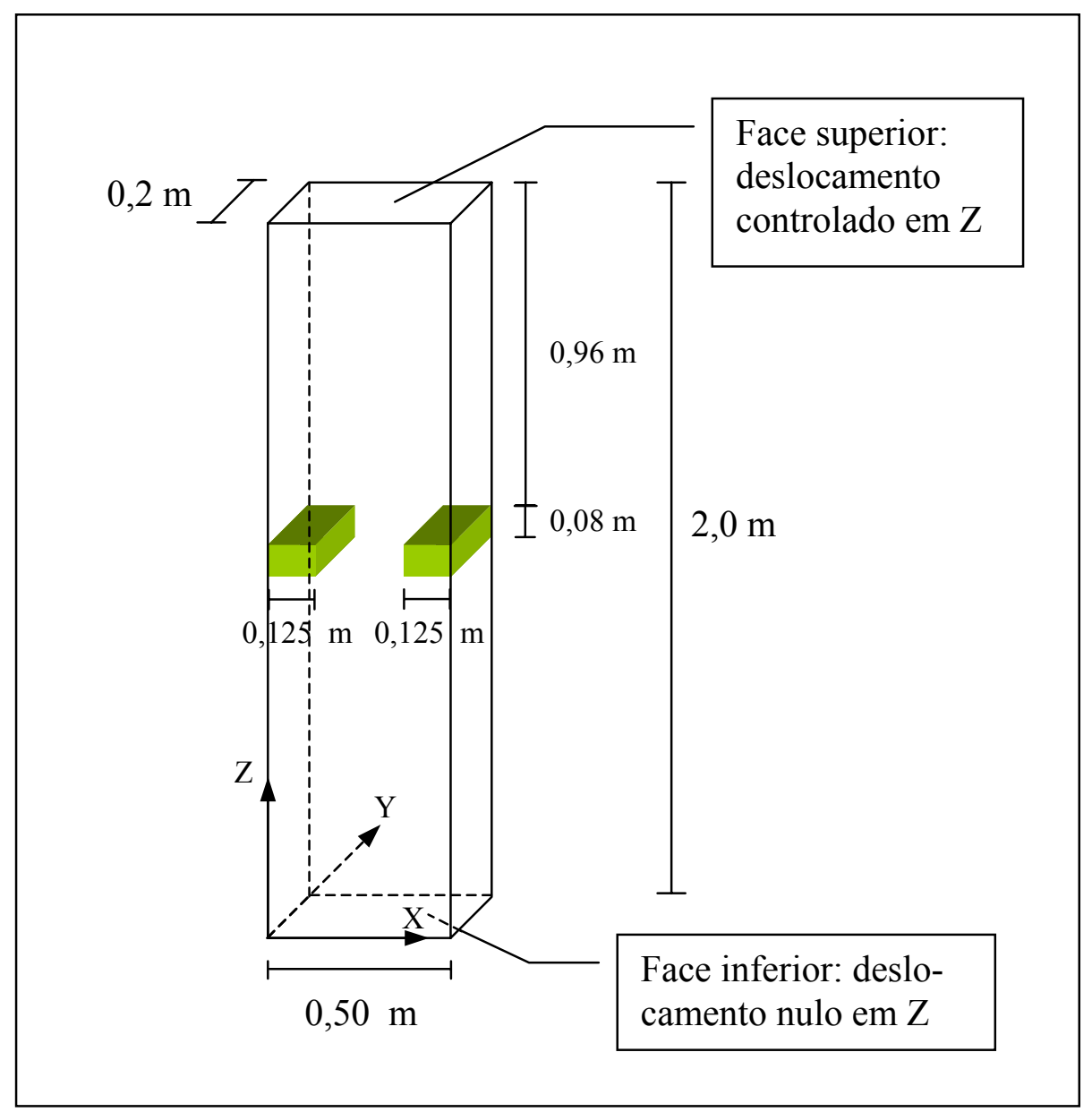

Figura 25 - Dimensões da barra e das regiões enfraquecidas.

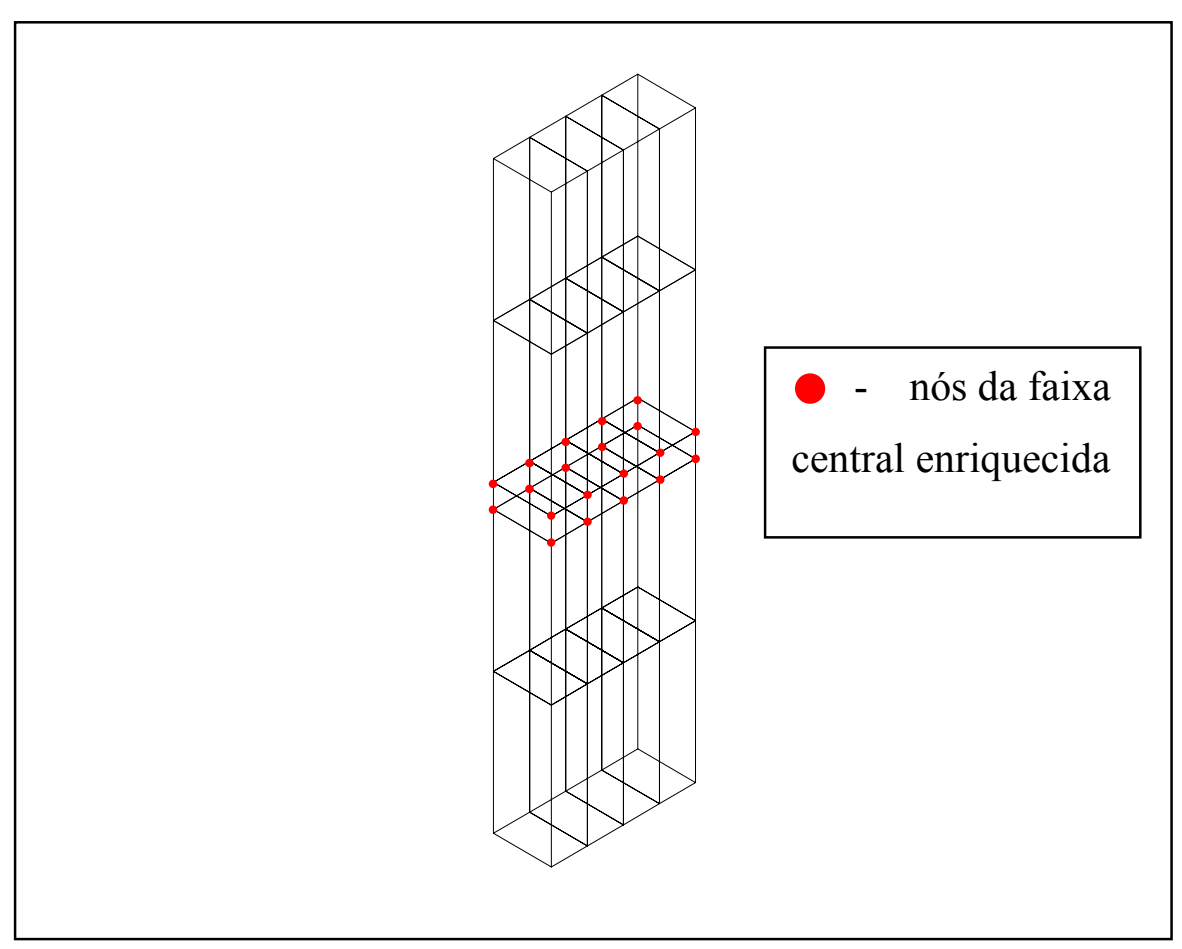

Figura 26 - Malha de elementos e faixa central enriquecida 
Impõe-se à face superior um deslocamento total de $2,425 \cdot 10^{-4} \mathrm{~m}$, ao longo de cinqüenta passos. O primeiro deles consiste num deslocamento de $1,2.10^{-4} \mathrm{~m}$ e os demais, de $2,5.10^{-6} \mathrm{~m}$. Na figura 27 , apresentam-se as curvas força $\mathrm{x}$ deslocamento para os quatro tipos de enriquecimento utilizados na análise.

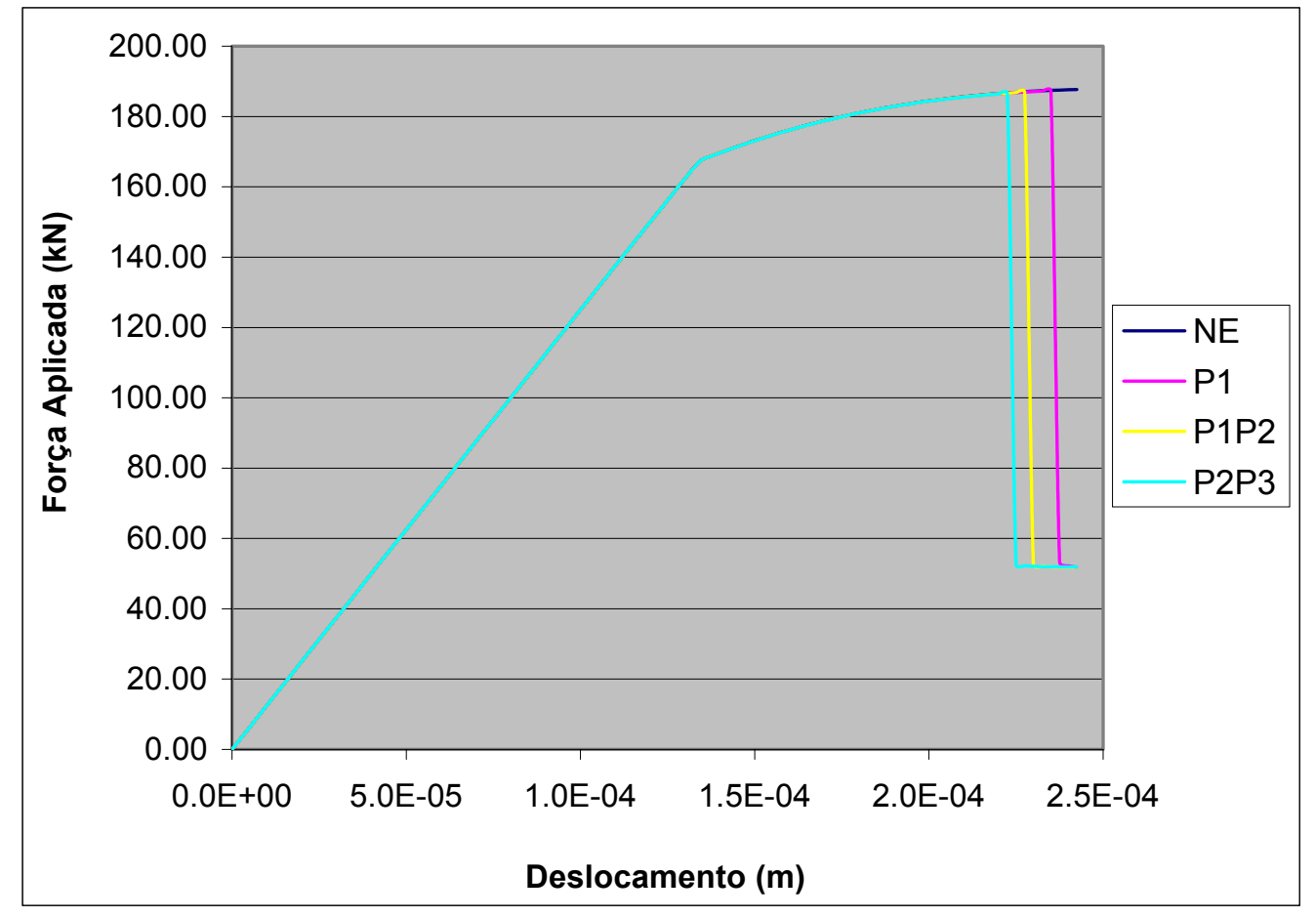

Figura 27 - Relação força x deslocamento

Verifica-se que o fenômeno da localização do dano (ao qual corresponde, na figura 27, a queda abrupta na força aplicada) ocorre em passos distintos de acordo com o tipo de enriquecimento adotado. Para a aproximação NE, a localização não chega a ocorrer. Para os enriquecimentos P1, P1P2 e P2P3, a localização ocorre, respectivamente, quando se impõem à peça os seguintes deslocamentos: $2,375.10^{-4} \mathrm{~m}, 2,3.10^{-4} \mathrm{~m}$ e $2,25.10^{-4} \mathrm{~m}$. Nota-se, além disso, que as curvas força $\mathrm{x}$ deslocamento para os diferentes tipos de enriquecimento, até o início da localização, praticamente se sobrepõem.

A figura 28 apresenta o dano num plano perpendicular ao eixo $Y$, na metade da espessura da peça, no último passo de deslocamento aplicado, para os enriquecimentos $\mathrm{P} 1$, P1P2 e P2P3. Percebe-se nitidamente como a localização do dano ocorre de forma distinta de acordo com o enriquecimento adotado: na medida em que a aproximação é enriquecida, a zona de localização tende a expandir-se no centro da peça. 


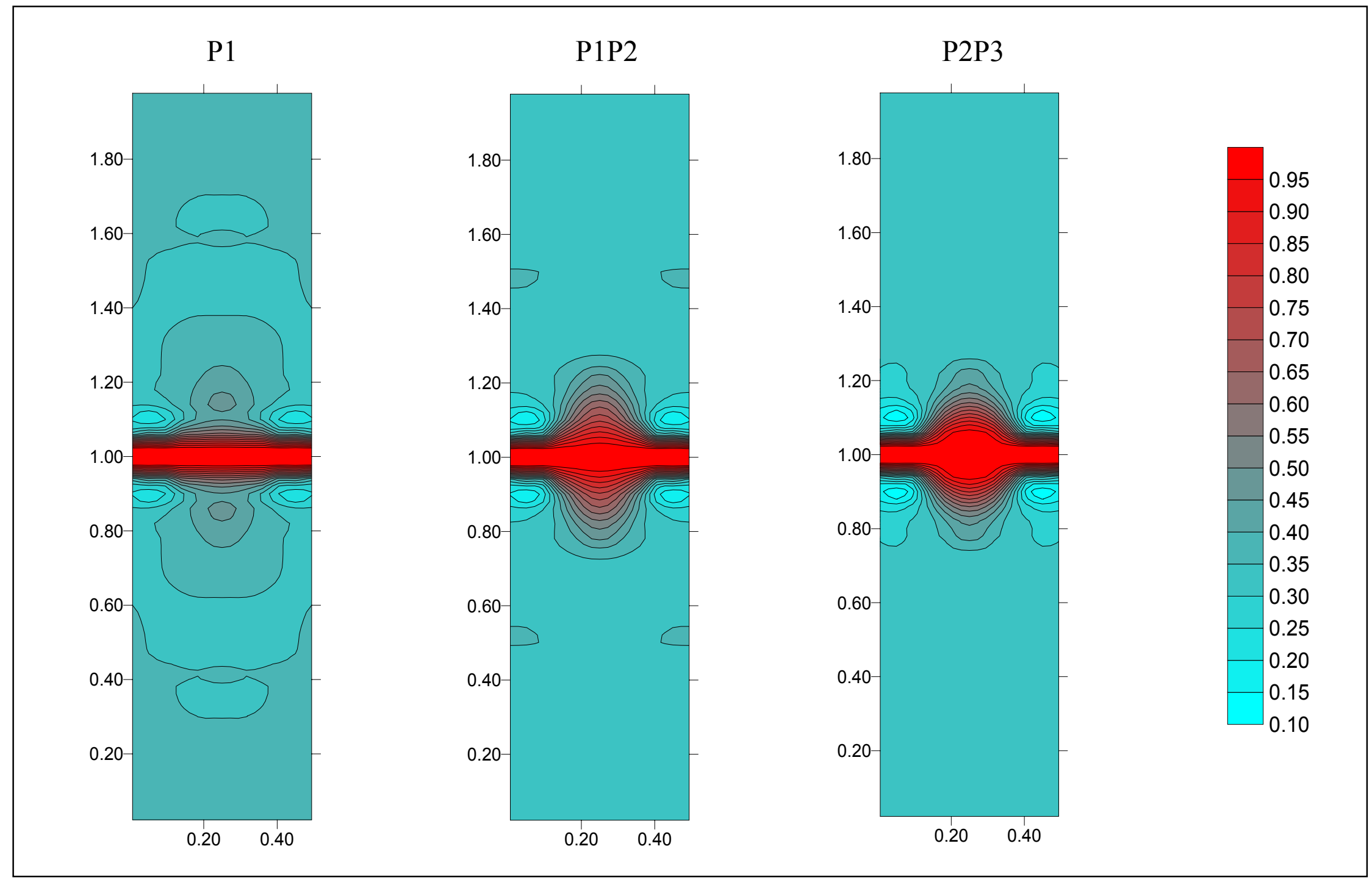

Figura 28 - Localização do dano (passo 50). 
Naturalmente, não é o objetivo desta pesquisa abordar em profundidade o problema da localização de deformações. O objetivo aqui é o de avaliar se o enriquecimento proporcionado pelo MEFG permite definir de modo mais claro a região de evolução mais localizada do dano, o que parece, de fato, acontecer.

\subsection{Exemplo 4}

Este exemplo foi extraído de WELLS et al (2002). A estrutura analisada é um bloco, em cuja face superior está uma placa infinitamente rígida sobre a qual se aplica a carga. As dimensões do bloco e da placa são mostradas na figura 29. As faces inferior e laterais do bloco tem deslocamento prescrito em todas as direções.

O modelo constitutivo do material do bloco é o elastoplástico perfeito com critério de plastificação de von Mises. As propriedades mecânicas do material idealizado são:

$$
\begin{aligned}
& E=1,0 M P a=1,0 \mathrm{~N} / \mathrm{mm}^{2} \\
& v=0,49 \\
& \sigma_{t}=0,01 \quad M P a=0,01 \mathrm{~N} / \mathrm{mm}^{2}
\end{aligned}
$$

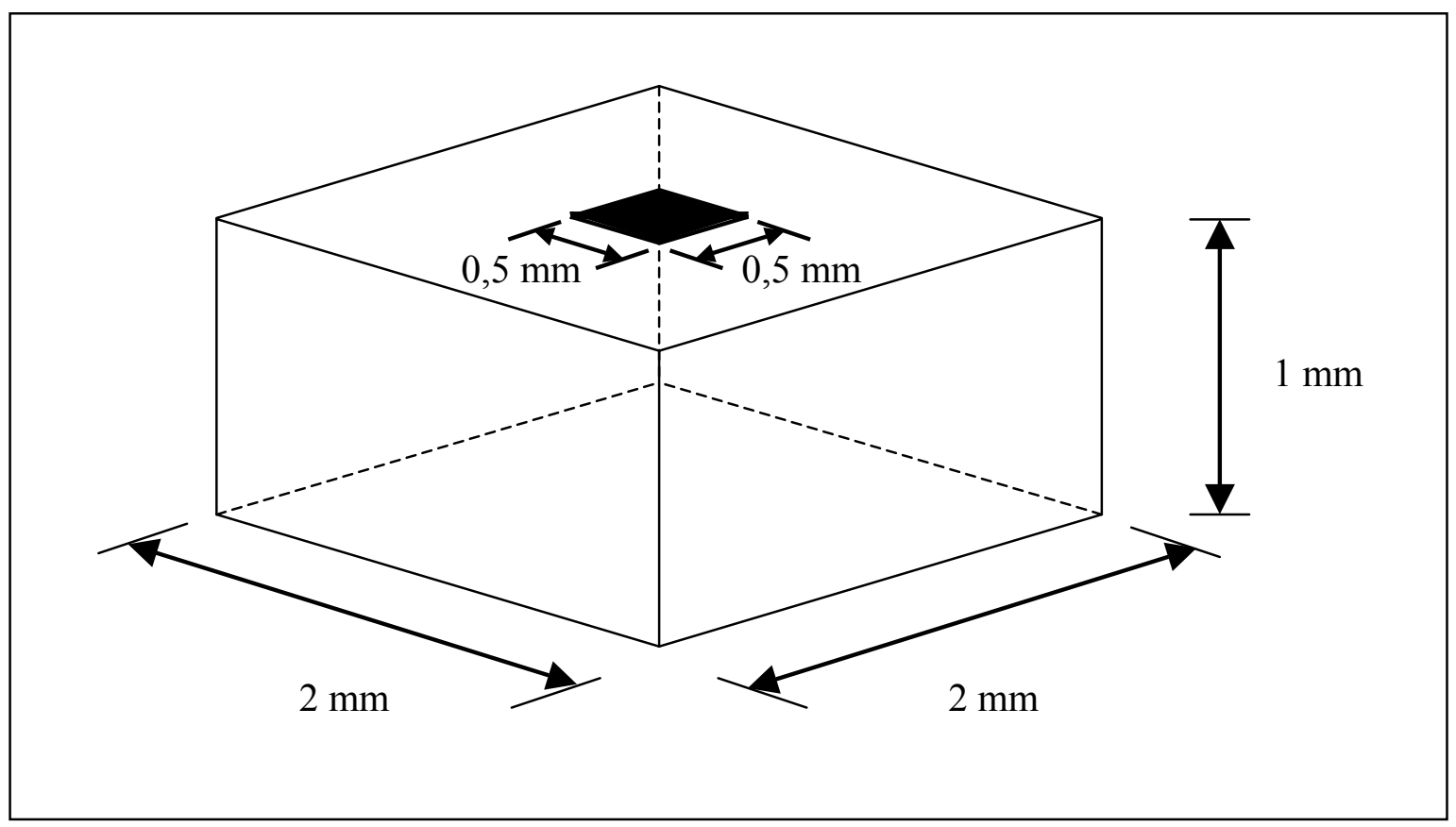

Figura 29 - Dimensões do bloco e da placa rígida.

Fazendo-se uso da dupla simetria da estrutura, analisa-se apenas uma quarta parte da mesma. A carga é aplicada de modo indireto, por meio de deslocamento vertical prescrito na região sob a placa. A figura 30 mostra a estrutura analisada e as respectivas condições de contorno. 
Foram utilizadas duas malhas de elementos hexaédricos: a primeira com 64 elementos e a segunda com 384. Para ambas as discretizações apresenta-se a relação obtida entre o deslocamento na direção $\mathrm{Z}$ na região sob a placa e a força necessária para produzilo (ambos em valor absoluto).

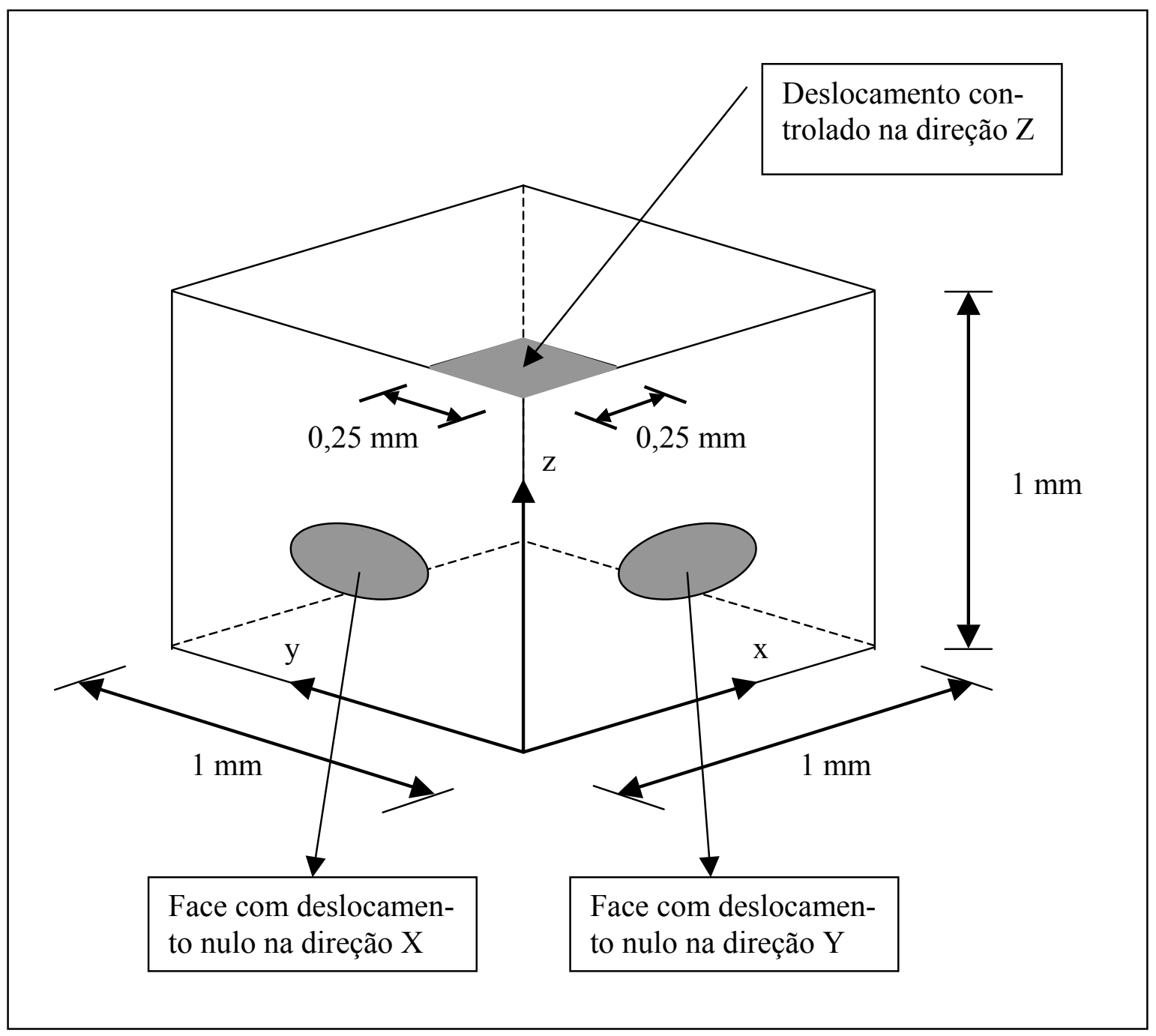

Figura 30 - Estrutura analisada utilizando dupla simetria.

Nas figuras 31 e 32 é possível perceber nitidamente o efeito do travamento volumétrico, quando se usa baixo grau de aproximação (a sigla NE indica que a aproximação linear para os deslocamentos não foi enriquecida), pois em ambos os gráficos, a carga limite não é atingida.

Verifica-se também que o enriquecimento tal como proposto neste trabalho permite superar esse efeito, uma vez que já para o enriquecimento polinomial de primeiro grau (P1) a estrutura atinge a carga limite. Além disso, nota-se uma boa eficiência do método de enriquecimento polinomial, visto que mesmo para uma malha pouco refinada (como a de 64 elementos), o valor da carga limite obtida aproxima-se bastante do apresentado por 
WELLS et al (2002), na medida em que se utilizam enriquecimentos polinomiais de segundo e terceiro graus.

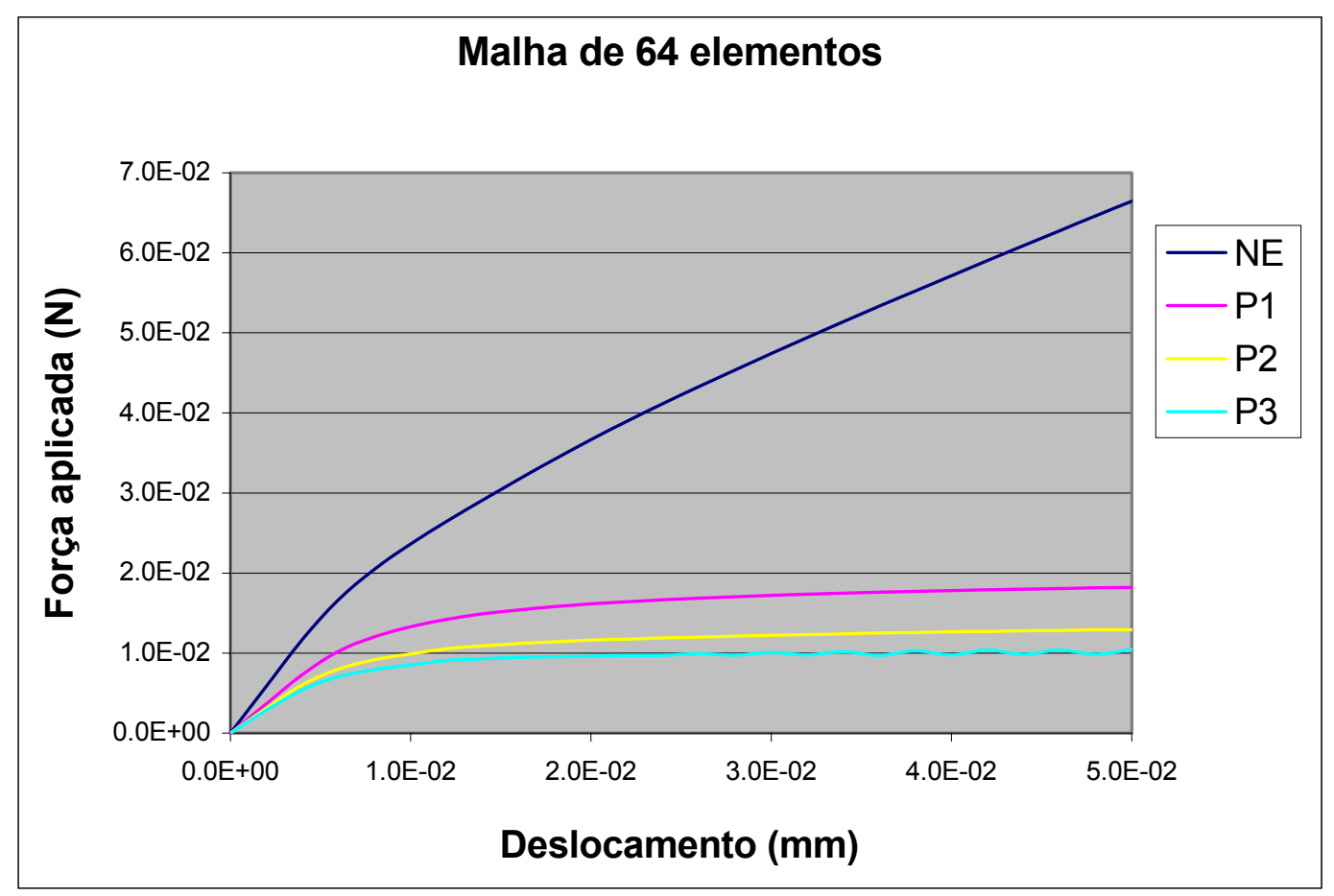

Figura 31 - Curva Força x Deslocamento para malha de 64 elementos.

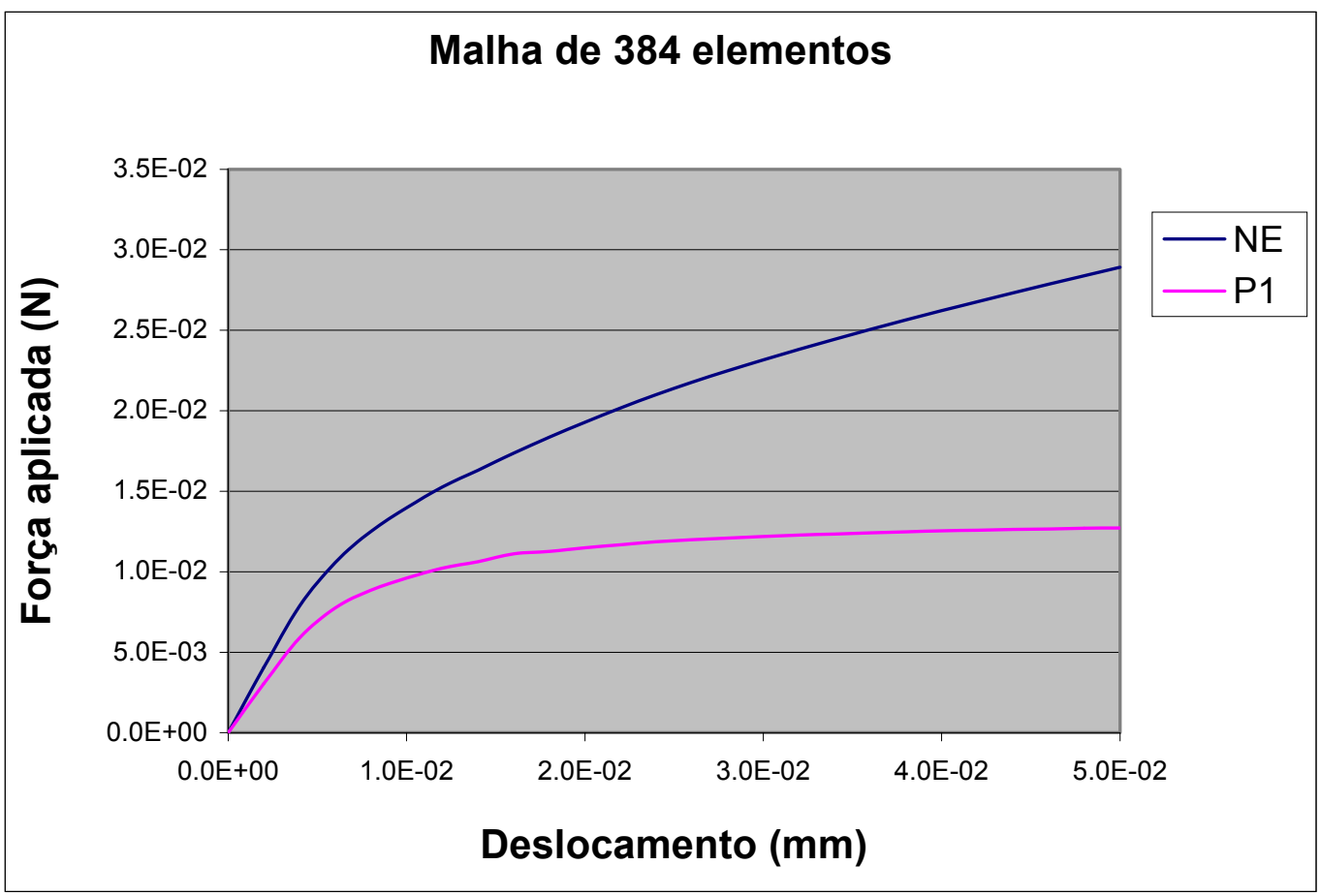

Figura 32 - Curva Força x Deslocamento para malha de 384 elementos. 


\subsection{Exemplo 5}

A estrutura analisada é uma chapa de espessura unitária com dois recortes cilíndricos, mostrada na figura 33. Indicam-se também na figura as faces nas quais se impõem deslocamentos controlados na direção do eixo $y$.

O modelo constitutivo do material que forma a chapa é o de Lemaitre - com dano e plasticidade, conforme apresentado no item 4.3.2.5 do capítulo 4. As propriedades mecânicas do material são:

$$
\begin{aligned}
& E=210 \mathrm{GPa}=2,1 \cdot 10^{5} \mathrm{~N} / \mathrm{mm}^{2} \\
& v=0,3 \\
& K=10,5 \mathrm{GPa}=1,05 \cdot 10^{4} \mathrm{~N} / \mathrm{mm}^{2} \\
& \sigma_{t}=620 \mathrm{MPa}=620 \mathrm{~N} / \mathrm{mm}^{2} \\
& D_{C}=0,40, E_{D}=0,0 \text { e } E_{R}=0,2
\end{aligned}
$$

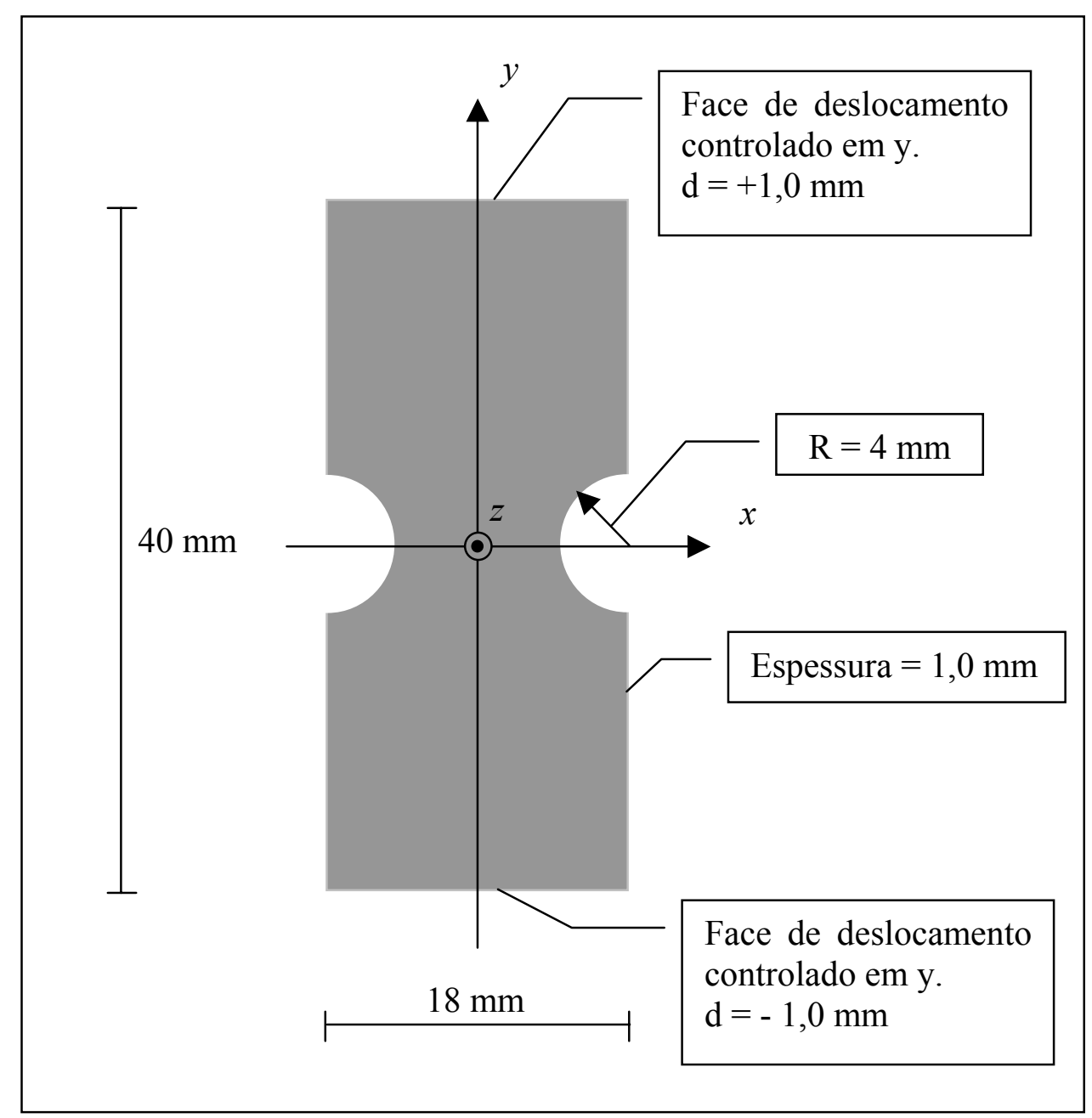

Figura 33 - Dimensões da chapa analisada. 
A análise estrutural é realizada apenas sobre um quarto da estrutura - utilizando-se os seguintes vínculos de simetria:

- num plano normal ao eixo $z$ (na metade da espessura), impõe-se deslocamento nulo na direção do eixo $z$.

- num plano normal ao eixo $x$, passando por $x=0$, impõe-se deslocamento nulo na direção do eixo $x$.

A figura 34 mostra a estrutura analisada efetivamente, num plano $x-y$ em $z=0$, fazendo-se uso dos vínculos de simetria.

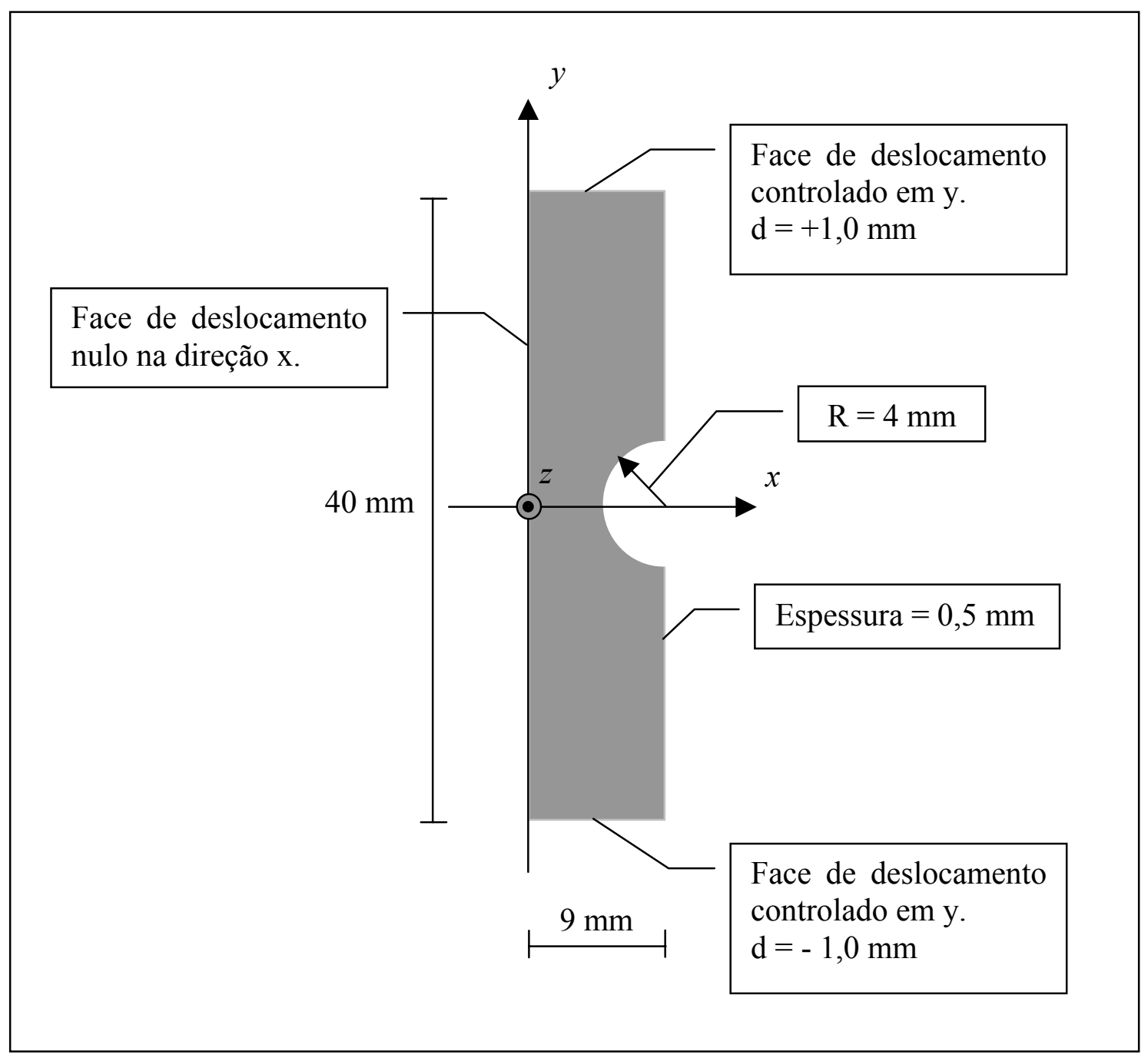

Figura 34 - Estrutura efetivamente analisada - vínculos de simetria

O deslocamento controlado é aplicado ao longo de 100 passos. Foram utilizadas duas malhas de elementos hexaédricos: a primeira com 62 elementos (malha A) e a segunda com 82 (malha B). As malhas utilizadas são mostradas na figura 35, sendo que na espessura há apenas uma camada de elementos. Como se pode ver nessa figura, a malha B 
é mais refinada na região central da peça (que apresenta os mais elevados gradientes de tensão), em comparação à malha $\mathrm{A}$ : com isso, podem-se confrontar resultados levando-se em conta também o efeito do refinamento $\mathbf{h}$.

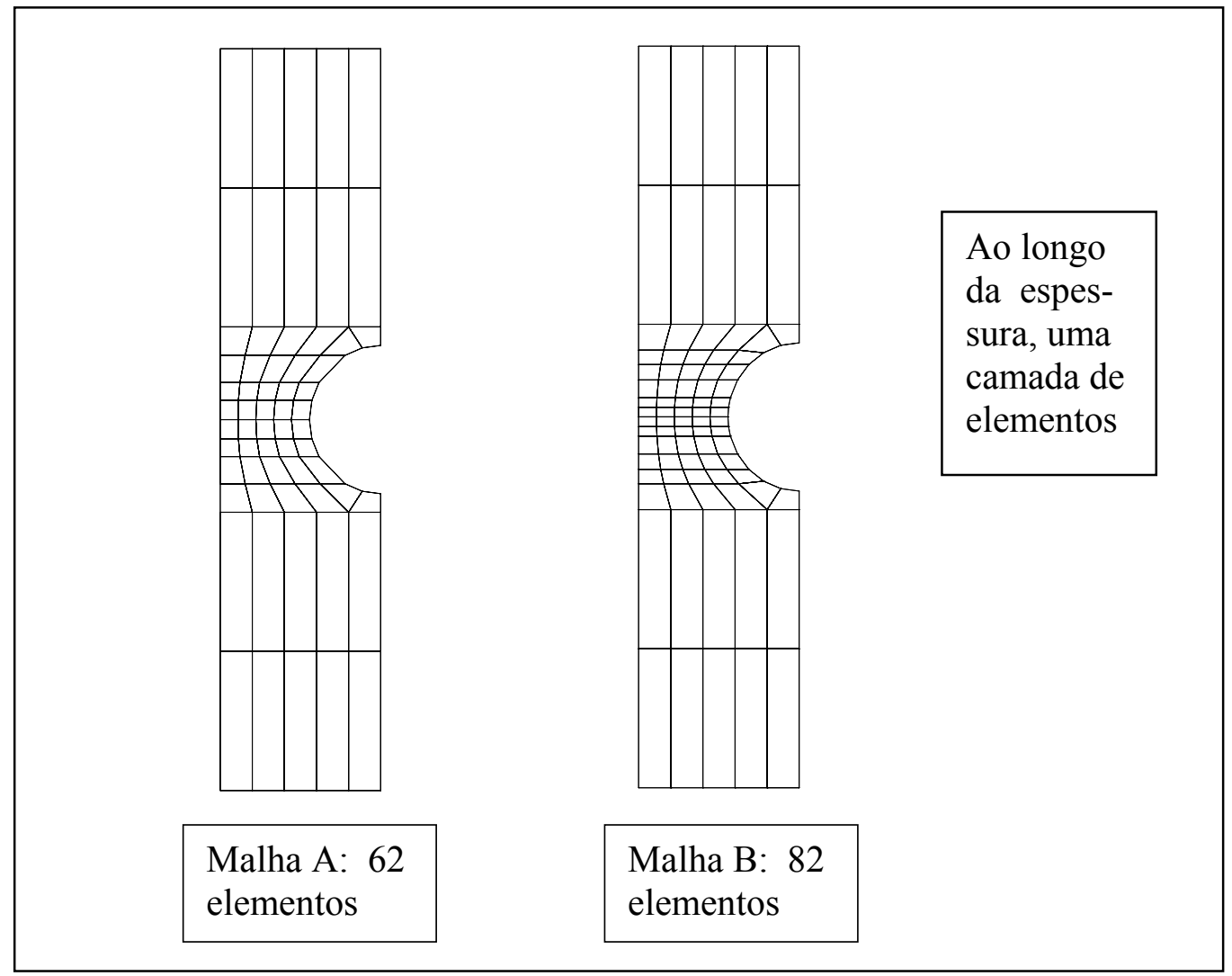

Figura 35 - Malhas de elementos utilizadas na análise.

Duas simulações foram realizadas: na primeira, não se considerou o dano, apenas a plasticidade, adotando-se o modelo constitutivo descrito no item 3.3.2.2 do capítulo 3; na segundo, utilizou-se o modelo de Lemaitre.

$1^{\text {a }}$ simulação - Elastoplasticidade com encruamento isótropo linear.

Para ambas as malhas mostradas na figura 35, a análise foi realizada utilizando três tipos de aproximação:

- Sem enriquecimento (NE).

- Com enriquecimento polinomial de primeiro grau em todos os nós (P1).

- Com enriquecimento polinomial de segundo grau nos nós da faixa central da chapa e de primeiro grau nos demais nós (adotando-se a sigla P2 para esta aproximação). Os nós que pertencem à faixa central cuja aproximação foi enriquecida com polinômio de segundo grau são mostrados na figura 36. 


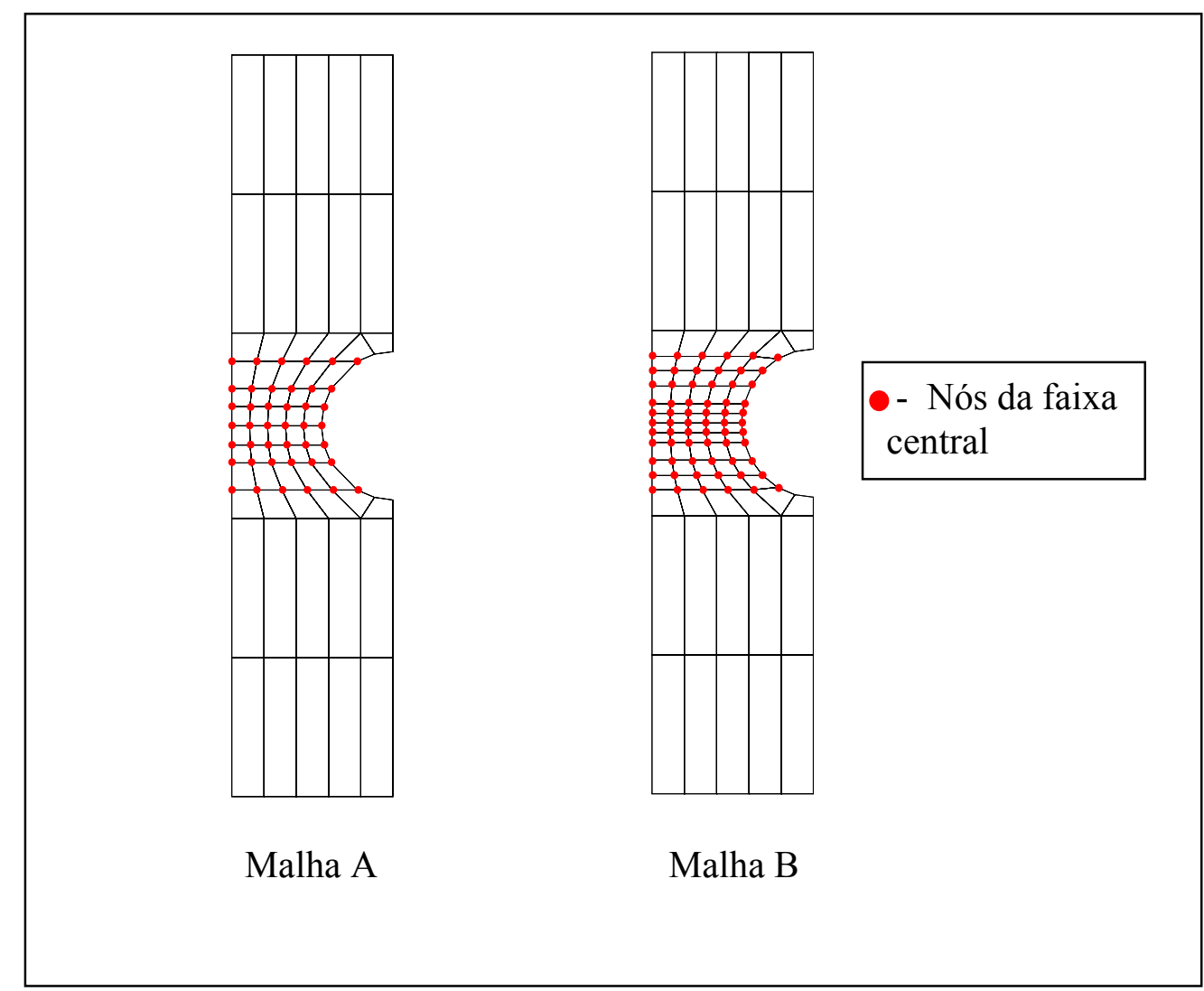

Figura 36 - Nós da faixa central com enriquecimento polinomial de segundo grau

Apresentam-se a seguir os gráficos: valor da força aplicada nas faces contra deslocamento prescrito na direção $y$.

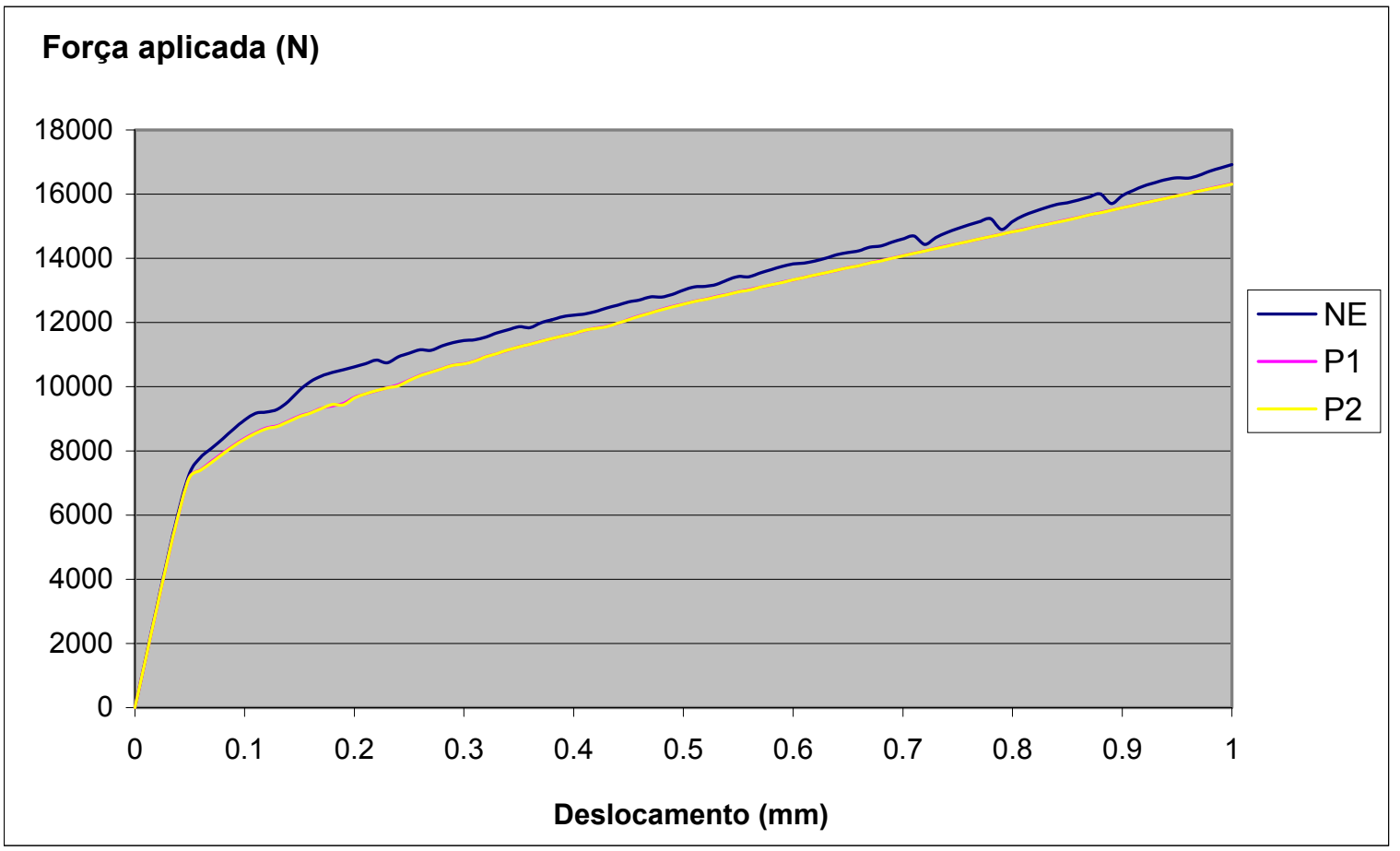

Figura 37 - Força aplicada x deslocamento (malha A) 


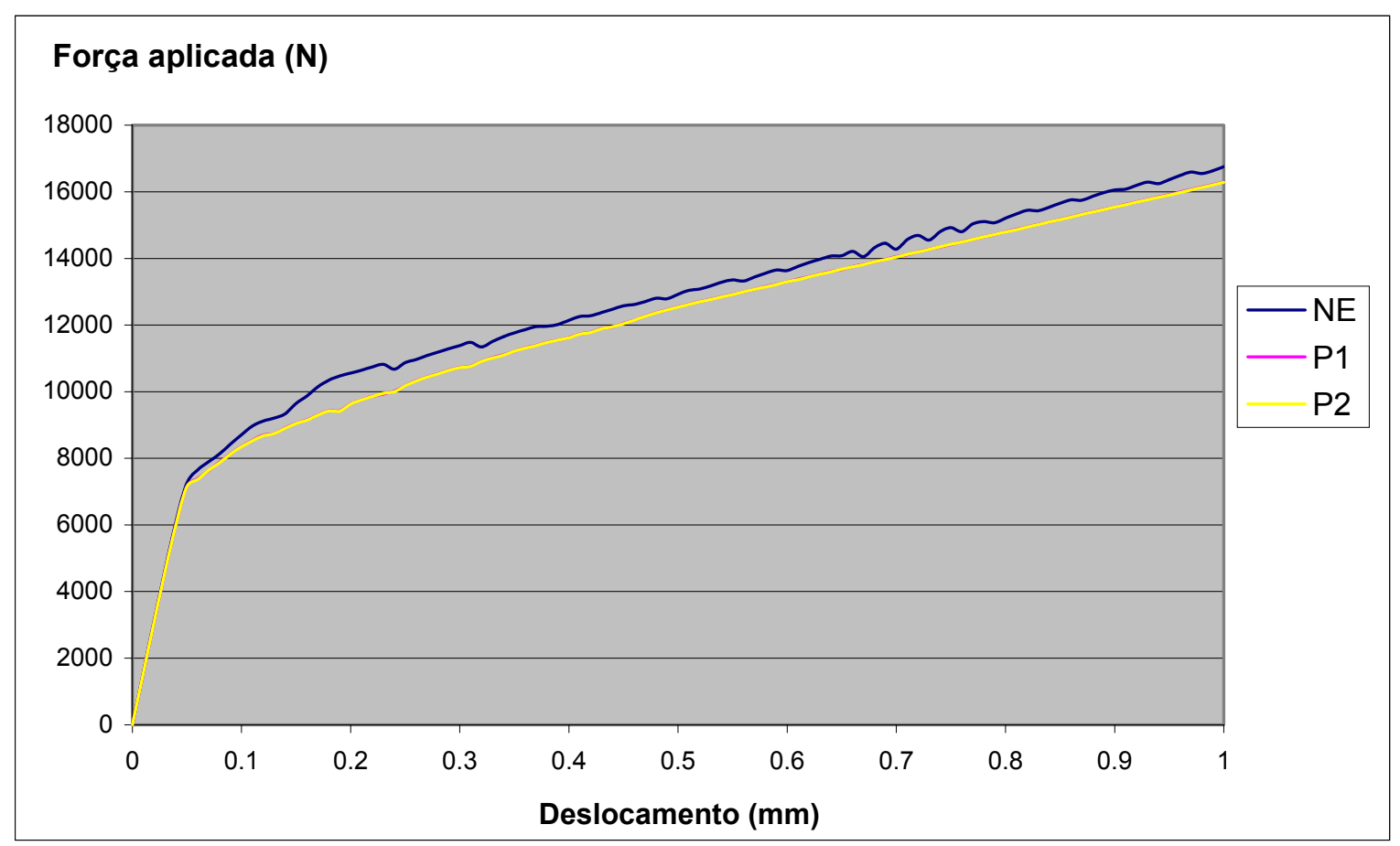

Figura 38 - Força aplicada x deslocamento (malha B)

Nas figuras 37 e 38, pode-se ver nitidamente o efeito de regularização da resposta global (carga $\mathrm{x}$ deslocamento) que se obtém com o enriquecimento da aproximação (tanto para a malha menos refinada como para a mais refinada). Nota-se que há pouca alteração na resposta global ao se melhorar a aproximação na faixa central (passando do enriquecimento $\mathrm{P} 1$ para o $\mathrm{P} 2$ ).

Nas figuras seguintes, apresentam-se (para ambas as malhas) os mapas da distribuição da tensão de von Mises (tensão equivalente) no $100^{\circ}$ passo (nível de deslocamento: 1,0 mm), considerando-se os três tipos de aproximação. Esses valores correspondem aos obtidos num plano normal a $z$ que dista $2,346.10^{-2} \mathrm{~mm}$ do plano que passa pela metade da espessura.

Das figuras 39 e 40, pode-se constatar que, para ambas as malhas, há uma diferença muito acentuada na resposta obtida sem enriquecimento (NE), de um lado, e com enriquecimentos P1 e P2, de outro. As tensões mínimas e máximas obtidas com enriquecimentos P1 e P2 são muito próximas entre si e divergem bastante dos respectivos valores obtidos quando não se faz enriquecimento (NE). Certamente a resposta sem enriquecimento sofre dos efeitos de travamento já discutidos anteriormente. Embora discreto, o efeito do enriquecimento P2 sobre o P1 faz-se notar pela maior regularização das curvas de isotensão na região central, que é exatamente a de maiores gradientes de tensão. 


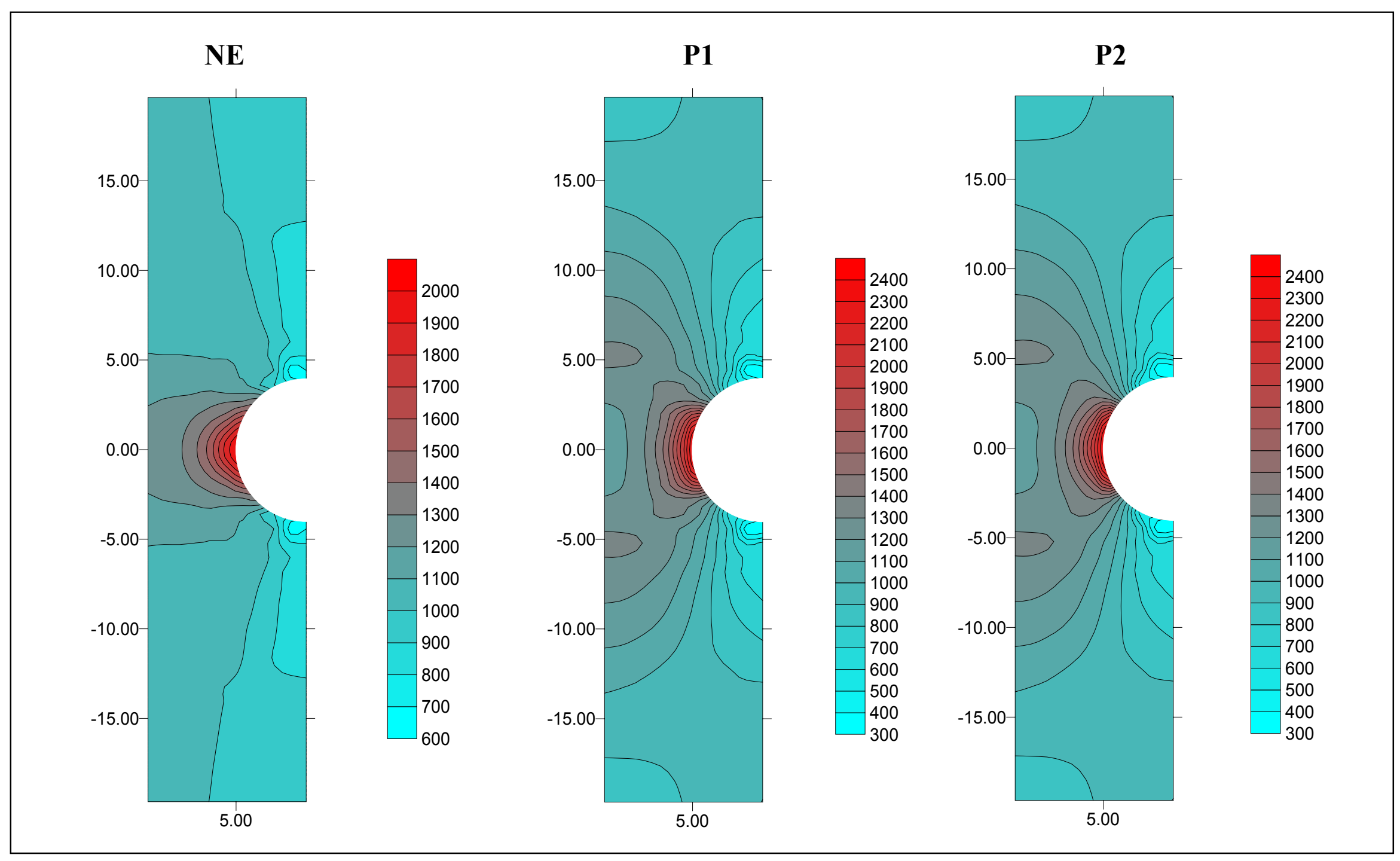

Figura 39 - Mapa da tensão equivalente (malha A), em $\mathrm{N} / \mathrm{mm}^{2}$. 


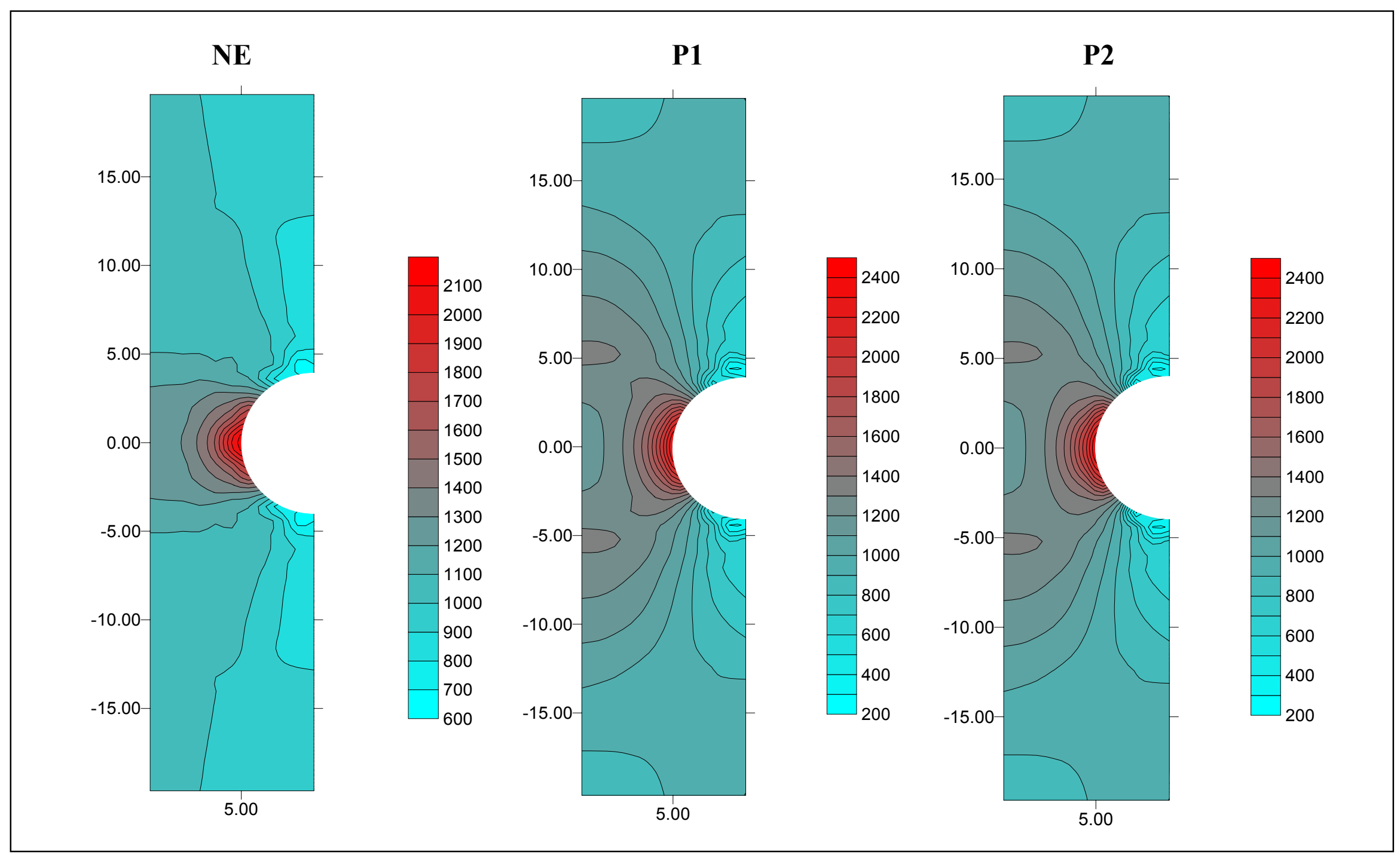

Figura 40 - Mapa da tensão equivalente (malha B), em $\mathrm{N} / \mathrm{mm}^{2}$. 
$2^{\mathrm{a}}$ simulação - Modelo de Lemaitre.

Para ambas as malhas mostradas na figura 35, a análise foi realizada utilizando-se os três tipos de aproximação descritos na $1^{\mathrm{a}}$ etapa.

Os gráficos com o valor da força aplicada nas faces de deslocamento prescrito na direção $y$ são apresentados a seguir.

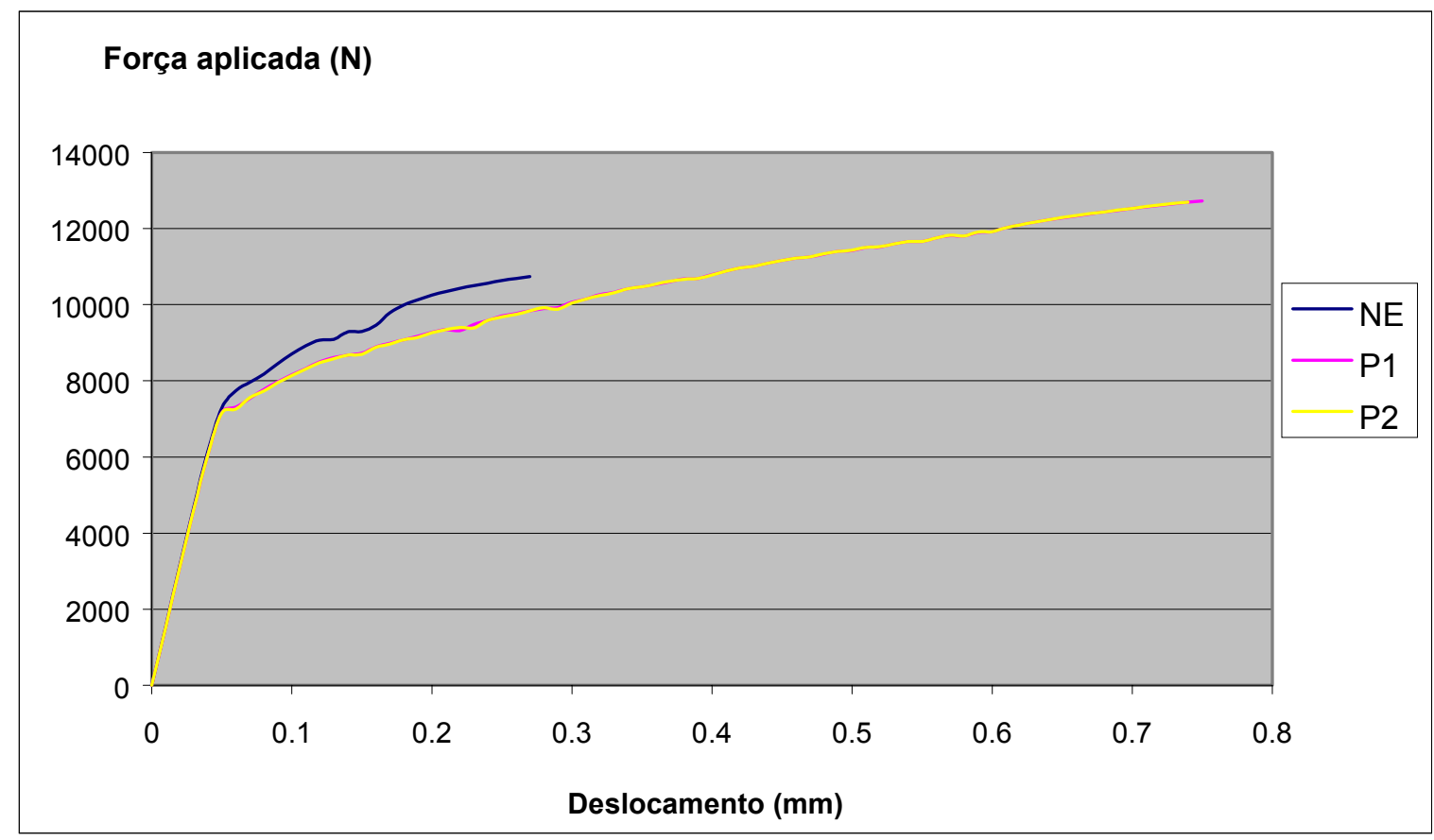

Figura 41 - Força aplicada x deslocamento (malha A)

\section{Força aplicada (N)}

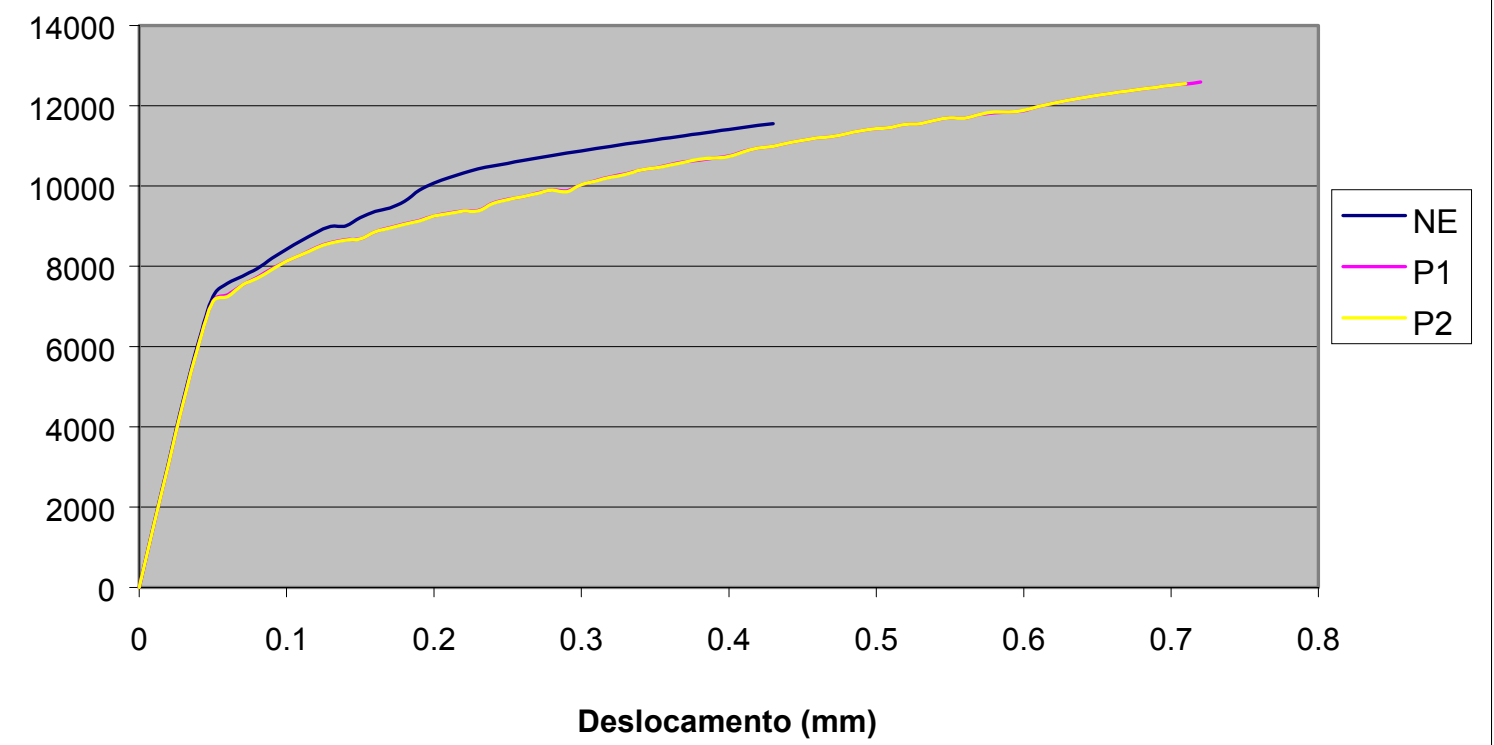

Figura 42 - Força aplicada x deslocamento (malha B) 
Da análise das figuras 41 e 42, percebe-se que, para ambas as malhas, o dano crítico é atingido mais rapidamente quando se usa a aproximação polinomial mais pobre (NE). Isso ocorre pois nesse caso a estrutura apresenta uma rigidez excessiva, com conseqüente aumento das deformações plásticas e do dano. Utilizando-se a malha A (menos refinada), o dano atingiu o valor máximo (limitado a 0,99) nos passo 27 (aproximação NE), 75 (P1) e 74 (P2). Já com a utilização da malha B (mais refinada), o valor máximo do dano foi atingido nos passos 43 (NE), 72 (P1) e 71 (P2).

Relativamente à malha $\mathrm{A}$, os mapas de tensão equivalente e de dano são apresentados nas figuras 43 e 44, correspondentes ao passo 27, para as três aproximações e nas figuras 45 e 46, correspondentes ao passo 74, apenas para os enriquecimentos P1 e P2.

Nas figuras 43 e 44, nota-se uma qualidade muito ruim da resposta quando se utiliza aproximação não-enriquecida $(\mathrm{NE})$, tanto em termos da tensão equivalente como do dano, quando comparada à obtida com o uso das aproximações enriquecidas P1 e P2. Na figura 44, verifica-se inclusive a obtenção de dano máximo em posições incorretas.

A melhoria da qualidade da resposta obtida ao se utilizar o enriquecimento P2, em relação ao P1, pode ser vista nas figuras 45 e 46 . Na região central, percebe-se claramente a melhor definição das curvas de tensão de von Mises e de dano, respectivamente.

Os mapas de tensão equivalente e de dano referentes à malha $\mathrm{B}$ são apresentados nas figuras 47 e 48 , no passo 43 para as três aproximações e nas figuras 49 e 50, no passo 71, apenas com os enriquecimentos P1 e P2.

Os mesmos comentários feitos com relação à malha A são válidos: a aproximação NE conduz a resultados insatisfatórios (figuras 47 e 48) e há uma regularização da resposta em termos de tensões e do dano ao se passar do enriquecimento P1 para o P2 (figuras 49 e $50)$.

Quando se usa a malha B, porém, nota-se que o efeito de regularização da resposta pelo uso de aproximação com enriquecimento P2, comparado ao uso do enriquecimento $\mathrm{P} 1$, é bem menos pronunciado do que o verificado ao se utilizar a malha A. Isso certamente deve-se ao fato de a malha B já apresentar maior refinamento na região central (do tipo h), tornando o efeito do enriquecimento polinomial (do tipo p) mais notável na malha menos refinada $(\mathrm{A})$. 


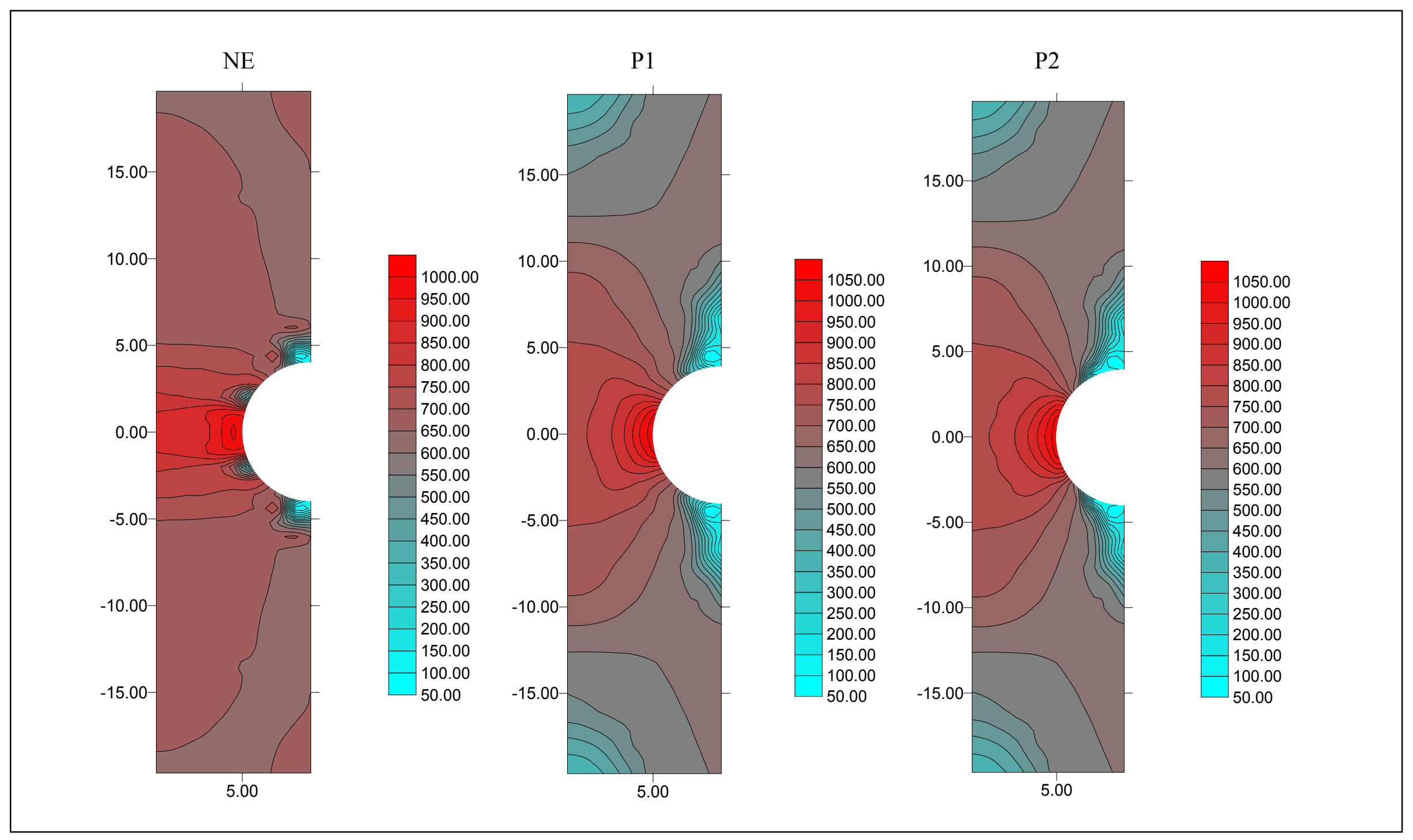

Figura 43 - Malha A - Tensão equivalente em $\mathrm{N} / \mathrm{mm}^{2}$ - Passo 27 


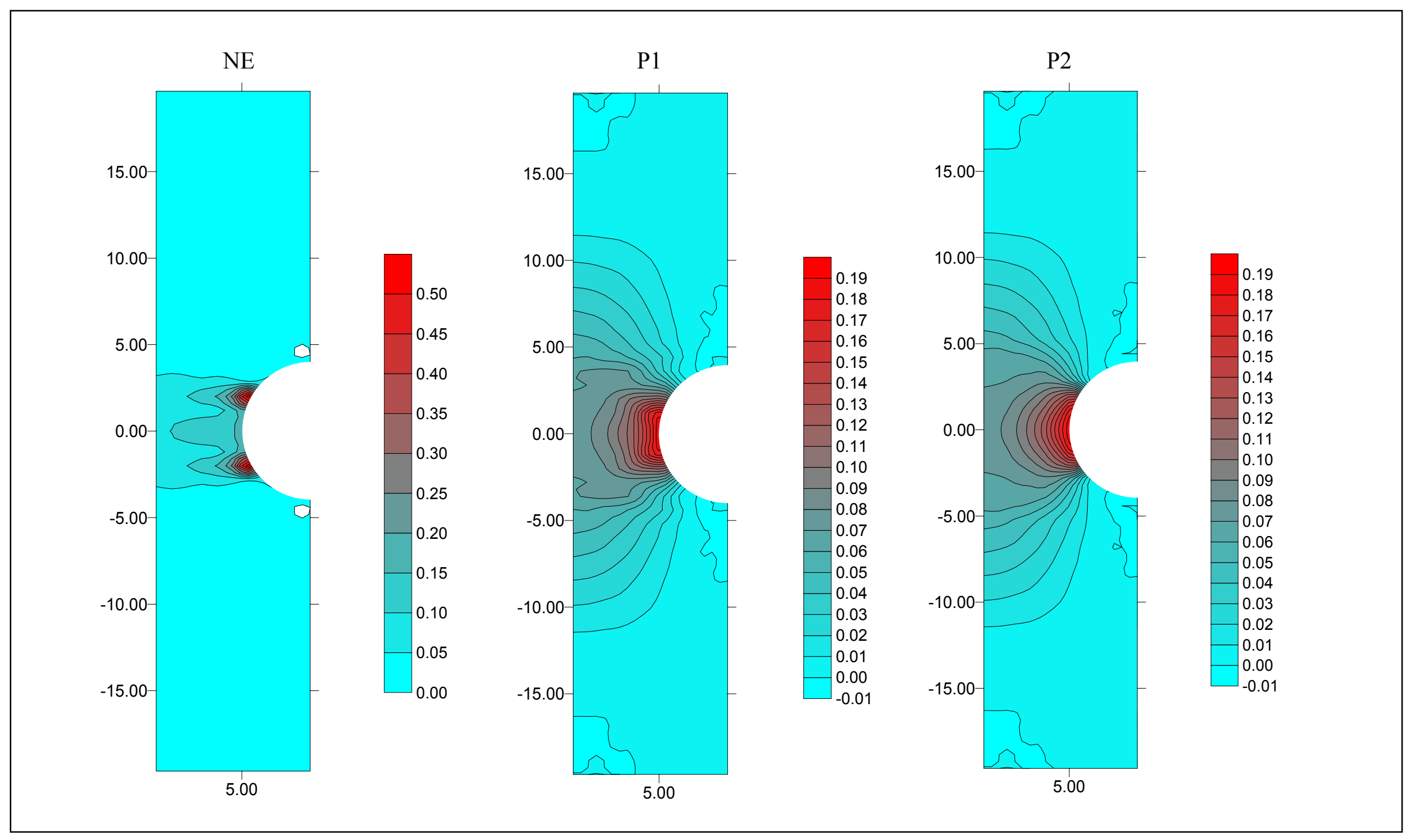

Figura 44 - Malha A - Dano - Passo 27 


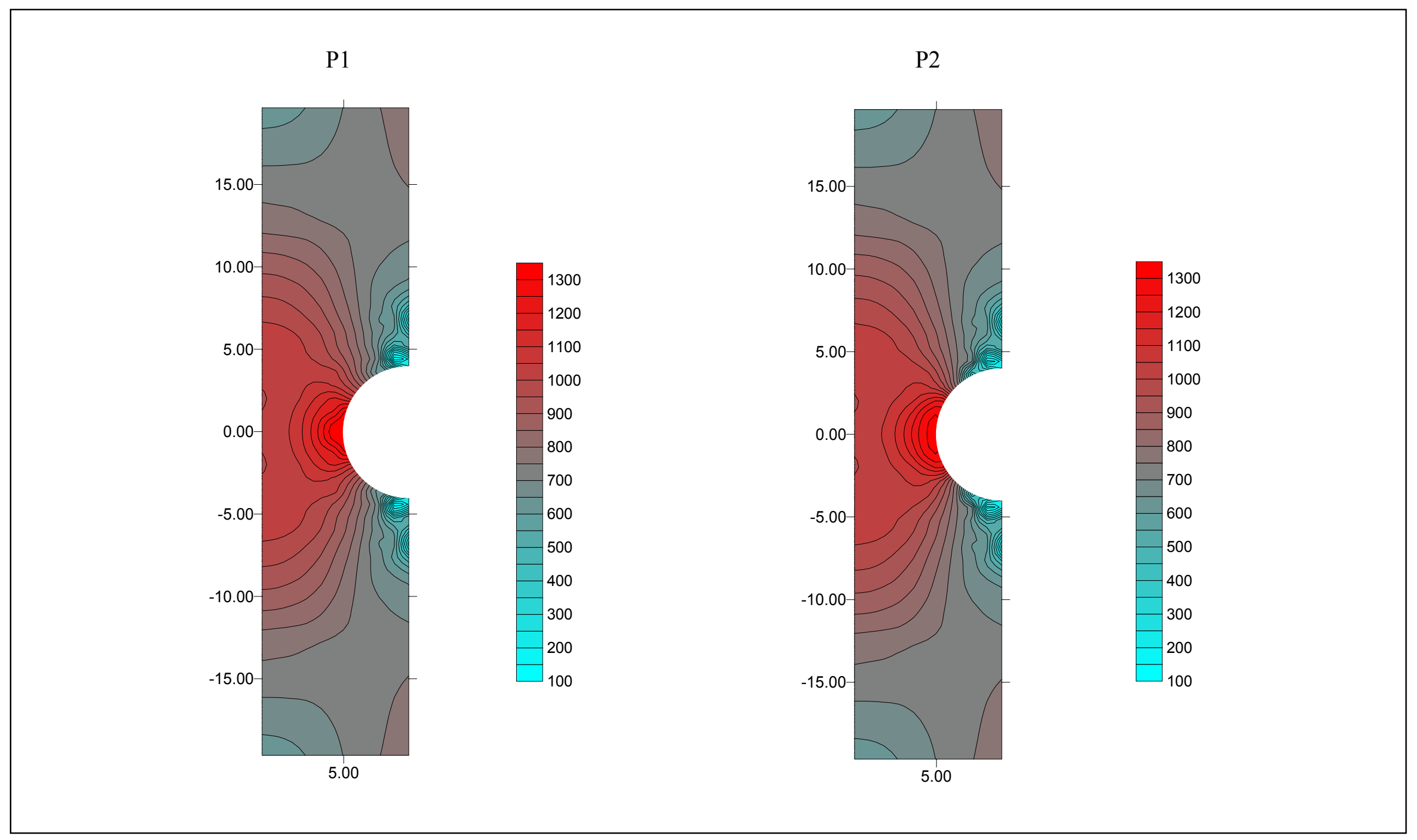

Figura 45 - Malha A - Tensão equivalente em $\mathrm{N} / \mathrm{mm}^{2}$ - Passo 74 


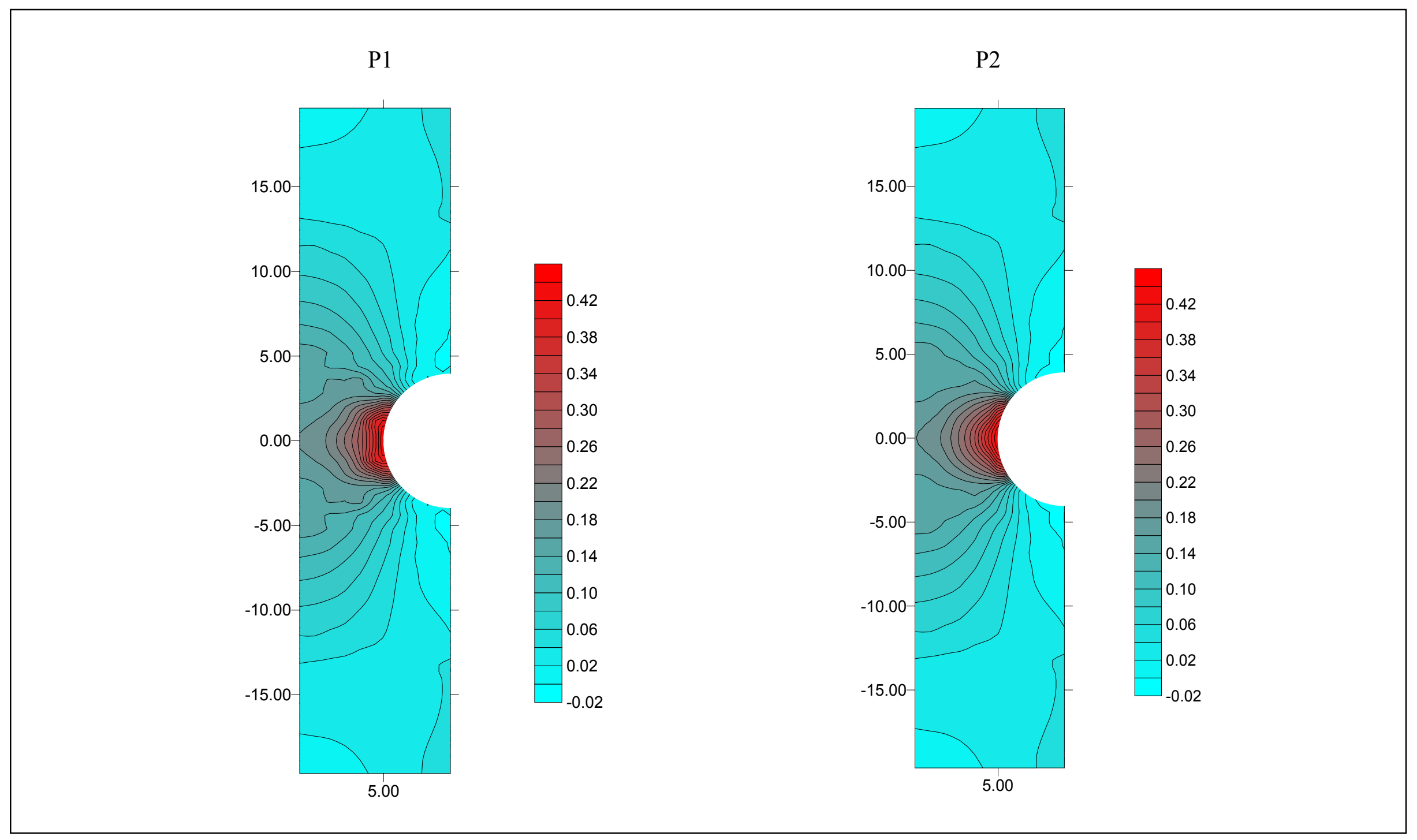

Figura 46 - Malha A - Dano - Passo 74 


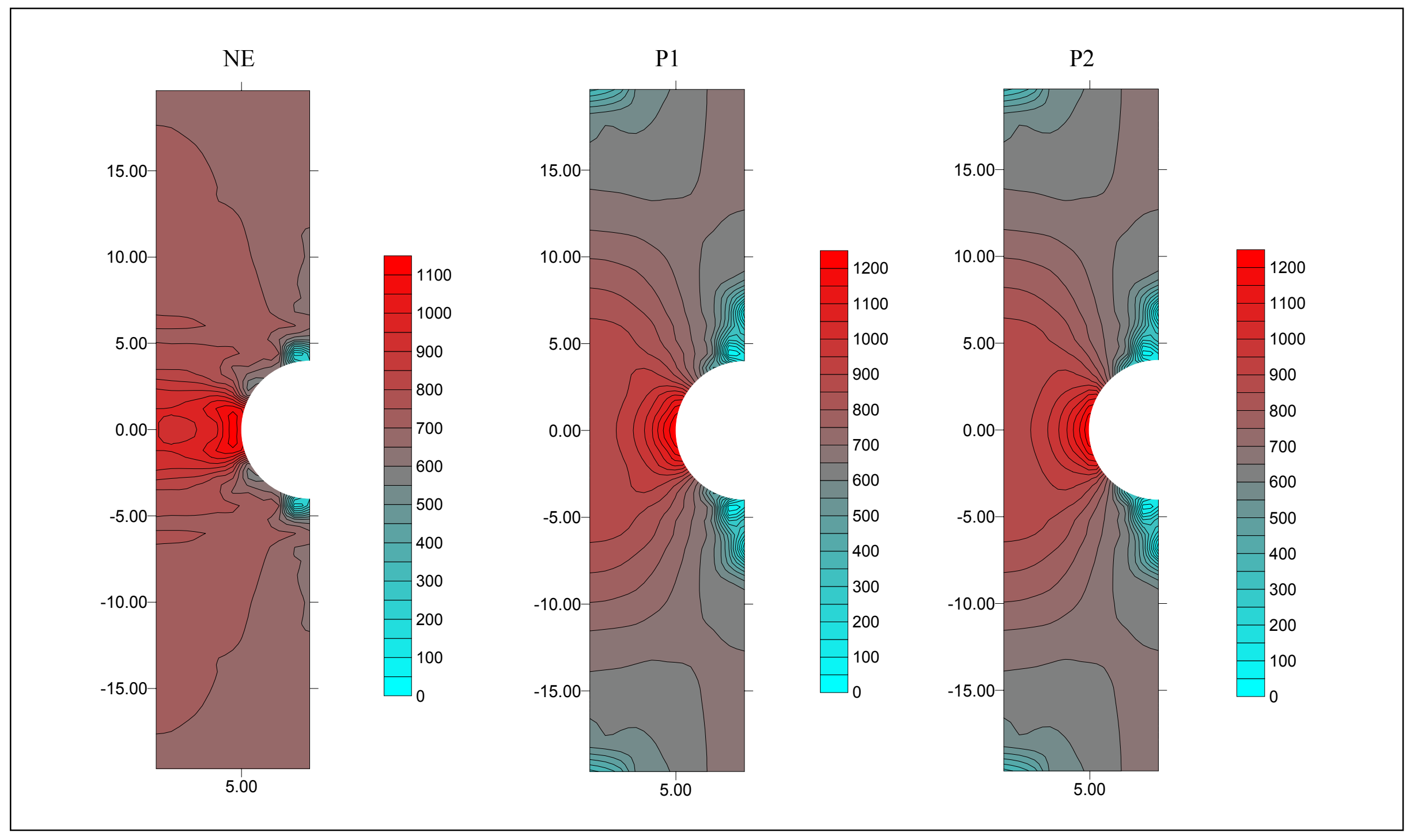

Figura 47 - Malha B - Tensão equivalente em $\mathrm{N} / \mathrm{mm}^{2}$ - Passo 43 


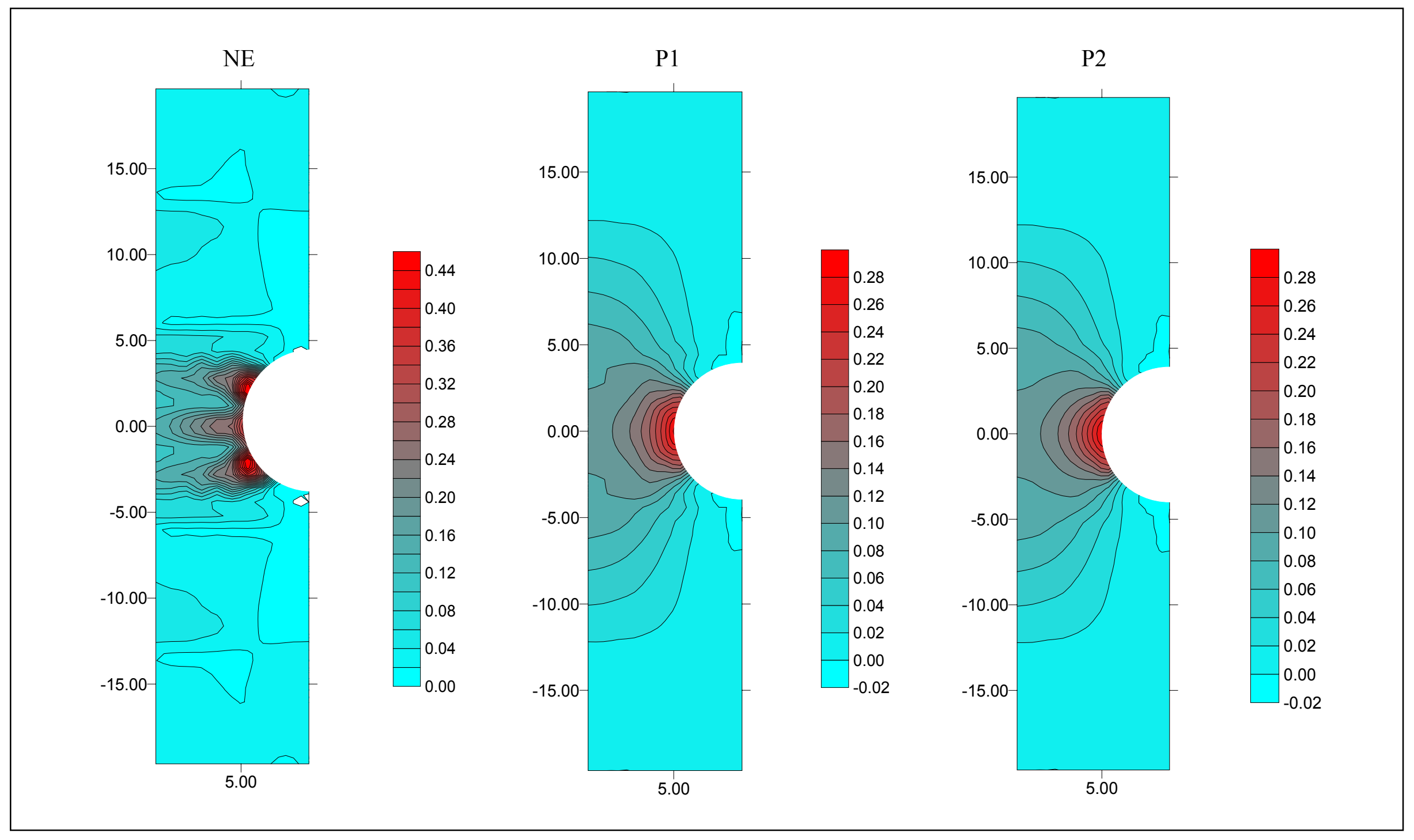

Figura 48 - Malha B - Dano - Passo 43 


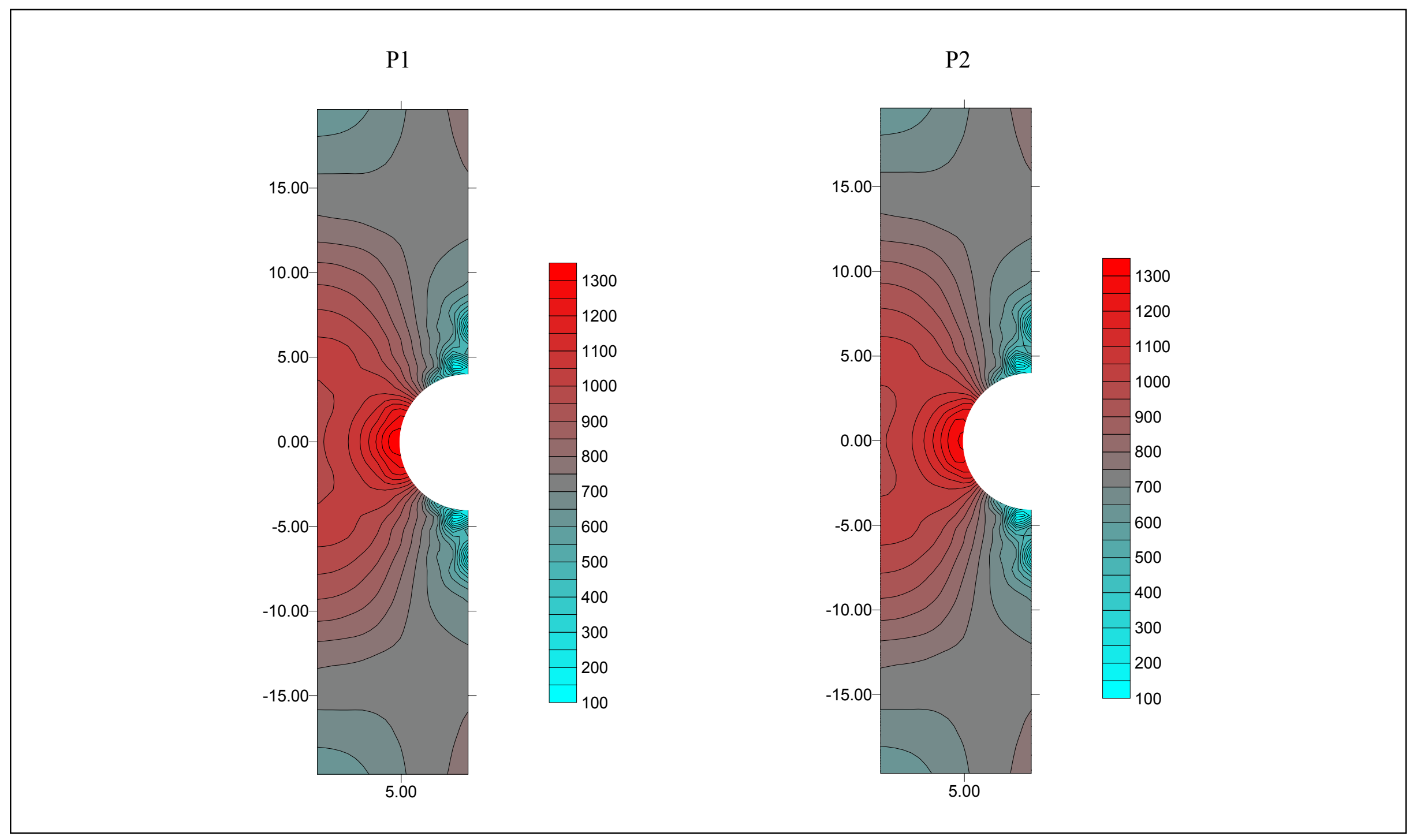

Figura 49 - Malha B - Tensão equivalente em $\mathrm{N} / \mathrm{mm}^{2}$ - Passo 71 


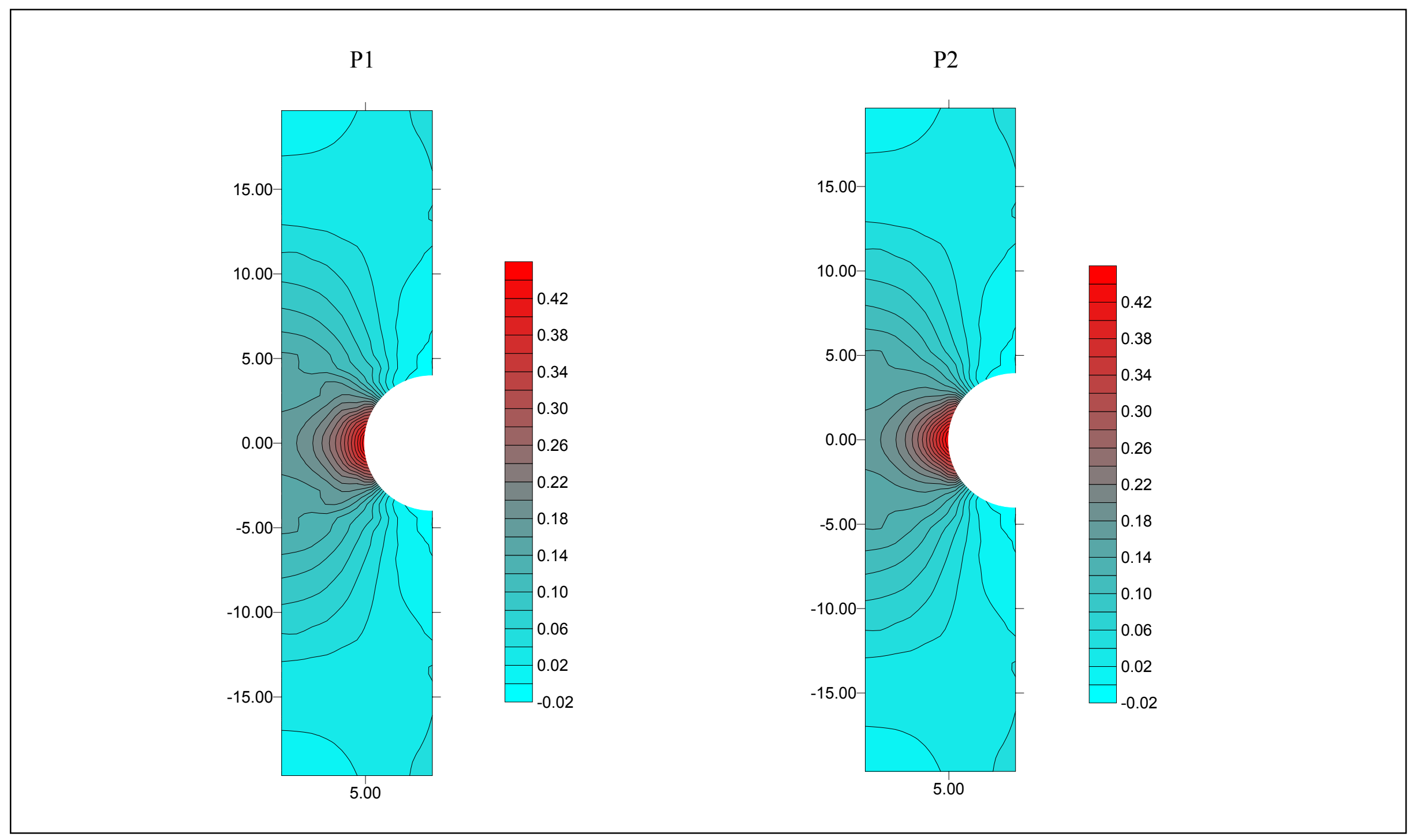

Figura 50 - Malha B - Dano - Passo 71 


\section{CONCLUSÕES E CONSIDERAÇÕES FINAIS}

Neste capítulo, busca-se, por um lado, apresentar as contribuições do trabalho desenvolvido e a compatibilidade entre os objetivos inicialmente propostos e o resultado final; por outro, apresentar sugestões para pesquisas futuras na aplicação do MEFG em análise não-linear, objeto principal deste trabalho.

A pesquisa em formulações não-convencionais do Método dos Elementos Finitos, em especial as que utilizam conceitos dos métodos sem malha, pode ser considerada um campo de investigação relativamente recente, mas que tem gerado uma produção acadêmica crescente. Este trabalho insere-se, portanto, no objetivo mais amplo de consolidar essa linha de pesquisa no Departamento de Estruturas da EESC/USP, a qual teve como primeiro trabalho a tese de doutorado apresentada por BARROS (2002).

A ênfase maior deste trabalho consistiu na exploração das vantagens que o MEFG oferece quando comparado ao MEF convencional, em especial a possibilidade de enriquecimento local da aproximação, sem perda de conformidade entre os elementos. A simulação numérica, em campo não-linear, com modelos de dano e plasticidade, é original. Nesse caso, explorou-se o MEFG para a melhor definição da distribuição das tensões equivalentes (de von Mises) e do dano, com o uso do enriquecimento local em regiões de elevados gradientes dessas grandezas.

Pode-se ressaltar como contribuição particular a implementação computacional realizada, adaptada a análises tridimensionais, a qual pode servir de base a futuros desenvolvimentos. Na apresentação deste trabalho, procurou-se detalhar a geração das matrizes de rigidez e dos vetores de forças nodais, visando enfatizar a semelhança com o MEF convencional e a possibilidade de alterar, sem grandes dificuldades, códigos preexistentes para adaptá-los à moldura do MEFG.

A partir das simulações numéricas apresentadas no Capítulo 6, puderam-se extrair algumas conclusões, apresentadas a seguir:

- o enriquecimento da aproximação inicial, na forma proposta pelo MEFG, permite uma melhoria na qualidade da resposta, sem a necessidade de alteração da malha de elementos. Como vantagem em relação à versão $\mathbf{p}$ do $\mathrm{MEF}$, ressalta-se a possibilidade do uso de funções enriquecedoras de diferentes graus polinomiais, conforme a região do domínio, sem perda de conformidade entre os elementos.

- pôde-se evidenciar a viabilidade de superar, com a utilização do MEFG, o travamento volumétrico em problemas de plasticidade. 
- a característica do MEFG de permitir de modo bastante conveniente o enriquecimento local da aproximação faz desse método uma alternativa bastante competitiva na simulação do fenômeno de localização. Com isso, podem-se capturar os elevados gradientes de deformação que ocorrem de forma localizada, sem que haja um aumento muito substancial do custo computacional.

- o enriquecimento polinomial de grau mais elevado em regiões de maior concentração de tensões, nos moldes do MEFG, permitiu uma melhor definição das regiões de plastificação e da distribuição do dano. Isto pôde ser claramente demonstrado no exemplo numérico apresentado no item 6.5.

- de um modo geral, é bastante significativo o custo computacional de geração das matrizes de rigidez de elementos com enriquecimento polinomial de grau elevado, o que pode limitar, no momento, aplicações práticas em larga escala.

Como propostas para desenvolvimentos futuros, podem-se citar:

- Busca de maior eficiência computacional: os procedimentos descritos neste trabalho, em especial a geração de matrizes de rigidez de elementos enriquecidos e a solução de sistemas lineares de grande porte, apresentam custo computacional elevado. Quanto à geração das matrizes de rigidez, no caso de se tratar de modelos de dano, pode-se propor um estudo mais aprofundado da influência da ordem de quadratura na distribuição do dano, visando verificar a possibilidade de redução do custo da integração numérica. Já com relação à resolução dos sistemas de equações com matriz não-simétrica, que aparecem quando se utilizam modelos de dano e plasticidade, pode-se propor a pesquisa de subrotinas de cálculo mais eficientes para a solução de sistemas não-simétricos.

- Estudo do fenômeno da localização de deformações e de dano. Em BARROS (2002) foi mostrado como a técnica de enriquecimento polinomial pode ser usada em problemas de localização do dano numa pequena região do domínio; abre-se, também a possibilidade de aplicação do MEFG a problemas desse tipo utilizando-se funções nãopolinomiais.

- Estudo e implementação, no contexto do MEFG, da transição dano-fratura. O processo de enriquecimento segundo o MEFG apresenta características muito favoráveis a essa simulação: por um lado, pode-se definir de modo mais preciso e conveniente a zona de localização de deformações; por outro, pode-se introduzir no problema uma descontinuidade no campo de deslocamentos por meio de funções do tipo Heaviside, dispensando a necessidade de alteração na malha. 
- O uso das funções de forma lagrangianas do MEF, combinado com a utilização de funções enriquecidoras polinomiais, conduz à geração de sistemas lineares singulares, o que requer a utilização de sub-rotinas de solução de sistemas compatíveis. Uma proposta de desenvolvimento consiste em gerar uma aproximação inicial não-polinomial, de modo a evitar a singularidade da matriz de rigidez. 


\section{REFERÊNCIAS BIBLIOGRÁFICAS}

ÁLVARES, M. S. (1993). Estudo de um modelo de dano para o concreto: formulação, identificação paramétrica e aplicação com o emprego do método dos elementos finitos. Dissertação (Mestrado) - Escola de Engenharia de São Carlos, Universidade de São Paulo, São Carlos.

ANSYS RELEASE 5.5.1 (1998). Theory Manual. $3^{\text {rd }}$ edition. SAS IP, Inc.

BARROS, F. B. (2002). Métodos sem malha e método dos elementos finitos generalizados em análise não-linear de estruturas. Tese (Doutorado) - Escola de Engenharia de São Carlos, Universidade de São Paulo, São Carlos.

BAUSCHINGER, J. (1886). On the change of the elastic limit and strength of iron and steel by elongation, shortening, heating, cooling and repeated loading. Mittheilung aus dem Mechanisch-Technisches Laboratorium der K. Technische Hochschule in München, v. 13.

BAŽANT, Z. P. ; BELYTSCHKO, T. ; CHANG, T. P. (1984). Continuum theory for strain-softening. Journal of Engineering Mechanics, v. 110, n. 12, p. 1666-1692.

BELYTSCHKO, T. ; LU, Y. ; GU, L. (1994). Element-free Galerkin methods. International Journal for Numerical Methods in Engineering, v. 37, p. 229-256.

BELYTSCHKO, T. ; MOËS, N. (2002). Extended finite element method for cohesive crack growth. Engineering Fracture Mechanics, v. 69, p. 813-833.

BENALLAL, A. ; BILLARDON, R. ; DOGHRI, I. (1988). Integration algorithm and the corresponding consistent tangent operator for fully coupled elastoplastic and damage equations. Communications in Applied Numerical Methods, v. 4, n. 6, p. 731-740.

BUSSAMRA, F. L. S. (1999). Elementos finitos híbridos-Trefftz: um modelo elastoplástico tridimensional. Tese (Doutorado) - Escola Politécnica, Universidade de São Paulo, São Paulo.

CIPOLlinA, A. ; LOPEZ-INOJOSA, A. ; FLOREZ-LÓPEZ, J. (1995). Simplified damage mechanics approach to nonlinear analysis of frames. Computers and Structures, v. 54, n. 6, p. 1113-1126.

COMI, C. (1999). Computational modelling of gradient-enhanced damage in quasi-brittle materials. Mechanics of Cohesive-Frictional Materials, v. 4, n. 1, p. 17-36.

COOK, R. (1987). A plane hybrid element with rotational d.o.f. and adjustable stiffness. International Journal for Numerical Methods in Engineering, v. 24, p. 1499-1508.

COWPER, G. R. (1973). Gaussian quadrature formulas for triangles. International Journal for Numerical Methods in Engineering, v. 7, p. 405-408. 
DOGHRI, I. (1995). Numerical implementation and analysis of a class of metal plasticity models coupled with ductile damage. International Journal for Numerical Methods in Engineering, v. 38, n. 20, p. 3403-3431.

DOLBOW, J. ; MOËS, N. ; BELYTSCHKO, T. (2000). Discontinuous enrichment in finite elements with a partition of unity method. Finite Elements in Analysis and Design, v. 36, p. 235-260.

DRIEMEIER, L. (1995). Considerações sobre a fadiga em metais e o comportamento do concreto sob solicitação cíclica. Dissertação (Mestrado) - Escola de Engenharia de São Carlos, Universidade de São Paulo, São Carlos.

DRIEMEIER, L. (1999). Contribuição ao estudo da localização de deformações com modelos constitutivos de dano e plasticidade. Tese (Doutorado) - Escola de Engenharia de São Carlos, Universidade de São Paulo, São Carlos.

DRUCKER, D. C. (1951). A more fundamental approach to plastic stress-strain relations. In: U. S. NATIONAL CONGRESS ON APPLIED MECHANICS, $1^{\text {st }}$, New York, ASME. Proceedings. p. 487-491.

DUARTE, C. A. (1996). The hp-cloud method. PhD thesis, The University of Texas at Austin.

DUARTE, C. A. ; ODEN, J. T. (1996). An h-p adaptive method using clouds. Computer Methods in Applied Mechanics and Engineering, v. 139, p. 237-262.

DUARTE, C. A. ; BABUSKA, I. ; ODEN, J. T. (2000). Generalized finite element methods for three-dimensional structural mechanics problems. Computers \& Structures, v. 77 , n. 2, p. 215-232.

DUFF, I. S. ; REID, J. K. (1983). The multifrontal solution of indefinite sparse symmetric linear systems. ACM Transactions on Mathematical Software, v. 9, n. 3, p. 302-325.

FRÉMOND, M. ; NEDJAR, B. (1996). Damage, gradient of damage and principle of virtual power. International Journal of Solids and Structures, v. 33, n. 8, p. 1083-1103.

GURSON, A. L. (1977). Continuum theory of ductile rupture by void nucleation and growth. Journal of Engineering Materials and Technology, Transactions of the ASME, v. 99 , n. 1, p. 2-15.

JINYUN, Y. (1984). Symmetric Gaussian quadrature formulae for tetrahedronal regions. Computer Methods in Applied Mechanics and Engineering, v. 43, p. 349-353.

KACHANOV, L. M. (1958). Time of the rupture process of nonlinear solid mechanics. Otd. Tekh. Nauk., v. 8, p. 28-31. 
KACHANOV, L. M. (1984). On the brittle fracture of a thin plastic interlayer in creep conditions. In: MECHANICS of material behaviour, The Daniel C. Drucker Anniversary Volume. Urbana-Champaign, IL, USA, Elsevier. p. 191-199 (Studies in Applied Mechanics).

LA BORDERIE, C. ; MAZARS, J. ; PIJAUDIER-CABOT, G. (1991). Response of plain and reinforced concrete structures under cyclic loadings. Cachan, France, Laboratoire de Mécanique et Technologie, Rapport Interne n. 123.

LANCASTER, P. ; SAULKAUSKAS, K. (1981). Surfaces generated by moving least square methods. Mathematics of Computation, v. 37, n. 155, p. 141-158.

LEMAITRE, J. (1985). A continuous damage mechanics model for ductile fracture. Journal of Engineering Materials and Technology, Transactions of the ASME, v. 107, n. 1, p. 83-89.

LEMAITRE, J. ; CHABOCHE, J. L. (1985). Mécanique des materiaux solides. Paris, Dunod.

LEMAITRE, J. ; CHABOCHE, J. L. (1990). Mechanics of solid materials. Cambrige University Press.

LISZKA, T. ; ORKISZ, J. (1980). The finite difference method at arbitrary irregular grids and its applications in applied mechanics. Computers \& Structures, v. 11, n. 3-4, p. 83-95.

MAZARS, J. (1984). Application de la mécanique de l'éndommagement au comportement non lineaire e à la rupture du béton de structure. Thèse de Doctorat d'État, Université de Paris VI.

MELAN, E. (1938). Zur Plastizität des raümlichen Kontinuums. Ing. Arch. , v. 9, p. 116126.

MELENK, J. M. (1995). On generalized finite element methods. PhD thesis, University of Maryland, College Park.

MELENK, J. M. ; BABUSKA, I. (1996). The partition of unity finite element method: Basic theory and applications. Computer Methods in Applied Mechanics and Engineering, v. 39, p. 289-314.

MURAKAMI, S. ; OHNO, N. (1981). Constitutive equation of creep based on the concept of a creep-hardening surface. Res. Mechanica Letters, v. 1, n. 8, p. 357-362.

NAYROLES, B. ; TOUZOT, G. ; VILLON, P. (1992). Generalizing the finite element method: diffuse approximation and diffuse elements. Computational Mechanics, v. 10, p. 307-318. 
NÓBREGA, S. H. S. (1997). Sobre o desenvolvimento de elementos finitos de casca: uma classe de formulações mistas. Tese (Doutorado) - Escola Politécnica, Universidade de São Paulo, São Paulo.

ODEN, J. T. ; DUARTE, C. A. ; ZIENKIEWICZ, O. C. (1998). A new cloud-based hp finite element method. Computer Methods in Applied Mechanics and Engineering, v. 153, p. 117-126.

OÑATE, E. ; IDELSOHN, S. ; ZIENKIEWICZ, O. C. ; TAYLOR, R. L. ; SACCO, C. (1996). A stabilized finite point method for analysis of fluid mechanics problems. Computer Methods in Applied Mechanics and Engineering, v. 139, p. 315-346.

PERRONE, N. ; KAO, R. (1975). A general finite difference method for arbitrary meshes. Computers \& Structures, v. 5, p. 45-58.

PIJAUDIER-CABOT, G. ; BAŽANT, Z. P. (1987). Nonlocal damage theory. Journal of Engineering Mechanics, v. 113, n. 10, p. 1512-1533.

PILKEY, W. D. ; WUNDERLICH, W. (1994). Mechanics of structures : variational and computational methods. Boca Raton, CRC Press.

PITUBA, J. J. C. (2003). Sobre a formulação de um modelo de dano para o concreto. Tese (Doutorado) - Escola de Engenharia de São Carlos, Universidade de São Paulo, São Carlos.

PRAGER, W. (1949). Recent developments in the mathematical theory of plasticity. $J$. Appl. Phys., v. 20, n. 3, p. 235-241.

PRANDTL, L. (1924). Spannungsverteilung in plastischen Koerpern. In: INTERNATIONAL CONGRESS ON APPLIED MECHANICS, $1^{\text {st }}$, Delft. Proceedings. p. $43-54$.

RABOTNOV, Y. N. (1969). Creep problems in structural members. New York, Elsevier (North-Holland series in applied mathematics and mechanics, v. 7).

REUSS, A (1930). Berucksichtigung der elastischen formanderung in der plastizitats theorie. Z. Angew. Math. Mech., v. 10, p. 266.

RIZZI, E. (1995). Sulla localizzazione delle deformazioni in materiali e strutture. Tesi di Dottorato di Ricerca in Ingegneria delle Strutture, Politecnico di Milano.

SAINT-VENANT, B. (1870). Memoire sur l'établissement des équations differentielles des mouvements interieurs opérés dans les corps solides ductiles au delà des limites où l'élasticité pourrait les ramener à leur premier état. Compt. Rend., v. 70, p. 473-480.

SIMO, J. C. ; HUGHES, T. J. R. (1997). Computational Inelasticity. New York, SpringerVerlag. 
STROUBOULIS, T. ; BABUSKA, I. ; COPPS, K. (2000). The design and analysis of the generalized finite element method. Computer Methods in Applied Mechanics and Engineering, v. 181, n. 1-3, p. 43-69.

TRESCA, H. (1864). Sur l'ecoulement des corps solides soumis à des fortes pressions. Compt. Rend., v. 59, p. 754.

VON MISES, R. (1913). Mechanik der festen Koerper in plastisch deformablen Zustant. Goettinger Nachr., Math.-Phys. Kl., p. 582-592.

VON MISES, R. (1928). Mechanik der plastischen Formänderung von Kristallen. $Z$. Angew. Math. Mech., v. 8, p. 161-185.

WELLS, G. N. ; SLUYS, L. J. (2001). A new method for modelling cohesive cracks using finite elements. International Journal for Numerical Methods in Engineering, v. 50, p. 2667-2682.

WELlS, G. N. ; SLUYS, L. J. ; de BORST, R. (2002). A p-adaptive scheme for overcoming volumetric locking during plastic flow. Computer Methods in Applied Mechanics and Engineering, v. 191, n. 29-30, p. 3153-3164. 


\section{Apêndice A - Dependência linear quando funções de forma (PU) e} funções enriquecedoras são ambas polinomiais.

Como exemplo de ocorrência de dependência linear, admita-se que se esteja construindo a aproximação enriquecida num elemento finito tetraédrico que apresenta como partição de unidade funções de forma do tipo trilinear lagrangianas, cuja base associada é:

$$
P_{1}=\{1, x, y, z\}
$$

Uma propriedade importante da aproximação em questão é que os monômios da base $P_{1}$ podem ser reproduzidos por combinações lineares das funções de forma que eles geram. De fato, tomando-se como referência um ponto $\mathrm{X}$ qualquer no interior do tetraedro, são válidas as relações:

$$
\begin{aligned}
& \sum_{\alpha=1}^{4} \phi_{\alpha}(X)=1 \\
& \sum_{\alpha=1}^{4} a_{\alpha} \phi_{\alpha}(X)=x \\
& \sum_{\alpha=1}^{4} b_{\alpha} \phi_{\alpha}(X)=y \\
& \sum_{\alpha=1}^{4} c_{\alpha} \phi_{\alpha}(X)=z
\end{aligned}
$$

sendo $\{x, y, z\}$ o vetor que contém as coordenadas do ponto no interior do tetraedro e $\phi_{\alpha}$ as funções de forma geradas a partir da base $P_{1}$.

Admita-se também que o enriquecimento seja feito com a própria base $P_{1}$. A família de funções enriquecidas terá, portanto, 16 funções componentes resumidas na representação que segue:

$$
\mathfrak{J}_{n=4}^{p=2}=\left\{\phi_{\alpha}, \phi_{\alpha} x, \phi_{\alpha} y, \phi_{\alpha} z, \alpha=1, \ldots, 4\right\}
$$

Entretanto, a base polinomial correspondente à família de funções enriquecidas, e que poderia gerá-la diretamente, possui apenas 10 termos:

$$
P_{2}=\left\{1, x, y, z, x^{2}, x y, x z, y^{2}, y z, z^{2}\right\}
$$

Também os monômios da base $P_{2}$ podem ser gerados por combinações lineares das funções da família enriquecida $\mathfrak{J}_{n=4}^{p=2}$. No caso dos monômios não-lineares, tem-se: 


$$
\begin{aligned}
& \sum_{\alpha=1}^{4} a_{\alpha}\left(\phi_{\alpha} x\right)=x \sum_{\alpha=1}^{4} a_{\alpha} \phi_{\alpha}=x^{2} \\
& \sum_{\alpha=1}^{4} b_{\alpha}\left(\phi_{\alpha} x\right)=x \sum_{\alpha=1}^{4} b_{\alpha} \phi_{\alpha}=x y \\
& \sum_{\alpha=1}^{4} c_{\alpha}\left(\phi_{\alpha} x\right)=x \sum_{\alpha=1}^{4} c_{\alpha} \phi_{\alpha}=x z \\
& \sum_{\alpha=1}^{4} b_{\alpha}\left(\phi_{\alpha} y\right)=y \sum_{\alpha=1}^{4} b_{\alpha} \phi_{\alpha}=y^{2} \\
& \sum_{\alpha=1}^{4} c_{\alpha}\left(\phi_{\alpha} y\right)=y \sum_{\alpha=1}^{4} c_{\alpha} \phi_{\alpha}=y z \\
& \sum_{\alpha=1}^{4} c_{\alpha}\left(\phi_{\alpha} z\right)=z \sum_{\alpha=1}^{4} c_{\alpha} \phi_{\alpha}=z^{2}
\end{aligned}
$$

Por outro lado, das 16 funções de forma da família $\mathfrak{I}_{n=4}^{p=2}, 6$ são redundantes, ou seja, o conjunto total de funções de forma desta família é linearmente dependente. Tal característica tem relação com o fato de que a base $P_{2}$ é formada por apenas 10 componentes.

Para verificar a dependência linear, nota-se em primeiro lugar que:

$$
\sum_{\alpha=1}^{4}\left(\phi_{\alpha} x\right)=x \sum_{\alpha=1}^{4}\left(\phi_{\alpha}\right)=x
$$

Comparando-se as eqs. (A.3) e (A.14), escreve-se:

$$
\sum_{\alpha=1}^{4}\left(\phi_{\alpha} x\right)-\sum_{\alpha=1}^{4}\left(a_{\alpha} \phi_{\alpha}\right)=0
$$

A eq. (A.15) exibe solução não-trivial se $a_{\alpha}=x$. Portanto as funções $\phi_{\alpha}$ e $\left(\phi_{\alpha} x\right)$ são linearmente dependentes. Por processo análogo, provam-se as dependências lineares entre $\phi_{\alpha}$ e $\left(\phi_{\alpha} y\right)$ e entre $\phi_{\alpha}$ e $\left(\phi_{\alpha} z\right)$.

Tendo-se em vista, agora, a relação:

$$
\sum_{\alpha=1}^{4} a_{\alpha}\left(\phi_{\alpha} y\right)=y \sum_{\alpha=1}^{4} a_{\alpha} \phi_{\alpha}=y x
$$

e comparando-a com a eq. (A.9), chega-se a:

$$
\sum_{\alpha=1}^{4} a_{\alpha}\left(\phi_{\alpha} y\right)-\sum_{\alpha=1}^{4} b_{\alpha}\left(\phi_{\alpha} x\right)=0
$$


Verifica-se, assim, a dependência linear entre $\left(\phi_{\alpha} x\right)$ e $\left(\phi_{\alpha} y\right)$, pois a eq. (A.17) apresenta solução $a_{\alpha}=b_{\alpha}$ (com $a_{\alpha}$ e $b_{\alpha}$ não-nulos). De forma análoga, prova-se a dependência linear entre $\left(\phi_{\alpha} x\right)$ e $\left(\phi_{\alpha} z\right)$ e entre $\left(\phi_{\alpha} y\right)$ e $\left(\phi_{\alpha} z\right)$. 


\section{Apêndice B - Coordenadas adimensionais de volume no tetraedro}

Deduz-se neste apêndice a expressão das coordenadas adimensionais de volume (ou naturais) de um tetraedro.

Sendo $x, y$ e $z$ as coordenadas de um ponto $P$ qualquer do tetraedro, $x_{i}, y_{i}$ e $z_{i}$ as coordenadas dos nós e $\xi_{i}$ as coordenadas adimensionais de volume, com $i=1, \ldots, 4$, escrevem-se:

$$
\begin{aligned}
& x=\sum_{i=1}^{4} x_{i} \xi_{i} \\
& y=\sum_{i=1}^{4} y_{i} \xi_{i} \\
& z=\sum_{i=1}^{4} z_{i} \xi_{i}
\end{aligned}
$$

Deve haver a seguinte relação de dependência entre as coordenadas adimensionais para torná-las uma partição de unidade:

$$
\sum_{i=1}^{4} \xi_{i}=1
$$

A reunião das equações (B.1), (B.2), (B.3) e (B.4) resulta no sistema:

$$
\left[\begin{array}{cccc}
1 & 1 & 1 & 1 \\
x_{1} & x_{2} & x_{3} & x_{4} \\
y_{1} & y_{2} & y_{3} & y_{4} \\
z_{1} & z_{2} & z_{3} & z_{4}
\end{array}\right]\left\{\begin{array}{l}
\xi_{1} \\
\xi_{2} \\
\xi_{3} \\
\xi_{4}
\end{array}\right\}=\left\{\begin{array}{l}
1 \\
x \\
y \\
z
\end{array}\right\}
$$

Resolvendo-se o sistema definido pela eq. (B.5), obtém-se a expressão geral para as coordenadas adimensionais:

$$
\xi_{i}=V_{0}^{i}+a_{i} x+b_{i} y+c_{i} z, \quad i=1, \ldots, 4
$$

Os termos $V_{0}^{i}, a_{i}, b_{i}$ e $c_{i}$ da eq. (B.6) são dados, fazendo-se uma permutação cíclica dos índices $i, j, k, l$ correspondentes aos números 1, 2, 3 e 4, pelas expressões seguintes:

$$
V_{0}^{i}=\frac{1}{6 V}\left|\begin{array}{lll}
x_{j} & x_{k} & x_{l} \\
y_{j} & y_{k} & y_{l} \\
z_{j} & z_{k} & z_{l}
\end{array}\right|
$$




$$
\begin{aligned}
& a_{i}=\frac{-1}{6 V}\left|\begin{array}{ccc}
1 & 1 & 1 \\
y_{j} & y_{k} & y_{l} \\
z_{j} & z_{k} & z_{l}
\end{array}\right| \\
& b_{i}=\frac{1}{6 V}\left|\begin{array}{ccc}
1 & 1 & 1 \\
x_{j} & x_{k} & x_{l} \\
z_{j} & z_{k} & z_{l}
\end{array}\right| \\
& c_{i}=\frac{-1}{6 V}\left|\begin{array}{lll}
1 & 1 & 1 \\
x_{j} & x_{k} & x_{l} \\
y_{j} & y_{k} & y_{l}
\end{array}\right|
\end{aligned}
$$

Sendo que $6 \mathrm{~V}$ corresponde a seis vezes o volume do tetraedro, o que se obtém calculando o determinante dado a seguir:

$$
6 V=\left|\begin{array}{cccc}
1 & 1 & 1 & 1 \\
x_{1} & x_{2} & x_{3} & x_{4} \\
y_{1} & y_{2} & y_{3} & y_{4} \\
z_{1} & z_{2} & z_{3} & z_{4}
\end{array}\right|
$$

Para o ponto $P$ no interior do tetraedro, como ilustra a figura B.1, a coordenada adimensional de volume $\xi_{i}$ dá o valor da razão entre o volume do tetraedro definido pelos pontos $P, j, k, l$ e o volume total $(V)$. É esse, portanto, o significado geométrico das coordenadas adimensionais de volume.

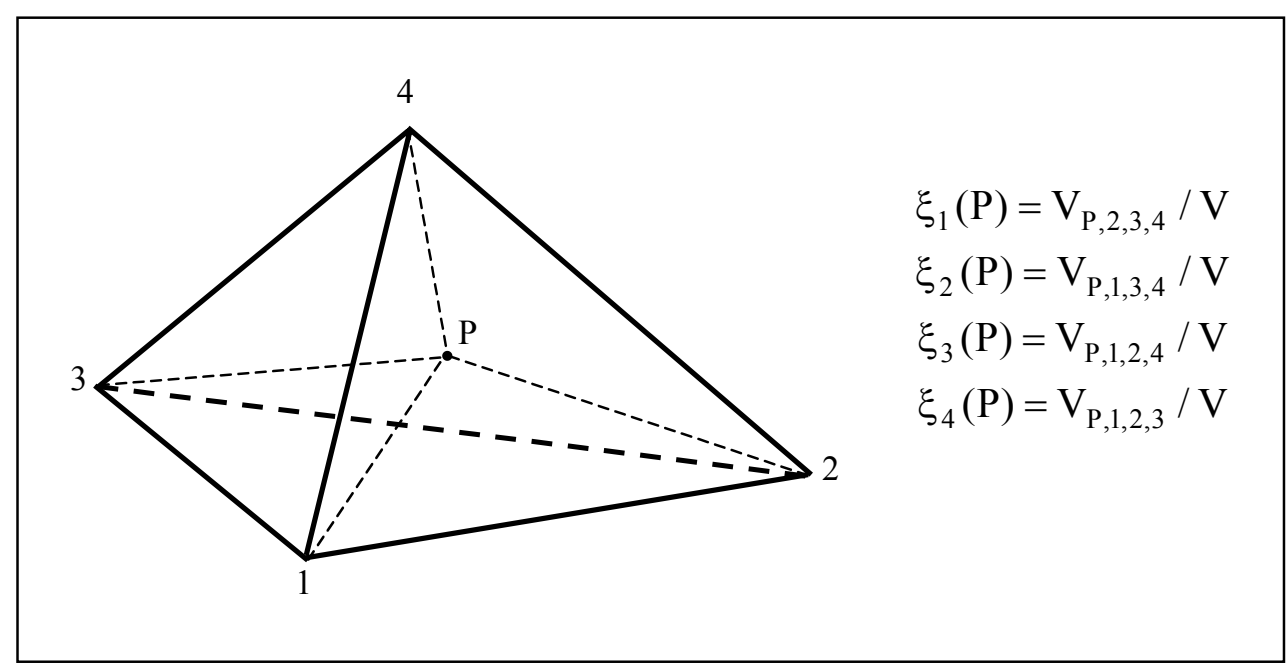

Figura B.1 - Significado geométrico das coordenadas adimensionais de volume 


\section{Apêndice C - Coordenadas adimensionais de área no triângulo}

Deduz-se neste apêndice a expressão das coordenadas adimensionais de área (ou naturais) de um triângulo. Admite-se um sistema de coordenadas cartesianas $(r, s)$, sendo $r_{i}$ e $s_{i}$ as coordenadas nodais e $\zeta_{i}$ as coordenadas adimensionais de área, para $i=1, \ldots, 3$.

Num ponto $P$ qualquer do triângulo, escreve-se:

$$
\begin{aligned}
& r=\sum_{i=1}^{3} r_{i} \zeta_{i} \\
& s=\sum_{i=1}^{3} s_{i} \zeta_{i}
\end{aligned}
$$

A relação de dependência entre as coordenadas adimensionais, que as torna uma partição de unidade, é dada por:

$$
\sum_{i=1}^{3} \zeta_{i}=1
$$

As equações (C.1), (C.2) e (C.3) formam o sistema:

$$
\left[\begin{array}{ccc}
1 & 1 & 1 \\
r_{1} & r_{2} & r_{3} \\
s_{1} & s_{2} & s_{3}
\end{array}\right]\left\{\begin{array}{l}
\zeta_{1} \\
\zeta_{2} \\
\zeta_{3}
\end{array}\right\}=\left\{\begin{array}{l}
1 \\
r \\
s
\end{array}\right\}
$$

A expressão geral para as coordenadas adimensionais de área, obtida da solução do sistema linear dado pela eq. (C.4) é:

$$
\zeta_{i}=a_{i}^{0}+\omega_{i} r+\eta_{i} s, \quad(i=1, \ldots, 3)
$$

Os termos $a_{i}^{0}, \omega_{i}$ e $\eta_{i}$ da eq. (C.5) são dados, fazendo-se uma permutação cíclica dos índices $i, j$ e $k$, correspondentes aos números 1, 2 e 3, pelas expressões seguintes:

$$
\begin{aligned}
& a_{i}^{0}=\left(r_{j} s_{k}-r_{k} s_{j}\right) / 2 A \\
& \omega_{i}=\left(s_{j}-s_{k}\right) / 2 A \\
& \eta_{i}=\left(r_{k}-r_{j}\right) / 2 A
\end{aligned}
$$

Sendo $2 A$ o dobro da área do triângulo, calculado pelo determinante:

$$
2 A=\left|\begin{array}{ccc}
1 & 1 & 1 \\
r_{1} & r_{2} & r_{3} \\
S_{1} & S_{2} & S_{3}
\end{array}\right|
$$


Para o ponto $P$ no interior do triângulo, como ilustra a figura C.1, a coordenada adimensional de área $\zeta_{i}$ dá o valor da razão entre a área do triângulo definido pelos pontos $P, j, k$ e a área total $(A)$. É esse, portanto, o significado geométrico das coordenadas adimensionais de área.

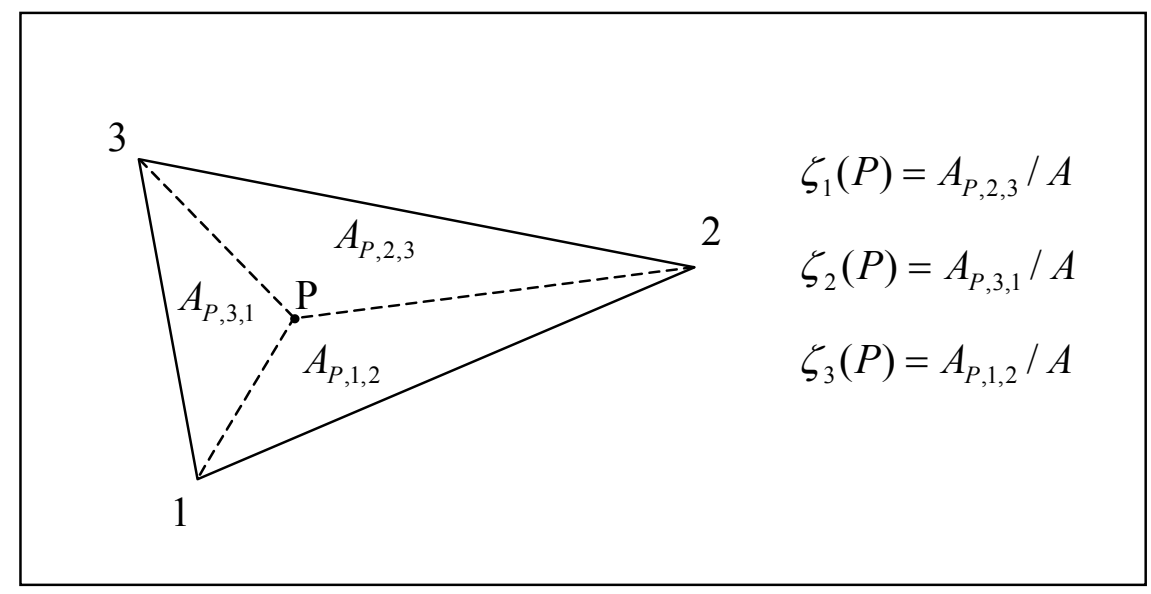

Figura C.1 - Significado geométrico das coordenadas adimensionais de área 


\section{Apêndice D - Cálculo e expressão final do vetor de forças nodais do tetraedro sem enriquecimento.}

Apresenta-se neste apêndice o cálculo do vetor de forças nodais do tetraedro sem enriquecimento; este cálculo é feito utilizando-se a eq. (26), desconsiderando-se as forças de volume:

$$
f_{e}=\int_{A} \phi^{T} \phi^{p} \bar{p}_{e} d A
$$

Para as faces contadas de 1 a 4 , as matrizes de aproximação $\phi=\phi^{p}$ são dadas, respectivamente, pelas eqs. (60a), (61a), (62a) e (63a).

Para as faces contadas de 1 a 4, o vetor de parâmetros nodais de carregamento do elemento $\left(\bar{p}_{e}\right)$ é dado, respectivamente, pelas eqs. (60b), (61b), (62b) e (63b).

No cálculo de $f_{e}$, usa-se a fórmula de integração na área do triângulo em função das coordenadas naturais:

$$
\int_{A} \zeta_{1}^{m} \zeta_{2}^{n} \zeta_{3}^{p} d A=2 A \frac{m ! n ! p !}{(m+n+p+2) !}
$$

Os integrandos da eq. (D.1) são formados por produtos de monômios do tipo $\zeta_{i}^{1} \zeta_{j}^{1}$. Utilizando-se a eq. (D.2), é possível extrair a seguinte relação:

$$
\int_{A} \zeta_{i}^{1} \zeta_{j}^{1} d A= \begin{cases}A / 12 & \text { se } i \neq j \\ A / 6 & \text { se } i=j\end{cases}
$$

Fazendo-se uso da relação definida por (D.3), calculam-se as parcelas do vetor de forças nodais referentes a cada um das faces, que são apresentadas na página seguinte: 
Face 1:

$\frac{A_{\text {face } 1}}{12}\left\{\begin{array}{c}2 \bar{p} x_{1}+\bar{p} x_{2}+\bar{p} x_{3} \\ 2 \bar{p} y_{1}+\bar{p} y_{2}+\bar{p} y_{3} \\ 2 \bar{p} z_{1}+\bar{p} z_{2}+\bar{p} z_{3} \\ \bar{p} x_{1}+2 \bar{p} x_{2}+\bar{p} x_{3} \\ \bar{p} y_{1}+2 \bar{p} y_{2}+\bar{p} y_{3} \\ \bar{p} z_{1}+2 \bar{p} z_{2}+\bar{p} z_{3} \\ \bar{p} x_{1}+\bar{p} x_{2}+2 \bar{p} x_{3} \\ \bar{p} y_{1}+\bar{p} y_{2}+2 \bar{p} y_{3} \\ \bar{p} z_{1}+\bar{p} z_{2}+2 \bar{p} z_{3} \\ 0 \\ 0 \\ 0\end{array}\right\}$

Face 3:

$$
\frac{A_{\text {face } 3}}{12}\left\{\begin{array}{c}
0 \\
0 \\
0 \\
2 \bar{p} y_{2}+\bar{p} y_{3}+\bar{p} y_{4} \\
\bar{p} z_{2}+\bar{p} z_{3}+\bar{p} z_{4} \\
\bar{p} x_{2}+2 \bar{p} x_{3}+\bar{p} x_{4} \\
\bar{p} y_{2}+2 \bar{p} y_{3}+\bar{p} y_{4} \\
\bar{p} z_{2}+2 \bar{p} z_{3}+\bar{p} z_{4} \\
\bar{p} x_{2}+\bar{p} x_{3}+2 \bar{p} x_{4} \\
\bar{p} y_{2}+\bar{p} y_{3}+2 \bar{p} y_{4} \\
\bar{p} z_{2}+\bar{p} z_{3}+2 \bar{p} z_{4}
\end{array}\right\}
$$

Face 2:

$\frac{A_{\text {face } 2}}{12}\left\{\begin{array}{c}2 \bar{p} x_{1}+\bar{p} x_{2}+\bar{p} x_{4} \\ 2 \bar{p} y_{1}+\bar{p} y_{2}+\bar{p} y_{4} \\ 2 \bar{p} z_{1}+\bar{p} z_{2}+\bar{p} z_{4} \\ \bar{p} x_{1}+2 \bar{p} x_{2}+\bar{p} x_{4} \\ \bar{p} y_{1}+2 \bar{p} y_{2}+\bar{p} y_{4} \\ \bar{p} z_{1}+2 \bar{p} z_{2}+\bar{p} z_{4} \\ 0 \\ 0 \\ 0 \\ \bar{p} x_{1}+\bar{p} x_{2}+2 \bar{p} x_{4} \\ \bar{p} y_{1}+\bar{p} y_{2}+2 \bar{p} y_{4} \\ \bar{p} z_{1}+\bar{p} z_{2}+2 \bar{p} z_{4}\end{array}\right\}$

Face 4:

$\frac{A_{\text {face } 4}}{12}\left\{\begin{array}{c}2 \bar{p} x_{1}+\bar{p} x_{3}+\bar{p} x_{4} \\ 2 \bar{p} y_{1}+\bar{p} y_{3}+\bar{p} y_{4} \\ 2 \bar{p} z_{1}+\bar{p} z_{3}+\bar{p} z_{4} \\ 0 \\ 0 \\ 0 \\ \bar{p} x_{1}+2 \bar{p} x_{3}+\bar{p} x_{4} \\ \bar{p} y_{1}+2 \bar{p} y_{3}+\bar{p} y_{4} \\ \bar{p} z_{1}+2 \bar{p} z_{3}+\bar{p} z_{4} \\ \bar{p} x_{1}+\bar{p} x_{3}+2 \bar{p} x_{4} \\ \bar{p} y_{1}+\bar{p} y_{3}+2 \bar{p} y_{4} \\ \bar{p} z_{1}+\bar{p} z_{3}+2 \bar{p} z_{4}\end{array}\right\}$




\section{Apêndice E - Expansão das submatrizes do tetraedro em função do}

\section{enriquecimento}

Como visto no item 2.3.3.1.2, no processo de enriquecimento da aproximação cada termo da matriz do elemento tetraédrico sem enriquecimento dá origem à sua própria submatriz, pela adição de linhas e colunas referentes aos novos parâmetros. Apresenta-se a seguir a expressão geral da submatriz derivada de cada um dos nove termos de uma submatriz $3 \times 3$ genérica do elemento não-enriquecido:

1) Termo: $A(3 i-2,3 j-2)$

Para $k_{i}=0, n f\left(u_{i}\right)$

Para $k_{j}=0, n f\left(u_{j}\right)$

$$
\begin{aligned}
A\left(3 i-2+k_{i}, 3 j-2+k_{j}\right)=\int_{V} & {\left[\left(a_{i} f_{u_{i}}^{k_{i}}+\xi_{i} \frac{\partial f_{u_{i}}^{k_{i}}}{\partial x}\right)\left(a_{j} f_{u_{j}}^{k_{j}}+\xi_{j} \frac{\partial f_{u_{j}}^{k_{j}}}{\partial x}\right) c t_{1}+\right.} \\
& +\left(b_{i} f_{u_{i}}^{k_{i}}+\xi_{i} \frac{\partial f_{u_{i}}^{k_{i}}}{\partial y}\right)\left(b_{j} f_{u_{j}}^{k_{j}}+\xi_{j} \frac{\partial f_{u_{j}}^{k_{j}}}{\partial y}\right) c t_{3}+ \\
& \left.+\left(c_{i} f_{u_{i}}^{k_{i}}+\xi_{i} \frac{\partial f_{u_{i}}^{k_{i}}}{\partial z}\right)\left(c_{j} f_{u_{j}}^{k_{j}}+\xi_{j} \frac{\partial f_{u_{j}}^{k_{j}}}{\partial z}\right) c t_{3}\right] d V
\end{aligned}
$$

2) Termo: $A(3 i-2,3 j-1)$

Para $k_{i}=0, n f\left(u_{i}\right)$

Para $k_{j}=0, n f\left(v_{j}\right)$

$$
\begin{aligned}
A\left(3 i-2+k_{i}, 3 j-1+k_{j}\right)=\int_{V} & {\left[\left(a_{i} f_{u_{i}}^{k_{i}}+\xi_{i} \frac{\partial f_{u_{i}}^{k_{i}}}{\partial x}\right)\left(b_{j} f_{v_{j}}^{k_{j}}+\xi_{j} \frac{\partial f_{v_{j}}^{k_{j}}}{\partial y}\right) c t_{2}+\right.} \\
& \left.+\left(b_{i} f_{u_{i}}^{k_{i}}+\xi_{i} \frac{\partial f_{u_{i}}^{k_{i}}}{\partial y}\right)\left(a_{j} f_{v_{j}}^{k_{j}}+\xi_{j} \frac{\partial f_{v_{j}}^{k_{j}}}{\partial x}\right) c t_{3}\right] d V
\end{aligned}
$$


3) Termo: $A(3 i-2,3 j)$

Para $k_{i}=0, n f\left(u_{i}\right)$

Para $k_{j}=0, n f\left(w_{j}\right)$

$$
\begin{aligned}
A\left(3 i-2+k_{i}, 3 j+k_{j}\right)=\int_{V} & {\left[\left(a_{i} f_{u_{i}}^{k_{i}}+\xi_{i} \frac{\partial f_{u_{i}}^{k_{i}}}{\partial x}\right)\left(c_{j} f_{w_{j}}^{k_{j}}+\xi_{j} \frac{\partial f_{w_{j}}^{k_{j}}}{\partial z}\right) c t_{2}+\right.} \\
& \left.+\left(c_{i} f_{u_{i}}^{k_{i}}+\xi_{i} \frac{\partial f_{u_{i}}^{k_{i}}}{\partial z}\right)\left(a_{j} f_{w_{j}}^{k_{j}}+\xi_{j} \frac{\partial f_{w_{j}}^{k_{j}}}{\partial x}\right) c t_{3}\right] d V
\end{aligned}
$$

4) Termo: $A(3 i-1,3 j-2)$

Para $k_{i}=0, n f\left(v_{i}\right)$

Para $k_{j}=0, n f\left(u_{j}\right)$

$$
\begin{aligned}
A\left(3 i-1+k_{i}, 3 j-2+k_{j}\right)=\int_{V} & {\left[\left(b_{i} f_{v_{i}}^{k_{i}}+\xi_{i} \frac{\partial f_{v_{i}}^{k_{i}}}{\partial y}\right)\left(a_{j} f_{u_{j}}^{k_{j}}+\xi_{j} \frac{\partial f_{u_{j}}^{k_{j}}}{\partial x}\right) c t_{2}+\right.} \\
& \left.+\left(a_{i} f_{v_{i}}^{k_{i}}+\xi_{i} \frac{\partial f_{v_{i}}^{k_{i}}}{\partial x}\right)\left(b_{j} f_{u_{j}}^{k_{j}}+\xi_{j} \frac{\partial f_{u_{j}}^{k_{j}}}{\partial y}\right) c t_{3}\right] d V
\end{aligned}
$$

5) Termo: $A(3 i-1,3 j-1)$

Para $k_{i}=0, n f\left(v_{i}\right)$

Para $k_{j}=0, n f\left(v_{j}\right)$

$$
\begin{aligned}
A\left(3 i-1+k_{i}, 3 j-1+k_{j}\right)=\int_{V} & {\left[\left(a_{i} f_{v_{i}}^{k_{i}}+\xi_{i} \frac{\partial f_{v_{i}}^{k_{i}}}{\partial x}\right)\left(a_{j} f_{v_{j}}^{k_{j}}+\xi_{j} \frac{\partial f_{v_{j}}^{k_{j}}}{\partial x}\right) c t_{3}+\right.} \\
& +\left(b_{i} f_{v_{i}}^{k_{i}}+\xi_{i} \frac{\partial f_{v_{i}}^{k_{i}}}{\partial y}\right)\left(b_{j} f_{v_{j}}^{k_{j}}+\xi_{j} \frac{\partial f_{v_{j}}^{k_{j}}}{\partial y}\right) c t_{1}+ \\
& \left.+\left(c_{i} f_{v_{i}}^{k_{i}}+\xi_{i} \frac{\partial f_{v_{i}}^{k_{i}}}{\partial z}\right)\left(c_{j} f_{v_{j}}^{k_{j}}+\xi_{j} \frac{\partial f_{v_{j}}^{k_{j}}}{\partial z}\right) c t_{3}\right] d V
\end{aligned}
$$


6) Termo: $A(3 i-1,3 j)$

Para $k_{i}=0, n f\left(v_{i}\right)$

Para $k_{j}=0, n f\left(w_{j}\right)$

$$
\begin{aligned}
A\left(3 i-1+k_{i}, 3 j+k_{j}\right)=\int_{V} & {\left[\left(b_{i} f_{v_{i}}^{k_{i}}+\xi_{i} \frac{\partial f_{v_{i}}^{k_{i}}}{\partial y}\right)\left(c_{j} f_{w_{j}}^{k_{j}}+\xi_{j} \frac{\partial f_{w_{j}}^{k_{j}}}{\partial z}\right) c t_{2}+\right.} \\
& \left.+\left(c_{i} f_{v_{i}}^{k_{i}}+\xi_{i} \frac{\partial f_{v_{i}}^{k_{i}}}{\partial z}\right)\left(b_{j} f_{w_{j}}^{k_{j}}+\xi_{j} \frac{\partial f_{w_{j}}^{k_{j}}}{\partial y}\right) c t_{3}\right] d V
\end{aligned}
$$

7) Termo: $A(3 i, 3 j-2)$

Para $k_{i}=0, n f\left(w_{i}\right)$

Para $k_{j}=0, n f\left(u_{j}\right)$

$$
\begin{aligned}
A\left(3 i+k_{i}, 3 j-2+k_{j}\right)=\int_{V} & {\left[\left(c_{i} f_{w_{i}}^{k_{i}}+\xi_{i} \frac{\partial f_{w_{i}}^{k_{i}}}{\partial z}\right)\left(a_{j} f_{u_{j}}^{k_{j}}+\xi_{j} \frac{\partial f_{u_{j}}^{k_{j}}}{\partial x}\right) c t_{2}+\right.} \\
& \left.+\left(a_{i} f_{w_{i}}^{k_{i}}+\xi_{i} \frac{\partial f_{w_{i}}^{k_{i}}}{\partial x}\right)\left(c_{j} f_{u_{j}}^{k_{j}}+\xi_{j} \frac{\partial f_{u_{j}}^{k_{j}}}{\partial z}\right) c t_{3}\right] d V
\end{aligned}
$$

8) Termo: $A(3 i, 3 j-1)$

Para $k_{i}=0, n f\left(w_{i}\right)$

Para $k_{j}=0, n f\left(v_{j}\right)$

$$
\begin{aligned}
A\left(3 i+k_{i}, 3 j-1+k_{j}\right)=\int_{V} & {\left[\left(c_{i} f_{w_{i}}^{k_{i}}+\xi_{i} \frac{\partial f_{w_{i}}^{k_{i}}}{\partial z}\right)\left(b_{j} f_{v_{j}}^{k_{j}}+\xi_{j} \frac{\partial f_{v_{j}}^{k_{j}}}{\partial y}\right) c t_{2}+\right.} \\
& \left.+\left(b_{i} f_{w_{i}}^{k_{i}}+\xi_{i} \frac{\partial f_{w_{i}}^{k_{i}}}{\partial y}\right)\left(c_{j} f_{v_{j}}^{k_{j}}+\xi_{j} \frac{\partial f_{v_{j}}^{k_{j}}}{\partial z}\right) c t_{3}\right] d V
\end{aligned}
$$


9) Termo: $A(3 i, 3 j)$

Para $k_{i}=0, n f\left(w_{i}\right)$

Para $k_{j}=0, n f\left(w_{j}\right)$

$$
\begin{aligned}
A\left(3 i+k_{i}, 3 j+k_{j}\right)=\int_{V} & {\left[\left(a_{i} f_{w_{i}}^{k_{i}}+\xi_{i} \frac{\partial f_{w_{i}}^{k_{i}}}{\partial x}\right)\left(a_{j} f_{w_{j}}^{k_{j}}+\xi_{j} \frac{\partial f_{w_{j}}^{k_{j}}}{\partial x}\right) c t_{3}+\right.} \\
& +\left(b_{i} f_{w_{i}}^{k_{i}}+\xi_{i} \frac{\partial f_{w_{i}}^{k_{i}}}{\partial y}\right)\left(b_{j} f_{w_{j}}^{k_{j}}+\xi_{j} \frac{\partial f_{w_{j}}^{k_{j}}}{\partial y}\right) c t_{3}+ \\
& \left.+\left(c_{i} f_{w_{i}}^{k_{i}}+\xi_{i} \frac{\partial f_{w_{i}}^{k_{i}}}{\partial z}\right)\left(c_{j} f_{w_{j}}^{k_{j}}+\xi_{j} \frac{\partial f_{w_{j}}^{k_{j}}}{\partial z}\right) c t_{1}\right] d V
\end{aligned}
$$

Os termos da matriz sem enriquecimento correspondem ao valor das expressões para $k_{i}=0$ e $k_{j}=0$, adotando-se valor unitário para a função enriquecedora e, obviamente, nulo para suas derivadas parciais. Como esperado, os valores obtidos correspondem exatamente aos das eqs. (55). 


\section{Apêndice F - Aproximação dos deslocamentos e contribuição de} cada face ao vetor de forças nodais do hexaedro enriquecido.

Apresentam-se a seguir as matrizes de aproximação dos deslocamentos $[\phi]_{3 x(12+n p a r)}$ referentes a cada face do tetraedro:

\section{Face 1:}

$$
\begin{aligned}
& {[\phi]=\left[\begin{array}{ccccccccccccc}
\zeta_{1} & \zeta_{1} f_{u_{1}}^{1} & \cdots & \zeta_{1} f_{u_{1}}^{n f\left(u_{1}\right)} & 0 & 0 & \cdots & 0 & 0 & 0 & \cdots & 0 \\
0 & 0 & \cdots & 0 & \zeta_{1} & \zeta_{1} f_{v_{1}}^{1} & \cdots & \zeta_{1} f_{v_{1}}^{n f\left(v_{1}\right)} & 0 & 0 & \cdots & 0 \\
0 & 0 & \cdots & 0 & 0 & 0 & \cdots & 0 & \zeta_{1} & \zeta_{1} f_{w_{1}}^{1} & \cdots & \zeta_{1} f_{w_{1}}^{n f\left(w_{1}\right)}
\end{array}\right.} \\
& \begin{array}{cccccccccccc}
\zeta_{2} & \zeta_{2} f_{u_{2}}^{1} & \cdots & \zeta_{2} f_{u_{2}}^{n f\left(u_{2}\right)} & 0 & 0 & \cdots & 0 & 0 & 0 & \cdots & 0 \\
0 & 0 & \cdots & 0 & \zeta_{2} & \zeta_{2} f_{v_{2}}^{1} & \cdots & \zeta_{2} f_{v_{2}}^{n f\left(v_{2}\right)} & 0 & 0 & \cdots & 0 \\
0 & 0 & \cdots & 0 & 0 & 0 & \cdots & 0 & \zeta_{2} & \zeta_{2} f_{w_{2}}^{1} & \cdots & \zeta_{2} f_{w_{2}}^{n f\left(w_{2}\right)}
\end{array} \\
& \begin{array}{cccccccccccc}
\zeta_{3} & \zeta_{3} f_{u_{3}}^{1} & \cdots & \zeta_{3} f_{u_{3}}^{n f\left(u_{3}\right)} & 0 & 0 & \cdots & 0 & 0 & 0 & \cdots & 0 \\
0 & 0 & \cdots & 0 & \zeta_{3} & \zeta_{3} f_{v_{3}}^{1} & \cdots & \zeta_{3} f_{v_{3}}^{n f\left(v_{3}\right)} & 0 & 0 & \cdots & 0 \\
0 & 0 & \cdots & 0 & 0 & 0 & \cdots & 0 & \zeta_{3} & \zeta_{3} f_{w_{3}}^{1} & \cdots & \zeta_{3} f_{w_{3}}^{n f\left(w_{3}\right)}
\end{array} \\
& {\left[\begin{array}{lll}
0 & 0 & 0 \\
0 & 0 & 0 \\
0 & 0 & 0
\end{array}\right]}
\end{aligned}
$$

Face 2:

$$
\begin{aligned}
& {[\phi]=\left[\begin{array}{cccccccccccc}
\zeta_{1} & \zeta_{1} f_{u_{1}}^{1} & \cdots & \zeta_{1} f_{u_{1}}^{n f\left(u_{1}\right)} & 0 & 0 & \cdots & 0 & 0 & 0 & \cdots & 0 \\
0 & 0 & \cdots & 0 & \zeta_{1} & \zeta_{1} f_{v_{1}}^{1} & \cdots & \zeta_{1} f_{v_{1}}^{n f\left(v_{1}\right)} & 0 & 0 & \cdots & 0 \\
0 & 0 & \cdots & 0 & 0 & 0 & \cdots & 0 & \zeta_{1} & \zeta_{1} f_{w_{1}}^{1} & \cdots & \zeta_{1} f_{w_{1}}^{n f\left(w_{1}\right)}
\end{array}\right.} \\
& \begin{array}{cccccccccccc}
\zeta_{2} & \zeta_{2} f_{u_{2}}^{1} & \cdots & \zeta_{2} f_{u_{2}}^{n f\left(u_{2}\right)} & 0 & 0 & \cdots & 0 & 0 & 0 & \cdots & 0 \\
0 & 0 & \cdots & 0 & \zeta_{2} & \zeta_{2} f_{v_{2}}^{1} & \cdots & \zeta_{2} f_{v_{2}}^{n f\left(v_{2}\right)} & 0 & 0 & \cdots & 0 \\
0 & 0 & \cdots & 0 & 0 & 0 & \cdots & 0 & \zeta_{2} & \zeta_{2} f_{w_{2}}^{1} & \cdots & \zeta_{2} f_{w_{2}}^{n f\left(w_{2}\right)}
\end{array}
\end{aligned}
$$

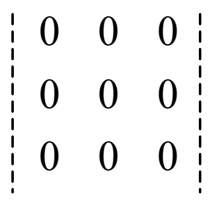

$$
\begin{aligned}
& {\left[\begin{array}{cccccccccccc}
\zeta_{3} & \zeta_{3} f_{u_{4}}^{1} & \cdots & \zeta_{3} f_{u_{4}}^{n f\left(u_{4}\right)} & 0 & 0 & \cdots & 0 & 0 & 0 & \cdots & 0 \\
0 & 0 & \cdots & 0 & \zeta_{3} & \zeta_{3} f_{v_{4}}^{1} & \cdots & \zeta_{3} f_{v_{4}}^{n f\left(v_{4}\right)} & 0 & 0 & \cdots & 0 \\
0 & 0 & \cdots & 0 & 0 & 0 & \cdots & 0 & \zeta_{3} & \zeta_{3} f_{w_{4}}^{1} & \cdots & \zeta_{3} f_{w_{4}}^{n f\left(w_{4}\right)}
\end{array}\right]}
\end{aligned}
$$




\section{Face 3:}

$$
\begin{aligned}
& {[\phi]=\left[\begin{array}{ccc:c}
0 & 0 & 0 & 0 \\
0 & 0 & 0 & 0 \\
0 & 0 & 0 & 0
\end{array}\right.} \\
& \begin{array}{cccccccccccc}
\zeta_{1} & \zeta_{1} f_{u_{2}}^{1} & \cdots & \zeta_{1} f_{u_{2}}^{n f\left(u_{2}\right)} & 0 & 0 & \cdots & 0 & 0 & 0 & \cdots & 0 \\
0 & 0 & \cdots & 0 & \zeta_{1} & \zeta_{1} f_{v_{2}}^{1} & \cdots & \zeta_{1} f_{v_{2}}^{n f\left(v_{2}\right)} & 0 & 0 & \cdots & 0 \\
0 & 0 & \cdots & 0 & 0 & 0 & \cdots & 0 & \zeta_{1} & \zeta_{1} f_{w_{2}}^{1} & \cdots & \zeta_{1} f_{w_{2}}^{n f\left(w_{2}\right)}
\end{array} \\
& \begin{array}{cccccccccccc}
\zeta_{2} & \zeta_{2} f_{u_{3}}^{1} & \cdots & \zeta_{2} f_{u_{3}}^{n f\left(u_{3}\right)} & 0 & 0 & \cdots & 0 & 0 & 0 & \cdots & 0 \\
0 & 0 & \cdots & 0 & \zeta_{2} & \zeta_{2} f_{v_{3}}^{1} & \cdots & \zeta_{2} f_{v_{3}}^{n f\left(v_{3}\right)} & 0 & 0 & \cdots & 0 \\
0 & 0 & \cdots & 0 & 0 & 0 & \cdots & 0 & \zeta_{2} & \zeta_{2} f_{w_{3}}^{1} & \cdots & \zeta_{2} f_{w_{3}}^{n f\left(w_{3}\right)}
\end{array} \\
& {\left[\begin{array}{ccccccccccccc}
\zeta_{3} & \zeta_{3} f_{u_{4}}^{1} & \cdots & \zeta_{3} f_{u_{4}}^{n f\left(u_{4}\right)} & 0 & 0 & \cdots & 0 & 0 & 0 & \cdots & 0 \\
0 & 0 & \cdots & 0 & \zeta_{3} & \zeta_{3} f_{v_{4}}^{1} & \cdots & \zeta_{3} f_{v_{4}}^{n f\left(v_{4}\right)} & 0 & 0 & \cdots & 0 \\
0 & 0 & \cdots & 0 & 0 & 0 & \cdots & 0 & \zeta_{3} & \zeta_{3} f_{w_{4}}^{1} & \cdots & \zeta_{3} f_{w_{4}}^{n f\left(w_{4}\right)}
\end{array}\right]}
\end{aligned}
$$

\section{Face 4:}

$$
\begin{aligned}
{[\phi] } & =\left[\begin{array}{ccccccccccccc}
\zeta_{1} & \zeta_{1} f_{u_{1}}^{1} & \cdots & \zeta_{1} f_{u_{1}}^{n f\left(u_{1}\right)} & 0 & 0 & \cdots & 0 & 0 & 0 & \cdots & 0 \\
0 & 0 & \cdots & 0 & \zeta_{1} & \zeta_{1} f_{v_{1}}^{1} & \cdots & \zeta_{1} f_{v_{1}}^{n f\left(v_{1}\right)} & 0 & 0 & \cdots & 0 \\
0 & 0 & \cdots & 0 & 0 & 0 & \cdots & 0 & \zeta_{1} & \zeta_{1} f_{w_{1}}^{1} & \cdots & \zeta_{1} f_{w_{1}}^{n f\left(w_{1}\right)} \\
0 & 0 & 0 & & & & & & & & & & \\
0 & 0 & 0 & & & & & & & & & & \\
0 & 0 & 0 & & & & & & & & & & \\
\vdots & \zeta_{2} & \zeta_{2} f_{u_{3}}^{1} & \cdots & \zeta_{2} f_{u_{3}}^{n f\left(u_{3}\right)} & 0 & 0 & \cdots & 0 & 0 & 0 & \cdots & 0 \\
0 & 0 & \cdots & 0 & \zeta_{2} & \zeta_{2} f_{v_{3}}^{1} & \cdots & \zeta_{2} f_{v_{3}}^{n f\left(v_{3}\right)} & 0 & 0 & \cdots & 0 \\
0 & 0 & \cdots & 0 & 0 & 0 & \cdots & 0 & \zeta_{2} & \zeta_{2} f_{w_{3}}^{1} & \cdots & \zeta_{2} f_{w_{3}}^{n f\left(w_{3}\right)} \\
\vdots & \zeta_{3} & \zeta_{3} f_{u_{4}}^{1} & \cdots & \zeta_{3} f_{u_{4}}^{n f\left(u_{4}\right)} & 0 & 0 & \cdots & 0 & 0 & 0 & \cdots & 0 \\
0 & 0 & \cdots & 0 & \zeta_{3} & \zeta_{3} f_{v_{4}}^{1} & \cdots & \zeta_{3} f_{v_{4}}^{n f\left(v_{4}\right)} & 0 & 0 & \cdots & 0 \\
0 & 0 & \cdots & 0 & 0 & 0 & \cdots & 0 & \zeta_{3} & \zeta_{3} f_{w_{4}}^{1} & \cdots & \zeta_{3} f_{w_{4}}^{n f\left(w_{4}\right)}
\end{array}\right]
\end{aligned}
$$


O cálculo do vetor de forças nodais do tetraedro enriquecido é feito utilizando-se a eq. (26), sendo desconsideradas as forças de volume:

$$
f_{e}=\int_{A} \phi^{T} \phi^{p} \bar{p}_{e} d A
$$

Para as faces de 1 a 4, as matrizes de aproximação do carregamento $\left(\phi^{p}\right)$ são dadas, respectivamente, pelas eqs. (60a), (61a), (62a) e (63a).

Para as faces de 1 a 4 , o vetor de parâmetros nodais de carregamento $\left(\bar{p}_{e}\right)$ é dado, respectivamente, pelas eqs. (60b), (61b), (62b) e (63b).

Utilizando-se a eq. (F.1), calculam-se as parcelas do vetor de forças nodais referentes a cada face, apresentadas na seqüência. As áreas das faces de numeração 1, 2, 3 e 4 do tetraedro, conforme a convenção da figura 4, são indicadas por $A_{1}, A_{2}, A_{3}$ e $A_{4}$, respectivamente. 
Face 1:

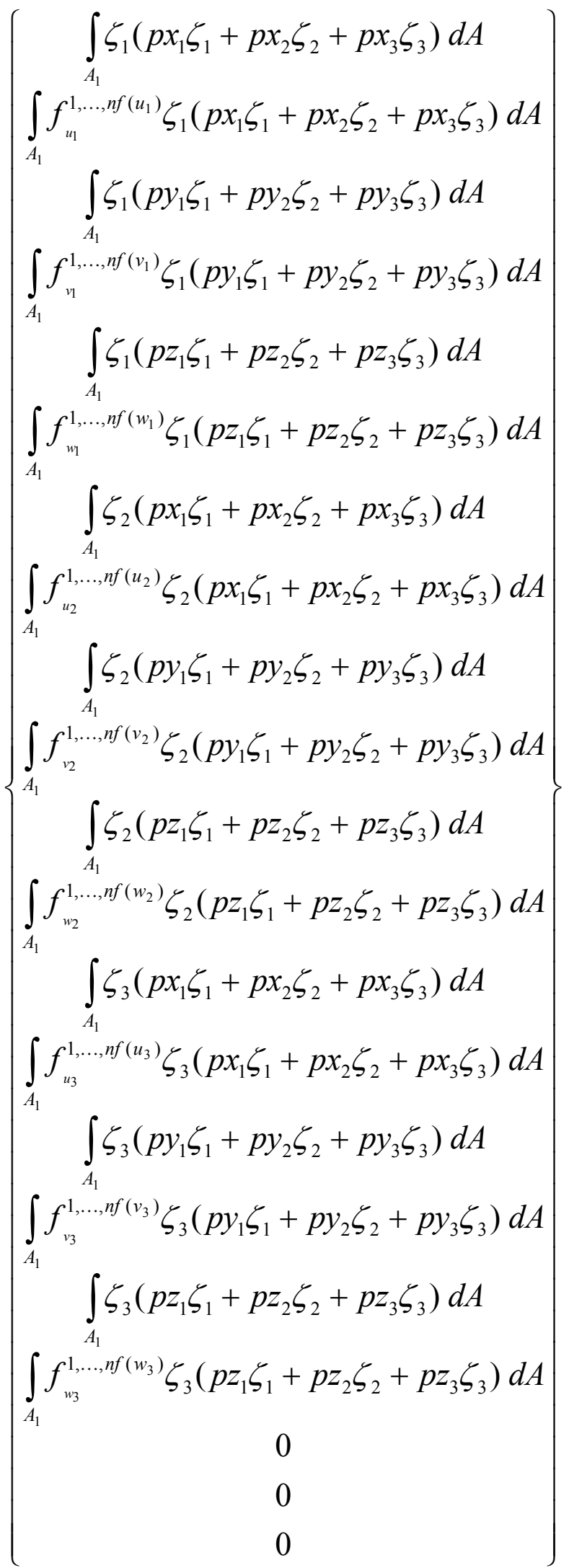


Face 2:

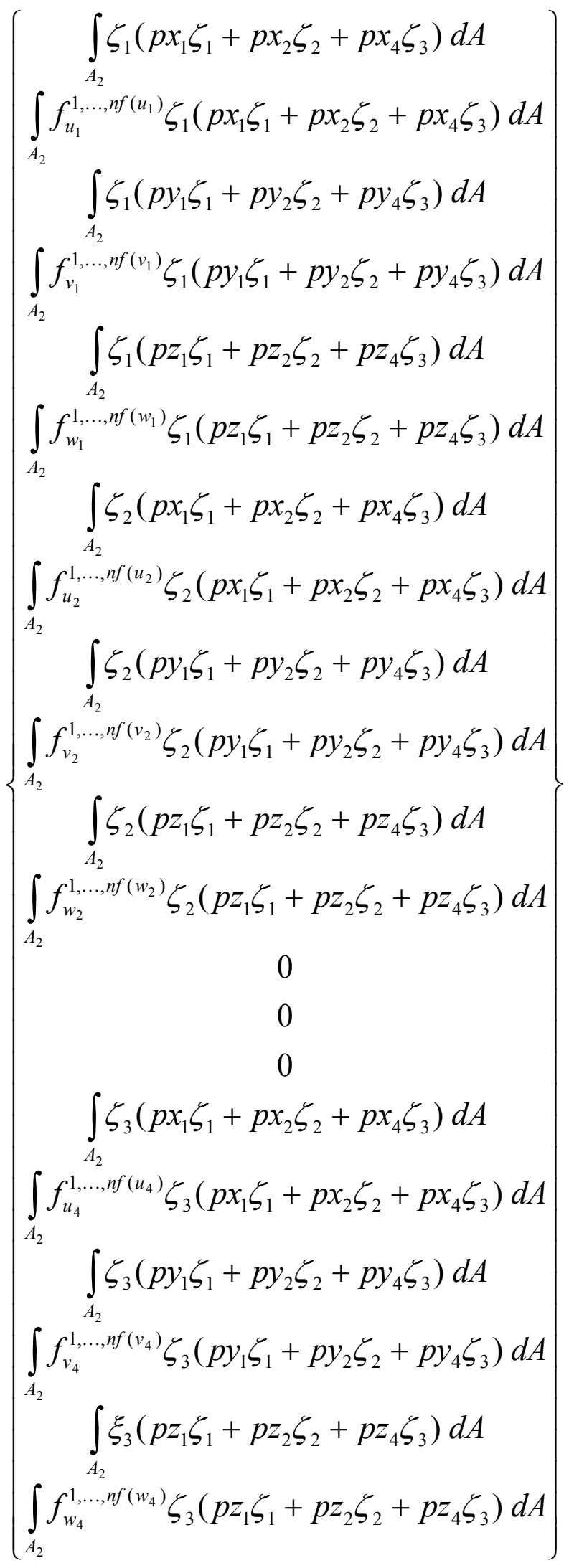


Face 3:

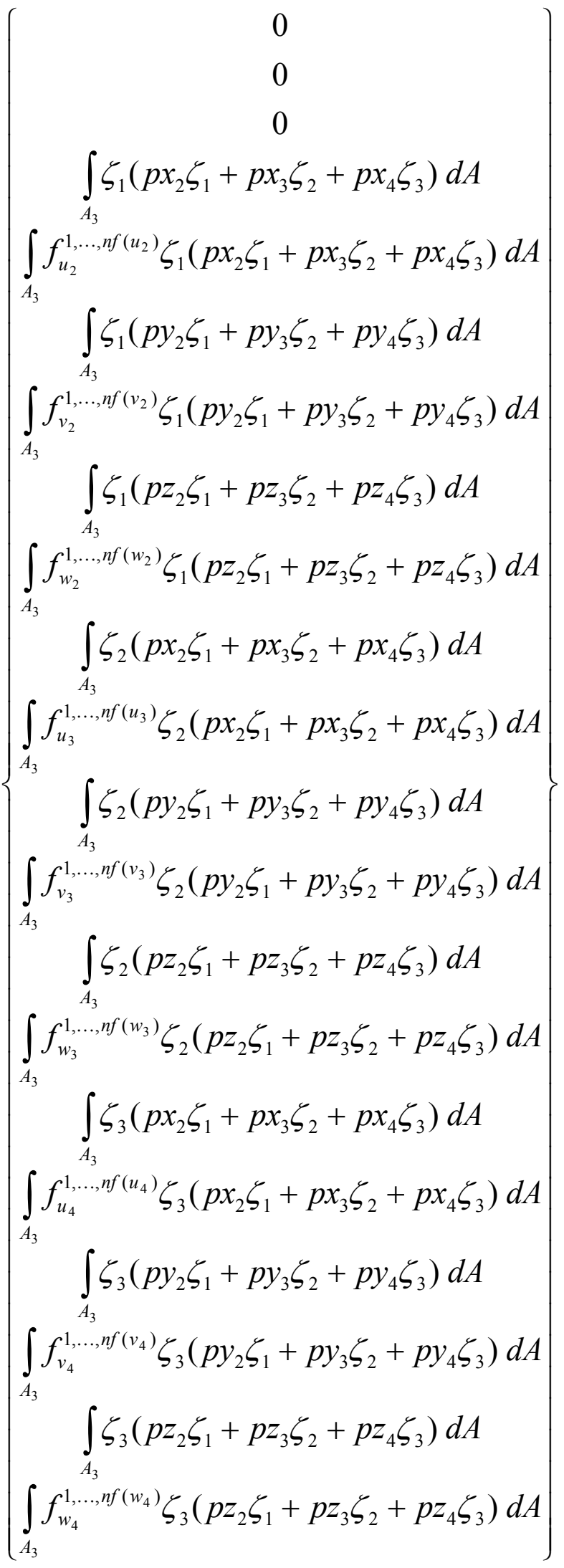


Face 4:

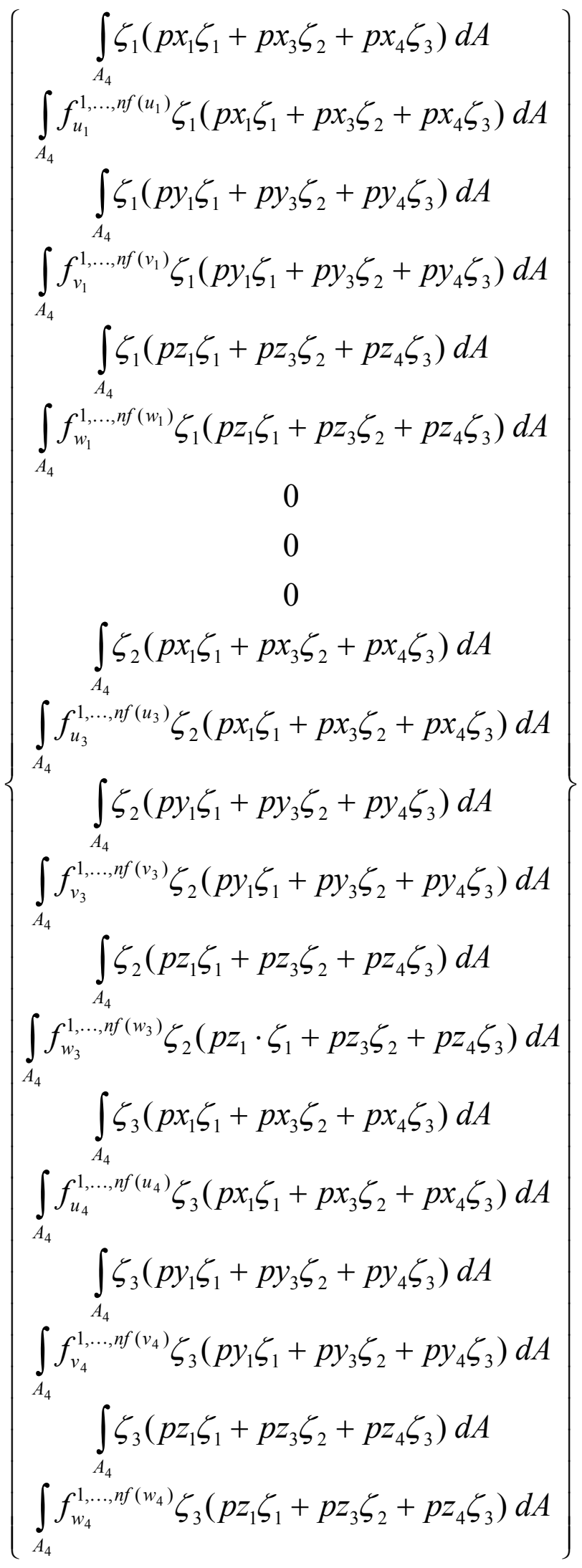




\section{Apêndice G - Contribuição de cada face do hexaedro ao vetor de forças nodais do elemento}

Apresenta-se a seguir a contribuição de cada face do hexaedro ao vetor de forças nodais do elemento. A área de cada face é dada por $A_{i}, i=1, \ldots, 6$.

\section{$\underline{\text { Face 1: }}$}

$$
\left\{\begin{array}{ll}
\int_{A_{1}} N_{1}\left(N_{1} p x_{1}+N_{2} p x_{2}+N_{3} p x_{3}+N_{4} p x_{4}\right) d A \\
\int_{A_{1}} N_{1}\left(N_{1} p y_{1}+N_{2} p y_{2}+N_{3} p y_{3}+N_{4} p y_{4}\right) d A \\
\int_{A_{1}} N_{1}\left(N_{1} p z_{1}+N_{2} p z_{2}+N_{3} p z_{3}+N_{4} p z_{4}\right) d A \\
\int_{A_{1}}^{A_{1}} N_{2}\left(N_{1} p x_{1}+N_{2} p x_{2}+N_{3} p x_{3}+N_{4} p x_{4}\right) d A \\
\int_{A_{1}} N_{2}\left(N_{1} p y_{1}+N_{2} p y_{2}+N_{3} p y_{3}+N_{4} p y_{4}\right) d A \\
\int_{A_{1}}^{A_{1}} N_{2}\left(N_{1} p z_{1}+N_{2} p z_{2}+N_{3} p z_{3}+N_{4} p z_{4}\right) d A \\
\int_{A_{1}} N_{3}\left(N_{1} p x_{1}+N_{2} p x_{2}+N_{3} p x_{3}+N_{4} p x_{4}\right) d A \\
\int_{A_{1}} N_{3}\left(N_{1} p y_{1}+N_{2} p y_{2}+N_{3} p y_{3}+N_{4} p y_{4}\right) d A \\
\int_{A_{1}}^{A_{1}} N_{3}\left(N_{1} p z_{1}+N_{2} p z_{2}+N_{3} p z_{3}+N_{4} p z_{4}\right) d A \\
\int_{A_{1}} N_{4}\left(N_{1} p x_{1}+N_{2} p x_{2}+N_{3} p x_{3}+N_{4} p x_{4}\right) d A \\
\int_{A_{1}} N_{4}\left(N_{1} p y_{1}+N_{2} p y_{2}+N_{3} p y_{3}+N_{4} p y_{4}\right) d A \\
\int_{A_{1}} N_{4}\left(N_{1} p z_{1}+N_{2} p z_{2}+N_{3} p z_{3}+N_{4} p z_{4}\right) d A
\end{array}\right\}
$$


Face 2:

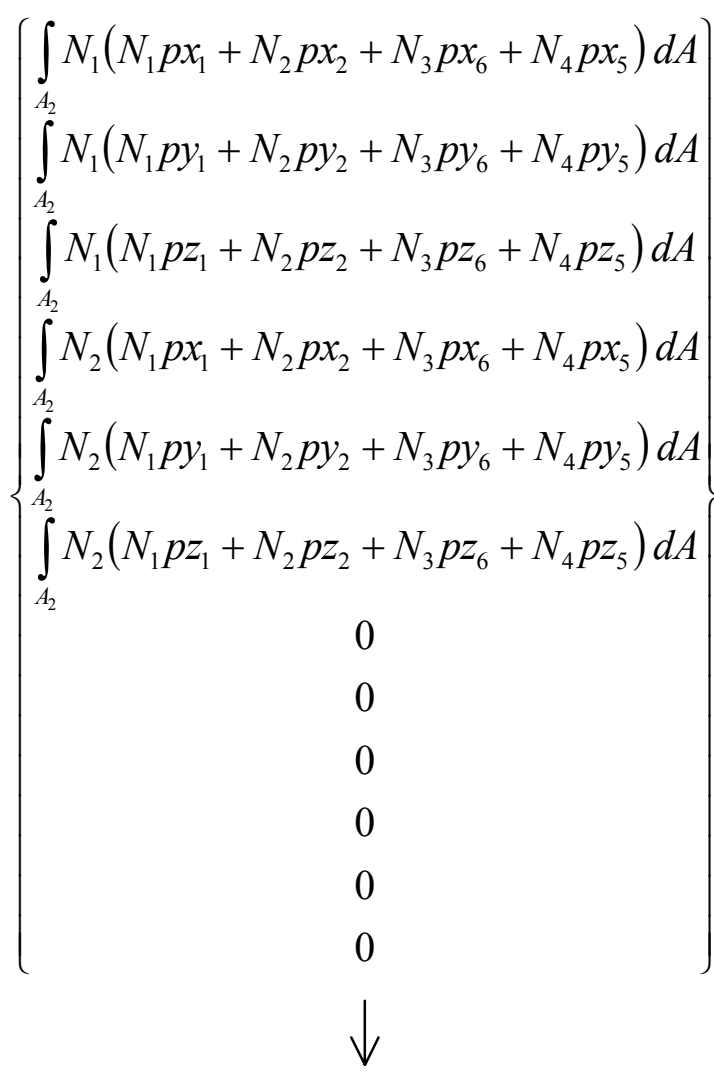

Face 3:

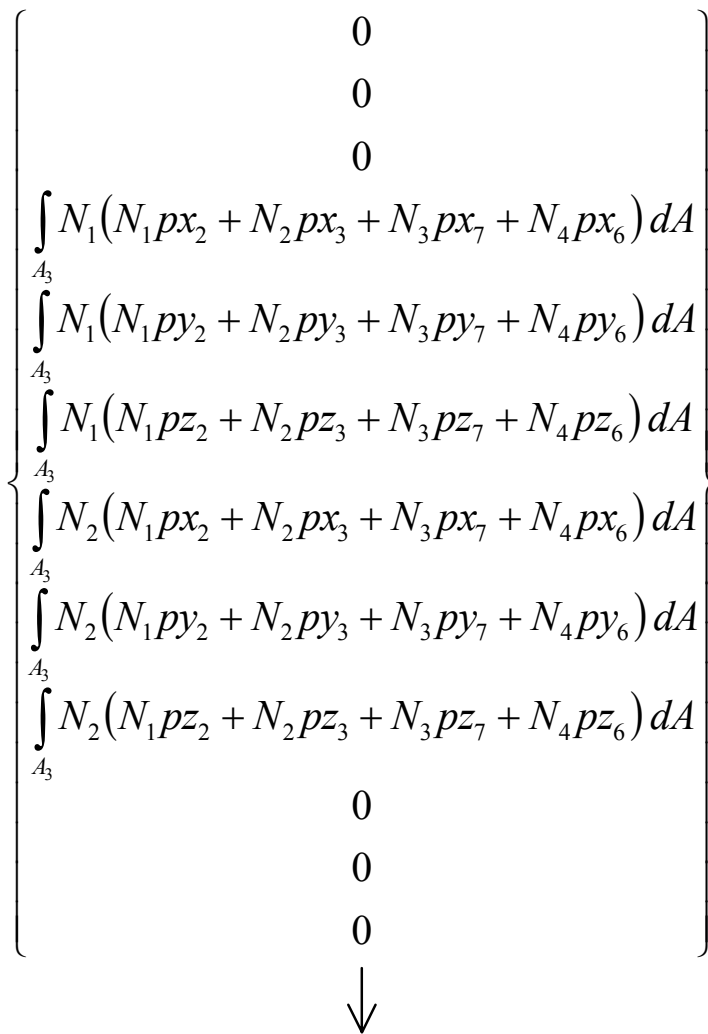

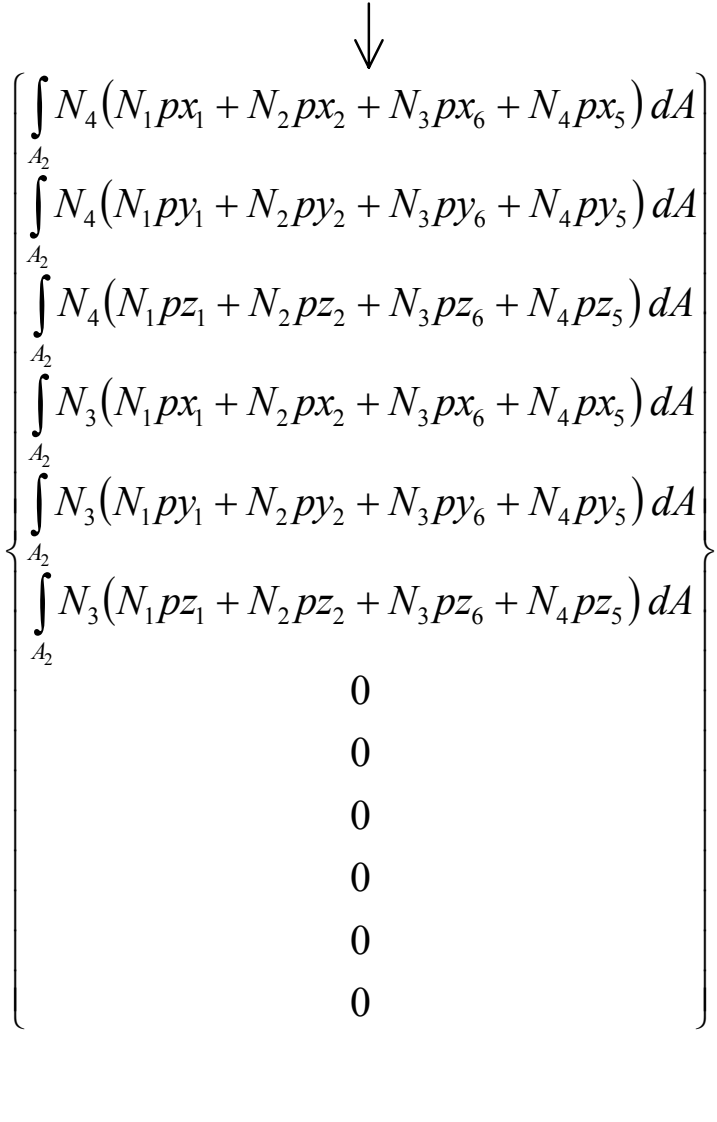

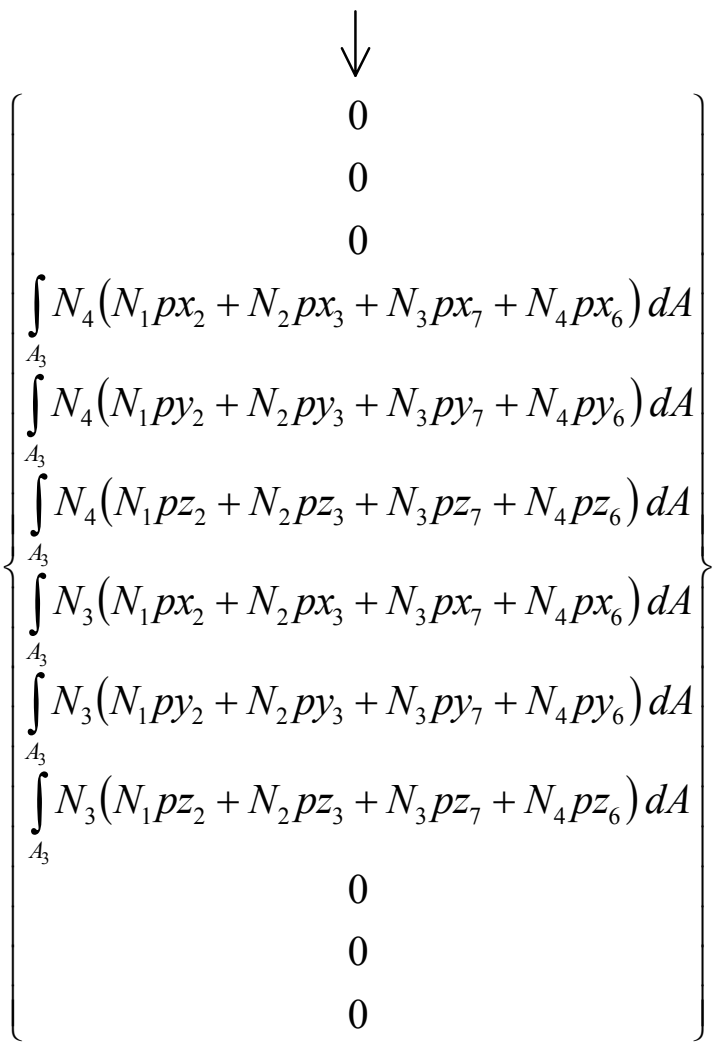




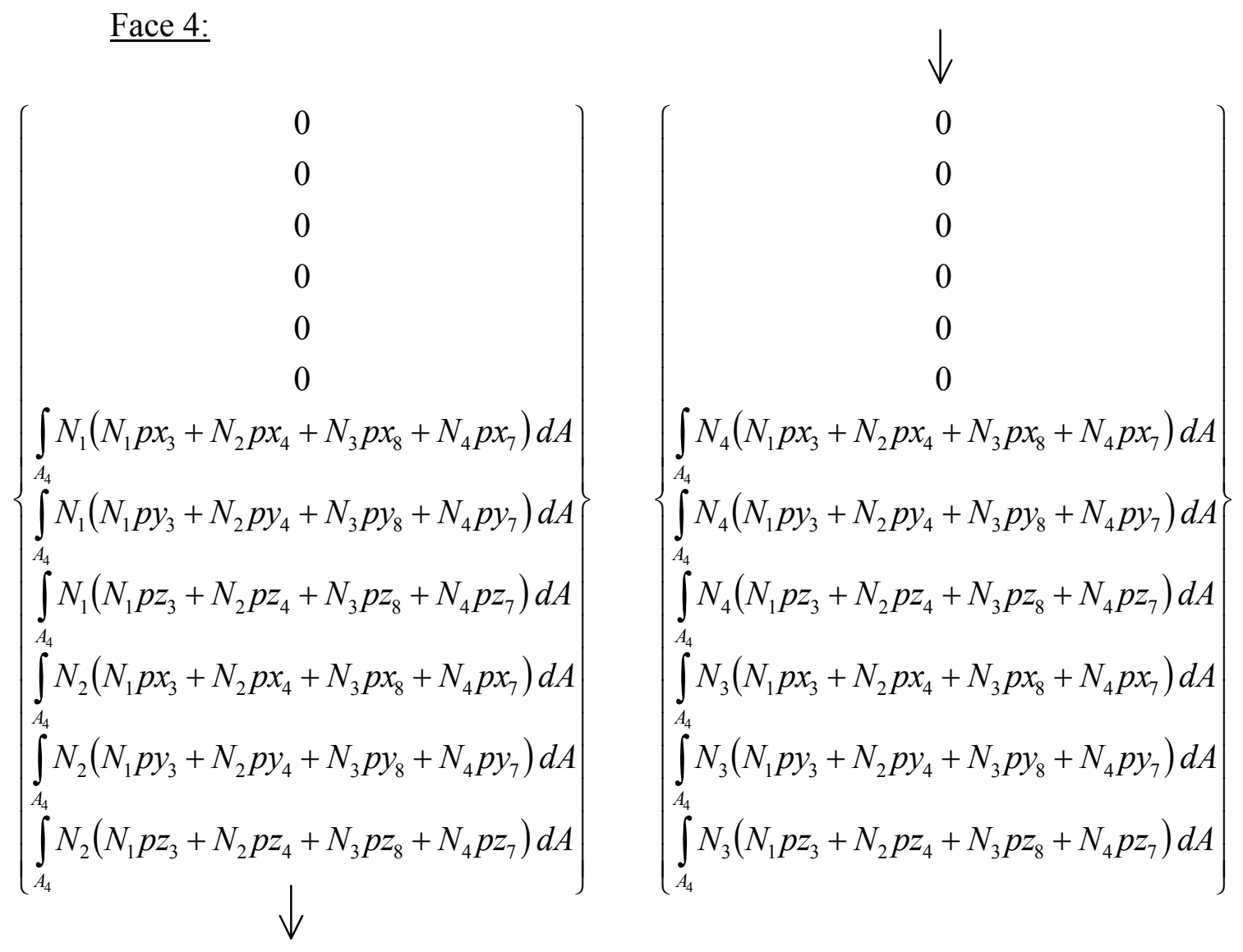

Face 5:

$$
\left\{\begin{array}{c}
\int_{A_{5}} N_{2}\left(N_{1} p x_{4}+N_{2} p x_{1}+N_{3} p x_{5}+N_{4} p x_{8}\right) d A \\
\int_{A_{5}} N_{2}\left(N_{1} p y_{4}+N_{2} p y_{1}+N_{3} p y_{5}+N_{4} p y_{8}\right) d A \\
\int_{A_{5}} N_{2}\left(N_{1} p z_{4}+N_{2} p z_{1}+N_{3} p z_{5}+N_{4} p z_{8}\right) d A \\
0 \\
0 \\
0 \\
0 \\
\int_{A_{5}} N_{1}\left(N_{1} p x_{4}+N_{2} p x_{1}+N_{3} p x_{5}+N_{4} p x_{8}\right) d A \\
\int_{A_{5}} N_{1}\left(N_{1} p y_{4}+N_{2} p y_{1}+N_{3} p y_{5}+N_{4} p y_{8}\right) d A \\
\int_{A_{5}} N_{1}\left(N_{1} p z_{4}+N_{2} p z_{1}+N_{3} p z_{5}+N_{4} p z_{8}\right) d A \\
\downarrow
\end{array}\right\}\left\{\begin{array}{c}
\int_{A_{5}} N_{3}\left(N_{1} p x_{4}+N_{2} p x_{1}+N_{3} p x_{5}+N_{4} p x_{8}\right) d A \\
\int_{A_{5}} N_{3}\left(N_{1} p y_{4}+N_{2} p y_{1}+N_{3} p y_{5}+N_{4} p y_{8}\right) d A \\
\int_{A_{5}} N_{3}\left(N_{1} p z_{4}+N_{2} p z_{1}+N_{3} p z_{5}+N_{4} p z_{8}\right) d A \\
0 \\
0 \\
0 \\
0 \\
0 \\
\int_{A_{5}}
\end{array}\right\}
$$




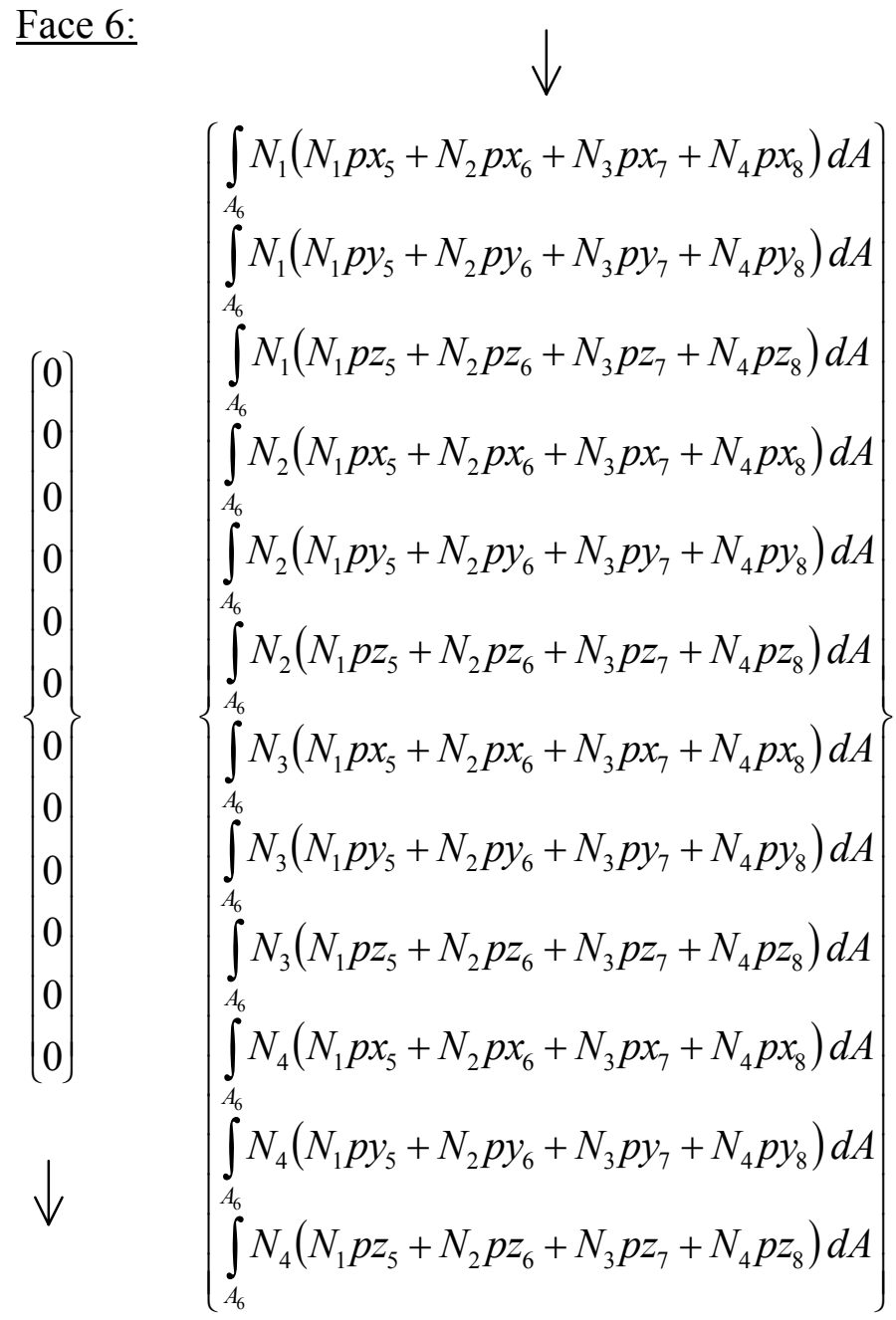

O vetor de forças nodais do elemento $\left(f_{e}\right)$ é dado pela soma das contribuições das faces carregadas, mostradas anteriormente.

Para permitir o uso da integração por pontos de Gauss, alteram-se as variáveis de integração das coordenadas $r$ e $s$ para as coordenadas adimensionais $\zeta$ e $\vartheta$. A expressão utilizada na integração, com a alteração das variáveis de integração mencionada anteriormente, é a seguinte:

$$
f_{e}=\int_{-1}^{+1+1} \int_{-1}^{T} \phi^{T} \phi^{p} \bar{p}_{e}\left|J_{p}\right| d \xi d \eta
$$

$\mathrm{Na}$ eq. (G.1), $J_{p}$ é o Jacobiano da transformação de coordenadas no plano indicada, dado pela eq. (80). 


\section{Apêndice H - Expansão das submatrizes do hexaedro em função do}

\section{enriquecimento}

Como visto no item 2.3.3.2.2, cada termo da submatriz original $3 \times 3$ do hexaedro não-enriquecido gera sua própria submatriz, sendo que os termos desta apresentam todos uma mesma expressão geral, que é apresentada a seguir:

1) Termo: $A(3 i-2,3 j-2)$

Para $k_{i}=0, n f\left(u_{i}\right)$

Para $k_{j}=0, n f\left(u_{j}\right)$

$$
\begin{aligned}
A\left(3 i-2+k_{i}, 3 j-2+k_{j}\right)=\int_{V} & {\left[\left(\frac{\partial N_{i}}{\partial x} f_{u_{i}}^{k_{i}}+N_{i} \frac{\partial f_{u_{i}}^{k_{i}}}{\partial x}\right)\left(\frac{\partial N_{j}}{\partial x} f_{u_{j}}^{k_{j}}+N_{j} \frac{\partial f_{u_{j}}^{k_{j}}}{\partial x}\right) c t_{1}+\right.} \\
& +\left(\frac{\partial N_{i}}{\partial y} f_{u_{i}}^{k_{i}}+N_{i} \frac{\partial f_{u_{i}}^{k_{i}}}{\partial y}\right)\left(\frac{\partial N_{j}}{\partial y} f_{u_{j}}^{k_{j}}+N_{j} \frac{\partial f_{u_{j}}^{k_{j}}}{\partial y}\right) c t_{3}+ \\
& \left.+\left(\frac{\partial N_{i}}{\partial z} f_{u_{i}}^{k_{i}}+N_{i} \frac{\partial f_{u_{i}}^{k_{i}}}{\partial z}\right)\left(\frac{\partial N_{j}}{\partial z} f_{u_{j}}^{k_{j}}+N_{j} \frac{\partial f_{u_{j}}^{k_{j}}}{\partial z}\right) c t_{3}\right] d V
\end{aligned}
$$

2) Termo: $A(3 i-2,3 j-1)$

Para $k_{i}=0, n f\left(u_{i}\right)$

Para $k_{j}=0, n f\left(v_{j}\right)$

$$
\begin{aligned}
A\left(3 i-2+k_{i}, 3 j-1+k_{j}\right)=\int_{V} & {\left[\left(\frac{\partial N_{i}}{\partial x} f_{u_{i}}^{k_{i}}+N_{i} \frac{\partial f_{u_{i}}^{k_{i}}}{\partial x}\right)\left(\frac{\partial N_{j}}{\partial y} f_{v_{j}}^{k_{j}}+N_{j} \frac{\partial f_{v_{j}}^{k_{j}}}{\partial y}\right) c t_{2}+\right.} \\
& \left.+\left(\frac{\partial N_{i}}{\partial y} f_{u_{i}}^{k_{i}}+N_{i} \frac{\partial f_{u_{i}}^{k_{i}}}{\partial y}\right)\left(\frac{\partial N_{j}}{\partial x} f_{v_{j}}^{k_{j}}+N_{j} \frac{\partial f_{v_{j}}^{k_{j}}}{\partial x}\right) c t_{3}\right] d V
\end{aligned}
$$


3) Termo: $A(3 i-2,3 j)$

Para $k_{i}=0, n f\left(u_{i}\right)$

Para $k_{j}=0, n f\left(w_{j}\right)$

$$
\begin{aligned}
A\left(3 i-2+k_{i}, 3 j+k_{j}\right)=\int_{V} & {\left[\left(\frac{\partial N_{i}}{\partial x} f_{u_{i}}^{k_{i}}+N_{i} \frac{\partial f_{u_{i}}^{k_{i}}}{\partial x}\right)\left(\frac{\partial N_{j}}{\partial z} f_{w_{j}}^{k_{j}}+N_{j} \frac{\partial f_{w_{j}}^{k_{j}}}{\partial z}\right) c t_{2}+\right.} \\
+ & \left.\left(\frac{\partial N_{i}}{\partial z} f_{u_{i}}^{k_{i}}+N_{i} \frac{\partial f_{u_{i}}^{k_{i}}}{\partial z}\right)\left(\frac{\partial N_{j}}{\partial x} f_{w_{j}}^{k_{j}}+N_{j} \frac{\partial f_{w_{j}}^{k_{j}}}{\partial x}\right) c t_{3}\right] d V
\end{aligned}
$$

4) Termo: $A(3 i-1,3 j-2)$

Para $k_{i}=0, n f\left(v_{i}\right)$

Para $k_{j}=0, n f\left(u_{j}\right)$

$$
\begin{aligned}
A\left(3 i-1+k_{i}, 3 j-2+k_{j}\right)=\int_{V} & {\left[\left(\frac{\partial N_{i}}{\partial y} f_{v_{i}}^{k_{i}}+N_{i} \frac{\partial f_{v_{i}}^{k_{i}}}{\partial y}\right)\left(\frac{\partial N_{j}}{\partial x} f_{u_{j}}^{k_{j}}+N_{j} \frac{\partial f_{u_{j}}^{k_{j}}}{\partial x}\right) c t_{2}+\right.} \\
& \left.+\left(\frac{\partial N_{i}}{\partial x} f_{v_{i}}^{k_{i}}+N_{i} \frac{\partial f_{v_{i}}^{k_{i}}}{\partial x}\right)\left(\frac{\partial N_{j}}{\partial y} f_{u_{j}}^{k_{j}}+N_{j} \frac{\partial f_{u_{j}}^{k_{j}}}{\partial y}\right) c t_{3}\right] d V
\end{aligned}
$$

5) Termo: $A(3 i-1,3 j-1)$

Para $k_{i}=0, n f\left(v_{i}\right)$

Para $k_{j}=0, n f\left(v_{j}\right)$

$$
\begin{aligned}
A\left(3 i-1+k_{i}, 3 j-1+k_{j}\right)=\int_{V} & {\left[\left(\frac{\partial N_{i}}{\partial x} f_{v_{i}}^{k_{i}}+N_{i} \frac{\partial f_{v_{i}}^{k_{i}}}{\partial x}\right)\left(\frac{\partial N_{j}}{\partial x} f_{v_{j}}^{k_{j}}+N_{j} \frac{\partial f_{v_{j}}^{k_{j}}}{\partial x}\right) c t_{3}+\right.} \\
& +\left(\frac{\partial N_{i}}{\partial y} f_{v_{i}}^{k_{i}}+N_{i} \frac{\partial f_{v_{i}}^{k_{i}}}{\partial y}\right)\left(\frac{\partial N_{j}}{\partial y} f_{v_{j}}^{k_{j}}+N_{j} \frac{\partial f_{v_{j}}^{k_{j}}}{\partial y}\right) c t_{1}+ \\
& \left.+\left(\frac{\partial N_{i}}{\partial z} f_{v_{i}}^{k_{i}}+N_{i} \frac{\partial f_{v_{i}}^{k_{i}}}{\partial z}\right)\left(\frac{\partial N_{j}}{\partial z} f_{v_{j}}^{k_{j}}+N_{j} \frac{\partial f_{v_{j}}^{k_{j}}}{\partial z}\right) c t_{3}\right] d V
\end{aligned}
$$


6) Termo: $A(3 i-1,3 j)$

Para $k_{i}=0, n f\left(v_{i}\right)$

Para $k_{j}=0, n f\left(w_{j}\right)$

$$
\begin{aligned}
A\left(3 i-1+k_{i}, 3 j+k_{j}\right)=\int_{V} & {\left[\left(\frac{\partial N_{i}}{\partial y} f_{v_{i}}^{k_{i}}+N_{i} \frac{\partial f_{v_{i}}^{k_{i}}}{\partial y}\right)\left(\frac{\partial N_{j}}{\partial z} f_{w_{j}}^{k_{j}}+N_{j} \frac{\partial f_{w_{j}}^{k_{j}}}{\partial z}\right) c t_{2}+\right.} \\
& \left.+\left(\frac{\partial N_{i}}{\partial z} f_{v_{i}}^{k_{i}}+N_{i} \frac{\partial f_{v_{i}}^{k_{i}}}{\partial z}\right)\left(\frac{\partial N_{j}}{\partial y} f_{w_{j}}^{k_{j}}+N_{j} \frac{\partial f_{w_{j}}^{k_{j}}}{\partial y}\right) c t_{3}\right] d V
\end{aligned}
$$

7) Termo: $A(3 i, 3 j-2)$

Para $k_{i}=0, n f\left(w_{i}\right)$

Para $k_{j}=0, n f\left(u_{j}\right)$

$$
\begin{aligned}
A\left(3 i+k_{i}, 3 j-2+k_{j}\right)=\int_{V} & {\left[\left(\frac{\partial N_{i}}{\partial z} f_{w_{i}}^{k_{i}}+N_{i} \frac{\partial f_{w_{i}}^{k_{i}}}{\partial z}\right)\left(\frac{\partial N_{j}}{\partial x} f_{u_{j}}^{k_{j}}+N_{j} \frac{\partial f_{u_{j}}^{k_{j}}}{\partial x}\right) c t_{2}+\right.} \\
& \left.+\left(\frac{\partial N_{i}}{\partial x} f_{w_{i}}^{k_{i}}+N_{i} \frac{\partial f_{w_{i}}^{k_{i}}}{\partial x}\right)\left(\frac{\partial N_{j}}{\partial z} f_{u_{j}}^{k_{j}}+N_{j} \frac{\partial f_{u_{j}}^{k_{j}}}{\partial z}\right) c t_{3}\right] d V
\end{aligned}
$$

8) Termo: $A(3 i, 3 j-1)$

Para $k_{i}=0, n f\left(w_{i}\right)$

Para $k_{j}=0, n f\left(v_{j}\right)$

$$
\begin{aligned}
A\left(3 i+k_{i}, 3 j-1+k_{j}\right)=\int_{V} & {\left[\left(\frac{\partial N_{i}}{\partial z} f_{w_{i}}^{k_{i}}+N_{i} \frac{\partial f_{w_{i}}^{k_{i}}}{\partial z}\right)\left(\frac{\partial N_{j}}{\partial y} f_{v_{j}}^{k_{j}}+N_{j} \frac{\partial f_{v_{j}}^{k_{j}}}{\partial y}\right) c t_{2}+\right.} \\
& \left.+\left(\frac{\partial N_{i}}{\partial y} f_{w_{i}}^{k_{i}}+N_{i} \frac{\partial f_{w_{i}}^{k_{i}}}{\partial y}\right)\left(\frac{\partial N_{j}}{\partial z} f_{v_{j}}^{k_{j}}+N_{j} \frac{\partial f_{v_{j}}^{k_{j}}}{\partial z}\right) c t_{3}\right] d V
\end{aligned}
$$


9) Termo: $A(3 i, 3 j)$

Para $k_{i}=0, n f\left(w_{i}\right)$

Para $k_{j}=0, n f\left(w_{j}\right)$

$$
\begin{aligned}
A\left(3 i+k_{i}, 3 j+k_{j}\right)=\int_{V} & {\left[\left(\frac{\partial N_{i}}{\partial x} f_{w_{i}}^{k_{i}}+N_{i} \frac{\partial f_{w_{i}}^{k_{i}}}{\partial x}\right)\left(\frac{\partial N_{j}}{\partial x} f_{w_{j}}^{k_{j}}+N_{j} \frac{\partial f_{w_{j}}^{k_{j}}}{\partial x}\right) c t_{3}+\right.} \\
& +\left(\frac{\partial N_{i}}{\partial y} f_{w_{i}}^{k_{i}}+N_{i} \frac{\partial f_{w_{i}}^{k_{i}}}{\partial y}\right)\left(\frac{\partial N_{j}}{\partial y} f_{w_{j}}^{k_{j}}+N_{j} \frac{\partial f_{w_{j}}^{k_{j}}}{\partial y}\right) c t_{3}+ \\
& \left.+\left(\frac{\partial N_{i}}{\partial z} f_{w_{i}}^{k_{i}}+N_{i} \frac{\partial f_{w_{i}}^{k_{i}}}{\partial z}\right)\left(\frac{\partial N_{j}}{\partial z} f_{w_{j}}^{k_{j}}+N_{j} \frac{\partial f_{w_{j}}^{k_{j}}}{\partial z}\right) c t_{1}\right] d V
\end{aligned}
$$

Os termos originais das submatrizes sem enriquecimento correspondem ao valor das expressões anteriores quando $k_{i}=0, k_{j}=0$, adotando-se valor unitário para a função enriquecedora e nulo para suas derivadas parciais. Como esperado, os valores obtidos correspondem exatamente aos das eqs. (76). 


\section{Apêndice I - Matrizes de aproximação dos deslocamentos nas faces}

\section{do hexaedro enriquecido}

As matrizes de aproximação dos deslocamentos nas faces do hexaedro enriquecido

$[\phi]_{3 x(24+n p a r)}$ são as seguintes:

$\underline{\text { Face 1: }}$

$$
\begin{aligned}
& \phi=\left[\begin{array}{cccccccccccc}
N_{1} & N_{1} f_{u_{1}}^{1} & \cdots & N_{1} f_{u_{1}}^{n f\left(u_{1}\right)} & 0 & 0 & \cdots & 0 & 0 & 0 & \cdots & 0 \\
0 & 0 & \cdots & 0 & N_{1} & N_{1} f_{v_{1}}^{1} & \cdots & N_{1} f_{v_{1}}^{n f\left(v_{1}\right)} & 0 & 0 & \cdots & 0 \\
0 & 0 & \cdots & 0 & 0 & 0 & \cdots & 0 & N_{1} & N_{1} f_{w_{1}}^{1} & \cdots & N_{1} f_{w_{1}}^{n f\left(w_{1}\right)}
\end{array}\right. \\
& \begin{array}{cccccccccccc}
N_{2} & N_{2} f_{u_{2}}^{1} & \cdots & N_{2} f_{u_{2}}^{n f\left(u_{2}\right)} & 0 & 0 & \cdots & 0 & 0 & 0 & \cdots & 0 \\
0 & 0 & \cdots & 0 & N_{2} & N_{2} f_{v_{2}}^{1} & \cdots & N_{2} f_{v_{2}}^{n f\left(v_{2}\right)} & 0 & 0 & \cdots & 0 \\
0 & 0 & \cdots & 0 & 0 & 0 & \cdots & 0 & N_{2} & N_{2} f_{w_{2}}^{1} & \cdots & N_{2} f_{w_{2}}^{n f\left(w_{2}\right)}
\end{array} \\
& \begin{array}{cccccccccccc}
N_{3} & N_{3} f_{u_{3}}^{1} & \cdots & N_{3} f_{u_{3}}^{n f\left(u_{3}\right)} & 0 & 0 & \cdots & 0 & 0 & 0 & \cdots & 0 \\
0 & 0 & \cdots & 0 & N_{3} & N_{3} f_{v_{3}}^{1} & \cdots & N_{3} f_{v_{3}}^{n f\left(v_{3}\right)} & 0 & 0 & \cdots & 0 \\
0 & 0 & \cdots & 0 & 0 & 0 & \cdots & 0 & N_{3} & N_{3} f_{w_{3}}^{1} & \cdots & N_{3} f_{w_{3}}^{n f\left(w_{3}\right)}
\end{array} \\
& \begin{array}{cccccccccccc}
N_{4} & N_{4} f_{u_{4}}^{1} & \cdots & N_{4} f_{u_{4}}^{n f\left(u_{4}\right)} & 0 & 0 & \cdots & 0 & 0 & 0 & \cdots & 0 \\
0 & 0 & \cdots & 0 & N_{4} & N_{4} f_{v_{4}}^{1} & \cdots & N_{4} f_{v_{4}}^{n f\left(v_{4}\right)} & 0 & 0 & \cdots & 0 \\
0 & 0 & \cdots & 0 & 0 & 0 & \cdots & 0 & N_{4} & N_{4} f_{w_{4}}^{1} & \cdots & N_{4} f_{w_{4}}^{n f\left(w_{4}\right)}
\end{array} \\
& {\left[\begin{array}{lll:lll:lll:lll}
0 & 0 & 0 & 0 & 0 & 0 & 0 & 0 & 0 & 0 & 0 & 0 \\
0 & 0 & 0 & 0 & 0 & 0 & 0 & 0 & 0 & 0 & 0 & 0 \\
0 & 0 & 0 & 0 & 0 & 0 & 0 & 0 & 0 & 0 & 0 & 0
\end{array}\right]}
\end{aligned}
$$




\section{Face 2:}

$$
\begin{aligned}
& \phi=\left[\begin{array}{cccccccccccc}
N_{1} & N_{1} f_{u_{1}}^{1} & \cdots & N_{1} f_{u_{1}}^{n f\left(u_{1}\right)} & 0 & 0 & \cdots & 0 & 0 & 0 & \cdots & 0 \\
0 & 0 & \cdots & 0 & N_{1} & N_{1} f_{v_{1}}^{1} & \cdots & N_{1} f_{v_{1}}^{n f\left(v_{1}\right)} & 0 & 0 & \cdots & 0 \\
0 & 0 & \cdots & 0 & 0 & 0 & \cdots & 0 & N_{1} & N_{1} f_{w_{1}}^{1} & \cdots & N_{1} f_{w_{1}}^{n f\left(w_{1}\right)}
\end{array}\right. \\
& \begin{array}{cccccccccccc}
N_{2} & N_{2} f_{u_{2}}^{1} & \cdots & N_{2} f_{u_{2}}^{n f\left(u_{2}\right)} & 0 & 0 & \cdots & 0 & 0 & 0 & \cdots & 0 \\
0 & 0 & \cdots & 0 & N_{2} & N_{2} f_{v_{2}}^{1} & \cdots & N_{2} f_{v_{2}}^{n f\left(v_{2}\right)} & 0 & 0 & \cdots & 0 \\
0 & 0 & \cdots & 0 & 0 & 0 & \cdots & 0 & N_{2} & N_{2} f_{w_{2}}^{1} & \cdots & N_{2} f_{w_{2}}^{n f\left(w_{2}\right)}
\end{array} \\
& \mid \begin{array}{lll:lll:}
0 & 0 & 0 & 0 & 0 & 0 \\
0 & 0 & 0 & 0 & 0 & 0 \\
0 & 0 & 0 & 0 & 0 & 0
\end{array} \\
& \begin{array}{cccccccccccc}
N_{4} & N_{4} f_{u_{5}}^{1} & \cdots & N_{4} f_{u_{5}}^{n f\left(u_{5}\right)} & 0 & 0 & \cdots & 0 & 0 & 0 & \cdots & 0 \\
0 & 0 & \cdots & 0 & N_{4} & N_{4} f_{v_{5}}^{1} & \cdots & N_{4} f_{v_{5}}^{n f\left(v_{5}\right)} & 0 & 0 & \cdots & 0 \\
0 & 0 & \cdots & 0 & 0 & 0 & \cdots & 0 & N_{4} & N_{4} f_{w_{5}}^{1} & \cdots & N_{4} f_{w_{5}}^{n f\left(w_{5}\right)}
\end{array} \\
& \begin{array}{cccccccccccc}
N_{3} & N_{3} f_{u_{6}}^{1} & \cdots & N_{3} f_{u_{6}}^{n f\left(u_{6}\right)} & 0 & 0 & \cdots & 0 & 0 & 0 & \cdots & 0 \\
0 & 0 & \cdots & 0 & N_{3} & N_{3} f_{v_{6}}^{1} & \cdots & N_{3} f_{v_{6}}^{n f\left(v_{6}\right)} & 0 & 0 & \cdots & 0 \\
0 & 0 & \cdots & 0 & 0 & 0 & \cdots & 0 & N_{3} & N_{3} f_{w_{6}}^{1} & \cdots & N_{3} f_{w_{6}}^{n f\left(w_{6}\right)}
\end{array} \\
& {\left[\begin{array}{lll:lll}
0 & 0 & 0 & 0 & 0 & 0 \\
0 & 0 & 0 & 0 & 0 & 0 \\
0 & 0 & 0 & 0 & 0 & 0
\end{array}\right]}
\end{aligned}
$$




\section{Face 3:}

$$
\begin{aligned}
& \phi=\left[\begin{array}{lll}
0 & 0 & 0 \\
0 & 0 & 0 \\
0 & 0 & 0
\end{array}\right. \\
& \begin{array}{cccccccccccc}
N_{1} & N_{1} f_{u_{2}}^{1} & \cdots & N_{1} f_{u_{2}}^{n f\left(u_{2}\right)} & 0 & 0 & \cdots & 0 & 0 & 0 & \cdots & 0 \\
0 & 0 & \cdots & 0 & N_{1} & N_{1} f_{v_{2}}^{1} & \cdots & N_{1} f_{v_{2}}^{n f\left(v_{2}\right)} & 0 & 0 & \cdots & 0 \\
0 & 0 & \cdots & 0 & 0 & 0 & \cdots & 0 & N_{1} & N_{1} f_{w_{2}}^{1} & \cdots & N_{1} f_{w_{2}}^{n f\left(w_{2}\right)}
\end{array} \\
& \begin{array}{cccccccccccc}
N_{2} & N_{2} f_{u_{3}}^{1} & \cdots & N_{2} f_{u_{3}}^{n f\left(u_{3}\right)} & 0 & 0 & \cdots & 0 & 0 & 0 & \cdots & 0 \\
0 & 0 & \cdots & 0 & N_{2} & N_{2} f_{v_{3}}^{1} & \cdots & N_{2} f_{v_{3}}^{n f\left(v_{3}\right)} & 0 & 0 & \cdots & 0 \\
0 & 0 & \cdots & 0 & 0 & 0 & \cdots & 0 & N_{2} & N_{2} f_{w_{3}}^{1} & \cdots & N_{2} f_{w_{3}}^{n f\left(w_{3}\right)}
\end{array} \\
& \begin{array}{lll:lll::}
0 & 0 & 0 & 0 & 0 & 0 \\
0 & 0 & 0 & 0 & 0 & 0 \\
0 & 0 & 0 & 0 & 0 & 0
\end{array}: \\
& \begin{array}{cccccccccccc}
N_{4} & N_{4} f_{u_{6}}^{1} & \cdots & N_{4} f_{u_{6}}^{n f\left(u_{6}\right)} & 0 & 0 & \cdots & 0 & 0 & 0 & \cdots & 0 \\
0 & 0 & \cdots & 0 & N_{4} & N_{4} f_{v_{6}}^{1} & \cdots & N_{4} f_{v_{6}}^{n f\left(v_{6}\right)} & 0 & 0 & \cdots & 0 \\
0 & 0 & \cdots & 0 & 0 & 0 & \cdots & 0 & N_{4} & N_{4} f_{w_{6}}^{1} & \cdots & N_{4} f_{w_{6}}^{n f\left(w_{6}\right)}
\end{array} \\
& \begin{array}{cccccccccccc}
N_{3} & N_{3} f_{u_{7}}^{1} & \cdots & N_{3} f_{u_{7}}^{n f\left(u_{7}\right)} & 0 & 0 & \cdots & 0 & 0 & 0 & \cdots & 0 \\
0 & 0 & \cdots & 0 & N_{3} & N_{3} f_{v_{7}}^{1} & \cdots & N_{3} f_{v_{7}}^{n f\left(v_{7}\right)} & 0 & 0 & \cdots & 0 \\
0 & 0 & \cdots & 0 & 0 & 0 & \cdots & 0 & N_{3} & N_{3} f_{w_{7}}^{1} & \cdots & N_{3} f_{w_{7}}^{n f\left(w_{7}\right)}
\end{array} \\
& {\left[\begin{array}{lll}
0 & 0 & 0 \\
0 & 0 & 0 \\
0 & 0 & 0
\end{array}\right]}
\end{aligned}
$$




\section{Face 4:}

$$
\begin{aligned}
& \phi=\left[\begin{array}{lll:lll:l}
0 & 0 & 0 & 0 & 0 & 0 & 0 \\
0 & 0 & 0 & 0 & 0 & 0 & : \\
0 & 0 & 0 & 0 & 0 & 0 &
\end{array}\right. \\
& \begin{array}{cccccccccccc}
N_{1} & N_{1} f_{u_{3}}^{1} & \cdots & N_{1} f_{u_{3}}^{n f\left(u_{3}\right)} & 0 & 0 & \cdots & 0 & 0 & 0 & \cdots & 0 \\
0 & 0 & \cdots & 0 & N_{1} & N_{1} f_{v_{3}}^{1} & \cdots & N_{1} f_{v_{3}}^{n f\left(v_{3}\right)} & 0 & 0 & \cdots & 0 \\
0 & 0 & \cdots & 0 & 0 & 0 & \cdots & 0 & N_{1} & N_{1} f_{w_{3}}^{1} & \cdots & N_{1} f_{w_{3}}^{n f\left(w_{3}\right)}
\end{array} \\
& {\left[\begin{array}{cccccccccccc}
N_{2} & N_{2} f_{u_{4}}^{1} & \cdots & N_{2} f_{u_{4}}^{n f\left(u_{4}\right)} & 0 & 0 & \cdots & 0 & 0 & 0 & \cdots & 0 \\
0 & 0 & \cdots & 0 & N_{2} & N_{2} f_{v_{4}}^{1} & \cdots & N_{2} f_{v_{4}}^{n f\left(v_{4}\right)} & 0 & 0 & \cdots & 0 \\
0 & 0 & \cdots & 0 & 0 & 0 & \cdots & 0 & N_{2} & N_{2} f_{w_{4}}^{1} & \cdots & N_{2} f_{w_{4}}^{n f\left(w_{4}\right)}
\end{array}\right.} \\
& \begin{array}{lll:lll:}
0 & 0 & 0 & 0 & 0 & 0 \\
0 & 0 & 0 & 0 & 0 & 0 \\
0 & 0 & 0 & 0 & 0 & 0
\end{array} \\
& \begin{array}{cccccccccccc}
N_{4} & N_{4} f_{u_{7}}^{1} & \cdots & N_{4} f_{u_{7}}^{n f\left(u_{7}\right)} & 0 & 0 & \cdots & 0 & 0 & 0 & \cdots & 0 \\
0 & 0 & \cdots & 0 & N_{4} & N_{4} f_{v_{7}}^{1} & \cdots & N_{4} f_{v_{7}}^{n f\left(v_{7}\right)} & 0 & 0 & \cdots & 0 \\
0 & 0 & \cdots & 0 & 0 & 0 & \cdots & 0 & N_{4} & N_{4} f_{w_{7}}^{1} & \cdots & N_{4} f_{w_{7}}^{n f\left(w_{7}\right)}
\end{array} \\
& {\left[\begin{array}{cccccccccccc}
N_{3} & N_{3} f_{u_{8}}^{1} & \cdots & N_{3} f_{u_{8}}^{n f\left(u_{8}\right)} & 0 & 0 & \cdots & 0 & 0 & 0 & \cdots & 0 \\
0 & 0 & \cdots & 0 & N_{3} & N_{3} f_{v_{8}}^{1} & \cdots & N_{3} f_{v_{8}}^{n f\left(v_{8}\right)} & 0 & 0 & \cdots & 0 \\
0 & 0 & \cdots & 0 & 0 & 0 & \cdots & 0 & N_{3} & N_{3} f_{w_{8}}^{1} & \cdots & N_{3} f_{w_{8}}^{n f\left(w_{8}\right)}
\end{array}\right]}
\end{aligned}
$$




\section{Face 5:}

$$
\begin{aligned}
& \phi=\left[\begin{array}{cccccccccccc}
N_{2} & N_{2} f_{u_{1}}^{1} & \cdots & N_{2} f_{u_{1}}^{n f\left(u_{1}\right)} & 0 & 0 & \cdots & 0 & 0 & 0 & \cdots & 0 \\
0 & 0 & \cdots & 0 & N_{2} & N_{2} f_{v_{1}}^{1} & \cdots & N_{2} f_{v_{1}}^{n f\left(v_{1}\right)} & 0 & 0 & \cdots & 0 \\
0 & 0 & \cdots & 0 & 0 & 0 & \cdots & 0 & N_{2} & N_{2} f_{w_{1}}^{1} & \cdots & N_{2} f_{w_{1}}^{n f\left(w_{1}\right)}
\end{array}\right.
\end{aligned}
$$

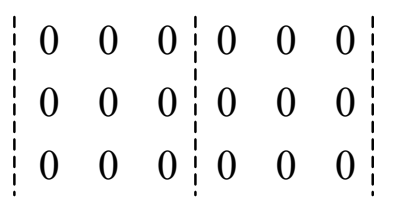

$$
\begin{aligned}
& \begin{array}{cccccccccccc}
N_{1} & N_{1} f_{u_{4}}^{1} & \cdots & N_{1} f_{u_{4}}^{n f\left(u_{4}\right)} & 0 & 0 & \cdots & 0 & 0 & 0 & \cdots & 0 \\
0 & 0 & \cdots & 0 & N_{1} & N_{1} f_{v_{4}}^{1} & \cdots & N_{1} f_{v_{4}}^{n f\left(v_{4}\right)} & 0 & 0 & \cdots & 0 \\
0 & 0 & \cdots & 0 & 0 & 0 & \cdots & 0 & N_{1} & N_{1} f_{w_{4}}^{1} & \cdots & N_{1} f_{w_{4}}^{n f\left(w_{4}\right)}
\end{array} \\
& \begin{array}{cccccccccccc}
N_{3} & N_{3} f_{u_{5}}^{1} & \cdots & N_{3} f_{u_{5}}^{n f\left(u_{5}\right)} & 0 & 0 & \cdots & 0 & 0 & 0 & \cdots & 0 \\
0 & 0 & \cdots & 0 & N_{3} & N_{3} f_{v_{5}}^{1} & \cdots & N_{3} f_{v_{5}}^{n f\left(v_{5}\right)} & 0 & 0 & \cdots & 0 \\
0 & 0 & \cdots & 0 & 0 & 0 & \cdots & 0 & N_{3} & N_{3} f_{w_{5}}^{1} & \cdots & N_{3} f_{w_{5}}^{n f\left(w_{5}\right)}
\end{array}
\end{aligned}
$$

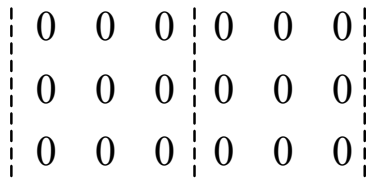

$$
\begin{aligned}
& {\left[\begin{array}{cccccccccccc}
N_{4} & N_{4} f_{u_{8}}^{1} & \cdots & N_{4} f_{u_{8}}^{n f\left(u_{8}\right)} & 0 & 0 & \cdots & 0 & 0 & 0 & \cdots & 0 \\
0 & 0 & \cdots & 0 & N_{4} & N_{4} f_{v_{8}}^{1} & \cdots & N_{4} f_{v_{8}}^{n f\left(v_{8}\right)} & 0 & 0 & \cdots & 0 \\
0 & 0 & \cdots & 0 & 0 & 0 & \cdots & 0 & N_{4} & N_{4} f_{w_{8}}^{1} & \cdots & N_{4} f_{w_{8}}^{n f\left(w_{8}\right)}
\end{array}\right]}
\end{aligned}
$$




\section{Face 6:}

$$
\begin{aligned}
& \phi=\left[\begin{array}{lll:lll:lll:lll:l}
0 & 0 & 0 & 0 & 0 & 0 & 0 & 0 & 0 & 0 & 0 & 0 & : \\
0 & 0 & 0 & 0 & 0 & 0 & 0 & 0 & 0 & 0 & 0 & 0 & 0 \\
0 & 0 & 0 & 0 & 0 & 0 & 0 & 0 & 0 & 0 & 0 & 0 & 0
\end{array}\right. \\
& \begin{array}{cccccccccccc}
N_{1} & N_{1} f_{u_{5}}^{1} & \cdots & N_{1} f_{u_{5}}^{n f\left(u_{5}\right)} & 0 & 0 & \cdots & 0 & 0 & 0 & \cdots & 0 \\
0 & 0 & \cdots & 0 & N_{1} & N_{1} f_{v_{5}}^{1} & \cdots & N_{1} f_{v_{5}}^{n f\left(v_{5}\right)} & 0 & 0 & \cdots & 0 \\
0 & 0 & \cdots & 0 & 0 & 0 & \cdots & 0 & N_{1} & N_{1} f_{w_{5}}^{1} & \cdots & N_{1} f_{w_{5}}^{n f\left(w_{5}\right)}
\end{array} \\
& \begin{array}{cccccccccccc}
N_{2} & N_{2} f_{u_{6}}^{1} & \cdots & N_{2} f_{u_{6}}^{n f\left(u_{6}\right)} & 0 & 0 & \cdots & 0 & 0 & 0 & \cdots & 0 \\
0 & 0 & \cdots & 0 & N_{2} & N_{2} f_{v_{6}}^{1} & \cdots & N_{2} f_{v_{6}}^{n f\left(v_{6}\right)} & 0 & 0 & \cdots & 0 \\
0 & 0 & \cdots & 0 & 0 & 0 & \cdots & 0 & N_{2} & N_{2} f_{w_{6}}^{1} & \cdots & N_{2} f_{w_{6}}^{n f\left(w_{6}\right)}
\end{array} \\
& {\left[\begin{array}{cccccccccccc}
N_{3} & N_{3} f_{u_{7}}^{1} & \cdots & N_{3} f_{u_{7}}^{n f\left(u_{7}\right)} & 0 & 0 & \cdots & 0 & 0 & 0 & \cdots & 0 \\
0 & 0 & \cdots & 0 & N_{3} & N_{3} f_{v_{7}}^{1} & \cdots & N_{3} f_{v_{7}}^{n f\left(v_{7}\right)} & 0 & 0 & \cdots & 0 \\
0 & 0 & \cdots & 0 & 0 & 0 & \cdots & 0 & N_{3} & N_{3} f_{w_{7}}^{1} & \cdots & N_{3} f_{w_{7}}^{n f\left(w_{7}\right)}
\end{array}\right.} \\
& {\left[\begin{array}{cccccccccccc}
N_{4} & N_{4} f_{u_{8}}^{1} & \cdots & N_{4} f_{u_{8}}^{n f\left(u_{8}\right)} & 0 & 0 & \cdots & 0 & 0 & 0 & \cdots & 0 \\
0 & 0 & \cdots & 0 & N_{4} & N_{4} f_{v_{8}}^{1} & \cdots & N_{4} f_{v_{8}}^{n f\left(v_{8}\right)} & 0 & 0 & \cdots & 0 \\
0 & 0 & \cdots & 0 & 0 & 0 & \cdots & 0 & N_{4} & N_{4} f_{w_{8}}^{1} & \cdots & N_{4} f_{w_{8}}^{n f\left(w_{8}\right)}
\end{array}\right]}
\end{aligned}
$$




\section{Apêndice $\mathbf{J}$ - Contribuição de cada face ao vetor de forças nodais}

\section{do hexaedro enriquecido}

$\underline{\text { Face 1: }}$

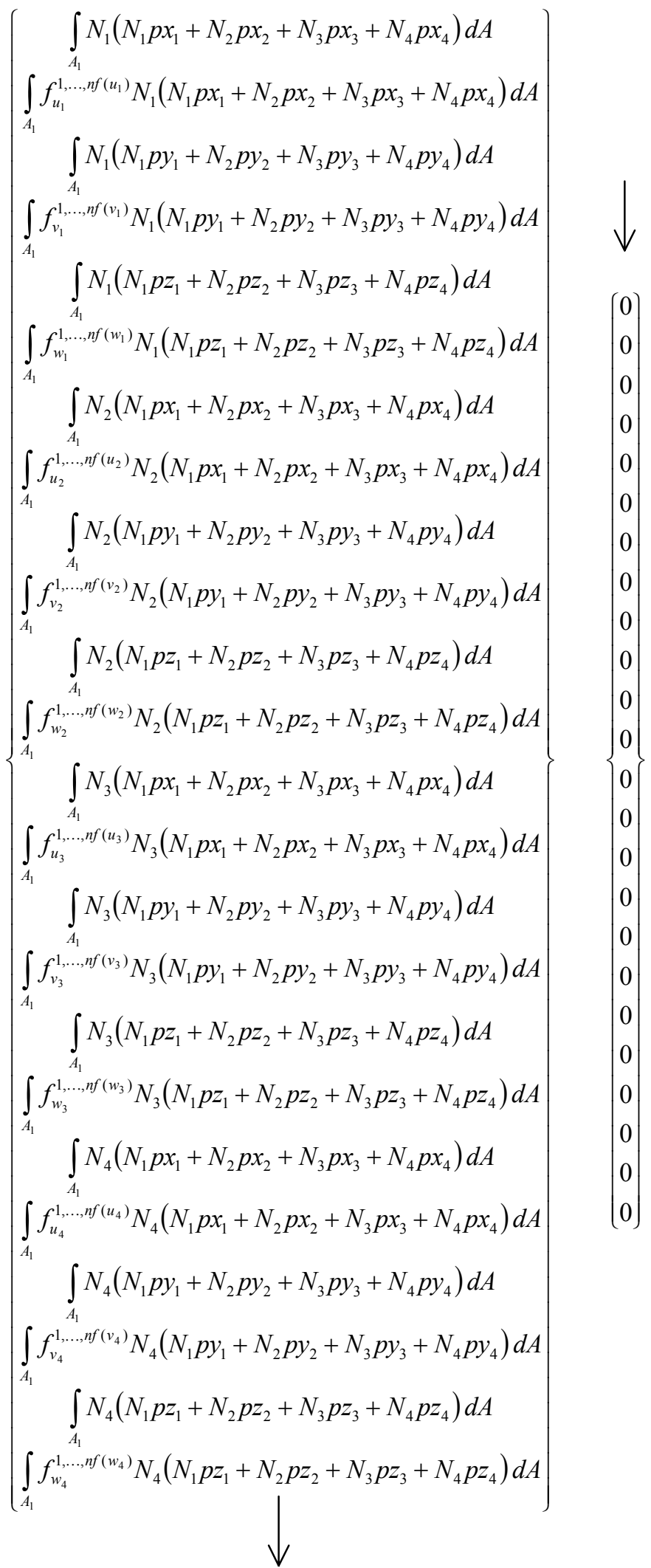




\section{Face 2:}

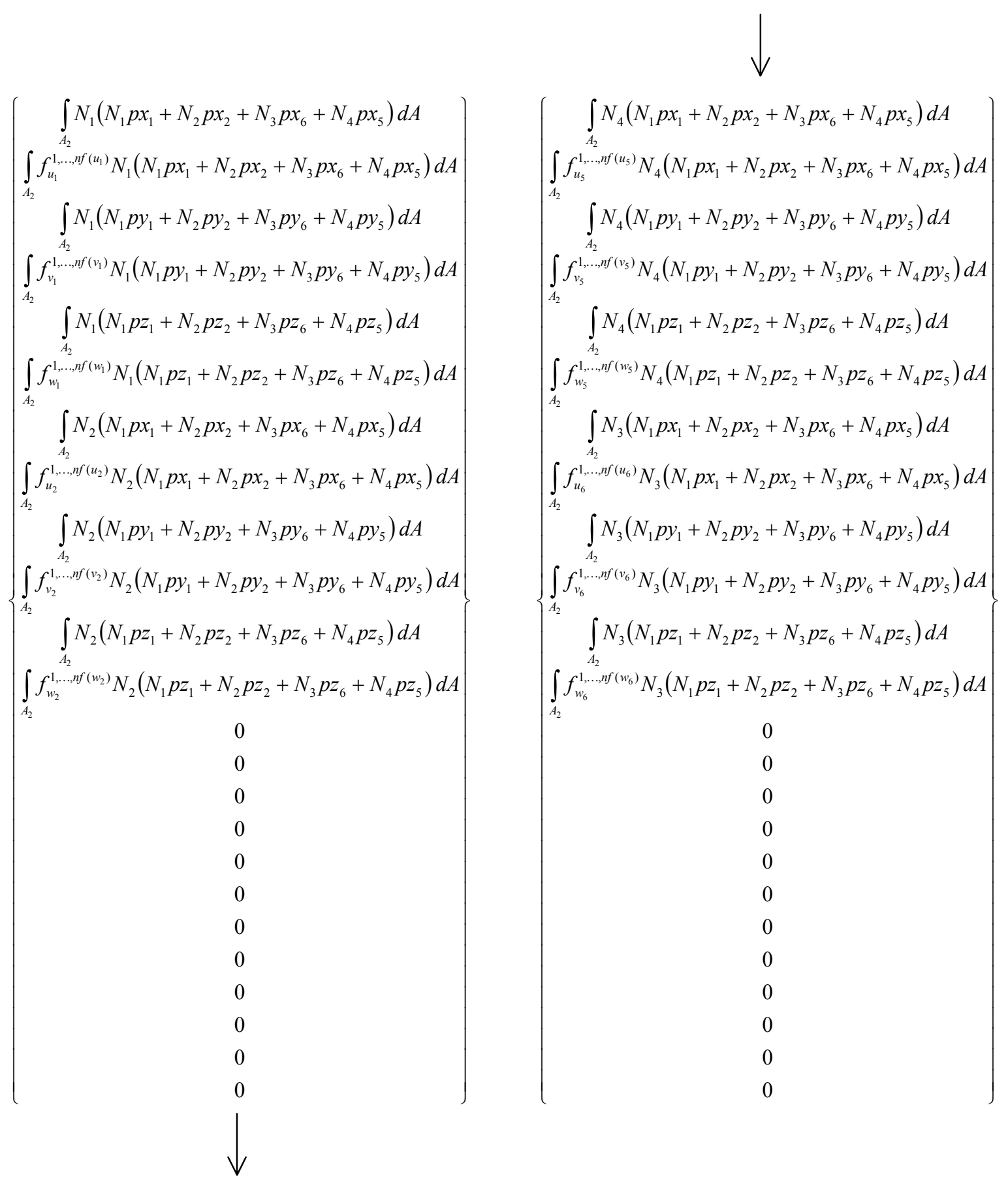




\section{Face 3:}

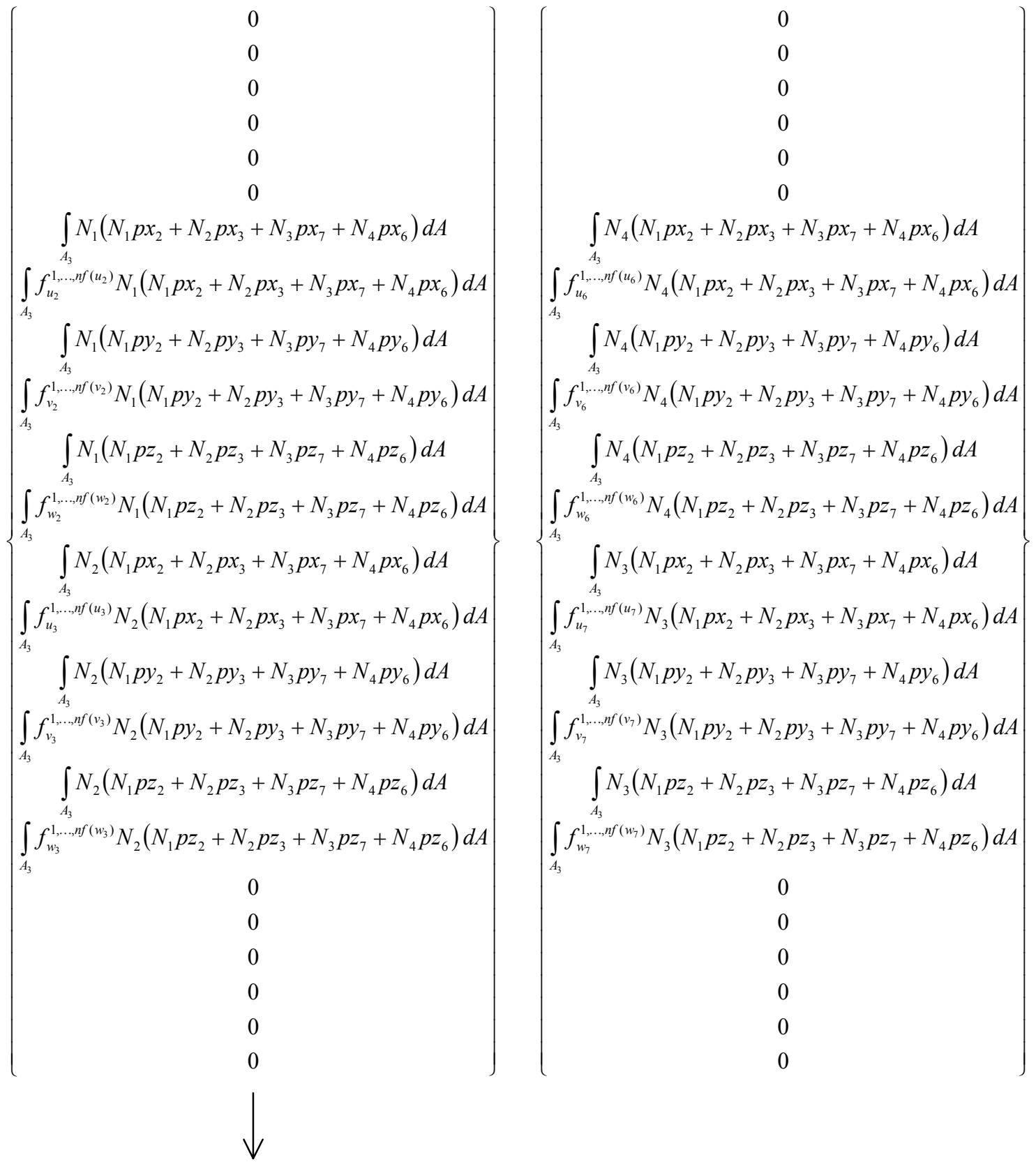




\section{Face 4:}

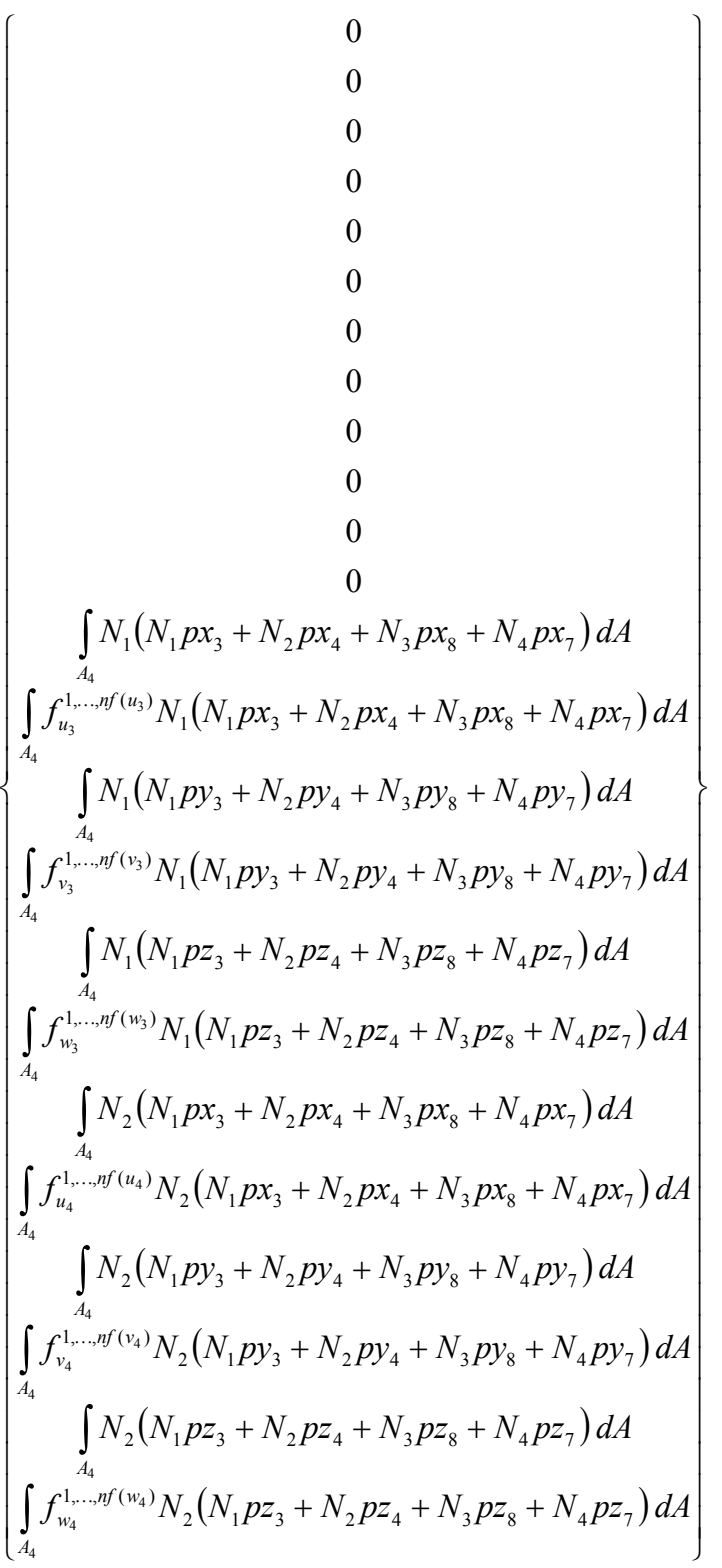

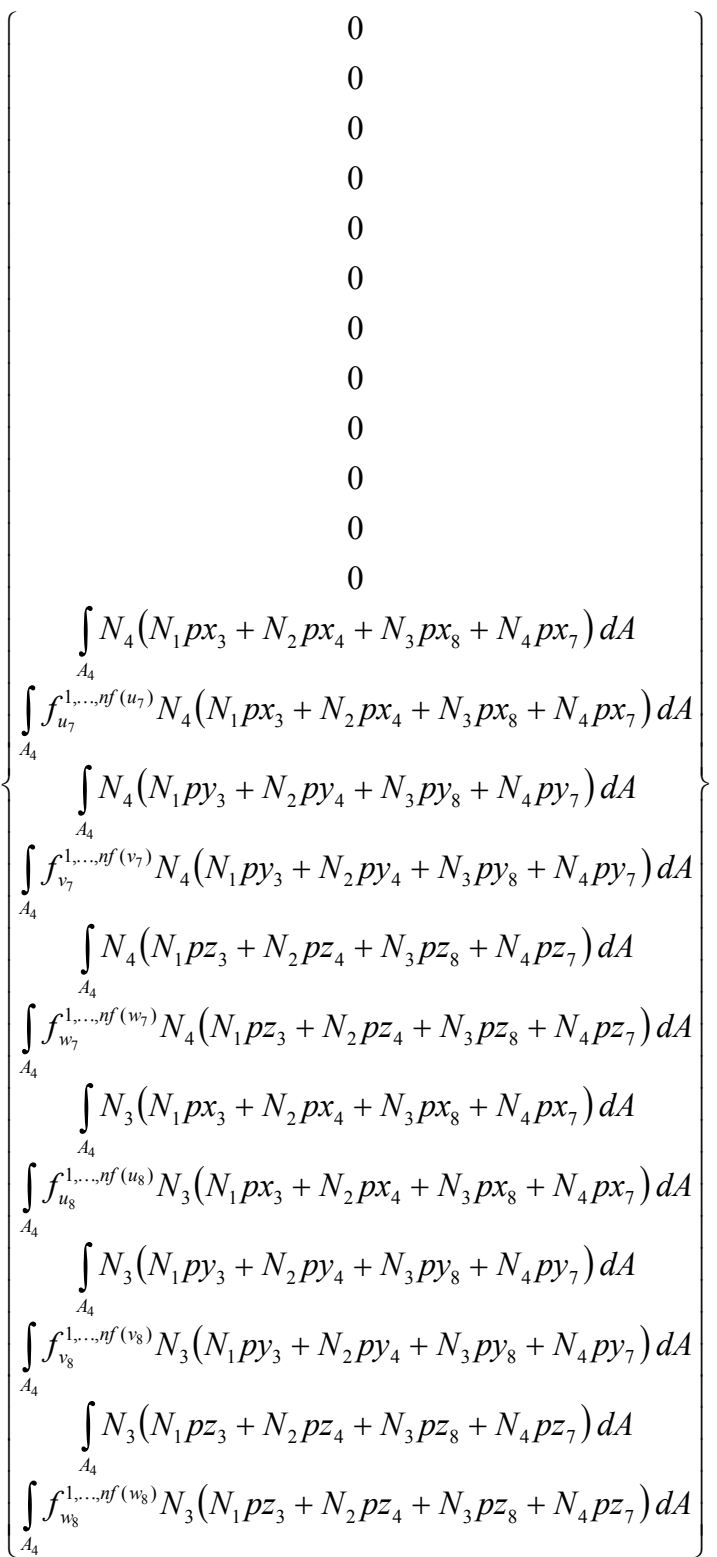




\section{Face 5:}
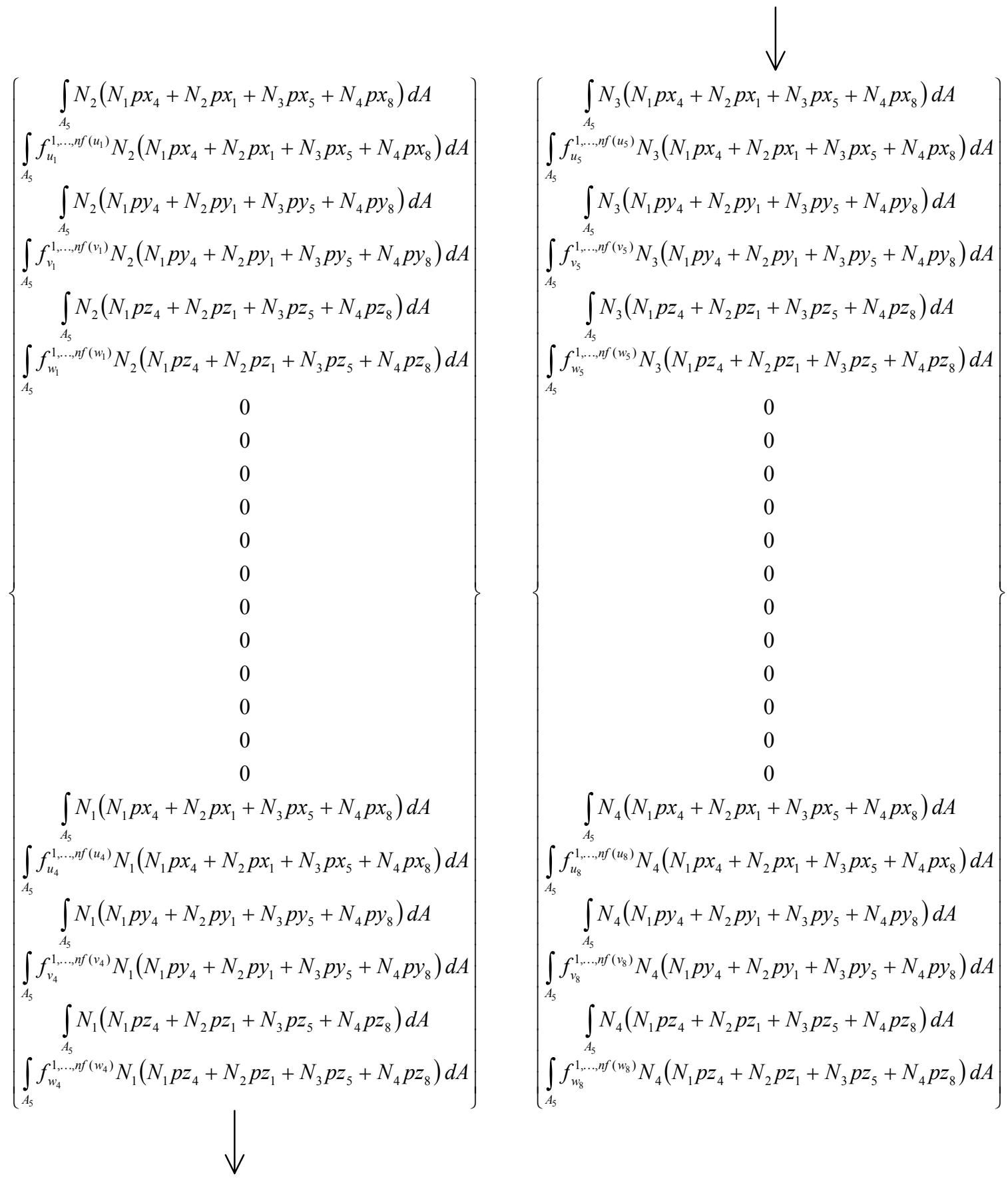
Face 6:

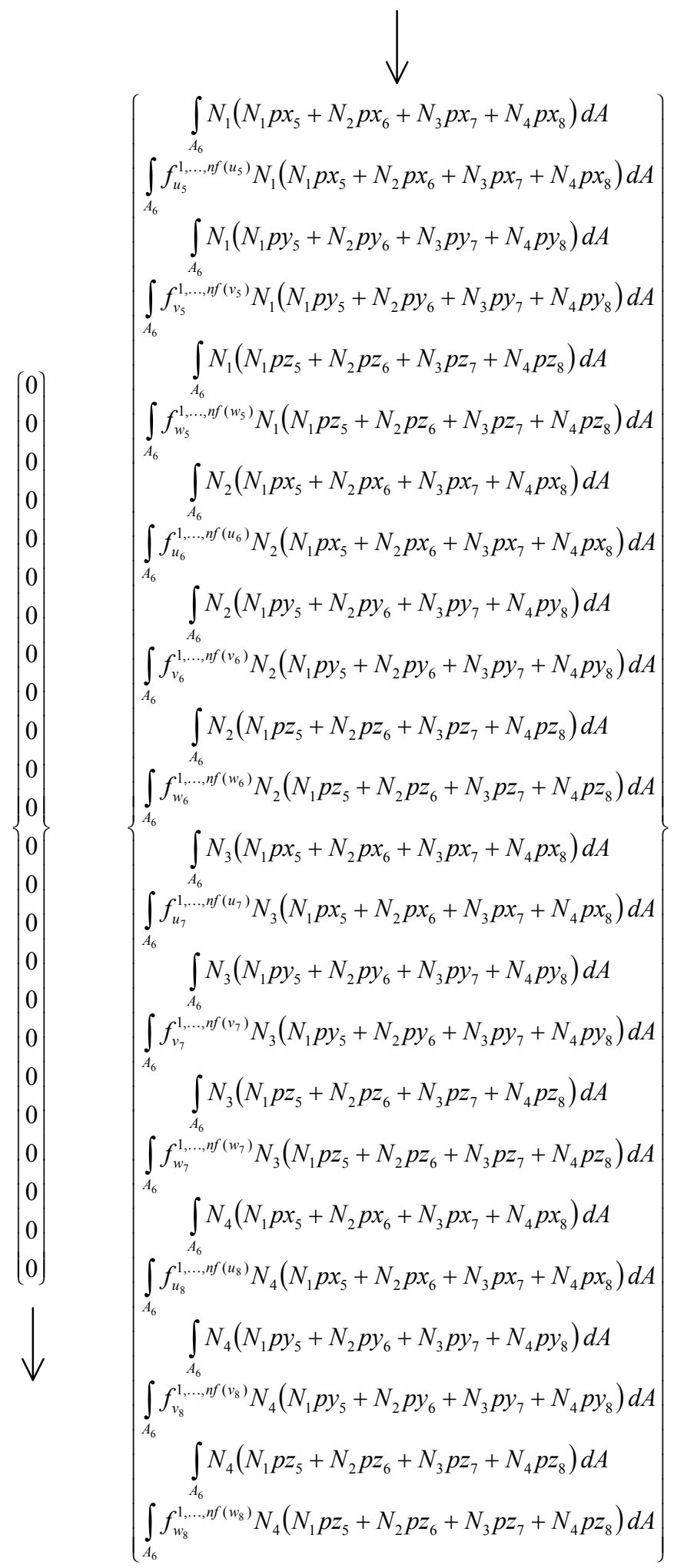

$\mathrm{Da}$ mesma forma que para o elemento hexaédrico não-enriquecido, deve-se proceder a uma alteração nas coordenadas de integração, para possibilitar o uso de pontos de Gauss. 


\section{Apêndice K - Matriz da relação constitutiva tangente $\left(D_{6 x 6}^{e p}\right)-$}

\section{elastoplasticidade com encruamento isótropo linear}

Seja a expressão do tensor algorítmico tangente para o modelo elastoplástico com critério de plastificação de von Mises e encruamento isótropo linear, dada pela eq. (177):

$$
C_{n+1}^{e p}=C-\frac{4 \mu^{2} \Delta \lambda}{\left\|S_{n+1}^{p r}\right\|} \Pi+\frac{4 \mu^{2} \Delta \lambda}{3\left\|S_{n+1}^{p r}\right\|}(\mathrm{I} \otimes \mathrm{I})+\left[\frac{4 \mu^{2} \Delta \lambda}{\left\|S_{n+1}^{p r}\right\|}-\frac{2 \mu}{\left(1+\frac{K}{3 \mu}\right)}\right] n_{n+1} \otimes n_{n+1}
$$

Por simplicidade, adota-se a seguinte expressão:

$$
C_{n+1}^{e p}=C-C_{n+1}^{p}
$$

Sendo a parcela do tensor relativa ao efeito da plastificação $C_{n+1}^{p}$ dada por:

$$
C_{n+1}^{p}=K P_{1} \Pi+K P_{2}(\mathrm{I} \otimes \mathrm{I})+K P_{3}\left(n_{n+1} \otimes n_{n+1}\right)
$$

E sendo os parâmetros:

$$
\begin{aligned}
& K P_{1}=\frac{4 \mu^{2} \Delta \lambda}{\left\|S_{n+1}^{p r}\right\|} \\
& K P_{2}=-\frac{4 \mu^{2} \Delta \lambda}{3\left\|S_{n+1}^{p r}\right\|}=-\frac{K P_{1}}{3} \\
& K P_{3}=\frac{2 \mu}{\left(1+\frac{K}{3 \mu}\right)}-\frac{4 \mu^{2} \Delta \lambda}{\left\|S_{n+1}^{p r}\right\|}
\end{aligned}
$$

Busca-se obter a matriz tangente $D^{e p}$, de dimensão $6 \times 6$, que corresponda ao tensor $C_{n+1}^{e p}$ e relacione por meio da equação $\delta \sigma=D^{e p} \delta \varepsilon$ os incrementos nas componentes de tensão e deformação agrupados nos vetores $\delta \sigma^{T}=\left\{\begin{array}{llllll}\delta \sigma_{x} & \delta \sigma_{y} & \delta \sigma_{z} & \delta \tau_{x y} & \delta \tau_{x z} & \delta \tau_{y z}\end{array}\right\}$ e $\delta \varepsilon^{T}=\left\{\begin{array}{llllll}\delta \varepsilon_{x} & \delta \varepsilon_{y} & \delta \varepsilon_{z} & \delta \gamma_{x y} & \delta \gamma_{x z} & \delta \gamma_{y z}\end{array}\right\}$, respectivamente.

Sendo $D^{e l}$ a matriz da relação constitutiva elástica linear correspondente ao tensor $C$ e $D^{p}$ a matriz tangente correspondente ao tensor $C_{n+1}^{p}$, a matriz $D^{e p}$ é dada por:

$$
D^{e p}=D^{e l}-D^{p}
$$

A matriz $D^{e l}$ é precisamente a matriz $D$ dada pela eq. (16), no capítulo 2 . 
Para o cálculo da matriz $D^{p}$ aplica-se cada uma das três parcelas do tensor $C_{n+1}^{p}$ ao tensor de deformações $\delta \varepsilon$. Por simplicidade de notação nas deduções seguintes, utilizamse $\sigma$ e $\varepsilon$ em referência aos incrementos $\delta \sigma$ e $\delta \varepsilon$, respectivamente.

1) $\sigma^{(1)}=K P_{1} \Pi \varepsilon=K P_{1} \varepsilon=K P_{1}\left[\begin{array}{ccc}\varepsilon_{x} & \varepsilon_{x y} & \varepsilon_{x z} \\ \varepsilon_{x y} & \varepsilon_{y} & \varepsilon_{y z} \\ \varepsilon_{x z} & \varepsilon_{y z} & \varepsilon_{z}\end{array}\right]$.

Desse modo, as componentes do tensor de tensões são:

$$
\begin{aligned}
& \sigma_{x}^{(1)}=K P_{1} \varepsilon_{x} \\
& \sigma_{y}^{(1)}=K P_{1} \varepsilon_{y} \\
& \sigma_{z}^{(1)}=K P_{1} \varepsilon_{z} \\
& \tau_{x y}^{(1)}=K P_{1} \varepsilon_{x y}=\frac{K P_{1}}{2} \gamma_{x y} \\
& \tau_{x z}^{(1)}=K P_{1} \varepsilon_{x z}=\frac{K P_{1}}{2} \gamma_{x z} \\
& \tau_{y z}^{(1)}=K P_{1} \varepsilon_{y z}=\frac{K P_{1}}{2} \gamma_{y z}
\end{aligned}
$$

A matriz constitutiva relativa à primeira parcela do tensor $C_{n+1}^{p}$ é, portanto:

$$
D_{(1)}^{p}=\left[\begin{array}{cccccc}
K P_{1} & 0 & 0 & 0 & 0 & 0 \\
& K P_{1} & 0 & 0 & 0 & 0 \\
& & K P_{1} & 0 & 0 & 0 \\
& & & \frac{K P_{1}}{2} & 0 & 0 \\
& \text { sim. } & & & \frac{K P_{1}}{2} & 0 \\
& & & & & \frac{K P_{1}}{2}
\end{array}\right]
$$

2) $\sigma^{(2)}=K P_{2}(\mathrm{I} \otimes \mathrm{I}) \varepsilon=K P_{2}(\mathrm{I} \cdot \varepsilon) \mathrm{I}=K P_{2}(\operatorname{tr} \varepsilon) \mathrm{I}=K P_{2}\left(\varepsilon_{x}+\varepsilon_{y}+\varepsilon_{z}\right)\left[\begin{array}{lll}1 & 0 & 0 \\ 0 & 1 & 0 \\ 0 & 0 & 1\end{array}\right]$

Desse modo, as componentes do tensor de tensões são:

$$
\begin{aligned}
& \sigma_{x}^{(2)}=K P_{2} \varepsilon_{x}+K P_{2} \varepsilon_{y}+K P_{2} \varepsilon_{z} \\
& \sigma_{y}^{(2)}=K P_{2} \varepsilon_{x}+K P_{2} \varepsilon_{y}+K P_{2} \varepsilon_{z} \\
& \sigma_{z}^{(2)}=K P_{2} \varepsilon_{x}+K P_{2} \varepsilon_{y}+K P_{2} \varepsilon_{z} \\
& \tau_{x y}^{(2)}=0
\end{aligned}
$$




$$
\begin{aligned}
& \tau_{x z}^{(2)}=0 \\
& \tau_{y z}^{(2)}=0
\end{aligned}
$$

A matriz constitutiva relativa à segunda parcela do tensor $C_{n+1}^{p}$ é, portanto:

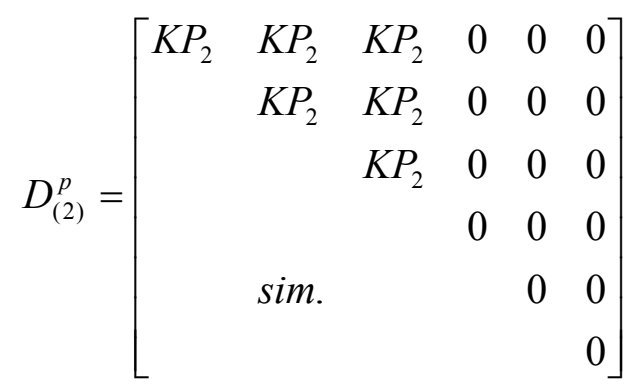

3) $\sigma^{(3)}=K P_{3}\left(n_{n+1} \otimes n_{n+1}\right) \varepsilon=K P_{3}\left(n_{n+1} \cdot \varepsilon\right) n_{n+1}=K P_{3} \operatorname{tr}\left(n_{n+1}^{T} \varepsilon\right) n_{n+1}$

Sendo o tensor $n_{n+1}$ constituído pelas componentes:

$$
n_{n+1}=\left[\begin{array}{ccc}
n_{1} & n_{4} & n_{5} \\
n_{4} & n_{2} & n_{6} \\
n_{5} & n_{6} & n_{3}
\end{array}\right]
$$

E sendo $\operatorname{tr}\left(n_{n+1}^{T} \varepsilon\right)=n_{1} \varepsilon_{x}+n_{2} \varepsilon_{y}+n_{3} \varepsilon_{z}+n_{4} \gamma_{x y}+n_{5} \gamma_{x z}+n_{6} \gamma_{y z}$

Chega-se a:

$$
\sigma^{(3)}=K P_{3} \operatorname{tr}\left(n_{n+1}^{T} \varepsilon\right)\left[\begin{array}{ccc}
n_{1} & n_{4} & n_{5} \\
n_{4} & n_{2} & n_{6} \\
n_{5} & n_{6} & n_{3}
\end{array}\right]
$$

As componentes do tensor de tensões $\sigma^{(3)}$ são:

$$
\begin{aligned}
& \sigma_{x}^{(3)}=K P_{3}\left(n_{1}^{2} \varepsilon_{x}+n_{1} n_{2} \varepsilon_{y}+n_{1} n_{3} \varepsilon_{z}+n_{1} n_{4} \gamma_{x y}+n_{1} n_{5} \gamma_{x z}+n_{1} n_{6} \gamma_{y z}\right) \\
& \sigma_{y}^{(3)}=K P_{3}\left(n_{1} n_{2} \varepsilon_{x}+n_{2}^{2} \varepsilon_{y}+n_{2} n_{3} \varepsilon_{z}+n_{2} n_{4} \gamma_{x y}+n_{2} n_{5} \gamma_{x z}+n_{2} n_{6} \gamma_{y z}\right) \\
& \sigma_{z}^{(3)}=K P_{3}\left(n_{1} n_{3} \varepsilon_{x}+n_{2} n_{3} \varepsilon_{y}+n_{3}^{2} \varepsilon_{z}+n_{3} n_{4} \gamma_{x y}+n_{3} n_{5} \gamma_{x z}+n_{3} n_{6} \gamma_{y z}\right) \\
& \tau_{x y}^{(3)}=K P_{3}\left(n_{1} n_{4} \varepsilon_{x}+n_{2} n_{4} \varepsilon_{y}+n_{3} n_{4} \varepsilon_{z}+n_{4}^{2} \gamma_{x y}+n_{4} n_{5} \gamma_{x z}+n_{4} n_{6} \gamma_{y z}\right) \\
& \tau_{x z}^{(3)}=K P_{3}\left(n_{1} n_{5} \varepsilon_{x}+n_{2} n_{5} \varepsilon_{y}+n_{3} n_{5} \varepsilon_{z}+n_{4} n_{5} \gamma_{x y}+n_{5}^{2} \gamma_{x z}+n_{5} n_{6} \gamma_{y z}\right) \\
& \tau_{y z}^{(3)}=K P_{3}\left(n_{1} n_{6} \varepsilon_{x}+n_{2} n_{6} \varepsilon_{y}+n_{3} n_{6} \varepsilon_{z}+n_{4} n_{6} \gamma_{x y}+n_{5} n_{6} \gamma_{x z}+n_{6}{ }^{2} \gamma_{y z}\right)
\end{aligned}
$$

A matriz constitutiva relativa à terceira parcela do tensor $C_{n+1}^{p}$ é, portanto: 


$$
D_{(3)}^{p}=\left[\begin{array}{cccccc}
K P_{3} n_{1}^{2} & K P_{3} n_{1} n_{2} & K P_{3} n_{1} n_{3} & K P_{3} n_{1} n_{4} & K P_{3} n_{1} n_{5} & K P_{3} n_{1} n_{6} \\
& K P_{3} n_{2}{ }^{2} & K P_{3} n_{2} n_{3} & K P_{3} n_{2} n_{4} & K P_{3} n_{2} n_{5} & K P_{3} n_{2} n_{6} \\
& & K P_{3} n_{3}{ }^{2} & K P_{3} n_{3} n_{4} & K P_{3} n_{3} n_{5} & K P_{3} n_{3} n_{6} \\
& & & K P_{3} n_{4}{ }^{2} & K P_{3} n_{4} n_{5} & K P_{3} n_{4} n_{6} \\
& & & & K P_{3} n_{5}^{2} & K P_{3} n_{5} n_{6} \\
& & & & & K P_{3} n_{6}{ }^{2}
\end{array}\right]
$$

A matriz constitutiva $D^{p}$, que também é simétrica, é dada pela soma das matrizes $D_{(1)}^{p}, D_{(2)}^{p}$ e $D_{(3)}^{p}$. Seus termos acima da diagonal principal são:

$$
\begin{aligned}
& D_{11}^{p}=K P_{1}+K P_{2}+K P_{3} n_{1}^{2} \\
& D_{12}^{p}=K P_{2}+K P_{3} n_{1} n_{2} \\
& D_{13}^{p}=K P_{2}+K P_{3} n_{1} n_{3} \\
& D_{14}^{p}=K P_{3} n_{1} n_{4} \\
& D_{15}^{p}=K P_{3} n_{1} n_{5} \\
& D_{16}^{p}=K P_{3} n_{1} n_{6} \\
& D_{22}^{p}=K P_{1}+K P_{2}+K P_{3} n_{2}{ }^{2} \\
& D_{23}^{p}=K P_{2}+K P_{3} n_{2} n_{3} \\
& D_{24}^{p}=K P_{3} n_{2} n_{4} \\
& D_{25}^{p}=K P_{3} n_{2} n_{5} \\
& D_{26}^{p}=K P_{3} n_{2} n_{6} \\
& D_{33}^{p}=K P_{1}+K P_{2}+K P_{3} n_{3}{ }^{2} \\
& D_{34}^{p}=K P_{3} n_{3} n_{4} \\
& D_{35}^{p}=K P_{3} n_{3} n_{5} \\
& D_{36}^{p}=K P_{3} n_{3} n_{6} \\
& D_{44}^{p}=\frac{K P_{1}}{2}+K P_{3} n_{4}^{2} \\
& D_{45}^{p}=K P_{3} n_{4} n_{5} \\
& D_{46}^{p}=K P_{3} n_{4} n_{6} \\
& D_{55}^{p}=\frac{K P_{1}}{2}+K P_{3} n_{5}^{2}
\end{aligned}
$$




$$
\begin{aligned}
& D_{56}^{p}=K P_{3} n_{5} n_{6} \\
& D_{66}^{p}=\frac{K P_{1}}{2}+K P_{3} n_{6}{ }^{2}
\end{aligned}
$$

Conhecidas as matrizes $D^{e l}$ e $D^{p}$, utiliza-se a relação (K.7) para a obtenção da matriz $D^{e p}$. 


\section{Apêndice L - Lei de evolução do dano em forma incremental}

Seja a expressão da lei de evolução do dano dada pela eq. (263):

$$
\dot{D}=\dot{\lambda} \frac{D_{C}}{\left(\varepsilon_{R}-\varepsilon_{D}\right)(1-D)}\left[\frac{2(1+v)}{3}+3(1-2 v)\left(\frac{\sigma_{H}}{\sigma_{e q}}\right)^{2}\right]
$$

Na qual:

$D_{C}, \varepsilon_{R}$ e $\varepsilon_{D}$ são parâmetros característicos do material obtidos em ensaios uniaxiais.

$\sigma_{H}=\frac{1}{3} \operatorname{tr} \sigma$ é a tensão média ou hidrostática.

$\sigma_{e q}=\sqrt{3 J 2}=\sqrt{\frac{3}{2}}\|S\|$ é a tensão equivalente ou tensão de von Mises.

Em passo finito, a eq. (L.1) pode ser expressa na forma:

$$
D_{n+1}=D_{n}+\Delta \lambda\left[\frac{2 D_{C}(1+v)}{3\left(\varepsilon_{R}-\varepsilon_{D}\right)\left(1-D_{n+1}\right)}+\frac{3 D_{C}(1-2 v)}{\left(\varepsilon_{R}-\varepsilon_{D}\right)\left(1-D_{n+1}\right)} \frac{\frac{1}{9}\left(\operatorname{tr} \sigma_{n+1}\right)^{2}}{\frac{3}{2}\left\|S_{n+1}\right\|^{2}}\right]
$$

Retoma-se aqui a expressão do tensor de tensões $\sigma_{n+1}$ dada no item 4.3.2.5:

$\sigma_{n+1}=\left(1-D_{n+1}\right) C\left(\varepsilon_{n+1}-\varepsilon_{n+1}^{p}\right)$

Aplicando-se o tensor constitutivo elástico à eq. (L.3), obtém-se:

$$
\begin{aligned}
\sigma_{n+1} & =\left(1-D_{n+1}\right)[\lambda(\mathrm{I} \otimes \mathrm{I})+2 \mu \Pi]\left(\varepsilon_{n+1}-\varepsilon_{n+1}^{p}\right) \Rightarrow \\
\sigma_{n+1} & =\left(1-D_{n+1}\right)\left[\lambda(\mathrm{I} \otimes \mathrm{I}) \varepsilon_{n+1}+2 \mu \Pi \varepsilon_{n+1}-\lambda(\mathrm{I} \otimes \mathrm{I}) \varepsilon_{n+1}^{p}-2 \mu \Pi \varepsilon_{n+1}^{p}\right] \Rightarrow \\
\sigma_{n+1} & =\left(1-D_{n+1}\right)\left[\lambda t r \varepsilon_{n+1} \mathrm{I}+2 \mu \varepsilon_{n+1}-\lambda t r \varepsilon_{n+1}^{p} \mathrm{I}-2 \mu \varepsilon_{n+1}^{p}\right] \Rightarrow \\
\sigma_{n+1} & =\left(1-D_{n+1}\right)\left[\lambda t r \varepsilon_{n+1} \mathrm{I}+2 \mu \varepsilon_{n+1}-2 \mu \varepsilon_{n+1}^{p}\right]
\end{aligned}
$$

Calcula-se também $\operatorname{tr} \sigma_{n+1}$ :

$$
\begin{aligned}
& \operatorname{tr} \sigma_{n+1}=\left(1-D_{n+1}\right)\left[\lambda \operatorname{tr} \varepsilon_{n+1} \operatorname{tr} \mathrm{I}+2 \mu \operatorname{tr} \varepsilon_{n+1}-2 \mu \operatorname{tr} \varepsilon_{n+1}^{p}\right] \Rightarrow \\
& \operatorname{tr} \sigma_{n+1}=\left(1-D_{n+1}\right)\left[3 \lambda \operatorname{tr} \varepsilon_{n+1}+2 \mu \operatorname{tr} \varepsilon_{n+1}\right]=\left(1-D_{n+1}\right)(3 \lambda+2 \mu) \operatorname{tr} \varepsilon_{n+1}
\end{aligned}
$$

Substituindo-se a eq. (L.5) na eq. (L.2), chega-se a:

$$
D_{n+1}=D_{n}+\Delta \lambda\left[\frac{2 D_{C}(1+v)}{3\left(\varepsilon_{R}-\varepsilon_{D}\right)\left(1-D_{n+1}\right)}+\frac{2 D_{C}(1-2 v)\left(1-D_{n+1}\right)(3 \lambda+2 \mu)^{2}\left(\operatorname{tr} \varepsilon_{n+1}\right)^{2}}{9\left(\varepsilon_{R}-\varepsilon_{D}\right)\left\|S_{n+1}\right\|^{2}}\right]
$$


Agrupando-se as constantes de cada um dos termos entre parênteses, tem-se:

$D_{n+1}=D_{n}+\Delta \lambda\left[\frac{k_{1}}{\left(1-D_{n+1}\right)}+\frac{k_{2}\left(1-D_{n+1}\right)\left(\operatorname{tr} \varepsilon_{n+1}\right)^{2}}{\left\|S_{n+1}\right\|^{2}}\right]$

Sendo:

$k_{1}=\frac{2 D_{C}(1+v)}{3\left(\varepsilon_{R}-\varepsilon_{D}\right)}$

$k_{2}=\frac{2 D_{C}(1-2 v)(3 \lambda+2 \mu)^{2}}{9\left(\varepsilon_{R}-\varepsilon_{D}\right)}$

A eq. (L.7) pode ser escrita de modo compacto, como se mostra abaixo:

$D_{n+1}=D_{n}+\Delta \lambda f_{D}$

Sendo:

$f_{D}=\frac{k_{1}}{\left(1-D_{n+1}\right)}+\frac{k_{2}\left(1-D_{n+1}\right)\left(\operatorname{tr} \varepsilon_{n+1}\right)^{2}}{\left\|S_{n+1}\right\|^{2}}$

Substituindo-se a eq. (L.10) na eq. (L.11), tem-se:

$$
f_{D}=\frac{k_{1}}{\left(1-D_{n}-\Delta \lambda f_{D}\right)}+\frac{k_{2}\left(1-D_{n}-\Delta \lambda f_{D}\right)\left(\operatorname{tr} \varepsilon_{n+1}\right)^{2}}{\left\|S_{n+1}\right\|^{2}}
$$




\section{Apêndice M - Cálculo do tensor algorítmico tangente segundo o modelo de Lemaitre}

Seja a expressão do tensor de tensões em forma incremental $\sigma_{n+1}$ dada pela eq. (282):

$\sigma_{n+1}=C \varepsilon_{n+1}-D_{n+1} C \varepsilon_{n+1}-C \varepsilon_{n}^{p}+D_{n+1} C \varepsilon_{n}^{p}-\Delta \lambda C n_{n+1}$

E o tensor algorítmico tangente, dado pela eq. (281), tem por expressão:

$$
C_{n+1}^{e p, d}=\frac{\partial \sigma_{n+1}}{\partial \varepsilon_{n+1}}
$$

A derivada parcial indicada na eq. (M.2) deve ser feita em relação a cada um dos cinco termos que compõem o tensor de tensões $\sigma_{n+1}$. É o que se apresenta na seqüência:

1) Em relação ao primeiro termo:

$$
\frac{\partial\left(C \varepsilon_{n+1}\right)}{\partial \varepsilon_{n+1}}=C
$$

2) Em relação ao segundo termo:

$$
\frac{\partial\left(-D_{n+1} C \varepsilon_{n+1}\right)}{\partial \varepsilon_{n+1}}=-\left[D_{n+1} \frac{\partial\left(C \varepsilon_{n+1}\right)}{\partial \varepsilon_{n+1}}+C \varepsilon_{n+1} \otimes \frac{\partial D_{n+1}}{\partial \varepsilon_{n+1}}\right]=-\left[D_{n+1} C+C \varepsilon_{n+1} \otimes \frac{\partial D_{n+1}}{\partial \varepsilon_{n+1}}\right]
$$

Aplicando-se o tensor elástico linear $C=\lambda(\mathrm{I} \otimes \mathrm{I})+2 \mu \Pi$, dado pela eq. (122), ao tensor de deformações $\varepsilon_{n+1}$, obtém-se:

$$
C \varepsilon_{n+1}=\lambda\left(\mathrm{I} \cdot \varepsilon_{n+1}\right) \mathrm{I}+2 \mu \Pi \varepsilon_{n+1}=\lambda\left(\operatorname{tr} \varepsilon_{n+1}\right) \mathrm{I}+2 \mu \varepsilon_{n+1}
$$

E substituindo-se a eq. (M.5) na eq. (M.4), chega-se a:

$$
\frac{\partial\left(-D_{n+1} C \varepsilon_{n+1}\right)}{\partial \varepsilon_{n+1}}=-D_{n+1} C-\lambda\left(\operatorname{tr} \varepsilon_{n+1}\right) \mathrm{I} \otimes \frac{\partial D_{n+1}}{\partial \varepsilon_{n+1}}-2 \mu \varepsilon_{n+1} \otimes \frac{\partial D_{n+1}}{\partial \varepsilon_{n+1}}
$$

3) Em relação ao terceiro termo:

$$
\frac{\partial\left(-C \varepsilon_{n}^{p}\right)}{\partial \varepsilon_{n+1}}=0
$$

4) Em relação ao quarto termo:

$$
\frac{\partial\left(D_{n+1} C \varepsilon_{n}^{p}\right)}{\partial \varepsilon_{n+1}}=D_{n+1} \frac{\partial\left(C \varepsilon_{n}^{p}\right)}{\partial \varepsilon_{n+1}}+C \varepsilon_{n}^{p} \otimes \frac{\partial D_{n+1}}{\partial \varepsilon_{n+1}}
$$

Fazendo-se uso da eq. (M.7), obtém-se: 
$\frac{\partial\left(D_{n+1} C \varepsilon_{n}^{p}\right)}{\partial \varepsilon_{n+1}}=C \varepsilon_{n}^{p} \otimes \frac{\partial D_{n+1}}{\partial \varepsilon_{n+1}}$

Aplicando-se o tensor elástico linear $C$ a $\varepsilon_{n}^{p}$ e sendo $\operatorname{tr} \varepsilon_{n}^{p}=0$, obtém-se:

$C \varepsilon_{n}^{p}=\lambda\left(\mathrm{I} \cdot \varepsilon_{n}^{p}\right) \mathrm{I}+2 \mu \Pi \varepsilon_{n}^{p}=\lambda\left(\operatorname{tr} \varepsilon_{n}^{p}\right) \mathrm{I}+2 \mu \varepsilon_{n}^{p}=2 \mu \varepsilon_{n}^{p}$

Substituindo-se a eq. (M.10) na eq. (M.9), obtém-se finalmente:

$$
\frac{\partial\left(D_{n+1} C \varepsilon_{n}^{p}\right)}{\partial \varepsilon_{n+1}}=2 \mu \varepsilon_{n}^{p} \otimes \frac{\partial D_{n+1}}{\partial \varepsilon_{n+1}}
$$

5) Em relação ao quinto termo:

$$
\frac{\partial\left(-\Delta \lambda C n_{n+1}\right)}{\partial \varepsilon_{n+1}}=-\left[\Delta \lambda \frac{\partial\left(C n_{n+1}\right)}{\partial \varepsilon_{n+1}}+C n_{n+1} \otimes \frac{\partial \Delta \lambda}{\partial \varepsilon_{n+1}}\right]
$$

Da aplicação do tensor $C$ a $n_{n+1}$ resulta, sendo $\operatorname{tr} n_{n+1}=0$ :

$$
C n_{n+1}=\lambda\left(\mathrm{I} \cdot n_{n+1}\right) \mathrm{I}+2 \mu \prod n_{n+1}=\lambda\left(\operatorname{tr} n_{n+1}\right) \mathrm{I}+2 \mu n_{n+1}=2 \mu n_{n+1}
$$

E substituindo-se (M.13) em (M.12), chega-se à expressão:

$$
\frac{\partial\left(-\Delta \lambda C n_{n+1}\right)}{\partial \varepsilon_{n+1}}=-2 \mu \Delta \lambda \frac{\partial n_{n+1}}{\partial \varepsilon_{n+1}}-2 \mu n_{n+1} \otimes \frac{\partial \Delta \lambda}{\partial \varepsilon_{n+1}}
$$

Somando-se as expressões dadas pelas eqs. (M.3), (M.6), (M.7), (M.11) e (M.14), obtém-se, finalmente, o tensor algorítmico tangente $C_{n+1}^{e p, d}$ :

$$
\begin{aligned}
C_{n+1}^{e p, d}= & \left(1-D_{n+1}\right) C-\lambda\left(\operatorname{tr} \varepsilon_{n+1}\right) \mathrm{I} \otimes \frac{\partial D_{n+1}}{\partial \varepsilon_{n+1}}-2 \mu \varepsilon_{n+1} \otimes \frac{\partial D_{n+1}}{\partial \varepsilon_{n+1}}+2 \mu \varepsilon_{n}^{p} \otimes \frac{\partial D_{n+1}}{\partial \varepsilon_{n+1}}- \\
& -2 \mu \Delta \lambda \frac{\partial n_{n+1}}{\partial \varepsilon_{n+1}}-2 \mu n_{n+1} \otimes \frac{\partial \Delta \lambda}{\partial \varepsilon_{n+1}}
\end{aligned}
$$




\section{Apêndice $\mathbf{N}$ - Dedução da expressão dos tensores $\frac{\partial n_{n+1}}{\partial \varepsilon_{n+1}}, \frac{\partial \Delta \lambda}{\partial \varepsilon_{n+1}}$ e} $\frac{\partial D_{n+1}}{\partial \varepsilon_{n+1}}$

1) Cálculo de $\frac{\partial n_{n+1}}{\partial \varepsilon_{n+1}}$.

Por definição, admite-se válida a relação $n_{n+1}^{p r}=\frac{S_{n+1}^{p r}}{\left\|S_{n+1}^{p r}\right\|}$. Através da eq. (271), pôdese demonstrar também que $n_{n+1}=n_{n+1}^{p r}$. Portanto, a derivada procurada pode ser escrita como mostrado abaixo:

$$
\begin{aligned}
& \frac{\partial n_{n+1}}{\partial \varepsilon_{n+1}}=\frac{\partial\left(\frac{1}{\left\|S_{n+1}^{p r}\right\|} S_{n+1}^{p r}\right)}{\partial \varepsilon_{n+1}}=\frac{1}{\left\|S_{n+1}^{p r}\right\|} \frac{\partial S_{n+1}^{p r}}{\partial \varepsilon_{n+1}}+S_{n+1}^{p r} \otimes \frac{\partial\left(\frac{1}{\left\|S_{n+1}^{p r}\right\|}\right)}{\partial \varepsilon_{n+1}}= \\
& =\frac{1}{\left\|S_{n+1}^{p r}\right\|} \frac{\partial S_{n+1}^{p r}}{\partial \varepsilon_{n+1}}+S_{n+1}^{p r} \otimes \frac{-1}{\left\|S_{n+1}^{p r}\right\|^{2}} \frac{\partial\left\|S_{n+1}^{p r}\right\|}{\partial \varepsilon_{n+1}}=\frac{1}{\left\|S_{n+1}^{p r}\right\|} \frac{\partial S_{n+1}^{p r}}{\partial \varepsilon_{n+1}}-\frac{1}{\left\|S_{n+1}^{p r}\right\|^{2}} S_{n+1}^{p r} \otimes \frac{\partial\left\|S_{n+1}^{p r}\right\|}{\partial \varepsilon_{n+1}}
\end{aligned}
$$

Verifica-se então a necessidade de calcular as seguintes derivadas parciais: $\frac{\partial S_{n+1}^{p r}}{\partial \varepsilon_{n+1}} \mathrm{e}$ $\frac{\partial\left\|S_{n+1}^{p r}\right\|}{\partial \varepsilon_{n+1}}$

1.1) Cálculo de $\frac{\partial S_{n+1}^{p r}}{\partial \varepsilon_{n+1}}$.

Utilizando-se as eqs. (265) e (154), chega-se à seguinte expressão para o tensor desviador de tensões de previsão:

$$
S_{n+1}^{p r}=\left(1-D_{n}\right) 2 \mu\left[\varepsilon_{n+1}-\frac{1}{3}\left(\operatorname{tr} \varepsilon_{n+1}\right) \mathrm{I}-\varepsilon_{n}^{p}\right]
$$

A eq. (N.2) pode ser escrita, utilizando-se tensores de quarta ordem aplicados a $\varepsilon_{n+1}$ e a $\varepsilon_{n}^{p}$, na forma seguinte:

$$
S_{n+1}^{p r}=\left(1-D_{n}\right) 2 \mu\left[\Pi-\frac{1}{3}(\mathrm{I} \otimes \mathrm{I})\right] \varepsilon_{n+1}-\left(1-D_{n}\right) 2 \mu \Pi \varepsilon_{n}^{p}
$$

Portanto, a derivada parcial procurada resulta: 
$\frac{\partial S_{n+1}^{p r}}{\partial \varepsilon_{n+1}}=\left(1-D_{n}\right) 2 \mu\left[\Pi-\frac{1}{3}(\mathrm{I} \otimes \mathrm{I})\right]$

1.2) Cálculo de $\frac{\partial\left\|S_{n+1}^{p r}\right\|}{\partial \varepsilon_{n+1}}$.

Da própria definição de norma de um tensor, tem-se:

$$
\left\|S_{n+1}^{p r}\right\|=\left(S_{n+1}^{p r} \cdot S_{n+1}^{p r}\right)^{\frac{1}{2}}
$$

Substituindo-se a eq. (N.5) na derivada procurada, obtém-se:

$$
\frac{\partial\left\|S_{n+1}^{p r}\right\|}{\partial \varepsilon_{n+1}}=\frac{\partial\left(S_{n+1}^{p r} \cdot S_{n+1}^{p r}\right)^{\frac{1}{2}}}{\partial \varepsilon_{n+1}}=\frac{1}{2} \frac{1}{\left(S_{n+1}^{p r} \cdot S_{n+1}^{p r}\right)^{\frac{1}{2}}}\left[2 \frac{\partial S_{n+1}^{p r}}{\partial \varepsilon_{n+1}} S_{n+1}^{p r}\right]=\frac{1}{\left\|S_{n+1}^{p r}\right\|}\left[\frac{\partial S_{n+1}^{p r}}{\partial \varepsilon_{n+1}} S_{n+1}^{p r}\right]
$$

Substituindo-se a eq. (N.4) na eq. (N.6), chega-se, finalmente, à expressão:

$$
\begin{aligned}
& \frac{\partial\left\|S_{n+1}^{p r}\right\|}{\partial \varepsilon_{n+1}}=\frac{\left(1-D_{n}\right) 2 \mu}{\left\|S_{n+1}^{p r}\right\|}\left[\Pi-\frac{1}{3}(\mathrm{I} \otimes \mathrm{I})\right] S_{n+1}^{p r}=\frac{\left(1-D_{n}\right) 2 \mu}{\left\|S_{n+1}^{p r}\right\|}\left[S_{n+1}^{p r}-\frac{1}{3}\left(\mathrm{I} \cdot S_{n+1}^{p r}\right) \mathrm{I}\right] \Rightarrow \\
& \frac{\partial\left\|S_{n+1}^{p r}\right\|}{\partial \varepsilon_{n+1}}=\frac{\left(1-D_{n}\right) 2 \mu}{\left\|S_{n+1}^{p r}\right\|} S_{n+1}^{p r}=\left(1-D_{n}\right) 2 \mu n_{n+1}
\end{aligned}
$$

Retoma-se então o cálculo de $\frac{\partial n_{n+1}}{\partial \varepsilon_{n+1}}$, substituindo-se as eqs. (N.4) e (N.7) na eq. (N.1), o que resulta em:

$$
\frac{\partial n_{n+1}}{\partial \varepsilon_{n+1}}=\frac{\left(1-D_{n}\right) 2 \mu}{\left\|S_{n+1}^{p r}\right\|}\left[\Pi-\frac{1}{3}(\mathrm{I} \otimes \mathrm{I})-n_{n+1} \otimes n_{n+1}\right]
$$

A eq. (N.8) pode ser reescrita na forma:

$$
\frac{\partial n_{n+1}}{\partial \varepsilon_{n+1}}=K N_{1} \Pi+K N_{2}(\mathrm{I} \otimes \mathrm{I})+K N_{3}\left(n_{n+1} \otimes n_{n+1}\right)
$$

Sendo:

$$
\begin{aligned}
& K N_{1}=\frac{2 \mu\left(1-D_{n}\right)}{\left\|S_{n+1}^{p r}\right\|} \\
& K N_{2}=-\frac{2 \mu\left(1-D_{n}\right)}{3\left\|S_{n+1}^{p r}\right\|}=-\frac{1}{3} K N_{1} \\
& K N_{3}=-\frac{2 \mu\left(1-D_{n}\right)}{\left\|S_{n+1}^{p r}\right\|}=-K N_{1}
\end{aligned}
$$


2) Cálculo de $\frac{\partial \Delta \lambda}{\partial \varepsilon_{n+1}}$.

Seja a expressão de $\Delta \lambda$, dada pela eq. (278):

$$
\Delta \lambda=\frac{f_{n+1}^{p r}}{\left(2 \mu+\frac{2}{3} K\right)}-\frac{k_{1}\left(f_{n+1}^{p r}\right)^{2}}{\left(1-D_{n}\right)^{2}\left(2 \mu+\frac{2}{3} K\right)^{2}}-\frac{k_{2}\left(\operatorname{tr} \varepsilon_{n+1}\right)^{2}\left(f_{n+1}^{p r}\right)^{2}}{\left(2 \mu+\frac{2}{3} K\right)^{2}\left[\left\|S_{n+1}^{p r}\right\|-\frac{2 \mu f_{n+1}^{p r}}{\left(2 \mu+\frac{2}{3} K\right)}\right]^{2}}
$$

Nos itens 2.1, 2.2 e 2.3 apresentam-se as derivadas parciais em relação a $\varepsilon_{n+1}$ de cada uma das três parcelas de $\Delta \lambda$.

2.1) Em relação à primeira parcela de $\Delta \lambda$ :

$$
\frac{1}{\left(2 \mu+\frac{2}{3} K\right)} \frac{\partial f_{n+1}^{p r}}{\partial \varepsilon_{n+1}}
$$

Sendo a expressão de $f_{n+1}^{p r}$ :

$$
f_{n+1}^{p r}=\left\|S_{n+1}^{p r}\right\|-\sqrt{\frac{2}{3}}\left(\sigma_{t}+K \alpha_{n}\right)\left(1-D_{n}\right)
$$

Conclui-se, utilizando-se a eq. (N.7):

$\frac{\partial f_{n+1}^{p r}}{\partial \varepsilon_{n+1}}=\frac{\partial\left\|S_{n+1}^{p r}\right\|}{\partial \varepsilon_{n+1}}=\left(1-D_{n}\right) 2 \mu n_{n+1}$

Substituindo-se (N.16) em (N.14), obtém-se finalmente:

$$
\frac{1}{\left(2 \mu+\frac{2}{3} K\right)} \frac{\partial f_{n+1}^{p r}}{\partial \varepsilon_{n+1}}=\frac{\left(1-D_{n}\right) 2 \mu}{\left(2 \mu+\frac{2}{3} K\right)} n_{n+1}
$$

2.2) Em relação à segunda parcela de $\Delta \lambda$ :

$$
-\frac{k_{1}}{\left(1-D_{n}\right)^{2}\left(2 \mu+\frac{2}{3} K\right)^{2}} \frac{\partial\left(f_{n+1}^{p r}\right)^{2}}{\partial \varepsilon_{n+1}}
$$

Calcula-se $\frac{\partial\left(f_{n+1}^{p r}\right)^{2}}{\partial \varepsilon_{n+1}}$, fazendo-se uso da eq. (N.16):

$$
\frac{\partial\left(f_{n+1}^{p r}\right)^{2}}{\partial \varepsilon_{n+1}}=2 f_{n+1}^{p r} \frac{\partial f_{n+1}^{p r}}{\partial \varepsilon_{n+1}}=4 \mu\left(1-D_{n}\right) f_{n+1}^{p r} n_{n+1}
$$

Substituindo-se a eq. (N.19) na eq. (N.18), chega-se à expressão: 


$$
-\frac{k_{1}}{\left(1-D_{n}\right)^{2}\left(2 \mu+\frac{2}{3} K\right)^{2}} 4 \mu\left(1-D_{n}\right) f_{n+1}^{p r} n_{n+1}=\frac{-4 \mu k_{1} f_{n+1}^{p r}}{\left(1-D_{n}\right)\left(2 \mu+\frac{2}{3} K\right)^{2}} n_{n+1}
$$

2.3) Em relação à terceira parcela de $\Delta \lambda$ :

$$
\frac{-\frac{k_{2}}{\left(2 \mu+\frac{2}{3} K\right)^{2}} \partial\left\{\left(\operatorname{tr} \varepsilon_{n+1}\right)^{2}\left(f_{n+1}^{p r}\right)^{2}\left[\left\|S_{n+1}^{p r}\right\|-\frac{2 \mu f_{n+1}^{p r}}{\left(2 \mu+\frac{2}{3} K\right)}\right]^{-2}\right\}}{\partial \varepsilon_{n+1}}
$$

Para o cálculo da derivada parcial dada pela eq. (N.21), deve-se usar a regra da cadeia. Faz-se necessário, portanto, conhecer a expressão das três derivadas parciais seguintes:

$$
\text { 2.3.1) } \frac{\partial\left(\operatorname{tr} \varepsilon_{n+1}\right)^{2}}{\partial \varepsilon_{n+1}}
$$

Pode-se utilizar o produto interno $\left(\mathrm{I} \cdot \varepsilon_{n+1}\right)$ em lugar do traço $\operatorname{tr} \varepsilon_{n+1}$. Dessa forma, a expressão anterior resulta em:

$$
\begin{aligned}
\frac{\partial\left(\mathrm{I} \cdot \varepsilon_{n+1}\right)^{2}}{\partial \varepsilon_{n+1}} & =2\left(\mathrm{I} \cdot \varepsilon_{n+1}\right) \frac{\partial\left(\mathrm{I} \cdot \varepsilon_{n+1}\right)}{\partial \varepsilon_{n+1}}=2\left(\operatorname{tr} \varepsilon_{n+1}\right)\left[\frac{\partial \mathrm{I}}{\partial \varepsilon_{n+1}} \varepsilon_{n+1}+\frac{\partial \varepsilon_{n+1}}{\partial \varepsilon_{n+1}} \mathrm{I}\right]= \\
& =2\left(\operatorname{tr} \varepsilon_{n+1}\right)[\Pi \mathrm{I}]=2\left(\operatorname{tr} \varepsilon_{n+1}\right) \mathrm{I}
\end{aligned}
$$

2.3.2) $\frac{\partial\left(f_{n+1}^{p r}\right)^{2}}{\partial \varepsilon_{n+1}}$,já expressa pela eq. (N.19).

$2.3 .3) \frac{\partial\left[\left\|S_{n+1}^{p r}\right\|-\frac{2 \mu f_{n+1}^{p r}}{\left(2 \mu+\frac{2}{3} K\right)}\right]^{-2}}{\partial \varepsilon_{n+1}}$. Esta expressão equivale a:

$$
-2\left[\left\|S_{n+1}^{p r}\right\|-\frac{2 \mu f_{n+1}^{p r}}{\left(2 \mu+\frac{2}{3} K\right)}\right]^{-3}\left[\frac{\partial\left\|S_{n+1}^{p r}\right\|}{\partial \varepsilon_{n+1}}-\frac{2 \mu}{\left(2 \mu+\frac{2}{3} K\right)} \frac{\partial f_{n+1}^{p r}}{\partial \varepsilon_{n+1}}\right]
$$

Substituindo-se na eq. (N.23) as expressões de $\frac{\partial\left\|S_{n+1}^{p r}\right\|}{\partial \varepsilon_{n+1}}$ e $\frac{\partial f_{n+1}^{p r}}{\partial \varepsilon_{n+1}}$, dadas pelas eqs. (N.7) e (N.16), respectivamente, obtém-se a derivada procurada: 


$$
\frac{\partial\left[\left\|S_{n+1}^{p r}\right\|-\frac{2 \mu f_{n+1}^{p r}}{\left(2 \mu+\frac{2}{3} K\right)}\right]^{-2}}{\partial \varepsilon_{n+1}}=\frac{-8 \mu K\left(1-D_{n}\right)}{3\left(2 \mu+\frac{2}{3} K\right)\left[\left\|S_{n+1}^{p r}\right\|-\frac{2 \mu f_{n+1}^{p r}}{\left(2 \mu+\frac{2}{3} K\right)}\right]^{3}} n_{n+1}
$$

Aplicando-se a regra da cadeia, obtém-se a expressão da derivada parcial da terceira parcela de $\Delta \lambda$ em relação a $\varepsilon_{n+1}$ :

$$
\begin{aligned}
& \frac{-2 k_{2}\left(\operatorname{tr} \varepsilon_{n+1}\right)\left(f_{n+1}^{p r}\right)^{2}}{\left(2 \mu+\frac{2}{3} K\right)^{2}\left[\left\|S_{n+1}^{p r}\right\|-\frac{2 \mu f_{n+1}^{p r}}{\left(2 \mu+\frac{2}{3} K\right)}\right]^{2}} \mathrm{I}-\frac{4 \mu k_{2}\left(\operatorname{tr} \varepsilon_{n+1}\right)^{2}\left(1-D_{n}\right) f_{n+1}^{p r}}{\left(2 \mu+\frac{2}{3} K\right)^{2}\left[\left\|S_{n+1}^{p r}\right\|-\frac{2 \mu f_{n+1}^{p r}}{\left(2 \mu+\frac{2}{3} K\right)}\right]^{2}} n_{n+1}+ \\
& +\frac{8 \mu K k_{2}\left(\operatorname{tr} \varepsilon_{n+1}\right)^{2}\left(1-D_{n}\right)\left(f_{n+1}^{p r}\right)^{2}}{3\left(2 \mu+\frac{2}{3} K\right)^{3}\left[\left\|S_{n+1}^{p r}\right\|-\frac{2 \mu f_{n+1}^{p r}}{\left(2 \mu+\frac{2}{3} K\right)}\right]^{3}} n_{n+1}
\end{aligned}
$$

Somando-se as expressões dadas pelas eqs. (N.17), (N.20) e (N.25), obtém-se:

$$
\begin{gathered}
\frac{\partial \Delta \lambda}{\partial \varepsilon_{n+1}}=\frac{2 \mu\left(1-D_{n}\right)}{\left(2 \mu+\frac{2}{3} K\right)} n_{n+1}-\frac{4 \mu k_{1} f_{n+1}^{p r}}{\left(1-D_{n}\right)\left(2 \mu+\frac{2}{3} K\right)^{2}} n_{n+1}-\frac{2 k_{2}\left(\operatorname{tr} \varepsilon_{n+1}\right)\left(f_{n+1}^{p r}\right)^{2}}{\left(2 \mu+\frac{2}{3} K\right)^{2}\left[\left\|S_{n+1}^{p r}\right\|-\frac{2 \mu f_{n+1}^{p r}}{\left(2 \mu+\frac{2}{3} K\right)}\right]^{2} \mathrm{I}-} \\
-\frac{4 \mu k_{2}\left(\operatorname{tr} \varepsilon_{n+1}\right)^{2}\left(1-D_{n}\right) f_{n+1}^{p r}}{\left(2 \mu+\frac{2}{3} K\right)^{2}\left[\left\|S_{n+1}^{p r}\right\|-\frac{2 \mu f_{n+1}^{p r}}{\left(2 \mu+\frac{2}{3} K\right)}\right]^{2}} n_{n+1}+\frac{8 \mu K k_{2}\left(\operatorname{tr} \varepsilon_{n+1}\right)^{2}\left(1-D_{n}\right)\left(f_{n+1}^{p r}\right)^{2}}{3\left(2 \mu+\frac{2}{3} K\right)^{3}\left[\left\|S_{n+1}^{p r}\right\|-\frac{2 \mu f_{n+1}^{p r}}{\left(2 \mu+\frac{2}{3} K\right)}\right]_{n+1}}
\end{gathered}
$$

A expressão de $\frac{\partial \Delta \lambda}{\partial \varepsilon_{n+1}}$ dada por (N.26) pode ser escrita na seguinte forma compacta:

$$
\frac{\partial \Delta \lambda}{\partial \varepsilon_{n+1}}=K L_{1} \mathrm{I}+K L_{2} n_{n+1}
$$

Sendo: 


$$
\begin{aligned}
K L_{1}= & \frac{-2 k_{2}\left(\operatorname{tr} \varepsilon_{n+1}\right)\left(f_{n+1}^{p r}\right)^{2}}{\left(2 \mu+\frac{2}{3} K\right)^{2}\left[\left\|S_{n+1}^{p r}\right\|-\frac{2 \mu f_{n+1}^{p r}}{\left(2 \mu+\frac{2}{3} K\right)}\right]^{2}} \quad \text { (N.28) } \\
K L_{2}= & \frac{2 \mu}{\left(2 \mu+\frac{2}{3} K\right)}\left[\left(1-D_{n}\right)-\frac{2 k_{1} f_{n+1}^{p r}}{\left(1-D_{n}\right)\left(2 \mu+\frac{2}{3} K\right)}\right]+ \\
+ & \frac{4 \mu k_{2}\left(\operatorname{tr} \varepsilon_{n+1}\right)^{2} f_{n+1}^{p r}\left(1-D_{n}\right)}{2}\left[\| \frac{2 K f_{n+1}^{p r}}{3} K\right)^{2}\left[\left\|S_{n+1}^{p r}\right\|-\frac{2 \mu f_{n+1}^{p r}}{\left(2 \mu+\frac{2}{3} K\right)}\right]^{2}\left[-1+\frac{2}{3\left(2 \mu+\frac{2}{3} K\right)\left[S_{n+1}^{p r} \|-\frac{2 \mu f_{n+1}^{p r}}{\left(2 \mu+\frac{2}{3} K\right)}\right]}\right]
\end{aligned}
$$

3) Cálculo de $\frac{\partial D_{n+1}}{\partial \varepsilon_{n+1}}$.

Seja a expressão para a evolução do dano em passo finito, dada pela eq. (280):

$$
D_{n+1}=D_{n}+\frac{k_{1} f_{n+1}^{p r}}{\left(1-D_{n}\right)\left(2 \mu+\frac{2}{3} K\right)}+\frac{k_{2}\left(1-D_{n}\right)\left(\operatorname{tr} \varepsilon_{n+1}\right)^{2} f_{n+1}^{p r}}{\left(2 \mu+\frac{2}{3} K\right)\left[\left\|S_{n+1}^{p r}\right\|-\frac{2 \mu f_{n+1}^{p r}}{\left(2 \mu+\frac{2}{3} K\right)}\right]^{2}}
$$

Nos itens 3.1, 3.2 e 3.3, apresenta-se o cálculo da derivada parcial em relação a $\varepsilon_{n+1}$ de cada uma das três parcelas da eq. (N.30).

3.1) $\frac{\partial D_{n}}{\partial \varepsilon_{n+1}}=0$

3.2) $\frac{k_{1}}{\left(1-D_{n}\right)\left(2 \mu+\frac{2}{3} K\right)} \frac{\partial f_{n+1}^{p r}}{\partial \varepsilon_{n+1}}$

Substituindo-se a eq. (N.16) na eq. (N.32), chega-se a:

$$
\frac{k_{1}}{\left(1-D_{n}\right)\left(2 \mu+\frac{2}{3} K\right)}\left(1-D_{n}\right) 2 \mu n_{n+1}=\frac{2 \mu k_{1}}{\left(2 \mu+\frac{2}{3} K\right)} n_{n+1}
$$

3.3) Deve-se calcular: 
$\frac{k_{2}\left(1-D_{n}\right)}{\left(2 \mu+\frac{2}{3} K\right)} \frac{\partial\left\{\left(\operatorname{tr} \varepsilon_{n+1}\right)^{2} f_{n+1}^{p r}\left[\left\|S_{n+1}^{p r}\right\|-\frac{2 \mu f_{n+1}^{p r}}{\left(2 \mu+\frac{2}{3} K\right)}\right]^{-2}\right\}}{\partial \varepsilon_{n+1}}$

Utiliza-se a regra da cadeia para a derivação da eq. (N.34), sendo que as derivadas

parciais $\frac{\partial\left(\operatorname{tr} \varepsilon_{n+1}\right)^{2}}{\partial \varepsilon_{n+1}}, \quad \frac{\partial f_{n+1}^{p r}}{\partial \varepsilon_{n+1}} \mathrm{e} \frac{\partial\left[\left\|S_{n+1}^{p r}\right\|-\frac{2 \mu f_{n+1}^{p r}}{\left(2 \mu+\frac{2}{3} K\right)}\right]^{-2}}{\partial \varepsilon_{n+1}}$ têm suas expressões definidas pelas eqs. (N.22), (N.16) e (N.24), respectivamente. Como resultado, obtém-se:

$$
\begin{aligned}
& \frac{2 k_{2}\left(1-D_{n}\right)\left(\operatorname{tr} \varepsilon_{n+1}\right) f_{n+1}^{p r}}{\left(2 \mu+\frac{2}{3} K\right)\left[\left\|S_{n+1}^{p r}\right\|-\frac{2 \mu f_{n+1}^{p r}}{\left(2 \mu+\frac{2}{3} K\right)}\right]^{2}} \mathrm{I}+\frac{2 \mu k_{2}\left(1-D_{n}\right)^{2}\left(\operatorname{tr} \varepsilon_{n+1}\right)^{2}}{\left(2 \mu+\frac{2}{3} K\right)\left[\left\|S_{n+1}^{p r}\right\|-\frac{2 \mu f_{n+1}^{p r}}{\left(2 \mu+\frac{2}{3} K\right)}\right]^{2}} n_{n+1}- \\
& -\frac{8 \mu K k_{2}\left(1-D_{n}\right)^{2}\left(t r \varepsilon_{n+1}\right)^{2} f_{n+1}^{p r}}{3\left(2 \mu+\frac{2}{3} K\right)^{2}\left[\left\|S_{n+1}^{p r}\right\|-\frac{2 \mu f_{n+1}^{p r}}{\left(2 \mu+\frac{2}{3} K\right)}\right]^{3}} n_{n+1}
\end{aligned}
$$

Portanto, somando-se as expressões dadas pelas eqs. (N.31), (N.33) e (N.35), temse:

$$
\begin{aligned}
& \frac{\partial D_{n+1}}{\partial \varepsilon_{n+1}}=\frac{2 \mu k_{1}}{\left(2 \mu+\frac{2}{3} K\right)} n_{n+1}+\frac{2 k_{2}\left(1-D_{n}\right)\left(\operatorname{tr} \varepsilon_{n+1}\right) f_{n+1}^{p r}}{\left(2 \mu+\frac{2}{3} K\right)\left[\left\|S_{n+1}^{p r}\right\|-\frac{2 \mu f_{n+1}^{p r}}{\left(2 \mu+\frac{2}{3} K\right)}\right]^{2}} \mathrm{I}+ \\
& \left.+\frac{2 \mu k_{2}\left(1-D_{n}\right)^{2}\left(\operatorname{tr} \varepsilon_{n+1}\right)^{2}}{\left(2 \mu+\frac{2}{3} K\right)\left[\left\|S_{n+1}^{p r}\right\|-\frac{2 \mu f_{n+1}^{p r}}{\left(2 \mu+\frac{2}{3} K\right)}\right]^{2}} n_{n+1}-\frac{8 \mu K k_{2}\left(1-D_{n}\right)^{2}\left(t r \varepsilon_{n+1}\right)^{2} f_{n+1}^{p r}}{3\left(2 \mu+\frac{2}{3} K\right)^{2}\left[\left\|S_{n+1}^{p r}\right\|-\frac{2 \mu f_{n+1}^{p r}}{2}\right]_{n+1}^{3}} n^{\left(2 \mu+\frac{2}{3} K\right)}\right]^{2}
\end{aligned}
$$

A eq. (N.36) pode ser escrita em forma compacta como mostrado a seguir: 


$$
\frac{\partial D_{n+1}}{\partial \varepsilon_{n+1}}=K D_{1} \mathrm{I}+K D_{2} n_{n+1}
$$

Sendo:

$$
\begin{aligned}
K D_{1}= & \frac{2 k_{2}\left(1-D_{n}\right)\left(t r \varepsilon_{n+1}\right) f_{n+1}^{p r}}{\left(2 \mu+\frac{2}{3} K\right)\left[\left\|S_{n+1}^{p r}\right\|-\frac{2 \mu f_{n+1}^{p r}}{\left(2 \mu+\frac{2}{3} K\right)}\right]^{2}} \\
K D_{2}= & \frac{2 \mu k_{1}}{\left(2 \mu+\frac{2}{3} K\right)}+\frac{2 \mu k_{2}\left(1-D_{n}\right)^{2}\left(t r \varepsilon_{n+1}\right)^{2}}{\left(2 \mu+\frac{2}{3} K\right)\left[\left\|S_{n+1}^{p r}\right\|-\frac{2 \mu f_{n+1}^{p r}}{\left(2 \mu+\frac{2}{3} K\right)}\right]^{2}}- \\
& -\frac{8 \mu K k_{2}\left(1-D_{n}\right)^{2}\left(t r \varepsilon_{n+1}\right)^{2} f_{n+1}^{p r}}{3\left(2 \mu+\frac{2}{3} K\right)^{2}\left[\left\|S_{n+1}^{p r}\right\|-\frac{2 \mu f_{n+1}^{p r}}{\left(2 \mu+\frac{2}{3} K\right)}\right]^{3}}
\end{aligned}
$$




\section{Apêndice O - Matriz da relação constitutiva tangente $\left(D_{6 \times 6}^{e p}\right)-$}

\section{modelo de Lemaitre}

Apresenta-se a dedução, a partir do tensor algorítmico tangente $C_{n+1}^{e p, d}$, da matriz da relação constitutiva tangente $D^{e p, d}$, de dimensão $6 \times 6$, que relaciona, segundo o modelo de dano dúctil de Lemaitre, o vetor dos incrementos nas componentes de tensão $\delta \sigma^{T}=\left\{\begin{array}{llllll}\delta \sigma_{x} & \delta \sigma_{y} & \delta \sigma_{z} & \delta \tau_{x y} & \delta \tau_{x z} & \delta \tau_{y z}\end{array}\right\}$ ao vetor dos incrementos nas componentes de deformação $\delta \varepsilon^{T}=\left\{\begin{array}{llllll}\delta \varepsilon_{x} & \delta \varepsilon_{y} & \delta \varepsilon_{z} & \delta \gamma_{x y} & \delta \gamma_{x z} & \delta \gamma_{y z}\end{array}\right\}$.

Como se vê na eq. (287), o tensor algorítmico tangente $C_{n+1}^{e p, d}$ é dado por :

$$
C_{n+1}^{e p, d}=C_{n+1}^{e l, d}-C_{n+1}^{p, d}
$$

Portanto, a matriz $D^{e p, d}$ também pode ser desmembrada na forma seguinte:

$$
D^{e p, d}=D^{e l, d}-D^{p, d}
$$

A matriz $D^{e l, d}$, correspondente ao tensor $C_{n+1}^{e l, d}=\left(1-D_{n+1}\right) C$, é a matriz dos módulos elásticos lineares, multiplicada por $\left(1-D_{n+1}\right)$. Sua expressão é:

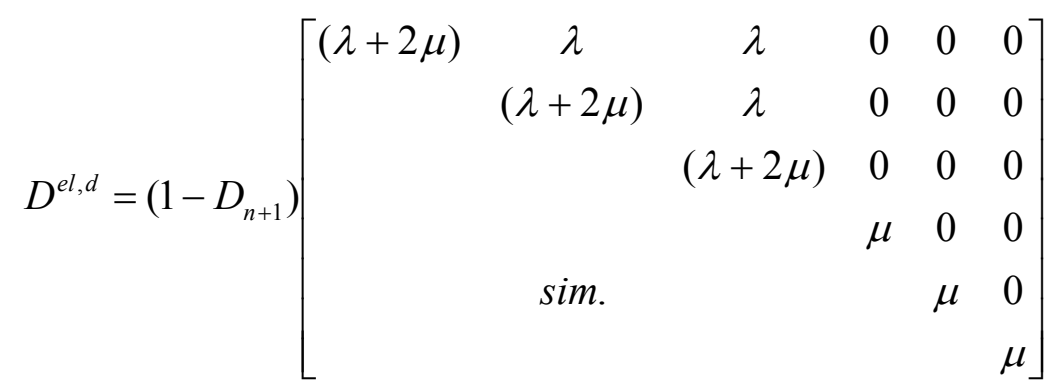

Sendo $\lambda$ e $\mu$ as constantes de Lamé, dadas pelas eqs. (17a) e (17b).

A matriz $D^{p, d}$ é obtida aplicando-se o tensor $C_{n+1}^{p, d}$, definido pela eq. (289), ao tensor de deformações de segunda ordem $\delta \varepsilon_{n+1}$.

$$
\begin{aligned}
C_{n+1}^{p, d}= & D P_{1} \Pi+D P_{2}(\mathrm{I} \otimes \mathrm{I})+D P_{3}\left(\varepsilon_{n+1} \otimes \mathrm{I}\right)+D P_{4}\left(\varepsilon_{n}^{p} \otimes \mathrm{I}\right)+D P_{5}\left(\mathrm{I} \otimes n_{n+1}\right)+ \\
& +D P_{6}\left(n_{n+1} \otimes \mathrm{I}\right)+D P_{7}\left(\varepsilon_{n+1} \otimes n_{n+1}\right)+D P_{8}\left(\varepsilon_{n}^{p} \otimes n_{n+1}\right)+D P_{9}\left(n_{n+1} \otimes n_{n+1}\right) \\
\delta \varepsilon_{n+1}= & {\left[\begin{array}{lll}
\delta \varepsilon_{x} & \delta \varepsilon_{x y} & \delta \varepsilon_{x z} \\
\delta \varepsilon_{x y} & \delta \varepsilon_{y} & \delta \varepsilon_{y z} \\
\delta \varepsilon_{x z} & \delta \varepsilon_{y z} & \delta \varepsilon_{z}
\end{array}\right] }
\end{aligned}
$$


Deve-se ressaltar que as componentes $\gamma_{i j}$ do vetor de incrementos de deformação $\delta \varepsilon$ têm como valor o dobro das correspondentes componentes $\varepsilon_{i j}, i \neq j$, no tensor de incrementos de deformação dado pela eq. (O.4).

A seguir, aplica-se cada uma das nove parcelas do tensor constituivo $C_{n+1}^{p, d}$ ao tensor dos incrementos nas deformações $\delta \varepsilon_{n+1}$, cujo índice é omitido, por comodidade. Da relação entre os vetores $\delta \sigma$ e $\delta \varepsilon$, obtém-se a respectiva matriz de relação constitutiva tangente $D_{(i, i=1, \ldots, 9)}^{p, d}$. Por simplicidade de notação, as componentes dos vetores de incrementos de deformação e de tensão são escritas com omissão do símbolo $\delta$.

1) $\sigma^{(1)}=D P_{1} \Pi \varepsilon=D P_{1} \varepsilon=\left[\begin{array}{ccc}D P_{1} \varepsilon_{x} & D P_{1} \varepsilon_{x y} & D P_{1} \varepsilon_{x z} \\ D P_{1} \varepsilon_{x y} & D P_{1} \varepsilon_{y} & D P_{1} \varepsilon_{y z} \\ D P_{1} \varepsilon_{x z} & D P_{1} \varepsilon_{y z} & D P_{1} \varepsilon_{z}\end{array}\right]$

As componentes de tensão são:

$\sigma_{x}^{(1)}=D P_{1} \varepsilon_{x}$

$\sigma_{y}^{(1)}=D P_{1} \varepsilon_{y}$

$\sigma_{z}^{(1)}=D P_{1} \varepsilon_{z}$

$\tau_{x y}^{(1)}=D P_{1} \varepsilon_{x y}=\frac{D P_{1}}{2} \gamma_{x y}$

$\tau_{x z}^{(1)}=D P_{1} \varepsilon_{x z}=\frac{D P_{1}}{2} \gamma_{x z}$

$\tau_{y z}^{(1)}=D P_{1} \varepsilon_{y z}=\frac{D P_{1}}{2} \gamma_{y z}$

A matriz da relação constitutiva referente a esta parcela do tensor $C_{n+1}^{p, d}$ é, portanto:

$D_{(1)}^{p, d}=\left[\begin{array}{cccccc}D P_{1} & 0 & 0 & 0 & 0 & 0 \\ 0 & D P_{1} & 0 & 0 & 0 & 0 \\ 0 & 0 & D P_{1} & 0 & 0 & 0 \\ 0 & 0 & 0 & \frac{D P_{1}}{2} & 0 & 0 \\ 0 & 0 & 0 & 0 & \frac{D P_{1}}{2} & 0 \\ 0 & 0 & 0 & 0 & 0 & \frac{D P_{1}}{2}\end{array}\right]$

2) $\sigma^{(2)}=D P_{2}(\mathrm{I} \otimes \mathrm{I}) \varepsilon=D P_{2}(\mathrm{I} \cdot \varepsilon) \mathrm{I}=D P_{2}(\operatorname{tr} \varepsilon) \mathrm{I} \Rightarrow$ 


$$
\sigma^{(2)}=\left[\begin{array}{ccc}
D P_{2}\left(\varepsilon_{x}+\varepsilon_{y}+\varepsilon_{z}\right) & 0 & 0 \\
0 & D P_{2}\left(\varepsilon_{x}+\varepsilon_{y}+\varepsilon_{z}\right) & 0 \\
0 & 0 & D P_{2}\left(\varepsilon_{x}+\varepsilon_{y}+\varepsilon_{z}\right)
\end{array}\right]
$$

Assim, as componentes de tensão são:

$$
\begin{aligned}
& \sigma_{x}^{(2)}=D P_{2} \varepsilon_{x}+D P_{2} \varepsilon_{y}+D P_{2} \varepsilon_{z} \\
& \sigma_{y}^{(2)}=D P_{2} \varepsilon_{x}+D P_{2} \varepsilon_{y}+D P_{2} \varepsilon_{z} \\
& \sigma_{z}^{(2)}=D P_{2} \varepsilon_{x}+D P_{2} \varepsilon_{y}+D P_{2} \varepsilon_{z} \\
& \tau_{x y}^{(2)}=0 \\
& \tau_{x z}^{(2)}=0 \\
& \tau_{y z}^{(2)}=0
\end{aligned}
$$

E a matriz da relação constitutiva referente a esta parcela do tensor $C_{n+1}^{p, d}$ é:

$$
D_{(2)}^{p, d}=\left[\begin{array}{cccccc}
D P_{2} & D P_{2} & D P_{2} & 0 & 0 & 0 \\
D P_{2} & D P_{2} & D P_{2} & 0 & 0 & 0 \\
D P_{2} & D P_{2} & D P_{2} & 0 & 0 & 0 \\
0 & 0 & 0 & 0 & 0 & 0 \\
0 & 0 & 0 & 0 & 0 & 0 \\
0 & 0 & 0 & 0 & 0 & 0
\end{array}\right]
$$

3) $\sigma^{(3)}=D P_{3}(\varepsilon \otimes \mathrm{I}) \varepsilon=D P_{3}(\mathrm{I} \cdot \varepsilon) \varepsilon=D P_{3}(\operatorname{tr} \varepsilon) \varepsilon=D P_{3}^{\prime} \varepsilon \Rightarrow$

$$
\sigma^{(3)}=\left[\begin{array}{ccc}
D P_{3}^{\prime} \varepsilon_{x} & D P_{3}^{\prime} \varepsilon_{x y} & D P_{3}^{\prime} \varepsilon_{x z} \\
D P_{3}^{\prime} \varepsilon_{x y} & D P_{3}^{\prime} \varepsilon_{y} & D P_{3}^{\prime} \varepsilon_{y z} \\
D P_{3}^{\prime} \varepsilon_{x z} & D P_{3}^{\prime} \varepsilon_{y z} & D P_{3}^{\prime} \varepsilon_{z}
\end{array}\right]
$$

Sendo $D P_{3}^{\prime}=D P_{3}(\operatorname{tr} \varepsilon)$.

As componentes de tensão são:

$$
\begin{aligned}
& \sigma_{x}^{(3)}=D P_{3}^{\prime} \varepsilon_{x} \\
& \sigma_{y}^{(3)}=D P_{3}^{\prime} \varepsilon_{y} \\
& \sigma_{z}^{(3)}=D P_{3}^{\prime} \varepsilon_{z} \\
& \tau_{x y}^{(3)}=D P_{3}^{\prime} \varepsilon_{x y}=\frac{D P_{3}^{\prime}}{2} \gamma_{x y} \\
& \tau_{x z}^{(3)}=D P_{3}^{\prime} \varepsilon_{x z}=\frac{D P_{3}^{\prime}}{2} \gamma_{x z}
\end{aligned}
$$


$\tau_{y z}^{(3)}=D P_{3}^{\prime} \varepsilon_{y z}=\frac{D P_{3}^{\prime}}{2} \gamma_{y z}$

Portanto, a matriz da relação constitutiva referente a esta parcela do tensor $C_{n+1}^{p, d}$ é:

$$
D_{(3)}^{p, d}=\left[\begin{array}{cccccc}
D P_{3}^{\prime} & 0 & 0 & 0 & 0 & 0 \\
0 & D P_{3}^{\prime} & 0 & 0 & 0 & 0 \\
0 & 0 & D P_{3}^{\prime} & 0 & 0 & 0 \\
0 & 0 & 0 & \frac{D P_{3}^{\prime}}{2} & 0 & 0 \\
0 & 0 & 0 & 0 & \frac{D P_{3}^{\prime}}{2} & 0 \\
0 & 0 & 0 & 0 & 0 & \frac{D P_{3}^{\prime}}{2}
\end{array}\right]
$$

4) $\sigma^{(4)}=D P_{4}\left(\varepsilon_{n}^{p} \otimes \mathrm{I}\right) \varepsilon=D P_{4}(\mathrm{I} \cdot \varepsilon) \varepsilon_{n}^{p}=D P_{4}(\operatorname{tr} \varepsilon) \varepsilon_{n}^{p} \Rightarrow$

$$
\sigma^{(4)}=D P_{4}\left(\varepsilon_{x}+\varepsilon_{y}+\varepsilon_{z}\right)\left[\begin{array}{ccc}
\varepsilon_{x}^{p, n} & \varepsilon_{x y}^{p, n} & \varepsilon_{x z}^{p, n} \\
\varepsilon_{x y}^{p, n} & \varepsilon_{y}^{p, n} & \varepsilon_{y z}^{p, n} \\
\varepsilon_{x z}^{p, n} & \varepsilon_{y z}^{p, n} & \varepsilon_{z}^{p, n}
\end{array}\right]
$$

As componentes do tensor de tensões são:

$$
\begin{aligned}
& \sigma_{x}^{(4)}=\left(D P_{4} \varepsilon_{x}^{p, n}\right) \varepsilon_{x}+\left(D P_{4} \varepsilon_{x}^{p, n}\right) \varepsilon_{y}+\left(D P_{4} \varepsilon_{x}^{p, n}\right) \varepsilon_{z} \\
& \sigma_{y}^{(4)}=\left(D P_{4} \varepsilon_{y}^{p, n}\right) \varepsilon_{x}+\left(D P_{4} \varepsilon_{y}^{p, n}\right) \varepsilon_{y}+\left(D P_{4} \varepsilon_{y}^{p, n}\right) \varepsilon_{z} \\
& \sigma_{z}^{(4)}=\left(D P_{4} \varepsilon_{z}^{p, n}\right) \varepsilon_{x}+\left(D P_{4} \varepsilon_{z}^{p, n}\right) \varepsilon_{y}+\left(D P_{4} \varepsilon_{z}^{p, n}\right) \varepsilon_{z} \\
& \tau_{x y}^{(4)}=D P_{4} \varepsilon_{x y}^{p, n} \varepsilon_{x}+D P_{4} \varepsilon_{x y}^{p, n} \varepsilon_{y}+D P_{4} \varepsilon_{x y}^{p, n} \varepsilon_{z}=\frac{D P_{4} \gamma_{x y}^{p, n}}{2} \varepsilon_{x}+\frac{D P_{4} \gamma_{x y}^{p, n}}{2} \varepsilon_{y}+\frac{D P_{4} \gamma_{x y}^{p, n}}{2} \varepsilon_{z} \\
& \tau_{x z}^{(4)}=D P_{4} \varepsilon_{x z}^{p, n} \varepsilon_{x}+D P_{4} \varepsilon_{x z}^{p, n} \varepsilon_{y}+D P_{4} \varepsilon_{x z}^{p, n} \varepsilon_{z}=\frac{D P_{4} \gamma_{x z}^{p, n}}{2} \varepsilon_{x}+\frac{D P_{4} \gamma_{x z}^{p, n}}{2} \varepsilon_{y}+\frac{D P_{4} \gamma_{x z}^{p, n}}{2} \varepsilon_{z} \\
& \tau_{y z}^{(4)}=D P_{4} \varepsilon_{y z}^{p, n} \varepsilon_{x}+D P_{4} \varepsilon_{y z}^{p, n} \varepsilon_{y}+D P_{4} \varepsilon_{y z}^{p, n} \varepsilon_{z}=\frac{D P_{4} \gamma_{y z}^{p, n}}{2} \varepsilon_{x}+\frac{D P_{4} \gamma_{y z}^{p, n}}{2} \varepsilon_{y}+\frac{D P_{4} \gamma_{y z}^{p, n}}{2} \varepsilon_{z}
\end{aligned}
$$

A matriz constitutiva relativa a esta parcela do tensor $C_{n+1}^{p, d}$ é:

$$
D_{(4)}^{p, d}=\left[\begin{array}{cccccc}
D P_{4} \varepsilon_{x}^{p, n} & D P_{4} \varepsilon_{x}^{p, n} & D P_{4} \varepsilon_{x}^{p, n} & 0 & 0 & 0 \\
D P_{4} \varepsilon_{y}^{p, n} & D P_{4} \varepsilon_{y}^{p, n} & D P_{4} \varepsilon_{y}^{p, n} & 0 & 0 & 0 \\
D P_{4} \varepsilon_{z}^{p, n} & D P_{4} \varepsilon_{z}^{p, n} & D P_{4} \varepsilon_{z}^{p, n} & 0 & 0 & 0 \\
\frac{D P_{4} \gamma_{x y}^{p, n}}{2} & \frac{D P_{4} \gamma_{x y}^{p, n}}{2} & \frac{D P_{4} \gamma_{x y}^{p, n}}{2} & 0 & 0 & 0 \\
\frac{D P_{4} \gamma_{x z}^{p, n}}{2} & \frac{D P_{4} \gamma_{x z}^{p, n}}{2} & \frac{D P_{4} \gamma_{x z}^{p, n}}{2} & 0 & 0 & 0 \\
\frac{D P_{4} \gamma_{y z}^{p, n}}{2} & \frac{D P_{4} \gamma_{y z}^{p, n}}{2} & \frac{D P_{4} \gamma_{y z}^{p, n}}{2} & 0 & 0 & 0
\end{array}\right]
$$


5) $\sigma^{(5)}=D P_{5}\left(\mathrm{I} \otimes n_{n+1}\right) \varepsilon=D P_{5}\left(n_{n+1} \cdot \varepsilon\right) \mathrm{I}=D P_{5}\left[\operatorname{tr}\left(n_{n+1}^{T} \varepsilon\right)\right] \mathrm{I} \Rightarrow$

$$
\sigma^{(5)}=\left[\begin{array}{ccc}
D P_{5}\left[\operatorname{tr}\left(n_{n+1}^{T} \varepsilon\right)\right] & 0 & 0 \\
0 & D P_{5}\left[\operatorname{tr}\left(n_{n+1}^{T} \varepsilon\right)\right] & 0 \\
0 & 0 & D P_{5}\left[\operatorname{tr}\left(n_{n+1}^{T} \varepsilon\right)\right]
\end{array}\right]
$$

Seja o tensor $n_{n+1}$ formado pelas componentes:

$n_{n+1}=\left[\begin{array}{ccc}n_{1} & n_{4} & n_{5} \\ n_{4} & n_{2} & n_{6} \\ n_{5} & n_{6} & n_{3}\end{array}\right]$

Assim, $\operatorname{tr}\left(n_{n+1}^{T} \varepsilon\right)=n_{1} \varepsilon_{x}+n_{2} \varepsilon_{y}+n_{3} \varepsilon_{z}+2 n_{4} \varepsilon_{x y}+2 n_{5} \varepsilon_{x z}+2 n_{6} \varepsilon_{y z} \Rightarrow$

$$
\operatorname{tr}\left(n_{n+1}^{T} \varepsilon\right)=n_{1} \varepsilon_{x}+n_{2} \varepsilon_{y}+n_{3} \varepsilon_{z}+n_{4} \gamma_{x y}+n_{5} \gamma_{x z}+n_{6} \gamma_{y z}
$$

Portanto, as componentes de $\sigma^{(5)}$ são:

$$
\begin{aligned}
& \sigma_{x}^{(5)}=D P_{5} n_{1} \varepsilon_{x}+D P_{5} n_{2} \varepsilon_{y}+D P_{5} n_{3} \varepsilon_{z}+D P_{5} n_{4} \gamma_{x y}+D P_{5} n_{5} \gamma_{x z}+D P_{5} n_{6} \gamma_{y z} \\
& \sigma_{y}^{(5)}=D P_{5} n_{1} \varepsilon_{x}+D P_{5} n_{2} \varepsilon_{y}+D P_{5} n_{3} \varepsilon_{z}+D P_{5} n_{4} \gamma_{x y}+D P_{5} n_{5} \gamma_{x z}+D P_{5} n_{6} \gamma_{y z} \\
& \sigma_{z}^{(5)}=D P_{5} n_{1} \varepsilon_{x}+D P_{5} n_{2} \varepsilon_{y}+D P_{5} n_{3} \varepsilon_{z}+D P_{5} n_{4} \gamma_{x y}+D P_{5} n_{5} \gamma_{x z}+D P_{5} n_{6} \gamma_{y z} \\
& \tau_{x y}^{(5)}=0 \\
& \tau_{x z}^{(5)}=0 \\
& \tau_{y z}^{(5)}=0
\end{aligned}
$$

Em forma matricial, a relação constitutiva é dada por:

$$
D_{(5)}^{p, d}=\left[\begin{array}{cccccc}
D P_{5} n_{1} & D P_{5} n_{2} & D P_{5} n_{3} & D P_{5} n_{4} & D P_{5} n_{5} & D P_{5} n_{6} \\
D P_{5} n_{1} & D P_{5} n_{2} & D P_{5} n_{3} & D P_{5} n_{4} & D P_{5} n_{5} & D P_{5} n_{6} \\
D P_{5} n_{1} & D P_{5} n_{2} & D P_{5} n_{3} & D P_{5} n_{4} & D P_{5} n_{5} & D P_{5} n_{6} \\
0 & 0 & 0 & 0 & 0 & 0 \\
0 & 0 & 0 & 0 & 0 & 0 \\
0 & 0 & 0 & 0 & 0 & 0
\end{array}\right]
$$

6) $\sigma^{(6)}=D P_{6}\left(n_{n+1} \otimes \mathrm{I}\right) \varepsilon=D P_{6}(\mathrm{I} \cdot \varepsilon) n_{n+1}=D P_{6}(\operatorname{tr} \varepsilon) n_{n+1}$. Sendo $n_{n+1}$ dado por

(O.14), pode-se escrever:

$$
\sigma^{(6)}=D P_{6}\left(\varepsilon_{x}+\varepsilon_{y}+\varepsilon_{z}\right)\left[\begin{array}{ccc}
n_{1} & n_{4} & n_{5} \\
n_{4} & n_{2} & n_{6} \\
n_{5} & n_{6} & n_{3}
\end{array}\right]
$$

As componentes do tensor $\sigma^{(6)}$ são, portanto: 


$$
\begin{aligned}
& \sigma_{x}^{(6)}=D P_{6} n_{1} \varepsilon_{x}+D P_{6} n_{1} \varepsilon_{y}+D P_{6} n_{1} \varepsilon_{z} \\
& \sigma_{y}^{(6)}=D P_{6} n_{2} \varepsilon_{x}+D P_{6} n_{2} \varepsilon_{y}+D P_{6} n_{2} \varepsilon_{z} \\
& \sigma_{z}^{(6)}=D P_{6} n_{3} \varepsilon_{x}+D P_{6} n_{3} \varepsilon_{y}+D P_{6} n_{3} \varepsilon_{z} \\
& \tau_{x y}^{(6)}=D P_{6} n_{4} \varepsilon_{x}+D P_{6} n_{4} \varepsilon_{y}+D P_{6} n_{4} \varepsilon_{z} \\
& \tau_{x z}^{(6)}=D P_{6} n_{5} \varepsilon_{x}+D P_{6} n_{5} \varepsilon_{y}+D P_{6} n_{5} \varepsilon_{z} \\
& \tau_{y z}^{(6)}=D P_{6} n_{6} \varepsilon_{x}+D P_{6} n_{6} \varepsilon_{y}+D P_{6} n_{6} \varepsilon_{z}
\end{aligned}
$$

A expressão da matriz constitutiva desta parcela é:

$$
D_{(6)}^{p, d}=\left[\begin{array}{cccccc}
D P_{6} n_{1} & D P_{6} n_{1} & D P_{6} n_{1} & 0 & 0 & 0 \\
D P_{6} n_{2} & D P_{6} n_{2} & D P_{6} n_{2} & 0 & 0 & 0 \\
D P_{6} n_{3} & D P_{6} n_{3} & D P_{6} n_{3} & 0 & 0 & 0 \\
D P_{6} n_{4} & D P_{6} n_{4} & D P_{6} n_{4} & 0 & 0 & 0 \\
D P_{6} n_{5} & D P_{6} n_{5} & D P_{6} n_{5} & 0 & 0 & 0 \\
D P_{6} n_{6} & D P_{6} n_{6} & D P_{6} n_{6} & 0 & 0 & 0
\end{array}\right]
$$

7) $\sigma^{(7)}=D P_{7}\left(\varepsilon \otimes n_{n+1}\right) \varepsilon=D P_{7}\left(n_{n+1} \cdot \varepsilon\right) \varepsilon=D P_{7}\left[\operatorname{tr}\left(n_{n+1}^{T} \varepsilon\right)\right] \varepsilon=D P_{7}^{\prime} \varepsilon \Rightarrow$

$$
\sigma^{(7)}=\left[\begin{array}{ccc}
D P_{7}^{\prime} \varepsilon_{x} & D P_{7}^{\prime} \varepsilon_{x y} & D P_{7}^{\prime} \varepsilon_{x z} \\
D P_{7}^{\prime} \varepsilon_{x y} & D P_{7}^{\prime} \varepsilon_{y} & D P_{7}^{\prime} \varepsilon_{y z} \\
D P_{7}^{\prime} \varepsilon_{x z} & D P_{7}^{\prime} \varepsilon_{y z} & D P_{7}^{\prime} \varepsilon_{z}
\end{array}\right]
$$

Sendo $D P_{7}^{\prime}=D P_{7} \operatorname{tr}\left(n_{n+1}^{T} \varepsilon\right)$ e $\operatorname{tr}\left(n_{n+1}^{T} \varepsilon\right)$ dado pela eq. (O.15). As componentes do tensor $\sigma^{(7)}$ são, portanto:

$$
\begin{aligned}
& \sigma_{x}^{(7)}=D P_{7}^{\prime} \varepsilon_{x} \\
& \sigma_{y}^{(7)}=D P_{7}^{\prime} \varepsilon_{y} \\
& \sigma_{z}^{(7)}=D P_{7}^{\prime} \varepsilon_{z} \\
& \tau_{x y}^{(7)}=D P_{7}^{\prime} \varepsilon_{x y}=\frac{D P_{7}^{\prime}}{2} \gamma_{x y} \\
& \tau_{x z}^{(7)}=D P_{7}^{\prime} \varepsilon_{x z}=\frac{D P_{7}^{\prime}}{2} \gamma_{x z} \\
& \tau_{y z}^{(7)}=D P_{7}^{\prime} \varepsilon_{y z}=\frac{D P_{7}^{\prime}}{2} \gamma_{y z}
\end{aligned}
$$

E a expressão da matriz constitutiva desta parcela é, portanto: 
$D_{(7)}^{p, d}=\left[\begin{array}{cccccc}D P_{7}^{\prime} & 0 & 0 & 0 & 0 & 0 \\ 0 & D P_{7}^{\prime} & 0 & 0 & 0 & 0 \\ 0 & 0 & D P_{7}^{\prime} & 0 & 0 & 0 \\ 0 & 0 & 0 & \frac{D P_{7}^{\prime}}{2} & 0 & 0 \\ 0 & 0 & 0 & 0 & \frac{D P_{7}^{\prime}}{2} & 0 \\ 0 & 0 & 0 & 0 & 0 & \frac{D P_{7}^{\prime}}{2}\end{array}\right]$

8) $\sigma^{(8)}=D P_{8}\left(\varepsilon_{n}^{p} \otimes n_{n+1}\right) \varepsilon=D P_{8}\left(n_{n+1} \cdot \varepsilon\right) \varepsilon_{n}^{p}=D P_{8}\left[\operatorname{tr}\left(n_{n+1}^{T} \varepsilon\right)\right] \varepsilon_{n}^{p} \Rightarrow$

$$
\sigma^{(8)}=D P_{8}\left[\operatorname{tr}\left(n_{n+1}^{T} \varepsilon\right)\right]\left[\begin{array}{lll}
\varepsilon_{x}^{p, n} & \varepsilon_{x y}^{p, n} & \varepsilon_{x z}^{p, n} \\
\varepsilon_{x y}^{p, n} & \varepsilon_{y}^{p, n} & \varepsilon_{y z}^{p, n} \\
\varepsilon_{x z}^{p, n} & \varepsilon_{y z}^{p, n} & \varepsilon_{z}^{p, n}
\end{array}\right]
$$

Sendo $\operatorname{tr}\left(n_{n+1}^{T} \varepsilon\right)$ dado pela eq. (O.15), as componentes do tensor $\sigma^{(8)}$ são:

$$
\begin{aligned}
\sigma_{x}^{(8)} & =D P_{8} \varepsilon_{x}^{p, n}\left(n_{1} \varepsilon_{x}+n_{2} \varepsilon_{y}+n_{3} \varepsilon_{z}+n_{4} \gamma_{x y}+n_{5} \gamma_{x z}+n_{6} \gamma_{y z}\right) \\
\sigma_{y}^{(8)} & =D P_{8} \varepsilon_{y}^{p, n}\left(n_{1} \varepsilon_{x}+n_{2} \varepsilon_{y}+n_{3} \varepsilon_{z}+n_{4} \gamma_{x y}+n_{5} \gamma_{x z}+n_{6} \gamma_{y z}\right) \\
\sigma_{z}^{(8)} & =D P_{8} \varepsilon_{z}^{p, n}\left(n_{1} \varepsilon_{x}+n_{2} \varepsilon_{y}+n_{3} \varepsilon_{z}+n_{4} \gamma_{x y}+n_{5} \gamma_{x z}+n_{6} \gamma_{y z}\right) \\
\tau_{x y}^{(8)} & =D P_{8} \varepsilon_{x y}^{p, n}\left(n_{1} \varepsilon_{x}+n_{2} \varepsilon_{y}+n_{3} \varepsilon_{z}+n_{4} \gamma_{x y}+n_{5} \gamma_{x z}+n_{6} \gamma_{y z}\right)= \\
& =D P_{8} \frac{\gamma_{x y}^{p, n}}{2}\left(n_{1} \varepsilon_{x}+n_{2} \varepsilon_{y}+n_{3} \varepsilon_{z}+n_{4} \gamma_{x y}+n_{5} \gamma_{x z}+n_{6} \gamma_{y z}\right) \\
\tau_{x z}^{(8)} & =D P_{8} \varepsilon_{x z}^{p, n}\left(n_{1} \varepsilon_{x}+n_{2} \varepsilon_{y}+n_{3} \varepsilon_{z}+n_{4} \gamma_{x y}+n_{5} \gamma_{x z}+n_{6} \gamma_{y z}\right)= \\
& =D P_{8} \frac{\gamma_{x z}^{p, n}}{2}\left(n_{1} \varepsilon_{x}+n_{2} \varepsilon_{y}+n_{3} \varepsilon_{z}+n_{4} \gamma_{x y}+n_{5} \gamma_{x z}+n_{6} \gamma_{y z}\right) \\
\tau_{y z}^{(8)} & =D P_{8} \varepsilon_{y z}^{p, n}\left(n_{1} \varepsilon_{x}+n_{2} \varepsilon_{y}+n_{3} \varepsilon_{z}+n_{4} \gamma_{x y}+n_{5} \gamma_{x z}+n_{6} \gamma_{y z}\right)= \\
& =D P_{8} \frac{\gamma_{y z}^{p, n}}{2}\left(n_{1} \varepsilon_{x}+n_{2} \varepsilon_{y}+n_{3} \varepsilon_{z}+n_{4} \gamma_{x y}+n_{5} \gamma_{x z}+n_{6} \gamma_{y z}\right)
\end{aligned}
$$

$\mathrm{Na}$ forma matricial, tem-se: 


$$
D_{(8)}^{p, d}=\left[\begin{array}{lllllll}
D P_{8} \varepsilon_{x}^{p, n} n_{1} & D P_{8} \varepsilon_{x}^{p, n} n_{2} & D P_{8} \varepsilon_{x}^{p, n} n_{3} & D P_{8} \varepsilon_{x}^{p, n} n_{4} & D P_{8} \varepsilon_{x}^{p, n} n_{5} & D P_{8} \varepsilon_{x}^{p, n} n_{6} \\
D P_{8} \varepsilon_{y}^{p, n} n_{1} & D P_{8} \varepsilon_{y}^{p, n} n_{2} & D P_{8} \varepsilon_{y}^{p, n} n_{3} & D P_{8} \varepsilon_{y}^{p, n} n_{4} & D P_{8} \varepsilon_{y}^{p, n} n_{5} & D P_{8} \varepsilon_{y}^{p, n} n_{6} \\
D P_{8} \varepsilon_{z}^{p, n} n_{1} & D P_{8} \varepsilon_{z}^{p, n} n_{2} & D P_{8} \varepsilon_{z}^{p, n} n_{3} & D P_{8} \varepsilon_{z}^{p, n} n_{4} & D P_{8} \varepsilon_{z}^{p, n} n_{5} & D P_{8} \varepsilon_{z}^{p, n} n_{6} \\
\frac{D P_{8} \gamma_{x y}^{p, n} n_{1}}{2} & \frac{D P_{8} \gamma_{x y}^{p, n} n_{2}}{2} & \frac{D P_{8} \gamma_{x y}^{p, n} n_{3}}{2} & \frac{D P_{8} \gamma_{x y}^{p, n} n_{4}}{2} & \frac{D P_{8} \gamma_{x y}^{p, n} n_{5}}{2} & \frac{D P_{8} \gamma_{x y}^{p, n} n_{6}}{2} \\
\frac{D P_{8} \gamma_{x z}^{p, n} n_{1}}{2} & \frac{D P_{8} \gamma_{x z}^{p, n} n_{2}}{2} & \frac{D P_{8} \gamma_{x z}^{p, n} n_{3}}{2} & \frac{D P_{8} \gamma_{x z}^{p, n} n_{4}}{2} & \frac{D P_{8} \gamma_{x z}^{p, n} n_{5}}{2} & \frac{D P_{8} \gamma_{x z}^{p, n} n_{6}}{2} \\
\frac{D P_{8} \gamma_{y z}^{p, n} n_{1}}{2} & \frac{D P_{8} \gamma_{y z}^{p, n} n_{2}}{2} & \frac{D P_{8} \gamma_{y z}^{p, n} n_{3}}{2} & \frac{D P_{8} \gamma_{y z}^{p, n} n_{4}}{2} & \frac{D P_{8} \gamma_{y z}^{p, n} n_{5}}{2} & \frac{D P_{8} \gamma_{y z}^{p, n} n_{6}}{2}
\end{array}\right]
$$

9) $\sigma^{(9)}=D P_{9}\left(n_{n+1} \otimes n_{n+1}\right) \varepsilon=D P_{9}\left(n_{n+1} \cdot \varepsilon\right) n_{n+1}=D P_{9}\left[\operatorname{tr}\left(n_{n+1}^{T} \varepsilon\right)\right] n_{n+1} \Rightarrow$

$$
\sigma^{(9)}=D P_{9}\left[\operatorname{tr}\left(n_{n+1}^{T} \varepsilon\right)\right]\left[\begin{array}{ccc}
n_{1} & n_{4} & n_{5} \\
n_{4} & n_{2} & n_{6} \\
n_{5} & n_{6} & n_{3}
\end{array}\right]
$$

Sendo $\operatorname{tr}\left(n_{n+1}^{T} \varepsilon\right)$ definido por (O.15). As componentes do tensor $\sigma^{(9)}$ são:

$$
\begin{aligned}
& \sigma_{x}^{(9)}=D P_{9} n_{1}\left(n_{1} \varepsilon_{x}+n_{2} \varepsilon_{y}+n_{3} \varepsilon_{z}+n_{4} \gamma_{x y}+n_{5} \gamma_{x z}+n_{6} \gamma_{y z}\right) \\
& \sigma_{y}^{(9)}=D P_{9} n_{2}\left(n_{1} \varepsilon_{x}+n_{2} \varepsilon_{y}+n_{3} \varepsilon_{z}+n_{4} \gamma_{x y}+n_{5} \gamma_{x z}+n_{6} \gamma_{y z}\right) \\
& \sigma_{z}^{(9)}=D P_{9} n_{3}\left(n_{1} \varepsilon_{x}+n_{2} \varepsilon_{y}+n_{3} \varepsilon_{z}+n_{4} \gamma_{x y}+n_{5} \gamma_{x z}+n_{6} \gamma_{y z}\right) \\
& \tau_{x y}^{(9)}=D P_{9} n_{4}\left(n_{1} \varepsilon_{x}+n_{2} \varepsilon_{y}+n_{3} \varepsilon_{z}+n_{4} \gamma_{x y}+n_{5} \gamma_{x z}+n_{6} \gamma_{y z}\right) \\
& \tau_{x z}^{(9)}=D P_{9} n_{5}\left(n_{1} \varepsilon_{x}+n_{2} \varepsilon_{y}+n_{3} \varepsilon_{z}+n_{4} \gamma_{x y}+n_{5} \gamma_{x z}+n_{6} \gamma_{y z}\right) \\
& \tau_{y z}^{(9)}=D P_{9} n_{6}\left(n_{1} \varepsilon_{x}+n_{2} \varepsilon_{y}+n_{3} \varepsilon_{z}+n_{4} \gamma_{x y}+n_{5} \gamma_{x z}+n_{6} \gamma_{y z}\right)
\end{aligned}
$$

E a matriz constitutiva referente à nona parcela do tensor $C_{n+1}^{p, d}$ é:

$$
D_{(9)}^{p, d}=\left[\begin{array}{cccccc}
D P_{9} n_{1}^{2} & D P_{9} n_{1} n_{2} & D P_{9} n_{1} n_{3} & D P_{9} n_{1} n_{4} & D P_{9} n_{1} n_{5} & D P_{9} n_{1} n_{6} \\
D P_{9} n_{1} n_{2} & D P_{9} n_{2}{ }^{2} & D P_{9} n_{2} n_{3} & D P_{9} n_{2} n_{4} & D P_{9} n_{2} n_{5} & D P_{9} n_{2} n_{6} \\
D P_{9} n_{1} n_{3} & D P_{9} n_{2} n_{3} & D P_{9} n_{3}^{2} & D P_{9} n_{3} n_{4} & D P_{9} n_{3} n_{5} & D P_{9} n_{3} n_{6} \\
D P_{9} n_{1} n_{4} & D P_{9} n_{2} n_{4} & D P_{9} n_{3} n_{4} & D P_{9} n_{4}^{2} & D P_{9} n_{4} n_{5} & D P_{9} n_{4} n_{6} \\
D P_{9} n_{1} n_{5} & D P_{9} n_{2} n_{5} & D P_{9} n_{3} n_{5} & D P_{9} n_{4} n_{5} & D P_{9} n_{5}^{2} & D P_{9} n_{5} n_{6} \\
D P_{9} n_{1} n_{6} & D P_{9} n_{2} n_{6} & D P_{9} n_{3} n_{6} & D P_{9} n_{4} n_{6} & D P_{9} n_{5} n_{6} & D P_{9} n_{6}^{2}
\end{array}\right]
$$

Somando-se as nove matrizes $D_{(i, i=1, \ldots, 9)}^{p, d}$, definidas por $(\mathrm{O} .6),(\mathrm{O} .8),(\mathrm{O} .10),(\mathrm{O} .12)$, (O.16), (O.18), (O.20), (O.22), (O.24), obtém-se finalmente a matriz $D^{p, d}$, cujos termos são apresentados a seguir: 


$$
\begin{aligned}
& D_{11}^{p, d}=D P_{1}+D P_{2}+D P_{3}^{\prime}+D P_{4} \varepsilon_{x}^{p, n}+D P_{5} n_{1}+D P_{6} n_{1}+D P_{7}^{\prime}+D P_{8} \varepsilon_{x}^{p, n} n_{1}+D P_{9} n_{1}^{2} \\
& D_{12}^{p, d}=D P_{2}+D P_{4} \varepsilon_{x}^{p, n}+D P_{5} n_{2}+D P_{6} n_{1}+D P_{8} \varepsilon_{x}^{p, n} n_{2}+D P_{9} n_{1} n_{2} \\
& D_{13}^{p, d}=D P_{2}+D P_{4} \varepsilon_{x}^{p, n}+D P_{5} n_{3}+D P_{6} n_{1}+D P_{8} \varepsilon_{x}^{p, n} n_{3}+D P_{9} n_{1} n_{3} \\
& D_{14}^{p, d}=D P_{5} n_{4}+D P_{8} \varepsilon_{x}^{p, n} n_{4}+D P_{9} n_{1} n_{4} \\
& D_{15}^{p, d}=D P_{5} n_{5}+D P_{8} \varepsilon_{x}^{p, n} n_{5}+D P_{9} n_{1} n_{5} \\
& D_{16}^{p, d}=D P_{5} n_{6}+D P_{8} \varepsilon_{x}^{p, n} n_{6}+D P_{9} n_{1} n_{6} \\
& D_{21}^{p, d}=D P_{2}+D P_{4} \varepsilon_{y}^{p, n}+D P_{5} n_{1}+D P_{6} n_{2}+D P_{8} \varepsilon_{y}^{p, n} n_{1}+D P_{9} n_{1} n_{2} \\
& D_{22}^{p, d}=D P_{1}+D P_{2}+D P_{3}^{\prime}+D P_{4} \varepsilon_{y}^{p, n}+D P_{5} n_{2}+D P_{6} n_{2}+D P_{7}^{\prime}+D P_{8} \varepsilon_{y}^{p, n} n_{2}+D P_{9} n_{2}^{2} \\
& D_{23}^{p, d}=D P_{2}+D P_{4} \varepsilon_{y}^{p, n}+D P_{5} n_{3}+D P_{6} n_{2}+D P_{8} \varepsilon_{y}^{p, n} n_{3}+D P_{9} n_{2} n_{3} \\
& D_{24}^{p, d}=D P_{5} n_{4}+D P_{8} \varepsilon_{y}^{p, n} n_{4}+D P_{9} n_{2} n_{4} \\
& D_{25}^{p, d}=D P_{5} n_{5}+D P_{8} \varepsilon_{y}^{p, n} n_{5}+D P_{9} n_{2} n_{5} \\
& D_{26}^{p, d}=D P_{5} n_{6}+D P_{8} \varepsilon_{y}^{p, n} n_{6}+D P_{9} n_{2} n_{6} \\
& D_{31}^{p, d}=D P_{2}+D P_{4} \varepsilon_{z}^{p, n}+D P_{5} n_{1}+D P_{6} n_{3}+D P_{8} \varepsilon_{z}^{p, n} n_{1}+D P_{9} n_{1} n_{3} \\
& D_{32}^{p, d}=D P_{2}+D P_{4} \varepsilon_{z}^{p, n}+D P_{5} n_{2}+D P_{6} n_{3}+D P_{8} \varepsilon_{z}^{p, n} n_{2}+D P_{9} n_{2} n_{3} \\
& D_{33}^{p, d}=D P_{1}+D P_{2}+D P_{3}^{\prime}+D P_{4} \varepsilon_{z}^{p, n}+D P_{5} n_{3}+D P_{6} n_{3}+D P_{7}^{\prime}+D P_{8} \varepsilon_{z}^{p, n} n_{3}+D P_{9} n_{3}^{2} \\
& D_{34}^{p, d}=D P_{5} n_{4}+D P_{8} \varepsilon_{z}^{p, n} n_{4}+D P_{9} n_{3} n_{4} \\
& D_{35}^{p, d}=D P_{5} n_{5}+D P_{8} \varepsilon_{z}^{p, n} n_{5}+D P_{9} n_{3} n_{5} \\
& D_{36}^{p, d}=D P_{5} n_{6}+D P_{8} \varepsilon_{z}^{p, n} n_{6}+D P_{9} n_{3} n_{6} \\
& D_{41}^{p, d}=\frac{D P_{4}}{2} \gamma_{x y}^{p, n}+D P_{6} n_{4}+\frac{D P_{8}}{2} \gamma_{x y}^{p, n} n_{1}+D P_{9} n_{1} n_{4} \\
& D_{42}^{p, d}=\frac{D P_{4}}{2} \gamma_{x y}^{p, n}+D P_{6} n_{4}+\frac{D P_{8}}{2} \gamma_{x y}^{p, n} n_{2}+D P_{9} n_{2} n_{4} \\
& D_{43}^{p, d}=\frac{D P_{4}}{2} \gamma_{x y}^{p, n}+D P_{6} n_{4}+\frac{D P_{8}}{2} \gamma_{x y}^{p, n} n_{3}+D P_{9} n_{3} n_{4} \\
& D_{44}^{p, d}=\frac{D P_{1}}{2}+\frac{D P_{3}^{\prime}}{2}+\frac{D P_{7}^{\prime}}{2}+\frac{D P_{8}}{2} \gamma_{x y}^{p, n} n_{4}+D P_{9} n_{4}{ }^{2} \\
& D_{45}^{p, d}=\frac{D P_{8}}{2} \gamma_{x y}^{p, n} n_{5}+D P_{9} n_{4} n_{5} \\
& D_{46}^{p, d}=\frac{D P_{8}}{2} \gamma_{x y}^{p, n} n_{6}+D P_{9} n_{4} n_{6}
\end{aligned}
$$




$$
\begin{aligned}
& D_{51}^{p, d}=\frac{D P_{4}}{2} \gamma_{x z}^{p, n}+D P_{6} n_{5}+\frac{D P_{8}}{2} \gamma_{x z}^{p, n} n_{1}+D P_{9} n_{1} n_{5} \\
& D_{52}^{p, d}=\frac{D P_{4}}{2} \gamma_{x z}^{p, n}+D P_{6} n_{5}+\frac{D P_{8}}{2} \gamma_{x z}^{p, n} n_{2}+D P_{9} n_{2} n_{5} \\
& D_{53}^{p, d}=\frac{D P_{4}}{2} \gamma_{x z}^{p, n}+D P_{6} n_{5}+\frac{D P_{8}}{2} \gamma_{x z}^{p, n} n_{3}+D P_{9} n_{3} n_{5} \\
& D_{54}^{p, d}=\frac{D P_{8}}{2} \gamma_{x z}^{p, n} n_{4}+D P_{9} n_{4} n_{5} \\
& D_{55}^{p, d}=\frac{D P_{1}}{2}+\frac{D P_{3}^{\prime}}{2}+\frac{D P_{7}^{\prime}}{2}+\frac{D P_{8}}{2} \gamma_{x z}^{p, n} n_{5}+D P_{9} n_{5}^{2} \\
& D_{56}^{p, d}=\frac{D P_{8}}{2} \gamma_{x z}^{p, n} n_{6}+D P_{9} n_{5} n_{6} \\
& D_{61}^{p, d}=\frac{D P_{4}}{2} \gamma_{y z}^{p, n}+D P_{6} n_{6}+\frac{D P_{8}}{2} \gamma_{y z}^{p, n} n_{1}+D P_{9} n_{1} n_{6} \\
& D_{62}^{p, d}=\frac{D P_{4}}{2} \gamma_{y z}^{p, n}+D P_{6} n_{6}+\frac{D P_{8}}{2} \gamma_{y z}^{p, n} n_{2}+D P_{9} n_{2} n_{6} \\
& D_{66}^{p, d}=\frac{D P_{1}}{2}+\frac{D P_{3}^{\prime}}{2}+\frac{D P_{7}^{\prime}}{2}+\frac{D P_{8}}{2} \gamma_{y z}^{p, n} n_{6}+D P_{9} n_{6}^{2} \\
& D_{63}^{p, d}=\frac{D P_{4}}{2} \gamma_{y z}^{p, n}+D P_{6} n_{6}+\frac{D P_{8}}{2} \gamma_{y z}^{p, n} n_{3}+D P_{9} n_{3} n_{6} \\
& \gamma_{y z}^{p, n} n_{5}+D P_{9} n_{5} n_{6} \\
& \frac{D P_{8}}{2} \gamma_{y z}^{p, n} n_{4}+D P_{9} n_{4} n_{6} \\
& D_{6}
\end{aligned}
$$

Conhecidas as matrizes constitutivas tangentes $D^{e l, d}$ e $D^{p, d}$, tem-se a seguinte expressão para a matriz $D^{e p, d}$ :

$$
D^{e p, d}=\left\{\begin{array}{lr}
D^{e l, d} & \text { caso } \Delta \lambda=0 \\
D^{e l, d}-D^{p, d} & \text { caso } \Delta \lambda>0
\end{array}\right.
$$

